UNIVERSIDADE DE SÃO PAULO

ESCOLA POLITÉCNICA

WILLIAM ALEXANDRE LABECCA DE CASTRO

Matrizes operacionais e formalismo coadjunto em equações diferenciais fracionais

São Paulo 

WILLIAM ALEXANDRE LABECCA DE CASTRO

Matrizes operacionais e formalismo coadjunto em equações diferenciais fracionais

Tese apresentada à Escola Politécnica da Universidade de São Paulo para obtenção do Título de Doutor em Ciências.

São Paulo

2015 

WILLIAM ALEXANDRE LABECCA DE CASTRO

\section{Matrizes operacionais e formalismo coadjunto em equações diferenciais fracionais}

Tese apresentada à Escola Politécnica da Universidade de São Paulo para obtenção do Título de Doutor em Ciências.

Área de Concentração:

Engenharia de Sistemas

Orientador:

Prof. Dr. José Roberto Castilho Piqueira

São Paulo 
Este exemplar foi revisado e corrigido em relação à versão original, sob responsabilidade única do autor e com a anuência de seu orientador.

São Paulo, de de

Assinatura do autor:

Assinatura do orientador:

Catalogação-na-publicação

Castro, William Alexandre Labecca de

Matrizes operacionais e formalismo coadjunto em equações diferenciais fracionais / W. A. L. Castro -- versão corr. -- São Paulo, 2015. $347 \mathrm{p}$.

Tese (Doutorado) - Escola Politécnica da Universidade de São Paulo. Departamento de Engenharia de Telecomunicações e Controle.

1.Equações diferenciais 2.Séries de Fourier 3.Matrizes operacionais 4.Cálculo fracional I.Universidade de São Paulo. Escola Politécnica. Departamento de Engenharia de Telecomunicações e Controle II.t. 


\section{AGRADECIMENTOS}

Ao Prof. Dr. José Roberto Castilho Piqueira, Ilustre Diretor da Escola Politécnica da USP, sou imensamente grato pela preciosa orientação, inestimável apoio e pela sincera confiança depositada em minha pessoa.

Ao meu grande amigo Prof. Dr. José Osvaldo de Souza Guimarães, responsável pela minha vinda à Escola Politécnica, agradeço pelo companheirismo e pelas inúmeras e agradáveis horas de frutíferas discussões, das quais extraí tantos conhecimentos.

Gostaria também de expressar minha gratidão ao Prof. Dr. José Jaime da Cruz, pelas valiosas sugestões.

E, finalmente, à CAPES, pelo fundamental apoio financeiro, sem o qual a consecução dessa Tese não teria sido possível. 



\section{RESUMO}

O método das matrizes operacionais é expandido para o corpo complexo a ordens arbitrárias pela abordagem de Riemann-Liouville e Caputo com ênfase nas séries de Fourier complexas. Elabora-se uma adaptação do formalismo bra-ket de Dirac à linguagem tensorial no espaço de Hilbert de funções com expansões finitas para uso específico na teoria de equações diferenciais e matrizes operacionais, denominado "Formalismo Coadjunto". Estende-se o tratamento aos operadores fracionais de Weyl para períodos genéricos a fim de determinar as matrizes operacionais de derivação e integração de ordem arbitrária na base complexa de Fourier. Introduz-se um novo método de resolução de equações diferenciais ordinárias lineares e fracionais não-homogêneas, denominado "Modelagem Operacional", que permite a obtenção de soluções de equações de alta ordem com grande precisão sem a necessidade de imposição de condições iniciais ou de contorno. O método apresentado é aperfeiçoado por meio de um novo tipo de expansão, que denominamos "Séries Associadas de Fourier", a qual apresenta convergência mais rápida que a série de Fourier original numa restrição de domínio, possibilitando soluções de EDOs e EDFs de alta ordem com maior precisão e ampliando a esfera de casos passíveis de resolução.

Palavras-chave: Matrizes operacionais. Equações Diferenciais. Cálculo fracionário. Séries de Fourier. 



\section{ABSTRACT}

Operational matrices method is expanded to complex field and arbitrary orders by using the Riemann-Liouville and Caputo approach with emphasis on complex Fourier series. Dirac's bra-ket notation is associated to tensor procedures in Hilbert spaces for finite function expansions to be applied specifically to differential equations and operational matrices, being called "Coadjoint Formalism". This treatment is extended to Weyl fractional operators for generic periods in order to establish the integral and derivative operational matrices of fractional order to complex Fourier basis. A new method to solve linear non-homogeneous ODEs and FDEs, called "Operational Modelling" is introduced. It yields high precision solutions on high order differential equations without assumption of boundary or initial conditions. The presented method is improved by a new kind of function expansion, called "Fourier Associated Series", which yields a faster convergence than original Fourier in a restrict domain, enabling to obtain solutions of high order ODEs and FDEs with excellent precision and broadening the set of solvable equations.

Keywords: Operational Matrices. Differential equations. Fractional calculus. Fourier series. 



\section{Lista de Figuras}

Sistema de eixos oblíquos . . . . . . . . . . . . . . p. 11

2 Comparação entre a função original e sua expansão na base não-ortogonal

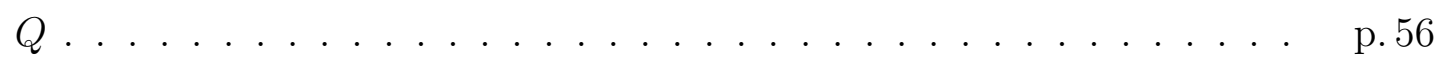

3 Gráficos real e imaginário da base de Fourier transformada $\tilde{\epsilon}_{1}(x) \ldots$. . p. 65

4 Gráficos real e imaginário da base de Fourier transformada $\tilde{\epsilon}_{2}(x) \ldots$. . p. 66

5 Gráficos real e imaginário da base de Fourier transformada $\tilde{\epsilon}_{3}(x) \ldots$. . p. 66

6 Esquema geométrico de representação SF-SA . . . . . . . . . . . . . . p. 110

$7 \quad$ Derivação operacional de $\phi_{1}(x)=x^{2} \operatorname{com} \ell=12 \ldots \ldots$. . . . . . 113

$8 \quad$ Integração operacional de $\phi_{1}(x)=x^{2} \operatorname{com} \ell=12 \ldots \ldots$. . . . . . 114

$9 \quad$ Derivação operacional de $\phi_{2}(x)=e^{x} \operatorname{com} \ell=12 \ldots \ldots$. . . . . . 114

10 Integração operacional de $\phi_{2}(x)=e^{x} \operatorname{com} \ell=12 \ldots \ldots$. . . . . . 114

11 Derivação operacional de $\phi_{3}(x)=\exp \left(-\frac{x^{2}}{2}\right) \operatorname{com} \ell=12 \ldots \ldots$ p. . . . 115

12 Integração operacional de $\phi_{3}(x)=\exp \left(-\frac{x^{2}}{2}\right) \operatorname{com} \ell=12 \ldots \ldots$ p. 115

13 Derivação operacional de $\phi_{4}(x)=\cos \left(x^{2}\right) \operatorname{com} \ell=12 \ldots \ldots$. . . . . . 115

14 Integração operacional de $\phi_{4}(x)=\cos \left(x^{2}\right) \operatorname{com} \ell=12 \ldots \ldots$ p. 116

15 Derivação operacional de $\phi_{5}(x)=e^{-x^{2}} \cos (7 x) \operatorname{com} \ell=12 \ldots$. . . p. 116

16 Integração operacional de $\phi_{5}(x)=e^{-x^{2}} \cos (7 x) \operatorname{com} \ell=12 \ldots \ldots$ p. 116

17 Gráfico da expansão e das integrais direta e operacional de exp $x$. . . . p. 117

18 Gráfico da função solução $f_{2}(x) \ldots \ldots$. . . . . . . . . . . . . p. 132

19 Gráfico da superposição entre $\mathcal{D}_{e q}^{3} f_{2}(x)$ e $\phi(x) \ldots \ldots$. . . . . . . p. 132

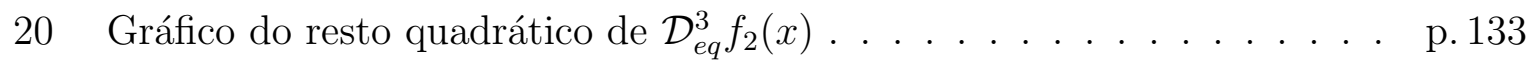

21 Gráfico da função solução $f_{12}(x) \ldots \ldots$. . . . . . . . . . . . p. 134 
22 Gráfico da superposição entre $\mathcal{D}_{e q}^{3} f_{12}(x)$ e $\phi(x) \ldots \ldots$. . . . . . p. 135

23 Gráfico do resto quadrático de $\mathcal{D}_{\text {eq }}^{3} f_{12}(x) \ldots \ldots \ldots \ldots$ p. . . . . . . . . . . . . .

24 Séries de Fourier original e associada da função sinal . . . . . . . . . p. 138

25 Séries de Fourier original e associada da função identidade $\quad$. . . . . . . p. 139

26 Séries de Fourier original e associada da função quadrática . . . . . . . p. 140

27 Séries de Fourier original e associada da função cúbica $\quad$. . . . . . . . p. 141

28 Séries de Fourier original e associada da função quártica . . . . . . . . . p. 142

29 Séries de Fourier original e associada da função exponencial . . . . . . . p p. 143

30 Séries de Fourier original e associada da wavelet Mexican Hat . . . . . p. 144

31 Séries de Fourier original e associada da wavelet de Morlet na restrição p. 144

32 Ilustração da convergência mais rápida da série associada . . . . . . . p p. 146

33 Solução NBV da EDO exponencial do caso $3 \operatorname{com} \ell=15, L=\frac{5 \pi}{7} \quad \cdots \quad$ p. 148

34 Resto quadrático da solução NBV para o caso 3 com range 15 e $L=\frac{5 \pi}{7} \quad$ p. 148

35 Gráficos de $\mathcal{D}_{e q} f_{2}(x)$ e $e^{x}$ para séries associadas com EC, NC e NF, $L=\frac{5 \pi}{7}$ p. 149

36 Gráficos de $\mathcal{D}_{e q} f_{3}(x)$ e $e^{x}$ para séries associadas com EC, NC e NF, $L=\frac{5 \pi}{7}$ p. 149

37 Restos quadráticos de $\mathcal{D}_{\text {eq }} f_{2}(x)$ para $\mathrm{SA}$ com EC, NC e NF, $L=\frac{5 \pi}{7}$. . p. 149

38 Restos quadráticos de $\mathcal{D}_{\text {eq }} f_{3}(x)$ para $\mathrm{SA}$ com EC, NC e NF, $L=\frac{5 \pi}{7}$. . p. 150

39 Restos quadráticos de $\mathcal{D}_{\text {eq }} f_{15}(x)$ para SA com EC, NC e NF, $L=\frac{5 \pi}{7}$. . p. 150

40 Interpolação polinomial da distribuição de Cauchy . . . . . . . . . . . . p. 154

41 Solução da EDO na base de Fourier original com range 5 e $L=\frac{5 \pi}{8}$. . p. 154

42 Solução na base original com range 5 e $L=\frac{5 \pi}{8}$ usando os nodos de Chebyshev . . . . . . . . . . . . . . . . . p. 155

43 Solução na base original com range 5 e $L=\frac{5 \pi}{8}$ usando os nodos de Fourierp. 155

44 Solução na base original com range 9 e $L=\frac{5 \pi}{8} \ldots \ldots$. . . . . . . 156

45 Solução na base original com range 9 e $L=\frac{5 \pi}{8}$ usando os nodos de Chebyshev . . . . . . . . . . . . . . . . p. 156

46 Solução na base original com range 9 e $L=\frac{5 \pi}{8}$ usando os nodos de Fourierp. 157 
47 Solução na base associada com range 9 e $L=\frac{5 \pi}{8} \quad \ldots \ldots$. . . . . . . . 157

48 Solução na base associada com range 9 e $L=\frac{5 \pi}{8}$ usando os nodos de Fourier . . . . . . . . . . . . . . . . . . . . . p. 158

49 Solução na base associada com range 9 e $L=\frac{5 \pi}{8}$ usando os nodos de Chebyshev . . . . . . . . . . . . . . . . . p. 158

50 Resto quadrático da solução original com nodos de Fourier, $\ell=9$ e $L=\frac{5 \pi}{8}$ p. 159

51 Resto quadrático da solução associada com nodos de Chebyshev, $\ell=9$

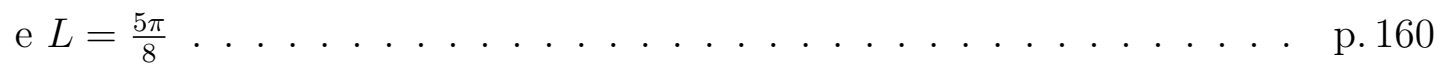

52 Resto quadrático da solução associada e nodos de Chebyshev, $\ell=15$ e

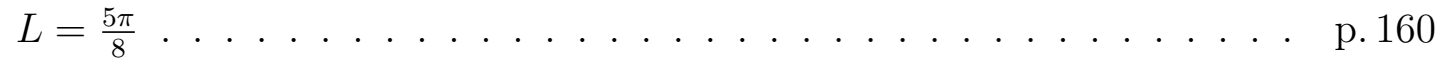

53 Resto quadrático da solução original com nodos de Fourier, $\ell=15$ e $L=\frac{5 \pi}{8}$ p. 160

54 Resto quadrático da solução associada e nodos de Fourier, $\ell=15$ e $L=\frac{5 \pi}{8}$ p. 161

55 Resto quadr. da sol. NBV assoc. p/ MHW com nodos de Cheb., $\ell=7 \quad$ p. 161

56 Resto quadr. da sol. NBV assoc. p/ MHW com nodos de Cheb., $\ell=15$ p. 161

57 Partes real e imaginária e curva paramétrica complexa da função $\phi$. . p. 164

58 Mapas paramétricos cartesiano e polar da função $f(x, y)=z(x, y)$. . . p. 165

59 Mapas paramétricos cartesiano e polar da função $f(x, y)=e^{z(x, y)}$. . . . p. 166

60 Mapas paramétricos polares das funções $z, z^{2}$ e $z^{3} \ldots \ldots$. . . . . p. 166

61 Gráfico da parte real da solução aproximada da EDO de $7^{\mathrm{a}}$ ordem $\quad$. . . $\quad$ p. 167

62 Gráfico da parte imaginária da solução aproximada da EDO de $7^{\mathrm{a}}$ ordem p. 168

63 Curva paramétrica complexa da solução aproximada da EDO de $7^{\mathrm{a}}$ ordemp. 168

64 Comparação gráfica entre as curvas paramétricas de $\mathcal{D}_{\text {eq }} f_{15}(x)$ e $\phi(x)$. p. 169

65 Gráfico da parte real da função-resto $\mathcal{R}_{15}(x) \ldots \ldots$. . . . . . . . . p. 169

66 Gráfico da parte imaginária da função-resto $\mathcal{R}_{15}(x) \ldots \ldots$. . . . . . . . 169

67 Curva paramétrica complexa da função resto $\mathcal{R}_{15}(x) \ldots \ldots$. . . . . . p. 170

68 Gráficos de superfície das partes real, imaginária e absoluta de $f_{15}(x)$. p. 170

69 Mapas paramétricos cartesiano e polar da solução aproximada $f_{15}(x)$. p. 170 
70 Gráficos comparativos da expansão e das integrais real e operacional por IFRL da função exponencial . . . . . . . . . . . . . . . . . . . . . . . p.177

$71 \quad$ Gráficos de $\mathcal{I}_{1}(x), \mathcal{I}_{2}(x), \mathcal{I}_{3}(x) \ldots \ldots \ldots \ldots$ p. 198

72 Solução NBV SF-EC da EDO $(\nu=0) \ldots \ldots$. . . . . . . . . . . . . p. 199

73 Solução NBV SF-NF da EDO $(\nu=0) \ldots \ldots$. . . . . . . . . . . . . 199

74 Solução NBV SA-EC da EDO $(\nu=0) \ldots \ldots$. . . . . . . . . . . . . 199

75 Solução NBV SA-NF da EDO $(\nu=0) \ldots \ldots$. . . . . . . . . . . 199

76 Solução NBV SA-NC da EDO $(\nu=0) \ldots \ldots$. . . . . . . . . . 200

77 Solução NBV SF-EC da EDF $(\nu=\pi) \ldots \ldots \ldots$ p. 200

78 Solução NBV SF-NF da EDF $(\nu=\pi) \ldots \ldots$. . . . . . . . . . 200

79 Solução NBV SA-EC da $\operatorname{EDF}(\nu=\pi) \ldots \ldots \ldots$ p. 201

80 Solução NBV SA-NF da $\operatorname{EDF}(\nu=\pi) \ldots \ldots \ldots$ p. . . . . . . . . . . . . .

81 Solução NBV SA-NC da $\operatorname{EDF}(\nu=\pi) \ldots \ldots$. . . . . . . . . . 201

82 Solução NBV SF-EC da EDO $(\nu=1) \ldots \ldots$. . . . . . . . . . . . 202

83 Solução NBV SF-NF da EDO $(\nu=1) \ldots \ldots$. . . . . . . . . . . . 202

84 Solução NBV SA-EC da EDO $(\nu=1) \ldots \ldots$. . . . . . . . . . . 202

85 Solução NBV SA-NF da EDO $(\nu=1) \ldots \ldots$. . . . . . . . . . . 203

86 Solução NBV SA-NC da EDO $(\nu=1) \ldots \ldots$. . . . . . . . . . 203

87 Solução NBV SF-EC da EDF $(\nu=0.4) \ldots \ldots$. . . . . . . . . . . 203

88 Solução NBV SF-NF da EDF $(\nu=0.4) \ldots \ldots$. . . . . . . . . . 203

89 Solução NBV SA-EC da $\operatorname{EDF}(\nu=0.4) \ldots \ldots$. . . . . . . . . . . p. 204

90 Solução NBV SA-NF da EDF $(\nu=0.4) \ldots \ldots$. . . . . . . . . . p. 204

91 Solução NBV SA-NC da EDF $(\nu=0.4) \ldots \ldots$. . . . . . . . . . 204

92 Gráfico da parte real da solução $f_{15}$ da $\operatorname{EDF}(7 \nu) \ldots \ldots$. . . . . . . 206

93 Gráfico da parte imaginária da solução $f_{15}$ da $\operatorname{EDF}(7 \nu) \ldots \ldots$. . . . 206

94 Gráfico da curva paramétrica complexa da solução $f_{15}$ da $\operatorname{EDF}(7 \nu)$. . p. 206

95 Gráficos complexos de superfície da solução $f_{15}$ da $\operatorname{EDF}(7 \nu) \quad \ldots$. . p 207 
96 Mapas paramétricos cartesiano e polar da solução $f_{15}$ da $\operatorname{EDF}(7 \nu)$. . p. 207

97 Gráfico da parte real da função-resto $\mathcal{R}_{15}(x) \ldots \ldots$. . . . . . . . . . p. 207

98 Gráfico da parte imaginária da função-resto $\mathcal{R}_{15}(x) \ldots \ldots$. . . . . . . p. 207

99 Curva paramétrica complexa da função resto $\mathcal{R}_{15}(x) \ldots \ldots$. . . . . . p. 208

100 Parte real da solução NBV-SA-NC da EDF de Calor . . . . . . . . . . p. 211

101 Parte imaginária da solução NBV-SA-NC da EDF de Calor . . . . . . . p. 211

102 Curva paramétrica complexa da solução NBV-SA-NC da EDF de Calor p. 212

103 Comparação entre as curvas paramétricas de $\mathcal{D}_{e q} f_{15}$ e $\phi \ldots \ldots$. . . . p. 212

104 Parte real do resto local $R_{15}(x)$ na solução da EDF de Calor $\ldots$. . . . . p. 212

105 Parte imaginária do resto local $R_{15}(x)$ na solução da EDF de Calor . p. 213

106 Curva paramétrica complexa do resto local $R_{15}(x)$ na solução da EDF de Calor . . . . . . . . . . . . . . . . . . . . . p. . . . . . . . . . . . . . .

107 Gráficos complexos de superfície da solução NBV-SA-NC da EDF de Calorp. 213

108 Mapas paramétricos da solução NBV-SA-NC da EDF de Calor . . . . . p. 214

109 Representação esquemática das relações entre diversos tipos de espaços p. 239

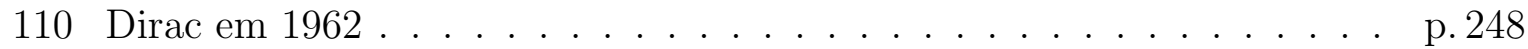

111 As duas páginas do tratado de Lacroix que versam sobre derivada fracionalp. 262

112 Magnus Gösta Mittag-Leffler (1846-1927) . . . . . . . . . . . . . . p. 266

113 Gráfico da função gama no domínio real . . . . . . . . . . . . . . p. 269

114 Gráfico da função duplo fatorial no domínio real . . . . . . . . . . . . . p. 272

115 Função gama recíproca no domínio real . . . . . . . . . . . . . p. 276

116 Valor absoluto da função gama com argumento complexo . . . . . . . . p. 279

117 Função beta no domínio real . . . . . . . . . . . . . . . . . p. 287

118 Função binomial com parâmetro $\alpha$ real positivo . . . . . . . . . . . . . p. 290

119 Função binomial com parâmetro $\alpha$ real negativo . . . . . . . . . . . . p. 290

120 Função binomial com parâmetro $\alpha$ imaginário . . . . . . . . . . . . . . p. 291

121 Parte real da função binomial com parâmetro $\alpha$ imaginário . . . . . . p. p. 291 
122 Função binomial com parâmetro $\alpha$ complexo . . . . . . . . . . . . . . . p. 292

123 Parte real da função binomial com parâmetro $\alpha$ complexo . . . . . . . . p. 292

124 Função binomial generalizada no domínio real positivo . . . . . . . . . p. 293

125 Função binomial generalizada no domínio real negativo . . . . . . . . . p. 293

126 Função binomial generalizada no domínio real . . . . . . . . . . . . . p. 294

127 Caminho de integração . . . . . . . . . . . . . . . p. 306

128 Funções piso, teto e dente-de-serra . . . . . . . . . . . . . . p. . 315

129 Definições esquerda e direita da derivada fracional . . . . . . . . . . p. 316 


\section{Lista de Tabelas}

1 Funções analisadas na comparação MOD/MOI × D/J . . . . . . . . . . p. 113

2 Erros nas expansões de algumas funções para $\ell=12 \ldots \ldots$. . . . . 118

3 Erros nas derivadas operacionais de algumas funções para $\ell=12$. . . p. 119

4 Erros nas integrais operacionais de algumas funções para $\ell=12 \quad \ldots \quad$ p. 119

$5 \quad$ Erros nas expansões de algumas funções para $\ell=15 \ldots$. . . . . . . . p. 119

6 Erros nas derivadas operacionais de algumas funções para $\ell=15 \ldots$. . p. 119

7 Erros nas integrais operacionais de algumas funções para $\ell=15 \quad \ldots \quad$ p. 119

8 Erros quadráticos das expansões SF/SA de algumas funções nas restrições correspondentes . . . . . . . . . . . . . . . . . . p. 138

9 Erros quadráticos da solução NBV-SA para a exponencial com diferentes

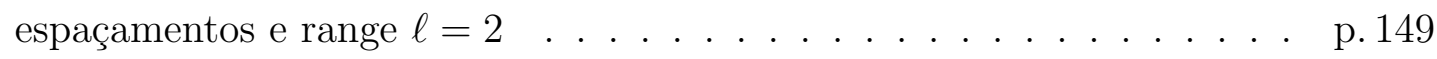

10 Erros quadráticos da solução NBV-SA para a exponencial com diferentes espaçamentos e range $\ell=3 \ldots \ldots \ldots$. . . . . . . . . . 150

11 Erros quadráticos da solução NBV-SA para a exponencial com diferentes espaçamentos e range $\ell=15 \ldots \ldots \ldots$. . . . . . . . . . 150

12 Erros absolutos totais da exponencial na comparação BVP-NBV e imposição de condições à esquerda e à direita em função do range . . . . . p. 152

13 Variação do erro absoluto total na solução NBV da exponencial em função do range . . . . . . . . . . . . . . . . . . . . . . . . p. 152

14 Principais transformadas de Fourier . . . . . . . . . . . . . . . p. 329 



\section{LISTA DE ABREVIATURAS E SIGLAS}

DFC Derivada Fracional de Caputo

DFCL Derivada Fracional de Caputo-Liouville

DFCR Derivada Fracional de Caputo-Riemann

DFCW Derivada Fracional de Caputo-Weyl

DFW Derivada Fracional de Weyl

DFWM Derivada Fracional de Weyl-Marchaud

DFL Derivada Fracional de Liouville

DFR Derivada Fracional de Riemann

DFRL Derivada Fracional de Riemann-Liouville

EC Espaçamento Constante

EDO Equação diferencial ordinária

EDF Equação diferencial fracionária

EDP Equação diferencial parcial

EDPF Equação diferencial parcial fracionária

IP Produto interno ou espaço com produto interno

IFL Integral Fracional de Liouville

IFR Integral Fracional de Riemann

IFRL Integral Fracional de Riemann-Liouville

IFW Integral Fracional de Weyl 
MOD Matriz Operacional de Derivação

$\operatorname{MOD}(v)$ Matriz Operacional de Derivação fracionária de ordem $v$

MOI Matriz Operacional de Integração (antiderivação)

$\operatorname{MOI}(v)$ Matriz Operacional de Integração fracionária de ordem $v$

MOp Matriz Operacional

NBV Método de Modelagem Operacional sem condições de contorno

NC Nodos de Chebyshev

NF Nodos de Fourier

NIV Método de solução por Modelagem Operacional sem condições iniciais

QI Funções de quadrado integrável

SA Série Associada de Fourier

SF Série de Fourier (complexa)

TD Transposição de domínio

TF Transformada de Fourier (simetrizada)

TL Transformada de Laplace 


\section{LISTA DE SÍMBOLOS}

$\equiv \quad$ Equivalência

〜 $\quad$ Equivalência de notações

$\simeq \quad$ Isomorfismo

$\bar{z}, z^{*} \quad$ Complexo-conjugado de $z$

$(\cdot)^{\prime} \quad$ Operação de transposição

$(\cdot)^{T} \quad$ Operação de transposição

$(\cdot)^{*} \quad$ Operação de conjugação complexa

$(\cdot)^{\dagger} \quad$ Operação de conjugação hermitiana

$(\cdot)^{\star} \quad$ Operação de dualidade

V Espaço vetorial genérico

$\mathbb{V}^{*} \quad$ Espaço complexo-conjugado de $\mathbb{V}$

$\mathbb{V}^{\star} \quad$ Espaço dual de $\mathbb{V}$

$\mathbb{V}^{n} \quad$ Espaço vetorial finito de dimensão $n$

K Corpo genérico

$\mathcal{H}$ Espaço de Hilbert de funções

$\mathbb{M}_{n}(\mathbb{K}) \quad$ Espaço das matrizes quadradas de ordem $n$ sobre o corpo $\mathbb{K}$

$\mathfrak{R}\{\cdot\} \quad$ Parte real de um número complexo

$\mathfrak{J}\{\cdot\} \quad$ Parte imaginária de um número complexo

$|\cdot| \quad$ Módulo ou valor absoluto 
$\|\cdot\| \quad$ Norma euclidiana

$\|\cdot\|_{2} \quad$ Norma euclidiana

$\|\cdot\|_{p} \quad$ Norma $\mathcal{L}^{p}$

$\|\cdot\|_{\infty} \quad$ Norma máxima

$d(u, v) \quad$ Métrica ou distância entre $u$ e $v$

$\langle$,$\rangle \quad Produto interno usual$

$\langle\mid\rangle \quad$ Produto interno de Dirac

$C^{0}[a, b]$ Classe de funções contínuas num intervalo $[a, b]$

$C^{m}[a, b]$ Classe de funções contínuas $m$-diferenciáveis num intervalo $[a, b]$

$\mathcal{L}^{1} \quad$ Espaço de funções integráveis e absolutamente integráveis no domínio

$\mathcal{L}^{2} \quad$ Espaço de funções integráveis a Lebesgue no domínio

$\mathcal{L}_{w}^{2} \quad$ Espaço de funções integráveis a Lebesgue com função-peso $w$ no domínio

$\mathcal{L}^{2}[a, b]$ Espaço de funções integráveis a Lebesgue num intervalo $[a, b]$

$\Gamma(z) \quad$ Função gama

$\gamma(z, w) \quad$ Função gama inferior incompleta

$\Gamma(z, w) \quad$ Função gama superior incompleta

$\gamma^{*}(z, w)$ Extensão holomórfica da função gama incompleta

$\psi(z) \quad$ Função digama

$\psi^{(k)}(z) \quad$ Funções poligama

$\mathrm{B}(p, q) \quad$ Função beta

$\mathrm{B}(z ; p, q)$ Função beta incompleta

$I_{z}(p, q) \quad$ Função beta regularizada 
$(n)_{k} \quad$ Símbolo de Pochhammer

$\left(\begin{array}{l}n \\ k\end{array}\right) \quad$ Coeficientes binomiais

$\kappa_{\alpha}(z) \quad$ Função binomial de parâmetro $\alpha$

$\kappa(z, w) \quad$ Função binomial generalizada

$E_{\alpha}(z) \quad$ Função de Mittag-Leffler

$E_{\alpha, \beta}(z) \quad$ Função de Wiman ou Mittag-Leffler de dois parâmetros

$E_{\alpha, \beta}^{\rho}(z) \quad$ Função de Prabhakar ou Mittag-Leffler de três parâmetros

$E_{z}(v, \lambda)$ Função de Mellin-Ross

$\lceil\cdot\rceil$ Função teto

$\lfloor\cdot\rfloor \quad$ Função piso

$[\cdot] \quad$ Valor inteiro

$\{\cdot\} \quad$ Valor fracionário

$\mathrm{O}_{n} \quad$ Espaço de Ordem $n$-dimensional

$\mathrm{O}_{n}(\mathrm{~V}) \quad$ Espaço de Ordem $n$-dimensional sobre o espaço vetorial (V)

$\mathbb{O}_{n}(\mathcal{H})$ Espaço de Ordem $n$-dimensional sobre o espaço de Hilbert

$\mathrm{O}_{n}(\mathbb{C}) \quad$ Espaço de Ordem $n$-dimensional sobre o corpo complexo

$\mathcal{K}_{v}(x) \quad$ Kernel fracional

$H(x) \quad$ Função de Heaviside

$\mathfrak{F}\{\cdot\} \quad$ Transformada de Fourier

$\mathcal{L}\{\cdot\} \quad$ Transformada de Laplace

$(f * g)_{\theta} \quad$ Produto de convolução cíclica de $f$ e $g$ no intervalo de período $2 \pi$

$(f * g)_{L} \quad$ Produto de convolução cíclica de $f$ e $g$ no no intervalo de período $2 L$ 
$(f * g)_{\widetilde{\mho}}$ Produto de convolução de $f$ e $g$ para as transformadas de Fourier

$(f * g)_{\mathcal{L}}$ Produto de convolução de $f$ e $g$ para as transformadas de Laplace

$f \star g \quad$ Integral de correlação cruzada das funções $f$ e $g$

$\mathfrak{D}_{n}(x) \quad$ Núcleo de Dirichlet complexo com ordem de expansão $n$

${ }_{0} D_{x}^{v} \equiv D^{v} \quad$ Diferintegral de Riemann-Liouville de ordem $v$

${ }_{-\infty} D_{x}^{v} \equiv D_{-\infty}^{v} \quad$ Diferintegral de Liouville de ordem $v$

${ }_{a} D_{x}^{v} \equiv D_{a}^{v} \quad$ Diferintegral de Riemann de ordem $v$

${ }_{0}^{c} D_{x}^{v} \equiv{ }^{c} D^{v} \quad$ Derivada fracional de Caputo de ordem $v$

${ }_{-\infty}^{c} D_{x}^{v} \equiv{ }^{c} D_{-\infty}^{v} \quad$ Derivada fracional de Caputo-Liouville de ordem $v$

${ }_{a}^{c} D_{x}^{v} \equiv{ }^{c} D_{a}^{v} \quad$ Derivada fracional de Caputo-Riemann de ordem $v$

${ }_{x}^{c} D_{\infty}^{v} \equiv{ }^{c} W_{\infty}^{v} \quad$ Derivada fracional de Caputo-Weyl de ordem $v$

${ }_{x} W_{\infty}^{v} \quad$ Derivada fracional de Weyl de ordem $v$

$\Psi_{ \pm}^{v}(x)$ Operador de integração fracional de Weyl de ordem $v$

${ }_{L} W_{ \pm}^{v} \quad$ Diferintegral de Weyl de ordem $v$ para funções de semiperíodo $L$

${ }_{L} D_{ \pm}^{\alpha} \quad$ Derivada de Weyl-Marchaud de ordem $\alpha$ para funções de semiperíodo $L$

$u^{i} \quad$ Coordenadas contravariantes do vetor $u$

$u_{i} \quad$ Coordenadas covariantes do vetor $\mathrm{u}$

|\rangle$_{\infty} \quad$ Ket no espaço de Hilbert de funções

$\left\langle\left.\right|_{\infty} \quad\right.$ Bra no espaço de Hilbert de funções

| $\rangle_{0_{n}} \quad$ Ket no espaço de Ordem $\mathbb{O}_{n}$

$\left\langle I_{0_{n}} \quad\right.$ Bra no espaço de Ordem $\mathrm{O}_{n}$

$\langle u \mid v\rangle_{[B]}$ Produto hermitiano das representações coordenadas na base [B] 
$\langle u \mid v\rangle_{0_{n}} \quad$ Produto dual em $\mathbb{O}_{n}$

$|\phi\rangle_{[B]} \quad$ Representação coordenada do ket $\phi$ na base $[B]$

$\delta_{i}^{k}, \delta_{k}^{i} \quad$ Delta de Kronecker

$\delta(x) \quad$ Função delta de Dirac

$|B|_{0_{n}} \quad$ Base B de $O_{n}$ na $1^{\mathrm{a}}$ representação

$B^{\dagger} \quad$ Base adjunta de $B$

$B^{\star} \quad$ Base dual de $B$

B $\quad$ Base coadjunta de $B$

$\left\langle\left.\underline{u}\right|_{[B]} \quad\right.$ Representação coordenada dual do vetor $u$ na base $[B]$

$|\underline{u}\rangle_{[B]} \quad$ Representação coordenada coadjunta do vetor $u$ na base $[B]$

|B ] $\quad 1^{\mathrm{a}}$ representação isomórfica coadjunta de uma base [B] contravariante

$\mid \dot{B}] \quad 1^{\text {a }}$ representação isomórfica coadjunta de uma base $[B]$ covariante

|B $\rangle \quad 2^{\mathrm{a}}$ representação isomórfica coadjunta de uma base $[B]$ contravariante

$|\dot{B}\rangle \quad 2^{\text {a }}$ representação isomórfica coadjunta de uma base $[B]$ covariante

$\stackrel{(m)}{\mathbb{P}} \quad$ Projetor no subespaço de ordem $m$

$\mathbb{P} \quad$ Autoprojetor no Espaço de Ordem

$\stackrel{(m)}{\mathbb{P}^{\dagger}} \quad$ Projetor adjunto no subespaço de ordem $m$

$\mathbb{P}^{\dagger} \quad$ Autoprojetor adjunto no Espaço de Ordem

$\overleftrightarrow{\gamma} \quad$ Operador tensorial métrico

$\overleftrightarrow{\gamma}^{\star} \quad$ Operador tensorial métrico recíproco

$\gamma_{i k} \quad$ Componente $i k$ do tensor métrico $\overleftrightarrow{\gamma}$

$\gamma^{i k} \quad$ Componente $i k$ do tensor métrico recíproco $\overleftrightarrow{\gamma}^{\star}$ 
$\left|f_{n}\right\rangle_{\infty} \quad$ Função $f$ aproximada por uma expansão em série de $n$ termos

$q \quad$ Razão periódica das séries de Fourier

q $\quad$ Razão periódica das séries associadas de Fourier

L Semiperíodo das séries de Fourier

$\tilde{L} \quad$ Semiperíodo das séries associadas de Fourier

$F_{R}(x) \quad$ Função de expansão híbrida

$\left|e_{m}\right\rangle_{\infty} \quad$ Componente $m$ da base complexa de Fourier na $2^{\mathrm{a}}$ representação

$|m\rangle_{\infty} \quad$ Componente $m$ da base complexa de Fourier renormalizada na $2^{\mathrm{a}}$ repr.

$\left|\epsilon_{k}\right\rangle \quad$ Componente $k$ da base polinomial canônica na $2^{\mathrm{a}}$ representação

$\langle C| \quad$ Covetor coeficiente ajustado na expansão de Fourier complexa

$\left|E_{m}\right\rangle_{\infty} \quad$ Componente $m$ da base ajustada de Fourier na $2^{\mathrm{a}}$ representação

$\mu_{r}(x) \quad$ Funções coeficientes de uma EDO ou EDF no método de modelagem

$\mathcal{D}_{e q} \quad$ Operador de uma equação diferencial no método de modelagem

$\mathcal{M} \quad$ Matriz anticoeficiente no método de modelagem

$\langle\Phi| \quad$ Covetor não-homogêneo de uma EDO ou EDF no método de modelagem

$\mathcal{R}_{n}(x) \quad$ Função resto local de uma expansão em série de $n$ termos

$\mathcal{R}_{n}^{2}(x) \quad$ Resto quadrático local de uma expansão em série de $n$ termos

$\mathcal{E}_{n} \quad$ Erro total no período para um expansão em série de $n$ termos

$\mathcal{E}_{n}^{2} \quad$ Erro quadrático total no período para um expansão em série de $n$ termos 


\section{Sumário}

1 INTRODUÇÃO $\quad$ p. 1

1.1 Apresentação do Tema . . . . . . . . . . . . . . . . p. 1

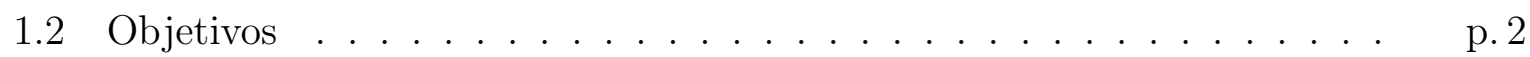

1.3 Motivação e Considerações Analíticas . . . . . . . . . . . . . . . p. 2

1.3.1 Uso de Matrizes Operacionais . . . . . . . . . . . . . p. 2

1.3.2 Formalismo bra-ket . . . . . . . . . . . . p. 4

1.3.3 Método Operacional Complexo de Fourier . . . . . . . . . . p. p.4

1.3.4 Abordadem do Cálculo Diferencial e Integral de Ordem Arbitrária p. 5

1.4 Estado da Arte . . . . . . . . . . . . . . . . p. 5

1.5 Conclusão da Apresentação f . . . . . . . . . . . . . . . p p 8

2 FUNDAMENTAÇÃO TEÓRICA p. 9

2.1 Formalismo Tensorial na Linguagem Bra-Ket . . . . . . . . . p. 9

2.1.1 Definições e Notação . . . . . . . . . . . . . . . . . . . p.9

Sistemas e Eixos . . . . . . . . . . . . . p. 9

Coordenadas Covariantes e Contravariantes . . . . . . p. 10

Descrição Geométrica . . . . . . . . . . . . . p. 10

2.1.2 Formalismo no Espaço de Ordem . . . . . . . . . . . . p. 11

2.1.2.1 Determinação dos Espaços de Atuação . . . . . . . . . p. 12

2.1.2.2 Espaços de Kets . . . . . . . . . . . . . . p. 14

Bases de Kets . . . . . . . . . . . . . . p. 15

2.1 .3 Abordagem Linear . . . . . . . . . . . . . . p. 16 
Equivalência de Notações . . . . . . . . . . . . . p. p. 16

2.1.3.1 Kets e Bras em $\mathcal{O}_{n} \ldots \ldots \ldots$ p. 16

Bras em $\mathcal{O}_{n} \ldots \ldots \ldots \ldots \ldots$ p. 18

2.1.3.2 Operações de Transformação entre Bras e Kets . . . p.18

2.1.3.3 Espaços Duais . . . . . . . . . . . . p. 20

Definição Formal de Bra . . . . . . . . . . . . p. 21

2.2 Reformulação Coadjunta . . . . . . . . . . . . . . . . . p. 22

2.2.1 Representações Isomórficas da Base . . . . . . . . . . . . . . p. 22

Bases e Transformações . . . . . . . . . . . . . . . . p. 22

2.2.2 Representações de um Vetor . . . . . . . . . . . . . . p. 24

2.2.3 Relação de Dualidade e Base Recíproca . . . . . . . . . . . p. 25

$2.2 .4 \operatorname{IPs} \mathrm{em} \mathcal{O}_{n} \ldots \ldots \ldots \ldots \ldots \ldots \ldots$ p. . . . . . . . . . . . .

Produto Hermitiano . . . . . . . . . . . . p. 26

Produto Dual ................ . . p. 26

2.2.5 Construção das Bases Correlatas . . . . . . . . . . . . p. 27

Base Adjunta . . . . . . . . . . . . . . . p. 27

Base Coadjunta . . . . . . . . . . . . p. 27

2.2.6 Representações Correlatas de um Ket . . . . . . . . . . . p. 27

Representação Adjunta . . . . . . . . . . . . . p. 27

Representação Coadjunta . . . . . . . . . . . p. p. 28

Representação Dual . . . . . . . . . . . . . . . . p. p. 28

IPs nas Diferentes Representações . . . . . . . . . . . p. 29

2.2.7 Determinação das Coordenadas . . . . . . . . . . . p. 30

Coordenadas Contravariantes . . . . . . . p. p. 30

Coordenadas Covariantes . . . . . . . . . p. 31

Correspondência Matricial . . . . . . . . . . . p. 31 
Relação entre as duas Representações Isomórficas da Base p. 32

2.2.8 Produto Externo em $\mathcal{O}_{n} \ldots \ldots \ldots \ldots$ p. 33

Natureza Tensorial do Produto Externo . . . . . . . . p.33

2.2 .9 Projetores em $\mathcal{O}_{n} \ldots \ldots \ldots \ldots \ldots$ p. 34

Autoprojetor . . . . . . . . . . . . p. . 35

Projetor Adjunto . . . . . . . . . . . . p. 35

2.2.10 Tensor Métrico . . . . . . . . . . . . . . . . . . . p. 36

Ortogonalidade de Bases . . . . . . . . . . . p. 36

Métrica................... p. 37

Tensor Recíproco . . . . . . . . . . . . . . . p. 38

2.2.11 Relação entre Bases e Coordenadas Covariantes e Contravariantes p.38

2.2.12 Relação entre os Projetores e o Tensor Métrico . . . . . . . . . p. p.41

2.2 .13 Operadores . . . . . . . . . . . . . . . . p. 41

2.3 Formalismo Coadjunto em $\mathcal{L}^{2} \ldots \ldots \ldots \ldots \ldots$ p. . . . . . . . . . . . . .

Espaço Primitivo . . . . . . . . . . . . . p. 44

2.3.1 Bases Correlatas em $\mathcal{O}_{n}(\mathcal{H}) \ldots \ldots \ldots \ldots$ p. 45

2.3.1.1 Obtenção dos Coeficientes . . . . . . . . . . p. 47

2.3.2 Autoprojetor em $\mathcal{O}_{n}(\mathcal{H}) \ldots \ldots \ldots \ldots$ p. 47

2.3.3 Relações entre Coordenadas Covariantes e Contravariantes em

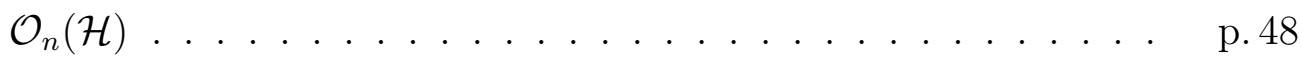

2.3.4 Operadores Métricos . . . . . . . . . . . . . . . p. 49

2.3.5 Expansão em Série de $n$ Termos . . . . . . . . . . . . . . p. 49

2.3.6 Aplicações Práticas do Formalismo . . . . . . . . . . . . p. p.50

2.3.7 Aplicação à Série Complexa de Fourier . . . . . . . . . . . . . . p.50

Translação de Range . . . . . . . . . . . . . . . p. 52

2.3.8 Aplicação aos Polinômios de Legendre . . . . . . . . . . . . . p. p.53

2.3.9 Exemplo em Base Não-ortogonal . . . . . . . . . . . . . p. 54 
2.3.10 Expansão em Base Não-Ortogonal . . . . . . . . . . . . . p.55

2.3.11 Mapeamentos Bijetores . . . . . . . . . . . p. 57

2.3.11.1 Uniformização de Domínios . . . . . . . . . . p. 58

2.3.11.2 Implementação do IP Híbrido . . . . . . . . . . . . p. 60

2.3.11.3 Mapeamentos tipo Fourier-Jacobi e Jacobi-Fourier . p p.60

Transposição de Domínio . . . . . . . . . . . . . p. 60

2.3.11.4 Esboço do Mapeamento Legendre-Fourier . . . . . . . p. 61

2.3.11.5 Mapeamento Hermite-Fourier . . . . . . . . . p. 61

2.3.11.6 Mapeamento Fourier-Hermite . . . . . . . . . p. 64

2.3.11.7 IP Híbrido Fourier-Hermite . . . . . . . . . p. 64

3 MATRIZES OPERACIONAIS NO FORMALISMO COADJUNTO p. 67

3.1 Matrizes Operacionais em Bases Genéricas . . . . . . . . . . . p. 67

3.1.1 Matrizes Operacionais de Ordens Superiores . . . . . . . . p 69

3.1.2 Notação Generalizada . . . . . . . . . . . . . . . . p. 70

3.1.3 Mudança de Base . . . . . . . . . . . . . . . p. 71

3.1.4 Determinação da MOD numa Base Polinomial Canônica . . . p. 72

3.1.5 Mudança de Base para MOp de Ordens Superiores . . . . . . p. p.73

3.1.6 Transformação do Covetor Coeficiente de MOps de Grau k Inteiro p.74

3.1.7 MOD de ordem genérica na base polinomial canônica . . . . . p. 75

3.1.8 Dedução da Matriz Sandwich . . . . . . . . . . . . . . p.79

3.1.9 Mudanças de Base em Domínios Distintos . . . . . . . . . . p. 81

3.2 Descrição do Método Proposto . . . . . . . . . . . . . . . p. 82

3.2.1 Séries e Bases Complexas de Fourier . . . . . . . . . . . p. 82

3.2.2 Convergência Pontual da Série Aproximativa . . . . . . . . . . p. 84

3.2.2.1 Núcleo de Dirichlet Complexo . . . . . . . . . . p. 84

3.2.2.2 Propriedades de $\mathfrak{D}_{\ell}(x) \ldots \ldots \ldots \ldots$ p. 86 
3.2.2.3 A Série Aproximativa como Integral de Convolução e de Correlação e Produto Interno . . . . . . . . . . . p. p. 88

3.2.2.4 Núcleo de Dirichlet no Formalismo de Dirac . . . . . . p. 89

3.2.2.5 Critério de Dirichlet . . . . . . . . . . . p. 90

3.2.3 Forma Explícita da MOD na Base de Fourier Complexa . . . . . p.90

3.2.3.1 Forma Explícita da MOD de Ordens Superiores na Base de Fourier Complexa . . . . . . . . . . . . . p. 91

3.2.4 Forma Explícita da MOI na Base de Fourier Complexa . . . . . p. p.93

Extensão a Matrizes de Dimensão Infinita . . . . . . . p. 96

3.2.5 Aperfeiçoamento do Método . . . . . . . . . . . . p. p. 97

3.2.5.1 Séries de Fourier Associadas . . . . . . . . . . p. 97

3.2.5.2 Função de Expansão Híbrida . . . . . . . . . . . . p. 99

3.2.6 Determinação do Erro da Série Associada . . . . . . . . . . . p. 101

Comentários sobre a interpretação geométrica das séries de Fourier originais e associadas . . . . . . . . p. 109

4 APLICAÇÕES DO MÉTODO p.111

4.1 Aproximação de Derivadas e Integrais pelo

Método Operacional de Fourier Complexo . . . . . . . . . . . . . . . p. 112

4.2 Resolução de EDOs Não-homogêneas pelo Método de Modelagem Operacional . . . . . . . . . . . . . . . . . . . . p. 121

4.2.1 Solução com Condições de Contorno . . . . . . . . . . . . . . p. 122

4.2.2 Problema com Condições Iniciais . . . . . . . . . . . . . . . . . p. 124

4.2.3 Método de Resolução sem Condições de Contorno (NBV) ou Ini-

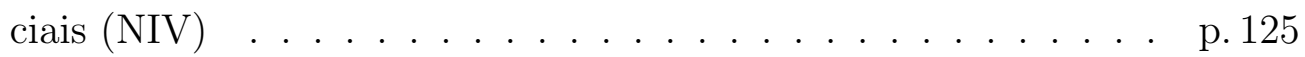

4.2.3.1 Fenômeno de Runge e Nodos de Chebyshev . . . . . . p. 127

Fenômeno de Gibbs . . . . . . . . . . . . . . . . . p. 128

Nodos de Fourier . . . . . . . . . . . . . . . . . . . p. 129

4.2 .4 Aplicação do Método . . . . . . . . . . . . . . . . . . p. 129 
4.2.4.1 Exemplos Numéricos . . . . . . . . . . . . . . p. 129

Exemplo didático . . . . . . . . . . . . . . p. 130

Exemplo mais significativo . . . . . . . . . . p. 133

4.3 Implementação das Séries Associadas . . . . . . . . . . . . . . p. 136

Comentários . . . . . . . . . . . . . . . p. 139

4.3.1 Discussão sobre a ausência de Parâmetros Impositivos . . . . . . p p. 145

Comparação BVP-NBV . . . . . . . . . . . . . . p. 151

4.4 Resolução pelo Método NBV . . . . . . . . . . . . . . . . p. 153

Comentários sobre os resultados . . . . . . . . . p. 159

4.4.1 EDO com Funções Coeficientes e Termo Independente Complexos p. 163

4.4.1.1 Recursos Gráficos Adicionais para Funções com Argumento Complexo . . . . . . . . . . . p. p. 164

4.4.1.2 Modelagem NBV da EDO de $7^{\mathrm{a}}$ Ordem . . . . . . p. 166

4.4.1.3 Comentários sobre os Resultados Obtidos na Resolução de EDOs . . . . . . . . . . . . . . . p. 171

\section{APLICAÇÕES FRACIONAIS E ANÁLISE DOS RESULTADOS p.173}

5.1 Matrizes Operacionais Fracionárias . . . . . . . . . . . . p. 173

5.1 .1 MOI de Fourier complexa pela IFRL . . . . . . . . . . . p. 174

5.1.2 MOI fracional de Fourier complexa pela IFRL . . . . . . . . p. 177

5.1.3 Operadores Fracionais de Weyl . . . . . . . . . . . p. 178

5.1.3.1 Integral Fracional de Weyl . . . . . . . . . . . . p. 179

5.1.3.2 Equivalência Weyl-Liouville . . . . . . . . . . p. 181

5.1.3.3 Propriedades do Operador Integral de Weyl . . . . . . p. 182

5.1.3.4 Propriedades da Integral Fracional de Weyl . . . . . . p. 185

5.1.3.5 Derivadas Fracionais de Weyl . . . . . . . . . p. 187

5.1.3.6 Propriedades da Derivada Fracional de Weyl . . . . . . p. 189 
5.1.3.7 MOps Fracionais de Weyl na Base Complexa de Fourier p. 191

5.2 Equações Diferenciais Fracionárias . . . . . . . . . . . . . . . . . p. 192

5.2.1 Solução Analítica da Equação de Bagley-Torvik . . . . . . . . . p. 193

5.2 .2 EDFs Ilustrativas . . . . . . . . . . . . . . . . . . . p. 195

5.2 .3 Resoluções Numéricas . . . . . . . . . . . . . . . . . . . . . p. 198

5.2.4 Aplicação a uma EDF Sequencial de Ordem $7 \nu$. . . . . . . p. 205

5.2.5 EDF Unidimensional de Condução do Calor com Fonte . . . . . p. 208

5.2.5.1 Solução NBV da EDF Unidimensional de Condução de Calor com Fonte . . . . . . . . . . . . p. 209

5.2 .5 .2 Conclusão . . . . . . . . . . . . . . . . p. 211

6 CONCLUSÃO P. 215

6.1 Uma Visão Geral . . . . . . . . . . . . . . . . . . . . . . . p. 215

6.1 .1 Contribuições da Tese . . . . . . . . . . . . . . p. 216

6.1.2 Limitações do Método Proposto . . . . . . . . . . . . . . . p.218

6.1.3 Prognósticos de Trabalho Futuro . . . . . . . . . . . . . . . . p. 219

6.1 .4 Considerações Finais . . . . . . . . . . . . . . . p. 220

$\begin{array}{ll}\text { Referências } & \text { p. } 221\end{array}$

Apêndice A - Fundamentos Matemáticos $\quad$ p. 231

A.1 Espaços Topológicos . . . . . . . . . . . . . . . . . . p. 231

A.2 Espaços Vetoriais Complexos . . . . . . . . . . . . . . . . p. 232

Espaço Complexo Conjugado . . . . . . . . . . . p. 232

A.3 Mapeamentos em Espaços Vetoriais . . . . . . . . . . . . . p. 233

Funcionais . . . . . . . . . . . . . . p. 233

Espaços Duais . . . . . . . . . . . . . . p. 233

Bases Duais . . . . . . . . . . . . . p. . 234 
A.4 Espaços Vetoriais Normados . . . . . . . . . . . . . . . . p. 234

Norma . . . . . . . . . . . . . . . p. 234

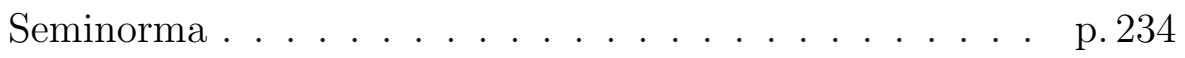

Tipos de Norma . . . . . . . . . . . . . p. 234

Espaços Normados . . . . . . . . . . . . . . . p. 235

A.5 Métrica . . . . . . . . . . . . . . . . . . . . p. 235

Pseudométrica . . . . . . . . . . . . . . p. 235

A.6 Espaços Métricos . . . . . . . . . . . . . . . . . . . p. 235

Sequências de Cauchy . . . . . . . . . . . . . p. 236

Espaços Métricos Completos . . . . . . . . . . p. 236

A.7 Espaços de Banach . . . . . . . . . . . . . . . . . p. 236

A.8 Produto Interno $\ldots \ldots \ldots \ldots \ldots \ldots \ldots$

Formas Sesquilineares . . . . . . . . . . . p. 237

Formas Hermitianas . . . . . . . . . . . . . . . p. 238

Formas Antiautoadjuntas . . . . . . . . . . p. 238

Espaços IP . . . . . . . . . . . . . . . . . . . . . . . . . . . p. 238

A.9 Espaços de Hilbert . . . . . . . . . . . . . . . . . . . . . . . p. 239

A.10 Espaços de Funções . . . . . . . . . . . . . . . . . . . . . . p. 240

Espaço de Lebesgue $\mathcal{L}^{2} \ldots \ldots$. . . . . . . . . . p. 240

Bases Ortonormais . . . . . . . . . . . p. 241

A.11 Espaço $\mathcal{L}_{w}^{2}$ e Integral de Lebesgue . . . . . . . . . . . . . . . . . . . p. 241

A.12 Isomorfismos em Diversos Tipos de Espaços . . . . . . . . . . . . . p. 243

Isomorfismos em Espaços Vetoriais . . . . . . . . p. 243

Isomorfismos em Espaços Métricos . . . . . . . . . . p. 244

Isomorfismos em Espaços Normados . . . . . . . . . . . p. 244

Isomorfismos em Espaços de Hilbert . . . . . . . . . . . . p. 244 
A.13 Variedades Diferenciáveis . . . . . . . . . . . . . . . . . p. 244

A.14 Definição Algébrica de Corpo . . . . . . . . . . . . . . . p. 245

Apêndice B - Notação de Dirac p. 247

B.1 Definição de Kets, Bras e Operadores . . . . . . . . . . . . . . p. 247

B.2 Produto Interno . . . . . . . . . . . . . . . . . . p. 249

B.3 Álgebra de Operadores . . . . . . . . . . . . . . . . . p. 251

B.4 Autovalores e Autoestados . . . . . . . . . . . . . . . . p. p. 253

B.5 Axioma da Associatividade do Produto . . . . . . . . . . p. 255

B.6 Processo de ortonormalização de Gram-Schmidt para vetores de estado . . . . . . . . . . . . . . p. 256

B.7 Extensão a Espaços de Funções . . . . . . . . . . . . . . . . p. 257

Produto Interno . . . . . . . . . . . . . p. . 257

Bases . . . . . . . . . . . . . . . p. 257

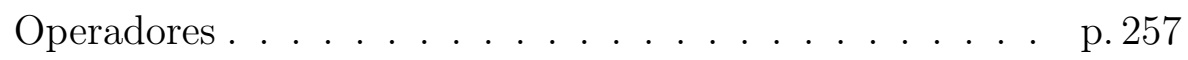

$\begin{array}{lr}\text { Apêndice C - Matrizes Operacionais } & \text { p. } 259\end{array}$

Apêndice D - Panorama Histórico do Cálculo Fracional p. 261

Funções de Mittag-Leffler . . . . . . . . . . . . p. p. 265

Apêndice E - Funções Especiais do Cálculo Fracional p. 267

E.1 Função Gama . . . . . . . . . . . . . . . . . . . . . p. 267

Representação Integral . . . . . . . . . . . . . p. 267

Extensão Analítica da Função Gama . . . . . . . . . . p. 268

Duplos Fatoriais . . . . . . . . . . . . p. 269

Valores Não-inteiros . . . . . . . . . . . . . . p. 270

Relação com as Transformadas de Laplace . . . . . . . p. 272

Expressão de Gauss . . . . . . . . . . . . . . . . p. 272 
Expressão de Euler . . . . . . . . . . . . . . . p. 274

Expressão de Weierstrass . . . . . . . . . . . p. 275

E.2 Fórmula Assintótica de Stirling . . . . . . . . . . . . . p. 276

E.3 Funções Gama Incompletas ． . . . . . . . . . . . . . . . . . p. 277

E.4 Relação entre as Funções Gama e Logarítmica . . . . . . . . . . . . . . p. 279

E.5 Funções Psi . . . . . . . . . . . . . . . . . . . . . . . . . . . . . . . p. 280

Função Digama . . . . . . . . . . . . . . . . . . p. 280

Propriedades . . . . . . . . . . . . . . . p. 280

Funções Psi Generalizadas . . . . . . . . . . . . . . p. 282

E.6 Função Beta . . . . . . . . . . . . . . . . . . . . . . . . . . p. 282

Forma Padrão . . . . . . . . . . . . . . . . . p. 282

Segunda Forma . . . . . . . . . . . . . . . p. 283

Forma Trigonométrica . . . . . . . . . . p. 283

Forma Racional . . . . . . . . . . . . . . . . . p. p. 284

Funções Beta Incompletas . . . . . . . . . . . . . . . p. 284

E.7 Relação entre a Função Beta e a Função Gama . . . . . . . . . . . . . . p. 285

E.8 Fórmula de Duplicação de Legendre . . . . . . . . . . . . . . . . p. 285

Extensão Analítica da Função Beta . . . . . . . . . . p. 286

Extensão das Funções Beta Incompletas . . . . . . . . . p. 286

E.9 Extensão dos Coeficientes Binomiais _ . . . . . . . . . . . p. 287

E.10 Funções Binomiais . . . . . . . . . . . . . . . . . . . . p. 289

E.11 Relação de Stifel Modificada . . . . . . . . . . . . . . . . . . . . p. 294

E.12 Expressão Generalizada do Binômio . . . . . . . . . . . . . . . . p. 295

E.13 Função de Mittag-Leffler . . . . . . . . . . . . . . . . . . . p. 296

Apêndice F - Fundamentos do Cálculo Fracional p. 299

F.1 Fórmula de Cauchy para Integrações Sucessivas _ . . . . . . . . . . . p. 299 
F.2 Extensão a Ordens Fracionárias . . . . . . . . . . . . . . . p. 304

F.3 Abordagem Fracional por Transformadas de Laplace . . . . . . . . . . p. 304

F.4 Abordagem Fracional Complexa pela Integral de Cauchy Generalizada . p. 305

F.5 Integrais Fracionais de Ordem Arbitrária . . . . . . . . . . . p. 308

Propriedades da Integral Fracional de Riemann . . . . . . p. 309

F.6 Exemplos de Integração Fracional . . . . . . . . . . . . . . . . . . . . p.311

F.7 Derivada de uma Integral Fracional . . . . . . . . . . . . . . . . p. 314

Funções Piso, Teto e Dente de Serra . . . . . . . . . . . . p. 314

F.8 Derivadas Fracionais . . . . . . . . . . . . . . . . . p. 315

Derivada Fracional de Riemann-Liouville . . . . . . . p. 316

Derivada Fracional de Caputo . . . . . . . . . . . p.319

Relação entre DFR e DFCR . . . . . . . . . . . . . p. 321

Relação entre derivadas e integrais fracionais . . . . . . p. 323

F.9 Transformadas, Convoluções e Correlações . . . . . . . . . . . . . . p. 326

F.9.1 Transformadas de Fourier . . . . . . . . . . . . p. 326

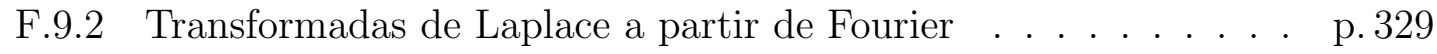

F.9.3 Convolução Complexa e Correlação Cruzada . . . . . . . . . . . p. 330

Produto de Convolução para a TL . . . . . . . . . . . p. 330

Produto de Convolução para a TF . . . . . . . . . . . . p. 331

Propriedades da Convolução . . . . . . . . . . . . . p. p. 331

Convolução Cíclica . . . . . . . . . . . . . . . p. p. 332

Correlação Cruzada . . . . . . . . . . . . . . . . . p. 333

F.10 Transformadas no Cálculo Fracional . . . . . . . . . . . . p. 333

TL da Integral Fracional de Riemann-Liouville . . . . . . p. 334

TL da Derivada Fracional de Riemann-Liouville . . . . . p. 335

TL da Derivada Fracional de Caputo . . . . . . . . . p. 336 
Cálculo das Transformadas de Laplace Inversas de Funções de Ordem Fracionária . . . . . . . . . . p. 337

TL da função de Mittag-Leffler de 3 Parâmetros . . . . . p. p.339

TF de Funções de Ordem Fracionária . . . . . . . . . . . p. 341

TF da IFL . . . . . . . . . . . . . . . . . . . . p. . 341

TF da DFL e DFCL . . . . . . . . . . . . . . p. 342

Apêndice G - Demonstração da Fórmula de Reflexão de Euler p. 345 


\section{INTRODUÇÃ O}

\subsection{Apresentação do Tema}

O tema da tese consiste na ampliação do uso efetivo das matrizes operacionais para casos mais genéricos, como o de funções periódicas estendidas ao campo complexo e ao cálculo fracionário utilizando, mais especificamente, as séries complexas de Fourier.

Como se sabe, a resolução de determinadas equações diferenciais frequentemente demanda considerável esforço, sendo que algumas não chegam a apresentar solução analítica por funções elementares ou sequer por séries infinitas com relações de recorrência.

Assim, métodos alternativos têm sido propostos com a finalidade de estabelecer processos numéricos de resolução para os casos mais difíceis. Alguns, mais consagrados, como os de Runge-Kutta e Adams-Bashforth (preditor-corretor), são utilizados com frequência nos fenômenos envolvendo problemas de valor inicial (NOUGIER, 1983), sendo, inclusive, parte integrante de softwares específicos de cálculo, como Mathematica ${ }^{\circledR}$ e Matlab ${ }^{\circledR}$, embora não se apliquem a problemas com condições de contorno (BVP). O método das variações (LARA; GADELLA, 2008; ZHU J.Q.; SHU; DUMBSER, 2008) e o de Galerkin (KARR; YAKUSHIN; NICOLOSI, 2000; BRENNER; SCOTT, 2008; ERN; GUERMOND, 2013) também são usados amiúde em soluções numéricas.

Há, entretanto, outros processos, como o de aproximações por famílias ortonormais de funções, que ainda não são utilizados de maneira suficientemente ampla. Ademais, têm sido implementados métodos mais modernos, como os que se utilizam de algoritmos genéticos. Com o crescente sucesso da computação evolutiva, outras vertentes têm se inserido nos tratamentos numéricos das equações diferenciais, como é o caso da determinação de matrizes operacionais no cálculo fracionário (CHALISHAJAR; KANTAWALA, 2008; KHADER, 2011). 


\subsection{Objetivos}

Os objetivos essenciais do trabalho são expostos, resumida e esquematicamente, a seguir:

- Reelaborar a apresentação teórica das matrizes operacionais no formalismo bra-ket de Dirac para englobar variáveis complexas em bases genéricas (i.e., não necessariamente ortogonais) com a finalidade de utilizar esse formalismo, de natureza tensorial, na resolução de equações diferenciais;

- Desenvolver as formas explícitas das matrizes operacionais clássicas de derivação e integração complexas de Fourier;

- Expandir o desenvolvimento anterior às matrizes operacionais de derivação e integração complexas em bases genéricas a ordens arbitrárias;

- Resolver equações diferenciais ordinárias lineares não-homogêneas com alto grau de precisão através do método operacional complexo de Fourier desenvolvido no trabalho, com vistas a uma extensão futura a EDOs não-lineares e EDPs;

- Aperfeiçoar o método elaborado para resolver equações diferenciais ordinárias nãohomogêneas de ordem arbitrária, i.e., as chamadas EDFs não-homogêneas, a fim de resolver problemas práticos;

- Aplicar os resultados obtidos em casos práticos, tais como a EDF de Bagley-Torvik e a EDF unidimensional de calor com presença de fonte.

\subsection{Motivação e Considerações Analíticas}

Nesta seção enumeramos as vantagens de cada uma das abordagens utilizadas no desenvolvimento do trabalho, ou seja, discutimos os motivos que conduzem à implementação da técnica das matrizes operacionais com a notação de Dirac para as séries complexas de Fourier e sua extensão às equações diferenciais de ordem arbitrária.

\subsubsection{Uso de Matrizes Operacionais}

De fato, as aproximações por funções ortogonais têm desempenhado um papel vital na Física e na Engenharia, além de fornecer contribuições ao desenvolvimento tecnológico e, 
de modo particular, à evolução da Análise Matemática. Entretanto, embora essa abordagem não seja recente, sua importância vem crescendo de maneira significativa nas últimas três décadas (RAZZAGHI; YOUSEFI, 2001; ELBARBARY, 2002; KHELLAT; YOUSEFI, 2006; DOHA; BHRAWY, 2008), à medida em que vem assumindo um papel fundamental na elaboração de novas técnicas, principalmente relacionadas à resolução de problemas de análise e de controle, por exemplo.

As técnicas envolvidas nessa abordagem tem visado construir algoritmos efetivos e adequados à utilização em problemas variacionais e na computação evolutiva.

A motivação subjacente a esse método reside no fato de ele tornar possível transformar equações diferenciais implícitas de um determinado problema em sistemas de equações algébricas, simplificando consideravelmente o processo de resolução, uma vez que a forma matricial é facilmente implementada na computação. Em outras palavras, um problema elaborado através do tratamento diferencial usual passa a ser descrito por um sistema de equações lineares, o que se ajusta à resolução computacional por processos numéricos.

O método encontra sua formalização na linguagem da álgebra linear, com a utilização de operadores lineares em forma de matrizes representativas dos processos de derivação e integração, denominadas matrizes operacionais. A resolução de equações diferenciais através do uso de tais matrizes operacionais tem sido implementada por alguns autores, em bases de polinômios ortogonais. Já foram determinadas matrizes de integração para as funções de Bessel (PARASKEVOPOULOS; SKLAVOUNOS; GEORGIOU, 1990), para as séries de Fourier trigonométricas (PARASKEVOPOULOS; SPARIS; MOUROUTSOS, 1985; RAZZAGHI; RAZZAGHI, 1988) e Laguerre (KING; PARASKEVOPOULOS, 1979; HWANG; SHIH, 1982) e para os polinômios de Chebyshev (PARASKEVOPOUlOS, 1983; HORNG; CHOU, 1985), Legendre (CHANG; WANG, 1982; PARASKEVOPOUlOS, 1985), Bernstein (SINGH; SINGH; SINGH, 2009) e Walsh (CHEN; HSIAO, 1975; CHEN; TSAY; WU, 1977). Também foram estabelecidas as matrizes operacionais de Kronecker (KILICMAN; ZHOUR, 2007), com base no produto de convolução de Kronecker.

Recentemente, Guimarães (2008) aliou a esse processo a computação evolutiva, desenvolvendo um tipo de algoritmo genético espectral que possibilitou a resolução de equações diferenciais não-lineares com séries de alta ordem. Além disso, Guimarães, Piqueira e Lobo Netto (2010) deduziram uma forma de se obter todas as matrizes operacionais de bases polinomiais pelo Teorema da Matriz Sanduíche.

Extensões das matrizes operacionais ao campo do cálculo fracional têm sido realizadas nos últimos anos como um recurso adicional e poderoso na abordagem de uma série cada 
vez mais ampla de problemas de Engenharia, Física, Química, Matemática, Computação, Biologia e até Economia.

\subsubsection{Formalismo bra-ket}

Entre as vantagens da abordagem pelo formalismo bra-ket de Dirac, podemos citar, antes de tudo, seu grande poder de concisão. De fato, as expressões matemáticas envolvidas no desenvolvimento da teoria assumem, em sua maior parte, formas muito mais sintéticas e elegantes.

Mas não é apenas o aspecto estético ou de apresentação que ganha com isso. Devido à clareza dessa linguagem no que diz respeito aos conceitos de espaço original e dual, derivados da álgebra linear, e à sua evidente adaptação aos espaços de Hilbert, seu uso parece ideal no tratamento de fenômenos inseridos nesses espaços. Consequentemente, consegue-se, além de maior clareza na apresentação das equações, também um melhor entendimento da teoria, como um todo.

Além disso, advém ainda uma importante consequência de seu uso, a saber, maior facilidade no desenvolvimento de tópicos avançados devido à perfeita adequação da linguagem.

\subsubsection{Método Operacional Complexo de Fourier}

Podemos citar também vantagens importantes relacionadas à abordagem complexa de Fourier. Em primeiro lugar, vem a necessidade de um tratamento mais adequado das funções periódicas, cujo comportamento se adapta muito melhor às bases de Fourier, o que deve traduzir-se em notáveis ganhos, ou seja, resultados mais precisos, com menor custo computacional, na resolução de equações diferenciais com soluções periódicas.

Ademais, há o evidente enriquecimento teórico advindo da expansão do método ao corpo complexo, cujos desdobramentos deverão ser estudados de forma cuidadosa posteriormente, à medida em que apareçam novos resultados no processo de reolução de EDOs por esse método.

Ressaltamos que a expansão dessas técnicas de resolução de equações diferenciais, fracionárias ou não, deverá acarretar em um significativo aumento de aplicações práticas e uso efetivo em diversas áreas de interesse na engenharia. 


\subsubsection{Abordadem do Cálculo Diferencial e Integral de Ordem Arbitrária}

Apesar de ainda não ser um assunto dos mais difundidos, o cálculo fracional apresenta algumas vantagens na abordagem de determinados fenômenos, especialmente na área de polímeros, em problemas biofísicos e em Termodinâmica (HILFER et al., 2000). Recentemente, vem encontrando maior variedade de aplicações práticas, como em controle nãolinear (MACHADO, 2011), sistemas difusivos e meios viscoelásticos (SABATIER; AGRAWAL; MACHADO, 2007), evolução temporal (HILFER et al., 2000) e até sistemas elétricos (OLIVEIRA; MAINARDI; VAZ JUNIOR, 2011), também encontrando uso em Mecânica Quântica (LASKIn, 2000b; OLIVEIRA; COSTA; VAZ JUnior, 2010), Física de Partículas e Teoria do Caos (LASKIN, 2000a; ZASLAVSKY, 2002), já tendo sido estabelecidos, inclusive, os fundamentos de uma dinâmica fracional de sistemas caóticos hamiltonianos (ZASLAVSKY, 2008).

Existe uma clara vantagem no uso da teoria das equações diferenciais de ordem abitrária, pois ela se presta a um duplo papel, já que os processos de resolução podem ser usados tanto em equações diferenciais fracionárias obtidas diretamente na formulação de determinados problemas, como através de transformações em algumas equações de ordem inteira para torná-las fracionárias, conduzindo a métodos alternativos de resolução. Isso proporciona uma ferramenta a mais nas técnicas de resolução de equações diferenciais de ordem inteira.

\subsection{Estado da Arte}

A utilização de matrizes operacionais na base de Fourier, ao que pudemos constatar, só foi implementada para a série real, não tendo sido encontrado nenhum exemplo desse uso para a base complexa. Os artigos relativos a essas formalizações são especificados e resumidos nesta seção.

Já no caso das matrizes operacionais no cálculo fracionário, embora a abordagem esteja sendo efetivada (CHALISHAJAR; KANTAWALA, 2008; KHADER, 2011), não encontramos referências sobre seu uso na base complexa de Fourier na solução de problemas desse tipo. No entanto, acreditamos que sua implementação para qualquer tipo de equação diferencial ordinária, homogênea ou não-homogênea, linear ou não-linear, com vistas a uma utilização racional e efetiva em funções periódicas a valores complexos seja altamente desejável, pois uma ampla quantidade de fenômenos físicos apresenta características 
periódicas e uma descrição matemática nesses termos, aliada a um tratamento complexo, se ajustaria consideravelmente melhor a tais fenômenos, ampliando o escopo e aplicabilidade do método. Acreditamos que essa formulação mais ampla proporcione efetivo ganho nas aplicações. Além disso, um evidente vínculo com a teoria das wavelets parece sugerir um caminho bastante promissor.

A respeito do uso de matrizes operacionais na base de Fourier, as fontes são escassas e nenhuma delas se refere à base complexa, utilizando-se somente das séries de Fourier reais. Vamos citar detalhadamente o que conseguimos encontrar sobre o assunto:

O artigo mais antigo encontrado sobre matrizes operacionais de Fourier é devido a Paraskevopoulos, Sparis e Mouroutsos (1985), onde se determina uma expressão geral para a matriz operacional de integração de Fourier em base real. Em Razzaghi e Razzaghi (1988) é apresentado um método direto para resolver problemas variacionais usando séries de Fourier, utilizando a definição da matriz operacional de integração de Fourier do artigo anterior. Endow (1989) fornece as equações de estado no problema de um regulador ótimo em termos das séries truncadas de Fourier e das MOIs associadas e esenvolve um algoritmo computacional para calcular os coeficientes de expansão das derivadas das variáveis de estado. Obs: Um outro artigo de 2005, de B.M. Mohan, listado abaixo, indica uma incorreção deste artigo, revisando-o.

Caiti e Cannata (1993) analisam e comentam as propriedades das matrizes operacionais de derivação e integração para as funções trigonométricas de Fourier. Razzaghi, Arabshahi e Lin (1995) discutem um método para identificação de parâmetros de EDOs não-lineares via séries de Fourier. A MOI de integração é usada também na versão para integração repetida para reduzir problemas de identificação a sistemas algébricos de resolução. Em Mohan e Kar (2005) mostra-se que a abordagem das séries de Fourier para o problema de controle ótimo no artigo de Endow está incorreta. Ebrahimi et al. (2007) desenvolvem um algoritmo computacional direto para estimativa de controle ótimo e trajetória de sistemas lineares singulares e expandem as variáveis de estado e o vetor de controle na base de Fourier real com coeficientes desconhecidos. Toutounian, Tohidi e Kilicman (2013) introduzem as matrizes operacionais de Fourier de derivação e de transmissão para resolver EDOs de alta ordem com coeficientes constantes e estendem o método para equações de pantógrafo generalizadas (tipo de ED de delay) com coeficientes variáveis usando nodos de colocação de Legendre-Gauss.

Na pesquisa bibliográfica, localizamos diversos artigos referentes à aplicação de $m a-$ trizes operacionais ao cálculo fracional, principalmente referentes aos últimos dois anos. 
A seguir, mostramos um resumo dessas referências.

Existe um ótimo apanhado geral (survey) do que havia sido realizado neste assunto até então, elaborado por Luchko (1999). Após a publicação desse survey, Podlubny (2000) sugere uma forma de representação matricial para análogos discretos de várias formas de diferenciação e integração fracionais. A intenção é implementar uma unificação entre a diferenciação numérica de ordem inteira e integrações repetidas usando um tipo específico de matrizes triangulares (triangular strip matrices), referidas em Bulgakov (1954) e Suprunenko e Tyshkevich (1966). Aplicada a soluções numéricas de EDOs, a abordagem unifica também a solução de equações diferenciais de ordem inteira ou fracionária com a das equações integrais fracionárias, conseguindo uma significativa simplificação.

No artigo já mencionado de Chalishajar e Kantawala (2008), os autores utilizam matrizes operacionais em bases de Walsh para resolver o problema de sistemas distribuídos no cálculo fracional e comparar os resultados com os obtidos por funções ortogonais em geral, especialmente, com os do método numérico de Wu e Chen (2004) para resolução de equações diferenciais fracionárias.

Khader (2011) generaliza a matriz operacional de Legendre para derivadas de ordem fracionária a fim de resolver equações diferenciais fracionárias não-lineares usando a definição da derivada fracional de Caputo. Compara o método com o ADM (Adomian Decomposition Method) (ADOMIAN, 1988).

Maleknejad, Basirat e Hashemizadeh (2011?) propõem um método aproximativo de resolução das equações integrais de Hammerstein através das matrizes operacionais de Bernstein. Em Rahmani, Rahimi e Mordad (2011) são usadas funções Block-Pulse e suas matrizes operacionais na solução da equação integro-diferencial de Volterra-Fredholm. Já Loghmani (2012) a soluciona por meio do uso das funções cardinais de Chebyshev. Ainda com relação a essa mesma equação, Maleknejad, Basirat e Hashemizadeh (2012) apresentam uma solução numérica de alta ordem via matrizes operacionais de Bernstein. Wang et al. (2012) apresentam um algoritmo (OPM) de simulação no domínio do tempo baseado em matrizes operacionais para a abordagem de sistemas de EDOs, EDFs, equações diferenciais algébricas e de alta ordem, com atuação similar a métodos de análise transiente avançada, como os métodos de Gear e trapezoidal.

Kazem, Abbasbandy e Kumar (2013) propõem funções de Legendre de ordem fracional na solução de EDFs. Baleanu, Alipour e Jafari (2013) obtêm uma solução analítica aproximada da equação diferencial quadrática de Riccati com derivadas fracionais de Riemann-Liouville usando as matrizes operacionais de Bernstein. Nesse mesmo ano são 
estabelecidas as matrizes operacionais de integração fracional dos polinômios transladados (shifted) de Legendre (ERJAEE, 2013) e Chebyshev (BHRAWY; ALOFI, 2013). Em 2014, Labecca, Guimarães e Piqueira (2014) determinam as matrizes operacionais de derivação e integração da base complexa de Fourier.

\subsection{Conclusão da Apresentação}

Tendo constatado, portanto, a grande variedade de utilizações de todas essas técnicas e a ausência de unificação e de um desenvolvimento apropriado do assunto, propomos neste trabalho uma sistematização e uma ampliação desse formalismo linear operacional na linguagem bra-ket para o cálculo fracional a variáveis complexas, com base nas definições integrais de Riemann-Liouville, Caputo e Weyl, enfatizando as séries de Fourier complexas e as transformadas de Fourier e Laplace, tendo como principal objetivo o direcionamento à solução de problemas práticos específicos passíveis de formulação por meio de equações diferenciais, com aplicação, em especial, na Engenharia e na Física.

Adicionalmente, procederíamos à sondagem de possíveis interpretações físicas das condições inerentes às transformadas de Laplace e de Fourier das diferintegrais, cuja esfera de ação prática pode se estender de forma bastante promissora. Com isso, acreditamos estar contribuindo para ampliar de modo significativo as técnicas numéricas de resolução das EDOs (com posterior extensão às EDPs), o que possibilitaria abordagens práticas mais poderosas em diversas áreas de grande importância hoje, como fluidodinâmica, viscoelasticidade, caos e automação e controle, por exemplo. 


\section{FUNDAMENTAÇ̃̃O TEÓRICA}

Iniciamos este capítulo pelo desenvolvimento formal da extensão da notação de Dirac para uso efetivo na teoria geral de equações diferenciais e, mais especificamente, à sua aplicação no método das matrizes operacionais. Nossa meta é desenvolver um método tensorial nessa linguagem com aplicações específicas na teoria de equações diferenciais.

Vamos começar com algumas definições importantes e convenções de notação.

\subsection{Formalismo Tensorial na Linguagem Bra-Ket}

\subsubsection{Definições e Notação}

Sistemas e Eixos Em se tratando de vetores comuns, isto é, inseridos no espaço euclidiano tridimensional $\mathbb{R}^{3}$, é intuitiva a compreensão de alguns conceitos relacionados a aspectos geométricos que emergem naturalmente. Um deles se refere ao sistema de eixos. Em um espaço físico tridimensional usual existe a necessidade de se determinar pelo menos três vetores linearmente independentes que atuem como base. Normalmente são eleitos vetores perpendiculares entre si, o que constitui um sistema ortogonal. Entretanto, essa condição não é obrigatória e, eventualmente, pode ser definida uma base não-ortogonal ou, como também é conhecida, um sistema de eixos oblíquos.

Em um sistema de eixos oblíquos não há apenas uma única maneira de determinarmos as componentes de um vetor, mas duas. Para um sistema de eixos cartesianos, isto é, ortogonais entre si, as componentes de um vetor são definidas pelas projeções ortogonais a cada eixo. Ao somarmos vetorialmente essas projeções reobtemos o vetor projetado. Embora isso seja óbvio num sistema cartesiano, o mesmo não ocorre num sistema nãoortogonal.

Portanto, visando-se a possibilidade de utilizar sistemas oblíquos, surge a necessidade 
de estabelecer critérios semelhantes aos consagrados no formalismo tensorial. Um deles diz respeito às notações dos diferentes tipos de coordenadas.

Coordenadas Covariantes e Contravariantes No caso de procedimento semelhante ao descrito acima, mas efetuado agora em um sistema oblíquo, as projeções obtidas, denominadas coordenadas covariantes, são tais que, ao serem vetorialmente somadas, o resultado dessa operação não fornece o vetor projetado. Define-se, então, outro tipo de coordenadas, chamadas de coordenadas contravariantes, com diferente regra de formação, obtidas pelas projeções paralelas aos eixos, produzindo novas componentes cuja soma vetorial resulte no vetor original.

Há, por conseguinte, duas projeções possíveis: a paralela e a ortogonal. Cada uma delas fornece componentes de natureza diversa, que recebem, portanto, denominações e notações distintas, as quais adotaremos, em conformidade com as convenções utilizadas no cálculo tensorial e na geometria diferencial. Em suma:

- Projeções paralelas fornecem coordenadas contravariantes, denotadas por índices sobrescritos: $u^{i}$;

- Projeções ortogonais fornecem coordenadas covariantes, denotadas por índices subscritos: $u_{i}$.

Na figura 1 são mostradas todas as coordenadas referidas acima para dois sistemas bidimensionais distintos: um cartesiano $\left\{\hat{e}_{1}, \hat{e}_{2}\right\}$ e um oblíquo $\left\{\hat{e}_{1}^{\prime}, \hat{e}_{2}^{\prime}\right\}$, nos quais as coordenadas covariantes aparecem como $\left(u_{1}, u_{2}\right)$ e $\left(\dot{u}_{1}, \dot{u}_{2}\right)$, respectivamente, e as contravariantes como $\left(u^{1}, u^{2}\right)$ e $\left(\dot{u}^{1}, \hat{u}^{2}\right)$.

Existe uma relação explícita entre as coordenadas covariantes e contravariantes, a ser deduzida posteriormente, obtida pela noção de funcionais lineares e expressa através do tensor métrico.

Descrição Geométrica Evidentemente, os conceitos discutidos nesta seção podem ser estendidos do espaço físico comum a espaços vetoriais mais gerais, desde que possuam produto interno definido. Isso acontece porque as noções de perpendicularidade e paralelismo fazem sentido em espaços IP, como é o caso dos espaços de Hilbert. Graças a isso, a formulação tradicional do cálculo tensorial pode ser estendido para um espaço de funções.

Ao tratarmos de espaços vetoriais de dimensão finita, como é o caso do espaço de ordem $\mathcal{O}_{n}$, definido adiante, é possível interpretar uma base como um sistema de co- 
Figura 1: Sistema de eixos oblíquos

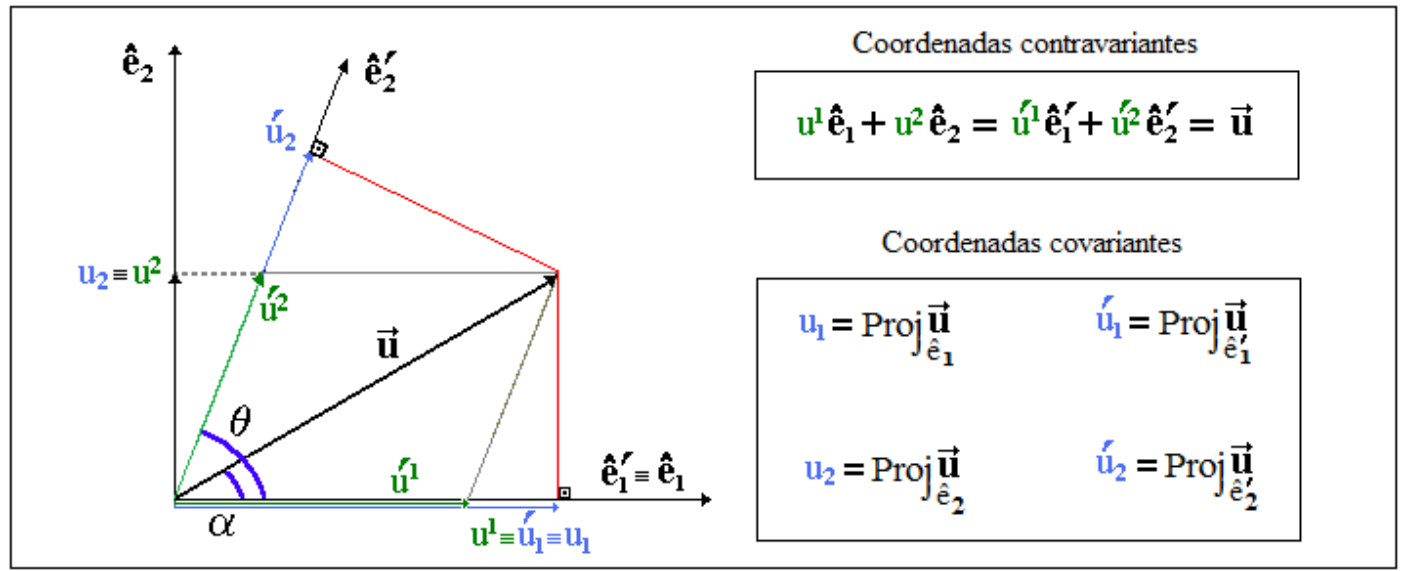

ordenadas num espaço $n$-dimensional, o que permite adotar uma descrição de natureza geométrica. Isso torna o tratamento mais intuitivo, além de fornecer elementos para um desenvolvimento formal afim ao da geometria diferencial.

Como o espaço de funções $\mathcal{L}^{2}$ é um espaço de Hilbert, a equivalência entre kets de $\mathcal{O}_{n}$ e vetores, entendidos como entes espaciais n-dimensionais, possibilita, pelo que foi dito acima, uma interpretação geométrica de caráter simbólico para os kets e, consequentemente, um tratamento tensorial adequado em notação de Dirac.

\subsubsection{Formalismo no Espaço de Ordem}

A primeira ideia a ser esclarecida diz respeito ao conceito de ket. Uma vez que este incorpora o significado de vetor, gozando das mesmas propriedades, é possível usá-lo não apenas em espaços de Hilbert, mas também em espaços de dimensão finita. Com isso, é possível implementar-se uma notação comum a qualquer tipo de espaço, com o intuito de unificar os tratamentos em uma abordagem apropriada à teoria das equações diferenciais. Esta é a motivação por trás da extensão do formalismo.

Antes, porém, de prosseguir neste caminho, é preciso especificar com rigor alguns conceitos fundamentais de suma importância, ainda que não-triviais, cuja compreensão e tratamento afastam-se um pouco do modus operandi tradicional.

O primeiro ponto diz respeito aos espaços de atuação de cada operação definida nesse formalismo estendido. Não é óbvio, por exemplo, que quaisquer operações definidas em um espaço discreto e finito possam ser efetuadas também num espaço contínuo infinito da mesma maneira e que sejam intercambiáveis entre eles. Para esclarecer este ponto, vamos discutir os espaços de atuação. 
Outro ponto significativo se refere à representação mais adequada de cada espaço em termos operacionais e à escolha da melhor abordagem do ponto de vista efetivo do cálculo. Essa discussão será efetuada na seção das representações isomórficas.

Iniciemos, portanto, pelos conceitos envolvidos nos espaços de atuação.

\subsubsection{Determinação dos Espaços de Atuação}

As operações envolvendo expressões de funções expandidas em série ocorrem em dois espaços distintos:

- O espaço de Hilbert $\mathcal{H}$, de dimensão infinita;

- O espaço de dimensão finita $n$, gerado na expansão em série de $n$ termos da função considerada.

Assim, trabalharemos constantemente em espaços de natureza diversa, o Espaço de Hilbert $\mathcal{H}$ e o outro referido acima, denotado por $\mathcal{O}_{n}$, que designaremos por Espaço de Ordem $n$ ou, simplesmente, por Espaço de Ordem, subentendendo-se, implicitamente, o $n$ que representa o número de termos da expansão. Adiantamos que as matrizes operacionais geradas em $\mathcal{O}_{n}$ pertencem a $\mathbb{M}_{n}(\mathbb{C})$.

Vale lembrar que $\mathcal{O}_{n}$ pode ser estendido a um espaço enumerável de dimensão infinita, o que será feito na seção referente a expansões de séries infinitas.

Além disso, pode-se especificar o espaço de ordem de acordo com a natureza de seus elementos: se estes pertencerem a um espaço vetorial $\mathrm{V}$, denotaremos o espaço assim constituído por $\mathcal{O}_{n}(\mathrm{~V})$.

Desse modo, se seus elementos fizerem parte do espaço de Hilbert, denotaremos esse espaço por $\mathcal{O}_{n}(\mathcal{H})$, ao passo que, se pertencerem ao corpo complexo, lembrando que todo corpo é um espaço vetorial sobre si mesmo, a notação será $\mathcal{O}_{n}(\mathbb{C})$. Todavia, para este trabalho uma definição um pouco mais específica e rigorosa é desejável.

Definição 1 : Dados um intervalo fechado $I=[a, b] \subset \mathbb{R}$ e uma função limitada $f: I \rightarrow \mathbb{C} \mid f \in \mathcal{C}^{0}[a, b]$, define-se o espaço de ordem $\mathcal{O}_{n}(\mathcal{H})$ como o espaço de dimensão n finita gerado pelas funções componentes do desenvolvimento em série, com $n$ termos, da função $f$ no intervalo $I$.

Esse espaço é, obviamente, um espaço vetorial de dimensão $n$, visto que é gerado por um conjunto de funções $\mathcal{L}^{2}$, geralmente ortogonais, mas não necessariamente, que 
obedecem às condições de espaço vetorial.

Em princípio, conforme já foi dito, não há por que os dois espaços, $\mathcal{H}$ e $\mathcal{O}_{n}$, "dialogarem" entre si, isto é, nada garante que as definições estabelecidas em um sejam semelhantes às do outro e nem se configura evidente o fato de que possam existir operações híbridas entre eles.

Por esse motivo, é sumamente importante discriminar com precisão em qual dos espaços determinadas operações estão sendo efetuadas e quais suas propriedades, definições e condições de existência, para que a exposição do método proposto não fique comprometida.

As principais operações entre os elementos de um espaço vetorial $\mathrm{V}$ sobre um corpo $\mathbb{K}$, como se sabe, são o produto de um escalar desse corpo por um vetor de $\mathrm{V}$ e o produto interno entre dois elementos de $\mathrm{V}$. Porém, também existem outras possibilidades, como, por exemplo, o produto vetorial, no caso de um espaço $\mathbb{R}^{3}$, ou outras mais amplas, como as relacionadas às definições de produtos externos, tensoriais ou diádicos, os quais, no entanto, serão abordados neste trabalho apenas de forma introdutória, na medida da necessidade.

As duas primeiras operações citadas acima são aplicações com correspondências tais que:

$$
\begin{array}{ll}
\mathbb{C} \times \mathcal{H} \longrightarrow \mathcal{H} & \mathcal{H} \times \mathcal{H} \longrightarrow \mathbb{C} \\
\mathbb{C} \times \mathcal{O}_{n}(\mathbb{C}) \longrightarrow \mathcal{O}_{n}(\mathbb{C}) & \mathcal{O}_{n}(\mathbb{C}) \times \mathcal{O}_{n}(\mathbb{C}) \longrightarrow \mathbb{C} \\
\mathbb{C} \times \mathcal{O}_{n}(\mathcal{H}) \longrightarrow \mathcal{O}_{n}(\mathcal{H}) & \mathcal{O}_{n}(\mathcal{H}) \times \mathcal{O}_{n}(\mathcal{H}) \longrightarrow \mathbb{C}
\end{array}
$$

Entretanto, também é possível definirmos aplicações mistas, isto é, que relacionem os dois espaços de naturezas diferentes, de modo que tenhamos:

$$
\mathcal{H} \times \mathcal{O}_{n}(\mathbb{C}) \longrightarrow \mathcal{O}_{n}(\mathcal{H}) \quad \mathcal{H} \times \mathcal{O}_{n}(\mathcal{H}) \longrightarrow \mathcal{O}_{n}(\mathbb{C})
$$

Denotaremos os kets, respectivamente, no espaço de Hilbert e no espaço de ordem, por:

$$
|\rangle_{\infty} \in \mathcal{H}, \quad|\rangle_{0_{n}} \in \mathcal{O}_{n}
$$

e, analogamente, para os bras:

$$
{ }_{\infty}\langle| \in \mathcal{H}, \quad{ }_{0_{n}}\langle| \in \mathcal{O}_{n}
$$


Dessa forma, uma função $\phi_{i}(x)$, componente de uma base numa expansão em série, pode ser grafada $\left|\phi_{i}\right\rangle_{\infty}$ e um vetor $u \in \mathcal{O}_{n}(\mathbb{C})$, com $n$ coordenadas $u_{i} \in \mathbb{C}$, como $|u\rangle_{0_{n}}$. Observe que também é possível representarmos vetores $\varphi \in \mathcal{O}_{n}(\mathcal{H})$ com componentes $\varphi_{i}(x)$ no espaço de Hilbert por $|\varphi\rangle_{0_{n}}$. Formalmente, teríamos que denotar esses dois últimos vetores por $|u\rangle_{0_{n}(\mathrm{C})} \mathrm{e}|\varphi\rangle_{0_{n}(\mathcal{H})}$, respectivamente, mas isso sobrecarregaria indevidamente a notação.

Para efeitos práticos, a notação inferior referente ao espaço de atuação pode até mesmo ser suprimida, desde que os conceitos estejam claros e não surjam ambiguidades. No entanto, nos casos em que a necessidade de clareza assim o exija, utilizaremos a notação completa.

Existe também a possibilidade de lidarmos com kets ou bras cujas componentes estejam em $\mathbb{C}$, o que induziria à notação $\left|A_{i}\right\rangle_{\mathbb{C}}$ para todo $A_{i} \in \mathbb{C}$, a qual será dispensada, por tratar-se apenas de um escalar complexo, cuja operacionalidade não requer este tipo de tratamento.

É digno de nota ainda que, no caso de bases ortonormais, pode ser adotada a prática usual de denotar-se um elemento de base de $\mathcal{O}_{n}(\mathcal{H})$ ou $\mathcal{O}_{n}(\mathbb{C})$ apenas pelo seu índice, ou seja, $\left|\phi_{i}\right\rangle_{\infty} \equiv|i\rangle_{\infty}$ e $\left|b_{i}\right\rangle_{0_{n}} \equiv|i\rangle_{0_{n}}$, uma vez que não há distinção entre as coordenadas covariantes e contravariantes.

\subsubsection{Espaços de Kets}

Um espaço de kets deve obedecer as propriedades de um espaço vetorial, quer dizer, os kets devem pertencer a um conjunto $\mathbb{V}$ munido de duas operações fundamentais: a adição $(+): \mathbb{V} \times \mathbb{V} \longrightarrow \mathbb{V}$ e a multiplicação por escalar $(\cdot): \mathbb{C} \times \mathbb{V} \longrightarrow \mathbb{V}$, de maneira que, para $\forall \lambda, \mu \in \mathbb{C}, \forall|\alpha\rangle,|\beta\rangle,|\gamma\rangle \in \mathbb{V}$, tenhamos:

Para a adição $(+)$ :

- $(A 0)$ fechamento: $|\alpha\rangle+|\beta\rangle \in \mathbb{V}$,

- $($ A1) comutatividade: $|\alpha\rangle+|\beta\rangle=|\beta\rangle+|\alpha\rangle$,

- $($ A2) associatividade : $(|\alpha\rangle+|\beta\rangle)+|\gamma\rangle=|\alpha\rangle+(|\beta\rangle+|\gamma\rangle)$,

- (A3) elemento neutro aditivo: $\exists|0\rangle \in \mathbb{V}:|\alpha\rangle+|0\rangle=|0\rangle+|\alpha\rangle=|\alpha\rangle$,

- $(A 4)$ elemento oposto: $\exists|-\alpha\rangle \in \mathbb{V}:|\alpha\rangle+|-\alpha\rangle=|-\alpha\rangle+|\alpha\rangle=|0\rangle$.

Para o produto por escalar $(\cdot)$ : 
- $(M 0)$ fechamento: $\lambda|\alpha\rangle \in \mathbb{V}$,

- $(M 1)$ distributividade à esquerda: $(\lambda+\mu)|\alpha\rangle=\lambda|\alpha\rangle+\mu|\alpha\rangle$,

- $(M 2)$ distributividade à direita : $\lambda(|\alpha\rangle+|\beta\rangle)=\lambda|\alpha\rangle+\lambda|\beta\rangle$,

- (M3) elemento neutro: $1|\alpha\rangle=|\alpha\rangle, 1=1+0 i \in \mathbb{C}$,

- $(M 4)$ associatividade : $\lambda(\mu|\alpha\rangle)=(\lambda \mu)|\alpha\rangle$.

Um espaço de kets obedecendo a essas condições tem a estrutura de um espaço vetorial complexo. Mas o espaço de ordem $\mathcal{O}_{n}(\mathbb{C})$ é um espaço vetorial de dimensão $n$, podendo, portanto, ser identificado com o conjunto de kets definido acima. O mesmo vale para $\mathcal{O}_{n}(\mathcal{H})$, o que significa que podemos expandir a noção de espaço vetorial a $\mathcal{O}_{n}(\mathrm{~V})$.

Naturalmente, uma vez que desejamos que as propriedades de espaços vetoriais sejam preservadas na mudança de notação de um espaço vetorial ordinário para o espaço de kets, devemos assumir que o mapeamento $\left.\Phi: \mathbb{V} \longrightarrow \mathcal{O}_{n}(\mathrm{~V})|\Phi(\alpha)=| \alpha\right\rangle$ seja um isomorfismo, isto é, seja bijetivo e satisfaça as condições: $\Phi(\alpha+\beta)=\Phi(\alpha)+\Phi(\beta), \Phi(\lambda \alpha)=\lambda \Phi(\alpha)$, ou seja, deve-se verificar que $|u+v\rangle=|u\rangle+|v\rangle$ e $|\lambda u\rangle=\lambda|u\rangle$.

Bases de Kets Seja $\mathcal{O}_{n}(\mathbb{C})$ um espaço de ordem $n$ sobre $\mathbb{C}$. Então, o conjunto de kets $E=\left\{\left|e_{1}\right\rangle, \ldots,\left|e_{n}\right\rangle\right\}$, grafado sinteticamente como $\left\{\left|e_{i}\right\rangle\right\}_{i=1}^{n}$, constitui uma base de $\mathcal{O}_{n}(\mathbb{C})$. Eventualmente, faremos menção a essa base denominando-a base original, em oposição à base do espaço dual, que será definida adiante. Deve-se notar que isso se aplica também para o caso de um um espaço de ordem atuando sobre um outro mais genérico, i.e., $\mathcal{O}_{n}(\mathrm{~V})$, o que nos permite estender o conceito a $\mathrm{V} \equiv \mathcal{H}$.

Decorre das propriedades de fechamento dos espaços vetoriais, $(A 0)$ e $(M 0)$, apresentadas no início dessa seção, que uma soma do tipo $\sum_{i}^{n} \alpha^{i}\left|e_{i}\right\rangle, \alpha_{i} \in \mathbb{C}$, pertence ao espaço $\mathcal{O}_{n}(\mathrm{~V})$, podendo, por conseguinte, ser escrito como um ket. Logo, qualquer elemento $|\alpha\rangle \in \mathcal{O}_{n}(\mathrm{~V})$ pode ser expresso em termos da base $E$ e de coeficientes $\alpha^{i} \in \mathbb{C}$ apropriados, como

$$
|\alpha\rangle=\sum_{i=1}^{n} \alpha^{i}\left|e_{i}\right\rangle
$$

Do ponto de vista prático, isso significa que a representação "natural" de um ket, que chamaremos preferencialmente de representação original, é aquela em que o ket é escrito em termos de suas coordenadas contravariantes $\alpha^{i}$. 


\subsubsection{Abordagem Linear}

Tendo em vista que o espaço de ordem $\mathcal{O}_{n}(\mathcal{H})$ é gerado pelos vetores de Hilbert constituintes de uma base de funções com dimensão finita idêntica ao número de termos da expansão em série, a abordagem linear através do uso de matrizes afigura-se como um caminho natural. Nesse sentido, adotaremos algumas convenções de notação baseadas no formalismo matricial linear.

Equivalência de Notações Seguindo a abordagem tradicional, assumiremos que qualquer ket pode, a priori, ser representado por uma matriz-coluna e vice-versa, quer dizer, qualquer representação vetorial em matriz-coluna pode ser entendida e denotada como um ket.

A mesma argumentação é válida para a relação entre um bra e uma matriz-linha, entendida aqui como um covetor, i.e., um elemento do espaço dual.

Assim, pode-se estabelecer uma equivalência básica de notações:

$$
|\rangle \sim[\vdots], \quad\langle| \sim[\cdots]
$$

\subsubsection{Kets e Bras em $\mathcal{O}_{n}$}

Conforme o que foi exposto, um ket em $\mathcal{O}_{n}(\mathbb{C})$, i.e., cujas componentes sejam escalares complexos, será representado por suas coordenadas contravariantes dispostas em um vetor-coluna, isto é, dada uma base $B$ qualquer, escreve-se a chamada representação coordenada como:

$$
|u\rangle_{0_{n},[B]} \simeq\left[\begin{array}{c}
u^{1} \\
\vdots \\
u^{n}
\end{array}\right]_{B}
$$

É importante não esquecer que a notação acima trata-se apenas de uma representação isomórfica para o ket em uma dada base, pois suas coordenadas variam de acordo com a base escolhida, quando, na realidade, todo ket deve ser invariante sob mudança de coordenadas.

De acordo com o que foi apresentado no parágrafo sobre "bases de kets", um ket $|u\rangle$ deve ser denotado como uma combinação dos kets constituintes $\left\{\left|b_{i}\right\rangle\right\}$ de uma base original $B$, através de uma série de constantes escalares complexas, que são suas coordenadas na base dada. Cada um destes kets componentes poderia ser, na verdade, tanto um escalar 
complexo $\lambda^{i} \in \mathbb{C}$, quanto uma função $\varphi_{i}(x)$, ou seja, um vetor no espaço de Hilbert, gerando, respectivamente, vetores em $\mathcal{O}_{n}(\mathbb{C})$ e $\mathcal{O}_{n}(\mathcal{H})$. Evidentemente, a dimensão do espaço considerado será especificada, em cada caso, pela natureza e extensão da base utilizada. Nesse último caso, por exemplo, se um ket nesse espaço apresentar coordenadas $\varphi_{i}(x) \in \mathcal{H}$, as quais também podem ser denotadas como kets, então sua notação deveria assumir a forma:

$$
|\varphi\rangle_{0_{n}} \simeq\left[\begin{array}{c}
\left|\varphi_{1}\right\rangle_{\infty} \\
\vdots \\
\left|\varphi_{n}\right\rangle_{\infty}
\end{array}\right]_{B}
$$

Será esclarecido mais tarde o porquê de a representação natural das funções $\mathcal{L}^{2}$ ser feita pelas coordenadas covariantes. Mas adiantando o que será exposto, o motivo está relacionado ao fato de estarmos lidando com bases de funções na expansão em série e à característica covariante das bases originais.

Voltando ao ponto referente à representação isomórfica, ressaltamos que é preciso compreender bem este pormenor, quer dizer, que tal notação nada mais é que uma representação baseada num isomorfismo para o que deve ser interpretado da seguinte forma:

$$
|u\rangle_{0_{n}(\mathrm{v})}=\sum_{i}^{n} u^{i}\left|b_{i}\right\rangle_{\mathrm{v}}
$$

onde os $\left|b_{i}\right\rangle$ são os vetores constituintes da base $B$. A esta forma de expressar o vetor, chamamos de representação invariante. Lembrando, ademais, que um escalar complexo não exige notação em ket, quer dizer, se $\mathrm{V} \equiv \mathbb{C}$, então não se grafa $\left|b_{i}\right\rangle_{\mathrm{C}}$ mas simplesmente $b_{i}$. Deve-se notar, por conseguinte, que, para uma outra base $F$, dada pelos vetores $\left\{\left|f_{i}\right\rangle\right\}_{i=1}^{n}$, teríamos $|u\rangle_{0_{n}(\mathrm{v}),[F]}=\sum_{i}^{n} v^{i}\left|f_{i}\right\rangle_{\mathrm{v}}$, o que nos leva ao ponto que queríamos esclarecer, ou seja, embora o ket $|u\rangle$ seja o mesmo, suas representações pelas coordenadas não são idênticas, i.e., $|u\rangle_{B} \neq|u\rangle_{F}$.

Consequentemente, em prol dessa mesma clareza, denotaremos, mas apenas quando se fizer necessário, os índices relativos às bases em que são feitas as representações. De forma geral, contudo, suprimiremos o excesso formalismo de notação, na esperança de que todas as sutilezas envolvidas sejam subentendidas pelo leitor, inclusive no que se refere aos espaços de atuação. Na prática, isso significa, por exemplo, que a representação por coordenadas do vetor $|u\rangle_{0_{n}(v),[B]}$, referido acima, será grafada simplesmente $|u\rangle_{[B]}$ ou até mesmo $|u\rangle$, se isso não ocasionar nenhuma confusão ou ambiguidade. 
Bras em $\mathcal{O}_{n} \quad$ De maneira análoga ao ket, um bra em $\mathcal{O}_{n}$, constituído de componentes escalares complexas (ou de elementos do espaço de Hilbert), terá uma representação coordenada em termos de suas componentes complexo-conjugadas dispostas em um vetorlinha:

$$
\left\langle\left. u\right|_{0_{n},[B]} \simeq\left[\begin{array}{lll}
\bar{u}^{1} & \cdots & \bar{u}^{n}
\end{array}\right]_{B}\right.
$$

A motivação a essa definição será melhor entendida posteriormente. Novamente, ressaltamos que, subentendidos os detalhes, poderemos grafar o bra acima apenas por $\left\langle\left. u\right|_{[B]},\left\langle\left. u\right|_{o_{n}}\right.\right.$ ou, simplesmente, $\langle u|$, de acordo com cada caso.

No caso específico de um vetor em $\mathcal{O}_{n}(\mathcal{H})$, a prática usual neste trabalho será grafar o bra na forma

$$
\left\langle\left.\varphi\right|_{0_{n},[B]} \simeq\left[\begin{array}{lll}
\left\langle\varphi_{1}\right| & \cdots & \left\langle\varphi_{n}\right|
\end{array}\right]_{B},\right.
$$

já que ele será obtido por adjunção do ket construído sobre a base original $\left|\varphi_{i}\right\rangle$. Esta questão será desenvolvida na próxima seção e seguintes.

\subsubsection{Operações de Transformação entre Bras e Kets}

Algumas operações podem ser realizadas sobre vetores assim definidos, como a conjugação, a transposição e a adjunção.

A conjugação ocorre do modo esperado, pois, como as operações são efetuadas em espaços complexos, faz sentido definirmos o complexo conjugado de vetores da maneira usual, isto é,

$$
\forall|\lambda\rangle \in \mathcal{O}_{n}(\mathbb{C}), \quad|\lambda\rangle^{*}=|\bar{\lambda}\rangle \simeq\left[\begin{array}{c}
\bar{\lambda}^{1} \\
\vdots \\
\bar{\lambda}^{n}
\end{array}\right], \quad \lambda^{1}, \cdots, \lambda^{n} \in \mathbb{C}
$$

Obs: Tanto o asterisco quanto a barra superior são notações usadas para a conjugação complexa no presente trabalho.

Entretanto, ao contrário do que ocorre no tratamento da mecânica quântica, por exemplo, a operação de conjugação de um ket no presente formalismo não é suficiente para gerar um bra. No caso de $\mathcal{O}_{n}$, faz-se necessário aplicar a transposição.

A operação de transposição diz respeito especificamente ao formalismo matricial, podendo também ser empregada a matrizes-linha e coluna, entendidas como representações de vetores e covetores. Um vetor "transforma-se" em um covetor pela operação de trans- 
posição e vice-versa, quer dizer,

$$
|\lambda\rangle^{\prime} \simeq\left[\begin{array}{c}
\lambda^{1} \\
\vdots \\
\lambda^{n}
\end{array}\right]^{T}=\left[\begin{array}{lll}
\lambda^{1} & \ldots & \lambda^{n}
\end{array}\right], \quad|\lambda\rangle^{\prime \prime} \simeq\left[\begin{array}{lll}
\lambda^{1} & \ldots & \lambda^{n}
\end{array}\right]^{T}=\left[\begin{array}{c}
\lambda^{1} \\
\vdots \\
\lambda^{n}
\end{array}\right]
$$

Como $\lambda^{i} \in \mathbb{C}$ e um escalar transposto é ele mesmo, i.e., $\left(\lambda^{i}\right)^{T}=\lambda^{i}$, basta mudar a disposição da matriz, de coluna para linha ou vice-versa. O mesmo vale para uma grandeza $\varphi(x) \in \mathcal{H}$. De fato, trata-se tão somente de uma variação de notação para a mesma grandeza física, cujo significado reside apenas na operacionalidade, embora esteja representado no espaço dual em vez de no original. Esse conceito será melhor explicado adiante. Obs: A notação da transposição poderá ser feita pela sobreposição da letra $T$ maiúscula, pela linha como índice superior ou por um til superposto à grandeza, indiferentemente.

Já a adjunção é uma conjunção de ambas as operações anteriores, ou seja, $M^{\dagger}=\bar{M}^{\prime}$ ou, em termos de matrizes-linha e coluna,

$$
|\lambda\rangle^{\dagger} \simeq\left[\begin{array}{c}
\lambda^{1} \\
\vdots \\
\lambda^{n}
\end{array}\right]^{\dagger}=\left[\begin{array}{lll}
\bar{\lambda}^{1} & \ldots & \bar{\lambda}^{n}
\end{array}\right] \simeq\left\langle\lambda\left|, \quad\left[\begin{array}{lll}
\lambda^{1} & \cdots & \lambda^{n}
\end{array}\right]^{\dagger}=\left[\begin{array}{c}
\bar{\lambda}^{1} \\
\vdots \\
\bar{\lambda}^{n}
\end{array}\right] \simeq\right| \bar{\lambda}\right\rangle
$$

Vê-se também, imediatamente, das regras de operação que $\left[\begin{array}{lll}\lambda^{1} & \cdots & \lambda^{n}\end{array}\right] \simeq\langle\bar{\lambda}|$.

Em suma, emerge aqui uma distinção importante entre o tratamento que estamos construindo e o procedimento usual. Nos espaços de Hilbert, como usados na mecânica quântica, obtemos um bra a partir da conjugação complexa de um ket, isto é, $\langle\alpha|=| \alpha\rangle^{*}$, enquanto no formalismo aqui desenvolvido para espaços de ordem, o bra é obtido por adjunção, i.e., $\left\langle\left.\alpha\right|_{0_{n}}=\mid \alpha\right\rangle_{0_{n}}^{\dagger}$. A conjugação em $\mathcal{O}_{n}$, por seu turno, não produz um bra, uma vez que a transposição torna-se uma condição necessária, devido ao isomorfismo estabelecido com a álgebra de matrizes. No entanto, para um escalar complexo ou um ket no espaço de Hilbert, i.e., $|\varphi\rangle_{\infty} \in \mathcal{H}$, a adjunção se reduz à conjugação, já que a transposição é inócua para esses elementos, mostrando que o resultado anterior constitui um caso particular da presente abordagem. Por essa razão fica justificada a definição adotada para o bra no parágrafo anterior, motivada por consistência matemática.

Mostraremos a seguir, de modo formal, que o espaço dos bras nada mais é que o conhecido espaço dual da álgebra linear. 


\subsubsection{Espaços Duais}

Se $\mathcal{V}$ é um espaço vetorial sobre $\mathbb{C}$ e $\mathcal{W}$ é um conjunto tal que

$$
\mathcal{W}=\{\varphi: \mathcal{V} \longrightarrow \mathbb{C} \mid \varphi \in \mathfrak{L}\}
$$

onde $\mathfrak{L}$ designa o conjunto de todas as aplicações lineares, então $\mathcal{W}$ apresenta uma estrutura de espaço vetorial se, para $\forall \varphi, \omega \in \mathcal{W}, \lambda \in \mathbb{C}, x \in \mathcal{V}$, definirmos:

$$
\begin{gathered}
(\varphi+\omega)(x):=\varphi(x)+\omega(x) \\
(\lambda \varphi)(x):=\bar{\lambda} \varphi(x)
\end{gathered}
$$

Os elementos de $\mathcal{W}$ são chamados de funcionais lineares e o conjunto $\mathcal{W}$ assim definido constitui o espaço dual de $\mathcal{V}$, sendo denotado por $\mathcal{V}^{\star}$. Além disso, se $E=\left\{\left|e_{i}\right\rangle\right\}_{i=1}^{n}$ é uma base de $\mathcal{O}_{n}(\mathcal{V})$, então,

$$
\forall|v\rangle \in \mathcal{O}_{n}(\mathcal{V}), \exists \varphi^{1}(|v\rangle), \ldots, \varphi^{n}(|v\rangle):|v\rangle=\sum_{i}^{n} \varphi^{i}(|v\rangle)\left|e_{i}\right\rangle
$$

Nessas condições, os escalares complexos $\varphi^{i}(|v\rangle)$ são as coordenadas contravariantes de $|v\rangle$ na base $E$, quer dizer, $\varphi^{i}(|v\rangle)=v^{i}$.

Enunciamos agora um importante teorema:

Teorema 1 : Seja $E=\left\{\left|e_{i}\right\rangle\right\}_{i=1}^{n}$ uma base de $\mathcal{O}_{n}(\mathcal{V})$ e $|v\rangle=\sum_{i}^{n}\left\langle e^{i} \mid v\right\rangle\left|e_{i}\right\rangle$.

Então, para os escalares definidos acimas, verifica-se que:

(i) $\varphi^{i}: \mathcal{O}_{n}(\mathcal{V}) \longrightarrow \mathbb{C}$ é um funcional linear tal que $\varphi^{i}\left(\left|e_{k}\right\rangle\right)=\delta_{k}^{i},(i, k=1, \ldots, n)$;

(ii) O conjunto $\left\{\varphi^{i}\right\}_{i=1}^{n}$ é uma base de $\mathcal{O}_{n}^{\star}(\mathcal{V})$, chamada base dual de $E$;

(iii) Se $\omega \in \mathcal{O}_{n}^{\star}(\mathcal{V})$, então $\omega(|v\rangle)=\sum_{i}^{n} \varphi^{i}(|v\rangle) \omega\left(\left|e_{i}\right\rangle\right)$;

(iv) $\forall|v\rangle \in \mathcal{O}_{n}(\mathcal{V}):|v\rangle \neq|0\rangle, \exists \omega \in \mathcal{O}_{n}^{\star}(\mathcal{V}): \omega(|v\rangle) \neq 0$.

Demonstração 1 : Suponha $|v\rangle=\sum_{i}^{n} v^{i}\left|e_{i}\right\rangle$ e $|u\rangle=\sum_{i}^{n} u^{i}\left|e_{i}\right\rangle$, ou seja, $\varphi^{i}(|v\rangle)=v^{i}$ e $\varphi^{i}(|u\rangle)=u^{i}$. Então, segue diretamente que $\varphi^{i}\left(\left|e_{j}\right\rangle\right)=\delta_{j}^{i}$ e, além disso,

$$
\begin{aligned}
|v\rangle+\lambda|u\rangle & =\sum_{i}^{n}\left(v^{i}+\lambda u^{i}\right)\left|e_{i}\right\rangle \Rightarrow \\
\varphi^{i}(|v\rangle+\lambda|u\rangle) & =v^{i}+\lambda u^{i}=\varphi^{i}(|v\rangle)+\lambda \varphi^{i}(|u\rangle),
\end{aligned}
$$

o que demonstra a afirmação $(i)$. A seguir, seja $\Phi=\sum_{i}^{n} \lambda_{i} \varphi^{i}=0 \in \mathcal{O}_{n}^{\star}(\mathcal{V})$. 
Daí, $\Phi\left(\left|e_{i}\right\rangle\right)=\sum_{k}^{n} \lambda_{k} \varphi^{k}\left(\left|e_{i}\right\rangle\right)=0 \Leftrightarrow \lambda_{i}=0, n \geq i \in \mathbb{N}$. Assim, para $\omega \in \mathcal{O}_{n}^{\star}(\mathcal{V})$ tem-se:

$$
\omega(|v\rangle)=\omega\left(\sum_{i}^{n} v^{i}\left|e_{i}\right\rangle\right)=\sum_{i}^{n} v^{i} \omega\left(\left|e_{i}\right\rangle\right)=\sum_{i}^{n} \varphi^{i}(|v\rangle) \omega\left(\left|e_{i}\right\rangle\right)
$$

Logo, o conjunto $\left\{\varphi^{i}\right\}_{i=1}^{n}$ gera $\mathcal{O}_{n}^{\star}(\mathcal{V})$ e, consequentemente, as afirmações $(i i)$ e (iii) ficam demonstradas. Finalmente, se $|v\rangle \neq|0\rangle$, então $\exists \varphi^{i}(|v\rangle) \neq|0\rangle$ em $|v\rangle=\sum_{i}^{n} \varphi^{i}(|v\rangle)\left|e_{i}\right\rangle$. Fazendo-se $\omega=\varphi^{i}$, decorre $(i v)$, o que finaliza a demonstração do teorema.

Definição Formal de Bra Tratando agora do caso específico de um bra atuando sobre um ket, temos:

$$
\begin{gathered}
\langle\omega|(|\alpha\rangle)=\omega_{\alpha} \in \mathbb{C} \Rightarrow \\
\Rightarrow\langle\omega|\left(\sum_{i=1}^{n} \alpha^{i}\left|e_{i}\right\rangle\right)=\sum_{i=1}^{n} \alpha^{i}\langle\omega|\left(\left|e_{i}\right\rangle\right)=\sum_{i=1}^{n} \omega_{i} \alpha^{i}
\end{gathered}
$$

Definindo-se então um conjunto de funcionais $\left\{\left\langle e^{k}\right|\right\}_{k=1}^{n}$ tais que satisfaçam a uma relação biunívoca com a base original $B$, dada por

$$
\left\langle e^{k}\right|\left(\left|e_{i}\right\rangle\right)=\delta_{i}^{k}
$$

segue das relações anteriores que

$$
\langle\omega|\left(\sum_{i=1}^{n} \alpha^{i}\left|e_{i}\right\rangle\right)=\sum_{i=1}^{n} \alpha^{i}\langle\omega|\left(\left|e_{i}\right\rangle\right)=\sum_{i=1}^{n} \omega_{i} \alpha^{i} \equiv \sum_{i=1}^{n}\left(\sum_{k=1}^{n} \omega_{k} \delta^{k}{ }_{i}\right) \alpha^{i}
$$

Mas, utilizando (2.7) no último termo da expressão acima, vem

$$
\langle\omega|\left(\sum_{i=1}^{n} \alpha^{i}\left|e_{i}\right\rangle\right)=\sum_{i=1}^{n}\left(\sum_{k=1}^{n} \omega_{k}\left\langle e^{k}\right|\left(\left|e_{i}\right\rangle\right)\right) \alpha^{i}=\sum_{k=1}^{n}\left\langle e^{k}\right| \omega_{k} \sum_{i=1}^{n}\left(\alpha^{i}\left|e_{i}\right\rangle\right)
$$

Daí, comparando o primeiro e o último termos, decorre, por completude da base, que

$$
\langle\omega|=\sum_{k=1}^{n}\left\langle e^{k}\right| \omega_{k}
$$

Isto significa que o conjunto de todos os funcionais lineares atuando sobre um espaço de kets $\mathcal{O}_{n}$ é, ele próprio, um espaço vetorial de mesma dimensão. Logo, esse espaço é exatamente o espaço dual de $\mathcal{O}_{n}$, sendo, por isso, denotado $\mathcal{O}_{n}^{\star}$. Os elementos desse espaço são denominados bras e denotados por $<\mid$. Entretanto, é um espaço vetorial de natureza 
diversa daquele, visto que apresenta caráter antilinear, ou seja,

$$
\left\langle\lambda \omega_{1}+\mu \omega_{2}\right|=\bar{\lambda}\left\langle\omega_{1}\right|+\bar{\mu}\left\langle\omega_{2}\right|, \quad \forall \lambda, \mu \in \mathbb{C}, \forall \omega_{1}, \omega_{2} \in \mathcal{O}_{n}^{\star}
$$

Por consistência com a notação convencional, denotaremos por

$$
B^{\star}=\left\{\left\langle e^{i}\right|\right\}_{i=1}^{n}
$$

a base de $\mathcal{O}_{n}^{\star}$, que recebe o nome de base dual ou recíproca.

De tudo que foi exposto, conclui-se que aplicação $\langle\omega|(|\alpha\rangle)=\omega_{\alpha} \in \mathbb{C}$ pode ser identificada com o produto interno no espaço de ordem $\mathcal{O}_{n}$, de modo que podemos escrever:

$$
\langle\omega|(|\alpha\rangle) \equiv\langle\omega|| \alpha\rangle=\langle\omega \mid \alpha\rangle
$$

onde o último termo deve ser entendido como um IP.

\subsection{Reformulação Coadjunta}

Tendo em vista as peculiaridades do tratamento tensorial exigido para extensão do formalismo de Dirac a sistemas de funções não-ortogonais, somos conduzidos a uma nova abordagem linear, que deve levar em conta algumas distinções importantes em relação ao tratamento linear tradicional e à formulação padrão do cálculo tensorial. A essa nova roupagem, denominamos Reformulação Coadjunta ou Formalismo Coadjunto. A justificativa desse nome será dada adiante. O primeiro aspecto notável diz respeito à construção de bases correlatas à base original, através das operações de transformação entre bras e kets. Antes, porém, vamos estender o conceito de representações de uma base.

\subsubsection{Representações Isomórficas da Base}

Conforme o que foi dito anteriormente, em termos de operacionalidade, ou seja, eficiência de cálculo e rapidez de processamento, é conveniente, em certas ocasiões, trabalhar com diferentes representações vetoriais.

Bases e Transformações Neste parágrafo introduziremos duas representações importantes para transformações lineares e bases, segundo o isomorfismo $\mathbb{M}_{n}(\mathcal{H}) \simeq \mathcal{O}_{n}\left(\mathcal{H}^{n}\right)$, as quais chamaremos de primeira e segunda representações isomórficas. 
Com a finalidade de justificar as diferentes representações, examinaremos um isomorfismo equivalente envolvido no caso de um espaço vetorial de dimensão finita.

Seja $\mathbb{V}^{n}(\mathbb{C})$ um espaço vetorial de dimensão $n$ sobre o corpo complexo e $|v\rangle \in \mathbb{V}^{n}(\mathbb{C})$.

Por outro lado, um vetor $\left|v_{i}\right\rangle \in \mathbb{C}^{n}$ pode ser descrito numa base genérica $[E]=$ $\left\{\left|e_{i}\right\rangle\right\}_{i=1}^{n}$ por suas componentes $v^{k}{ }_{i} \in \mathbb{C}$, sendo $\left|v_{i}\right\rangle=v^{1}{ }_{i}\left|e_{1}\right\rangle+\cdots+v^{n}{ }_{i}\left|e_{n}\right\rangle$, através da representação isomórfica:

$$
\left|v_{i}\right\rangle \simeq\left[\begin{array}{c}
v^{1} \\
\vdots \\
v^{n}{ }_{i}
\end{array}\right]_{E} \in \mathbb{C}^{n}
$$

Portanto, supondo que cada $\left|v_{i}\right\rangle$ seja um vetor componente de uma dada base $[V]$, então essa base pode ser descrita em termos da base $[E]$, conforme a representação mais usual, pela matriz quadrada

$$
[V]=\left[\begin{array}{lll}
\left|v_{1}\right\rangle & \cdots & \left|v_{n}\right\rangle
\end{array}\right] \in \mathrm{M}_{n}(\mathbb{C})
$$

ou, alternativamente, pela matriz-coluna de $n^{2}$ elementos pertencentes ao corpo $\mathbb{C}$, ou seja, pelo vetor

$$
|V\rangle=\left[\begin{array}{c}
\left|v_{1}\right\rangle \\
\vdots \\
\left|v_{n}\right\rangle
\end{array}\right] \in \mathbb{V}^{n}\left(\mathbb{C}^{n}\right)
$$

Assim, sumarizando, para o caso de bras e kets em espaços de dimensão finita, sejam as bases $[V]=\left\{\left|v_{i}\right\rangle\right\}_{i=1}^{n}$ e $\left[V^{\star}\right]=\left\{\left\langle v^{i}\right|\right\}_{i=1}^{n}$ original e recíproca de um espaço vetorial complexo finito $n$-dimensional $\mathbb{V}^{n}(\mathbb{C})$ e de seu dual $\mathbb{V}^{n \star}(\mathbb{C})$, respectivamente. Então, para uma base constituída pelos elementos $\left|v_{i}\right\rangle \in \mathbb{C}^{n}$, denominamos:

- $1^{\underline{a}}$ representação isomórfica: $\left.\left.\mid V\right] \in \mathbb{M}_{n}(\mathbb{C}): \mid V\right] \simeq\left[\begin{array}{lll}- & \left|v_{i}\right\rangle & -\end{array}\right]_{i=1, \ldots, n}$ - $2^{a}$ representação isomórfica: $|V\rangle \in \mathbb{V}^{n}\left(\mathbb{C}^{n}\right):|V\rangle \simeq\left[\begin{array}{c}\mid \\ \left|v_{i}\right\rangle \\ \mid\end{array}\right]_{i=1, \ldots, n}$

Obs: Em ambos os casos, os kets de base são tomados na representação original usual, isto é, como vetores-coluna.

Logo, fica justificado o isomorfismo $\mathbb{M}_{n}(\mathbb{C}) \simeq \mathbb{V}^{n}\left(\mathbb{C}^{n}\right)$. Expandindo o conceito exposto acima, podemos estabelecer uma base $[\Phi]=\left\{\left|\phi_{i}\right\rangle_{\infty}\right\}_{i=1}^{n}$, de forma que seus vetores 
componentes sejam descritos numa outra base $[\varepsilon]=\left\{\left|\epsilon_{k}\right\rangle_{\infty}\right\}_{i=1}^{n}$ pelas coordenadas $\phi_{i}^{k} \in \mathcal{H}$, quer dizer,

$$
\left|\phi_{i}\right\rangle_{\infty}=\sum_{k=1}^{n} \phi_{i}{ }^{k}\left|\epsilon_{k}\right\rangle_{\infty} \simeq\left[\begin{array}{c}
\phi_{i}{ }^{1} \\
\vdots \\
\phi_{i}{ }^{n}
\end{array}\right]_{\varepsilon} \in \mathcal{H}^{n}
$$

Daí, considerando os kets $\left|\phi_{i}\right\rangle_{\infty}$ como as componentes constituintes de um vetor $|\phi\rangle_{0_{n}} \in \mathcal{O}_{n}\left(\mathcal{H}^{n}\right)$, justifica-se o isomorfismo $\mathbb{M}_{n}(\mathcal{H}) \simeq \mathcal{O}_{n}\left(\mathcal{H}^{n}\right)$. Logo, as representações isomórficas de uma base de $\mathcal{O}_{n}(\mathcal{H})$ podem ser definidas como segue.

Definição 2 : Dadas uma base $[B]=\left\{\left|b_{i}\right\rangle_{\infty}\right\}_{i=1}^{n}$ de $\mathcal{O}_{n}(\mathcal{H})$ e uma base de referência $[\varepsilon]$, chamamos de primeira representação da base, à matriz $\mid B]_{0_{n}} \in$ $\mathrm{M}_{n}(\mathcal{H})$ cujas colunas são formadas pelos kets da base $[B]$ em $[\varepsilon]$, ou seja,

$$
\mid B]_{0_{n}} \simeq\left[\begin{array}{lll}
\left|b_{1}\right\rangle_{\infty} & \cdots & \left|b_{n}\right\rangle_{\infty}
\end{array}\right]_{\varepsilon}
$$

Definição 3 : Define-se a segunda representação de uma base $[B]$ em $\mathcal{O}_{n}(\mathcal{H})$ como um vetor-coluna $|B\rangle_{0_{n}} \in \mathcal{O}_{n}\left(\mathcal{H}^{n}\right)$, de modo que

$$
|B\rangle_{0_{n}} \simeq\left[\begin{array}{c}
\left|b_{1}\right\rangle_{\infty} \\
\vdots \\
\left|b_{n}\right\rangle_{\infty}
\end{array}\right]_{\varepsilon}
$$

Veremos que as bases também podem ser constituídas por escalares complexos, o que gera, para as representações de uma base $[B] \in \mathcal{O}_{n}(\mathbb{C})$, o isomorfismo $\mathbb{M}_{n}(\mathbb{C}) \simeq \mathcal{O}^{n}\left(\mathbb{C}^{n}\right)$. De modo geral, todos os isomorfismos acima serão utilizados no formalismo de Dirac para a expansão de funções $\mathcal{L}^{2}$.

Neste trabalho a segunda representação será padrão, reservando-se a primeira, que é a usualmente empregada em Álgebra Linear, para processos específicos, como mudanças de base, por exemplo.

\subsubsection{Representações de um Vetor}

Já foi dito que, dada uma base covariante constituída pelos elementos $\left|e_{i}\right\rangle_{\mathrm{V}}$, um vetor do espaço de ordem $\mathcal{O}_{n}(\mathbb{V})$ pode ser descrito pelo ket $|u\rangle_{0_{n}}=\sum_{i}^{n} u^{i}\left|e_{i}\right\rangle_{\mathrm{V}}$. Os espaços $\mathbb{V}$ de interesse para este trabalho são $\mathcal{H}, \mathbb{C}$ e $\mathbb{C}^{n}$. Entretanto, conforme o parágrafo anterior, utilizaremos duas representações para um ket: 
- Representação coordenada (contravariante):

$$
|u\rangle_{[B]} \simeq\left[\begin{array}{c}
u^{1} \\
\vdots \\
u^{n}
\end{array}\right]_{[B]}
$$

- Representação invariante:

$$
|u\rangle_{0_{n}}=\sum_{i}^{n} u^{i}\left|e_{i}\right\rangle_{\infty}
$$

\subsubsection{Relação de Dualidade e Base Recíproca}

Tendo como ponto de partida a base genérica original $B=\left\{\left|e_{i}\right\rangle \mid\right\}_{i=1}^{n}$ de $\mathcal{O}_{n}$, pode-se definir, de maneira análoga ao que ocorre no espaço original, uma base no espaço dos bras através de uma expressão chamada de relação de dualidade:

$$
\left\langle e^{i} \mid e_{k}\right\rangle:=\delta_{k}^{i}
$$

A base dos bras $\left\{\left\langle e^{i}\right|\right\}_{i=1}^{n}$, construída a partir da base de kets $B$ pela relação de dualidade, conforme foi dito, é denominada base recíproca e denotada por $B^{\star}$.

Além disso, é possível estabelecer uma outra operação de transformação entre bras e kets, denominada operação de dualidade e definida por:

$$
\left|e_{i}\right\rangle^{\star}=\left\langle e^{i}\right|, \quad\left\langle\left. e^{i}\right|^{\star}=\mid e_{i}\right\rangle
$$

E, de modo geral, para um elemento $|u\rangle \in \mathcal{O}_{n}$, teremos:

$$
|u\rangle^{\star}=\langle u|, \quad\left\langle\left.\underline{u}\right|^{\star}=\mid u\right\rangle,
$$

onde o ponto inferior é a notação usada para representar o resultado da operação de dualidade. Note-se, entretanto, com relação a essa notação, que $\left\langle\left. u\right|^{\star}=\mid \dot{u}\right\rangle$.

Usando a adjunção, podemos reescrever a relação de dualidade de uma outra maneira:

$$
\left\langle e^{i} \mid e_{k}\right\rangle^{\dagger}=\left\langle e_{k} \mid e^{i}\right\rangle=\left(\delta_{k}^{i}\right)^{\dagger} \equiv \delta_{k}^{i}
$$

Logo, tem-se

$$
\left\langle e_{k} \mid e^{i}\right\rangle=\delta_{k}^{i}
$$

que denominamos $2^{\mathrm{a}}$ relação de dualidade. 


\subsubsection{IPs em $\mathcal{O}_{n}$}

Nesse ponto, surge uma questão interessante: como há dois modos de se obter um bra a partir de um ket nesse formalismo, a saber, pelas operações de adjunção e de dualidade, então somos induzidos a distinguir duas espécies de IP, dos quais falaremos a seguir.

Produto Hermitiano Consideremos dois elementos do espaço original em suas representações coordenadas $|u\rangle_{[B]},|v\rangle_{[B]} \in \mathcal{O}_{n}$. Uma vez que a operação de adjunção produz $|u\rangle^{\dagger}=\langle u|$, então a superposição de um bra assim gerado sobre um ket fornece:

$$
\langle u \| v\rangle \simeq\left[\begin{array}{lll}
\bar{u}^{1} & \cdots & \bar{u}^{n}
\end{array}\right]\left[\begin{array}{c}
v^{1} \\
\vdots \\
v^{n}
\end{array}\right] \equiv \sum_{i=1}^{n} \bar{u}^{i} v^{i}
$$

Como foi mostrado na subseção precedente, essa aplicação, cujo resultado é um escalar complexo, pode ser identificada a um produto interno em $\mathcal{O}_{n}$, o que nos leva à definição seguinte, que denominaremos de IP hermitiano ou, simplesmente, produto hermitiano:

Definição 4 : O produto hermitiano no espaço de ordem $\mathcal{O}_{n}(\mathbb{C})$ é uma aplicação $\langle\mid\rangle: \mathcal{O}_{n}(\mathbb{C}) \times \mathcal{O}_{n}(\mathbb{C}) \longrightarrow \mathbb{C}$ tal que

$$
\langle u \mid v\rangle_{[B]}:=\sum_{i}^{n} \bar{u}^{i} v^{i}
$$

Produto Dual Por outro lado, se um bra $\langle\underline{u}|$ obtido pela operação de dualidade $|u\rangle^{\star}$ atuar sobre um ket, teremos

$$
\langle\underline{u} \| v\rangle \simeq\left[\begin{array}{lll}
\bar{u}_{1} & \cdots & \bar{u}_{n}
\end{array}\right]\left[\begin{array}{c}
v^{1} \\
\vdots \\
v^{n}
\end{array}\right] \equiv \sum_{i=1}^{n} \bar{u}_{i} v^{i}
$$

Assim, somos conduzidos à definição de um IP de natureza dual no espaço de ordem sobre $\mathbb{C}$, que será denominado produto dual:

Definição 5 : O produto dual no espaço de ordem $\mathcal{O}_{n}(\mathbb{C})$ é uma aplicação $\langle\mid\rangle: \mathcal{O}_{n}(\mathbb{C}) \times \mathcal{O}_{n}(\mathbb{C}) \longrightarrow \mathbb{C}$ tal que

$$
\langle u \mid v\rangle_{0_{n}}:=\sum_{i}^{n} \bar{u}_{i} v^{i}
$$




\subsubsection{Construção das Bases Correlatas}

Utilizando as propriedades de transformação entre bras e kets apresentadas anteriormente é possível construir outras bases na $2^{\mathrm{a}}$ representação, todas relacionadas à base original por meio das operações de transformação.

Base Adjunta Da adjunção dos elementos da base original $B=\left\{\left|e_{i}\right\rangle\right\}_{i=1}^{n}$, segue que $\left|e_{i}\right\rangle^{\dagger}=\left\langle e_{i}\right|$. Como a correspondência bra-ket nesse caso é biunívoca, o conjunto $\left\{\left\langle e_{i}\right|\right\}_{i=1}^{n}$ também constitui uma base de $\mathcal{O}_{n}$. Essa base é denominada base adjunta e denotada $B^{\dagger}$, pois é construída pela adjunção dos elementos da base original. Mais tarde, essa notação será adaptada às exigências das representações isomórficas.

Base Coadjunta Além das bases original, adjunta e recíproca, ainda é possível construirmos uma outra base adicional correlata, obtida pela adjunção da recíproca. Assim, da correspondência biunívoca $\left\langle\left. e^{i}\right|^{\dagger}=\mid e^{i}\right\rangle$, segue que o conjunto $\left\{\left|e^{i}\right\rangle\right\}_{i=1}^{n}$ é uma base. Como ela é dual da base adjunta, numa correspondência semelhante à de vetores e covetores, vamos chamá-la de base coadjunta e denotá-la por B (um ponto sob o símbolo).

\subsubsection{Representações Correlatas de um Ket}

Foi visto na seção 2.1.3.1 que um vetor pode ser escrito, em termos de uma base original $E=\left\{\left|e_{i}\right\rangle\right\}_{i=1}^{n}$, como $|u\rangle_{0_{n}}=\sum_{i}^{n} u^{i}\left|e_{i}\right\rangle$. Porém, como há quatro bases fundamentais sobre as quais podemos definir coordenadas, haverá quatro representações possíveis para um mesmo vetor. Passamos a descrevê-las.

Representação Adjunta Efetuando a adjunção de um ket, tem-se

$$
|u\rangle_{0_{n}}^{\dagger}=\left\langle\left. u\right|_{0_{n}}=\left(\sum_{i}^{n} u^{i}\left|e_{i}\right\rangle\right)^{\dagger}=\sum_{i}^{n}\left\langle e_{i}\right|\left(u^{i}\right)^{\dagger} \equiv \sum_{i}^{n}\left\langle e_{i}\right| \bar{u}^{i}\right.
$$

Portanto, podemos representar um vetor pelo seu covetor adjunto, de modo que:

$$
\left\langle\left. u\right|_{0_{n}}=\sum_{i}^{n}\left\langle e_{i}\right| \bar{u}^{i}\right.
$$

é denominada sua representação adjunta. Essa expressão condiz com a equação 2.5, quando denotada isomorficamente em termos das coordenadas, i.e., $\left\langle\left. u\right|_{B} \simeq\left[-\bar{u}_{i}-\right]_{B}\right.$. 
Representação Coadjunta $\mathrm{O}$ mesmo vetor deve poder ser escrito em termos da base coadjunta B, através de suas coordenadas covariantes. Logo, assume-se, para a representação coadjunta, a expressão:

$$
|u\rangle_{0_{n}}:=\sum_{i}^{n} u_{i}\left|e^{i}\right\rangle
$$

Representação Dual Da anterior advém a representação dual (ou recíproca) de um ket, obtida por outra adjunção:

$$
\left\langle\left. u\right|_{0_{n}}=\mid u\right\rangle_{0_{n}}^{\dagger}=\left(\sum_{i}^{n} u_{i}\left|e^{i}\right\rangle\right)^{\dagger}=\sum_{i}^{n}\left\langle e^{i}\right|\left(u_{i}\right)^{\dagger} \equiv \sum_{i}^{n}\left\langle e^{i}\right| \bar{u}_{i}
$$

Então, a representação dual deverá assumir a forma:

$$
\left\langle\left. u\right|_{o_{n}}=\sum_{i}^{n}\left\langle e^{i}\right| \bar{u}_{i}\right.
$$

Dito isto, explicitaremos as representações correlatas coordenadas em uma dada base $B$, bem como as respectivas notações, destacando que as que apresentarem coordenadas covariantes serão denotadas com um ponto inferior:

- Repr. original:

$$
|\mathrm{u}\rangle_{[\mathrm{B}]} \simeq\left[\begin{array}{c}
u^{1} \\
\vdots \\
u^{n}
\end{array}\right]_{\mathrm{B}}
$$

- Repr. adjunta:

$$
\left\langle\left.\mathrm{u}\right|_{[\mathrm{B}]} \simeq\left[\begin{array}{lll}
\bar{u}^{1} & \cdots & \bar{u}^{n}
\end{array}\right]_{\mathrm{B}}\right.
$$

- Repr. dual:

$$
\left\langle\left.\underline{u}\right|_{[B]} \simeq\left[\begin{array}{lll}
\bar{u}_{1} & \cdots & \bar{u}_{n}
\end{array}\right]_{B}\right.
$$

- Repr. coadjunta:

$$
|\underline{u}\rangle_{[B]} \simeq\left[\begin{array}{c}
u_{1} \\
\vdots \\
u_{n}
\end{array}\right]_{B}
$$

Em seguida, resumimos as transformações entre bras e kets de acordo com a notação dada:

$$
|\mathrm{u}\rangle^{*}=|\overline{\mathrm{u}}\rangle, \quad|\mathrm{u}\rangle^{\prime}=\langle\overline{\mathrm{u}}|, \quad| \mathrm{u}\rangle^{\dagger}=\langle\mathrm{u}|, \quad| \mathrm{u}\rangle^{\star}=\langle\underline{\mathrm{u}}|
$$


Já o IP em $\mathcal{O}_{n}$ se transforma segundo as expressões:

$$
\langle\mathrm{u} \mid \mathrm{v}\rangle^{*}=\langle\overline{\mathrm{u}} \mid \overline{\mathrm{v}}\rangle, \quad\langle\mathrm{u} \mid \mathrm{v}\rangle^{\prime}=\langle\overline{\mathrm{v}} \mid \overline{\mathrm{u}}\rangle, \quad\langle\mathrm{u} \mid \mathrm{v}\rangle^{\dagger}=\langle\mathrm{v} \mid \mathrm{u}\rangle, \quad\langle\mathrm{u} \mid \mathrm{v}\rangle^{\star}=\langle\mathrm{v} \mid \mathrm{u}\rangle
$$

Fechamos esta seção reforçando o fato de que as coordenadas covariantes assumem valores distintos das contravariantes em sistemas não-ortogonais, o que geraria vetores completamente diferentes se tais coordenadas não fossem escritas em suas bases correspondentes às respectivas representações.

Um outro aspecto crucial que deve ser mencionado aqui diz respeito a um detalhe dessa apresentação, que é o fato de que os formatos das transformações acima são válidos para elementos do espaço original $\mathcal{O}_{n}$. Contudo, se as aplicarmos a elementos do espaço dual $\mathcal{O}_{n}^{\star}$, o aspecto das equações sofre algumas mudanças naturais, de forma a manter a consistência matemática. Esse ponto será discutido em seções subsequentes, quando for abordada a expansão em série de funções.

IPs nas Diferentes Representações Vamos mostrar agora que só faz sentido definir o produto hermitiano na representação coordenada, já que apenas nesse caso ele apresenta resultado diferente do produto dual.

O produto hermitiano na representação coordenada é construído sobre o adjunto do ket, i.e.,

$$
|u\rangle_{[B]}^{\dagger}=\left[\begin{array}{lll}
\bar{u}^{1} & \cdots & \bar{u}^{n}
\end{array}\right]=\left\langle\left. u\right|_{[B]},\right.
$$

de forma que

$$
\langle u \mid v\rangle_{[B]}=\left[\begin{array}{lll}
\bar{u}^{1} & \ldots & \bar{u}^{n}
\end{array}\right]\left[\begin{array}{c}
u^{1} \\
\vdots \\
u^{n}
\end{array}\right]=\sum_{i}^{n} \bar{u}^{i} v^{i}
$$

enquanto o da representação invariante, dado

$$
|u\rangle^{\dagger}=\sum_{i}^{n}\left\langle e_{i}\right| \bar{u}^{i}=\langle u|,
$$

deve seguir:

$$
\langle u \mid v\rangle_{0_{n}}=\sum_{i, k}^{n}\left\langle e_{i}\left|\bar{u}^{i} v^{k}\right| e_{k}\right\rangle=\sum_{i, k}^{n} g_{i k} \bar{u}^{i} u^{k}=\sum_{k}^{n} \bar{u}_{k} v^{k},
$$

o que mostra que os resultados são distintos em cada caso.

Por outro lado, considerando a expressão para o dual de um ket na representação coordenada: 


$$
|u\rangle_{[B]}^{\star}=\left[\begin{array}{lll}
\bar{u}_{1} & \cdots & \bar{u}_{n}
\end{array}\right]=\left\langle\left. u\right|_{B},\right.
$$

o produto dual nesse caso será

$$
\langle\underline{u} \mid v\rangle_{[B]}=\left[\begin{array}{lll}
\bar{u}_{1} & \ldots & \bar{u}_{n}
\end{array}\right]\left[\begin{array}{c}
u^{1} \\
\vdots \\
u^{n}
\end{array}\right]=\sum_{i}^{n} \bar{u}_{i} v^{i}
$$

ao passo que, sendo o dual de um ket na representação invariante igual a

$$
|u\rangle^{\star}=\sum_{i}^{n}\left\langle e^{i}\right| \bar{u}_{i}=\langle\underline{u}|
$$

esse mesmo produto assumirá a forma

$$
\langle\underline{u} \mid v\rangle_{0_{n}}=\sum_{i, k}^{n}\left\langle e^{i}\left|\bar{u}_{i} v^{k}\right| e_{k}\right\rangle=\sum_{i, k}^{n} \delta_{k}^{i} \bar{u}_{i} u^{k}=\sum_{k}^{n} \bar{u}_{k} v^{k}
$$

Logo, na representação invariante, os dois IPs, hermitiano e dual, fornecem o mesmo resultado. Desse modo, pode-se escrever

$$
\langle\underline{u} \mid v\rangle_{[B]}=\langle u \mid v\rangle_{o_{n}}
$$

Além disso, vemos que o produto dual não depende da representação, quer dizer, é o mesmo para ambos os casos, na representação coordenada ou invariante. Por isso, não fizemos distinção na hora de defini-lo anteriormente.

Por conseguinte, é indiferente a métrica ser definida pelo produto hermitiano ou pelo produto dual, os quais, nesse caso, podem ser ambos chamados simplesmente de produto interno, ou seja,

$$
d(u, v):=\langle d u \mid d u\rangle=\sum_{i, k}^{n}\left\langle e_{i}\left|d \bar{u}^{i} d u^{k}\right| e_{k}\right\rangle=\sum_{i, k}^{n} g_{i k} d \bar{u}^{i} d u^{k}
$$

\subsubsection{Determinação das Coordenadas}

Coordenadas Contravariantes Usando a definição do produto interno em $\mathcal{O}_{n}$ e a relação de dualidade, obtém-se:

$$
\left\langle e^{k} \mid u\right\rangle_{0_{n}}=\left\langle e^{k}\left|\sum_{i}^{n} u^{i}\right| e_{i}\right\rangle=\sum_{i}^{n}\left\langle e^{k} \mid e_{i}\right\rangle u^{i}=\sum_{i}^{n} \delta_{i}^{k} u^{i}=u^{k}
$$


Logo, as coordenadas contravariantes do ket $|u\rangle_{0_{n}}$ são obtidas pelo produto interno em $\mathcal{O}_{n}$ entre os elementos da base recíproca e esse ket, i.e.,

$$
u^{k}=\left\langle e^{k} \mid u\right\rangle_{o_{n}}
$$

Coordenadas Covariantes Partindo da base adjunta $B^{\dagger}$ e da representação coadjunta do ket e usando a $2^{\mathrm{a}}$ relação de dualidade, efetuamos:

$$
\left\langle e_{i} \mid u\right\rangle_{0_{n}}=\left\langle e_{i}\left|\sum_{k}^{n} u_{k}\right| e^{k}\right\rangle=\sum_{k}^{n}\left\langle e_{i} \mid e^{k}\right\rangle u_{k}=\sum_{k}^{n} \delta_{i}{ }^{k} u_{k}=u_{i}
$$

Portanto, as coordenadas covariantes do ket $|u\rangle_{0_{n}}$ são obtidas pelo produto interno em $\mathcal{O}_{n}$ entre os elementos da base adjunta e esse ket, i.e.,

$$
u_{i}=\left\langle e_{i} \mid u\right\rangle_{0_{n}}
$$

Correspondência Matricial No tratamento linear, o produto de duas matrizes $A, B \in \mathbb{M}_{m}(\mathbb{C})$ pode ser escrito como

$$
[A B]_{k}^{i}:=A^{(i)} B_{(k)}
$$

onde $[A B]$ é a matriz obtida do produto entre $A$ e $B$, sendo que os índices superior e inferior do elemento $[A B]^{i}{ }_{k}$ da matriz $[A B]$ denotam, respectivamente, sua linha e sua coluna; $A^{(i)}$ designa a $i$-ésima linha de $A$ e $B_{(k)}$ designa a $k$-ésima coluna de $B$. Na verdade, a convenção que irá predominar no presente trabalho será atribuir a representação da linha ao primeiro índice e da coluna, ao segundo. Deve-se reparar que o índice inferior está deslocado à direita, revelando a precedência do outro. Mas o mesmo produto também pode ser escrito na forma:

$$
[A B]_{k}^{i}=\sum_{r=1}^{n} A_{r}^{i} B_{k}^{r}
$$

Daí, se $A$ for uma matriz-linha e $B$, uma matriz-coluna, a expressão acima reduz-se a um simples produto interno $\langle A, B\rangle=\sum_{r=1}^{n} A_{r} B^{r}$.

Por outro lado, se um vetor e um covetor de $\mathcal{O}_{n}$ forem respectivamente denotados por

$$
|v\rangle \simeq\left[\begin{array}{c}
v^{1} \\
\vdots \\
v^{n}
\end{array}\right] \quad \text { e } \quad\langle\underline{u}| \simeq\left[\begin{array}{lll}
\bar{u}_{1} & \cdots & \bar{u}_{n}
\end{array}\right]
$$

a superposição de um bra desse tipo sobre um ket pode ser interpretada como uma 
aplicação cuja correspondência matricial seria dada, em conformidade com o que foi exposto acima, por

$$
\langle\underline{u}|| v\rangle \simeq\left[\begin{array}{lll}
\bar{u}_{1} & \cdots & \bar{u}_{n}
\end{array}\right]\left[\begin{array}{c}
v^{1} \\
\vdots \\
v^{n}
\end{array}\right] \equiv \sum_{i=1}^{n} \bar{u}_{i} v^{i}
$$

que é idêntico ao produto dual definido em (2.15).

Estendendo esse conceito, podemos relacionar também o produto hermitiano com a notação matricial, fazendo:

$$
\langle u| \simeq\left[\begin{array}{lll}
\bar{u}^{1} & \cdots & \bar{u}^{n}
\end{array}\right]
$$

para obter

$$
\langle u|| v\rangle \simeq\left[\begin{array}{lll}
\bar{u}^{1} & \cdots & \bar{u}^{n}
\end{array}\right]\left[\begin{array}{c}
v^{1} \\
\vdots \\
v^{n}
\end{array}\right] \equiv \sum_{i=1}^{n} \bar{u}^{i} v^{i}
$$

que é idêntico à definição (2.14).

Relação entre as duas Representações Isomórficas da Base Dada uma base de referência $\left\{\left|\epsilon_{i}\right\rangle\right\}_{0_{n}}$, se reescrevermos

$$
\left|b_{i}\right\rangle=\sum_{k}^{n} M_{i}^{k}\left|\epsilon_{k}\right\rangle,
$$

como

$$
|B\rangle \simeq\left[\begin{array}{c}
\left|b_{1}\right\rangle \\
\vdots \\
\left|b_{n}\right\rangle
\end{array}\right]_{\varepsilon}=\left[\begin{array}{ccc}
M_{1}{ }^{1} & \cdots & M_{1}{ }^{n} \\
\vdots & \ddots & \vdots \\
M_{n}{ }^{1} & \cdots & M_{n}{ }^{n}
\end{array}\right]\left[\begin{array}{c}
\left|\epsilon_{1}\right\rangle \\
\vdots \\
\left|\epsilon_{n}\right\rangle
\end{array}\right]
$$

então

$$
|B\rangle \simeq M|\epsilon\rangle
$$

onde $M$ é a matriz cujas linhas são as componentes de $[B]$ na base $[\varepsilon]$. Por outro lado, também se pode grafar:

$$
\mid B] \simeq\left[\begin{array}{lll}
\left|b_{1}\right\rangle & \cdots & \left|b_{n}\right\rangle
\end{array}\right]_{\varepsilon}=\left[\begin{array}{lll}
\left|\epsilon_{1}\right\rangle & \cdots & \left|\epsilon_{n}\right\rangle
\end{array}\right]\left[\begin{array}{ccc}
M_{1}^{1} & \cdots & M_{n}{ }^{1} \\
\vdots & \ddots & \vdots \\
M_{1}{ }^{n} & \cdots & M_{n}{ }^{n}
\end{array}\right]
$$

o que implica

$$
\mid B] \simeq \mid \varepsilon] M^{\prime}
$$

onde $M^{\prime}$ representa a transposta da matriz $M$. Assim, podemos concluir que $\left.\mid B\right] \simeq M^{\prime}$, onde $M^{\prime}$ é a matriz que tem os vetores da base $B$ como colunas, i.e., $\left|b_{i}\right\rangle \simeq M_{[i]}^{\prime}$. 


\subsubsection{Produto Externo em $\mathcal{O}_{n}$}

Foi visto na seção B.5 que é possível, por meio do axioma da associatividade do produto, definir um produto externo pela superposição de um ket sobre um bra, o que gera um operador cuja representação matricial é equivalente à de uma diádica.

Definiremos um produto externo $\Phi: \mathcal{O}_{n}(\mathrm{~V}) \times \mathcal{O}_{n}(\mathrm{~V}) \longrightarrow \mathrm{M}_{n}(\mathrm{~V})$ por uma superposição de um ket sobre um bra obtido pela transposição de outro ket:

$$
|u\rangle\langle\bar{v}|
$$

Esse produto é, na verdade, uma diádica $\overleftrightarrow{T}$. Note-se que, em termos da representação por coordenadas, obtém-se, explicitamente, o seguinte:

$$
|u\rangle\langle\bar{v}| \simeq\left[\begin{array}{c}
u^{1} \\
\vdots \\
u^{n}
\end{array}\right]\left[\begin{array}{lll}
v^{1} & \cdots & v^{n}
\end{array}\right] \equiv\left[\begin{array}{ccc}
u^{1} v^{1} & \cdots & u^{1} v^{n} \\
\vdots & \ddots & \vdots \\
u^{n} v^{1} & \cdots & u^{n} v^{n}
\end{array}\right]
$$

Os elementos da matriz acima constituem, de fato, as coordenadas da diádica $|u\rangle\langle\bar{v}|$.

Natureza Tensorial do Produto Externo O produto externo de dois elementos de uma base genérica, conforme definido anteriormente, constitui um tensor covariante de segunda ordem.

De fato, não é dífícil mostrar isso. Para tanto, seja $\tau_{r s}=\left|e_{r}\right\rangle\left\langle\bar{e}_{s}\right|$. Efetuando uma mudança de coordenadas $M$ tal que $u^{s} \stackrel{M}{\longrightarrow} \hat{u}^{r}$, tem-se que $\hat{u}^{r}=\sum_{s}^{n} M_{s}^{r} u^{s}$ implica:

$$
\frac{\partial \hat{u}^{r}}{\partial u^{p}}=\sum_{s}^{n} M_{s}^{r} \frac{\partial u^{s}}{\partial u^{p}}=\sum_{s}^{n} M_{s}^{r} \delta_{p}^{s}=M_{p}^{r},
$$

com a transformação inversa sendo expressa por

$$
\hat{M}_{s}^{q}=\frac{\partial u^{q}}{\partial \hat{u}^{s}}
$$

Consideremos agora uma grandeza $\overleftrightarrow{T}$ dada em termos de dois elementos $|v\rangle$ e $|w\rangle$ tal que $\overleftrightarrow{T}=|w\rangle\langle\bar{v}|$. Uma vez que tal grandeza deve ser um invariante sob mudança de coordenadas, tem-se

$$
\sum_{p, q}^{n} w^{p} v^{q}\left|e_{p}\right\rangle\left\langle\bar{e}_{q}\left|=\sum_{r, s}^{n} \hat{w}^{r} \hat{v}^{s}\right| \hat{e}_{r}\right\rangle\left\langle\hat{e}_{s}^{*}\right|
$$

ou, equivalentemente,

$$
\sum_{p, q}^{n} w^{p} v^{q} \tau_{p q}=\sum_{r, s}^{n} \hat{w}^{r} \hat{v}^{s} \hat{\tau}_{r s}
$$


Portanto, como $w^{p}=\sum_{r}^{n} \hat{M}_{r}^{p} \hat{w}^{r}$ e $v^{q}=\sum_{s}^{n} \hat{M}_{s}^{q} \hat{v}^{s}$, então a relação acima torna-se

$$
\sum_{r, s}^{n} \hat{w}^{r} \hat{v}^{s} \sum_{p, q}^{n} \hat{M}_{r}^{p} \hat{M}_{s}^{q} \tau_{p q}=\sum_{r, s}^{n} \hat{w}^{r} \hat{v}^{s} \hat{\tau}_{r s}
$$

implicando, devido à completude da base,

$$
\hat{\tau}_{r s}=\sum_{p, q}^{n} \hat{M}_{r}^{p} \hat{M}_{s}^{q} \tau_{p q}
$$

Agora, substituindo a relação (2.22) nessa expressão, obtém-se

$$
\hat{\tau}_{r s}=\sum_{p, q}^{n} \frac{\partial u^{p}}{\partial \hat{u}^{r}} \frac{\partial u^{q}}{\partial \hat{u}^{s}} \tau_{p q},
$$

que corresponde à transformação de um tensor covariante de segunda ordem (KREYSZIG, 1968)(p.108). Com este resultado, comprovamos a natureza tensorial do produto externo.

\subsubsection{Projetores em $\mathcal{O}_{n}$}

Um tipo especial de produto externo de essencial importância é o projetor em $\mathcal{O}_{n}$, definido por

$$
\stackrel{(n)}{\mathbb{P}}:=\sum_{r}^{n}\left|e_{r}\right\rangle\left\langle e^{r}\right|
$$

Para entender sua utilidade, vamos examinar o efeito de sua aplicação sobre um vetor de $\mathcal{O}_{n}$. Seja $|u\rangle_{0_{n}} \in \mathcal{O}_{n}$. Então,

$$
\begin{gathered}
\stackrel{(m)}{\mathbb{P}}|u\rangle_{0_{n}}=\sum_{r}^{m}\left|e_{r}\right\rangle\left\langle e^{r}\right|\left(\sum_{k}^{n} u^{k}\left|e_{k}\right\rangle\right)=\sum_{r}^{m}\left|e_{r}\right\rangle \sum_{k}^{n} u^{k}\left\langle e^{r} \mid e_{k}\right\rangle= \\
=\sum_{r}^{m}\left|e_{r}\right\rangle \sum_{k}^{n} u^{k} \delta_{k}^{r}=\sum_{r}^{m} u^{r}\left|e_{r}\right\rangle
\end{gathered}
$$

Portanto, o que o projetor $\stackrel{(m)}{\mathbb{P}}$ faz é justamente calcular a projeção de um vetor de $\mathcal{O}_{n}$ no subespaço vetorial de dimensão $m<n$. Sua atuação sobre um elemento da base do subespaço de dimensão $m$ seleciona este elemento, tendo um efeito de operador identidade, ou seja,

$$
\stackrel{(m)}{\mathbb{P}}\left|e_{k}\right\rangle=\sum_{r}^{m}\left|e_{r}\right\rangle\left\langle e^{r} \mid e_{k}\right\rangle=\sum_{r}^{m}\left|e_{r}\right\rangle \delta_{k}^{r}=\left|e_{k}\right\rangle, \quad k \leq m \leq n
$$


Autoprojetor Além disso, a projeção de um vetor de $\mathcal{O}_{n}$ no próprio espaço $\mathcal{O}_{n}$ fornece o mesmo vetor, i.e., $\stackrel{(n)}{\mathbb{P}}|u\rangle_{0_{n}}=|u\rangle_{0_{n}}$, funcionando, portanto, também como um operador identidade. Denominamos este operador de autoprojetor e o denotaremos simplesmente por $\mathbb{P}$. Assim, $\mathbb{P}|u\rangle_{0_{n}}=|u\rangle_{0_{n}}$.

Projetor Adjunto Podemos, ademais, calcular o projetor adjunto $\stackrel{(n)}{\mathbb{P}^{\dagger}}$, cujo efeito é similar a $\stackrel{(n)}{\mathbb{P}}$ :

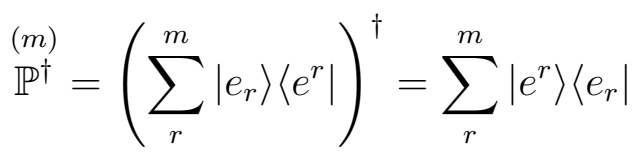

Assim, temos

$$
\stackrel{(m)}{\mathbb{P}^{\dagger}}|u\rangle=\sum_{r}^{m}\left|e^{r}\right\rangle\left\langle e_{r} \mid u\right\rangle=\sum_{r}^{m} u_{r}\left|e^{r}\right\rangle,
$$

o que significa que a ação do projetor adjunto sobre $|u\rangle$ fornece sua projeção no subespaço de dimensão $m$, mas na base coadjunta. Portanto,

$$
\stackrel{(m)}{\mathbb{P}^{\dagger}}\left|e^{k}\right\rangle=\left|e^{k}\right\rangle, \quad k \leq m \leq n
$$

$(n)$

O autoprojetor adjunto será, então, $\mathbb{P}^{\dagger} \equiv \mathbb{P}^{\dagger}$, de forma que vale a mesma relação obtida para o autoprojetor: $\mathbb{P}^{\dagger}|u\rangle_{0_{n}}=|u\rangle_{0_{n}}$.

Adicionalmente, pode-se pensar na retrooperação dos projetores, ou seja, sua atuação sobre elementos antepostos a ele. Por exemplo, a retrooperação do projetor sobre um elemento da base dual fornece esse mesmo elemento:

$$
\left\langle e^{i}\right| \stackrel{(m)}{\mathbb{P}}=\left\langle e^{i}\right|, \quad i \leq m \leq n
$$

pois

$$
\left\langle e^{i}\right| \stackrel{(m)}{\mathbb{P}}=\left\langle e^{i}\right|\left(\sum_{r}^{m}\left|e_{r}\right\rangle\left\langle e^{r}\right|\right)=\sum_{r}^{m}\left\langle e^{i} \mid e_{r}\right\rangle\left\langle e^{r}\right|=\sum_{r}^{m} \delta_{r}^{i}\left\langle e^{r}\right|=\left\langle e^{i}\right|
$$

Analogamente, efetuando a adjunção sobre a equação (2.24), obtém-se

$$
\left(\stackrel{(m)}{\mathbb{P}}\left|e_{k}\right\rangle\right)^{\dagger}=\left\langle e_{k}\right| \stackrel{(m)}{\mathbb{P}^{\dagger}}=\left\langle e_{k}\right|, \quad k \leq m \leq n
$$

Adiante, quando estivermos tratando de expansão em série de funções, veremos que o autoprojetor do espaço $\mathcal{O}_{n}$ atuando sobre uma função fornece sua expansão em $\mathcal{O}_{n}$. 


\subsubsection{Tensor Métrico}

Vimos, na seção precedente, várias aplicações dos projetores, mas não todas. Examinaremos agora a atuação do autoprojetor adjunto sobre uma componente da base original:

$$
\mathbb{P}^{\dagger}\left|e_{k}\right\rangle=\sum_{r}^{n}\left|e^{r}\right\rangle\left\langle e_{r} \mid e_{k}\right\rangle
$$

Nessa expressão surge uma grandeza ainda não definida: os elementos gerados pelo produto interno entre os vetores da base original. Denotá-los-emos por $g_{i k}$, de modo que

$$
g_{i k}:=\left\langle e_{i} \mid e_{k}\right\rangle
$$

e, consequentemente, reescrevemos:

$$
\mathbb{P}^{\dagger}\left|e_{k}\right\rangle=\sum_{r}^{n}\left|e^{r}\right\rangle g_{r k}
$$

Note-se que o espaço de atuação do IP não está sendo indicado, o que significa que pode ocorrer tanto no espaço finito $n$-dimensional quanto no espaço de Hilbert de funções.

Deve-se observar também que a aplicação $g: \mathcal{O}_{n}(\mathrm{~V}) \times \mathcal{O}_{n}(\mathrm{~V}) \longrightarrow \mathbb{C} \mid g\left(e_{i}, e_{k}\right)=g_{i k}$ constitui uma forma sesquilinear.

O produto externo dos elementos da base recíproca com coordenadas dadas por esses elementos constitui um tensor $\overleftrightarrow{g}=\sum_{i, k}^{n}\left|e^{i}\right\rangle g_{i k}\left\langle e^{k}\right|$ denominado, à semelhança da geometria diferencial, tensor métrico. Esse tensor pode, naturalmente, ser representado na base original $B=\left\{\left|e_{i}\right\rangle\right\}$, através de um isomorfismo $\overleftrightarrow{g} \simeq[g]_{B}$, por uma matriz $g \in \mathbb{M}_{n}(\mathbb{C})$, tal que

$$
g=\left[\begin{array}{ccc}
g_{11} & \cdots & g_{1 n} \\
\vdots & \ddots & \vdots \\
g_{n 1} & \cdots & g_{n n}
\end{array}\right], \quad g_{i j} \in \mathbb{C}
$$

Os elementos da matriz $g$ podem ser obtidos pela relação $g_{i k}=\left\langle e_{i}|\overleftrightarrow{g}| e_{k}\right\rangle$

Ortogonalidade de Bases Uma base para a qual valha a condição $g_{i k}=\zeta_{i} \delta_{i k}$, $\zeta_{i} \in \mathbb{R}$, será chamada de base ortogonal, ao passo que, aquelas para as quais valer $\zeta_{i}=1, \forall i \in \mathbb{N}, i \leq n$, serão denominadas bases ortonormais e, nesse caso, teremos, evidentemente, $g_{i k}=\delta_{i k}$. Bases ortogonais geram tensores diagonais, ou melhor, tensores com representação por matrizes diagonais. Já uma base ortonormal gera um tensor identidade. Entretanto, de maneira geral, poderemos trabalhar com bases oblíquas e não 
unitárias, para as quais os elementos do tensor métrico podem assumir quaisquer valores, inclusive complexos.

Uma propriedade importante do tensor métrico, além de ser um tensor covariante de segunda ordem, é que sua matriz de representação é hermitiana. De fato, como $g_{i k} \in \mathbb{C}$,

$$
g_{i k}^{\dagger}=\bar{g}_{i k}=\left\langle e_{i} \mid e_{k}\right\rangle^{\dagger}=\left\langle e_{k} \mid e_{i}\right\rangle=g_{k i} \Rightarrow \bar{g}=g^{\prime}
$$

ou, de modo equivalente,

$$
g^{\dagger}=g
$$

isto é, a matriz $g$ é hermitiana.

Com base na definição do tensor métrico, o produto hermitiano entre dois vetores $|u\rangle$ e $|v\rangle$ de $\mathcal{O}_{n}$ pode ser definido, alternativamente, como:

$$
\langle u \mid v\rangle_{0_{n}}:=\sum_{i}^{n} \sum_{k}^{n}\left\langle e_{i}\left|\bar{u}^{i} v^{k}\right| e_{k}\right\rangle=\sum_{i, k}^{n} g_{i k} \bar{u}^{i} v^{k}
$$

Métrica Como $\mathcal{O}_{n}$ é um espaço com produto interno e norma $\|\cdot\|$ gerada pelo IP: $\|x\|=\langle x \mid x\rangle^{1 / 2}$, a métrica será naturalmente induzida pela norma:

$$
d(u, v):=\|u-v\|=\langle u-v \mid u-v\rangle^{1 / 2}
$$

Logo, podemos escrever a métrica como

$$
d(u, v)=\left[\sum_{i, k}^{n} g_{i k} \overline{\left(u^{i}-v^{i}\right)}\left(u^{k}-v^{k}\right)\right]^{1 / 2}
$$

Do ponto de vista formal, uma métrica sobre um espaço vetorial $\mathbb{V}$ no corpo $\mathbb{K}$ é um funcional de $\mathbb{V} \times \mathbb{V}$ em $\mathbb{K}$, sendo usualmente entendida como um operador bilinear não-singular que atua comutativamente sobre pares de campos vetoriais produzindo um escalar (ou uma função, se a base for local, i.e., variar de ponto a ponto). Entretanto, a grandeza esperada para atuar como métrica, expressa por

$$
d(,): \mathcal{O}_{n}(\mathrm{~V}) \times \mathcal{O}_{n}(\mathrm{~V}) \longrightarrow \mathbb{R} \mid d(u, v)=\left[\sum_{i, k}^{n} g_{i k} \overline{\left(u^{i}-v^{i}\right)}\left(u^{k}-v^{k}\right)\right]^{1 / 2}
$$

não constitui um operador bilinear. Nesse caso, somos induzidos a estender a noção de métrica a um caso mais geral, definindo uma métrica sesquilinear. Pode-se mostrar que a grandeza acima satisfaz as condições de uma métrica sesquilinear, que são as seguintes: 
(i) $d(u, v)=\overline{d(v, u)}$;

(ii) $d(u, u)=0$;

(iii) $d(u, w) \leq d(u, v)+d(v, w) \quad$ (desigualdade triangular);

(iv) $d(u, v)>0, \forall u, v: u \neq v$.

Espera-se que a métrica assim definida seja não-degenerada, i.e., $\operatorname{det} g \neq 0$. Por fim, introduzimos, também, a notação diferencial da métrica, tradicional na Física:

$$
d s^{2}=\langle d u|\overleftrightarrow{g}| d u\rangle=\sum_{i, k}^{n} g_{i k} d \bar{u}^{i} d u^{k}
$$

Tensor Recíproco O mesmo procedimento de antes pode ser usado agora para examinar o efeito da atuação do projetor sobre uma componente da base coadjunta:

$$
\mathbb{P}\left|e^{k}\right\rangle=\sum_{r}^{n}\left|e_{r}\right\rangle\left\langle e^{r} \mid e^{k}\right\rangle
$$

Definindo um tensor recíproco, de componentes na representação matricial:

$$
g^{i k}:=\left\langle e^{i} \mid e^{k}\right\rangle
$$

podemos reescrever

$$
\mathbb{P}\left|e^{k}\right\rangle=\sum_{r}^{n}\left|e_{r}\right\rangle g^{r k}
$$

Enquanto o tensor métrico obedece à relação

$$
g_{i k}=\left\langle e_{i}|\overleftrightarrow{g}| e_{k}\right\rangle
$$

para o tensor recíproco, vale

$$
g^{i k}=\left\langle e^{i}\left|\overleftrightarrow{g^{k}}\right| e^{k}\right\rangle
$$

o que nos permite determinar os elementos da matriz $g^{\star}$ que representa esse tensor.

\subsubsection{Relação entre Bases e Coordenadas Covariantes e Con- travariantes}

Suponhamos que exista uma matriz $M$ que relacione as bases original $B$ e coadjunta $\dot{B}$ (covariante e contravariante, respectivamente), de modo que $\dot{B} \stackrel{M}{\longrightarrow} B$. Vamos mostrar que essa matriz existe e é, na verdade, a própria matriz $g$. Para isso, façamos $g_{i k}=\sum_{r}^{n} \delta_{i}^{r} g_{r k}$ 
de modo que obtenhamos, utilizando a relação de dualidade (2.13):

$$
\left\langle e_{i} \mid e_{k}\right\rangle=g_{i k}=\sum_{r}^{n} \delta_{i}^{r} g_{r k}=\sum_{r}^{n}\left\langle e_{i} \mid e^{r}\right\rangle g_{r k}
$$

Logo, a equação

$$
\left\langle e_{i} \mid e_{k}\right\rangle=\left\langle e_{i}\right|\left(\sum_{r}^{n}\left|e^{r}\right\rangle g_{r k}\right)
$$

implica, pela completude da base, que

$$
\left|e_{k}\right\rangle=\sum_{r}^{n}\left|e^{r}\right\rangle g_{r k}
$$

ou, em termos matriciais,

$$
\mid B]=\mid \dot{B}] g \quad \text { e } \quad|\mathrm{B}\rangle=\mathrm{g}^{\prime}|\dot{\mathrm{B}}\rangle
$$

na primeira e segunda representações, respectivamente. Constata-se, por conseguinte, que a matriz de transformação da base coadjunta à original é a matriz $g$, como havíamos adiantado.

De (2.34) e da invariança vetorial sob mudança de base, decorre a lei de transformação das coordenadas contravariantes às covariantes:

$$
\begin{gathered}
|u\rangle=\sum_{k}^{n} u^{k}\left|e_{k}\right\rangle=\sum_{k}^{n} u^{k} \sum_{r}^{n}\left|e^{r}\right\rangle g_{r k} \equiv \sum_{r}^{n} u_{r}\left|e^{r}\right\rangle \Rightarrow \\
u_{r}=\sum_{k}^{n} g_{r k} u^{k}
\end{gathered}
$$

ou, matricialmente,

$$
|\underline{u}\rangle_{\mid B]}=g|u\rangle_{\mid B]}
$$

Assim a matriz $g$, que leva da base coadjunta à original, transforma as coordenadas contravariantes em covariantes.

De (2.34) também vem outra expressão importante que revela a conexão entre o tensor métrico e o recíproco. Se efetuarmos um IP pela atuação de um ket $\left\langle e^{i}\right|$ à esquerda, segue

$$
\left\langle e^{i} \mid e_{k}\right\rangle=\delta^{i}{ }_{k}=\left\langle e^{i}\right|\left(\sum_{r}^{n}\left|e^{r}\right\rangle g_{r k}\right)=\sum_{r}^{n}\left\langle e^{i} \mid e^{r}\right\rangle g_{r k}=\sum_{r}^{n} g^{i r} g_{r k}
$$

Logo,

$$
\sum_{r}^{n} g^{i r} g_{r k}:=\delta_{k}^{i}
$$


Da adjunção de (2.38) decorre

$$
\sum_{r}^{n} g_{k r} g^{r i}:=\delta_{k}^{i}
$$

Isso significa que a matriz do tensor recíproco é a inversa da matriz do tensor métrico, quer dizer, denotando a primeira por $g^{\star}$, então $g^{\star}=g^{-1}$.

Procederemos de maneira análoga à anterior para determinar a transformação de base inversa:

$$
g^{i k}=\left\langle e^{i} \mid e^{k}\right\rangle=\sum_{r}^{n} g^{i r} \delta_{r}{ }^{k}=\sum_{r}^{n} g^{i r}\left\langle e_{r} \mid e^{k}\right\rangle \equiv\left(\sum_{r}^{n} g^{i r}\left\langle e_{r}\right|\right)\left|e^{k}\right\rangle
$$

obtendo, portanto,

$$
\left\langle e^{i}\right|=\sum_{r}^{n} g^{i r}\left\langle e_{r}\right|
$$

Efetuando uma adjunção nessa relação, obtém-se, devido à hermiticidade de $g$,

$$
\left|e^{i}\right\rangle=\sum_{r}^{n}\left|e_{r}\right\rangle g^{r i}
$$

ou, expressa matricialmente, na primeira e segunda representações:

$$
\mid \dot{B}]=\mid B] g^{\star} \quad \text { e } \quad|\dot{B}\rangle=\tilde{g}^{\star}|B\rangle
$$

Então, a matriz de transformação que leva a base original na coadjunta é $g^{\star}$.

Multiplicando a equação (2.36) por $g^{i r}$ e somando no segundo índice obtemos, usando (2.38):

$$
\sum_{r}^{n} g^{i r} u_{r}=\sum_{k}^{n} \sum_{r}^{n} g^{i r} g_{r k} u^{k}=\sum_{k}^{n} \delta_{k}^{i} u^{k}=u^{i}
$$

Logo, a transformação inversa a (2.36) é:

$$
u^{i}=\sum_{r}^{n} g^{i r} u_{r}
$$

$\mathrm{Ou}$

$$
|u\rangle_{\mid B]}=g^{\star}|\underline{u}\rangle_{\mid B]}
$$

Em outras palavras, os elementos de $g$ e de $g^{\star}$ atuam como operadores de transformação entre as coordenadas covariantes e as contravariantes.

Com os resultados obtidos nesta seção, podemos reescrever as equações (2.29) e (2.33), 
respectivamente, como

$$
\begin{array}{r}
\mathbb{P}^{\dagger}\left|e_{k}\right\rangle=\left|e_{k}\right\rangle \\
\mathbb{P}\left|e^{k}\right\rangle=\left|e^{k}\right\rangle
\end{array}
$$

Ao tomarmos as adjuntas dessas relações e considerarmos as outras anteriormente estabelecidas, constatamos que os projetores atuarão sempre como operadores e/ou retrooperadores identidade quando aplicados em componentes de bases, quaisquer que sejam.

\subsubsection{Relação entre os Projetores e o Tensor Métrico}

Se fizermos atuar o autoprojetor adjunto sobre o autoprojetor obteremos:

$$
\begin{gathered}
\mathbb{P}^{\dagger} \mathbb{P}=\left(\sum_{r}^{n}\left|e^{r}\right\rangle\left\langle e_{r}\right|\right)\left(\sum_{s}^{n}\left|e_{s}\right\rangle\left\langle e^{s}\right|\right) \equiv \sum_{r, s}^{n}\left|e^{r}\right\rangle\left\langle e_{r} \mid e_{s}\right\rangle\left\langle e^{s}\right|= \\
=\sum_{r, s}^{n}\left|e^{r}\right\rangle g_{r s}\left\langle e^{s}\right|=\overleftrightarrow{g}
\end{gathered}
$$

Logo, vê-se que o tensor métrico pode ser obtido da atuação de $\mathbb{P}^{\dagger}$ sobre $\mathbb{P}$ :

$$
\overleftrightarrow{g} \equiv \mathbb{P}^{\dagger} \mathbb{P}
$$

e funciona como um operador, que denominaremos operador métrico. Analogamente, define-se

$$
\overleftrightarrow{g^{\star}} \equiv \mathbb{P P}^{\dagger}
$$

denominado operador métrico recíproco. Além disso, a adjunção de (2.46) produz

$$
(\overleftrightarrow{g})^{\dagger} \equiv \mathbb{P}^{\dagger} \mathbb{P}=\overleftrightarrow{g}
$$

demonstrando, imediatamente, a hermiticidade do tensor métrico. Idem para (2.47) e o tensor recíproco. Adiante veremos que a atuação do operador métrico sobre uma função gera sua expansão em série.

\subsubsection{Operadores}

Já vimos que um tensor pode funcionar como um operador. Suponhamos que um vetor de $\mathcal{O}_{n}$ seja transformado em outro pela atuação de um operador T. Então,

$$
|v\rangle_{0_{n}}=T|u\rangle_{0_{n}}
$$


Usando o autoprojetor sobre $|v\rangle_{0_{n}}$, tem-se

$$
\begin{gathered}
\mathbb{P}|v\rangle=\mathbb{P}(T|u\rangle) \Rightarrow \\
\Rightarrow \sum_{i}^{n}\left|e_{i}\right\rangle\left\langle e^{i} \mid v\right\rangle_{W}=\sum_{i}^{n}\left|e_{i}\right\rangle\left\langle e^{i}|T| u\right\rangle_{W} \Rightarrow \\
\Rightarrow \sum_{i}^{n} v^{i}\left|e_{i}\right\rangle=\sum_{i}^{n}\left|e_{i}\right\rangle\left\langle e^{i}|T| \sum_{k}^{n} u^{k} \mid e_{k}\right\rangle=\sum_{i}^{n} \sum_{k}^{n}\left\langle e^{i}|T| e_{k}\right\rangle u^{k}\left|e_{i}\right\rangle
\end{gathered}
$$

Multiplicando os dois membros dessa expressão por $\left\langle e^{r}\right|$ à esquerda, segue

$$
\begin{aligned}
\left\langle e^{r}\left|\sum_{i}^{n} v^{i}\right| e_{i}\right\rangle=\left\langle e^{r}\left|\sum_{i}^{n} \sum_{k}^{n}\left\langle e^{i}|T| e_{k}\right\rangle u^{k}\right| e_{i}\right\rangle \Rightarrow \\
\Rightarrow \sum_{i}^{n} v^{i}\left\langle e^{r} \mid e_{i}\right\rangle=\sum_{i}^{n} \sum_{k}^{n}\left\langle e^{i}|T| e_{k}\right\rangle u^{k}\left\langle e^{r} \mid e_{i}\right\rangle \Rightarrow \\
\Rightarrow \sum_{i}^{n} \delta_{i}^{r} v^{i}=\sum_{i}^{n} \sum_{k}^{n} \delta^{r}\left\langle e^{i}|T| e_{k}\right\rangle u^{k} \Rightarrow \\
\Rightarrow v^{r}=\sum_{k}^{n}\left\langle e^{r}|T| e_{k}\right\rangle u^{k}
\end{aligned}
$$

Mas, matricialmente,

$$
v^{r}=\sum_{k}^{n} T_{k}^{r} u^{k}
$$

Logo, por comparação dos termos, decorre que um elemento do operador $T$ é obtido por:

$$
T_{k}^{r}=\left\langle e^{r}|T| e_{k}\right\rangle
$$

Agora, iremos estender esses conceitos aos espaços de funções.

\subsection{Formalismo Coadjunto em $\mathcal{L}^{2}$}

O objetivo a partir desse ponto é estender o que foi desenvolvido até agora para expansões em séries de funções. Naturalmente, vamos partir do caso mais genérico, ou seja, direcionado a funções quaisquer, desde que sejam, pelo menos, de classe $C^{0}$ e integráveis a Lebesgue. As séries envolvidas podem ter ou não as características usuais das séries conhecidas, quer dizer, não precisam, por exemplo, apresentar bases ortogonais.

Há alguns aspectos importantes a serem discutidos antes de adentrarmos na exposição propriamente dita. Em primeiro lugar, é preciso esclarecer que os vetores num espaço de 
Hilbert de funções complexas deverão ser funções analíticas numa determinada região de interesse. Um produto interno nesse espaço, por exemplo, deve ser definido por uma integração de duas funções analíticas num aberto $A \subseteq \mathbb{C}$ ao longo de um caminho $C$ qualquer, desde que $C \subset A$. Entretanto, vamos nos limitar, na maior parte desse trabalho, a funções complexas definidas apenas em domínios reais, visto que isso é suficiente para nosso objetivo.

Comecemos formalizando o conceito já bem conhecido de base e suas representações isomórficas em $\mathcal{O}_{n}(\mathcal{H})$.

Definição 6 : Dado um conjunto ordenado de funções $\left\{\phi_{i}(x)\right\}: \phi_{i} \in \mathcal{L}^{2}$, não necessariamente ortogonais, limitadas num intervalo fechado $I=[a, b] \subset \mathbb{R}$, com $\phi_{i}: I \rightarrow \mathbb{C} \mid \phi_{i} \in C^{0}[a, b]$, uma base $[\Phi]$ de $\mathcal{O}_{n}(\mathcal{H})$ é gerada pelo conjunto de kets $\left\{\left|\phi_{i}\right\rangle_{\infty}\right\}$.

Seja, portanto, $\left\{\phi_{i}(x)\right\}_{i=1}^{n}$ uma base genérica de $\mathcal{O}_{n}(\mathcal{H})$ expressa na $2^{\text {a }}$ representação por suas componentes $\left|\phi_{i}\right\rangle_{\infty}$ na forma de um vetor de base:

$$
|\phi\rangle_{0_{n}}=\left[\begin{array}{c}
\left|\phi_{1}\right\rangle \\
\left|\phi_{2}\right\rangle \\
\vdots \\
\left|\phi_{n}\right\rangle
\end{array}\right]
$$

Então, qualquer expansão em série de $n$ termos de uma função $f(x)$ considerada "bem-comportada", isto é, que satisfaça todas as exigências requeridas pela expansão na série arbitrada, poderá ser escrita como

$$
\left|f_{n}\right\rangle_{\infty}=\sum_{r}^{n} c^{r}\left|\phi_{r}\right\rangle_{\infty}
$$

O papel dos coeficientes $c^{r}$ é similar ao das coordenadas contravariantes numa base de $\mathcal{O}_{n}$, o que significa que também poderiam ser isomorficamente representados por um ket na base $\left\{\left|\phi_{i}\right\rangle\right\}$, ou seja, por um vetor coluna $\left[c^{r}\right]_{\phi}^{r=1, \ldots, n}$. Entretanto, vamos tratá-los de uma forma mais conveniente à abordagem funcional, definindo esse conjunto como um bra, denominado covetor coeficiente, o que nos possibilita reescrever a função em série numa forma sintética com a notação de Dirac. Formalizando o que foi dito: 
Definição 7 : Dada uma base na $2^{a}$ representação:

$$
|\phi\rangle_{0_{n}(\mathcal{H})}:=\left[\begin{array}{c}
\mid \\
\left|\phi_{r}\right\rangle_{\infty} \\
\mid
\end{array}\right]_{r=1, \ldots, n}
$$

a expansão de ordem $n$ de uma função $f \in C^{0}[a, b]$ limitada em $I=[a, b] \subset \mathbb{R}$ será dada por

$$
\left|f_{n}\right\rangle_{\infty}:=\sum_{r}^{n} c^{r(n)}\left|\phi_{r}\right\rangle_{\infty},
$$

onde o conjunto de escalares complexos $\left\{c^{r(n)}\right\}_{r=1}^{n}$ é formado pelos coeficientes contravariantes da expansão em série de $n$ termos na base dada.

Definição 8 : Dados os coeficientes contravariantes $\left\{c^{r(n)}\right\}_{r=1}^{n}$ da expansão em série de $n$ termos de uma função $f$ numa dada base, define-se o covetor dos coeficientes ou covetor coeficiente $\langle c| \in \mathcal{O}_{n}^{\star}(\mathbb{C})$ por:

$$
\langle c| \simeq\left[\begin{array}{lll}
c^{1(n)} & \cdots & c^{n(n)}
\end{array}\right]
$$

Uma vez que não ocorre de a ordem das matrizes ser alterada ao longo de cada problema específico, não há necessidade de manter o ìndice $n$ sobrescrito aos coeficientes, bastando escrever $c^{r}$, subentendendo-se que o conjunto de coeficientes é referente a uma certa ordem $n$ de expansão e não a qualquer outra.

Espaço Primitivo Um ponto importante a ser ressaltado aqui é que, nessa apresentação, conforme foi mencionado na subseção (2.2.6), referente às representações correlatas, o espaço dual passa a ser entendido como o "ambiente natural" dos coeficientes da função. Por isso, o espaço dual será chamado aqui de espaço primitivo do covetor coeficiente, já que a nomenclatura de espaço original é reservada ao espaço dos kets. Assim, as operações de transformação sobre $\langle c|$ fornecem:

$$
\left\langle\left. c\right|^{*}=\langle\bar{c}| \simeq\left[\begin{array}{lll}
\bar{c}^{1} & \ldots & \bar{c}^{n}
\end{array}\right], \quad\left\langle\left. c\right|^{\dagger}=\mid c\right\rangle \simeq\left[\begin{array}{c}
\bar{c}^{1} \\
\vdots \\
\bar{c}^{n}
\end{array}\right], \quad\left\langle\left. c\right|^{\prime}=\mid \bar{c}\right\rangle \simeq\left[\begin{array}{c}
c^{1} \\
\vdots \\
c^{n}
\end{array}\right]\right.
$$

Nesses termos, o IP deve ser reelaborado para adaptar-se a essa apresentação. Aqui cabe um esclarecimento importante. Devemos notar que, do ponto de vista formal, o covetor coeficiente e o vetor de base pertencem a espaços de naturezas distintas, i.e., 
$\langle c| \in \mathcal{O}_{n}^{\star}(\mathbb{C}) \simeq \mathbb{C}^{n}$ e $|\phi\rangle \in \mathcal{O}_{n}(\mathcal{H})$, o que deveria ser indicado na notação como $\left\langle\left. c\right|_{0_{n}^{\star}(\mathbb{C})} \mathrm{e}\right.$ $|\phi\rangle_{0_{n}(\mathcal{H})}$, grafia omitida por questão de concisão. Por esse motivo, o IP constituído entre eles é misto, sendo denotado por $\langle c \mid \phi\rangle_{\mathbf{C H}}$. Contudo, poderá ser grafado sinteticamente por $\langle c \mid \phi\rangle$, desde que sejam subentendidas e observadas todas as condições anteriores.

É claro que essa definição mista difere da concepção apresentada no desenvolvimento da reformulação coadjunta, pois lá foram estabelecidos dois produtos, o hermitiano e o dual, de acordo com o tipo de bra que participa da operação, sendo ambos definidos em um domínio diferente deste. Mais especificamente, aquele domínio é o produto cartesiano do espaço original por ele mesmo, enquanto neste participa também o espaço dual.

Definição 9 : O IP misto $\langle\mid\rangle_{\mathbf{C H}}$ é definido como uma aplicação

$$
\left.\langle\mid\rangle: \mathcal{O}_{n}^{\star}(\mathbb{C}) \times \mathcal{O}_{n}(\mathcal{H}) \longrightarrow \mathbb{C}\left|\langle c \mid \phi\rangle_{\mathbb{C H}}:=\sum_{r}^{n} c^{r}\right| \phi_{r}\right\rangle_{\infty}
$$

Obs: Na formulação da expansão em série de funções, esse IP misto será amiúde denotado sem o índice subscrito: $\langle\mid\rangle$, uma vez que constituirá o padrão, aparecendo com frequência.

Observadas tais condições, tem-se, portanto,

$$
\left|f_{n}\right\rangle_{\infty}=\langle c \mid \phi\rangle
$$

O interessante é que essa notação pode ser, inclusive, utilizada de forma simbólica para representar séries infinitas, ou seja, pode-se escrever

$$
|f\rangle_{\infty}=\langle c \mid \phi\rangle
$$

querendo-se sintetizar a série infinita

$$
|f\rangle_{\infty}=\sum_{r}^{\infty} c^{r}\left|\phi_{r}\right\rangle_{\infty}
$$

A soma infinita fica subentendida pela ausência do índice $n$ na função $f$.

\subsubsection{Bases Correlatas em $\mathcal{O}_{n}(\mathcal{H})$}

Podemos reproduzir os mesmos resultados obtidos na reformulação coadjunta para definir bases correlatas a uma base original $|\phi\rangle \simeq\left[\begin{array}{c}\left|\phi_{1}\right\rangle_{\infty} \\ \vdots \\ \left|\phi_{n}\right\rangle_{\infty}\end{array}\right]$ no espaço $\mathcal{O}_{n}(\mathcal{H})$. São elas: 
- Base adjunta: $\langle\phi| \simeq\left[\begin{array}{lll}\left\langle\left.\phi_{1}\right|_{\infty}\right. & \cdots & \left\langle\left.\phi_{n}\right|_{\infty}\right.\end{array}\right]$

- Base recíproca: $\langle\dot{\phi}| \simeq\left[\begin{array}{lll}\left\langle\left.\phi^{1}\right|_{\infty}\right. & \cdots & \left\langle\left.\phi^{n}\right|_{\infty}\right.\end{array}\right]$

- Base coadjunta: $|\dot{\phi}\rangle \simeq\left[\begin{array}{c}\left|\phi^{1}\right\rangle_{\infty} \\ \vdots \\ \left|\phi^{n}\right\rangle_{\infty}\end{array}\right]$

Obs: Deve-se notar que, como a base primitiva é covariante, a base coadjunta é contravariante e, por isso, o ponto vai em cima do símbolo e não em baixo, como no caso das covariantes obtidas a partir de kets no espaço original.

As componentes contravariantes das bases recíproca e coadjunta são obtidas das relações de dualidade em $\mathcal{H}$ :

$$
\left\langle\phi^{i} \mid \phi_{k}\right\rangle_{\infty}:=\delta_{k}^{i}, \quad\left\langle\phi_{r} \mid \phi^{s}\right\rangle_{\infty}:=\delta_{r}{ }^{s}
$$

É claro que, se o IP for definido em termos de uma função peso, as equações devem ser escritas com $\langle\mid\rangle_{W}$ em vez de $\langle\mid\rangle_{\infty}$.

Da mesma forma que na reformulação coadjunta em $\mathcal{O}_{n}$, também a função expandida em série pode escrita de quatro formas diferentes, duas covariantes e duas contravariantes, conforme a natureza da base escolhida, a saber:

- Original:

$$
|f\rangle=\sum_{r}^{\infty} c^{r}\left|\phi_{r}\right\rangle \equiv\langle c \mid \phi\rangle
$$

- Adjunta:

$$
\langle f|=\sum_{r}^{\infty}\left\langle\phi_{r}\right| \bar{c}^{r} \equiv\langle\phi \mid c\rangle
$$

- Recíproca:

$$
\langle f|=\sum_{r}^{\infty}\left\langle\phi^{r}\right| \bar{c}_{r} \equiv\langle\dot{\phi} \mid \mathrm{c}\rangle
$$

- Coadjunta:

$$
|f\rangle=\sum_{r}^{\infty} c_{r}\left|\phi^{r}\right\rangle \equiv\langle c \mid \dot{\phi}\rangle
$$

Apesar de havermos definido o covetor coeficiente, as equações acima possibilitam também, caso seja conveniente, estabelecer as representações da função em termos de seus coeficientes na base $|\phi\rangle$, que são suas coordenadas contravariantes nessa base: 
- Repr. original:

$$
|f\rangle_{\phi} \simeq\left[\begin{array}{c}
c^{1} \\
\vdots \\
c^{n}
\end{array}\right]
$$

- Repr. adjunta:

$$
\left\langle\left. f\right|_{\phi} \simeq\left[\begin{array}{lll}
\bar{c}^{1} & \cdots & \bar{c}^{n}
\end{array}\right]\right.
$$

- Repr. dual:

$$
\left\langle\left.\mathrm{f}\right|_{\phi} \simeq\left[\begin{array}{lll}
\bar{c}_{1} & \cdots & \bar{c}_{n}
\end{array}\right]\right.
$$

- Repr. coadjunta:

$$
|\mathrm{f}\rangle_{\phi} \simeq\left[\begin{array}{c}
c_{1} \\
\vdots \\
c_{n}
\end{array}\right]
$$

\subsubsection{Obtenção dos Coeficientes}

Usando a definição de IP com peso $W$ em $\mathcal{H}$ (sendo o IP de peso unitário grafado com subíndice $\infty$ ) e as relações de dualidade (2.53), é possível determinar os coeficientes de uma função em série:

$$
\left\langle\phi^{r} \mid f\right\rangle_{W}=\left\langle\phi^{r}\left|\sum_{k}^{\infty} c^{k}\right| \phi_{k}\right\rangle_{W}=\sum_{k}^{\infty} \delta_{k}^{r} c^{k}=c^{r}
$$

Logo,

$$
c^{r}=\left\langle\phi^{r} \mid f\right\rangle_{W}
$$

e, de

$$
\left\langle\phi_{r} \mid f\right\rangle_{W}=\left\langle\phi_{r}\left|\sum_{k}^{\infty} c_{k}\right| \phi^{k}\right\rangle_{W}=\sum_{k}^{\infty} \delta_{r}^{k} c_{k}=c_{r}
$$

vem

$$
c_{r}=\left\langle\phi_{r} \mid f\right\rangle_{W}
$$

\subsubsection{Autoprojetor em $\mathcal{O}_{n}(\mathcal{H})$}

De forma similar a $\mathcal{O}_{n}(\mathbb{C})$, também em $\mathcal{O}_{n}(\mathcal{H})$ se pode utilizar um projetor, definido como

$$
\mathbb{P}_{m}=\sum_{i}^{m}\left|\phi_{i}\right\rangle\left\langle\phi^{i}\right| \quad(m \leq n),
$$


bem como seu adjunto:

$$
\mathbb{P}_{m}^{\dagger}=\sum_{i}^{m}\left|\phi^{i}\right\rangle\left\langle\phi_{i}\right| \quad(m \leq n)
$$

O autoprojetor é simplesmente $\mathbb{P}_{n} \equiv \mathbb{P}$ e o autoprojetor adjunto, $\mathbb{P}^{\dagger}$. Podemos aplicar este último, por exemplo, sobre uma componente $\left|\phi_{k}\right\rangle$ da base $|\phi\rangle$, obtendo

$$
\mathbb{P}_{n}^{\dagger}\left|\phi_{k}\right\rangle=\sum_{i}^{n}\left|\phi^{i}\right\rangle\left\langle\phi_{i} \mid \phi_{k}\right\rangle_{W}
$$

Definindo, então, o elemento da métrica $\gamma_{i k}$ :

$$
\gamma_{i k}:=\left\langle\phi_{i} \mid \phi_{k}\right\rangle_{W}
$$

a aplicação do autoprojetor adjunto de $\mathcal{O}_{n}(\mathcal{H})$ sobre $\left|\phi_{k}\right\rangle$ torna-se

$$
\mathbb{P}^{\dagger}\left|\phi_{k}\right\rangle=\sum_{i}^{n}\left|\phi^{i}\right\rangle \gamma_{i k}
$$

Analogamente, aplicando-se o autoprojetor sobre uma componente da base $|\dot{\phi}\rangle$, obtemos

$$
\mathbb{P}\left|\phi^{k}\right\rangle=\sum_{i}^{n}\left|\phi_{i}\right\rangle\left\langle\phi^{i} \mid \phi^{k}\right\rangle_{W}:=\sum_{i}^{n}\left|\phi_{i}\right\rangle \gamma^{i k}
$$

onde

$$
\gamma^{i k}:=\left\langle\phi^{i} \mid \phi^{k}\right\rangle_{W}
$$

é um elemento da métrica recíproca em $\mathcal{O}_{n}(\mathcal{H})$.

\subsubsection{Relações entre Coordenadas Covariantes e Contravarian- tes em $\mathcal{O}_{n}(\mathcal{H})$}

Observando as equações (2.53) e (2.58), escrevemos:

$$
\left\langle\phi_{i} \mid \phi_{k}\right\rangle_{W}=\gamma_{i k}=\sum_{r}^{n} \delta_{i}{ }^{r} \gamma_{r k}=\sum_{r}^{n}\left\langle\phi_{i} \mid \phi^{r}\right\rangle_{W} \gamma_{r k} \equiv\left\langle\phi_{i}\right|\left(\sum_{r}^{n}\left|\phi^{r}\right\rangle_{W} \gamma_{r k}\right)
$$

Logo,

$$
\left|\phi_{k}\right\rangle=\sum_{r}^{n}\left|\phi^{r}\right\rangle \gamma_{r k}
$$

Procedendo de modo semelhante ao que foi efetuado na subseção 2.2.11, são estabe- 
lecidas as relações para a base recíproca e coadjunta:

$$
\left\langle\phi^{i}\left|=\sum_{r}^{n} \gamma^{i r}\left\langle\phi_{r}|, \quad| \phi^{i}\right\rangle=\sum_{r}^{n}\right| \phi_{r}\right\rangle \gamma^{r i}
$$

e para as coordenadas:

$$
u^{i}=\sum_{k}^{n} \gamma^{i k} u_{k}, \quad u_{i}=\sum_{k}^{n} \gamma_{i k} u^{k},
$$

além da relação de reciprocidade entre as métricas:

$$
\gamma^{\star}=\gamma^{-1}
$$

Matricialmente, as transformações entre as bases e as coordenadas são escritas:

$$
\begin{array}{ll}
\mid \dot{\phi}]=\mid \phi] \gamma^{\star}, & \mid \phi]=\mid \dot{\phi}] \gamma \\
|\dot{\phi}\rangle=\tilde{\gamma}^{\star}|\phi\rangle, & |\phi\rangle=\gamma^{\prime}|\dot{\phi}\rangle \\
|\underline{u}\rangle_{\mid \phi]}=\gamma|u\rangle_{\mid \phi]} & |u\rangle_{\mid \phi]}=\gamma^{\star}|\underline{u}\rangle_{\mid \phi]}
\end{array}
$$

\subsubsection{Operadores Métricos}

Assim como no caso de $\mathcal{O}_{n}(\mathbb{C})$, também aqui usamos os autoprojetores para definir o operador métrico $\overleftrightarrow{\gamma}$ e o operador métrico recíproco $\overleftrightarrow{\gamma}^{\star}$, respectivamente, como

$$
\overleftrightarrow{\gamma}=\mathbb{P}^{\dagger} \mathbb{P}, \quad \overleftrightarrow{\gamma^{\star}}=\mathbb{P P}^{\dagger}
$$

\subsubsection{Expansão em Série de $n$ Termos}

Com a ajuda do autoprojetor em $\mathcal{O}_{n}$, pode-se escrever de maneira sintética e elegante a expansão desenvolvida em $n$ termos de uma função qualquer, desde que essa obedeça às condições particulares exigidas pela base arbitrada. Então, nessas circunstâncias, tem-se, usando a equação (2.54)

$$
\left|f_{n}\right\rangle=\mathbb{P}|f\rangle=\sum_{r}^{n}\left|\phi_{r}\right\rangle\left\langle\phi^{r} \mid f\right\rangle_{W} \equiv \sum_{r}^{n} c^{r}\left|\phi_{r}\right\rangle
$$

que é idêntica à equação (2.49). Se, por outro lado, aplicarmos o operador métrico recíproco a uma função $|f\rangle$, obteremos a mesma expansão em termos dos coeficientes 
covariantes:

$$
\overleftrightarrow{\gamma}^{\star}|f\rangle \equiv \mathbb{P P}^{\dagger}|f\rangle=\sum_{r}^{n} \sum_{k}^{n}\left|\phi_{k}\right\rangle \gamma^{k r}\left\langle\phi_{r} \mid f\right\rangle_{W},
$$

onde, pela equação (2.55), identificamos os coeficientes covariantes, resultando:

$$
\overleftrightarrow{\gamma}^{\star}|f\rangle=\sum_{k}^{n} \sum_{r}^{n} \gamma^{k r} c_{r}\left|\phi_{k}\right\rangle
$$

e

$$
c^{k}=\sum_{r}^{n} \gamma^{k r}\left\langle\phi_{r} \mid f\right\rangle_{W},
$$

Naturalmente, como $\sum_{r}^{n} \gamma^{k r} c_{r}=c^{k}$, as expressões acima fornecem a mesma expansão obtida através do uso do autoprojetor. Entretanto, mais adiante, quando lidarmos com IP híbrido e mudanças de base, o operador métrico desempenhará um papel fundamental.

\subsubsection{Aplicações Práticas do Formalismo}

Vamos aplicar o formalismo descrito em casos concretos. O primeiro, naturalmente, é o foco de nosso interesse, a série de Fourier complexa. Após isto, faremos uma breve incursão nos polinômios de Legendre e na base polinomial canônica e, por fim, vamos expandir uma função numa outra base não-ortogonal e mensurar o erro quadrático, a fim de ilustrar a eficiência do processo.

\subsubsection{Aplicação à Série Complexa de Fourier}

Vamos definir, para a expansão em séries de Fourier complexas com $n=2 \ell+1$ termos, duas grandezas auxiliares:

- Razão periódica: $q=\pi / L$

- Extensão (range): $\ell \in \mathbb{N}$

Uma componente de índice $m$ da base complexa de Fourier, assume então a forma $e^{i m q x}$. Mas assumiremos, ainda, uma outra simplificação de notação que será de bastante utilidade para a concisão das equações. Grafaremos cada elemento da base por $e_{m}(x)$ ou, simplesmente, $\left|e_{m}\right\rangle_{\infty}$. Como o índice $m$ corre de $-\ell$ a $+\ell$, os índices negativos serão do tipo $\exp (-i k q x), k>0$ e, portanto, usando a notação de conjugação, podemos escrevê-lo 
como $\bar{e}_{m}(x)$ ou $\left|\bar{e}_{m}\right\rangle_{\infty}$. Assim, para $q=\pi / L$, temos:

$$
\begin{aligned}
\left|e_{m}\right\rangle_{\infty} & \equiv e_{m}(x)=\exp (i m q x) \\
\left|\bar{e}_{m}\right\rangle_{\infty} & \equiv \bar{e}_{m}(x)=\exp (-i m q x)
\end{aligned}
$$

Nessas circunstâncias, a base original na $2^{\mathrm{a}}$ representação para a série de Fourier complexa com $n=2 \ell+1$ termos é escrita $|e\rangle_{0_{n}}=\left[\left|e_{m}\right\rangle_{\infty}\right]_{m=-\ell}^{\ell}$, assumindo a forma explícita:

$$
|e\rangle_{0_{N}}=\left[\begin{array}{c}
\left|e_{-\ell}\right\rangle_{\infty} \\
\vdots \\
\left|e_{-1}\right\rangle_{\infty} \\
\left|e_{0}\right\rangle_{\infty} \\
\left|e_{1}\right\rangle_{\infty} \\
\vdots \\
\left|e_{\ell}\right\rangle_{\infty}
\end{array}\right] \equiv\left[\begin{array}{c}
\left|\bar{e}_{\ell}\right\rangle_{\infty} \\
\vdots \\
\left|\bar{e}_{1}\right\rangle_{\infty} \\
\left|e_{0}\right\rangle_{\infty} \\
\left|e_{1}\right\rangle_{\infty} \\
\vdots \\
\left|e_{\ell}\right\rangle_{\infty}
\end{array}\right]
$$

onde $\left|e_{0}\right\rangle_{\infty}=1$, naturalmente. Uma vez definida esta base, obtém-se facilmente a base adjunta:

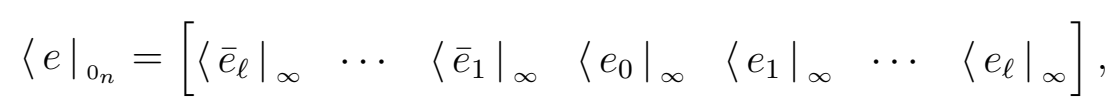

onde $\left\langle\left. e_{k}\right|_{\infty} \equiv \bar{e}_{k}(x)\right.$. Feito isto, aplicamos o operador métrico recíproco sobre a função escolhida para construir a expansão em série, obtendo, de acordo com a equação (2.68):

$$
\left|f_{n}\right\rangle_{\infty}=\overleftrightarrow{\gamma}^{\star}|f\rangle_{\infty}=\sum_{k, r=-\ell}^{\ell} \gamma^{k r} c_{r}\left|e_{k}\right\rangle_{\infty}=\sum_{k=-\ell}^{\ell} c^{k}\left|e_{k}\right\rangle_{\infty}
$$

Entretanto, ainda é preciso determinar a base recíproca para que possamos calcular os elementos do tensor recíproco na fórmula da expansão acima. Felizmente, como a base de Fourier é ortogonal, já que $\left\langle e_{i} \mid e_{k}\right\rangle=0, \forall i \neq k$, o tensor métrico é representado por uma matriz diagonal, de modo que

$$
\gamma_{r s}=\left\langle e_{r} \mid e_{s}\right\rangle_{W}=2 L \delta_{r s} \Longrightarrow \gamma^{i k}=\frac{1}{2 L} \delta^{i k}
$$

Logo,

$$
\left|e^{m}\right\rangle=\sum_{r=-\ell}^{\ell}\left|e_{r}\right\rangle \gamma^{r m} \Rightarrow\left\langle e^{m}\right|=\sum_{r=-\ell}^{\ell} \gamma^{m r}\left\langle e_{r}\right|=\sum_{r=-\ell}^{\ell} \frac{1}{2 L} \delta^{m r}\left\langle e_{r}\right|=\frac{1}{2 L}\left\langle e_{m}\right|,
$$

sendo

$$
\left|e^{m}\right\rangle=2 L\left|e_{m}\right\rangle
$$


a forma geral dos elementos da base recíproca de Fourier no semiperíodo $L$.

Mas deve-se notar que as componentes contravariantes da fórmula (2.72) são dadas por $c^{r}=\left\langle e^{r} \mid f\right\rangle_{\infty}$ e, assim, segue que os coeficientes serão

$$
c^{r}=\left\langle e^{r} \mid f\right\rangle=\frac{1}{2 L}\left\langle e_{r} \mid f\right\rangle=\frac{1}{2 L} \int_{-L}^{L} \bar{e}_{r}(x) f(x) d x,
$$

os quais devem ser dispostos no covetor coeficiente como

$$
\left\langle\left. c\right|_{o_{n}}=\left[\begin{array}{lllllllll}
c^{-\ell} & c^{-\ell+1} & \cdots & c^{-1} & c^{0} & c^{1} & \cdots & c^{\ell-1} & c^{\ell}
\end{array}\right]\right.
$$

Com isso, a função aproximada torna-se

$$
\left|f_{n}\right\rangle_{\infty}=\sum_{m=-\ell}^{\ell} c^{m}\left|e_{m}\right\rangle_{\infty} \equiv\langle c \mid e\rangle_{\mathrm{CH}},
$$

onde

$$
c^{m}=\left\langle e^{m} \mid f\right\rangle_{\infty}
$$

ou, na forma explícita:

$$
\left|f_{n}\right\rangle_{\infty}=\sum_{m=-\ell}^{\ell} \frac{1}{2 L} \int_{-L}^{L} \bar{e}_{m}(x) f(x) d x\left|e_{m}\right\rangle_{\infty}
$$

Logo, conseguimos, através desse formalismo, reproduzir o resultado tradicional:

$$
f_{n}(x)=\sum_{m=-\ell}^{\ell} e_{m}(x) \frac{1}{2 L} \int_{-L}^{L} \bar{e}_{m}(\xi) f(\xi) d \xi
$$

com o termo para $m=0$ incluso na expressão.

Translação de Range O tratamento matemático com a extensão tradicional de Fourier complexo, que abarca índices positivos e negativos, apresenta certas dificuldades relacionadas à computação. Assim, por conveniência operacional, a fim de que coeficientes e bases se adaptem aos índices matriciais no processamento dos dados, é interessante fazer um ajuste da extensão para valores apenas positivos, com um processo simples de redenominação indicial, que chamamos de translação de range.

Com esse intuito, dado um certo $\ell \in \mathbb{N}$ efetua-se a substituição de um conjunto de valores a outro, isto é, $\{k \in \mathbb{Z}:|k| \leq \ell\} \longrightarrow\left\{r \in \mathbb{N}^{*}: r=k+\ell+1\right\}$, o que implica $r \in[1, n] \subset \mathbb{N}, n=2 \ell+1$. Com isso, os coeficientes do covetor serão reescritos como

$$
c^{k} \rightarrow C^{r}=c^{r-\ell-1}, \quad(r=1, \ldots, n=2 \ell+1)
$$


na forma

$$
\left[\begin{array}{lllllllll}
C^{1} & C^{2} & \cdots & C^{\ell} & C^{\ell+1} & C^{\ell+2} & \cdots & C^{2 \ell} & C^{2 \ell+1}
\end{array}\right]=\left\langle\left. C\right|_{o_{n}},\right.
$$

o que nos remete a uma renotação dos elementos da base:

$$
E_{k}(x)=\exp [i q(k-\ell-1) x] \equiv\left|E_{k}\right\rangle_{\infty}, \quad(k=1, \ldots, 2 \ell+1)
$$

e, finalmente, à grafia da função aproximada:

$$
\left|f_{n}\right\rangle_{\infty}=\langle C \mid E\rangle_{\mathrm{CH}} \equiv \sum_{i=1}^{N} C^{i}\left|E_{i}\right\rangle_{\infty}, \quad(N=2 \ell+1)
$$

Chamaremos esse novo covetor $\left\langle\left. C\right|_{0_{n}} \text { de covetor coeficiente ajustado e a base } \mid E\right\rangle_{0_{n}}$, de base ajustada.

De fato, os elementos da base recíproca de qualquer base ortogonal $\left|b_{r}\right\rangle$ são dados pela fórmula geral $\left|b^{m}\right\rangle=\alpha_{m}^{-1}\left|b_{m}\right\rangle, \quad \alpha_{r}=\left\langle b_{r} \mid b_{r}\right\rangle$.

\subsubsection{Aplicação aos Polinômios de Legendre}

Apenas como ilustração, vamos aplicar esse formalismo à base dos polinômios de Legendre, que podem ser escritos como

$$
P_{n}(x)=\sum_{r=0}^{[n / 2]}(-1)^{r} \frac{(2 n-2 r) !}{2^{n} r !(n-r) !(n-2 r) !} x^{n-2 r}
$$

A relação de ortogonalidade fornece

$$
\gamma_{m n}=\left\langle P_{m} \mid P_{n}\right\rangle=\frac{2}{2 n+1} \delta^{m}{ }_{n}
$$

e, portanto, $\gamma^{m n}=\left(n+\frac{1}{2}\right) \delta_{n}^{m}$. Logo, a base de Legendre recíproca será dada pelos polinômios

$$
\left|P^{n}\right\rangle=\sum_{m=0}^{n-1}\left|P_{m}\right\rangle \gamma^{m n}=\sum_{m=0}^{n-1}\left|P_{m}\right\rangle\left(n+\frac{1}{2}\right) \delta_{n}^{m}=\left(n+\frac{1}{2}\right)\left|P_{n}\right\rangle
$$

Assim, uma função terá a seguinte expansão em série de Legendre:

$$
\left|f_{n}\right\rangle_{\infty}=\overleftrightarrow{\gamma}^{\star}|f\rangle_{\infty}=\sum_{k=0}^{n-1} \gamma^{k r} a_{r}\left|P_{k}\right\rangle_{\infty}=\sum_{k=0}^{n-1} a^{k}\left|P_{k}\right\rangle_{\infty}
$$

onde

$$
a^{n}=\left\langle P^{n} \mid f\right\rangle=\left(n+\frac{1}{2}\right) \int_{-1}^{1} P_{n}(x) f(x) d x
$$


Para calcular os coeficientes, basta substituir a forma do polinômio (2.76) na equação acima e calcular a integral

$$
\int_{-1}^{1} x^{n-2 r} f(x) d x
$$

para a função $f$ desejada. Evidentemente, também se pode usar a fórmula de Rodrigues para calcular os coeficientes. Listamos abaixo os cinco primeiros polinômios recíprocos de Legendre:

$$
\begin{gathered}
P^{0}(x)=\frac{1}{2} \\
P^{1}(x)=\frac{3 x}{2} \\
P^{2}(x)=\frac{5}{4}\left(3 x^{2}-1\right) \\
P^{3}(x)=\frac{7 x}{4}\left(5 x^{2}-3\right) \\
P^{4}(x)=\frac{9}{16}\left(35 x^{4}-30 x^{2}+3\right)
\end{gathered}
$$

De forma semelhante, podem-se determinar as expansões com seus respectivos coeficientes em qualquer base que se deseje, mesmo as não-ortogonais.

\subsubsection{Exemplo em Base Não-ortogonal}

Até agora, o que foi feito não constitui propriamente uma novidade, pois a base recíproca de uma ortogonal é simplesmente esta mesma dividida pelo fator de normalização, sendo portanto idêntica à base ortonormalizada. Com bases oblíquas, no entanto, o mecanismo não é tão simples.

Como exemplo de aplicação desse formalismo a uma base não-ortogonal, podemos optar pela canônica no domínio $D_{1}=[-1,1]$, que é o caso mais comum de não-ortogonalidade, sendo definida pelos polinômios

$$
\epsilon_{m}(x)=x^{m}, \quad m=0,1, \ldots, n-1
$$

A expressão analítica para os elementos do tensor métrico no domínio $D_{1}$ é dada pela fórmula:

$$
\gamma_{r s}=\frac{2 \operatorname{par}(r+s)}{r+s+1}
$$

sendo

$$
\operatorname{par}(k):=1-\bmod (k, 2)= \begin{cases}1, & \text { se } k \text { é par } \\ 0, & \text { se } k \text { é ímpar }\end{cases}
$$


o que fornece, para $n=5$, a seguinte matriz métrica:

$$
\gamma[\epsilon]=\left[\begin{array}{ccccc}
2 & 0 & 2 / 3 & 0 & 2 / 5 \\
0 & 2 / 3 & 0 & 2 / 5 & 0 \\
2 / 3 & 0 & 2 / 5 & 0 & 2 / 7 \\
0 & 2 / 5 & 0 & 2 / 7 & 0 \\
2 / 5 & 0 & 2 / 7 & 0 & 2 / 9
\end{array}\right]
$$

Assim, a matriz de seu tensor recíproco será:

$$
\gamma^{-1}[\epsilon]=\left[\begin{array}{ccccc}
\frac{225}{128} & 0 & -\frac{525}{64} & 0 & \frac{945}{128} \\
0 & \frac{75}{8} & 0 & -\frac{105}{8} & 0 \\
-\frac{525}{64} & 0 & \frac{2205}{32} & 0 & \frac{4725}{64} \\
0 & -\frac{105}{8} & 0 & \frac{175}{8} & 0 \\
\frac{945}{128} & 0 & \frac{4725}{64} & 0 & \frac{11025}{128}
\end{array}\right]
$$

Usando o formalismo acima também se pode estabelecer a base recíproca da canônica, cujos cinco primeiros polinômios, para esse específico valor de $n$, estão listados a seguir:

$$
\begin{aligned}
& \epsilon^{0}(x)=225 / 128-\left(525 x^{2}\right) / 64+\left(945 x^{4}\right) / 128 \\
& \epsilon^{1}(x)=(75 x) / 8-\left(105 x^{3}\right) / 8 \\
& \epsilon^{2}(x)=-525 / 64+\left(2205 x^{2}\right) / 32-\left(4725 x^{4}\right) / 64 \\
& \epsilon^{3}(x)=-(105 x) / 8+\left(175 x^{3}\right) / 8 \\
& \epsilon^{4}(x)=945 / 128-\left(4725 x^{2}\right) / 64+\left(11025 x^{4}\right) / 128
\end{aligned}
$$

É preciso lembrar que esse conjunto de polinômios é característico desse range. Se a ordem mudar, todos os polinômios mudam também, ao contrário das bases ortogonais, cujos polinômios anteriores se mantêm com o aumento da ordem de expansão. Aliás, esse é um dos inconvenientes das bases oblíquas, motivo pelo qual, normalmente se opta por trabalhar com as ortogonais.

\subsubsection{Expansão em Base Não-Ortogonal}

Vamos generalizar um pouco mais, tomando uma base diferente e expandindo uma função em série nessa base.

Seja uma base não-ortogonal definida no domínio $D=[-1,1]$ pelos elementos

$$
Q_{r}(x)=r x^{r-1}+i\left(1-\delta_{1 r}\right) x^{r-2}, \quad r \geq 1
$$


Então, a expansão de 10 termos da função $f(x)=e^{-2 \pi x^{2}}$ :

$$
\left|f_{n}\right\rangle_{\infty}=\overleftrightarrow{\gamma}^{\star}|f\rangle_{\infty}=\sum_{k=1}^{n} \sum_{r=1}^{n} \gamma^{k r} \mu_{r}\left|Q_{k}\right\rangle_{\infty}=\sum_{k=1}^{n} \mu^{k}\left|Q_{k}\right\rangle_{\infty}
$$

com

$$
\mu^{k}=\sum_{r=1}^{n} \gamma^{k r}\left\langle Q_{r} \mid f\right\rangle
$$

para $n=10$, fornece:

$$
\begin{aligned}
f_{10}(x)= & (2.03777+0 . i)+(0 .+1.04832 i)(i+2 x)-(2.09663+0 . i)\left(i x+3 x^{2}\right)-(0 .+0.661302 i)\left(i x^{2}+4 x^{3}\right)+ \\
& +(2.64521+0 . i)\left(i x^{3}+5 x^{4}\right)+(0 .+0.320826 i)\left(i x^{4}+6 x^{5}\right)-(1.92496+0 . i)\left(i x^{5}+7 x^{6}\right)- \\
- & (0 .+0.071728 i)\left(i x^{6}+8 x^{7}\right)+(0.573824+0 . i)\left(i x^{7}+9 x^{8}\right)-\left(0 .+1.41842 \times 10^{-12} i\right)\left(i x^{8}+10 x^{9}\right)
\end{aligned}
$$

O gráfico comparativo entre a função original e a parte real de sua expansão em série para 10 termos aparece a seguir, na figura (2).

Figura 2: Comparação entre a função original e sua expansão na base não-ortogonal $Q$

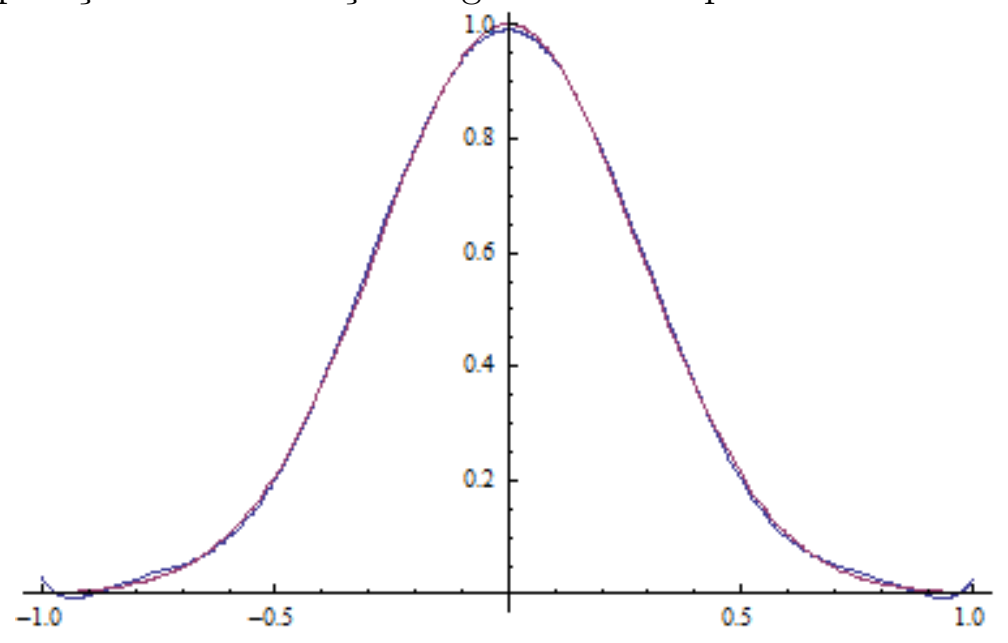

Como se vê, a aproximação é muito boa. O erro quadrático calculado vale $1.085 \times 10^{-4}$. Refazendo o processo para $n=15$, a precisão aumenta muito e o ajuste do gráfico fica praticamente perfeito. O erro nesse segundo caso passa a valer $1.015 \times 10^{-8}$. Concluímos, por conseguinte, que o formalismo funciona para bases oblíquas, produzindo excelentes resultados. 


\subsubsection{Mapeamentos Bijetores}

A ideia subjacente a mapeamentos deste tipo reside na possibilidade de, a partir de funções definidas em um determinado domínio, descrevê-las matematicamente de forma biunívoca em outro domínio qualquer.

Há duas possíveis situações para uma descrição dessa natureza: a transformação de um domínio finito em outro domínio finito, seja maior ou menor, e a transformação que relaciona domínios finitos e infinitos. Ambas devem ser bijetoras, para que as inversas existam. Além disso, ambas devem ser suaves, isto é, contínuas e diferenciáveis de classe $\mathbb{C}^{\infty}$, para que sejam apropriadas à utilização na teoria das equações diferenciais. Em outras palavras, tais mapeamentos, quando lineares, devem constituir difeomorfismos. No entanto, a linearidade não é uma condição essencial. De fato, mapeamentos não-lineares têm considerável aplicabilidade também.

Na verdade, poderíamos restringir um pouco tais exigências, limitando-nos a difeomorfismos $\mathbb{C}^{r}$, onde $r$ é a ordem da equação diferencial que se pretende resolver. Entretanto, visando o desenvolvimento posterior do método e sua consequente aplicação em equações diferenciais de ordem arbitrária, constitui melhor estratégia assumir o caso mais geral, evitando-se eventuais complicações que surjam na transformação da ordem das referidas equações. De qualquer maneira, consideraremos a aplicação de condições mais restritas se, e quando, se fizerem necessárias.

Evidentemente, no caso de aplicações entre domínios finitos, uma relação linear pode ser obtida facilmente. Entretanto, já no segundo caso, se fará necessária a utilização de uma relação não-linear, a fim de obter a compactificação do domínio e evitar infinitos em renormalizações. Para isso, usaremos o método de transposição de domínio (que designaremos pela abreviatura TD), descrito em Guimarães (2008), realizando o mesmo tipo de mapeamento lá efetuado.

Após desenvolver o esquema da uniformização de domínios e introduzir o conceito de produto híbrido, vamos aplicá-lo, inicialmente, a um mapeamento linear genérico entre o domínio da expansão de Fourier, $D_{L}=[-L, L]$, e o definido na expansão por polinômios do tipo Jacobi, isto é, com domínios $D_{1}=[-1,1]$, suficiente para a transposição desejada, que denominaremos Mapeamentos Fourier-Jacobi. Uma aplicação imediata é o mapeamento Fourier-Legendre e seu inverso, Legendre-Fourier. Em seguida, partiremos para um caso mais complicado, introduzindo uma TD não-linear para estabelecer o mapeamento Fourier-Hermite. 


\subsubsection{Uniformização de Domínios}

O processo de transposição de domínio consiste em efetuar o mapeamento descrito acima, a fim de possibilitar operações comuns entre bases definidas em domínios distintos. Além disso, mudanças de base mais genéricas no método de Dirac dependem dessa condição.

Existe, no entanto, um claro inconveniente resultante do processo de transposição de domínio. O problema reside no estabelecimento de uma função peso comum na formulação do IP entre duas bases distintas. Isto, como foi dito, é necessário para se poder utilizar o método de Dirac em mudanças de base mais gerais no espaço de Hilbert.

Esclarecendo este ponto, podemos perguntar o seguinte: no caso de IPs definidos em bases e domínios diferentes e com funções-peso distintas, um IP híbrido deverá ser estabelecido com qual das funções-peso?

Para responder a esta questão, é preciso usar um outro recurso, ao qual chamamos de uniformização dos domínios, e que será descrito a seguir.

Seja $\mid B]=\left\{\left|b_{k}(u)\right\rangle\right\}_{0(n)}$ uma base ortogonal qualquer, de dimensão $n$, num domínio $D_{L}=[-L, L]$. Então, se o IP em $D_{L}$ for definido com uma função peso $W(u), u \in D_{L}$, a métrica da referida base será constituída pelos elementos $g_{r s}^{(b)}=g\left(b_{r}, b_{s}\right) \equiv\left\langle b_{r} \mid b_{s}\right\rangle$, onde

$$
\left\langle b_{r} \mid b_{s}\right\rangle:=\int_{-L}^{L} b_{r}^{*}(u) b_{s}(u) W(u) d u
$$

Devido à ortogonalidade da base, podemos escrever $\left\langle b_{r} \mid b_{s}\right\rangle=\alpha_{r} \delta_{r s}$, onde $\alpha_{r}=1$ apenas no caso de uma base ortonormal. É possível, entretanto, implementar uma base renormalizada, i.e., ortonormal, a partir da base original, adotando, simplesmente,

$$
\hat{b}_{r}(u)=\frac{b_{r}(u)}{\sqrt{\alpha_{r}}}
$$

ou, no caso de domínios complexos,

$$
\hat{b}_{r}(u)=\frac{b_{r}(u)}{\sqrt{\left|\alpha_{r}\right|} \exp \left[\frac{i}{2} \arg \left(\alpha_{r}\right)+i \pi\right]}
$$

Esta base, definida com IP a peso $W(u)$, será ortonormal no domínio correspondente $D_{L}$, ou seja,

$$
\left\langle\hat{b}_{r} \mid \hat{b}_{s}\right\rangle=\int_{-L}^{L} \hat{b}_{r}^{*}(u) \hat{b}_{s}(u) W(u) d u=\delta_{r s}
$$


Em seguida, deve-se efetuar a transposição de domínio $u=u(x)$, para $x \in D_{T}$, onde $D_{T}$ é o domínio transposto. O novo período pode ser $[-1,1]$ ou $]-\infty, \infty[$, por exemplo, para funções do tipo Jacobi e de Hermite, respectivamente, ou, ainda, $[-T, T]$, para um novo período $T$ genérico e limitado.

Evidentemente, após a TD, o IP deverá ser reescrito para a nova variável, levando em conta o jacobiano da transformação $J(u, x)=\frac{d u}{d x}$, de modo que, denotando $\beta_{r}(x):=$ $\hat{b}_{r}(u(x))$ e $\omega(x):=W(u(x))$, tenhamos:

$$
\left\langle\beta_{s} \mid \beta_{r}\right\rangle=\int_{-T}^{T} \beta_{s}^{*}(x) \beta_{r}(x) \omega(x) J(u, x) d x
$$

A partir disso, definimos uma base $\left\{\left|\tilde{\beta}_{r}\right\rangle\right\}$, onde

$$
\tilde{\beta}_{r}(x):=\sqrt{J(u, x)} \beta_{r}(x),
$$

que denominaremos de base redimensionada, para a qual deve valer:

$$
\left\langle\tilde{\beta}_{r} \mid \tilde{\beta}_{s}\right\rangle=\int_{-T}^{T} \tilde{\beta}_{r}^{*}(x) \tilde{\beta}_{s}(x) \omega(x) d x=\delta_{r s}
$$

Em outros termos, a métrica fica preservada pela TD, pois foi efetuada apenas uma mudança de variável na integral que define o IP, o que implica ser a nova base $\left\{\left|\tilde{\beta}_{r}\right\rangle\right\}$ também ortonormal com relação ao IP definido com a nova função peso $\omega(x)$ no domínio $D_{T}$.

Apesar da conveniência dessa definição, ainda resta o problema da escolha de uma nova função peso que seja comum aos dois domínios.

Uma forma de resolver o problema é definir o que chamaremos de base uniforme. A ideia consiste em preservar a métrica identidade na imposição de um novo IP com peso unitário, isto é, definir funções $\hat{\beta}_{r}$ de forma que tenhamos $\left\langle\hat{\beta}_{r} \mid \hat{\beta}_{s}\right\rangle=\delta_{r s}$, onde

$$
\left\langle\hat{\beta}_{r} \mid \hat{\beta}_{s}\right\rangle=\int_{-T}^{T} \hat{\beta}_{r}^{*}(x) \hat{\beta}_{s}(x) d x
$$

A forma mais natural de conseguir isso é acoplar a função peso às novas funções, ou seja, definir as funções uniformizadas por:

$$
\hat{\beta}_{r}(x):=\sqrt{\omega(x)} \tilde{\beta}_{r}(x)
$$


Assim, podemos, finalmente, expressar as funções uniformes em termos das funções originais como:

$$
\hat{\beta}_{r}(x):=\left[\frac{W(u(x)) J(u, x)}{\alpha_{r}}\right]^{1 / 2} b_{r}(u(x))
$$

\subsubsection{Implementação do IP Híbrido}

Uma vez obtidas as funções uniformes após a TD, conforme expusemos acima, pode-se pensar também em uniformizar funções que permaneçam em seus domínios originais $D_{T}$, definindo uma renormalização a peso unitário, que consiste simplesmente em tomar novas funções definidas num IP de peso unitário, incorporando a função-peso original às funções de base, de forma análoga à expressão (2.84), mas, nesse caso, sem a TD, naturalmente. Sejam $\left\{f_{r}(x)\right\}_{0(n)}$ as funções no domínio original. Então, as funções P1-renormalizadas correspondentes serão:

$$
\phi_{r}(x):=\sqrt{\frac{W_{0}(x)}{\eta_{r}}} f_{r}(x),
$$

onde $\eta_{r}$ são os fatores de normalização e $W_{0}(x)$, a função-peso do IP entre as funções do domínio original.

Nessas condições, a implementação do IP híbrido é imediata, pois ambas as funções possuem agora função-peso unitária. Logo, o IP é definido com peso unitário ao longo de todo o domínio, isto é,

$$
\left\langle\phi_{r} \mid \hat{\beta}_{s}\right\rangle=\int_{-T}^{T} \phi_{r}^{*}(x) \hat{\beta}_{s}(x) d x
$$

Em suma, podemos escrever que, se as funções mantidas em seu domínio original $D_{T}$ forem $\left\{f_{r}(x)\right\}_{0(n)}$, tais que $\int_{-T}^{T} f_{r}^{*}(x) f_{s}(x) W_{0}(x) d x=\eta_{r} \delta_{r s}$ e as funções transformadas (antes da TD) são $\left\{b_{r}(x)\right\}_{0(n)}$, obedecendo, em seu domínio próprio, à condição $\int_{-L}^{L} b_{r}^{*}(x) b_{s}(x) W(x) d x=\alpha_{r} \delta_{r s}$, então um IP híbrido entre elas, após a TD: $u=u(x)$ e o processo de uniformização, pode ser definido pela expressão (2.87), onde as novas funções obedecem às expressões (2.85) e (2.86).

\subsubsection{Mapeamentos tipo Fourier-Jacobi e Jacobi-Fourier}

Transposição de Domínio Seja $\left\{P_{m}(x)\right\}_{0_{n}}$ uma base do tipo Jacobi, definida no domínio $D_{1}=[-1,1]$ e $\left\{e_{r}(u)\right\}$ a base de Fourier usual, no domínio $D_{L}=[-L, L]$. Então, utilizando as fórmulas estabelecidas na seção anterior para uma TD dada simplesmente 
por $x=u / L$, podemos expressar a uniformização da base $P$ no domínio $D_{L}$ como

$$
\hat{\pi}_{r}(u):=\left[\frac{W(x(u)) J(x, u)}{\alpha_{r}}\right]^{1 / 2} P_{r}(x(u))
$$

Como as bases são ortogonais, podemos escrever $\alpha_{r}=\left\langle P_{r} \mid P_{r}\right\rangle$ e o jacobiano da TD vale $1 / L$, o que conduz a

$$
\hat{\pi}_{r}(u):=\left[\left(\frac{1}{L}\right) \frac{W(x(u))}{\left\langle P_{r} \mid P_{r}\right\rangle}\right]^{1 / 2} P_{r}(x(u)), \quad u \in D_{L}
$$

Com isso, o IP híbrido será definido como

$$
\left\langle e_{m} \mid \hat{\pi}_{r}\right\rangle_{D_{L}}=\int_{-L}^{L} e_{m}^{*}(u) \hat{\pi}_{r}(u) d u
$$

Já o procedimento inverso permite transformar o domínio $D_{L}$ em $D_{1}$. Então, partindo da base de Fourier, cujos elementos são $e_{m}(u), u \in D_{L}$, pode-se efetuar a TD inversa, i.e., $u=x L$, gerando $J(u, x)=L$, o que nos permite escrever

$$
\hat{\epsilon}_{m}(x)=\frac{1}{\sqrt{2}} e_{m}(L x), \quad x \in D_{1}
$$

visto que $\left\langle e_{r} \mid e_{s}\right\rangle=2 L \delta_{r s}$ e a função peso é unitária no IP de Fourier, o que gera o IP híbrido:

$$
\left\langle P_{r} \mid \hat{\epsilon}_{m}\right\rangle_{D_{1}}=\int_{-1}^{1} P_{r}^{*}(x) \hat{\epsilon}_{m}(x) d x=\frac{1}{\sqrt{2}} \int_{-1}^{1} P_{r}(x) e_{m}(L x) d x
$$

\subsubsection{Esboço do Mapeamento Legendre-Fourier}

A aplicação direta das fórmulas acima nos conduzem à expressão dos elementos da base uniforme de Legendre:

$$
\hat{\pi}_{n}(u)=\sqrt{\frac{1}{L}\left(n+\frac{1}{2}\right)} P_{n}\left(\frac{u}{L}\right), \quad u \in D_{L},
$$

onde $P_{n}(x)$ são os polinômios de Legendre.

\subsubsection{Mapeamento Hermite-Fourier}

Podemos partir diretamente para as fórmulas estabelecidas no início da seção ou analisar mais detidamente cada passo, a fim de entender melhor o procedimento. Um aspecto importante é que o esquema de uniformização de domínios não precisa ser feito na ordem estipulada antes, conforme vamos ilustrar agora. Há mesmo a possibilidade de 
realizarmos apenas as partes do processo que forem estritamente necessárias.

Os polinômios de Hermite probabilísticos são dados por

$$
H e_{k}(x)=(-1)^{k} e^{x^{2} / 2}\left(\frac{d}{d x}\right)^{k} e^{-x^{2} / 2}, \quad\left(x \in D_{\infty}\right)
$$

onde $\left.D_{\infty}=\right]-\infty, \infty[=\mathbb{R}$.

O IP é calculado com a função peso $W(x)=e^{-x^{2} / 2}$, de forma que

$$
\left\langle H e_{m} \mid H e_{n}\right\rangle:=\int_{-\infty}^{\infty} H e_{m}^{*}(x) H e_{n}(x) W(x) d x=m ! \sqrt{2 \pi} \delta_{m n}
$$

Com a transposição de domínio (TD): $x=x(u)$, os novos polinômios obtidos, que chamaremos de polinômios de Hermite transformados, serão denotados por $\hat{H} e_{m}(u)$ e a função peso, por $\omega(u)$, onde $\hat{H} e_{m}(u):=H e_{m}[x(u)]$ e $\omega(u):=W[x(u)]$.

Entretanto, o IP deve ser reescrito levando em conta a TD, o que acarreta a introdução do jacobiano da transformação, $J(x, u)=\frac{d x}{d u}$, na integral e, com isso, a nova função peso obtida pelo produto entre o jacobiano e a anterior função peso transformada, definido por $\hat{W}(u)=\omega(u) J(x, u)$, garante a ortogonalidade da base com os mesmos valores, ou seja:

$$
\left\langle\hat{H} e_{m} \mid \hat{H} e_{n}\right\rangle:=\int_{-L}^{L} \hat{H} e_{m}^{*}(u) \hat{H} e_{n}(u) \hat{W}(u) d u=m ! \sqrt{2 \pi} \delta_{m n}
$$

Como estamos usando uma TD do tipo definido em [Guimarães], isto é, $x(u)=$ $\tan \left(\frac{q u}{2}\right)$, então o jacobiano assumirá a forma:

$$
J(x, u)=\frac{d x}{d u}=\frac{q}{2} \sec ^{2}\left(\frac{q u}{2}\right)
$$

Com vistas à unificação de tratamento do IP, possibilitando a definição de um IP híbrido entre as bases de Hermite e Fourier, vamos introduzir os polinômios de Hermite renormalizados a peso unitário ou P1-renormalizados:

$$
\phi_{m}(x)=\left[\frac{W(x)}{m ! \sqrt{2 \pi}}\right]^{1 / 2} H e_{m}(x)
$$

Com esses novos polinômios, agora ortonormais, o IP pode ser redefinido com peso unitário, fornecendo uma métrica identidade:

$$
\left\langle\phi_{m} \mid \phi_{n}\right\rangle:=\int_{-\infty}^{\infty} \phi_{m}^{*}(x) \phi_{n}(x) d x=\delta_{m n}
$$


Isso simplifica o processo de implementação de um IP híbrido, pois as funções ortonormais transformadas carregam a função peso incorporada em si mesmas.

Efetuando, então, a TD para as funções definidas acima, onde denotamos $\hat{\phi}_{n}(u)=$ $\phi_{n}[x(u)]$, obtém-se:

$$
\left\langle\hat{\phi}_{m} \mid \hat{\phi}_{n}\right\rangle=\int_{-L}^{L} \hat{\phi}_{m}^{*}(u) \hat{\phi}_{n}(u) J(x, u) d u
$$

Por fim, vamos definir o que designaremos por funções de Hermite uniformes, como

$$
\hat{h}_{n}(u)=J^{1 / 2}(x, u) \hat{\phi}_{n}(u)
$$

o que fornece

$$
\left\langle\hat{h}_{m} \mid \hat{h}_{n}\right\rangle=\int_{-L}^{L} \hat{h}_{m}^{*}(u) \hat{h}_{n}(u) d u=\delta_{m n}
$$

Nessas condições, as funções de Hermite uniformes são dadas por:

$$
\hat{h}_{n}(u)=\left[\frac{\hat{W}(u)}{n ! \sqrt{2 \pi}}\right]^{1 / 2} \hat{H} e_{n}(u) .
$$

Para a TD escolhida, a forma explícita da função acima definida será

$$
\hat{h}_{n}(u)=\sqrt{\frac{q}{n !}}\left(\frac{e}{8 \pi}\right)^{1 / 4} \exp \left[-\frac{1}{4} \sec ^{2}\left(\frac{q u}{2}\right)\right] \sec \left(\frac{q u}{2}\right) \hat{H} e_{n}(u)
$$

Por outro lado, a forma explícita da função de Hermite transformada será:

$$
\hat{H} e_{n}(u)=\left(\frac{-2}{q}\right)^{n} \exp \left[\frac{1}{2} \sec ^{2}\left(\frac{q u}{2}\right)\right] \cos ^{2 n}\left(\frac{q u}{2}\right)\left(\frac{d}{d u}\right)^{n} \exp \left[-\frac{1}{2} \sec ^{2}\left(\frac{q u}{2}\right)\right]
$$

Como se vê, esse mapeamento gera funções complicadas, cujo processamento simbólico pode demandar excesso de recursos do sistema. Emerge, então, a necessidade de estabelecer critérios que visem simplificar os resultados do procedimento.

Uma solução possível repousa na tentativa de se utilizar a transposição de domínio em Fourier complexo, cujo comportamento da base é "amigável" o suficiente para permitir uma transformação do tipo descrito com resultados visivelmente mais simples que o obtido acima.

Vamos, por conseguinte, implementar o processo inverso, isto é, executar uma TD na base complexa de Fourier a fim de expandir o domínio original $D_{L}$ ao domínio das funções de Hermite, ou seja, a $D_{\infty}$. 


\subsubsection{Mapeamento Fourier-Hermite}

A base complexa de Fourier renormalizada é dada pelos kets $\left|\hat{e}_{k}\right\rangle_{\infty}$, onde $q=\frac{\pi}{L}$ em

$$
\hat{e}_{k}(u)=\frac{1}{\sqrt{2 L}} \exp (i k q u), \quad\left(u \in D_{L}=[-L, L]\right)
$$

de maneira que $\left\langle\hat{e}_{s} \mid \hat{e}_{r}\right\rangle=\delta_{r s}$. Vamos estabelecer a TD: $u(x)=\frac{2}{q} \arctan x$. Disso, segue que o jacobiano da transformação será

$$
J(u, x)=\frac{2 / q}{1+x^{2}} .
$$

Definindo os elementos da base transformada por

$$
\epsilon_{k}(x):=\hat{e}_{k}[u(x)]
$$

não é difícil verificar que os elementos de base podem ser reescritos como

$$
\epsilon_{r}(x)=\frac{1}{\sqrt{2 L}}\left(\frac{1+i x}{1-i x}\right)^{r}, \quad\left(x \in D_{\infty}=\right]-\infty, \infty[)
$$

Dessa maneira, a base redimensionada, dada pela expressão (2.81), ou seja, $\tilde{\epsilon}_{r}(x)=\sqrt{J(u(x))} \epsilon_{r}(x)$, assumirá a forma:

$$
\tilde{\epsilon}_{r}(x)=\sqrt{\frac{2}{q}} \frac{\epsilon_{r}(x)}{\sqrt{1+x^{2}}}, \quad\left(x \in D_{\infty}\right)
$$

Visto que a função-peso, nesse caso, já é unitária, a base uniforme será a própria base redimensionada, isto é, $\hat{\epsilon}(x)=\tilde{\epsilon}(x)$. Abrindo, então, essa expressão em sua forma explícita, obtemos:

$$
\hat{\epsilon}_{r}(x)=\frac{1}{\sqrt{\pi}} \frac{(1+i x)^{r-1 / 2}}{(1-i x)^{r+1 / 2}}
$$

Por conseguinte, podemos estabelecer a métrica de Fourier uniforme pelo produto interno entre os elementos constituintes da base uniforme. Evidentemente, por construção, a métrica deverá ser a identidade. Logo,

$$
\hat{\gamma}_{r s}:=\left\langle\hat{\epsilon_{r}} \mid \hat{\epsilon_{s}}\right\rangle=\int_{-\infty}^{\infty} \hat{\epsilon}_{r}^{*}(x) \hat{\epsilon_{s}}(x) d x=\delta_{r s}
$$

\subsubsection{IP Híbrido Fourier-Hermite}

A partir desse procedimento, podemos definir um IP híbrido das bases uniformes. No caso, por exemplo, das funções de Hermite, não havendo necessidade de transposição 
de domínio, já que esta foi efetuada na base de Fourier, bastaria tomarmos as funções renormalizadas a peso unitário $\phi_{m}(x)$ definidas na expressão (2.88) ou, explicitamente,

$$
\phi_{m}(x)=(-1)^{m}\left[\frac{1}{m ! \sqrt{2 \pi}}\right]^{1 / 2} e^{x^{2} / 4}\left(\frac{d}{d x}\right)^{m} e^{-x^{2} / 2}
$$

Assim, o produto interno híbrido entre as funções de Fourier uniformes e as de Hermite P1-renormalizadas é dado por

$$
\left\langle\phi_{r} \mid \hat{\epsilon}_{s}\right\rangle=\int_{-\infty}^{\infty} \phi_{r}^{*}(x) \hat{\epsilon}_{s}(x) d x
$$

A título de exemplo, vamos apresentar os gráficos de algumas das funções das bases de Fourier transformada e uniforme, bem com as funções $\phi_{m}$ de Hermite P1-renormalizadas, expressas na eq. (2.88).

Conforme mostramos acima, as funções da base transformada de Fourier são dadas por

$$
\tilde{\epsilon}_{k}(x)=\frac{1}{\sqrt{2 L}}\left(\frac{1+i x}{1-i x}\right)^{k}
$$

Seus gráficos são apresentados abaixo, nas figuras (3), (4) e (5).

Figura 3: Gráficos real e imaginário da base de Fourier transformada $\tilde{\epsilon}_{1}(x)$
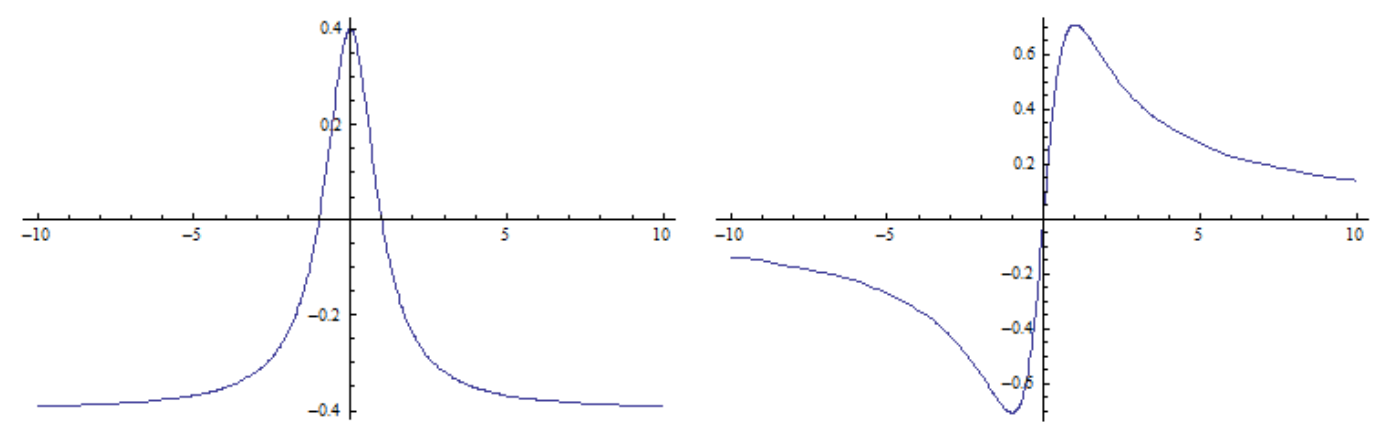

Apresentamos agora as seis primeiras funções de Hermite P1-renormalizadas, que aparecem listadas a seguir:

$$
\begin{array}{cc}
\phi_{0}(x)=\frac{e^{\frac{-x^{2}}{4}}}{(2 \pi)^{1 / 4}} & \phi_{1}(x)=\frac{x e^{x} \frac{-x^{2}}{4}}{(2 \pi)^{1 / 4}} \\
\phi_{2}(x)=\frac{e^{\frac{-x^{2}}{4}}}{(8 \pi)^{1 / 4}}\left(x^{2}-1\right) & \phi_{3}(x)=\frac{e^{x \frac{-x^{2}}{4}}}{\sqrt{3}(8 \pi)^{1 / 4}}\left(x^{3}-3 x\right) \\
\phi_{4}(x)=\frac{e^{\frac{-x^{2}}{4}}}{2 \sqrt{3}(8 \pi)^{1 / 4}}\left(x^{4}-6 x^{2}+3\right) & \phi_{5}(x)=\frac{e^{\frac{-x^{2}}{4}}}{2 \sqrt{15}(8 \pi)^{1 / 4}}\left(x^{5}-10 x^{3}+15 x\right)
\end{array}
$$


Figura 4: Gráficos real e imaginário da base de Fourier transformada $\tilde{\epsilon}_{2}(x)$
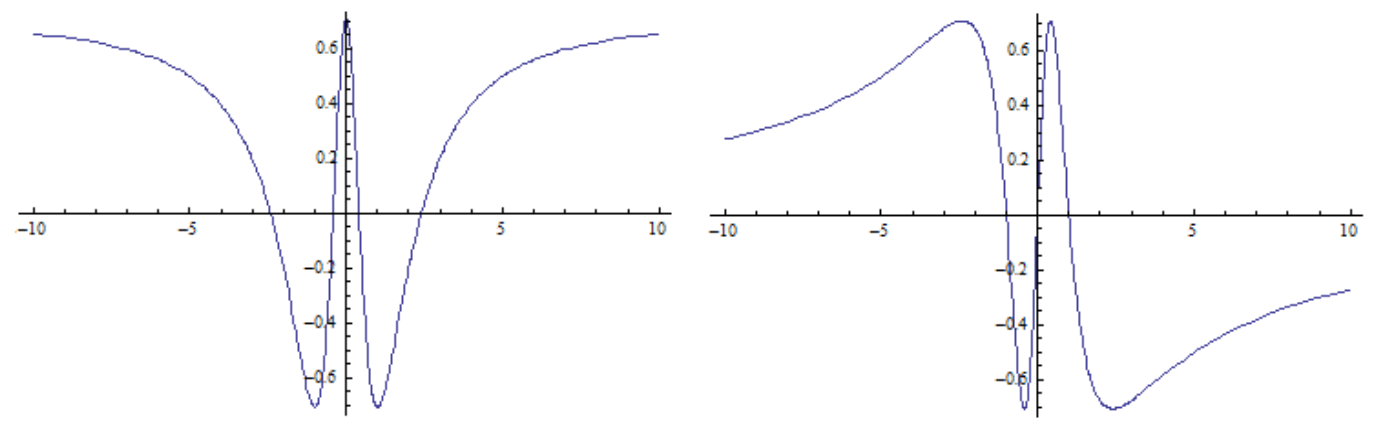

Figura 5: Gráficos real e imaginário da base de Fourier transformada $\tilde{\epsilon}_{3}(x)$
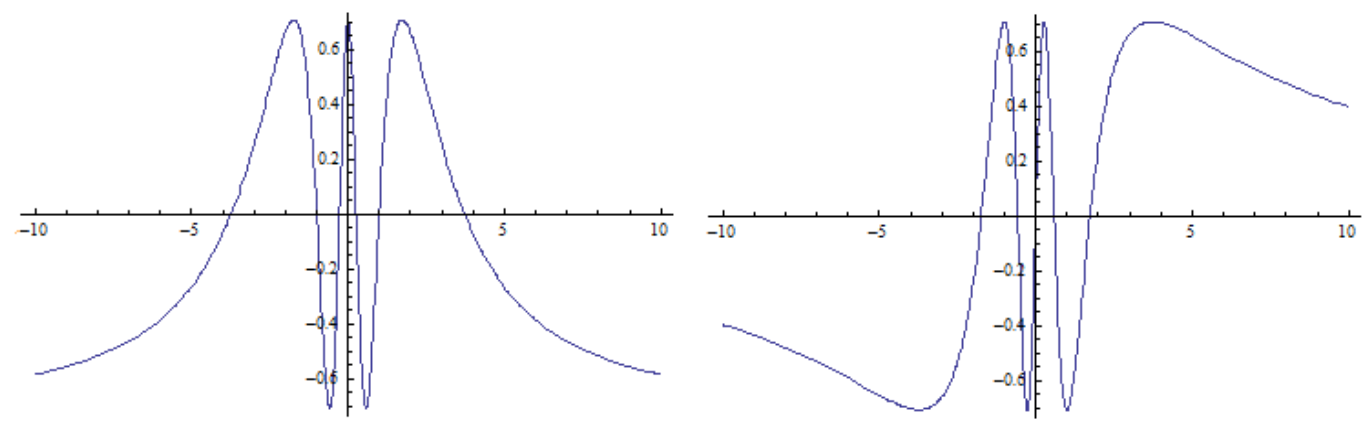

Por fim, podemos estabelecer o IP híbrido, de acordo com a eq.(2.90).

A matriz do tensor métrico do IP híbrido Fourier-Hermite para um desenvolvimento de ordem 5 é a seguinte:

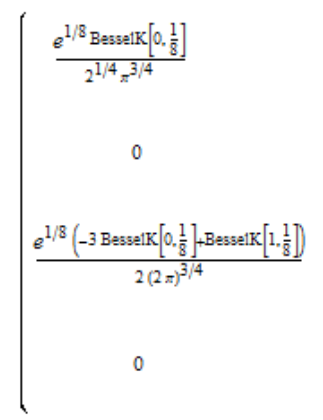

$$
\begin{aligned}
& \frac{e^{1 / 8}\left(-3 \text { BesselK }\left[0, \frac{1}{8}\right]+B \text { BessealK }\left[1 \cdot \frac{1}{8}\right)\right.}{22^{1 / 4} \pi^{3 / 4}} \quad \frac{e^{1 / 8}\left(10 \text { BesseatK }\left[0 \cdot \frac{1}{8}\right]-3 \text { BesselK }\left[1 \cdot \frac{1}{8}\right)\right.}{32^{1 / 4} \pi^{3 / 4}} \\
& \frac{t e^{1 / 8}\left(5 \text { BesselK }\left[0 \cdot \frac{1}{8}\right]-\text { BesselK }\left[1-\frac{1}{8}\right)\right.}{22^{1 / 4} \pi^{3 / 4}} \quad-\frac{1}{3} t^{1 / 8}\left(\frac{2}{\pi}\right)^{3 / 4}\left(11 \text { BesselK }\left[0, \frac{1}{8}\right]-3 \text { BesselK }\left[1, \frac{1}{8}\right]\right) \\
& -\frac{3 e^{1 / 8}\left(-3 \text { BesselK }\left[0 \cdot \frac{1}{8}\right]+\text { BesselK }\left[1 \cdot \frac{1}{8}\right]\right)}{2(2 \pi)^{3 / 4}} \quad \frac{e^{1 / 8}\left(-97 \text { BesselK }\left[0 \cdot \frac{1}{8}\right]+27 \text { BessealK }\left[1, \frac{1}{8}\right]\right)}{6(2 \pi)^{3 / 4}} \\
& -\frac{t e^{1 / 8}\left(11 \text { BesselK }\left[0, \frac{1}{8}\right]-3 \text { BesselK }\left[1 \cdot \frac{1}{8}\right)\right]}{\sqrt{3}(2 \pi)^{3 / 4}} \quad \frac{2 t 2^{1 / 4} e^{1 / 8}\left(31 \text { BesselK }\left[0 \cdot \frac{1}{8}\right]-9 \text { BesselK }\left[1, \frac{1}{8}\right)\right.}{3 \sqrt{3} \pi^{3 / 4}}
\end{aligned}
$$

$\frac{e^{1 / 8}\left(-221 \text { Besse1K }\left[0 . \frac{1}{8}\right]+63 \text { BesselK }\left[1, \frac{1}{8}\right)\right.}{302^{1 / 4} \pi^{3 / 4}}$

$\frac{t e^{1 / 8}\left(541 \text { BesselK }\left[0 \cdot \frac{1}{8}\right]-153 \text { BesselK }\left[1 \cdot \frac{1}{8}\right)\right.}{302^{1 / 4} \pi^{3 / 4}}$

$\frac{e^{1 / 8}\left(1367 \text { BesselK }\left[0 . \frac{1}{8}\right]-381 \text { BessetK }\left[1, \frac{1}{8}\right]\right)}{30(2 \pi)^{3 / 4}}$

$-\frac{t e^{1 / 8}\left(1867 \operatorname{Bessel|K}\left[0, \frac{1}{8}\right]-531 \text { BesselK }\left[1, \frac{1}{8}\right]\right.}{15 \sqrt{3}(2 \pi)^{3 / 4}}$ 


\section{MATRIZES OPERACIONAIS NO FORMALISMO COADJUNTO}

Desenvolvemos aqui a exposição formal das matrizes operacionais de derivação e integração no presente tratamento.

\subsection{Matrizes Operacionais em Bases Genéricas}

Conforme foi explicado em outro ponto do trabalho, a utilização das matrizes operacionais parte do pressuposto de existência de matrizes que atuem sobre elementos de espaços vetoriais de forma equivalente a operadores de derivação e antiderivação, recebendo, por esse motivo, o nome de matrizes operacionais.

Assumindo a ação de um operador linear desse tipo, como por exemplo, $\mathcal{D} \equiv \frac{d}{d x}$, atuando sobre uma função, que constitui um vetor em espaços de Hilbert, então sua aplicação sobre uma função $f(x)$, Lebesgue-integrável, aproximada por uma série de $n$ termos, denotada $f_{n}(x)$, escreve-se:

$$
\mathcal{D}\left|f_{n}\right\rangle_{\infty}=\mathcal{D}\langle C \mid \phi\rangle_{\mathbb{C H}}
$$

Ora, como a atuação da derivada sobre o covetor $\left\langle\left. C\right|_{0_{n}}\right.$ fornece valores nulos, visto que seus elementos são escalares complexos, então, a operação deverá ser efetivada apenas no vetor cujas componentes dependam da variável de derivação, recaindo exclusivamente sobre as coordenadas da base de expansão. Em outros termos, o operador só atua sobre a base, o que acarreta

$$
\mathcal{D}\left|f_{n}\right\rangle_{\infty}=\langle C|\mathcal{D}| \phi\rangle_{\mathbb{C H}} \equiv\left\langle C \mid \phi^{\prime}\right\rangle_{\mathbf{C H}},
$$

Assumimos agora uma descrição em termos de matrizes operacionais, denotando por 
$D$ a matriz operacional de derivação que atua sobre essa base e que abreviaremos pela sigla $M O D$, por concisão.

Assim, como $\left|f_{n}^{\prime}\right\rangle_{\infty} \equiv \mathcal{D}\left|f_{n}\right\rangle_{\infty}$ e $\left|\phi^{\prime}\right\rangle_{o_{n}} \equiv \mathcal{D}|\phi\rangle_{0_{n}}=D|\phi\rangle_{0_{n}}$, segue que

$$
\left|f_{n}^{\prime}\right\rangle_{\infty}=\left\langle C \mid \phi^{\prime}\right\rangle_{\mathrm{CH}}
$$

onde

$$
\left|\phi^{\prime}\right\rangle_{0_{n}}=D|\phi\rangle_{0_{n}}
$$

sendo $D$ entendida agora como uma matriz quadrada de ordem $n$, ou seja, $D \in \mathbb{M}_{n}(\mathbb{C})$.

Entretanto, como nosso objetivo é descrever o resultado obtido em termos da própria base escolhida, se chamarmos o novo covetor dos coeficientes de $\left|f_{n}^{\prime}\right\rangle_{\infty}$ na base $|\phi\rangle_{0_{n}}$ de $\left\langle\left. C_{\mathrm{D}}\right|_{0_{n}}\right.$, então

$$
\left|f_{n}^{\prime}\right\rangle_{\infty}=\langle C|D| \phi\rangle_{\mathrm{CH}}:=\left\langle C_{\mathrm{D}} \mid \phi\right\rangle_{\mathrm{CH}},
$$

o que implica:

$$
\left\langle C_{\mathrm{D}}\right|=\langle C| D
$$

Para determinarmos a matriz operacional de derivação que atua sobre os coeficientes, deve-se efetuar a operação de adjunção na expressão acima, i.e.,

$$
\left|C_{\mathrm{D}}\right\rangle=D^{\dagger}|C\rangle
$$

Este resultado, obtido de modo mais simples, amplia para o domínio complexo o resultado anteriormente obtido por Guimarães, Piqueira e Lobo Netto (2010) para variáveis reais:

$$
V^{T}=Z^{T} C^{T}
$$

identificando-se $V \equiv\left\langle C_{\mathrm{D}}\right|$ e $Z \equiv D$. Observamos, no entanto, que a operação acima também pode ser realizada, no presente formalismo, diretamente pela equação (3.3), sem a necessidade da adjunção, podendo-se interpretá-la como uma retrooperação sobre o covetor.

Analogamente, a matriz operacional de integração, abreviada por MOI, deverá obedecer aos mesmos procedimentos. Aplicando, a critério de exemplo, um operador de integração indefinida $\mathcal{J}$ (antiderivada) sobre a função aproximada, tem-se

$$
\mathcal{J}\left|f_{n}\right\rangle_{\infty} \equiv\left|\mathcal{J} f_{n}\right\rangle_{\infty}=\left|\int^{x} f_{n}(\xi) d \xi\right\rangle_{\infty}
$$


e, portanto, como a aplicação de $\mathcal{J}$ recai sobre a base, se chamarmos de $M_{I}$ a MOI que representa o operador $\mathcal{J}$, segue:

$$
\mathcal{J}\left|f_{n}\right\rangle_{\infty}=\left\langle C \mid \int^{x} \phi(\xi) d \xi\right\rangle_{\mathbb{C H}}=\langle C \mid \mathcal{J} \phi\rangle=\left\langle C\left|M_{I}\right| \phi\right\rangle_{\mathbb{C H}}:=\left\langle C_{I} \mid \phi\right\rangle,
$$

sendo $\left\langle\left. C_{I}\right|_{0_{n}} \text { o covetor coeficiente de } \mid \mathcal{J} f_{n}\right\rangle_{\infty}$ na base $|\phi\rangle_{0_{n}}$, o que fornece, para o vetor dos coeficientes da matriz de integração:

$$
\left\langle C_{I}\right|=\langle C| M_{I}
$$

ou, conforme Guimarães (2008),

$$
\left|C_{I}\right\rangle=M_{I}^{\dagger}|C\rangle
$$

\subsubsection{Matrizes Operacionais de Ordens Superiores}

É possível estender o processo de determinação de matrizes operacionais para derivadas de ordens superiores de uma maneira simples. Consideremos a segunda derivada de $f(x)$ numa base genérica $|\phi\rangle_{0_{n}}$. Dada a linearidade da atuação do operador de derivação e a constância dos valores das componentes do covetor coeficiente, a segunda derivada de $f(x)$ fornece:

$$
\begin{gathered}
\left|f_{n}^{\prime \prime}\right\rangle_{\infty} \equiv \mathcal{D}\left|f_{n}^{\prime}\right\rangle_{\infty}=\left\langle C|D| \phi^{\prime}\right\rangle_{\mathrm{CH}}= \\
=\left\langle C\left|D^{2}\right| \phi\right\rangle_{\mathrm{CH}}=\left\langle C \mid \phi^{\prime \prime}\right\rangle_{\mathrm{CH}}=\left\langle C_{\mathrm{D}^{2}} \mid \phi\right\rangle_{\mathrm{CH}},
\end{gathered}
$$

sendo $\left\langle C_{\mathrm{D}^{2}}\right|$ o covetor dos coeficientes de $\left|f_{n}^{\prime \prime}\right\rangle_{\infty}$ na base $|\phi\rangle_{0_{n}}$.

Na sequência, se repetirmos mais uma vez o processo, a $M O D$ de terceira ordem será dada por

$$
\left|f_{n}^{\prime \prime \prime}\right\rangle_{\infty} \equiv \mathcal{D}\left|f_{n}^{\prime \prime}\right\rangle_{\infty}=\left\langle C\left|D^{3}\right| \phi\right\rangle_{\mathbf{C H}}
$$

Por conseguinte, é facil constatar que esse procedimento, aplicado sucessivamente, conduz ao resultado estendido:

$$
\left|f_{n}^{(k)}\right\rangle_{\infty} \equiv \mathcal{D}^{k}\left|f_{n}\right\rangle_{\infty}=\left\langle C\left|D^{k}\right| \phi\right\rangle_{\mathrm{CH}}
$$

o que leva imediatamente à generalização da fórmula (3.3) para o covetor coeficiente da derivada de ordem $k$ da função $f(x)$ expandida na base $|\phi\rangle_{0_{n}} \operatorname{com} n$ termos:

$$
\left\langle C_{\mathrm{D}^{k}}\right|=\langle C| D^{k}, \quad(k \in \mathbb{N})
$$

Voltando agora ao caso de um operador integral, não é difícil constatar que o proce- 
dimento é similar e, novamente, em decorrência da linearidade e da constância do covetor coeficiente, obteremos, para uma aplicação recursiva do operador $\mathcal{J}$, o seguinte:

$$
\mathcal{J}^{k}\left|f_{n}\right\rangle_{\infty}=\int^{x} \cdots \int^{x} f_{n}(\xi)(d \xi)^{k}=\left\langle C\left|M_{I}^{k}\right| \phi\right\rangle_{\mathbb{C H}}:=\left\langle C_{I^{k}} \mid \phi\right\rangle_{\mathbb{C H}}
$$

e, consequentemente, a extensão da equação (3.5) ao caso geral fornece

$$
\left\langle C_{I^{k}}\right|=\langle C| M_{I}^{k} \quad(k \in \mathbb{N}),
$$

onde $\left\langle C_{I^{k}}\right|$ é o covetor coeficiente da integral indefinida de ordem $k$ da função $f(x)$ expandida na base $|\phi\rangle_{0_{n}}$.

\subsubsection{Notação Generalizada}

Em vista do que nos propomos, adiantamos aqui uma notação que será necessária na empreitada que se realizará posteriormente, a saber, a extensão desse tratamento ao cálculo fracional.

Essa nova notação visa incorporar de forma consistente, numa única grafia, os resultados referentes tanto às matrizes de derivação quanto às de integração. Um operador de derivação $\mathcal{D}$ aplicado recursivamente é usualmente grafado como $\mathcal{D}^{k}$. Seguindo este costume, designaremos o operador de integração pelo mesmo símbolo que o de derivação, mas com sinal negativo no índice de "derivação", i.e.,

$$
\mathcal{J} \equiv \mathcal{D}^{-1}
$$

Assim, na notação generalizada, um operador de integração aplicado recursivamente $k$ vezes será escrito:

$$
\mathcal{J}^{k} \equiv \mathcal{D}^{-k}
$$

Similarmente, as matrizes operacionais de derivação e integração passam a assumir uma forma geral única de grafia: $D^{k},(k \in \mathbb{Z})$. Munidos desse recurso, podemos reescrever a aplicação de um operador desse tipo sobre uma função aproximada em série $f_{n}(x)$ como

$$
\left|f_{n}^{(k)}\right\rangle_{\infty}=D^{k}\left|f_{n}\right\rangle_{\infty}=\left\langle C\left|D^{k}\right| \phi\right\rangle_{\mathrm{CH}}, \quad(k \in \mathbb{Z})
$$

Com isso, as relações (3.7) e (3.8) podem ser denotadas da seguinte forma:

$$
\left\langle C_{\mathrm{D}^{k}}\right|=\langle C| D^{k}, \quad(k \in \mathbb{Z})
$$




\subsubsection{Mudança de Base}

Nesta seção analisa-se o efeito de uma mudança de base na forma das matrizes operacionais. A análise é efetuada para um operador diferencial mas, conforme foi mostrado anteriormente, vale também para um operador de antiderivação. Logo, o resultado será aplicável a uma MOp genérica.

$\mathrm{Na}$ expansão em série de um função podemos utilizar bases polinomiais de diversas naturezas, como as de Hermite, Lagrange, Laguerre ou Chebyshev, por exemplo. No caso de possuirmos a forma explícita de uma matriz operacional numa dada base e precisarmos efetuar uma mudança de base, faz-se necessário conhecer a matriz de transformação a fim de se determinar explicitamente a forma da MOp nessa nova base.

Sejam, portanto, $|\phi\rangle_{0_{n}}$ e $|\xi\rangle_{0_{n}}$ as $2^{\text {as }}$ representações de $[\phi]$ e $[\xi]$, duas bases quaisquer de funções no espaço de ordem $\mathcal{O}_{n}(\mathcal{H})$. Então, uma função aproximada por uma série de $n$ termos $\left|f_{n}\right\rangle_{\infty}$, que é um invariante no espaço de ordem, deve ser descrita em termos de ambas as bases, obedecendo a essa condição de invariança:

$$
\left|f_{n}\right\rangle_{[\phi]}=\left|f_{n}\right\rangle_{[\xi]}
$$

quer dizer,

$$
\left|f_{n}\right\rangle_{\infty}=\left\langle C_{\phi} \mid \phi\right\rangle=\left\langle C_{\xi} \mid \xi\right\rangle
$$

onde os produtos internos acima são IPs mistos, bem como todos os seguintes.

Seja $M \in \mathbb{M}_{n}(\mathbb{C})$ a matriz de mudança de base que leva $|\phi\rangle_{0_{n}}$ em $|\xi\rangle_{0_{n}}$, i.e.,

$$
|\xi\rangle_{0_{n}}=M|\phi\rangle_{0_{n}}
$$

ou, equivalentemente, $|\phi\rangle_{0_{n}}=M^{-1}|\xi\rangle_{0_{n}}$. Disso e da equação (3.12), vem que

$$
\begin{gathered}
\left\langle C_{\xi} \mid \xi\right\rangle=\left\langle C_{\xi}|M| \phi\right\rangle=\left\langle C_{\phi} \mid \phi\right\rangle \Longrightarrow \\
\left\langle C_{\phi}\right|=\left\langle C_{\xi}\right| M,
\end{gathered}
$$

isto é, o covetor coeficiente na nova base assume a forma

$$
\left\langle C_{\xi}\right|=\left\langle C_{\phi}\right| M^{-1}
$$

Então, se $D_{\phi}$ é a MOp na base $[\phi]$, a partir do desenvolvimento seguinte:

$$
\left|f_{n}^{\prime}\right\rangle_{\infty}=\left\langle C_{\phi}\left|D_{\phi}\right| \phi\right\rangle=\left\langle C_{\xi}\left|M D M^{-1}\right| \xi\right\rangle:=\left\langle C_{\xi}\left|D_{\xi}\right| \xi\right\rangle
$$


segue, por unicidade, que a MOp na nova base será

$$
D_{\xi}=M D_{\phi} M^{-1}
$$

Vemos, portanto, que a MOp na nova base é obtida por uma simples transformação de similaridade. Assim, o covetor coeficiente da função derivada na base $[\xi]$ se torna:

$$
\left\langle C_{D_{\xi}}\right|:=\left\langle C_{\xi}\right| D_{\xi}=\left\langle C_{\phi}\right| M^{-1}\left(M D M^{-1}\right)=\left\langle C_{\phi}\right| D M^{-1}=\left\langle C_{D_{\phi}}\right| M^{-1},
$$

fornecendo a transformação

$$
\left\langle C_{D_{\xi}}\right|=\left\langle C_{D_{\phi}}\right| M^{-1}
$$

\subsubsection{Determinação da MOD numa Base Polinomial Canônica}

Agora, uma nova questão emerge: será possível determinar facilmente essa matriz $D$ em qualquer base? A resposta está condicionada à espécie de série escolhida para se efetuar a expansão. Entretanto, de maneira geral, isto não é uma tarefa difícil. Desenvolveremos este cálculo, inicialmente, para a base polinomial canônica, que não é uma base ortogonal.

Assim, seja a base polinomial canônica $|\epsilon\rangle_{0_{n}}$, cuja forma explícita é:

$$
|\epsilon\rangle_{0_{n}}=\left[\begin{array}{c}
1 \\
x \\
\vdots \\
x^{n-1}
\end{array}\right]
$$

Suponhamos agora que a função $f(x)$ possa ser aproximada em termos dos elementos da base na forma

$$
\left|f_{n}\right\rangle_{\infty}=\sum_{i=1}^{n} c^{i}\left|\epsilon_{i}\right\rangle_{\infty}
$$

onde

$$
\left|\epsilon_{j}\right\rangle_{\infty} \equiv \epsilon_{j}(x)=x^{j-1}
$$

Disso, segue

$$
\epsilon_{j}^{\prime}(x)=(j-1) x^{j-2} \quad \Longrightarrow \quad \epsilon_{j+1}^{\prime}(x)=j x^{j-1}
$$

$\mathrm{Ou}$

$$
\left|\epsilon_{j+1}^{\prime}\right\rangle_{\infty}=j\left|\epsilon_{j}\right\rangle_{\infty}=\sum_{\ell=1}^{n} \ell \delta_{j}^{\ell}\left|\epsilon_{\ell}\right\rangle_{\infty}
$$


Agora, reescrevendo a equação (3.2) como

$$
\left|\epsilon_{k}^{\prime}\right\rangle=\sum_{\ell=1}^{n} D_{k}^{\ell}\left|\epsilon_{\ell}\right\rangle,
$$

obtém-se

$$
\left|\epsilon_{j+1}^{\prime}\right\rangle=\sum_{\ell=1}^{n} \ell \delta_{j}{ }^{\ell}\left|\epsilon_{\ell}\right\rangle=\sum_{\ell=1}^{n} D_{j+1}^{\ell}\left|\epsilon_{\ell}\right\rangle
$$

o que implica, finalmente, $D_{j+1}^{\ell}=\ell \delta_{j}^{\ell}$, ou

$$
D_{i}^{j}=j \delta_{i-1}^{j} \quad(i, j=1, \ldots, n)
$$

onde o índice inferior, nesse caso, representa a linha e o superior, a coluna do elemento da matriz. Portanto, a MOD correspondente à primeira derivada em uma base polinomial canônica é dada explicitamente por

$$
D=\left(\begin{array}{cccccccc}
0 & 0 & 0 & 0 & 0 & \cdots & 0 & 0 \\
1 & 0 & 0 & 0 & 0 & \cdots & 0 & 0 \\
0 & 2 & 0 & 0 & 0 & \cdots & 0 & 0 \\
0 & 0 & 3 & 0 & 0 & \cdots & 0 & 0 \\
0 & 0 & 0 & 4 & 0 & \cdots & 0 & 0 \\
0 & 0 & 0 & 0 & 5 & \cdots & 0 & 0 \\
\vdots & \vdots & \vdots & \vdots & \vdots & \ddots & \vdots & \vdots \\
0 & 0 & 0 & 0 & 0 & \cdots & (n-1) & 0
\end{array}\right)
$$

\subsubsection{Mudança de Base para MOp de Ordens Superiores}

A expressão (3.10), anteriormente obtida, é válida para uma base genérica, contudo, quando estamos tratando de outras bases específicas, devemos usar as equações (3.13) e (3.15) para obter o resultado almejado, isto é, para determinarmos a forma explícita da MOp de ordem $k \in \mathbb{Z}$ após uma operação de mudança de base. Então, escrevendo a MOp de $k$-ésima ordem de uma dada função $f_{n}(x)$ aproximada em série, temos

$$
\left|f_{n}^{(k)}\right\rangle_{\infty} \equiv D_{\phi}^{k}\left|f_{n}\right\rangle_{\infty}=\left\langle C_{\phi}\left|D_{\phi}^{k}\right| \phi\right\rangle=\left\langle C_{\xi}\left|M D_{\phi}^{k} M^{-1}\right| \xi\right\rangle
$$

Mas, da comparação com

$$
\left|f_{n}^{(k)}\right\rangle_{\infty}:=\left\langle C_{\xi}\left|D_{\xi}^{k}\right| \xi\right\rangle
$$


segue

$$
D_{\xi}^{k}=M D_{\phi}^{k} M^{-1}, \quad(k \in \mathbb{Z})
$$

Note que podemos, de fato, interpretar essa notação como um produto de matrizes, hipótese essa que produz o mesmo resultado, como consequência da equação (3.16), pois

$$
D_{\xi}=M D_{\phi} M^{-1} \Rightarrow D_{\xi}^{k}=\overbrace{\left(M D_{\phi} M^{-1}\right) \cdots\left(M D_{\phi} M^{-1}\right)}^{k}=M D_{\phi}^{k} M^{-1}
$$

Consequentemente, a MOp de $k$-ésima ordem de $f_{n}(x)$, expressa numa base polinomial genérica, após uma mudança de base $M:[\phi] \stackrel{M}{\longrightarrow}[\xi]$, assume a forma expressa em (3.19).

\subsubsection{Transformação do Covetor Coeficiente de MOps de Grau $k$ Inteiro}

Efetua-se aqui uma generalização da fórmula (3.17) para a mudança de base $M$ : $[\phi] \stackrel{M}{\longrightarrow}[\xi]$. Pela equação (3.11) podemos escrever:

$$
\left\langle C_{\mathrm{D}_{\phi}^{k}}\right|=\left\langle C_{\phi}\right| D_{\phi}^{k}
$$

Usando a equação (3.14) na fórmula acima:

$$
\left\langle C_{\mathrm{D}_{\phi}^{k}}\right|=\left\langle C_{\xi}\right| M D_{\phi}^{k}
$$

Multiplicando ambos os lados dessa expressão por $M^{-1}$ à direita obtém-se

$$
\left\langle C_{\mathrm{D}_{\phi}^{k}}\right| M^{-1}=\left\langle C_{\xi}\right| M D_{\phi}^{k} M^{-1}
$$

Mas o produto de matrizes do lado direito, de acordo com (3.19), produz $D_{\xi}^{k}$, fornecendo

$$
\left\langle C_{\mathrm{D}_{\phi}^{k}}\right| M^{-1}=\left\langle C_{\xi}\right| D_{\xi}^{k}
$$

Agora, a equação (3.11) pode ser usada novamente para identificar-se o lado direito da expressão com $\left\langle C_{\mathrm{D}_{\xi}^{k}}\right|$, resultando

$$
\left\langle C_{\mathrm{D}_{\xi}^{k}}\right|=\left\langle C_{\mathrm{D}_{\phi}^{k}}\right| M^{-1}, \quad(k \in \mathbb{Z}),
$$

que constitui a generalização desejada. 


\subsubsection{MOD de ordem genérica na base polinomial canônica}

Como a MOD de ordem genérica nesse caso é dada simplesmente pelo sucessivo produto da primeira derivada da matriz $D$ por si mesma, expressa na equação (3.18), tudo o que temos a fazer é efetuar esse cálculo diretamente. Para segunda ordem, por exemplo, denotando $D^{2}=\mathrm{P}$, temos:

$$
\begin{gathered}
\mathrm{P}_{i}^{k}=\sum_{\ell=1}^{n} D_{i}^{\ell} D_{\ell}^{k}=\sum_{\ell=1}^{n} \ell \delta_{i-1}^{\ell} k \delta_{\ell-1}^{k}=(i-1) k \delta_{i-2}^{k} \Rightarrow \\
\Rightarrow \mathrm{P}_{i}^{k}=(i-1) k \delta_{i-2}^{k}
\end{gathered}
$$

Logo, os valores não-nulos de $D^{2}$ são

$$
\mathrm{P}_{m+1}^{m-1}=m(m-1) \equiv A_{m, 2}
$$

$\mathrm{Ou}$

$$
\mathrm{P}_{j}^{j-2}=(j-1)(j-2)
$$

É essencial reparar que a linha do elemento de matriz nesse caso é indicada pelo índice inferior ( $1^{\circ}$ índice) e a coluna, pelo superior ( $2^{\circ}$ índice). Isso se deve ao fato de a transformação ter sido efetuada sobre a base, cujas componentes têm caráter covariante. Assim, o índice mais próximo da grandeza se refere à linha, não importando se seja inferior ou superior. Em vista disso, deve-se ter cuidado em observar a correta posição do índice precedente. Portanto, a MOD de segunda ordem nessa base será

$$
D^{2}=\left(\begin{array}{ccccccc}
0 & 0 & 0 & 0 & 0 & \cdots & 0 \\
0 & 0 & 0 & 0 & 0 & \cdots & 0 \\
2 \cdot 1 & 0 & 0 & 0 & 0 & \cdots & 0 \\
0 & 3 \cdot 2 & 0 & 0 & 0 & \cdots & 0 \\
0 & 0 & 4 \cdot 3 & 0 & 0 & \cdots & 0 \\
\vdots & \vdots & \vdots & \ddots & \vdots & \ddots & \vdots \\
0 & 0 & 0 & \cdots & (n-1)(n-2) & \cdots & 0
\end{array}\right)
$$

Procedendo da mesma maneira mais uma vez para obter a terceira derivada $D^{3}=\mathrm{Q}$, chega-se a

$$
\mathrm{Q}_{i}{ }^{k}=\sum_{\ell=1}^{n} D_{i}^{\ell} \mathrm{P}_{\ell}{ }^{k}=\sum_{\ell=1}^{n} \ell \delta_{i-1}^{\ell}(\ell-1) k \delta_{\ell-2}^{k}=(i-1)(i-2) k \delta_{i-3}^{k},
$$


com os valores não-nulos de $D^{3}$ sendo dados pelos elementos

$$
\mathrm{Q}_{m+1}^{m-2}=m(m-1)(m-2)=A_{m, 3}
$$

$\mathrm{Ou}$

$$
\mathrm{Q}_{j}{ }^{j-3}=(j-1)(j-2)(j-3)
$$

Então, explicitamente, tem-se

$$
D^{3}=\left(\begin{array}{ccccccc}
0 & 0 & 0 & 0 & 0 & \cdots & 0 \\
0 & 0 & 0 & 0 & 0 & \ldots & 0 \\
0 & 0 & 0 & 0 & 0 & \ldots & 0 \\
3 \cdot 2 \cdot 1 & 0 & 0 & 0 & 0 & \ldots & 0 \\
0 & 4 \cdot 3 \cdot 2 & 0 & 0 & 0 & \ldots & 0 \\
\vdots & \vdots & \ddots & \vdots & \vdots & \ddots & \vdots \\
0 & 0 & \cdots & (n-1)(n-2)(n-3) & 0 & \cdots & 0
\end{array}\right)
$$

Consequentemente, não é difícil estabelecer que os elementos não-nulos para a MOD de ordem $k$ genérica serão

$$
\left[D^{k}\right]_{m+1}^{m-k+1}=A_{m, k}=m(m-1)(m-2) \cdots(m-k+1),
$$

onde o índice $m$ vai de $k$ até $(n-1)$.

Exemplo 1 : A ideia agora é expor os conceitos acima discutidos num exemplo simples concreto. Seja $f_{n}(x)$ uma função tal que

$$
f_{n}(x)=\sum_{k=1}^{n} \frac{x^{k-1}}{k}
$$

Então, expressando-se a função com 5 termos, tomando-se $n=5$, tem-se

$$
f_{5}(x)=1+\frac{x}{2}+\frac{x^{2}}{3}+\frac{x^{3}}{4}+\frac{x^{4}}{5}
$$

O covetor dos coeficientes na base canônica é então escrito:

$$
\langle C|=\left[\begin{array}{lllll}
1 & \frac{1}{2} & \frac{1}{3} & \frac{1}{4} & \frac{1}{5}
\end{array}\right]
$$


enquanto a MOD assume a forma

$$
D=\left(\begin{array}{lllll}
0 & 0 & 0 & 0 & 0 \\
1 & 0 & 0 & 0 & 0 \\
0 & 2 & 0 & 0 & 0 \\
0 & 0 & 3 & 0 & 0 \\
0 & 0 & 0 & 4 & 0
\end{array}\right)
$$

e o covetor coeficiente da derivada da função torna-se

$$
\left\langle C_{D}\right|=\langle C| D=\left[\begin{array}{lllll}
\frac{1}{2} & \frac{2}{3} & \frac{3}{4} & \frac{4}{5} & 0
\end{array}\right]
$$

Como ilustração do esquema de mudança de base, vamos assumir uma base, não necessariamente ortogonal, $|\phi\rangle_{0_{5}}$ tal que

$$
|\phi\rangle=\left[\begin{array}{c}
1 \\
1+2 x \\
x+3 x^{2} \\
x^{2}+4 x^{3} \\
x^{3}+5 x^{4}
\end{array}\right]
$$

Daí, como os elementos de $[\phi]$ são $\phi_{j}(x)=j x^{j-1}+\left(1-\delta_{j}^{1}\right) x^{j-2}$, vê-se que essa base pode ser escrita em termos da canônica como

$$
\begin{gathered}
\left|\phi_{1}\right\rangle=1\left|\epsilon_{1}\right\rangle, \\
\left|\phi_{k}\right\rangle=k\left|\epsilon_{k}\right\rangle+1\left|\epsilon_{k-1}\right\rangle \quad\left(k \in \mathbb{N}^{*} \backslash\{1\}\right)
\end{gathered}
$$

Comparando com a equação de mudança de base

$$
\left|\phi_{k}\right\rangle=\sum_{\ell=1}^{n} M_{k}^{\ell}\left|\epsilon_{\ell}\right\rangle,
$$

segue que os elementos não-nulos são $M_{k}{ }^{k-1}=1$ e $M_{k}{ }^{k}=k$ e, portanto, $a$ matriz de mudança de base da canônica para $[\phi]$ assume a forma explícita:

$$
M=\left(\begin{array}{lllll}
1 & 0 & 0 & 0 & 0 \\
1 & 2 & 0 & 0 & 0 \\
0 & 1 & 3 & 0 & 0 \\
0 & 0 & 1 & 4 & 0 \\
0 & 0 & 0 & 1 & 5
\end{array}\right)
$$


Consequentemente, sua inversa será

$$
M^{-1}=\frac{1}{120}\left(\begin{array}{rrrrr}
120 & 0 & 0 & 0 & 0 \\
-60 & 60 & 0 & 0 & 0 \\
20 & -20 & 40 & 0 & 0 \\
-5 & 5 & -10 & 30 & 0 \\
1 & -1 & 2 & -6 & 24
\end{array}\right)
$$

Logo, usando a equação (3.15):

$$
\begin{gathered}
\left\langle C_{\phi}\right|=\langle C| M^{-1}=\left[\begin{array}{lllll}
1 & \frac{1}{2} & \frac{1}{3} & \frac{1}{4} & \frac{1}{5}
\end{array}\right] M^{-1} \Rightarrow \\
\left\langle C_{\phi}\right|=\left[\begin{array}{lllll}
\frac{5737}{7200} & \frac{1463}{7200} & \frac{337}{3600} & \frac{21}{400} & \frac{1}{25}
\end{array}\right]
\end{gathered}
$$

Por conseguinte, na base genérica dada, a MOD é escrita na forma

$$
D_{\phi}=M D M^{-1}=\left(\begin{array}{rrrrr}
0 & 0 & 0 & 0 & 0 \\
2 & 0 & 0 & 0 & 0 \\
-2 & 3 & 0 & 0 & 0 \\
1 & -1 & 4 & 0 & 0 \\
-\frac{1}{3} & \frac{1}{3} & -\frac{2}{3} & 5 & 0
\end{array}\right)
$$

com o covetor coeficiente da primeira derivada sendo imediatamente obtido por:

$$
\left\langle C_{D_{\phi}}\right|=\left\langle C_{D}\right| M^{-1}=\left[\begin{array}{lllll}
\frac{31}{120} & \frac{29}{120} & \frac{11}{60} & \frac{1}{5} & 0
\end{array}\right]
$$

A expansão da função na nova base assume a forma:

$$
\begin{gathered}
\left|f_{5}\right\rangle_{[\phi]}=\sum_{k=1}^{5} c_{\phi}^{k}\left|\phi_{k}\right\rangle \Rightarrow \\
f_{5}(x)=\frac{5737}{7200}+\frac{1463}{7200}(1+2 x)+\frac{337}{3600}\left(x+3 x^{2}\right)+\frac{21}{400}\left(x^{2}+4 x^{3}\right)+\frac{1}{25}\left(x^{3}+5 x^{4}\right)
\end{gathered}
$$

Já para a segunda derivada, tem-se

$$
D^{2}=\left(\begin{array}{ccccc}
0 & 0 & 0 & 0 & 0 \\
0 & 0 & 0 & 0 & 0 \\
2 & 0 & 0 & 0 & 0 \\
0 & 6 & 0 & 0 & 0 \\
0 & 0 & 12 & 0 & 0
\end{array}\right)
$$


e, pela equação (3.11),

$$
\left\langle C_{D^{2}}\right|=\langle C| D^{2}=\left[\begin{array}{lllll}
\frac{2}{3} & \frac{3}{2} & \frac{12}{5} & 0 & 0
\end{array}\right]
$$

Após a mudança de base, chega-se a

$$
D_{\phi}^{2}=\left(\begin{array}{ccccc}
0 & 0 & 0 & 0 & 0 \\
0 & 0 & 0 & 0 & 0 \\
2 & 0 & 0 & 0 & 0 \\
0 & 6 & 0 & 0 & 0 \\
0 & 0 & 12 & 0 & 0
\end{array}\right)
$$

e, pela eq.(3.20), a

$$
\left\langle C_{D_{\phi}^{2}}\right|=\left\langle C_{D^{2}}\right| M^{-1}=\left[\begin{array}{lllll}
\frac{19}{60} & \frac{7}{20} & \frac{4}{5} & 0 & 0
\end{array}\right]
$$

\subsubsection{Dedução da Matriz Sandwich}

Esse nome foi primeiramente usado no trabalho de Guimarães (2008) para um resultado original ali obtido e que se refere à descrição de uma matriz de derivação em uma base polinomial qualquer, a partir de uma outra base conhecida e da matriz de mudança de base entre elas, comparando-as com uma base de referência que atua como intermediária.

O que faremos aqui é uma generalização daquele resultado, para o corpo complexo e para inclusão de bases não polinomiais.

Consideremos uma função $f(x)$ que satisfaça as condições necessárias para ser linearmente expressa em termos de determinadas séries. Chamemos de $[\varphi]$ a base da série original em que a função é expandida e de $[\alpha]$ e $[\beta]$ outras duas bases nas quais desejamos expressar a mesma função. Então, a aproximação por $n$ termos da referida função será escrita:

1. Na base original $|\varphi\rangle_{0_{n}}$ :

$$
\left|f_{n}\right\rangle_{\infty}=\langle C \mid \varphi\rangle
$$

2. Numa base genérica $|\alpha\rangle_{o_{n}}$ :

$$
\left|f_{n}\right\rangle_{\infty}=\left\langle C_{\alpha} \mid \alpha\right\rangle
$$

3. Numa base específica desejada $|\beta\rangle_{0_{n}}$ :

$$
\left|f_{n}\right\rangle_{\infty}=\left\langle C_{\beta} \mid \beta\right\rangle
$$


Nesses termos, podemos nos perguntar qual é a expressão que leva a matriz operacional de derivação (ou integração) descrita numa base genérica a uma outra, específica, mais conveniente.

Sabemos que uma base pode ser descrita em termos de outra por uma matriz de mudança de base, então, se $A$ e $B\left(A, B \in \mathbb{M}_{n}(\mathbb{C})\right)$ são as matrizes de mudança de base de $|\varphi\rangle$ para $|\alpha\rangle$ e $|\beta\rangle$, respectivamente, podemos escrever:

$$
\left\{\begin{array}{c}
|\alpha\rangle_{0_{n}}=A|\varphi\rangle_{0_{n}} \\
|\beta\rangle_{0_{n}}=B|\varphi\rangle_{0_{n}}
\end{array}\right.
$$

Entretanto, da equivalência de representações da função $f(x)$, vem

$$
\begin{gathered}
\langle C \mid \varphi\rangle=\left\langle C_{\alpha} \mid \alpha\right\rangle=\left\langle C_{\beta} \mid \beta\right\rangle \Rightarrow \\
\langle C \mid \varphi\rangle=\left\langle C_{\alpha}|A| \varphi\right\rangle=\left\langle C_{\beta}|B| \varphi\right\rangle
\end{gathered}
$$

o que, devido à linearidade e completude da base, fornece:

$$
\langle C|=\left\langle C_{\alpha}\right| A=\left\langle C_{\beta}\right| B
$$

ou, alternativamente,

$$
\left\{\begin{array}{l}
\left\langle C_{\alpha}\right|=\langle C| A^{-1} \\
\left\langle C_{\beta}\right|=\langle C| B^{-1}
\end{array}\right.
$$

Então, expressando-se a derivada em termos de cada base, tem-se

$$
\begin{gathered}
\left|f_{n}^{\prime}\right\rangle_{\infty}=\left\langle C \mid \phi^{\prime}\right\rangle=\left\langle C_{\alpha} \mid \alpha^{\prime}\right\rangle=\left\langle C_{\beta} \mid \beta^{\prime}\right\rangle \Rightarrow \\
\left|f_{n}^{\prime}\right\rangle_{\infty}=\langle C|D| \phi\rangle=\left\langle C_{\alpha}\left|D_{\alpha}\right| \alpha\right\rangle=\left\langle C_{\beta}\left|D_{\beta}\right| \beta\right\rangle
\end{gathered}
$$

onde $D, D_{\alpha}$ e $D_{\beta}$ são as matrizes operacionais de derivação nas bases canônica, genérica e específica, respectivamente. Substituindo-se as equações (3.22) e (3.23) na expressão acima, chega-se a

$$
\left\langle C\left|A^{-1} D_{\alpha} A\right| \phi\right\rangle=\left\langle C\left|B^{-1} D_{\beta} B\right| \phi\right\rangle
$$

ou, por completude,

$$
A^{-1} D_{\alpha} A=B^{-1} D_{\beta} B
$$


o que leva finalmente ao resultado

$$
D_{\beta}=B \Omega B^{-1}
$$

onde

$$
\Omega=A^{-1} D_{\alpha} A
$$

\subsubsection{Mudanças de Base em Domínios Distintos}

Há uma questão mais complicada a ser discutida, que diz respeito à possibilidade de se efetuar mudanças de base entre funções definidas em domínios diferentes, já que a forma explícita do IP em espaços de Hilbert depende do domínio de definição das funções que constituem a base.

Vamos discorrer um pouco sobre isso do ponto de vista do formalismo tensorial.

Através do uso do operador autoprojetor podemos descrever a expansão finita de ordem $n$ de uma função $\mathcal{L}_{w}^{2}$ genérica $f(x)$ em uma base específica $\left\{\left|b_{r}\right\rangle_{\infty}\right\}$, pela aproximação:

$$
|f\rangle_{\infty \| \mathcal{O}_{n}} \equiv\left|f_{n}\right\rangle_{\infty}=\mathbb{P}_{n}|f\rangle_{\infty}=\sum_{s}^{n}\left|b_{s}\right\rangle_{\infty}\left\langle b^{s} \mid f\right\rangle_{\infty}
$$

sendo os coeficientes da séries iguais a

$$
C^{s}=\left\langle b^{s} \mid f\right\rangle_{\infty}
$$

Seja agora $\left\{\left|\xi_{r}\right\rangle_{\infty}\right\}$ uma base genérica. A mesma função pode ser expandida numa nova série em termos dessa base como

$$
\left|f_{n}\right\rangle_{\infty}=\langle\alpha \mid \xi\rangle_{o_{n}} \equiv \sum_{r}^{n} \alpha^{r}\left|\xi_{r}\right\rangle_{\infty}
$$

Dessa forma, sejam $\alpha^{r}$ os coeficientes da expansão da função $f(x)$ na base genérica de ordem $n$. Então,

$$
\left|f_{n}\right\rangle_{\infty}=\sum_{r}^{n} \alpha^{r}\left|\xi_{r}\right\rangle_{\infty} \Rightarrow \alpha^{r}=\sum_{s}^{n} \gamma^{r s}\left\langle\xi_{s} \mid f\right\rangle_{\infty}
$$

onde $\gamma^{r s}=\left\langle\xi^{r} \mid \xi^{s}\right\rangle_{\infty}$ constitui um elemento da métrica recíproca base genérica.

Mas usando a equação obtida na atuação do autoprojetor na base específica, podemos 
escrever os coeficientes da expansão de $|f\rangle_{\infty}$ na base genérica como

$$
\begin{gathered}
\alpha^{k}=\sum_{m}^{n} \gamma^{k m}\left\langle\xi_{m} \mid f\right\rangle_{\infty}= \\
=\sum_{m}^{n} \gamma^{k m}\left\langle\xi_{m} \mid f_{n}\right\rangle_{\infty}= \\
=\sum_{m}^{n} \gamma^{k m}\left\langle\xi_{m}\left|\mathbb{P}_{n}\right| f_{n}\right\rangle_{\infty}= \\
=\sum_{m}^{n} \gamma^{k m}\left\langle\xi_{m}\left|\left(\sum_{s}^{n}\left|b_{s}\right\rangle\left\langle b^{s}\right|\right)\right| f\right\rangle_{\infty}= \\
=\sum_{m}^{n} \sum_{s}^{n} \gamma^{k m}\left\langle\xi_{m} \mid b_{s}\right\rangle\left\langle b^{s} \mid f\right\rangle_{\infty}
\end{gathered}
$$

onde $\gamma^{k m}=\left\langle\xi^{k} \mid \xi^{m}\right\rangle_{\infty}$ é um elemento da métrica recíproca da base genérica e $\left\langle\xi_{m} \mid b_{s}\right\rangle$ é o IP híbrido entre as duas bases.

Entretanto, de

$$
\left\langle b^{s} \mid f\right\rangle_{\infty}=\sum_{r}^{n} g^{s r}\left\langle b_{r} \mid f\right\rangle_{\infty}=C^{s}
$$

segue que os coeficientes na nova base serão dados, em termos da base específica, pela expressão:

$$
\alpha^{k}=\sum_{m}^{n} \sum_{s}^{n} \gamma^{k m}\left\langle\xi_{m} \mid b_{s}\right\rangle C^{s}
$$

\subsection{Descrição do Método Proposto}

\subsubsection{Séries e Bases Complexas de Fourier}

Nosso foco nessa altura é explorar as possibilidades das matrizes operacionais complexas de Fourier na resolução de equações diferenciais ordinárias de ordem inteira e fracional. Logo, vamos nos concentrar nesse tema a partir desse ponto.

Repassando brevemente o que foi visto, uma função seccionalmente diferenciável e periódica $f(x)$, de semiperíodo $L$, absolutamente integrável no intervalo limitado pelo período, pode ser expressa por meio de uma série de Fourier complexa como

$$
f(x)=\sum_{m=-\infty}^{\infty} c^{m} e_{m}(x)
$$


onde

$$
c^{m}=\frac{1}{2 L} \int_{-L}^{L} \bar{e}_{m}(\xi) f(\xi) d \xi
$$

são os coeficientes da série e

$$
e_{m}(x)=\exp (i m q x), \quad q=\frac{\pi}{L}
$$

são as componentes da base.

No caso de uma definição de período não simétrica em relação à origem, num intervalo $[a, b]$, por exemplo, sendo $L=\frac{b-a}{2}$, a expressão dos coeficientes $c^{m}$ assumiria a forma

$$
c^{m}=\frac{1}{2 L} \int_{a}^{b} \bar{e}_{m}(\xi) f(\xi) d \xi
$$

Em pontos de descontinuidade, o terorema de Fourier garante que a série tende ao valor $\frac{1}{2}\left[\lim _{x \rightarrow 0^{+}} f(x)+\lim _{x \rightarrow 0^{-}} f(x)\right]$. Entretanto, nesse caso, a convergência não poderá ser uniforme. Esse fato, estudado pelo físico estadunidense J. W. Gibbs (1839-1903), é o responsável pela ocorrência de oscilações de maior amplitude nas adjacências imediatas das descontinuidades, ou seja, um tipo de overshoot nessas regiões, efeito esse chamado de fenômeno de Gibbs, desempenhando papel correspondente ao fenômeno de Runge, que ocorre nas interpolações polinomiais.

Com seu auxílio, podemos expressar a função aproximada como

$$
f_{n}(x)=\sum_{m=-\ell}^{\ell} e_{m}(x) \frac{1}{2 L} \int_{-L}^{L} \bar{e}_{m}(\xi) f(\xi) d \xi
$$

Nesse caso, a base para a série de Fourier complexa $|e\rangle_{0_{n}}$, com $n=2 \ell+1$ termos, assume a forma:

$$
|e\rangle_{0_{n}}=\left[\begin{array}{c}
e^{-i \ell q x} \\
\vdots \\
e^{-i q x} \\
1 \\
e^{i q x} \\
\vdots \\
e^{i \ell q x}
\end{array}\right]
$$

Sendo a métrica ortogonal $\gamma_{i k}=2 L \delta_{i k}$, a base renormalizada de Fourier é obtida simplesmente por

$$
\left|\hat{e}_{r}\right\rangle_{\infty}=\frac{1}{2 L}\left|e_{r}\right\rangle_{\infty}
$$


e a recíproca por

$$
\left\langle\left. e^{m}\right|_{\infty}=\frac{1}{2 L}\left\langle\left. e_{m}\right|_{\infty}\right.\right.
$$

Nessas condições, o posicionamento indicial passa a ser irrelevante, uma vez que as coordenadas covariantes e contravariantes são iguais. Logo, pode-se adotar uma notação mais conhecida para bras e kets, através apenas de seus índices, i.e., $\left|\hat{e}_{m}\right\rangle \rightarrow|m\rangle$ e $\langle m \mid n\rangle=\delta_{m n}$.

Assim,

$$
\left|f_{n}\right\rangle_{\infty}=\sum_{m=-\ell}^{\ell} c^{m}|m\rangle_{\infty} \equiv\langle c \mid e\rangle_{0_{n}}
$$

Finalmente, o processo de derivação da função expandida gera

$$
\left|f_{n}^{\prime}\right\rangle_{\infty}=\sum_{m=-\ell}^{\ell} c^{m}\left|m^{\prime}\right\rangle_{\infty}
$$

Antes de efetuarmos o cálculo da forma explícita da matriz operacional de derivação na base complexa de Fourier, discorreremos um pouco sobre o núcleo de Dirichlet e a questão da convergência.

\subsubsection{Convergência Pontual da Série Aproximativa}

\subsubsection{Núcleo de Dirichlet Complexo}

O objetivo precípuo da expansão em série de Fourier complexa de uma função $f(x)$ : $\mathbb{R} \rightarrow \mathbb{C}$ é que essa soma convirja para a função à medida em que sua extensão $\ell$ cresça indefinidamente. Para isso, é preciso analisar as condições para as quais isso de fato ocorre. Tomando a série da função aproximada

$$
f_{n}(x)=\sum_{k=-\ell}^{\ell} c^{k} \exp (i k q x), \quad(n=2 \ell+1)
$$

e denotando, por concisão, $\exp (i k q x)=e_{k}(x)$, podemos expressar a soma que representa a função aproximada como

$$
s_{n}(x)=\sum_{|k| \leq \ell} c^{k} e_{k}(x)
$$

Obviamente, a soma acima é a própria função expandida, i.e., $s_{n}(x)=f_{n}(x)$. Lembramos, porém, que esta série pode ser reescrita, por conveniência computacional, na forma

$$
s_{n}(x)=\sum_{k=1}^{n} C^{k} E_{k}(x),
$$


onde $C^{k}=c^{k-\ell-1}$ e $E_{k}(x)=\exp [i q(k-\ell-1) x] \equiv e_{k-\ell-1}(x),(k=1, \ldots, 2 \ell+1)$. Iremos, entretanto, trabalhar com a primeira forma, por questão de simplicidade. Desse modo, o resto local $\mathcal{R}_{n}$, definido pela diferença entre a soma de extensão $\ell$ e amplitude $n=2 \ell+1$ e o valor da função original será

$$
\mathcal{R}_{n}(x)=s_{n}(x)-f(x)
$$

ou, em pontos de descontinuidade,

$$
\mathcal{R}_{n}(x)=s_{n}(x)-\frac{f(x+0)+f(x-0)}{2},
$$

sendo

$$
f(x \pm 0)=\lim _{x \rightarrow 0^{ \pm}} f(x)
$$

A estimativa desse valor, fornecerá uma medida adequada da convergência pontual da série. Em outras termos, para que se garanta a convergência da soma para o valor da função num ponto $x \in D_{f}$, espera-se que

$$
\lim _{n \rightarrow \infty} s_{n}(x)=f(x)
$$

Expandindo os coeficientes de Fourier na expressão da soma, tem-se

$$
\begin{aligned}
s_{n}(x) & =\sum_{|k| \leq \ell} \frac{1}{2 L} \int_{-L}^{L} f(y) e_{k}(-y) d y e_{k}(x)= \\
& =\int_{-L}^{L}\left[\frac{1}{2 L} \sum_{|k| \leq \ell} e_{k}(x-y)\right] f(y) d y
\end{aligned}
$$

A expressão entre colchetes constitui o kernel $K(x, y)$ de uma transformação integral, denominado Núcleo de Dirichlet complexo $\mathfrak{D}_{\ell}(x-y)$ e definido:

$$
\mathfrak{D}_{\ell}(x-y)=\frac{1}{2 L} \sum_{|k| \leq \ell} e_{k}(x-y)
$$

ou, em termos do período $T$,

$$
\mathfrak{D}_{\ell}(x)=\frac{1}{T} \sum_{|k| \leq \ell} \exp (i k q x), \quad(k \in \mathbb{Z})
$$


Escrito em termos da notação crescente, o núcleo de Dirichlet assume a forma

$$
\mathfrak{D}_{n}(x)=\frac{1}{T} \sum_{k=1}^{n} E_{k}(x)
$$

$\mathrm{Ou}$

$$
\mathfrak{D}_{n}(x)=\frac{1}{T} \sum_{k=1}^{n} \exp [i q(k-\ell-1) x], \quad(n=2 \ell+1)
$$

Assim, a soma $s_{n}(x)$ pode ser expressa na forma

$$
s_{n}(x)=\int_{-L}^{L} \mathfrak{D}_{n}(x-y) f(y) d y
$$

\subsubsection{Propriedades de $\mathfrak{D}_{\ell}(x)$}

1. $\mathfrak{D}_{\ell}(x)$ é par:

$$
\mathfrak{D}_{\ell}(-x)=\frac{1}{T} \sum_{|-k| \leq \ell} \exp (i(-k) q x)=\frac{1}{T} \sum_{|r| \leq \ell} \exp (\text { irqx })=\mathfrak{D}_{\ell}(x)
$$

2. $\mathfrak{D}_{\ell}(x)$ é contínuo:

Soma de funções contínuas é contínua, logo

$$
e^{i k q x} \text { cont. } \Rightarrow \sum e^{i k q x} \text { cont. } \Rightarrow \mathfrak{D}_{\ell}(x) \text { cont. }
$$

3. $\mathfrak{D}_{\ell}(x)$ é periódico de período $2 L$ :

$$
\begin{gathered}
\mathfrak{D}_{\ell}(x+2 L)= \\
=\frac{1}{2 L} \sum_{|k| \leq \ell} \exp [i k q(x+2 L)]=\frac{1}{2 L} \sum_{|k| \leq \ell} \exp (i k q x) \exp \left(i k \frac{\pi}{L} 2 L\right)= \\
=\frac{1}{2 L} \sum_{|k| \leq \ell} \exp (i k q x) \exp (2 \pi i k)=\frac{1}{2 L} \sum_{|k| \leq \ell} \exp (i k q x)= \\
=\mathfrak{D}_{\ell}(x)
\end{gathered}
$$

4. A integral de $\mathfrak{D}_{\ell}(x)$ no período é unitária:

$$
\int_{-L}^{L} \mathfrak{D}_{\ell}(x) d x=\int_{-L}^{L} \exp (i k q x) d x=\frac{1}{2 L} \sum_{|k| \leq \ell} \int_{-L}^{L} \exp (i k q x) d x=
$$




$$
=\frac{1}{2 L}\left(\left.\sum_{\substack{|k| \leq \ell \\ k \neq 0}} \frac{\exp (i k q x)}{i k q}\right|_{x=-L} ^{L}+2 L\right)=\frac{1}{2 L} \sum_{\substack{|k| \leq \ell \\ k \neq 0}} \frac{1}{i k q} \underbrace{\left(e^{i k \pi}-e^{-i k \pi}\right)}_{0}+1=1
$$

5. O complexo conjugado de $\mathfrak{D}_{\ell}(x)$ é igual à sua reflexão em torno da origem, i.e., $\mathfrak{D}_{\ell}^{*}(x)=\mathfrak{D}_{\ell}(-x):$

$$
\mathfrak{D}_{\ell}^{*}(x)=\frac{1}{T} \sum_{|k| \leq \ell} \overline{\exp (i k q x)}=\frac{1}{T} \sum_{|k| \leq \ell} \exp [i k q(-x)]=\mathfrak{D}_{\ell}(-x)
$$

6. $\mathfrak{D}_{\ell}(0)=\left(\ell+\frac{1}{2}\right) / L$ :

$$
\mathfrak{D}_{\ell}(x)=\frac{1}{2 L} \sum_{k=-\ell}^{\ell} 1=\frac{1}{2 L}(2 \ell+1)=\left(\ell+\frac{1}{2}\right) / L
$$

7. $\mathfrak{D}_{\ell}(x)$ pode ser expresso compactamente por

$$
\mathfrak{D}_{\ell}(x)=\frac{1}{2 L} \frac{\sin \left[\left(\ell+\frac{1}{2}\right) q x\right]}{\sin (q x / 2)}
$$

A demonstração da propriedade acima é um pouco mais trabalhosa. Primeiro, podemos separar a soma em duas partes:

$$
\mathfrak{D}_{\ell}(x)=\frac{1}{2 L}\left(\sum_{k=-\ell}^{-1}+\sum_{k=0}^{\ell}\right) e^{i k q x}
$$

Agora, chamando $z=e^{i q x}$, tem-se $e^{i k q x}=z^{k} \Rightarrow$

$$
\mathfrak{D}_{\ell}(x)=\frac{1}{2 L}\left(\sum_{k=1}^{\ell}(1 / z)^{k}+\sum_{k=0}^{\ell} z^{k}\right)
$$

Como

$$
\sum_{k=0}^{\ell} z^{k}=\frac{1-z^{k+1}}{1-z}
$$

$\mathrm{e}$

$$
\sum_{k=1}^{\ell}(1 / z)^{k}=(1 / z) \sum_{k=0}^{\ell-1}(1 / z)^{k}=(1 / z) \frac{1-(1 / z)^{\ell}}{1-(1 / z)}
$$

então

$$
\begin{gathered}
\mathfrak{D}_{\ell}(x)=\frac{1}{2 L}\left[\frac{1-z^{\ell+1}}{1-z}+\left(\frac{1}{z}\right) \frac{1-(1 / z)^{\ell}}{1-(1 / z)}\right] \Rightarrow \\
\mathfrak{D}_{\ell}(x)=\frac{1}{2 L}\left[\frac{1-e^{i q(\ell+1) x}}{1-e^{i q x}}+\left(\frac{1}{e^{i q x}}\right) \frac{1-e^{-i q \ell x}}{1-e^{-i q x}}\right]=
\end{gathered}
$$




$$
\begin{gathered}
=\frac{1}{2 L}\left(\frac{e^{-i q x / 2}-e^{i q(\ell+1 / 2) x}}{e^{-i q x / 2}-e^{i q x / 2}}+\frac{e^{-i q x / 2}-e^{-i q(\ell+1 / 2) x}}{e^{i q x / 2}-e^{-i q x / 2}}\right)= \\
=\frac{1}{2 L} \frac{1}{\left(e^{i q x / 2}-e^{-i q x / 2}\right)}\left(e^{-i q x / 2}-e^{-i q(\ell+1 / 2) x}-e^{-i q x / 2}+e^{i q(\ell+1 / 2) x}\right)= \\
=\frac{1}{2 L}\left(\frac{e^{i q(\ell+1 / 2) x}-e^{-i q(\ell+1 / 2) x}}{e^{i q x / 2}-e^{-i q x / 2}}\right) \Rightarrow \mathfrak{D}_{\ell}(x)=\frac{1}{2 L} \frac{\sin \left[\left(\ell+\frac{1}{2}\right) q x\right]}{\sin (q x / 2)}
\end{gathered}
$$

\subsubsection{A Série Aproximativa como Integral de Convolução e de Correlação e Produto Interno}

Como vimos, a soma correspondente à série de $n$ termos da função aproximada é escrita

$$
s_{n}(x)=\int_{-L}^{L} \mathfrak{D}_{n}(x-y) f(y) d y
$$

Mas como o intervalo é simétrico e a função $f$ é assumida periódica, então a soma pode ser expressa como uma integral de convolução cíclica de período $2 L$ :

$$
\begin{gathered}
s_{n}(x)=\mathfrak{D}_{n}(x) * f(x)=\left(\mathfrak{D}_{n} * f\right)(x) \Rightarrow \\
s_{n} \equiv \mathfrak{D}_{n} * f
\end{gathered}
$$

Por outro lado, fazendo $x-y \longrightarrow-y^{\prime}$ :

$$
\begin{gathered}
s_{n}(x)=\int_{-L}^{L} \mathfrak{D}_{n}\left(-y^{\prime}\right) f\left(x+y^{\prime}\right) d y^{\prime}=\int_{-L}^{L} \mathfrak{D}_{n}^{*}(y) f(x+y) d y \Rightarrow \\
s_{n}(x)=\left\langle\mathfrak{D}_{n}(y) \mid f(x+y)\right\rangle_{\infty}
\end{gathered}
$$

Logo, como $f(t) \star g(t)=\langle f(u) \mid g(t+u)\rangle_{\infty}$, então

$$
\begin{gathered}
s_{n}(x)=\mathfrak{D}_{n}(x) \star f(x)=\left(\mathfrak{D}_{n} \star f\right)(x) \Rightarrow \\
s_{n} \equiv \mathfrak{D}_{n} \star f
\end{gathered}
$$

Conclui-se, portanto, que a soma pode ser descrita tanto em termos de uma integral de convolução como de uma integral de correlação, apresentando, devido às propriedades do núcleo de Dirichlet, formas similares.

A equação (3.27) é uma das maneiras de expressar a soma como um produto interno de Dirac, mas não a única. Uma outra forma, que será útil na próxima seção, é obtida 
diretamente de (3.26):

$$
s_{n}(x)=\left\langle\mathfrak{D}_{n}^{*}(x-y) \mid f(y)\right\rangle_{\infty}
$$

Deve-se notar, entretanto, que a soma também pode ser descrita como um ket, já que se identifica com a função aproximada $\left|f_{n}(x)\right\rangle_{\infty \| 0(n)}$; portanto, pode-se denotá-la como $\left|s_{n}(x)\right\rangle_{\infty \| 0(n)}=\left\langle\mathfrak{D}_{n}^{*}(x-y) \mid f(y)\right\rangle_{\infty}$, o que não constitui inconsistência de notação, visto que $|f(y)\rangle \in \mathcal{H}$ e $\left\langle\mathfrak{D}_{n}^{*}(x-y)\right| \in \mathcal{H} \times \mathcal{H}$, o que significa que são grandezas de dimensões diferentes e, portanto, geram uma grandeza em $\mathcal{H}$. Ainda assim, haveria a possibilidade de escrevermos $\left|s_{n}(x)\right\rangle=\mathfrak{D}_{n}|f(x)\rangle$, ou seja, interpretando o núcleo de Dirichlet como um operador sobre um ket, ponto que será esclarecido posteriormente.

\subsubsection{Núcleo de Dirichlet no Formalismo de Dirac}

Da expressão da função aproximada na $2^{a}$ representação, segue

$$
\left|s_{n}(x)\right\rangle_{\infty \| 0(n)} \equiv\left|f_{n}(x)\right\rangle=\langle C \mid B(x)\rangle_{0(n)}=\sum_{r=1}^{n} C^{r(n)}\left|b_{r}(x)\right\rangle_{\infty}
$$

onde

$$
\left|b_{r}(x)\right\rangle_{\infty} \equiv E_{r}(x) \equiv e_{r-\ell-1}(x)=\exp [i q(r-\ell-1) x]
$$

são os elementos da base de Fourier nessa representação: $|B(x)\rangle=\left.\operatorname{col}\left[\left|b_{r}(x)\right\rangle\right]\right|_{r=1} ^{n}$.

Usando

$$
C^{r(n)}=\left\langle b^{r}(y) \mid f(y)\right\rangle_{\infty}=\sum_{s=1}^{n} \gamma^{r s}\left\langle b_{s}(y) \mid f(y)\right\rangle_{\infty}
$$

escrevemos

$$
\begin{gathered}
\left|s_{n}(x)\right\rangle_{\infty \| \mid 0(n)}=\sum_{r=1}^{n} \sum_{s=1}^{n} \gamma^{r s}\left\langle b_{s}(y) \mid f(y)\right\rangle_{\infty}\left|b_{r}(x)\right\rangle_{\infty} \Rightarrow \\
\left|s_{n}(x)\right\rangle=\left(\sum_{r=1}^{n} \sum_{s=1}^{n}\left|b_{r}(x)\right\rangle \gamma^{r s}\left\langle b_{s}(y)\right|\right)|f(y)\rangle
\end{gathered}
$$

Por outro lado, já vimos que

$$
\left|s_{n}(x)\right\rangle_{\infty \| 0(n)}=\left\langle\mathfrak{D}_{n}^{*}(x-y) \mid f(y)\right\rangle_{\infty}
$$

o que conduz a

$$
\left\langle\mathfrak{D}_{n}^{*}(x-y)\left|\equiv \sum_{r=1}^{n} \sum_{s=1}^{n}\right| b_{r}(x)\right\rangle \gamma^{r s}\left\langle b_{s}(y)\right|
$$

que é a forma do núcleo de Dirichlet complexo nesse formalismo. Conforme mencionamos antes, também é possível interpretá-lo como um operador, representado, nesse caso, por 
um tensor de segunda ordem, tomando-se

$$
\begin{gathered}
\mathfrak{D}_{n}(x) \simeq\left|\mathfrak{D}_{n}(x)\right\rangle={\overline{\left\langle\mathfrak{D}_{n}^{*}(x)\right.}}^{\dagger} \Rightarrow \\
\mathfrak{D}_{n}(x-y)=\sum_{r=1}^{n} \sum_{s=1}^{n}\left|\bar{b}_{s}(y)\right\rangle \gamma^{s r}\left\langle\bar{b}_{r}(x)\right|
\end{gathered}
$$

De fato, operando com a base complexa de Fourier, obtém-se

$$
\begin{gathered}
\mathfrak{D}_{n}(x-y)=\sum_{r=1}^{n} \sum_{s=1}^{n} E_{s}^{*}(y) \frac{1}{2 L} \delta^{s r} E_{r}(x)=\frac{1}{2 L} \sum_{r=1}^{n} E_{r}(-y) E_{r}(x) \Rightarrow \\
\mathfrak{D}_{n}(x-y)=\frac{1}{2 L} \sum_{r=1}^{n} E_{r}(x-y),
\end{gathered}
$$

que é exatamente a expressão (3.25) para $T=2 L$, conforme esperado.

\subsubsection{Critério de Dirichlet}

Usando o Lema de Riemann-Lebesgue e as propriedades do núcleo de Dirichlet é possível provar o chamado critério de Dirichlet, que garante a convergência de uma série de Fourier. A seguir, enunciamos o teorema.

Teorema 2 Seja $f$ uma função periódica limitada, contínua por partes e com um número finito de máximos e mínimos. Então

$$
\lim _{n \rightarrow \infty} s_{n}(x)=\frac{1}{2}\left[f\left(x^{+}\right)+f\left(x^{-}\right)\right]
$$

De modo particular, $s_{n}(x)$ converge pontualmente para $f(x)$ em todos os pontos onde $f$ for contínua. A demonstração pode ser encontrada em Figueiredo (2000).

\subsubsection{Forma Explícita da MOD na Base de Fourier Complexa}

A determinação da forma explícita da matriz operacional de derivação para a base complexa de Fourier não é difícil, sendo efetuada por cálculo direto. De maneira similar, argumenta-se que é possível determinar também uma outra matriz operacional que forneça, de fato, a antiderivada, ou a integral indefinida num dado intervalo, da função aproximada por uma série. Porém, enquanto a matriz de derivação obtida para a base complexa de Fourier assume uma simples forma diagonal, a matriz operacional de integração $M_{I}$ apresenta um aspecto mais elaborado, devido ao aparecimento de um termo não imediatamente redutível à base original. 
Para a obtenção da matriz operacional de derivação (MOD) da base complexa de Fourier, efetuamos a derivação da função aproximada $f_{n}(x)$ estabelecendo a ação da derivada sobre a base ajustada, ou seja,

$$
\frac{d}{d x} f_{n}(x)=\sum_{k=1}^{2 \ell+1} C^{k} E_{k}^{\prime}(x) .
$$

onde estamos indicando a derivada pela notação tradicional de linha. Então, de acordo $\operatorname{com}(2.75)$,

$$
E_{k}^{\prime}(x)=\sum_{j=1}^{n} i q(k-\ell-1) \delta_{k}^{j} E_{j}(x),
$$

Reescrevendo $\left|E^{\prime}\right\rangle=D|E\rangle$ na forma matricial $E_{k}^{\prime}(x)=\sum_{j=1}^{n} D_{k}^{j} E_{j}(x)$, chega-se a

$$
D_{k}^{j}=i q(k-\ell-1) \delta_{k}^{j}, \quad(k=1, \ldots, 2 \ell+1)
$$

Isto significa que a $M O D$ correspondente à primeira derivada de uma base complexa de Fourier padrão é dada por uma matriz diagonal:

$$
D=(i q) \operatorname{diag}\left(\begin{array}{lllllllll}
-\ell & -\ell+1 & \cdots & -1 & 0 & 1 & \cdots & \ell-1 & \ell
\end{array}\right)
$$

cuja forma explícita é:

$$
D=i q\left(\begin{array}{rrrrrrr}
-\ell & 0 & 0 & 0 & 0 & \cdots & 0 \\
0 & \ddots & 0 & 0 & 0 & \cdots & 0 \\
0 & 0 & -1 & 0 & 0 & \cdots & 0 \\
0 & 0 & 0 & 0 & 0 & \cdots & 0 \\
0 & 0 & 0 & 0 & 1 & \cdots & 0 \\
\vdots & \vdots & \vdots & \vdots & \vdots & \ddots & \vdots \\
0 & 0 & 0 & \cdots & 0 & 0 & \ell
\end{array}\right)
$$

\subsubsection{Forma Explícita da MOD de Ordens Superiores na Base de Fourier Complexa}

Vamos determinar agora a $M O D$ de $k$-ésima ordem para a base complexa de Fourier. Uma vez que basta somente tomarmos o produto da $M O D$ de primeira ordem por si mesma tantas vezes quantas forem necessárias para igualar a ordem da derivação desejada, a operação resulta simples, já que, neste caso, a matriz de derivação é diagonal.

Efetuando primeiramente o caso de segunda ordem, obtemos, de acordo com a equação 
(3.29) e redenominando $D^{2}=Q$ :

$$
\begin{gathered}
Q_{r}{ }^{s}=\sum_{j=1}^{n} D_{r}^{j} D_{j}{ }^{s}=(i q)^{2}(r-\ell-1)(s-\ell-1) \delta_{r}{ }^{s} \Rightarrow \\
Q_{r}{ }^{s}=-q^{2}(r-\ell-1)^{2} \delta_{r}{ }^{s}
\end{gathered}
$$

Isto implica que os valores não-nulos de $D^{2}$ são

$$
Q_{k}{ }^{k}=-q^{2}(k-\ell-1)^{2} \quad(k=1, \ldots, 2 \ell+1)
$$

Assim, uma vez que $(m-\ell)^{2}=(\ell-m)^{2}$, a $M O D$ de segunda ordem é dada por

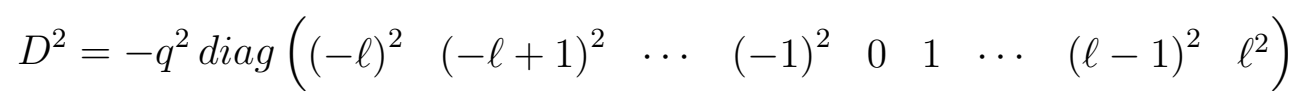

ou, explicitamente,

$$
D^{2}=-q^{2}\left(\begin{array}{ccccccc}
\ell^{2} & 0 & 0 & 0 & 0 & \cdots & 0 \\
0 & \ddots & 0 & 0 & 0 & \cdots & 0 \\
0 & 0 & 1 & 0 & 0 & \cdots & 0 \\
0 & 0 & 0 & 0 & 0 & \cdots & 0 \\
0 & 0 & 0 & 0 & 1 & \cdots & 0 \\
\vdots & \vdots & \vdots & \ddots & \vdots & \ddots & \vdots \\
0 & 0 & 0 & \cdots & 0 & \cdots & \ell^{2}
\end{array}\right)
$$

Repetindo o processo mais uma vez, obtém-se, para a terceira derivada, denotada $D^{3}=R$, o seguinte:

$$
\begin{aligned}
R_{r}{ }^{s} & =\sum_{j=1}^{n} D_{r}{ }^{j} Q_{j}{ }^{s}=\sum_{j=1}^{n} i q\left(-q^{2}\right)(r-\ell-1) \delta_{r}{ }^{j}(j-\ell-1)^{2} \delta_{j}{ }^{s}= \\
& =(i q)^{3}(r-\ell-1)(s-\ell-1)^{2} \delta_{r}{ }^{s}=(i q)^{3}(r-\ell-1)^{3} \delta_{r}{ }^{s},
\end{aligned}
$$

cujos valores não-nulos serão

$$
R_{k}^{k}=-i q^{3}(k-\ell-1)^{3}
$$

Portanto,

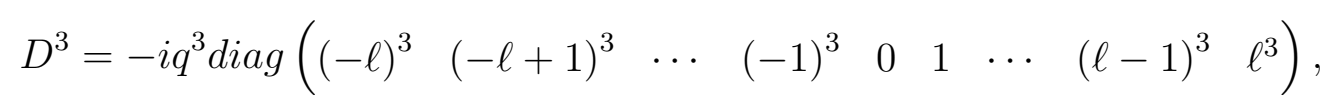


que é escrito explicitamente como

$$
D^{3}=(i q)^{3}\left(\begin{array}{rrrrrrr}
-\ell^{3} & 0 & 0 & 0 & 0 & \ldots & 0 \\
0 & \ddots & 0 & 0 & 0 & \ldots & 0 \\
0 & 0 & -1 & 0 & 0 & \ldots & 0 \\
0 & 0 & 0 & 0 & 0 & \ldots & 0 \\
0 & 0 & 0 & 0 & 1 & \ldots & 0 \\
\vdots & \vdots & \vdots & \vdots & \vdots & \ddots & \vdots \\
0 & 0 & 0 & \ldots & 0 & 0 & \ell^{3}
\end{array}\right)
$$

Assim, não é difícil verificar que os valores não-nulos da matriz $D^{m}$ devem ser dados por

$$
\left[D^{m}\right]_{j}^{j}=(i q)^{m}(j-\ell-1)^{m}
$$

e a $M O D$ de ordem $m$ será



ou, em termos dos elementos da matriz,

$$
\left[D^{m}\right]_{r}^{s}=(i q)^{m}(r-\ell-1)^{m} \delta_{r}^{s} \quad(m \in \mathbb{N})
$$

Essa última condição $(m \in \mathbb{N})$ é importante, pois o cálculo foi feito especificamente para derivadas, não sendo válido para o caso integral, que será visto a seguir.

\subsubsection{Forma Explícita da MOI na Base de Fourier Complexa}

Partindo novamente de uma função aproximada $f_{n}(x)$, com $n=2 \ell+1$ termos, efetuamos agora, a fim de determinar a matriz operacional de integração (MOI), uma integração indefinida:

$$
F_{n}(x):=\int f_{n}(x) d x=\sum_{k=1}^{2 \ell+1} C^{k} \int E_{k}(x) d x
$$

o que fornece

$$
F_{n}(x)=\sum_{k=1}^{\ell} C^{k} \frac{\exp [i q(k-\ell-1) x]}{i q(k-\ell-1)}+C^{\ell+1} x+\sum_{k=\ell+2}^{2 \ell+1} C^{k} \frac{\exp [i q(k-\ell-1) x]}{i q(k-\ell-1)}
$$

Verifica-se, entretanto, o aparecimento de um termo adicional, linear em $x$, estranho à base. Para resolver a questão, necessitamos expressá-lo em termos da base exponencial complexa de Fourier. Vamos desenvolver a série para o termo adicional dentro do mesmo 
período original de expansão da função. Com isso, obtém-se:

$$
x \cong \sum_{r=-\ell}^{\ell} \alpha^{r} e_{r}(x)
$$

onde

$$
\begin{aligned}
& \alpha^{r}=\frac{q}{2 \pi} \int_{-\pi / q}^{\pi / q} x \exp (-i r q x) d x=(-1)^{r} \frac{i}{r q}, \quad(r \neq 0) \\
& \alpha^{0}=\frac{q}{2 \pi} \int_{-\pi / q}^{\pi / q} x d x=\left.\frac{q}{2 \pi}\left(\frac{x^{2}}{2}\right)\right|_{-\pi / q} ^{\pi / q}=0 ;
\end{aligned}
$$

conduzindo-nos, portanto, a

$$
x \cong \sum_{r=-\ell}^{-1}(-1)^{r} \frac{i}{r q} \exp (i r q x)+\sum_{r=1}^{\ell}(-1)^{r} \frac{i}{r q} \exp (\text { irqx })
$$

É mais conveniente, porém, reescrever a expressão anterior com uma reindexação apropriada ao tratamento linear:

$$
\begin{aligned}
x & \cong \sum_{k=1}^{\ell}(-1)^{k-\ell-1} \frac{i}{(k-\ell-1) q} \exp [i q(k-\ell-1) x]+ \\
& +\sum_{k=\ell+2}^{2 \ell+1}(-1)^{k-\ell-1} \frac{i}{(k-\ell-1) q} \exp [i q(k-\ell-1) x]
\end{aligned}
$$

Assim, substituindo em $F_{n}(x)$ a expressão obtida, obtemos:

$$
\begin{aligned}
F_{n}(x)= & \sum_{k=1}^{\ell}\left[\left(\frac{1}{i} C^{k}+(-1)^{k-\ell-1} i C^{\ell+1}\right) \frac{1}{(k-\ell-1) q}\right] \exp [i q(k-\ell-1) x]+ \\
& +\sum_{k=\ell+2}^{2 \ell+1}\left[\left(\frac{1}{i} C^{k}+(-1)^{k-\ell-1} i C^{\ell+1}\right) \frac{1}{(k-\ell-1) q}\right] \exp [i q(k-\ell-1) x],
\end{aligned}
$$

ou ainda, redefinindo-se os coeficientes (usando a notação caligráfica $\mathcal{C}^{k}$ ):

$$
\mathcal{C}^{k}=\left(1-\delta_{\ell+1}^{k}\right)\left[\left((-1)^{k-\ell-1} C^{\ell+1}-C^{k}\right) \frac{i}{(k-\ell-1) q}\right]
$$

pode-se expressar a função integrada aproximada por:

$$
F_{n}(x)=\sum_{k=1}^{2 \ell+1} \mathcal{C}^{k} E_{k}(x)
$$


Entretanto, como $\langle\mathcal{C}|=\langle C| M_{I}$, então

$$
\mathcal{C}^{k}=\sum_{j=1}^{n} C^{j}\left(M_{I}\right)_{j}^{k}
$$

onde $C^{j}$ são os coeficientes contravariantes do covetor. Logo,

$$
\begin{aligned}
\mathcal{C}^{k}=C^{k} & \left(\frac{-i}{(k-\ell-1) q}\right)+C^{\ell+1}\left(\frac{(-1)^{k-\ell-1} i}{(k-\ell-1) q}\right), \quad(k \neq \ell+1) \Rightarrow \\
& \Rightarrow\left\{\begin{array}{l}
\left(M_{I}\right)_{k}^{k}=\frac{-i}{(k-\ell-1) q} \\
\left(M_{I}\right)_{\ell+1}^{k}=\frac{(-1)^{k-\ell-1} i}{(k-\ell-1) q}
\end{array}\right.
\end{aligned}
$$

Essas relações podem ser compactamente expressas por:

$$
\left(M_{I}\right)_{j}^{k}=\frac{\left(1-\delta_{\ell+1}^{k}\right) i}{(k-\ell-1) q}\left[\left((-1)^{k-\ell-1} \delta_{j}^{\ell+1}-\delta_{j}^{k}\right)\right], \quad(k=1, \ldots, 2 \ell+1) .
$$

Consequentemente. a forma explícita da MOI de primeira ordem para a base complexa de Fourier será então a seguinte:

$$
M_{I}=\frac{1}{i q}\left(\begin{array}{ccccccc}
-\frac{1}{\ell} & 0 & & \cdots & & 0 & 0 \\
0 & -\frac{1}{(\ell-1)} & & \cdots & & 0 & 0 \\
\vdots & \vdots & \ddots & & & \vdots & \vdots \\
\frac{(-1)^{\ell}}{\ell} & \frac{(-1)^{\ell-1}}{(\ell-1)} & \cdots & 0 & \cdots & -\frac{(-1)^{\ell-1}}{(\ell-1)} & -\frac{(-1)^{\ell}}{\ell} \\
\vdots & \vdots & & & \ddots & \vdots & \vdots \\
0 & 0 & & \cdots & & \frac{1}{(\ell-1)} & 0 \\
0 & 0 & & \cdots & & 0 & \frac{1}{\ell}
\end{array}\right)
$$

Percebe-se que a presença do termo central $C^{0}$ é o responsável pela complicação do cálculo da MOI. Caso esse termo não existisse ou pudesse ser desprezado, a linha intermediária sumiria e o resultado seria predito também pela fórmula geral da derivada, equação (3.30), com $m=-1$. Essa particularidade sugere que, desprezado o termo central, o resultado obtido acima deve ser, provavelmente, válido também para valores não-inteiros de $m$. De fato, como veremos, é o que ocorre.

A questão é se esse termo central pode, de alguma forma, ser desprezado sem prejuízo do resultado. O que será mostrado é que, em boa parte dos casos, a análise e o desenvol- 
vimento da função não ficam prejudicados, uma vez que este termo é responsável apenas pelo posicionamento do gráfico no eixo $O y$ e, por consequência, sua ausência produz apenas uma translação do gráfico nesse eixo, sem contudo alterar sua forma.

Além disso, existe uma propriedade adicional de funções periódicas no tratamento fracional que impõe a anulação do termo central. Isso será formalmente desenvolvido e analisado em detalhes posteriormente, na seção relativa aos operadores de Weyl.

Extensão a Matrizes de Dimensão Infinita É possível expandir o tratamento aqui apresentado a ordens infinitas, isto é, a expansões de séries infinitas. Já vimos que funções $\mathcal{L}^{1}$ podem ser desenvolvidas em séries complexas de Fourier como $|f\rangle=\langle c \mid e\rangle \equiv$ $\sum_{r=-\infty}^{\infty} c^{r}\left|e_{r}\right\rangle_{\infty}$. Então, a ação de um operador sobre uma função desse tipo fornece

$$
\mathcal{D}|f\rangle=\lim _{n \rightarrow \infty} \mathcal{D}\left|f_{n}\right\rangle=\lim _{n \rightarrow \infty}\left\langle c\left|D_{n}\right| e\right\rangle_{0_{n}}
$$

onde $D_{n}$ é a matriz operacional de dimensão $n$. Todavia, o produto da matriz operacional pelo vetor de base pode ser reescrito como um novo vetor de dimensão no espaço $\mathcal{O}_{n}$ :

$$
\left.\left.\left|\sum_{s}^{n}\left(D_{n}\right)_{r}^{s}\right| e_{s}\right\rangle_{\infty}\right\rangle_{0_{n}}
$$

onde $\operatorname{dim}(r)=n$. Assim, a aplicação do operador sobre a função torna-se

$$
\begin{gathered}
\left.\mathcal{D}|f\rangle=\lim _{n \rightarrow \infty}\left\langle c|| \sum_{s}^{n}\left(D_{n}\right)_{r}{ }^{s} \mid e_{s}\right\rangle_{\infty}\right\rangle_{0_{n}} \Rightarrow \\
\mathcal{D}|f\rangle=\lim _{n \rightarrow \infty} \sum_{r}^{n} \sum_{s}^{n} c^{r}\left(D_{n}\right)_{r}{ }^{s}\left|e_{s}\right\rangle_{\infty}
\end{gathered}
$$

enquanto sua atuação sobre a própria base fornece um vetor de dimensão infinita cuja $r$-ésima componente é:

$$
\left(D|e\rangle_{\infty}\right)_{r}=\lim _{n \rightarrow \infty} \sum_{s}^{n}\left(D_{n}\right)_{r}^{s}\left|e_{s}\right\rangle_{\infty}
$$

Evidentemente, isso vale não apenas para a base de Fourier complexa, mas para qualquer base na qual a função possa ser expandida. Contudo, no caso específico de Fourier, como a matriz de derivação é dada por $D_{r}{ }^{s}=i s q \delta_{r}{ }^{s}$, então a equação (3.33) assumirá a forma:

$$
\left(D|e\rangle_{\infty}\right)_{r}=\lim _{n \rightarrow \infty} \sum_{s=-n}^{n} i s q \delta_{r}{ }^{s}\left|e_{s}\right\rangle_{\infty}
$$


e, por conseguinte, a diferintegral de ordem $\nu$ torna-se:

$$
\left(D^{\nu}|e\rangle_{\infty}\right)_{r}=\sum_{s=-\infty}^{\infty}(i s q)^{\nu} \delta_{r}^{s}\left|e_{s}\right\rangle_{\infty},
$$

onde as matrizes $D^{\nu}$ são agora de dimensão infinita, as quais certamente convergem para $\nu<0$, i.e., para o caso de integrações fracionais.

\subsubsection{Aperfeiçoamento do Método}

\subsubsection{Séries de Fourier Associadas}

Além do método tradicional de uso das séries de Fourier complexas, analisamos casos de expansão de funções nas quais a implementação de uma formulação alternativa parece garantir uma precisão melhor em períodos menores que $2 \pi$.

O fato é que quando o método tradicional das derivadas operacionais complexas de Fourier falha para determinadas condições iniciais ou de contorno, tende no entanto a funcionar para um desenvolvimento ligeiramente diferente.

A ideia central consiste em tomarmos, para a expansão em série, períodos diferentes do período de definição da função. Se considerarmos a possibilidade de um desenvolvimento similar ao que seria implementado no domínio da frequência, isto é, se o novo período for definido por uma relação inversamente proporcional ao período original, essa expansão poderá apresentar uma precisão melhor que a da série original, caso o período original seja maior que $2 \pi$. Essa afirmação será formalmente demonstrada adiante.

O desenvolvimento tradicional das séries de Fourier complexas para uma função absolutamente integrável $f(x)$ seccionalmente diferenciável de semiperíodo $L$ é dado por

$$
|f\rangle=\sum_{k=-\infty}^{\infty} c^{k}\left|e_{k}\right\rangle
$$

onde os coeficientes são determinados pela expressão

$$
c^{k}=\left\langle e^{k} \mid f\right\rangle=\frac{1}{2 L} \int_{-L}^{L} \bar{e}_{k}(x) f(x) d x
$$

sendo $q=\frac{\pi}{L}$ definida como razão periódica. 
Neste caso, a base de Fourier de uma aproximação de extensão $\ell$ é dada pelo vetor

$$
|e\rangle=\left[\begin{array}{c}
\vdots \\
\left|e_{j}\right\rangle \\
\vdots
\end{array}\right]_{j=-\ell}^{\ell}
$$

sendo a série aproximada para $n=2 \ell+1$ termos:

$$
\left|f_{n}\right\rangle=\sum_{r=-\ell}^{\ell} c^{r}\left|e_{r}\right\rangle=\langle c \mid e\rangle
$$

onde o covetor $\langle c|$ é

$$
\langle c|=\left[\begin{array}{lllllll}
c^{-\ell} & \ldots & c^{-1} & c^{0} & c^{1} & \ldots & c^{\ell}
\end{array}\right]
$$

com $c^{k}$ definido acima.

Entretanto, se estabelecermos um desenvolvimento inspirado na ideia apresentada, ou seja, com base em expansões numa espécie de domínio de frequência, poderíamos, a partir de $\nu=1 / T$, onde $T=2 L$, postular a condição $2 L \rightarrow 1 /(2 L) \Rightarrow q \rightarrow 1 / q=L / \pi$. Com essa substituição, é possível então definir uma razão periódica associada $\tilde{q}=q^{-1}=L / \pi$. Nestas circunstâncias, a base associada será:

$$
|\tilde{e}\rangle=\left[\begin{array}{c}
\vdots \\
\left|\tilde{e}_{j}\right\rangle \\
\vdots
\end{array}\right]_{j=-\ell}^{\ell}
$$

Naturalmente, o período associado também será diferente, pois $\exp (i k L x / \pi)$ tem semiperíodo $\tilde{L}=\pi^{2} / L$. Definindo agora um fator de período $\rho$ tal que tenhamos $T=2 \pi \rho$, segue que $L=\rho \pi$ e $\tilde{L}=\pi / \rho$. Fatores de período maiores que a unidade representam períodos maiores que $2 \pi$ e menores que a unidade, períodos menores que esse valor, ou seja,

$$
\begin{aligned}
& \rho>1 \Leftrightarrow T>2 \pi \\
& \rho<1 \Leftrightarrow T<2 \pi \\
& \rho=1 \Leftrightarrow T=2 \pi
\end{aligned}
$$

Dada a base associada, anteriormente definida, a função será então desenvolvida pela 
série associada

$$
\left|f_{n}\right\rangle=\sum_{r=-\ell}^{\ell} \tilde{c}^{r}\left|\tilde{e}_{r}\right\rangle,
$$

na qual os coeficientes associados serão dados por:

$$
\tilde{c}^{r}=\frac{1}{2 \tilde{L}} \int_{-\tilde{L}}^{\tilde{L}} \tilde{e}_{r}^{*}(x) f(x) d x,
$$

sendo as grandezas

$$
\tilde{L}=\frac{\pi^{2}}{L} \quad \text { e } \quad \tilde{q}=\frac{L}{\pi}=\frac{1}{q}
$$

o semiperíodo associado e a razão periódica associada, respectivamente.

Entretanto, queremos que as duas funções $f_{n}$ e $\tilde{f}_{n}$ sejam representações da mesma função $f(x)$ no intervalo de período mais restrito

$$
\tau=[-L, L] \cap[-\tilde{L}, \tilde{L}]
$$

ou seja, $f_{n}(x)=\tilde{f}_{n}(x), x \in \tau$. Fora desse intervalo, porém, as funções podem ser completamente diferentes.

Chamaremos às séries assim definidas de Séries de Fourier Associadas ou, simplesmente, Séries Associadas.

Nestas condições, se tivermos uma função absolutamente integrável $f(x)$ seccionalmente diferenciável de semiperíodo $L=\rho \pi$, podemos utilizar a expansão em série associada para um semiperíodo $\tilde{L}=\pi / \rho$. Se $\rho>1$, então $T>2 \pi$ e $\tilde{T}<2 \pi$ na mesma proporção, já que $T \tilde{T}=4 \pi^{2}$.

\subsubsection{Função de Expansão Híbrida}

A questão que surge em consequência disso é a seguinte: considerando-se que a função seja definida num período original $T>2 \pi$, por exemplo, o período associado seria $\tilde{T}<$ $2 \pi<T$, e a expansão só deveria valer numa restrição $\tau=[-\tilde{L}, \tilde{L}]$ do domínio original; assim, qual o procedimento para completar o desenvolvimento da função nos intervalos extremos faltantes em relação ao período original?

Mostraremos que a expansão de menor período constitui sempre uma melhor aproximação da função original $f(x)$ para o intervalo correspondente a esse mesmo período, apresentando uma convergência mais rápida que a série de Fourier original, embora possa apresentar comportamento catastrófico fora desses limites. Nesse caso, bastaria tomar a melhor expansão para cada intervalo e "colar" as expressões para os respectivos desenvol- 
vimentos. Assim, essa função resultante da colagem das duas expansões, que chamaremos de função de expansão híbrida (ou simplesmente função híbrida), deverá apresentar uma aproximação melhor para o domínio original que qualquer um dos desenvolvimentos tomados separadamente.

Por exemplo, se a função é definida num período $T=2 \pi / 3<2 \pi$, então a melhor aproximação para esse intervalo é a própria série de Fourier. Se, por outro lado, o domínio de definição da função periódica $f(x)$ envolver um período $T=5 \pi / 2>2 \pi$, por exemplo, então, a série associada deverá apresentar uma aproximação melhor dentro do intervalo ] $-4 \pi / 5,4 \pi / 5$ [, enquanto a série de Fourier deve ser tomada nos extremos $I_{e s q}=\left[-\frac{5 \pi}{4},-\frac{4 \pi}{5}\right]$ e $I_{d i r}=\left[\frac{4 \pi}{5}, \frac{5 \pi}{4}\right]$. Entretanto, pode acontecer de as funções não assumirem o mesmo valor nos pontos de junção, i.e., $x_{c}= \pm \frac{4 \pi}{5}$, no presente caso, em se tratando de função par. Se assim for, será necessário determinar os pontos de junção através da

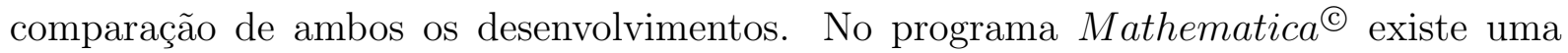
forma de resolver o problema pelo comando FindRoot $\left[f_{n}(x)==\tilde{f}_{n}(x), x\right]$. Assim, os pontos de junção serão dados pela solução encontrada $\pm x_{c}$, no caso de funções pares. Para funções sem simetria, devem ser calculados dois pontos de junção distintos, $x_{e}$ e $x_{d}$, esquerdo e direito, respectivamente. Nessas circunstâncias, os intervalos marginais serão dados por $I_{e s q}=\left[-L, x_{e}\right]$ e $I_{d i r}=\left[x_{d}, L\right]$, onde se deve ter $\left|x_{e}\right| \leq \tilde{L}$ e $\left|x_{d}\right| \leq \tilde{L}$.

Dessa forma, a função de expansão híbrida, que vamos denotar por $F_{R}(x)$ de semiperíodo $L$ em torno da origem será definida da seguinte maneira:

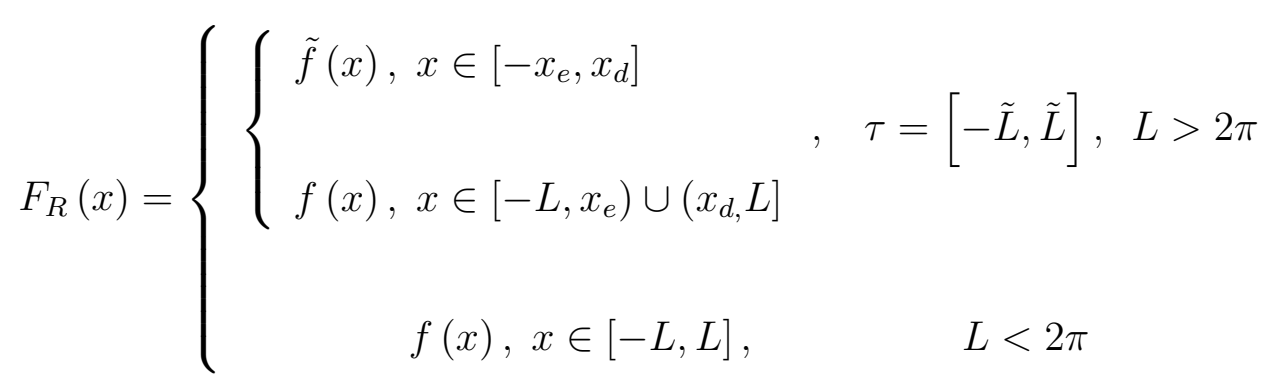

Entretanto, ocorre que o resultado do eventual afastamento entre a função original e sua expansão em série nos extremos do intervalo de restrição, devido ao fenômeno de Gibbs, acarreta um acentuado acréscimo no erro nessas regiões, uma espécie de efeito de borda. Para evitar isso, é preciso, em certos casos, restringir ainda mais o menor período a um intervalo apropriado que minimize esse erro adicional. É plausível inferir que esse distanciamento ocorre em funções ímpares ou assimétricas nas quais $f(-L)$ assuma um valor muito diferente de $f(L)$. Nesse caso, a expansão de Fourier gera descontinuidades periódicas. De fato, nas funções pares esse fenômeno não ocorre. 
Do ponto de vista da aplicação às matrizes operacionais, fica claro, pelo que foi exposto acima, o motivo pelo qual as condições de contorno assumem papel tão decisivo no processo de determinação da solução da equação diferencial.

Condições de contorno que obedeçam à exigência de simetria fornecerão, obviamente, possibilidades maiores de ajuste pela própria precisão da função em série.

Surge, todavia, um incoveniente adicional decorrente da definição adotada para a função $F_{R}(x)$. Em princípio, nada garante que o ponto de junção forneça um ajuste liso, isto é, que neste ponto não surja ao menos uma descontinuidade de primeira espécie (i.e., uma descontinuidade com limites laterais finitos e distintos) na derivada. É, inclusive, provável que essas descontinuidades na derivada ocorram com mais frequência que o caso contrário. Para corrigir isto, seria necessário utilizar uma forma de ajuste que alise a função híbrida em torno dos pontos de junção. Tal condição, entretanto, não foi implementada nesse trabalho, de maneira que a função híbrida não satisfaz, de fato, o requisito de ser uma função de classe $\mathcal{C}^{1}$.

É importante mencionar ainda que há dois conjuntos diferentes de pontos na plotagem dos gráficos, o primeiro é o par constituído pelos pontos limites da restrição do período, que são simétricos em relação à origem e o segundo é justamente o par dos pontos de junção $x_{c}$, não necessariamente simétricos. No código computacional escrito para o programa Mathematica ${ }^{\circledR}$, eles são denotados por $T$ e $T_{c}$, respectivamente.

Em seções posteriores, vamos mostrar quais os resultados das expansões em série de Fourier original e associada para algumas funções específicas, com o objetivo de ilustrar o que foi exposto aqui.

Além disso, as séries associadas serão usadas também na resolução de EDOs pelo método operacional original, i.e., com condições impostas, bem como pelo método NBV, ambos com espaçamento constante e com os nodos de Chebyshev.

Passamos agora a demonstrar a afirmação feita antes, sobre a convergência da série associada ser mais rápida que a da série de Fourier original.

\subsubsection{Determinação do Erro da Série Associada}

Definição 10 : Seja uma função $f(x) \in C^{0}[-L, L]$ definida no intervalo de período $2 L$; então, sua série de Fourier associada, com $n=2 \ell+1$ termos, definida em um novo período $2 \tilde{L}=\frac{2 \pi^{2}}{L}$, é escrita: 


$$
\tilde{f}_{\ell}(x)=\sum_{k=-\ell}^{\ell} \tilde{c}_{k} \tilde{e}_{k}(x)
$$

onde, para $\tilde{q}=\pi / \tilde{L}$, tem-se:

$$
\begin{aligned}
\tilde{e}_{k}(x) & =\exp (i k \tilde{q} x) \\
\tilde{c}_{k} & =\frac{1}{2 \tilde{L}} \int_{-\tilde{L}}^{\tilde{L}} \tilde{e}_{k}^{*}(u) f(u) d u
\end{aligned}
$$

Definição 11 : Se uma função $f$ admite uma expansão em série convergente com valor convergindo para $f$, define-se o resto local para uma expansão em série finita com $n$ termos como a diferença entre o valor da função (ou sua expansão em série infinita) e sua expansão finita nessa mesma série com $n$ termos, para um dado ponto $x$, ou seja, se $f(x)=\sum_{k=1}^{\infty} c_{k} b_{k}(x)$ em uma determinada base, dada pelas componentes $b_{k}(x)$, então:

$$
R_{n}(x):=f(x)-f_{n}(x)=\sum_{k=1}^{\infty} c_{k} b_{k}(x)-\sum_{k=1}^{n} c_{k} b_{k}(x)
$$

Especificamente para uma série de Fourier, tem-se

$$
R_{\ell}(x)=\sum_{k=-\infty}^{\infty} c_{k} b_{k}(x)-\sum_{k=-\ell}^{\ell} c_{k} b_{k}(x)
$$

Obs: $O$ resto local absoluto é simplesmente o valor absoluto do resto local acima definido, ou seja, $\left|R_{\ell}(x)\right|$.

Enunciamos e demonstramos, a seguir, dois teoremas originais referentes às séries associadas.

Teorema 3 : Seja uma função $f(x) \in C^{0}[-L, L]$ definida no intervalo de período $2 L$, com $L>\pi$, e série de Fourier convergente. Então, se existe a série de Fourier associada, a estimativa de seu máximo resto local absoluto, para um dado número de termos, é menor ou igual ao correspondente da série de Fourier original na restrição de período $2 \tilde{L}=\frac{2 \pi^{2}}{L}$.

\section{Demonstração :}

Consideremos dois intervalos reais $I_{P}=[-\tilde{L}, \tilde{L}] \subset \mathbb{R}$ e $I_{G}=[-L, L] \subset \mathbb{R}$, com $\tilde{L}<\pi<L$. Daí, segue que $I_{P} \subset I_{G}$.

Sejam $f(x)$ uma função contínua no intervalo $I_{G}$ e $A_{1}$ e $A_{2}$, conjuntos fechados definidos por $A_{1}=\left\{|f(x)|: x \in I_{P}\right\}$ e $A_{2}=\left\{|f(x)|: x \in I_{G}\right\}$, de onde segue que $A_{1} \subset A_{2}$, 
ambos com cota inferior igual a zero, pois

$$
0 \leq|f(x)|, \forall x \in \mathbb{R}
$$

Como $f(x)$ é, por hipótese, limitada em $I_{G}$, então $A_{1}$ e $A_{2}$ são compactos; logo, possuem máximo e mínimo locais, sendo

$$
\begin{aligned}
\inf A_{1} & =\min A_{1}=\min _{x \in I_{P}}|f(x)| \\
\inf A_{2} & =\min A_{2}=\min _{x \in I_{G}}|f(x)| \\
\sup A_{1} & =\max A_{1}=\max _{x \in I_{P}}|f(x)| \\
\sup A_{2} & =\max A_{2}=\max _{x \in I_{G}}|f(x)|
\end{aligned}
$$

Sejam, portanto,

$$
\min A_{1}=\tilde{m}, \min A_{2}=m, \max A_{1}=\tilde{M}, \quad \max A_{2}=M
$$

Então, pela propriedade de monotonicidade em conjuntos compactos, tem-se

$$
A_{1} \subset A_{2} \Rightarrow \sup A_{1} \leq \sup A_{2} \Rightarrow 0 \leq \tilde{M} \leq M
$$

Por outro lado, a expansão em série de Fourier complexa de uma determinada função $f: \mathbb{R} \rightarrow \mathbb{C}$, com $n$ termos, é escrita:

$$
f_{\ell}(x)=\sum_{k=-\ell}^{\ell} c_{k} e_{k}(x)
$$

A diferença entre a soma da série infinita e sua expansão $f_{\ell}(x)$ num certo ponto $x$ para $\ell \geq \ell_{0}=\ell(\varepsilon)$, chamada de resto local e denotado por $R_{\ell}(x)$, onde $\varepsilon>0$ é o erro máximo arbitrado, será

$$
\begin{aligned}
R_{\ell}(x) & :=\sum_{k=-\infty}^{\infty} c_{k} e_{k}(x)-\sum_{k=-\ell}^{\ell} c_{k} e_{k}(x) \Rightarrow \\
R_{\ell}(x) & =\sum_{k=-\infty}^{-\ell-1} c_{k} e_{k}(x)+\sum_{k=\ell+1}^{\infty} c_{k} e_{k}(x)
\end{aligned}
$$

Para cada índice $k$ há um índice oposto $(-k)$ de tal forma que, redenominando os 
índices, pode-se escrever

$$
R_{\ell}(x)=\sum_{k=\ell+1}^{\infty} c_{(-k)} \bar{e}_{k}(x)+\sum_{k=\ell+1}^{\infty} c_{k} e_{k}(x),
$$

sendo os coeficientes dados por

$$
c_{k}=\frac{1}{2 L} \int_{-L}^{L} f(u) e_{k}(-u) d u
$$

Assim, reescrevendo a expressão (3.43) em termos dos coeficientes, e lembrando que $e_{k}(x)=\exp (i k q x)$, obtemos

$$
\begin{gathered}
R_{\ell}(x)=\sum_{k=\ell+1}^{\infty} \exp (-i k q x) \frac{1}{2 L} \int_{-L}^{L} f(u) \exp (i k q u) d u+ \\
+\sum_{k=\ell+1}^{\infty} \exp (i k q x) \frac{1}{2 L} \int_{-L}^{L} f(u) \exp (-i k q u) d u \Rightarrow \\
R_{\ell}(x)=\sum_{k=\ell+1}^{\infty} \frac{1}{2 L} \int_{-L}^{L} f(u)\{\exp [i k q(u-x)]+\exp [-i k q(u-x)]\} d u \Rightarrow \\
R_{\ell}(x)=\sum_{k=\ell+1}^{\infty} \frac{1}{L} \int_{-L}^{L} f(u) \cos [i k q(u-x)] d u
\end{gathered}
$$

O segundo membro da expressão acima é uma somatória dos termos $R_{\ell \mu}(x)$ dados por

$$
R_{\ell \mu}(x):=\int_{-L}^{L} f(u) K_{\mu}(u, x) d u
$$

onde

$$
K_{\mu}(u, x)=\frac{1}{L} \cos [i \mu q(u-x)], \quad \mu \geq \ell+1 \in \mathbb{N}
$$

é o kernel da transformação obtida, de forma que

$$
R_{\ell}(x)=\sum_{\mu=\ell+1}^{\infty} R_{\ell \mu}(x)
$$

Então, o resto local absoluto na série de Fourier original é dado por

$$
\left|R_{\ell}(x)\right|=\left|\sum_{\mu=\ell+1}^{\infty} R_{\ell \mu}(x)\right| \leq \sum_{\mu=\ell+1}^{\infty}\left|R_{\ell \mu}(x)\right|
$$

Agora, arbitrando o majorante $\mathcal{M}_{\ell}(x)$ do resto local absoluto pelo último membro da 
desigualdade acima, escrevemos:

$$
\mathcal{M}_{\ell}(x)=\sum_{\mu=\ell+1}^{\infty}\left|R_{\ell \mu}(x)\right|
$$

Esse majorante será nossa estimativa para o máximo resto local absoluto da expansão em série, ou seja,

$$
\left|R_{\ell}(x)\right| \leq \mathcal{M}_{\ell}(x)
$$

De maneira análoga, a estimativa para o máximo resto local absoluto da função expandida na série de Fourier associada é dado pelo majorante

$$
\tilde{\mathcal{M}}_{\ell}(x)=\sum_{\mu=\ell+1}^{\infty}\left|\tilde{R}_{\ell \mu}(x)\right|
$$

onde

$$
\tilde{R}_{\ell \mu}(x)=\int_{-\tilde{L}}^{\tilde{L}} f(u) \tilde{K}_{\mu}(u, x) d u
$$

para o kernel associado:

$$
\left.\tilde{K}_{\mu}(u, x)=\frac{1}{\tilde{L}} \cos [i \mu \tilde{q}(u-x)]\right], \quad \mu \geq \ell+1
$$

Entretanto, para duas séries $\sum a_{n}(x)$ e $\sum b_{n}(x)$, com $0<a_{n}(x) \leq b_{n}(x)$ vale, pelo critério da comparação, que, se $\sum b_{n}(x)$ é convergente, então $\sum a_{n}(x)$ também converge e, além disso,

$$
\sum_{n=n_{0}}^{\infty} a_{n}(x) \leq \sum_{n=n_{0}}^{\infty} b_{n}(x)
$$

Assim, será preciso analisar os termos de cada uma das duas séries definidas pelos majorantes acima, i.e., $\tilde{\mathcal{M}}_{\ell}(x)$ e $\mathcal{M}_{\ell}(x)$, pois, caso valha $\left|\tilde{R}_{\ell \mu}(x)\right| \leq\left|R_{\ell \mu}(x)\right|$, então o primeiro majorante, pelo critério da comparação, será menor ou igual ao segundo, que é o que desejamos provar.

Mas $\mathcal{M}_{\ell}(x)$ é uma série convergente de termos positivos, pois é o valor absoluto de uma combinação de duas séries convergentes, provenientes das somas parciais da expansão por Fourier, que, por hipótese, converge. $\operatorname{Logo}, \mathcal{M}_{\ell}(x)$ constitui uma sequência monótona convergindo para zero à medida que $\ell$ tende a infinito, o que permite a utilização do critério de comparação.

Com a finalidade de estudar o comportamento do termo $\left|R_{\ell \mu}(x)\right|$, vamos introduzir uma partição regular de Riemann, isto é, com diâmetro constante $\left|\Delta u_{j}\right|=\Delta P$, de forma 
que, para o intervalo maior $I_{G}$ tenhamos $s$ sub-intervalos $\left[u_{j-1}, u_{j}\right]$ e, para o intervalo menor $I_{P}$, apenas $r$ subdivisões, com $r<s$. Nessas condições, tem-se $2 \tilde{L}=r \Delta P$ e $2 L=s \Delta P$.

Portanto, sejam $P_{1}$ e $P_{2}$ duas partições regulares de mesma norma, definidas em $I_{P}$ e $I_{G}$, respectivamente. Considere a partição $P_{1}$ do intervalo $I_{P}$ determinada pelos pontos $\hat{u}_{j}=-\tilde{L}+2\left(\frac{j}{r}\right) \tilde{L}$, para $j=0,1, \ldots, r$, e a partição $P_{2}$ do intervalo $I_{G}$, determinada pela mesma regra, mas com semiperíodo $L$ em vez de $\tilde{L}$, ou seja, $u_{j}=-L+2\left(\frac{j}{s}\right) L$, para $j=$ $0,1, \ldots, r, \ldots, s$. Note que $\Delta u_{j}=u_{j}-u_{j-1}=2 L / s=\Delta P$ e $\Delta \hat{u}_{j}=\hat{u}_{j}-\hat{u}_{j-1}=2 \tilde{L} / r=\Delta P$, isto é, $\Delta \hat{u}_{j}=\Delta u_{j}=\Delta P$, conforme esperado. Dessa forma, os termos das séries podem ser reescritos em termos dessas partições.

Usando-se a expressão (3.45) para se exprimir $R_{\ell \mu}(x)$ em termos da partição $P_{2}$, obtém-se:

$$
R_{\ell \mu}(x)=\sum_{j=1}^{s} f\left(u_{j}\right) \int_{u_{j-1}}^{u_{j}} K_{\mu}(u, x) d u+\sum_{j=1}^{s} \int_{u_{j-1}}^{u_{j}}\left[f(u)-f\left(u_{j}\right)\right] K_{\mu}(u, x) d u
$$

Daí,

$$
\left|R_{\ell \mu}(x)\right| \leq\left|\sum_{j=1}^{s} f\left(u_{j}\right) \int_{u_{j-1}}^{u_{j}} K_{\mu}(u, x) d u\right|+\left|\sum_{j=1}^{s} \int_{u_{j-1}}^{u_{j}}\left[f(u)-f\left(u_{j}\right)\right] K_{\mu}(u, x) d u\right|
$$

O primeiro termo do segundo membro pode ser majorado:

$$
\left|\sum_{j=1}^{s} f\left(u_{j}\right) \int_{u_{j-1}}^{u_{j}} K_{\mu}(u, x) d u\right| \leq \sum_{j=1}^{s}\left|f\left(u_{j}\right)\right|\left|\int_{u_{j-1}}^{u_{j}} K_{\mu}(u, x) d u\right|
$$

Porém, usando-se (3.46), tem-se

$$
\begin{gathered}
\left|\int_{u_{j-1}}^{u_{j}} K_{\mu}(u, x) d u\right|=\frac{1}{L}\left|\int_{u_{j-1}}^{u_{j}} \cos [i \mu q(u-x)] d u\right|= \\
=\frac{1}{L}\left|\frac{\sin [i \mu q(u-x)]}{i \mu q}\right|_{u_{j-1}}^{u_{j}} \leq \frac{2}{L|\mu| q}=\frac{2}{|\mu| \pi}
\end{gathered}
$$

ou seja,

$$
\left|\int_{u_{j-1}}^{u_{j}} K_{\mu}(u, x) d u\right| \leq \frac{2}{|\mu| \pi}
$$


Designemos agora:

$$
\begin{aligned}
& M_{j}=\sup \left\{|f(u)|: u \in\left[u_{j-1}, u_{j}\right]\right\} \\
& m_{j}=\inf \left\{|f(u)|: u \in\left[u_{j-1}, u_{j}\right]\right\}
\end{aligned}
$$

Lembrando das relações (3.41), é imediato que

$$
M_{j} \leq M \quad \text { e } \quad m_{j} \geq m
$$

Disso, segue que

$$
\left|f\left(u_{j}\right)\right| \leq M_{j} \leq M
$$

$\mathrm{e}$

$$
\left|f(u)-f\left(u_{j}\right)\right| \leq M_{j}-m_{j} \leq M-m, \quad \forall u \in\left[u_{j-1}, u_{j}\right]
$$

Munidos desses resultados, voltando à estimativa do primeiro termo, usando (3.52) e (3.54), podemos escrever:

$$
\left|\sum_{j=1}^{s} f\left(u_{j}\right) \int_{u_{j-1}}^{u_{j}} K_{\mu}(u, x) d u\right| \leq \frac{2 s M}{|\mu| \pi}
$$

Majorando agora o último termo da expressão (3.51), tem-se:

$$
\left|\sum_{j=1}^{s} \int_{u_{j-1}}^{u_{j}}\left[f(u)-f\left(u_{j}\right)\right] K_{\mu}(u, x) d u\right| \leq \sum_{j=1}^{s} \int_{u_{j-1}}^{u_{j}}\left|f(u)-f\left(u_{j}\right)\right|\left|K_{\mu}(u, x)\right| d u
$$

Utilizando o resultado (3.55) e notando que $\left|K_{\mu}(u, x)\right| \leq 1 / L$, obtemos, para o majorante do último termo, o seguinte resultado:

$$
\left|\sum_{j=1}^{s} \int_{u_{j-1}}^{u_{j}}\left[f(u)-f\left(u_{j}\right)\right] K_{\mu}(u, x) d u\right| \leq \frac{\Delta P}{L} \sum_{j=1}^{s}\left(M_{j}-m_{j}\right)=\frac{2}{s} \sum_{j=1}^{s}\left(M_{j}-m_{j}\right)
$$

Usando-se então a relação (3.55), pode-se reescrever o resultado acima como:

$$
\left|\sum_{j=1}^{s} \int_{u_{j-1}}^{u_{j}}\left[f(u)-f\left(u_{j}\right)\right] K_{\mu}(u, x) d u\right| \leq 2(M-m)
$$

Uma vez alcançados os resultados (3.56) e (3.57), basta substituí-los na expressão (3.51) para obter:

$$
\left|R_{\ell \mu}(x)\right| \leq \frac{2 s M}{|\mu| \pi}+2(M-m)
$$


Procedimento análogo pode ser implementado para se determinar o resultado correspondente para os coeficientes relativos à série associada, fornecendo:

$$
\left|\tilde{R}_{\ell \mu}(x)\right| \leq \frac{2 r \tilde{M}}{|\mu| \pi}+2(\tilde{M}-\tilde{m})
$$

Mas, como $r<s, \tilde{M} \leq M$ e $\tilde{m} \geq m$, então $\tilde{M}-\tilde{m} \leq M-m$ implica que, dado um $\varepsilon>0, \exists \ell_{0} \geq 1: \ell \geq \ell_{0}=\ell(\varepsilon)$ verifica-se, para as séries $\mathcal{M}_{\ell}(x)$ e $\tilde{\mathcal{M}}_{\ell}(x)$ dadas pelas expressões (3.47) e (3.48) que:

$$
\left|\tilde{R}_{\ell \mu}(x)\right| \leq\left|R_{\ell \mu}(x)\right| \Rightarrow \tilde{\mathcal{M}}_{\ell}(x) \leq \mathcal{M}_{\ell}(x)
$$

Com isso, fica portanto demonstrado que o máximo resto local absoluto da série de Fourier Associada é menor ou igual ao correspondente da série de Fourier original, para o caso de períodos maiores que $2 \pi$.

Teorema 4 : Seja uma função $f(x) \in C^{0}[-L, L]$ definida no intervalo de período $2 L$, com $L>\pi$. Então, o máximo erro absoluto da série de Fourier associada é menor ou igual ao correspondente da série original, com mesmo número de termos, na restrição $2 \tilde{L}=\frac{2 \pi^{2}}{L}$.

\section{Demonstração :}

De acordo com a equação (3.44) acima, o resto local é dado por

$$
R_{\ell}(x)=\sum_{\mu=\ell+1}^{\infty} \frac{1}{L} \int_{-L}^{L} f(u) \cos [i \mu q(u-x)] d u
$$

Podemos integrar essa expressão em $I_{P}=[-\tilde{L}, \tilde{L}] \subset \mathbb{R}$ para determinar o erro total $\varepsilon_{\ell}$ da expansão em série com $n=2 \ell+1$ termos no intervalo. Então

$$
\varepsilon_{\ell}=\int_{-\tilde{L}}^{\tilde{L}} R_{\ell}(x) d x=\sum_{\mu=\ell+1}^{\infty} \frac{1}{L} \int_{-\tilde{L}}^{\tilde{L}} \int_{-L}^{L} f(u) \cos [i \mu q(u-x)] d u d x
$$

Pelo teorema de Fubini, escreve-se

$$
\begin{aligned}
\varepsilon_{\ell} & =\sum_{\mu=\ell+1}^{\infty} \frac{1}{L} \int_{-L}^{L} \int_{-\tilde{L}}^{\tilde{L}} f(u) \cos [i \mu q(u-x)] d x d u= \\
& =\left.\sum_{\mu=\ell+1}^{\infty} \frac{1}{L} \int_{-L}^{L} f(u) \frac{\sin [i \mu q(u-x)]}{-i \mu q}\right|_{-\tilde{L}} ^{\tilde{L}} d u=
\end{aligned}
$$




$$
\begin{gathered}
=\sum_{\mu=\ell+1}^{\infty} \frac{1}{i \mu q L} \int_{-L}^{L} f(u)\{\sin [i \mu q(u+\tilde{L})]-\sin [i \mu q(u-\tilde{L})]\} d u \Rightarrow \\
\varepsilon_{\ell}=\sum_{\mu=\ell+1}^{\infty} \frac{2}{i \mu q L} \int_{-L}^{L} f(u) \sin (i \mu q \tilde{L}) \cos (i \mu q u) d u
\end{gathered}
$$

Lembrando que $q L=\pi$, pode-se escrever

$$
\varepsilon_{\ell}=\sum_{\mu=\ell+1}^{\infty} \frac{2}{i \pi \mu} \int_{-L}^{L} f(u) \sin (i \mu q \tilde{L}) \cos (i \mu q u) d u
$$

De forma análoga, o erro total no período para a expansão da série associada é

$$
\tilde{\varepsilon}_{\ell}=\sum_{\mu=\ell+1}^{\infty} \frac{2}{i \pi \mu} \int_{-\tilde{L}}^{\tilde{L}} f(u) \sin (i \mu \tilde{q} \tilde{L}) \cos (i \mu \tilde{q} u) d u
$$

Calculando o erro total absoluto na aproximação da série de Fourier original, obtém-se:

$$
\left|\varepsilon_{\ell}\right| \leq \sum_{\mu=\ell+1}^{\infty} \frac{2}{\pi|\mu|} \int_{-L}^{L}|f(u)| d u=\mathcal{E}_{\ell}
$$

onde $\mathcal{E}_{\ell}$ é o majorante do erro total absoluto da série de Fourier aproximada por $n=2 \ell+1$ termos no intervalo $I_{P}=[-\tilde{L}, \tilde{L}]$. De forma similar, seu correspondente na série associada será

$$
\tilde{\mathcal{E}}_{\ell}=\sum_{\mu=\ell+1}^{\infty} \frac{2}{\pi|\mu|} \int_{-\tilde{L}}^{\tilde{L}}|f(u)| d u
$$

Entretanto, em decorrência da condição $\tilde{L}<\pi<L$, tem-se

$$
\int_{-\tilde{L}}^{\tilde{L}}|f(u)| d u \leq \int_{-L}^{L}|f(u)| d u
$$

e, consequentemente, $\tilde{\mathcal{E}}_{\ell} \leq \mathcal{E}_{\ell}$. Logo, o máximo erro absoluto da série de Fourier associada na restrição é menor ou igual ao correspondente da série original com mesmo número de termos, como queríamos demonstrar.

\section{Comentários sobre a interpretação geométrica das séries de Fourier originais}

e associadas No mapeamento do círculo sobre a reta real, efetua-se a transformação $\theta \rightarrow x / \rho$, onde $\rho$ é o raio do círculo. Do ponto de vista geométrico, o inverso do raio corresponde à curvatura e, portanto, para círculos não-unitários, a curvatura será definida dessa maneira. Para uma relação entre funções periódicas de $2 \pi$ na reta real e o círculo unitário, tem-se, naturalmente, $\rho=1$ mas, para círculos com curvaturas não-unitárias, 
teremos $2 \pi \rightarrow 2 L / \rho$ e $i s \theta \rightarrow i s x / \rho \equiv i s q x, \forall s \in \mathbb{Z}$, sendo $q=1 / \rho$ a curvatura do círculo mapeado. Dessa forma, vemos que a razão periódica $q=\pi / L$ exerce papel análogo ao da curvatura do círculo não-unitário no mapeamento efetuado sobre a reta real. Assim, para períodos maiores que $2 \pi$, a curvatura será menor que a unidade, ao passo que, para períodos menores, a curvatura será maior que a unidade.

Figura 6: Esquema geométrico de representação SF-SA

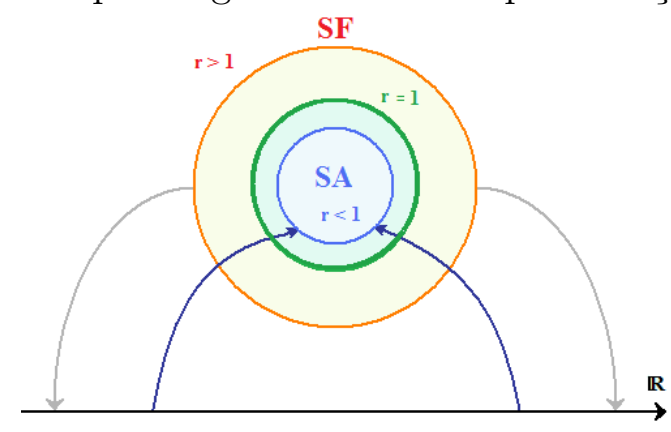

Todavia, se repartirmos a região de mapeamento dos círculos no plano entre os raios maiores e menores que a unidade, veremos que a região externa ao círculo unitário corresponde a curvaturas menores que a unidade e períodos maiores que $2 \pi$, ao passo que a região interior corresponderia a curvaturas maiores que a unidade e períodos inferiores a $2 \pi$. Logo, atribuindo às séries de Fourier originais e associadas seus respectivos domínios de aplicação, que correspondem às melhores precisões de cada uma delas, constatamos que o domínio de aplicação das séries de Fourier relaciona-se à região exterior, enquanto o das associadas, corresponde à região interior.

Além disso, mapeamentos da reta em círculos não-unitários têm relação direta com as séries associadas, do ponto de vista da forma, enquanto o mapeamento inverso fornece séries de Fourier originais, pois $x \rightarrow \rho \theta$ e $\theta \rightarrow q x$. Assim, podemos dizer que as séries de Fourier são construídas com base no parâmetro de curvatura $q$ no mapeamento do círculo na reta e as séries associadas são estabelecidas pelo parâmetro de raio $\rho \equiv \tilde{q}$ gerado no mapeamento inverso, i.e., da reta no círculo.

Na figura 6 mostra-se um esboço esquemático do que foi comentado. 


\section{APLICAÇÕES DO MÉTODO}

Este capítulo subdivide-se em quatro partes com distintos objetivos. Na primeira, o intuito é o de fazer uma estimativa do erro do método de matrizes operacionais na base de Fourier para os casos da derivação e integração e ver qual apresenta melhor precisão em relação ao método de derivação e integração usuais, comparando-os também ao erro obtido na própria expansão da função original, todos, evidentemente, com o mesmo número de termos.

Na segunda, desenvolvemos a ideia de como o método de modelagem pode ser usado para resolver uma equação diferencial dada, em dois casos: o primeiro, com poucos termos, explicitando praticamente todos os passos, apenas para exemplificar didaticamente o processo e torná-lo mais claro; o segundo, para um número maior de termos, deixando de lado os passos intermediários, cujos resultados são muito grandes para serem colocados ali, mostrando apenas os resultados finais. O objetivo desse segundo exemplo é o de mostrar que o método funciona consideravelmente bem conforme o número de termos aumenta.

Adicionalmente, na terceira parte, com o intuito de estudar e ilustrar o uso do ferramental desenvolvido, utilizaremos o formalismo das séries associadas de Fourier para expandir funções conhecidas, examinando a precisão obtida e comparando os resultados com as expansões dessas mesmas funções pelas séries de Fourier originais.

Por fim, uma vez comprovada a eficiência do método das séries associadas, iremos usálas no processo de resolução de EDOs lineares não-homogêneas pelo método das matrizes operacionais complexas de Fourier e da modelagem NBV/NIV. Com isso, pretendemos mostrar que as EDOs que não apresentam solução pelo método usual de Fourier são facilmente resolvidas, e com excelentes resultados, pelo método alternativo das séries associadas.

Espera-se, dessa maneira, atingir resultados que corroborem o uso de todas as formulações aqui expostas, discriminando o uso apropriado para cada caso particular. 


\subsection{Aproximação de Derivadas e Integrais pelo Método Operacional de Fourier Complexo}

Procedimento: Usaremos o mesmo algoritmo descrito em [cite gui1, gui2] para estimar o valor do erro da derivada obtida pela matriz operacional de derivação em comparação ao valor da derivada de uma função genérica $\phi(x)$ calculada diretamente.

Estimativa análoga é feita para os valores da integral calculada diretamente e a obtida pela matriz operacional de integração. Além disso, comparamos esses erros ao erro da expansão de Fourier complexa de mesma extensão (range). Adotamos o semiperíodo $L=\pi$ e expandimos várias funções com dois valores de extensão diferentes, primeiro para $\ell=12$, o que gera matrizes quadradas de ordem 25 e, em seguida, para $\ell=15$, produzindo matrizes de ordem 31. Apenas para lembrar, a extensão corresponde aos limites superior e inferior da soma na série para $\phi_{\ell}(x)=\sum_{k=-\ell}^{\ell} c^{k} b_{k}(x)$, o que perfaz um total de $N=2 \ell+1$ termos e gera matrizes operacionais de ordem $N$, conforme definido em seção prévia. Todos os cálculos, para ambas as extensões, são feitos com extrema rapidez no Mathematica $^{\circledR}$, não apresentando nenhuma espécie de problema de processamento.

As funções escolhidas para análise foram a quadrática, exponencial, gaussiana, senoidal com argumento quadrático e senoidal com amplitude gaussiana (tipo Morlet wavelet). Foram determinadas suas derivadas e primitivas e as correspondentes operacionais. Uma vez calculadas as derivadas e integrais de ambas as maneiras, isto é, diretamente e pelo método das matrizes operacionais, construímos os gráficos de suas partes reais e os comparamos. Em seguida, comentamos os resultados.

Dadas as expressões para os restos locais das partes reais referentes à expansão da função:

$$
\mathcal{R}\left(\phi_{s}\right)=\Re\left\{\phi(x)-\phi_{s}(x)\right\}
$$

à derivada operacional:

$$
\mathcal{R}(D \phi)=\Re\left\{\phi^{\prime}(x)-D \phi(x)\right\}
$$

e à integral operacional:

$$
\mathcal{R}(J \phi)=\Re\{\Phi(x)-J \phi(x)\},
$$

onde $\phi_{s}(x)$ é a expansão em série de Fourier, $D$ representa a matriz operacional de derivação, $\phi^{\prime}(x)$ é a derivada usual de $\phi(x), J \equiv M_{I}$ é a matriz operacional de integração e $\Phi(x)=\int \phi(x) d x$ é a integral da função calculada diretamente, passamos a definir os restos quadráticos locais pelos quadrados dos restos locais, i.e., $\mathcal{R}^{2}\left(\phi_{s}\right):=\left(\mathcal{R}\left(\phi_{s}\right)\right)^{2}$ e análogos, e os restos locais relativos da expansão, derivação e integração, respectivamente, 
por

$$
\begin{aligned}
E_{\text {rel }}\left(\phi_{s}\right) & :=\mathcal{R}\left(\phi_{s}\right) / I(\phi), \\
E_{\text {rel }}(D \phi) & :=\mathcal{R}(D \phi) / I(D \phi), \\
E_{\text {rel }}(J \phi) & :=\mathcal{R}(J \phi) / I(J \phi),
\end{aligned}
$$

onde

$$
\begin{aligned}
I(\phi) & =\int_{-L}^{L}|\phi(x)| d x \\
I(D \phi) & =\int_{-L}^{L}|D \phi(x)| d x \\
I(J \phi) & =\int_{-L}^{L}|J \phi(x)| d x
\end{aligned}
$$

A partir disso, construímos os gráficos correspondentes às funções-resto assim calculadas, mostradas nas figuras 7 a 16, para as funções seguintes:

Tabela 1: Funções analisadas na comparação MOD/MOI × D/J

\begin{tabular}{|c||c|}
\hline$\phi_{i}$ & função \\
\hline \hline$\phi_{1}$ & $x^{2}$ \\
\hline$\phi_{2}$ & $\exp x$ \\
\hline$\phi_{3}$ & $\exp \left(-x^{2} / 2\right)$ \\
\hline$\phi_{4}$ & $\cos \left(x^{2}\right)$ \\
\hline$\phi_{5}$ & $\exp \left(-x^{2}\right) \cos (7 x)$ \\
\hline
\end{tabular}

A seguir, os gráficos.

Gráficos dos restos locais de primeira ordem e quadrático das funções:

Figura 7: Derivação operacional de $\phi_{1}(x)=x^{2} \operatorname{com} \ell=12$
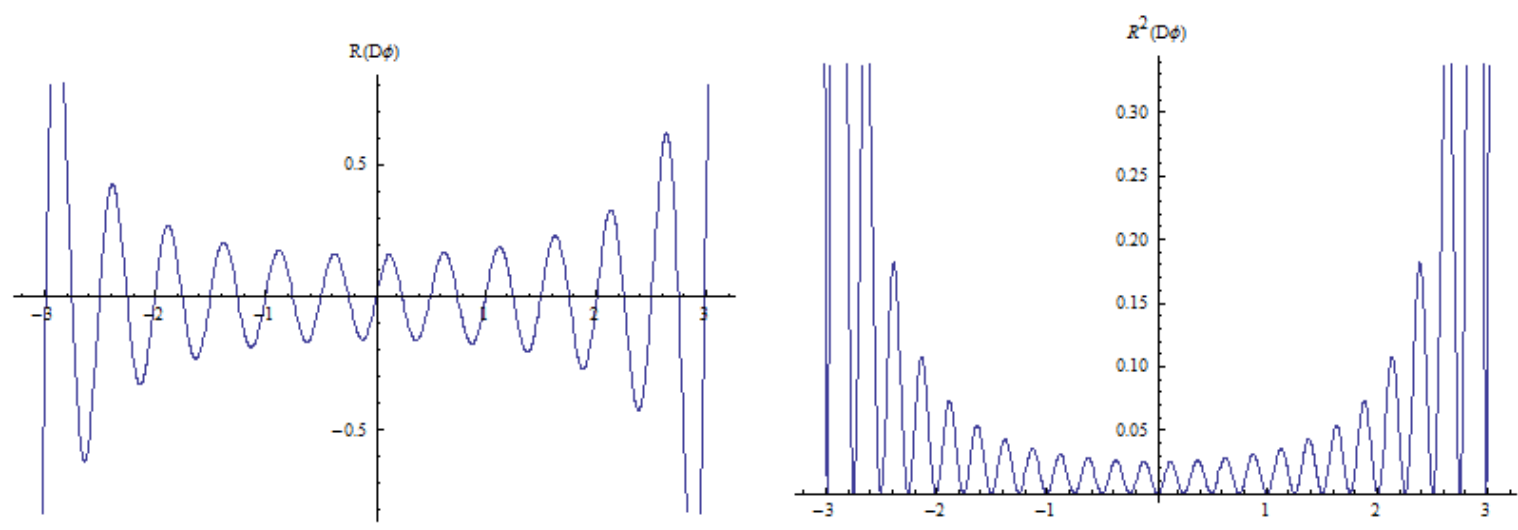
Figura 8: Integração operacional de $\phi_{1}(x)=x^{2} \operatorname{com} \ell=12$
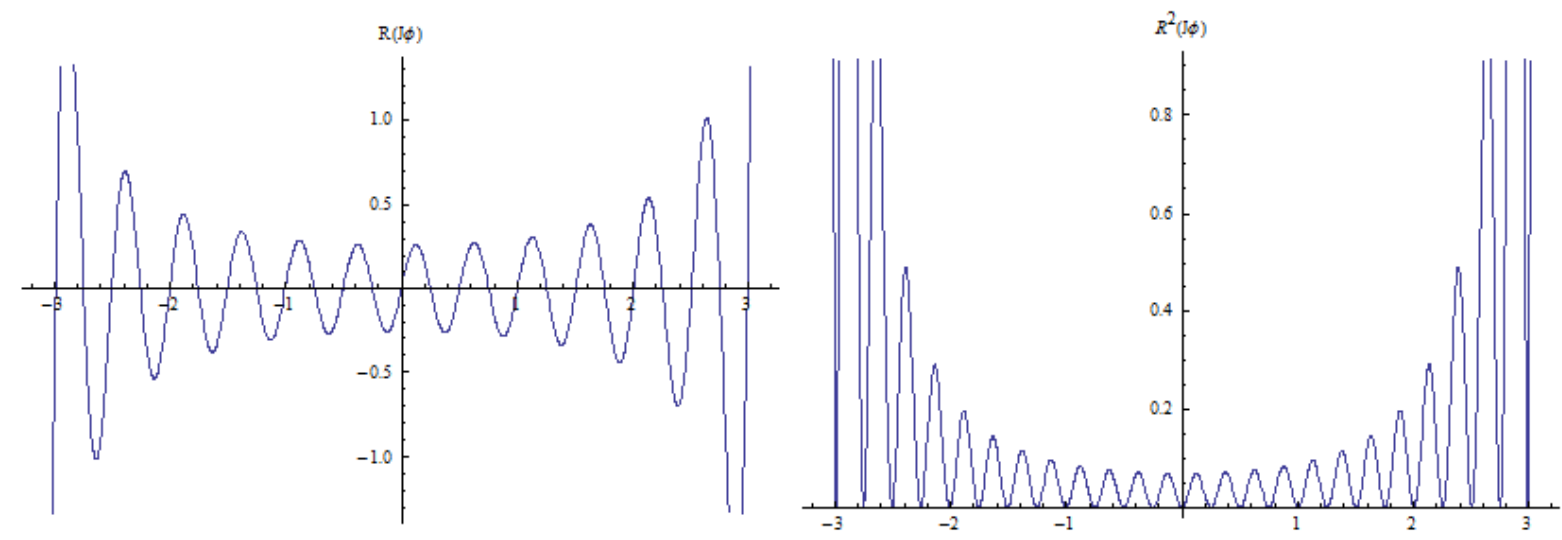

Figura 9: Derivação operacional de $\phi_{2}(x)=e^{x} \operatorname{com} \ell=12$
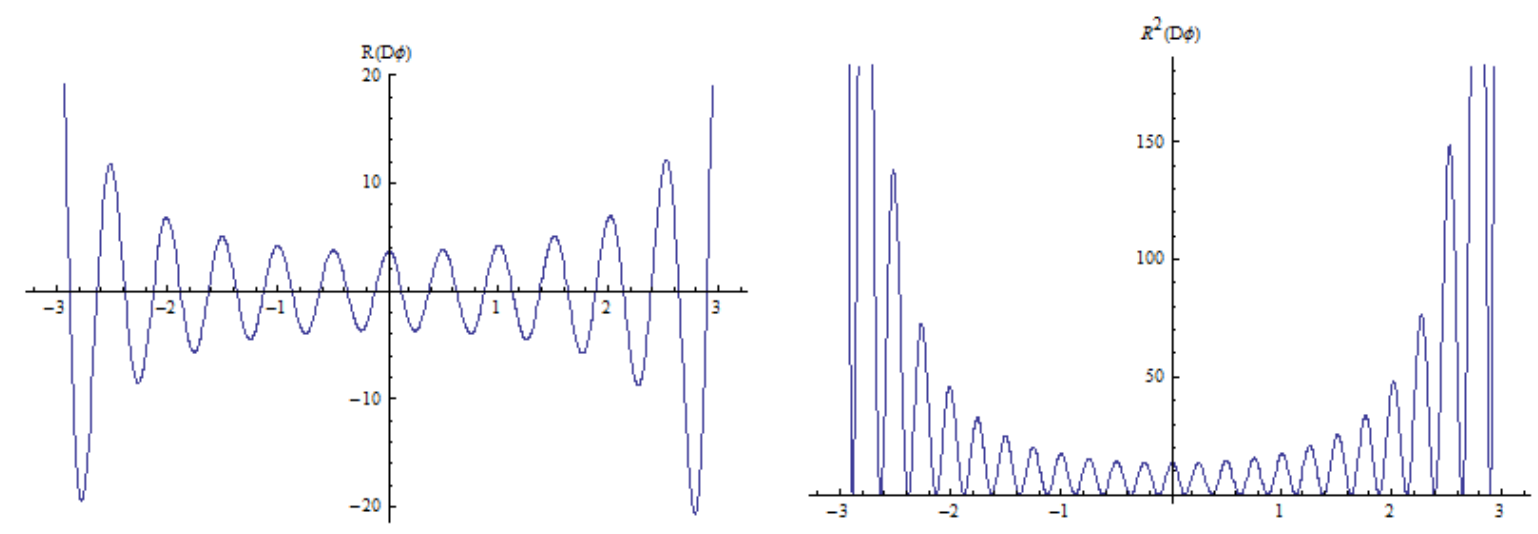

Figura 10: Integração operacional de $\phi_{2}(x)=e^{x} \operatorname{com} \ell=12$

Figura 11: Derivação operacional de $\phi_{3}(x)=\exp \left(-\frac{x^{2}}{2}\right) \operatorname{com} \ell=12$
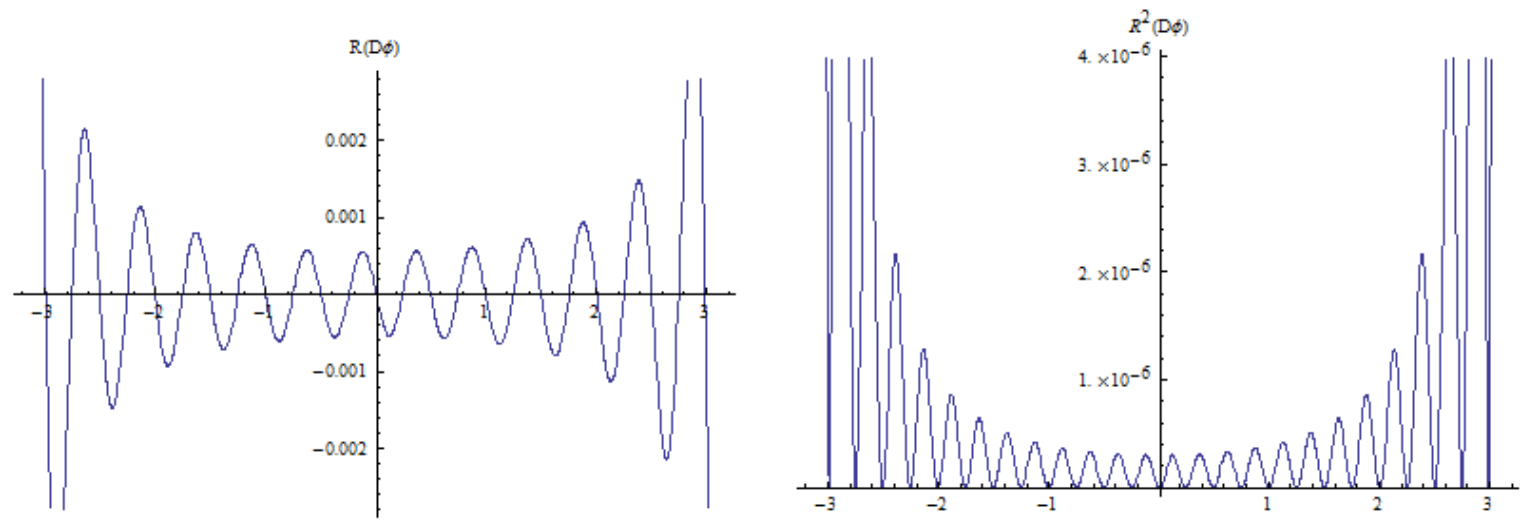

Figura 12: Integração operacional de $\phi_{3}(x)=\exp \left(-\frac{x^{2}}{2}\right) \operatorname{com} \ell=12$
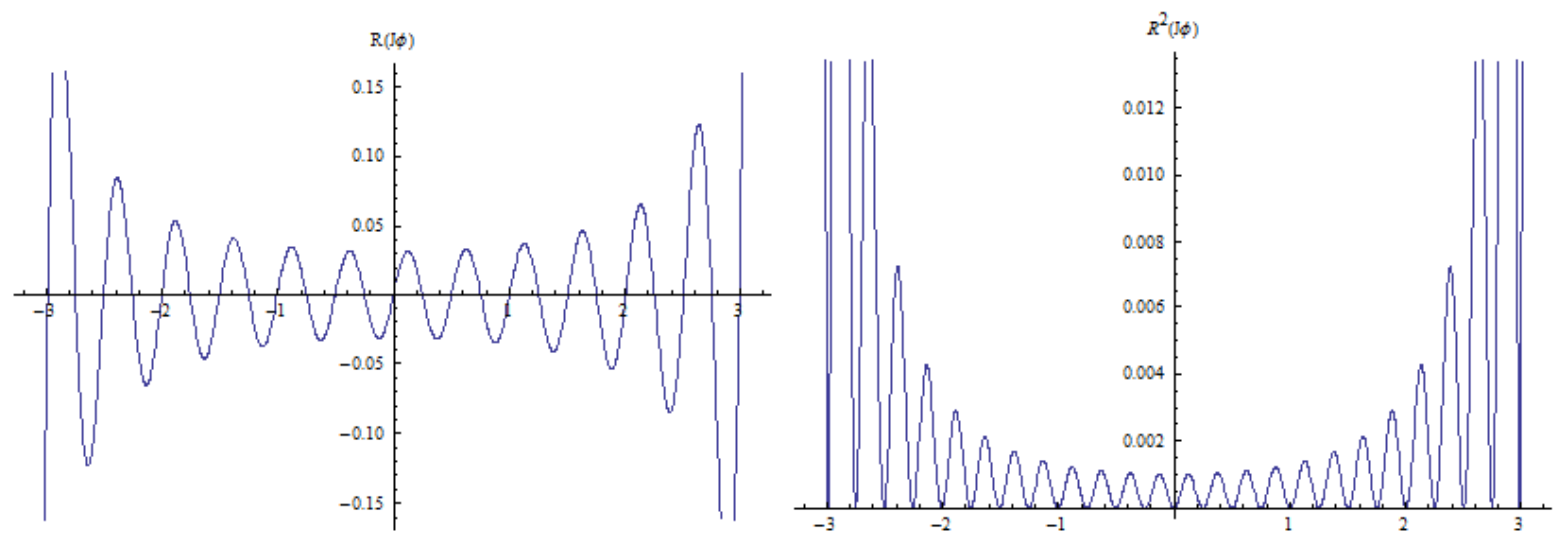

Figura 13: Derivação operacional de $\phi_{4}(x)=\cos \left(x^{2}\right) \operatorname{com} \ell=12$
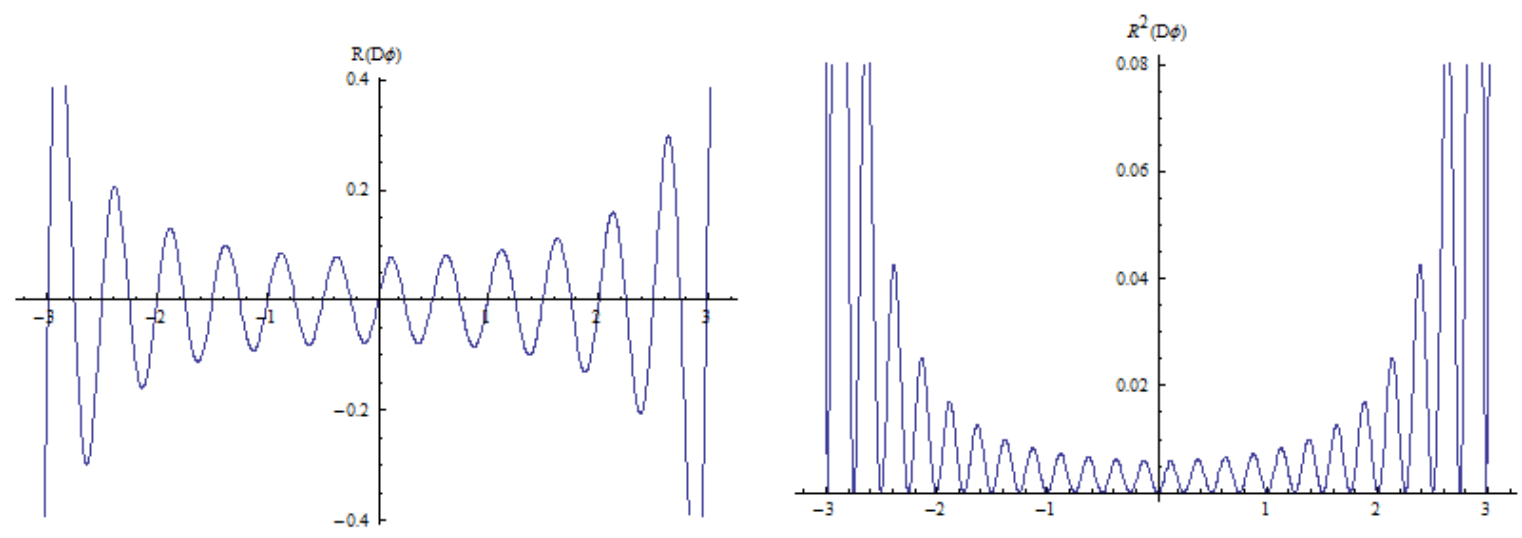
Figura 14: Integração operacional de $\phi_{4}(x)=\cos \left(x^{2}\right) \operatorname{com} \ell=12$
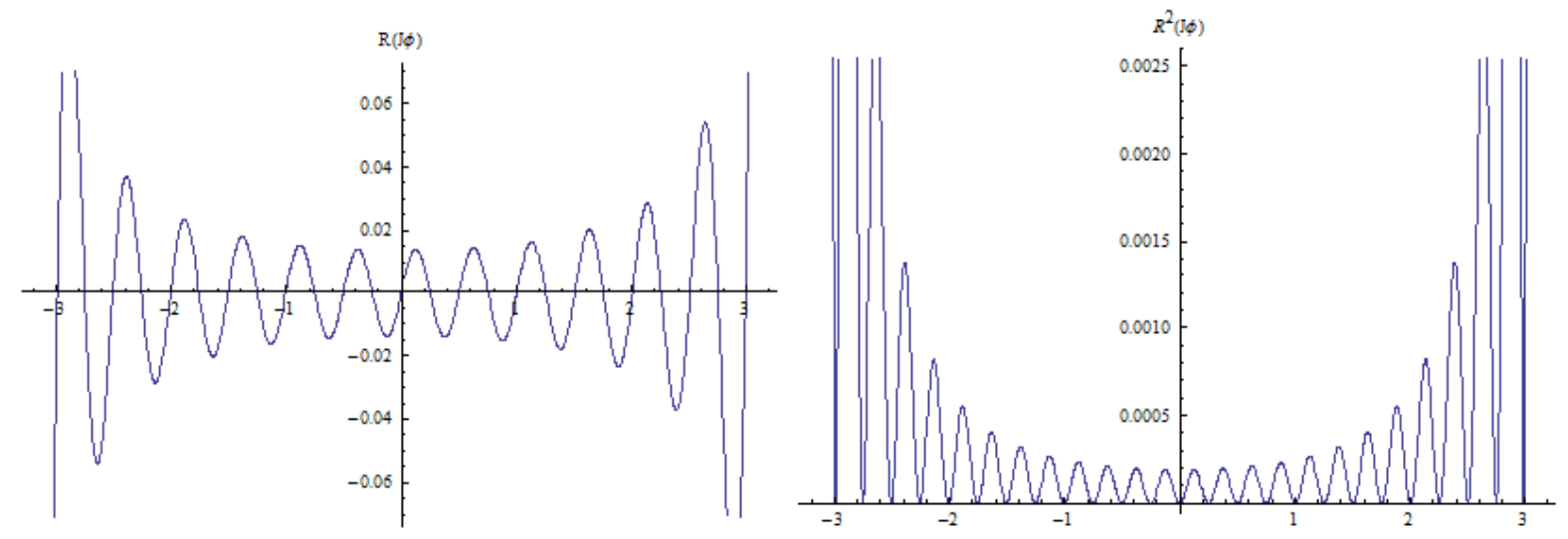

Figura 15: Derivação operacional de $\phi_{5}(x)=e^{-x^{2}} \cos (7 x) \operatorname{com} \ell=12$
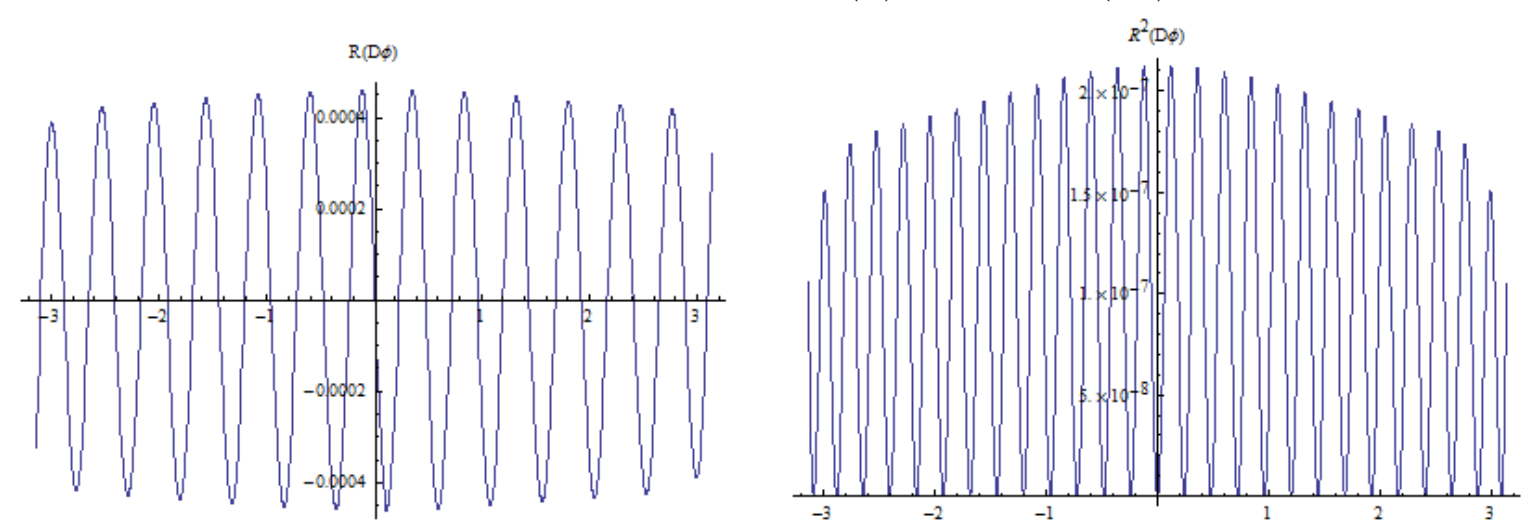

Figura 16: Integração operacional de $\phi_{5}(x)=e^{-x^{2}} \cos (7 x) \operatorname{com} \ell=12$
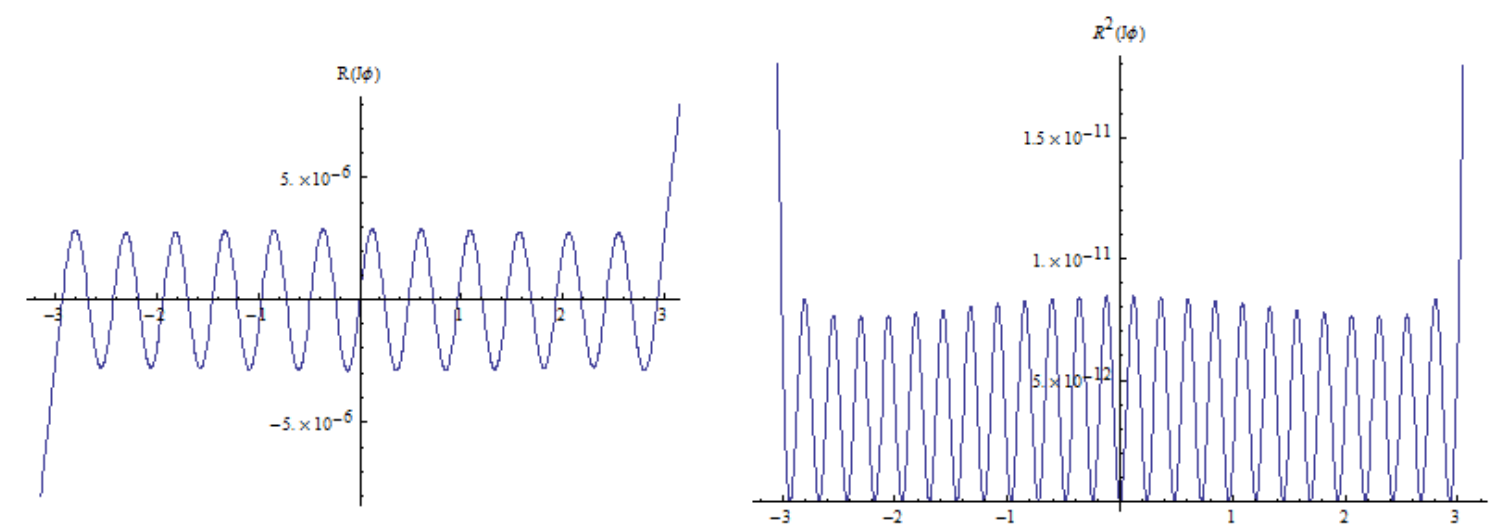

Comentários sobre os gráficos: Observando os gráficos anteriores, constata-se que a matriz operacional de derivação apresenta, em geral, resultados melhores que a de integração, com exceção dos dois últimos casos, em que ocorre o inverso, com grande vantagem para esta última.

Outra característica que se destaca na análise desses gráficos é o excelente resultado 
obtido para funções de natureza gaussiana, tanto na derivação quanto na integração operacional. Na função tipo Morlet wavelet, por exemplo, o resto local na derivação operacional para a expansão com range 12 , gira em torno de $10^{-4}$, enquanto na integração atinge a ordem de $10^{-6}$. Também a função $\cos \left(x^{2}\right)$ apresenta bons resultados para ambos os casos, com vantagem para a integração, da ordem de $10^{2}$.

A explicação para esse ótimos resultados está na classe dessas funções. Em primeiro lugar, as expansões de Fourier tendem, de maneira natural, a ser melhores para funções pares ou simétricas, que não apresentam descontinuidades nos extremos, atenuando o fenômeno de Gibbs. Em segundo, funções originalmente periódicas, como o cosseno, também adaptam-se melhor à expansão de Fourier, cuja base é constituída de senos e cossenos, ou de sua combinação, no caso complexo.

Para essas funções os gráficos das duas formas de integração, assim como os das derivadas, ajustam-se perfeitamente, ou seja, não é possível sequer perceber a presença de duas funções. O mesmo ocorre com os outros gráficos, excetuando-se os da função exponencial, cujos desvios atingem dimensões catastróficas, apontando para um problema de dimensão significativa nesse desenvolvimento.

É possível verificar o desvio catastrófico da integral operacional para o caso da função exponencial observando o gráfico seguinte:

Figura 17: Gráfico da expansão e das integrais direta e operacional de exp $x$

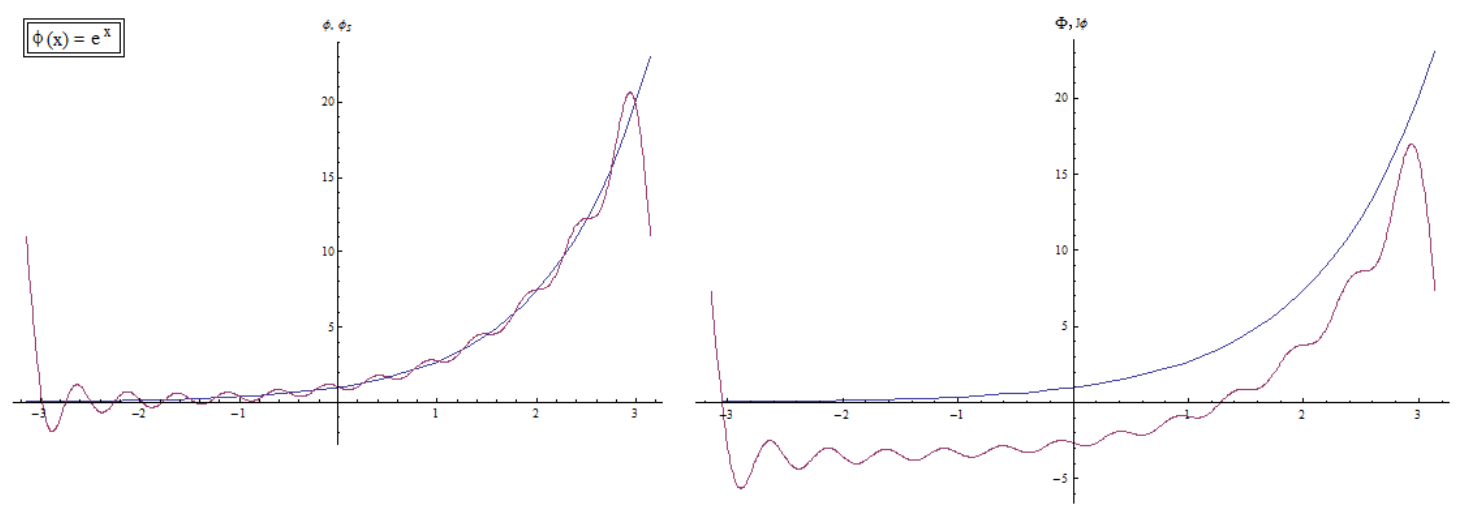

Nestes gráficos, correspondentes à expansão com range 12 e semiperíodo $\pi$, nota-se claramente o deslocamento translacional do gráfico da função para baixo (na altura do eixo $x$ em $x=0$ ) provocado pelo processo de integração operacional. Isto é consequência direta do cálculo efetuado pela abordagem clássica, acrescido do fato de a exponencial não ser muito adequada à expansão na base de Fourier, o que gera uma amplitude de oscilação excessiva. 
Posteriormente veremos que esse efeito translacional é parcialmente corrigido na primeira abordagem do cálculo fracional, devido à presença de termos não-nulos na coluna central da matriz operacional de integração calculada por Riemann-Liouville, os quais não aparecem no resultado clássico. No método de Weyl, contudo, o efeito desaparece por completo, pois os operadores são apropriadamente construídos para atuarem sobre bases de funções periódicas, como a de Fourier.

Vale notar ainda que não são todas as funções ímpares ou assimétricas que apresentam esse desvio. A função identidade, por exemplo, é um dos casos em que isso não ocorre. Sem entrarmos em maiores detalhes, a não-ocorrência desse fenômeno nessa função está ligada ao fato de ela cruzar a origem, embora seja ímpar.

Os gráficos das expansões não foram apresentados, mas será possível verificar, pelas tabelas dos erros anexadas adiante, que as aproximações são, via de regra, muito boas.

Passamos agora a estabelecer o cálculo dos erros totais para a construção das tabelas. Definem-se, para a função $\phi$ :

- erro total: $\mathcal{E}(\phi)=\int_{-L}^{L} \mathcal{R}\left(\phi_{s}(x)\right) d x$;

- erro total relativo: $\mathcal{E}_{\text {rel }}(\phi)=\frac{\mathcal{E}(\phi)}{I(\phi)}$;

- erro quadrático total: $\mathcal{E}^{2}(\phi)=\int_{-L}^{L} \mathcal{R}^{2}\left(\phi_{s}(x)\right) d x$

Os erros para as funções $D(\phi)$ e $J(\phi)$, são definidos de maneira análoga, com $\phi$ sendo substituído por $D \phi$ ou $J \phi$ nas equações escritas acima.

A seguir, as tebelas de erros para dois desenvolvimentos, com range 12 e 15 .

Tabela 2: Erros nas expansões de algumas funções para $\ell=12$

\begin{tabular}{|c||c|c|c|}
\hline Função $\phi$ & $\mathcal{E}(\phi)$ & $\mathcal{E}_{\text {rel }}(\phi)$ & $\mathcal{E}^{2}(\phi)$ \\
\hline \hline$x^{2}$ & 0.132204 & 0.00639569 & 0.00855134 \\
\hline $\exp x$ & 3.91463 & 0.169483 & 13.5493 \\
\hline $\exp \left(-\frac{1}{2} x^{2}\right)$ & $4.58997 \times 10^{-4}$ & $1.83421 \times 10^{-4}$ & $1.04914 \times 10^{-7}$ \\
\hline $\cos \left(x^{2}\right)$ & 0.0630572 & 0.014392 & 0.00187314 \\
\hline $\cos (7 x) \exp \left(-x^{2}\right)$ & $1.33369 \times 10^{-4}$ & $1.18196 \times 10^{-9}$ & $3.5043 \times 10^{-9}$ \\
\hline
\end{tabular}

Comentários sobre os erros: Como pode ser visto nas tabelas expostas acima, os erros nas funções polinomiais são muito grandes (apresentamos apenas a função quadrática a título de exemplo). Além disso, como foi mencionado no caso da função exp $x$, para funções ímpares ou assimétricas de maneira geral o erro pode se tornar catastrófico. A 
Tabela 3: Erros nas derivadas operacionais de algumas funções para $\ell=12$

\begin{tabular}{|c||c|c|c|}
\hline Função $\phi$ & $\mathcal{E}(D \phi)$ & $\mathcal{E}_{\text {rel }}(D \phi)$ & $\mathcal{E}^{2}(D \phi)$ \\
\hline \hline$x^{2}$ & 2.13452 & 0.108136 & 4.0191 \\
\hline $\exp x$ & 52.914 & 2.2909 & 2136.25 \\
\hline $\exp \left(-\frac{1}{2} x^{2}\right)$ & $7.4522 \times 10^{-3}$ & $3.75309 \times 10^{-3}$ & $5.04827 \times 10^{-5}$ \\
\hline $\cos \left(x^{2}\right)$ & 1.00455 & 0.0823767 & 0.822535 \\
\hline $\cos (7 x) \exp \left(-x^{2}\right)$ & $1.74608 \times 10^{-3}$ & $2.16761 \times 10^{-4}$ & $6.01282 \times 10^{-7}$ \\
\hline
\end{tabular}

Tabela 4: Erros nas integrais operacionais de algumas funções para $\ell=12$

\begin{tabular}{|c||c|c|c|}
\hline Função $\phi$ & $\mathcal{E}(J \phi)$ & $\mathcal{E}_{r e l}(J \phi)$ & $\mathcal{E}^{2}(J \phi)$ \\
\hline \hline$x^{2}$ & 3.50199 & 0.215708 & 10.8468 \\
\hline $\exp x$ & 23.76000 & 1.02868 & 98.4574 \\
\hline $\exp \left(-\frac{1}{2} x^{2}\right)$ & 0.425092 & 0.0723443 & 0.15939 \\
\hline $\cos \left(x^{2}\right)$ & 0.187812 & 0.0471337 & 0.0318655 \\
\hline $\cos (7 x) \exp \left(-x^{2}\right)$ & $1.22327 \times 10^{-5}$ & $7.42844 \times 10^{-5}$ & $3.21201 \times 10^{-11}$ \\
\hline
\end{tabular}

Tabela 5: Erros nas expansões de algumas funções para $\ell=15$

\begin{tabular}{|c||c|c|c|}
\hline Função $\phi$ & $\mathcal{E}(\phi)$ & $\mathcal{E}_{\text {rel }}(\phi)$ & $\mathcal{E}^{2}$ \\
\hline \hline$x^{2}$ & 0.0902838 & 0.00436769 & 0.00448758 \\
\hline $\exp x$ & 3.17082 & 0.13728 & 10.8287 \\
\hline $\exp \left(-\frac{1}{2} x^{2}\right)$ & $3.1823 \times 10^{-4}$ & $1.27169 \times 10^{-4}$ & $5.61061 \times 10^{-8}$ \\
\hline $\cos \left(x^{2}\right)$ & 0.04218 & $9.62704 \times 10^{-3}$ & $9.39641 \times 10^{-4}$ \\
\hline $\cos (7 x) \exp \left(-x^{2}\right)$ & $6.0318 \times 10^{-6}$ & $5.3456 \times 10^{-6}$ & $1.82088 \times 10^{-11}$ \\
\hline
\end{tabular}

Tabela 6: Erros nas derivadas operacionais de algumas funções para $\ell=15$

\begin{tabular}{|c||c|c|c|}
\hline Função $\phi$ & $\mathcal{E}(D \phi)$ & $\mathcal{E}_{\text {rel }}(D \phi)$ & $\mathcal{E}^{2}(D \phi)$ \\
\hline \hline$x^{2}$ & 1.79231 & 0.0907993 & 3.24181 \\
\hline $\exp x$ & 46.4096 & 2.00929 & 1734.16 \\
\hline $\exp \left(-\frac{1}{2} x^{2}\right)$ & $6.31975 \times 10^{-3}$ & $3.18276 \times 10^{-3}$ & $4.11323 \times 10^{-5}$ \\
\hline $\cos \left(x^{2}\right)$ & 0.825713 & 0.0677112 & 0.644114 \\
\hline $\cos (7 x) \exp \left(-x^{2}\right)$ & $1.14547 \times 10^{-4}$ & $1.42201 \times 10^{-5}$ & $1.10856 \times 10^{-8}$ \\
\hline
\end{tabular}

Tabela 7: Erros nas integrais operacionais de algumas funções para $\ell=15$

\begin{tabular}{|c||c|c|c|}
\hline Função $\phi$ & $\mathcal{E}(J \phi)$ & $\mathcal{E}_{\text {rel }}(J \phi)$ & $\mathcal{E}^{2}(J \phi)$ \\
\hline \hline$x^{2}$ & 2.89798 & 0.178503 & 8.7464 \\
\hline $\exp x$ & 23.6376 & 1.02339 & 95.8451 \\
\hline $\exp \left(-\frac{1}{2} x^{2}\right)$ & 0.356908 & 0.0607405 & 0.128561 \\
\hline $\cos \left(x^{2}\right)$ & 0.158994 & 0.0399015 & 0.0259105 \\
\hline $\cos (7 x) \exp \left(-x^{2}\right)$ & $1.94752 \times 10^{-6}$ & $1.18265 \times 10^{-5}$ & $4.58874 \times 10^{-12}$ \\
\hline
\end{tabular}


explicação para esse fenômeno tem relação com um fato típico da formulação da matriz operacional clássica, que deixa de considerar elementos importantes no cálculo. Esse desvio, porém, será completamente corrigido na abordagem fracional, com a introdução dos operadores fracionais de Weyl.

O fato, em resumo, é que o cálculo clássico das matrizes operacionais não especifica terminais, o que nos fornece primitivas e derivadas usuais. Já no processo fracional, a especificação dos terminais acrescenta termos que não existem na versão clássica e, como consequência, as matrizes obtidas têm outra forma. Do ponto de vista prático, ocorre no cálculo clássico, além do mais, uma translação das integrais no eixo y para determinadas funções, o que inviabiliza a análise do erro e gera esse afastamento catastrófico, embora isso só ocorra na matriz operacional de integração, devido à ausência das constantes adicionais que surgem somente na abordagem fracional. Por outro lado, a presença de tais constantes na integral fracional de Riemann e Riemann-Liouville, também tende a gerar translações desse tipo no caso das exponenciais. Logo, a natureza do processo de integração a ser empregado deve estar vinculado à classe da função sobre a qual se deseja operar.

Uma outra constatação interessante é o aumento significativo de precisão obtido com um ligeiro acréscimo de range (de 12 para 15), o que constitui uma grande vantagem, visto que os cálculos algébricos são muito rápidos e não ficam comprometidos por esse acréscimo de termos no desenvolvimento. Assim, o prognóstico de precisão é excepcionalmente bom para ranges consideravelmente maiores.

Uma característica notável surge na observação dos erros na função tipo Morlet, para a qual, em ambos os ranges, os erros totais de primeira e ordem e quadráticos da matriz operacional de integração foram menores que os da própria expansão de Fourier. Em outras palavras, o processo de integração pelo método de matriz operacional fornece maior precisão que a própria expansão em série da função original. Embora isso pareça surpreendente à primeira vista, encontra explicação na análise dos erros relativos, nos quais a matriz de integração apresenta precisão menor que a da expansão. Ainda assim, esse excelente resultado na MOI não deixa de causar certo estranhamento, considerando o que dissemos antes sobre a limitação da abordagem clássica. Esse fator é algo extremamente promissor para o papel exercido pela MOI fracional que deverá ser desenvolvida mais tarde.

Um ponto peculiar que surge como conclusão é que, ao contrário da prática usual em matrizes operacionais, na qual se costuma utilizar a matriz de integração no processo de 
resolução de equações diferenciais, na versão complexa de Fourier parece preferível usar a matriz de derivação, o que é exemplar, devido à sua extrema simplicidade. Ainda assim, deve-se observar que, embora se possa usar amiúde o método de derivação em vista de sua concisão, o processo de integração pode ser eventualmente utilizado nos casos em que o outro método não seja satisfatório, como parece ocorrer no caso da função tipo Morlet.

\subsection{Resolução de EDOs Não-homogêneas pelo Método de Modelagem Operacional}

O método aqui descrito possibilita a resolução de EDOs lineares não-homogêneas sujeitas tanto a condições iniciais (IVP) quanto a condições de contorno (BVP). Adicionalmente, implementamos uma solução original para equações dessa natureza, mas sem quaisquer parâmetros determinantes, quer signifiquem condições iniciais ou de contorno, através de um processo de modelagem da solução pela função do termo não-homogêneo da EDO, que denominamos método $N B V / N I V$. A exposição a seguir descreve todas as situações e esquematiza os processos de forma sistemática.

Suponhamos uma EDO não-homogênea de ordem $s \in \mathbb{N}$ do tipo:

$$
\sum_{r=0}^{s} \mu_{r}(x) \mathcal{D}^{r} y(x)=\phi(x),
$$

onde os coeficientes $\mu_{r}(x)$ são funções quaisquer e $\mathcal{D}^{r}=\frac{d^{r}}{d x^{r}}$.

Vamos assumir que exista uma solução aproximada para a EDO acima, expressa por uma função $\mathcal{L}^{1}$, desenvolvida em uma série com $n=2 \ell+1$ termos, denotada por $f_{\ell}(x)$ e chamada função candidata, com coeficientes não-conhecidos.

Então, podemos escrever:

$$
\left[\mu_{0}(x)+\sum_{r=1}^{s} \mu_{r}(x) \mathcal{D}^{r}\right] f_{\ell}(x)=\phi(x)
$$

O termo entre colchetes é um operador diferencial de ordem $s$, o qual será denominado operador da EDO e denotado por $\mathcal{D}_{e q}^{s}(x)$, cuja representação é uma matriz quadrada de ordem $n$, que chamaremos de matriz operacional da EDO, denotando-a por $\Delta(x)$. É importante deixar explícita a dependência da variável $x$ pois, embora as matrizes operacionais de derivação não sejam necessariamente variáveis, os coeficientes, em geral o serão. 
Fazendo a passagem às matrizes operacionais e adotando a notação $D^{0} \equiv I_{n}$, onde $I_{n}$ é a matriz identidade de ordem $n=2 \ell+1$, teremos então

$$
\Delta(x)=\sum_{r=0}^{s} \mu_{r}(x) D^{r} \Rightarrow \Delta(x) f_{\ell}(x)=\phi(x)
$$

Por outro lado, a função candidata pode ser expressa por uma série complexa de Fourier no formalismo de Dirac como

$$
\left|f_{\ell}\right\rangle_{\infty}=\langle C \mid B\rangle_{0_{n}}=\sum_{r=-\ell}^{\ell} C^{r}\left|b_{r}\right\rangle_{\infty}
$$

onde $|B\rangle_{0_{n}}$ é, nesse caso, a base de Fourier complexa, mas poderia ser, com efeito, qualquer outra que se deseje, obedecendo-se à readaptação indicial da somatória, i.e., $r=1, \cdots, n$, para uma função $\left|f_{n}\right\rangle_{\infty}$ expressa numa outra série.

Nessas condições, a EDO pode ser expressa na forma:

$$
\begin{gathered}
\mathcal{D}_{e q}^{s}(x)\left|f_{\ell}\right\rangle_{\infty}=\left\langle C\left|\mathcal{D}_{e q}^{s}\right| B\right\rangle_{0_{n}}=|\phi\rangle_{\infty} \Rightarrow \\
\Delta(x)\left|f_{\ell}\right\rangle_{\infty}=\langle C|\Delta| B\rangle_{0_{n}}
\end{gathered}
$$

O resultado da aplicação da matriz operacional da EDO sobre a base fornece um vetor de dimensão $n$, que denotaremos por $|\mathcal{V}\rangle_{0_{n}}=\Delta(x)|B\rangle_{0_{n}}$. Desse modo, a EDO pode ser reescrita simplesmente como

$$
\langle C \mid \mathcal{V}\rangle_{0_{n}}=|\phi\rangle_{\infty}
$$

ou, para $\langle C \mid \mathcal{V}\rangle_{0_{n}}: \equiv e d(x)$,

$$
e d(x)=\phi(x)
$$

A partir desse ponto, ocorre uma distinção relativa às condições dadas no problema. Vamos analisar separadamente cada caso a seguir.

\subsubsection{Solução com Condições de Contorno}

Vamos inicialmente estabelecer um conjunto de $n=2 \ell+1$ pontos arbitrariamente escolhidos

$$
\left.\left\{x_{j}\right\}: x_{j} \in\right]-L, L[, \quad j=0, \ldots, 2 \ell,
$$

onde $L$ é o semiperíodo da função original. A respeito da eleição dos pontos para construção da solução, há diversas possibilidades. Naturalmente, para uma primeira aborda- 
gem, escolhemos pontos definidos em intervalos iguais, isto é, com espaçamento constante.

No caso de uma EDO de segunda ordem, por exemplo, estabelecidas as condições de contorno $y_{c}\left(x_{0}\right)$ e $y_{c}\left(x_{2 \ell}\right)$, construiremos, por definição, um covetor $\left\langle\left. Y_{C}\right|_{0_{n}}\right.$, denominado covetor das condições, de maneira que sua primeira e última colunas sejam dadas pelas condições de contorno e as intermediárias, pelos valores assumidos pelo termo independente, i.e., a função $\phi(x)$ do termo não-homogêneo da EDO, no conjunto de pontos $\left\{x_{j}\right\}$ predeterminados, ou seja,

$$
\left\langle\left. Y_{C}\right|_{0_{n}}:=\left[\begin{array}{lllll}
y_{c}\left(x_{0}\right) & \phi\left(x_{1}\right) & \ldots & \phi\left(x_{2 \ell-1}\right) & y_{c}\left(x_{2 \ell}\right)
\end{array}\right]\right.
$$

A partir disso, com base no vetor $|\mathcal{V}\rangle$, é possível construir uma matriz numérica não-singular $\mathcal{M} \in \mathbb{M}_{n \times n}(\mathbb{C})$, denominada matriz anticoeficiente, tal que sua primeira e última colunas correspondam aos valores do vetor de base nos pontos extremos e as outras colunas assumam os valores do vetor $|\mathcal{V}\rangle$ nos pontos intermediários, isto é,

$$
\mathcal{M}=\left[\begin{array}{lllll}
\left|B\left(x_{0}\right)\right\rangle_{0_{n}} & \left|\mathcal{V}\left(x_{1}\right)\right\rangle_{0_{n}} & \ldots & \left|\mathcal{V}\left(x_{2 \ell-1}\right)\right\rangle_{0_{n}} & \left|B\left(x_{2 \ell}\right)\right\rangle_{0_{n}}
\end{array}\right]
$$

Entretanto, tomando-se a equação (4.1) e, lembrando que $\left|f_{\ell}\right\rangle_{\infty}=\langle C \mid B\rangle_{0_{n}}$, o covetor das condições pode ser expresso como

$$
\left\langle\left. Y_{C}\right|_{0_{n}}=\langle C|\left[\begin{array}{lllll}
\left|B\left(x_{0}\right)\right\rangle & \left|\mathcal{V}\left(x_{1}\right)\right\rangle_{0_{n}} & \cdots & \left|\mathcal{V}\left(x_{2 \ell-1}\right)\right\rangle_{0_{n}} & \left|B\left(x_{2 \ell}\right)\right\rangle_{0_{n}}
\end{array}\right]\right.
$$

ou, em termos da matriz anticoeficiente,

$$
\left\langle\left. Y_{C}\right|_{o_{n}}=\left\langle\left. C\right|_{0_{n}} \mathcal{M}\right.\right.
$$

Essa situação descreve, através de uma única equação, todas as condições do problema:

$$
\left\{\begin{array}{l}
f_{\ell}\left(x_{0}\right)=y_{c}\left(x_{0}\right) \\
\left\{e d\left(x_{j}\right)=\phi\left(x_{j}\right), \quad(j=1, \ldots, 2 \ell-1)\right. \\
f_{\ell}\left(x_{2 \ell}\right)=y_{c}\left(x_{2 \ell}\right)
\end{array}\right.
$$

Logo, o sistema linear $\left\langle Y_{C}\right|=\langle C| \mathcal{M}$ pode ser resolvido em termos do covetor coeficiente, conduzindo ao resultado:

$$
\langle C|=\left\langle Y_{C}\right| \mathcal{M}^{-1}
$$

Assim, uma vez determinado o covetor coeficiente e, por conseguinte, seus elementos, 
basta substituir os coeficientes encontrados na série da função candidata para obter a solução da EDO.

Para EDOs de ordens maiores, outras condições iniciais ou de contorno podem ser estabelecidas pelas derivadas de $1^{a}$ e demais ordens necessárias, devendo suas expressões serem dispostas na matriz $\mathcal{M}$. Discorreremos sobre isso na próxima seção.

\subsubsection{Problema com Condições Iniciais}

Estamos supondo uma EDO de ordem $s$. Assim, no caso de termos condições iniciais, podemos elegê-las até a derivada de ordem $s-1$, dispondo-as como componentes do covetor das condições, deixando os elementos restantes de $\left\langle Y_{C}\right|$ para os valores assumidos pela função do termo independente da EDO nos pontos arbitrariamente escolhidos.

Fixado o ponto $x_{0}$, geralmente no início do intervalo, que nesse caso não precisa ser simétrico, podendo correr de $x_{0}$ a $x_{0}+2 L$, dada a periodicidade da função expandida, estabelecem-se as condições iniciais.

Sejam, portanto, $y_{c}\left(x_{0}\right), y_{c}^{\prime}\left(x_{0}\right), \ldots, y_{c}^{(s-1)}\left(x_{0}\right)$ as ' $s$ ' condições iniciais dadas no problema.

Então, podemos escrevê-las da seguinte maneira:

$$
\left\{\begin{array}{c}
f_{\ell}\left(x_{0}\right)=y_{c}\left(x_{0}\right) \\
f_{\ell}^{\prime}\left(x_{0}\right)=y_{c}^{\prime}\left(x_{0}\right) \\
\vdots \\
f_{\ell}^{(s-1)}\left(x_{0}\right)=y_{c}^{(s-1)}\left(x_{0}\right) \\
\left\{e d\left(x_{j}\right)=\phi\left(x_{j}\right),(j=1, \ldots, n-s) ;\right.
\end{array}\right.
$$

É claro que, nesse caso, o conjunto de pontos deve ser redefindo para particionar o intervalo periódico em $n-s$ pontos, em vez de $n$. Naturalmente, isso tem um peso na precisão da solução, pois quanto maior o número de condições iniciais, Nessas circunstâncias, o covetor das condições passa a ser

$$
\left\langle Y_{C}\right|:=\left[\begin{array}{lllllll}
y_{c}\left(x_{0}\right) & y_{c}^{\prime}\left(x_{0}\right) & \cdots & y_{c}^{(s-1)}\left(x_{0}\right) & \phi\left(x_{1}\right) & \cdots & \phi\left(x_{n-s}\right)
\end{array}\right]
$$


Entretanto, agora as condições $y_{c}^{(r)}\left(x_{0}\right)$ são dadas pela relação

$$
y_{c}^{(r)}\left(x_{0}\right) \equiv\left|f_{\ell}^{(r)}\left(x_{0}\right)\right\rangle_{\infty}=\left\langle C\left|D^{r}\right| B\left(x_{0}\right)\right\rangle_{0_{n}}
$$

o que conduz a

$$
\begin{aligned}
& \left\langle\left. Y_{C}\right|_{0_{n}}=\left[\left\langle C\left|D^{0}\right| B\left(x_{0}\right)\right\rangle_{0_{n}} \quad\left\langle C\left|D^{1}\right| B\left(x_{0}\right)\right\rangle_{0_{n}} \quad \cdots\right.\right. \\
& \left.\cdots\left\langle C\left|D^{s-1}\right| B\left(x_{0}\right)\right\rangle_{0_{n}} \quad\left\langle C \mid \mathcal{V}\left(x_{1}\right)\right\rangle_{0_{n}} \quad \cdots \quad\left\langle C \mid \mathcal{V}\left(x_{n-s}\right)\right\rangle_{0_{n}}\right]
\end{aligned}
$$

Então, de maneira análoga à anterior, definimos a matriz $\mathcal{M} \in \mathbb{M}_{n}(\mathbb{C})$ por

$$
\mathcal{M}:=\left[\begin{array}{lllllll}
D^{0}\left|B\left(x_{0}\right)\right\rangle & D^{1}\left|B\left(x_{0}\right)\right\rangle & \cdots & D^{s-1}\left|B\left(x_{0}\right)\right\rangle & \left|\mathcal{V}\left(x_{1}\right)\right\rangle & \cdots & \left|\mathcal{V}\left(x_{n-s}\right)\right\rangle
\end{array}\right]
$$

Dessa forma, somos conduzidos à expressão

$$
\left\langle Y_{C}\right|=\langle C| \mathcal{M}
$$

e, novamente, à solução para o covetor coeficiente,

$$
\langle C|=\left\langle Y_{C}\right| \mathcal{M}^{-1}
$$

Dessa maneira, ambos os casos podem ser resolvidos pelo mesmo método, levando em conta o tipo de condições dadas no problema.

No entanto, curiosamente, mesmo no caso de não ser adotada nenhuma condição inicial ou de contorno, ainda é possível resolver a EDO, uma vez que os valores necessários para substituir tais condições podem ser tomados por simples comparação, ajustando-se os coeficientes da função candidata a fim de que satisfaçam a equação

$$
\mathcal{D}_{e q}^{s}\left|f_{\ell}\right\rangle=|\phi\rangle
$$

para os valores assumidos pela função do termo não-homogêneo nos pontos arbitrados, numa espécie de interpolação da função $\phi(x)$, por assim dizer. Desse modo, somos conduzidos a um terceiro método de resolução, descrito a seguir.

\subsubsection{Método de Resolução sem Condições de Contorno (NBV) ou Iniciais (NIV)}

Chamamos de método NBV (No Boundary Values) ao processo de obtenção da solução de uma EDO pelo algoritmo acima descrito, mas sem a adoção de condições de contorno. 
Analogamente, ao método NIV (No Initial Values), idêntico ao anterior, mas sem a necessidade de condições iniciais.

A justificativa para a possibilidade de resolução de uma EDO sem condições iniciais ou de contorno advém do fato de que a solução proposta no presente método é de caráter global e não localmente puntual, como no caso da resolução tradicional do cálculo.

Quando se determina a integral de uma função $f(x)$ qualquer, este processo fornece uma solução local $F(x)$ cuja constante de integração deve ser especificada de acordo com o valor que se deseja que a função $F$ assuma num dado ponto.

No método apresentado, todavia, a solução é global, no sentido de que vários pontos são dados simultaneamente e a função tomada como solução é "modelada" de acordo com os valores assumidos pela função do termo não-homogêneo nesses mesmos pontos, ao fazermos atuar $\mathcal{D}_{e q}^{s}(x)$ sobre $f_{\ell}(x)$ para realizar uma interpolação dos dados fornecidos pela função não-homogênea da EDO.

Uma vez estabelecida a função que interpola $\mathcal{D}_{e q}^{s}\left|f_{\ell}\right\rangle$, fica automaticamente determinado o covetor $\langle C|$ e, consequentemente, a solução da EDO. Por esse motivo, se houver um número suficiente de pontos para definir essa função-solução, a necessidade da inserção de condições determinantes, sejam de contorno ou iniciais, perde o sentido, tornando-se apenas uma ferramenta opcional. Na verdade, isto significa que tais condições ainda estão presentes, sendo impostas, todavia, pela própria forma do termo independente.

Na verdade, a imposição voluntária de condições pode até piorar a solução. O fato é que se os intervalos de igual espaçamento forem substituídos pelos nodos de Chebyshev ou de Fourier, as condições impostas podem acabar por desestruturar a equação-solução em série, pois os valores intermediários reconfiguram a solução para seu melhor ajuste, ao passo que as condições impostas forçam artificialmente uma situação de reajuste que pode não ser a melhor na configuração otimizada, caso tais condições não sejam bem escolhidas. Pode acontecer inclusive de não haver solução para determinadas condições.

Logo, afigura-se um procedimento aceitável, até mesmo aconselhável, quando as circunstâncias assim o permitirem, não fixar os pontos extremos num BVP, por exemplo, deixando-os livres para se adaptarem à solução de ótimo ajuste produzida pela utilização dos nodos apropriados.

Entretanto, isso exige uma reformulação do método na forma em que foi exposto. Este novo método passa a desempenhar um papel similar ao de uma interpolação, embora de forma indireta. 
As modificações necessárias à adaptação do método anterior descrito ao NBV/NIV estão contidas na reespecificação do covetor das condições, que passa a chamar-se covetor não-homogêneo $\langle\Phi|$, já que as condições passam a inexistir e todas as suas componentes devem ser dadas agora pelos valores assumidos pela função $\phi(x)$ no conjunto de pontos escolhidos. Dessa forma, basta definirmos

$$
\left\langle\left.\Phi\right|_{0_{n}}:=\left[\begin{array}{lllll}
\phi\left(x_{0}\right) & \phi\left(x_{1}\right) & \cdots & \phi\left(x_{2 \ell-1}\right) & \phi\left(x_{2 \ell}\right)
\end{array}\right] .\right.
$$

Nessas circunstâncias, a matriz anticoeficiente passa a ser:

$$
\mathcal{M}:=\left[\begin{array}{lllll}
\left|\mathcal{V}\left(x_{0}\right)\right\rangle & \left|\mathcal{V}\left(x_{1}\right)\right\rangle & \cdots & \left|\mathcal{V}\left(x_{2 \ell-1}\right)\right\rangle & \left|\mathcal{V}\left(x_{2 \ell}\right)\right\rangle
\end{array}\right]
$$

e a solução do sistema linear $\langle C| \mathcal{M}=\langle\Phi|$ fornece o covetor dos coeficientes, ou seja, $\langle C|=\langle\Phi| \mathcal{M}^{-1}$, o que corresponde à solução do sistema de equações

$$
\left\{e d\left(x_{j}\right)=\phi\left(x_{j}\right), \quad(j=0, \ldots, 2 \ell) .\right.
$$

Dessa forma, fica completamente determinada a solução procurada através da função candidata

$$
\left|f_{\ell}\right\rangle=\langle C \mid B\rangle
$$

\subsubsection{Fenômeno de Runge e Nodos de Chebyshev}

Voltando à questão da eleição dos pontos para a construção da matriz solução, uma pergunta cabível se refere à sua distribuição no intervalo de definição da função, ou seja, à opção de distanciamento entre eles. No caso de interpolações polinomiais realizadas a intervalos de espaçamento constante, ocorre o bem conhecido fenômeno de Runge, responsável pelo afastamento da função interpolada em relação à original com a presença de oscilações de grande amplitude nos extremos do intervalo e possíveis divergências nos polinômios interpoladores de ordem elevada.

Vamos nos deter um pouco sobre esse importante assunto.

Se a função a ser interpolada é contínua, então o Teorema da Aproximação de StoneWeirstrass garante que ela pode ser uniformemente aproximada num intervalo dado, em qualquer medida desejada, por uma função polinomial $P_{n}(x)$, de grau menor ou igual a $n$, ou seja,

$$
\lim _{n \rightarrow \infty}\left(\max _{a \leq x \leq b}\left|f(x)-P_{n}(x)\right|\right)=0
$$


Entretanto, o matemático alemão Carl D. T. Runge (1856-1927) descobriu que algumas interpolações com polinômios de alta ordem calculadas em pontos a intervalos regulares podem resultar em aproximações divergentes. Esse comportamento foi denominado fenômeno de Runge. O exemplo clássico é dado por uma função do tipo distribuição de Cauchy, $\phi(x)=\frac{1}{\pi}\left(1+x^{2}\right)^{-1}$. Ele trabalhou com a função $\pi \phi(5 x)$, demonstrando que uma interpolação com um polinômio $P_{n}(x)$ como o descrito no teorema acima, a pontos equidistantes no intervalo $[-1,1]$, provoca oscilações nas bordas do intervalo, tanto maiores quanto maior for o grau do polinômio, de forma que

$$
\lim _{n \rightarrow \infty}\left(\max _{-1 \leq x \leq 1}\left|f(x)-P_{n}(x)\right|\right)=+\infty
$$

Como forma de minimizar o problema, é preciso ir aumentando assintoticamente a densidade de pontos nas proximidades das bordas do intervalo. Em vista disso, o matemático russo Pafnuty L. Chebyshev (1821-1894) provou que, dentre todos os polinômios do tipo $H(x)=\prod_{k}\left(x-x_{k}\right)$, o que melhor realiza essa aproximação, apresentando o menor valor para $\max _{x \in I}|H(x)|$ no intervalo $I=[-1,1]$, é o polinômio no qual os pontos de eleição são dados por

$$
\xi_{i}=\cos \left(\frac{2 i-1}{2 n} \pi\right), \quad i=1, \ldots, n
$$

chamados nós (ou nodos) de Chebyshev. No caso de um intervalo genérico $[a, b]$, passa a valer:

$$
\xi_{i}=\frac{1}{2}(a+b)+\frac{1}{2}(b-a) \cos \left(\frac{2 i-1}{2 n} \pi\right), \quad i=1, \ldots, n
$$

Como o índice da somatória da série de Fourier complexa corre de $-\ell$ a $\ell$, então, após a translação de range para adaptação ao uso computacional, a expressão dos nodos de Chebyshev toma a forma:

$$
\xi_{i}=L \cos \left[\left(\frac{2 i-1}{2 \ell+1}\right) \frac{\pi}{2}\right], \quad i=1, \ldots, 2 \ell+1
$$

Para as séries de Fourier complexa usaremos os nodos de Chebyshev nessa formulação.

Fenômeno de Gibbs Já para o fenômeno de Gibbs, análogo do de Runge mas para séries de Fourier, a questão da minimização do efeito é mais complicado. Existem métodos que ajudam em sua atenuação, como as somas de Fejér e Riesz ou o uso da aproximação sigma. A teoria de wavelets apresenta uma vantagem nesse ponto, pois o fenômeno de Gibbs não ocorre com as transformadas wavelets de Haar. Mas não tencionamos complicar 
desnecessariamente a exposição com a introdução de mais conceitos.

Finalmente, argumentamos aqui que, embora o uso dos nodos de Chebyshev seja especificado para interpolações polinomiais, seria interessante pesquisar sua aplicação na solução do método proposto, a fim de estudar seu efeito sobre a precisão da solução obtida, comparando-a com a calculada através do conjunto de pontos com espaçamento constante, visando averiguar a possibilidade de minimização do fenômeno de Gibbs. Ademais, propomos ainda uma outra disposição para o estabelecimento do conjunto de pontos, de que trataremos a seguir.

Nodos de Fourier Ainda com relação a essa mesma questão, averiguaremos a possibilidade de utilizar um outro conjunto de pontos, também com espaçamento constante, mas com diferente distribuição, mais concentrada perto do meio do intervalo de definição da função e que chamaremos de Nodos de Fourier, embora as relações com as raízes nas séries de Fourier reais sejam longínquas. Serão definidos para um intervalo de período $2 L$ por:

$$
x_{i}:=2 L\left(\frac{i-\ell-1}{2 \ell+1}\right), \quad i=1, \ldots, n=2 \ell+1 .
$$

Naturalmente, como nos casos anteriores, o número de pontos é definido pela extensão $\ell$ da soma. Assim, há três conjuntos de pontos disponíveis para utilização nas soluções de EDOs ou EDFs, os nodos equidistantes ou pontos de espaçamento constante (EC), os nodos de Chebyshev (NC) e os nodos de Fourier (NF), conforme definimos acima.

\subsubsection{Aplicação do Método}

\subsubsection{Exemplos Numéricos}

Nesta seção aplicamos o método das matrizes operacionais complexas de Fourier a uma equação de terceira ordem não-homogênea do tipo

$$
\frac{d^{3} y}{d x^{3}}+\nu(x) \frac{d^{2} y}{d x^{2}}+\mu(x) \frac{d y}{d x}+\lambda(x) y=\phi(x)
$$

onde $\mu(x), \nu(x)$ e $\lambda(x)$ são funções polinomiais escolhidas e $\phi(x)$ é uma senoide de amplitude gaussiana:

$$
\phi(x)=\cos (7 x) e^{-x^{2}}
$$

A fim de exemplificar o uso do método, dois casos serão mostrados. O primeiro, apenas como uma ilustração didática do processo, tomando $\ell=2$, e semiperíodo $\pi / 7$, o 
que corresponde a uma função aproximada com apenas 5 termos. Em seguida, um outro exemplo é desenvolvido para a mesma equação, para semiperíodo $\pi$, com $\ell=12$, isto é, uma aproximação com 25 termos. O objetivo deste segundo exemplo é mostrar a precisão do método.

Exemplo didático Como primeiro exemplo, o método descrito, com extensão $\ell=2$ e rodando em um PC Dual-core com 2GB RAM e usando Mathematica 6 é aplicado à equação acima com as seguintes funções coeficientes:

$$
\nu(x)=x+9, \quad \mu(x)=-x-1, \quad \lambda(x)=x^{2}
$$

Conforme foi abordado na seção anterior, podemos reescrever a equação sinteticamente como $\mathcal{D}_{e q}^{3}(x) f_{2}(x)=\phi(x)$, onde $\mathcal{D}_{e q}^{3} \equiv D^{3}+(x+9) D^{2}-(x+1) D+x^{2}$ e $D \equiv \frac{d}{d x}$. A equação resultante será resolvida pelo método proposto, gerando como solução uma função aproximada $f_{2}(x)$. A precisão da solução obtida será avaliada através da comparação entre os dois membros da equação anterior, isto é, entre $\mathcal{D}_{e q}^{3}(x) f_{2}(x)$ e $\phi(x)$.

O resto local, definido por

$$
\mathcal{R}_{2}(x):=\Re\left\{\mathcal{D}_{e q}^{3}(x) f_{2}(x)-\phi(x)\right\}
$$

fornece então uma estimativa da precisão da solução obtida.

Além disso, definindo-se o resto quadrático como

$$
\mathcal{R}_{\ell}^{2}(x):=\left|\mathcal{R}_{\ell}(x)\right|^{2},
$$

obtemos um critério apropriado para estimar a precisão. Apresentamos, em seguida, ambos os gráficos, do resto e do resto quadrático.

Tomamos, para esse primeiro exemplo, um semiperíodo $L=\pi / 7$ com condições de contorno $y_{c}(-\pi / 7)=y_{c}(\pi / 7)=0$, apenas para ilustrar o funcionamento do método, conforme esclarecido anteriormente. Assim, o cálculo é efetuado com uma função aproximada de apenas 5 termos e uma condição livre, já que a equação é de $3^{\text {a }}$ ordem nas derivadas. Os valores intermediários, determinados por espaçamento constante, serão $x_{1}=-\frac{\pi}{14}, x_{2}=0, x_{3}=+\frac{\pi}{14}$.

Nestas circunstâncias, o covetor das condições

$$
\left\langle\left. Y_{C}\right|_{0_{n}}:=\left[\begin{array}{lllll}
y_{c}\left(x_{0}\right) & \phi\left(x_{1}\right) & \phi\left(x_{2}\right) & \phi\left(x_{3}\right) & y_{c}\left(x_{4}\right)
\end{array}\right]\right.
$$


assume a forma

$$
\left\langle Y_{C}\right|=\left[\begin{array}{lllll}
0 & 0 & 1 & 0 & 0
\end{array}\right]
$$

enquanto a matriz $\mathcal{M}$ será

$$
\mathcal{M}=\left(\begin{array}{lllll}
1 . & -1 . & 1 . & -1 . & 1 . \\
1719.97-2754.86 i & -348.429-429.954 i & 0.0503551 & -348.429+429.954 i & 1719.97+2754.86 i \\
-1764 .+2758 . i & -441 .+350 . i & 0 . & -441 .-350 . i & -1764 .-2758 . i \\
1807.93-2761.14 i & 351.571+451.945 i & 0.0503551 & 351.571-451.945 i & 1807.93+2761.14 i \\
1 . & -1 . & 1 . & -1 . & 1 .
\end{array}\right)
$$

Os coeficientes do covetor $\langle C|$ na função-série aproximada $f_{2}(x)=\langle C \mid B\rangle$ assumem os valores:

$$
\begin{array}{ll}
C_{1}=-(1.6109+2.51978 i) \times 10^{-6} ; & C_{4}=(-6.88813+5.46831 i) \times 10^{-4} ; \\
C_{2}=-(6.88668+5.46711 i) \times 10^{-4} ; & C_{5}=0 \\
C_{3}=\left(-1.37587 \times 10^{-3}+2.63941 \times 10^{-6} i\right) ; &
\end{array}
$$

fornecendo a solução:

$$
\begin{aligned}
f_{2}(x)= & \left(-1.37587 \times 10^{-3}+2.63941 \times 10^{-6} i\right)+ \\
& -(6.88668+5.46711 i) \times 10^{-4} \exp (-7 i x)+ \\
& -(6.88813-5.46831 i) \times 10^{-4} \exp (7 i x)- \\
& -(1.6109+2.51978 i) \times 10^{-6} \exp (-14 i x),
\end{aligned}
$$

cujo gráfico é apresentado na figura (18). Logo em seguida, na figura (19) mostrase o gráfico da superposição entre a função $\phi(x)$ e o lado esquerdo da EDO: ed $(x)=$ $\mathcal{D}_{e q}^{3}(x) f_{2}(x)$. O erro quadrático total $\mathcal{E}_{\ell}^{2}$ no período, definido por

$$
\mathcal{E}_{2}^{2}=\int_{-L}^{L}\left|\phi(x)-\mathcal{D}_{e q}^{3}(x) f_{2}(x)\right|^{2} d x
$$

assume o valor numérico $\mathcal{E}_{2}^{2} \cong 3.32346 \times 10^{-3}$.

Pode-se constatar que, mesmo neste exemplo tão simples, de caráter essencialmente didático, o resultado obtido pelo método descrito é bastante satisfatório, como demonstra o gráfico da figura (20), que representa a função resto quadrático para a solução $f_{2}(x)$ no período. 
Figura 18: Gráfico da função solução $f_{2}(x)$

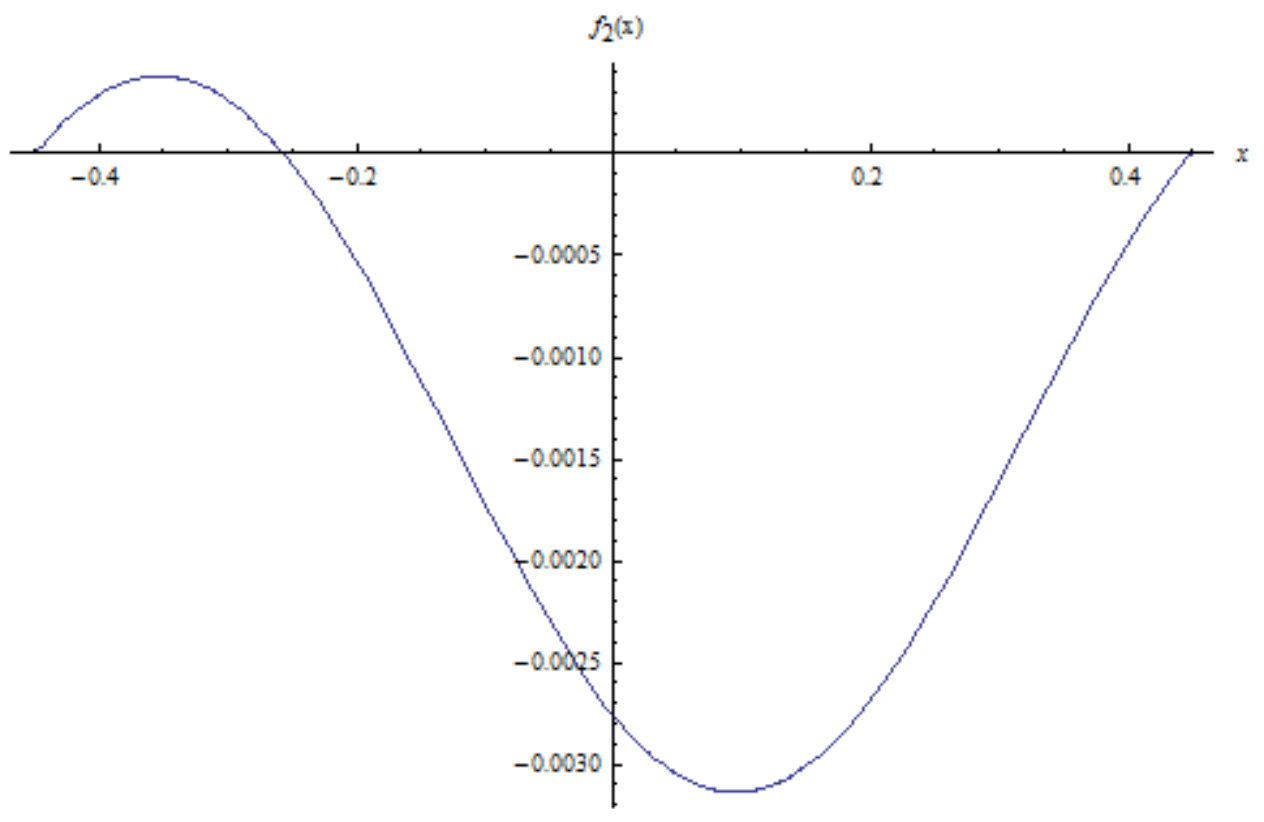

Figura 19: Gráfico da superposição entre $\mathcal{D}_{\text {eq }}^{3} f_{2}(x)$ e $\phi(x)$




Figura 20: Gráfico do resto quadrático de $\mathcal{D}_{e q}^{3} f_{2}(x)$

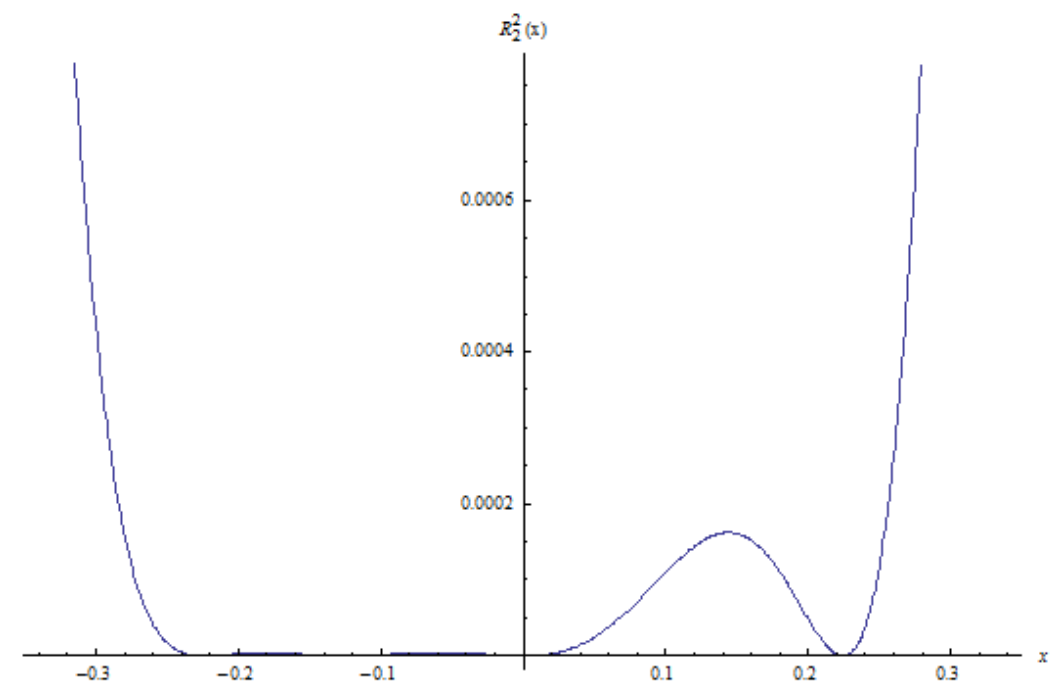

Exemplo mais significativo Agora, num segundo exemplo, cálculos mais apurados, com maior número de termos, são efetuados com a finalidade específica de estimar o poder de precisão do método. Neste caso, tomamos $L=\pi$ e range $\ell=12$ na mesma equação anterior, obtendo uma série com 25 termos. Consideramos as mesmas condições de contorno anteriores, i.e., $y(-\pi)=y(\pi)=0$.

É importante salientar que, curiosamente, para certos tipos de equações diferenciais, a melhor solução depende do número de termos da função-candidata de uma maneira não linear. Em outras palavras, para este tipo de equações, existe um valor máximo do número de termos para o qual a solução fica otimizada, quer dizer, qualquer número além desse valor específico irá inevitavelmente piorar a precisão da solução obtida.

A determinação do range de otimização parece não ser tarefa muito fácil no momento. Entretanto, algumas relações mais claras parecem emergir entre esse valor e o tipo de equação a ser solucionada.

Por exemplo, se o termo independente for altamente não-linear ou apresentar uma forma inapropriada na base de Fourier, isto é, não se ajustar bem à descrição matemática nessa base, aparece então a necessidade de determinar esse fator de otimização. Caso contrário, ou seja, se a função se adapta bem à descrição na base de Fourier ou é linear, certamente a precisão da solução crescerá diretamente com o número de termos da função candidata, conforme seria esperado. Além disso, há o fator de escolha das condições iniciais ou de contorno. É evidente que condições de contorno inapropriadas podem modificar o formato da solução obtida, chegando mesmo a impossibilitar algumas soluções. Todavia, essa questão exige estudos futuros mais cuidadosos a fim de obtermos uma descrição 
formal e pormenorizada para sua determinação.

Solucionando este segundo caso, a função aproximada $f_{12}(x)$ é dada a seguir, acompanhada de seu gráfico, apresentado na figura (21).

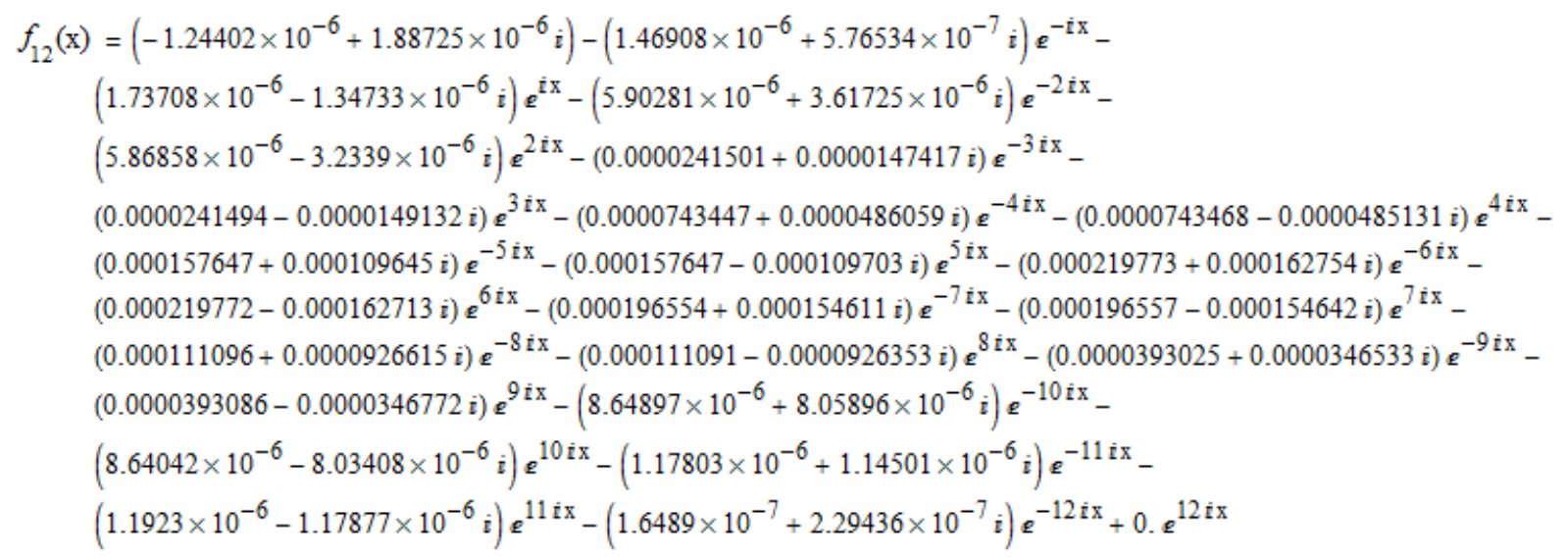

Figura 21: Gráfico da função solução $f_{12}(x)$

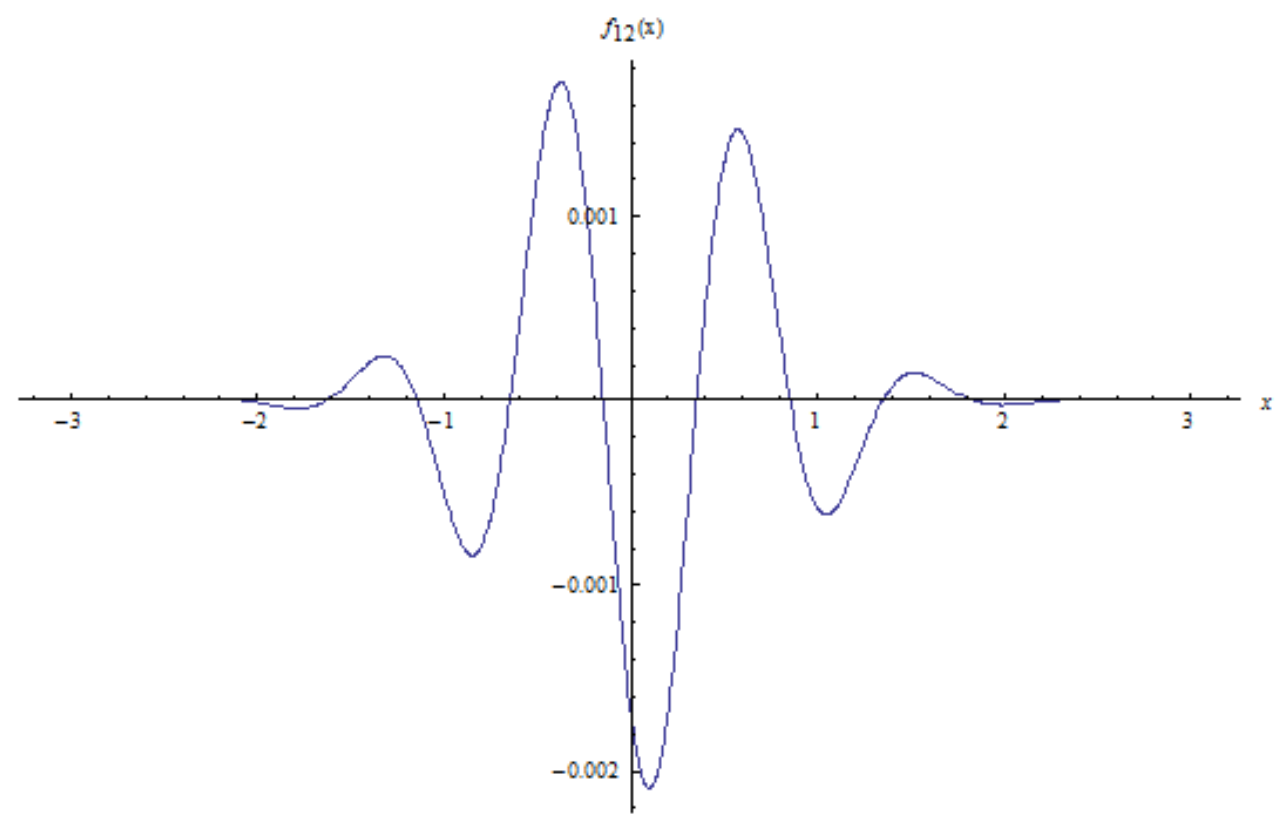

Uma análise gráfica comparativa da superposição entre $\mathcal{D}_{e q}^{3}(x) f_{12}(x)$ e $\phi(x)$ revela um ajuste praticamente perfeito entre elas, não sendo possível discernir as diferenças visualmente no gráfico mostrado na figura (22).

De fato, a observação da função resto quadrático local $\mathcal{R}_{12}^{2}(x)$, mostrado na figura (23), fornece uma ideia apropriada da excelente precisão atingida nesse ajuste. Podemos observar que o resto quadrático local gira em torno de $10^{-8}$, que é um valor consideravelmente pequeno, enquanto o erro quadrático total no período vale $4.33515 \times 10^{-8}$, o que 
Figura 22: Gráfico da superposição entre $\mathcal{D}_{e q}^{3} f_{12}(x)$ e $\phi(x)$

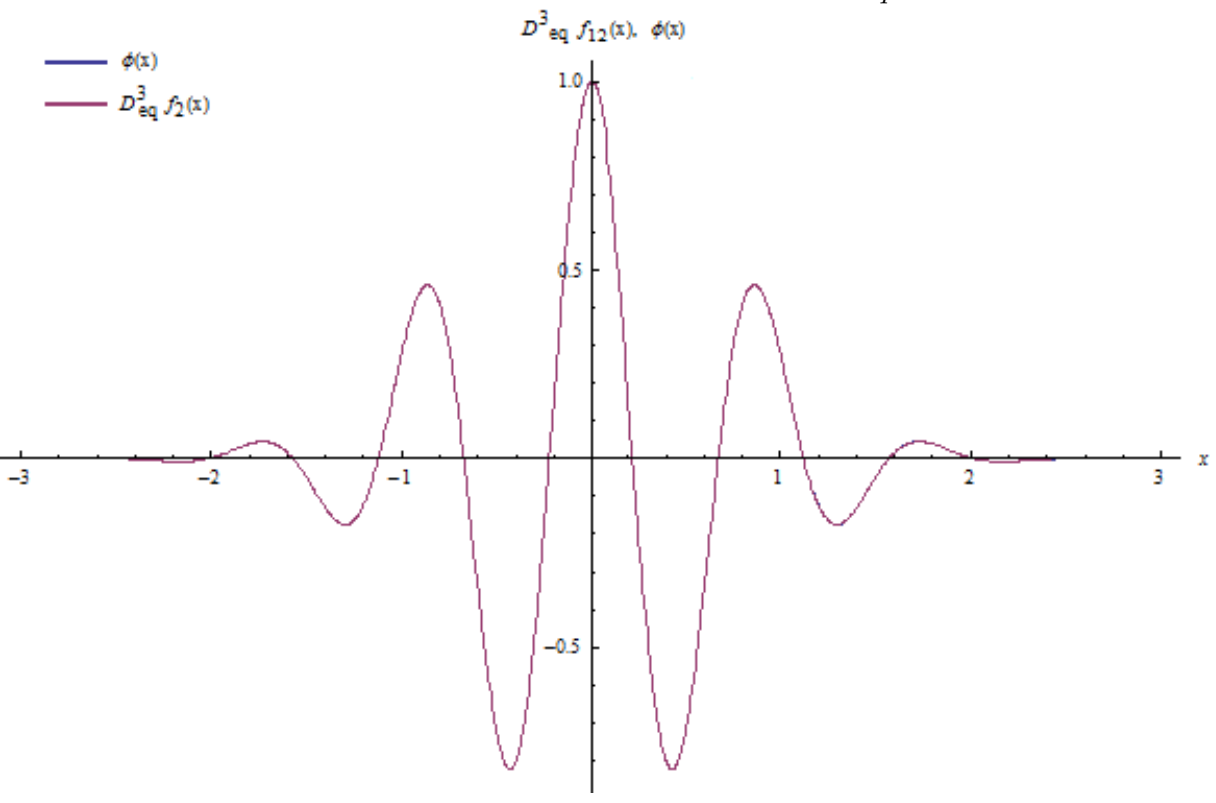

Figura 23: Gráfico do resto quadrático de $\mathcal{D}_{e q}^{3} f_{12}(x)$

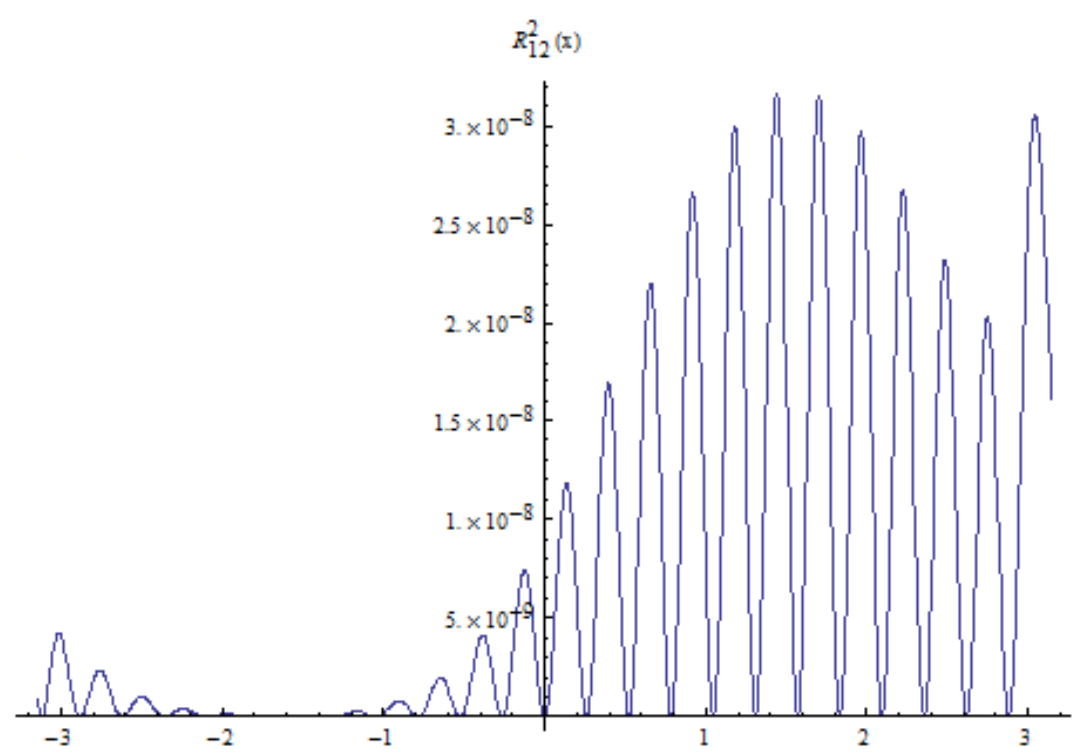


representa um resultado bastante satisfatório.

Também é interessante notar que, neste caso específico, o resto não cresce simetricamente nos extremos do intervalo, como normalmente acontece na expansão de funções periódicas. Uma possível razão para essa assimetria pode estar relacionada à propagação de erros no processo de derivação, mas isso constitui apenas uma hipótese, exigindo investigação posterior.

É preciso esclarecer que a precisão depende não apenas do número de termos, mas também da extensão do período da função. Em geral, quanto maior o período para dadas condições iniciais ou de contorno, tanto maior será o número de termos necessários no desenvolvimento para se conseguir uma desejada precisão.

Outra observação importante diz respeito às condições de contorno ou iniciais. Embora o método funcione muito bem para certas condições de contorno ou iniciais, pode falhar completamente para outros valores para os quais a equação parece não apresentar solução. Esse aspecto será bem explorado na próxima seção. Além disso, a mudança de período acarreta modificações radicais na validade da solução. Uma solução válida para um período pode não valer para outros, se não houver uma redefinição das condições de contorno e uma escolha apropriada do conjunto de pontos.

Em resumo, a existência de soluções para uma dada equação através do método aqui apresentado, além do número de termos no desenvolvimento, depende tanto das condições

de contorno (ou iniciais) quanto da definição do período, além da adequada escolha dos pontos nodais.

Nesse sentido, averiguamos a possibilidade de aprimorar a eficácia do método pela inserção de outros fatores, como a redefinição dos intervalos dos pontos de eleição para os nodos de Chebyshev e a introdução prática das séries de Fourier associadas, além do método NBV/NIV, que será exemplificado adiante.

Quanto à solução com condições iniciais, o procedimento é basicamente o mesmo que nas condições de contorno e, portanto, não vemos necessidade de exemplificá-lo separadamente.

\subsection{Implementação das Séries Associadas}

Agora, conforme a exposição feita no capítulo anterior, utilizaremos o método em sua forma alternativa, com as séries associadas, comparando os resultados entre as duas 
formulações. Acrescentamos a isso, a comparação entre as soluções obtidas das duas formas com os conjuntos de pontos a espaçamento constante e pelos nodos de Chebyshev.

Inicialmente, construímos os gráficos obtidos pelas expansões em série de Fourier original e associada para cada uma das funções abaixo:

1. Sign function:

$$
f(x)=\left\{\begin{array}{rl}
+1, & x>0 \\
0, & x=0 \\
-1, & x<0
\end{array}, \quad x \in[-L, L], \quad L=\frac{9 \pi}{7}, \quad \ell=13\right.
$$

2. Função identidade: $f(x)=x, \quad x \in[-L, L], \quad L=\frac{9 \pi}{7}, \quad \ell=13$;

3. Função quadrática: $f(x)=x^{2}, \quad x \in[-L, L], \quad L=\frac{9 \pi}{7}, \quad \ell=3$;

4. Função cúbica: $f(x)=x^{3}, \quad x \in[-L, L], \quad L=\frac{9 \pi}{7}, \quad \ell=16$;

5. Função quártica: $f(x)=x^{4}, \quad x \in[-L, L], \quad L=\frac{9 \pi}{7}, \quad \ell=7$;

6. Função exponencial: $f(x)=\exp x, \quad x \in[-L, L], \quad L=\frac{8 \pi}{7}, \quad \ell=12$;

7. Mexican Hat Wavelet:

$$
f(x)=\left(1-2 x^{2}\right) \exp \left(-x^{2}\right), \quad x \in[-L, L], \quad L=\frac{10 \pi}{7}, \quad \ell=5
$$

8. Morlet Wavelet:

$$
f(x)=\cos (\pi x \sqrt{2 / \ln 2}) \exp \left(-x^{2}\right), \quad x \in[-L, L], \quad L=\frac{13 \pi}{7}, \quad \ell=12
$$

A seguir, são mostrados os gráficos de todas as funções listadas acima, sendo que o gráfico em preto reproduz a função original sem expansão, enquanto a linha vermelha representa a série de Fourier e a azul, a série associada. Algumas das figuras apresentam, além do gráfico no período total, também um gráfico menor na restrição de período da série associada, no qual se pode observar melhor o comportamento de ambas as funções e o detalhe dos pontos de junção.

A função de expansão híbrida consiste, como já dissemos, na junção entre a série associada na restrição de período $\left[x_{e}, x_{d}\right]$ e a de Fourier original fora dele,i.e., $\left[-L, x_{e}\right) \cup$ $\left(x_{d}, L\right]$. Na verdade, os pontos de junção $x_{e}$ e $x_{d}$ pertencem ao interior do intervalo de restrição e são calculados pelas intersecções das funções aproximadas pelas duas séries mais próximas às bordas da restrição. 
Como não foi efetuado aqui o alisamento das junções, podemos notar, em alguns gráficos, os "bicos" correspondentes aos pontos não-deriváveis da função híbrida.

Também é exposta uma tabela dos erros integrais das duas séries, original e associada, na restrição de período. Esse erro é definido, para a função em série $f_{s}(x)$, como

$$
\epsilon_{c}^{2}\left(f_{s}\right):=\int_{x_{e}}^{x_{d}}\left|f(x)-f_{s}(x)\right|^{2} d x
$$

mesma definição que a do erro quadrático no período $\epsilon_{T}^{2}$, na qual, entretanto, os extremos de integração vão de $-L$ a $L$.

Figura 24: Séries de Fourier original e associada da função sinal

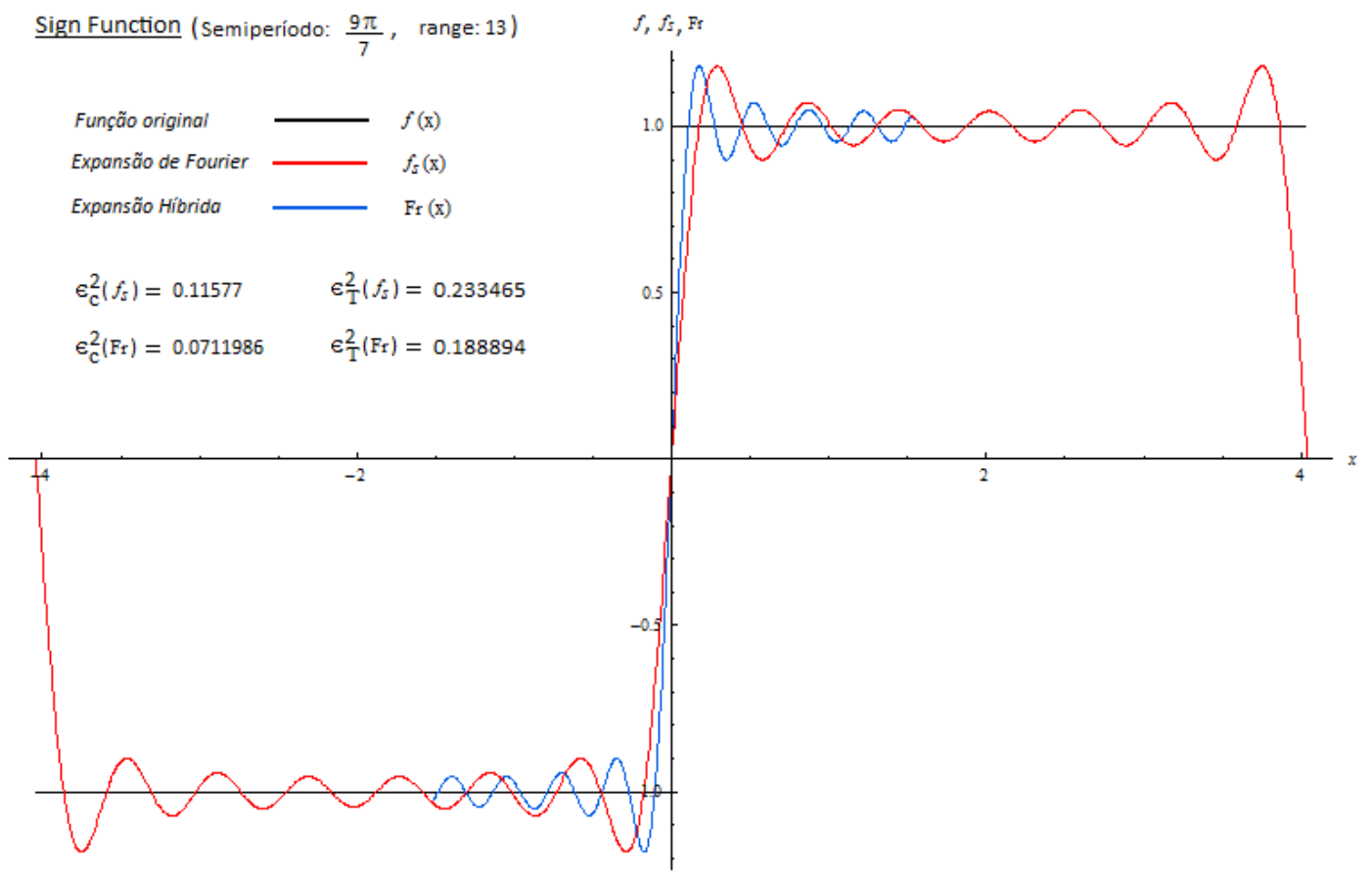

Tabela 8: Erros quadráticos das expansões SF/SA de algumas funções nas restrições correspondentes

\begin{tabular}{|c||c|c|}
\hline Função & $\epsilon_{c}^{2}(S F)$ & $\epsilon_{c}^{2}(S A)$ \\
\hline \hline Sinal & 0.11577 & 0.00711986 \\
\hline Identidade & 0.0194534 & 0.0117219 \\
\hline Quadrática & 0.230418 & 0.0649601 \\
\hline Cúbica & 4.75659 & 0.787174 \\
\hline Quártica & 11.8574 & 0.846174 \\
\hline Exponencial & 0.584564 & 0.159626 \\
\hline Mexican Hat & $5.01441 \times 10^{-3}$ & $9.31469 \times 10^{-6}$ \\
\hline Morlet & $3.04926 \times 10^{-2}$ & $4.96976 \times 10^{-3}$ \\
\hline
\end{tabular}


Figura 25: Séries de Fourier original e associada da função identidade

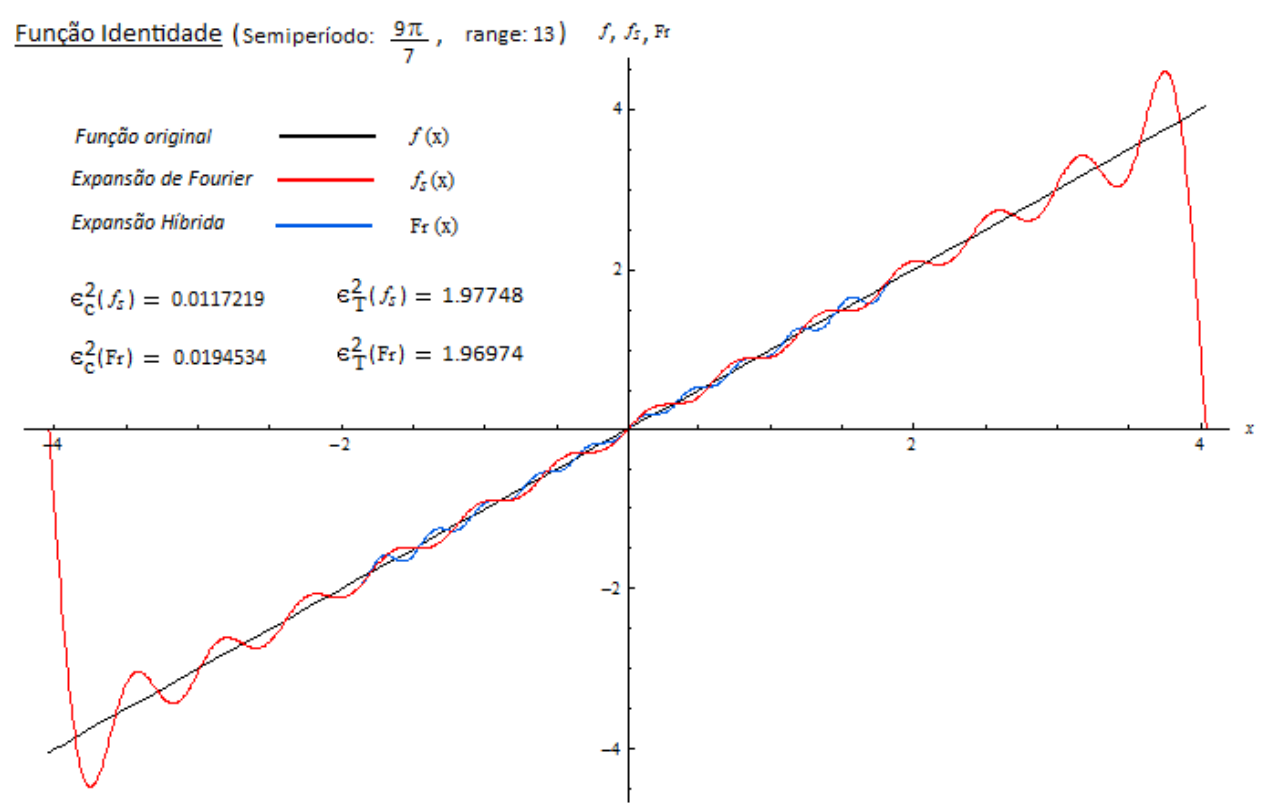

Comentários Os erros quadráticos totais das funções $F_{R}(x)$ e $f_{s}(x)$, que são uma forma de mensuração do afastamento em relação à função original, onde $f_{s}(x)$ é a expansão de Fourier e $F_{R}(x)$ é a função híbrida, foram calculados em todos os casos e os resultados obtidos, indicados nos gráficos por $\epsilon_{T}^{2}$, foram unanimemente mais precisos para a função $F_{R}(x)$.

Nos dois primeiros casos, por exemplo, a função sinal apresentou $\epsilon_{T}^{2}\left(F_{R}\right) \cong 0.18889 \mathrm{e}$ $\epsilon_{T}^{2}\left(f_{s}\right) \cong 0.23347$ e a função identidade: $\epsilon_{T}^{2}\left(F_{R}\right) \cong 1.9697$ e $\epsilon_{T}^{2}\left(f_{s}\right) \cong 1.9775$, mostrando ligeira vantagem de precisão para a função híbrida. Nos casos seguintes, essa maior precisão da função $F_{R}(x)$ é bastante significativa e pode ser vista diretamente pelo gráfico, sendo o gráfico de $f_{s}(x)$ representado pela curva vermelha e o de $F_{R}(x)$, pela curva azul.

Para alguns casos, como os das funções cúbica e quártica, a diferença entre os dois erros é extremamente acentuada, com uma vantagem de precisão notável para a série associada. Na Mexican Hat wavelet também ocorre um aumento de precisão significativo com o uso da série associada em vez da original, por volta de mil vezes, já que a precisão passa da ordem de $10^{-3}$ para $10^{-6}$.

Em resumo, o importante a ressaltar-se aqui é que, em todas as funções estudadas, a aproximação proposta por meio das séries associadas foi bem melhor que a obtida pela série de Fourier original, ilustrando na prática a demonstração formal feita no capítulo anterior.

Um outro ponto diz respeito à convergência mais rápida da série associada na res- 
Figura 26: Séries de Fourier original e associada da função quadrática

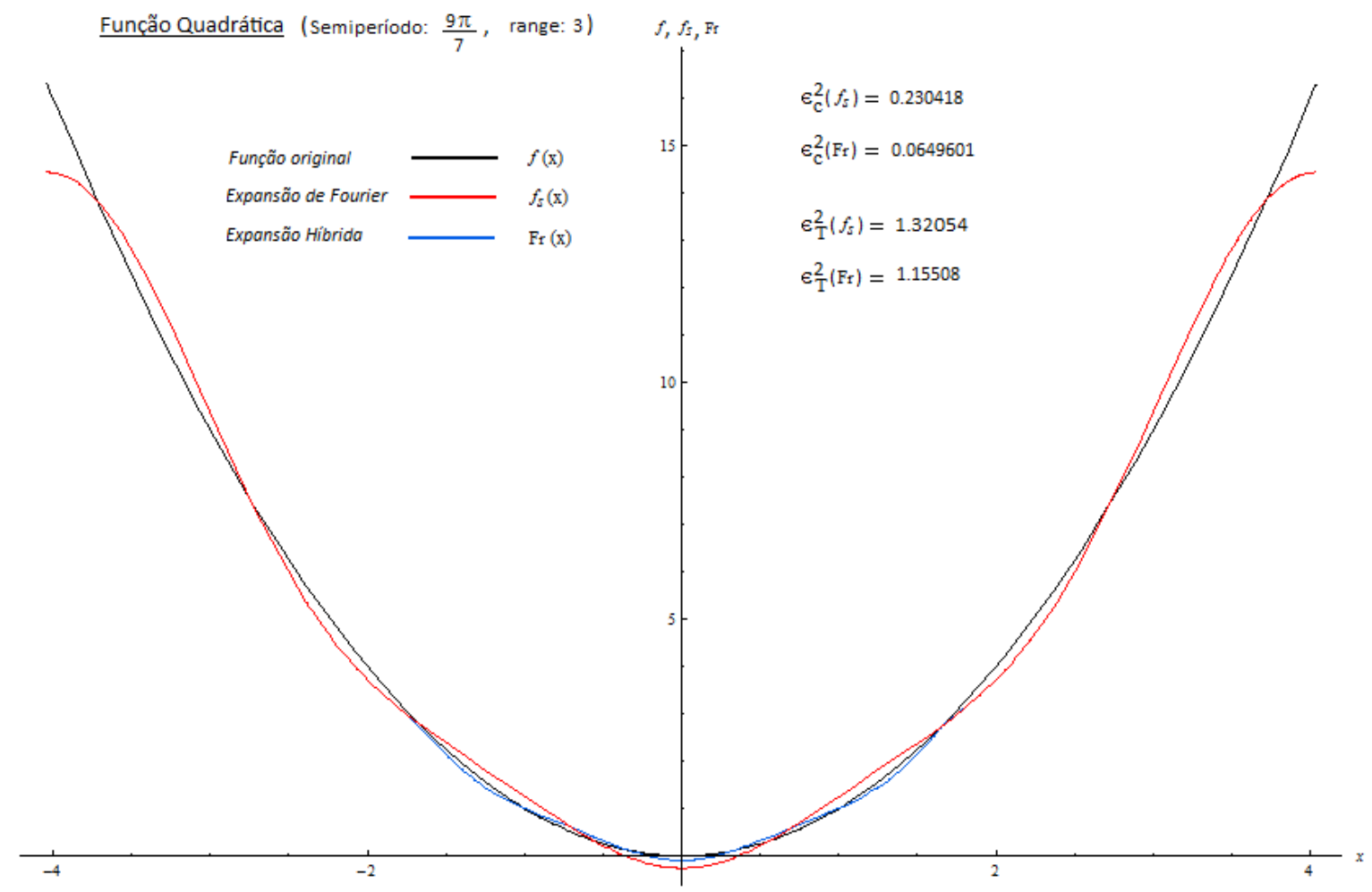

Detalhe da Restricão de Período

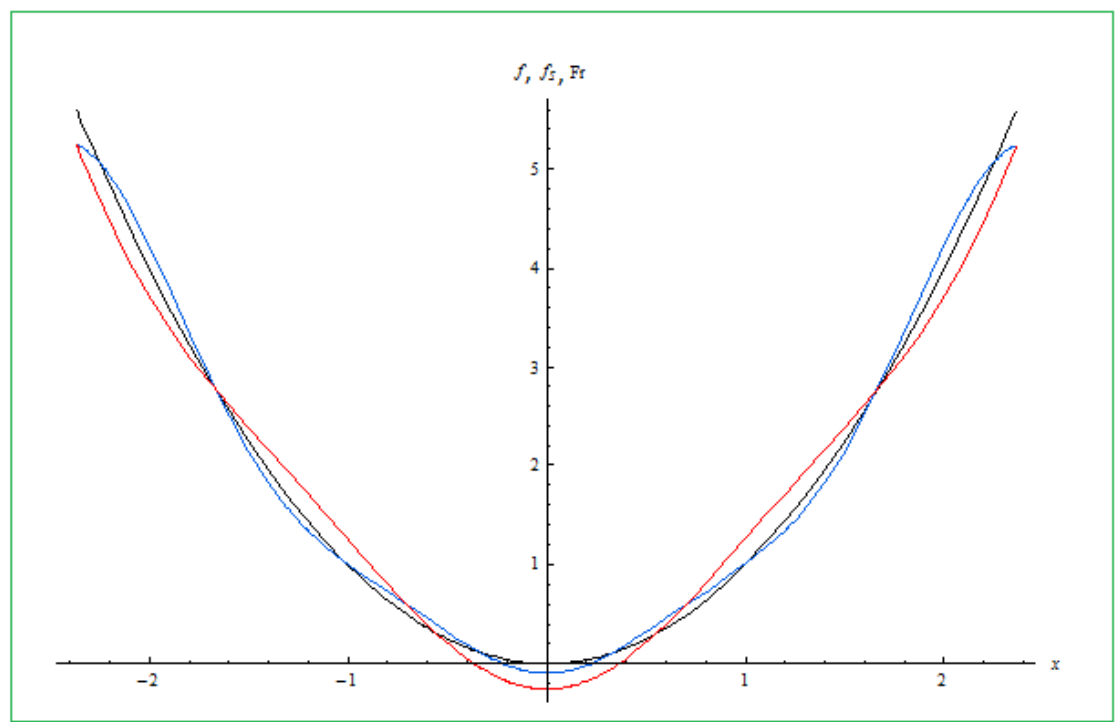


Figura 27: Séries de Fourier original e associada da função cúbica

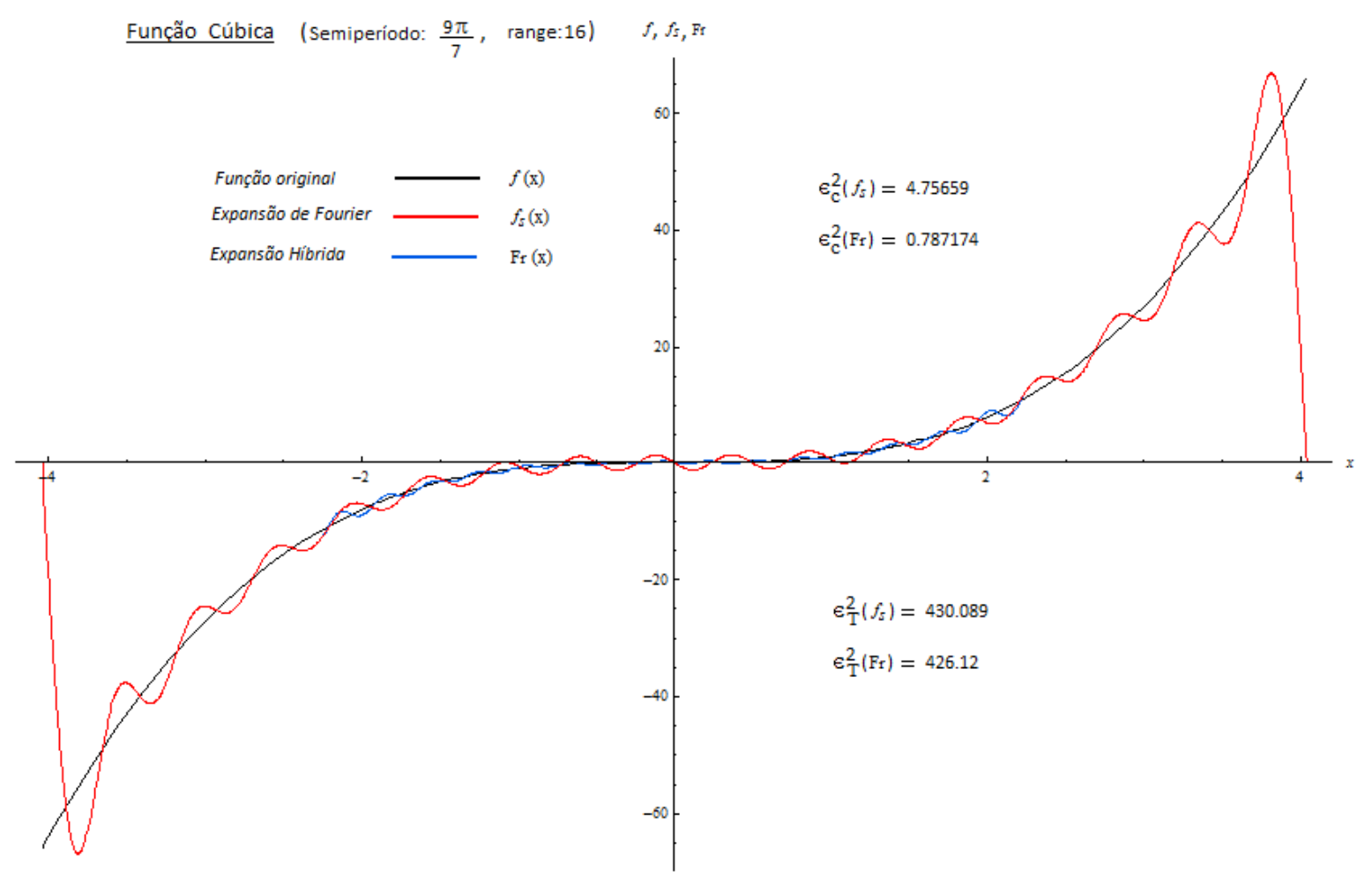

Detalhe da Restricão de Período

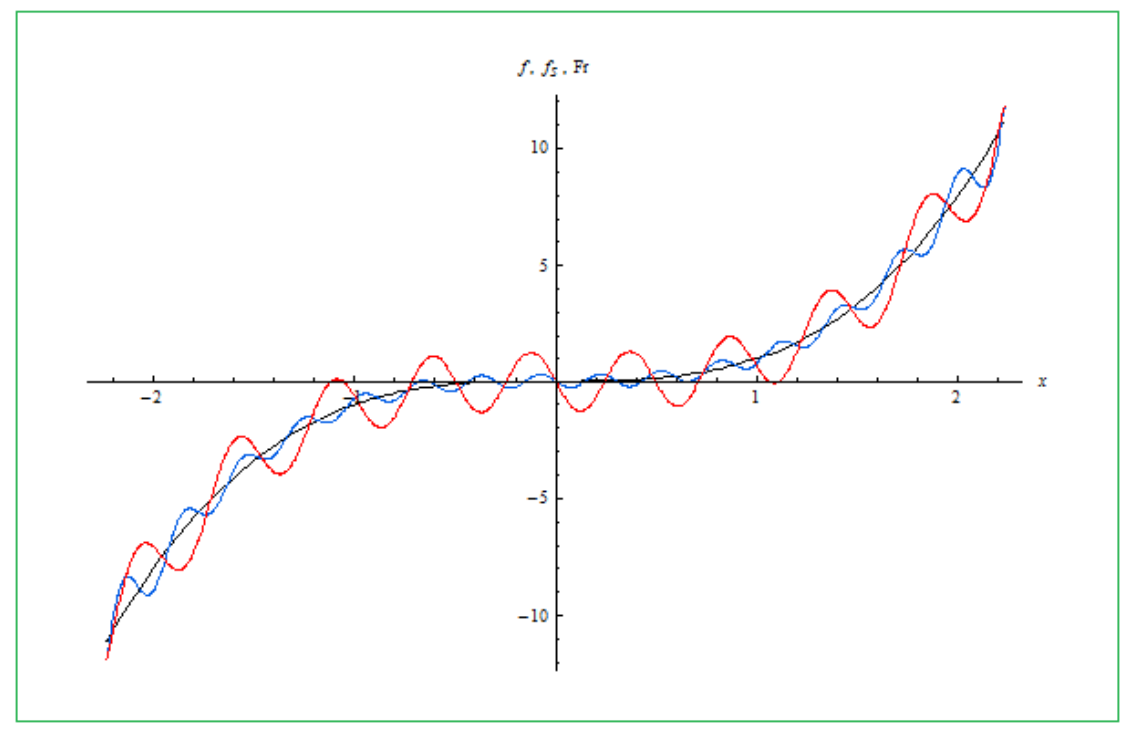


Figura 28: Séries de Fourier original e associada da função quártica

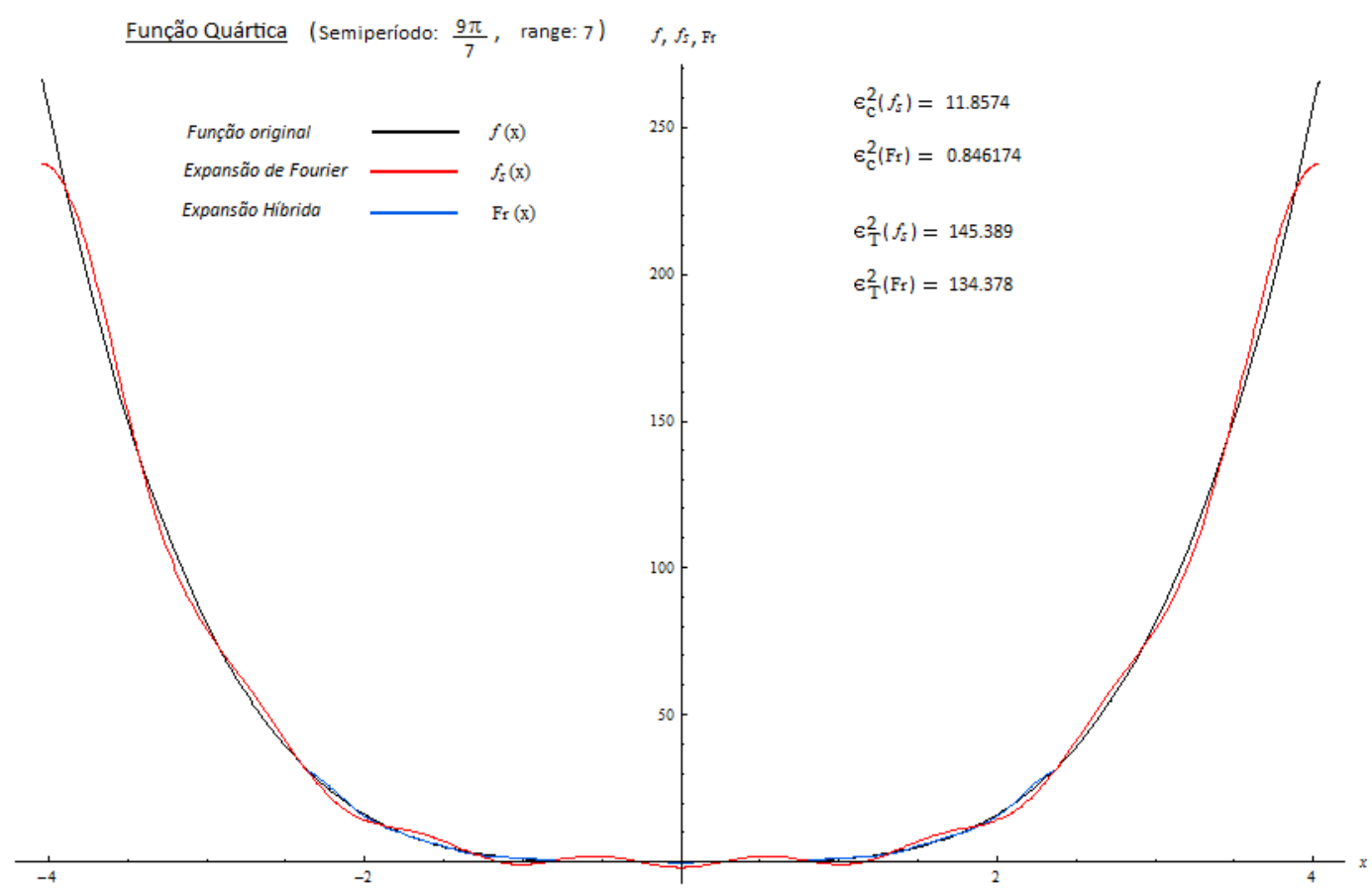

Detalhe da Restrição de Período

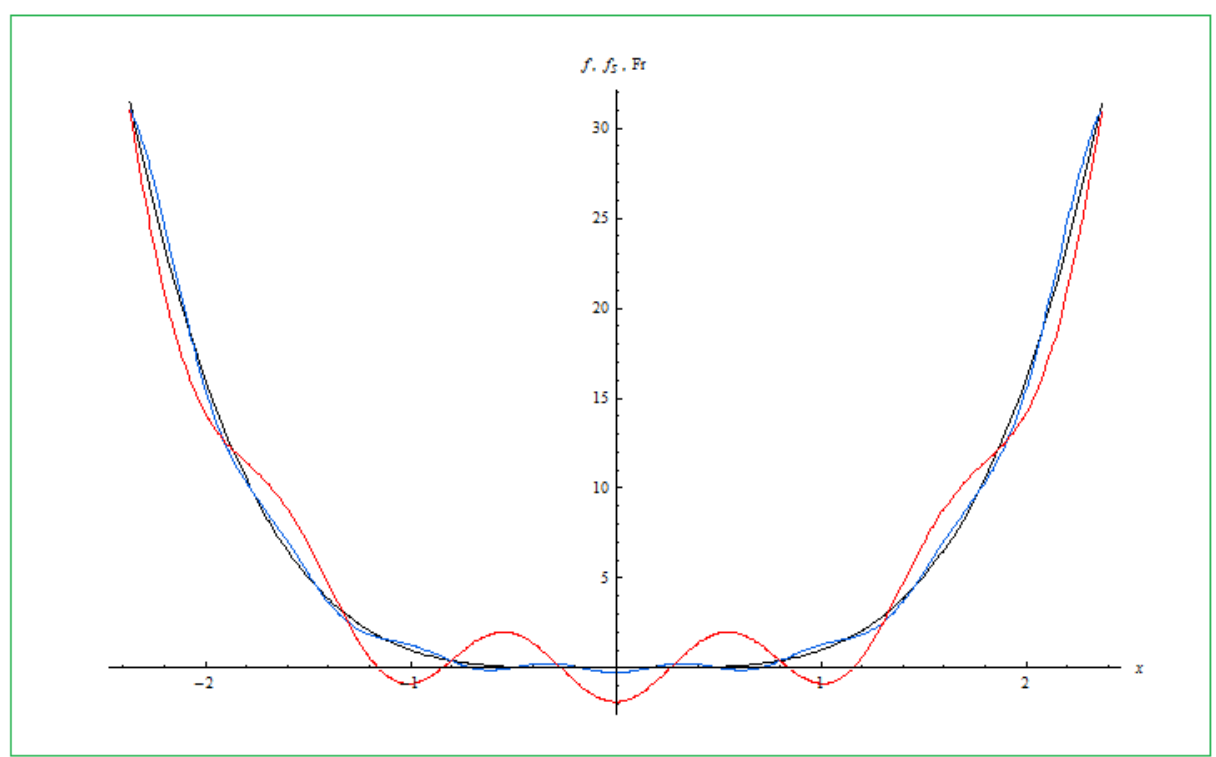


Figura 29: Séries de Fourier original e associada da função exponencial Função Exponencial (Semiperiodo: $\frac{8 \pi}{7}$, range:12) $f, f_{s}, \mathrm{Fr}_{\mathrm{f}}$

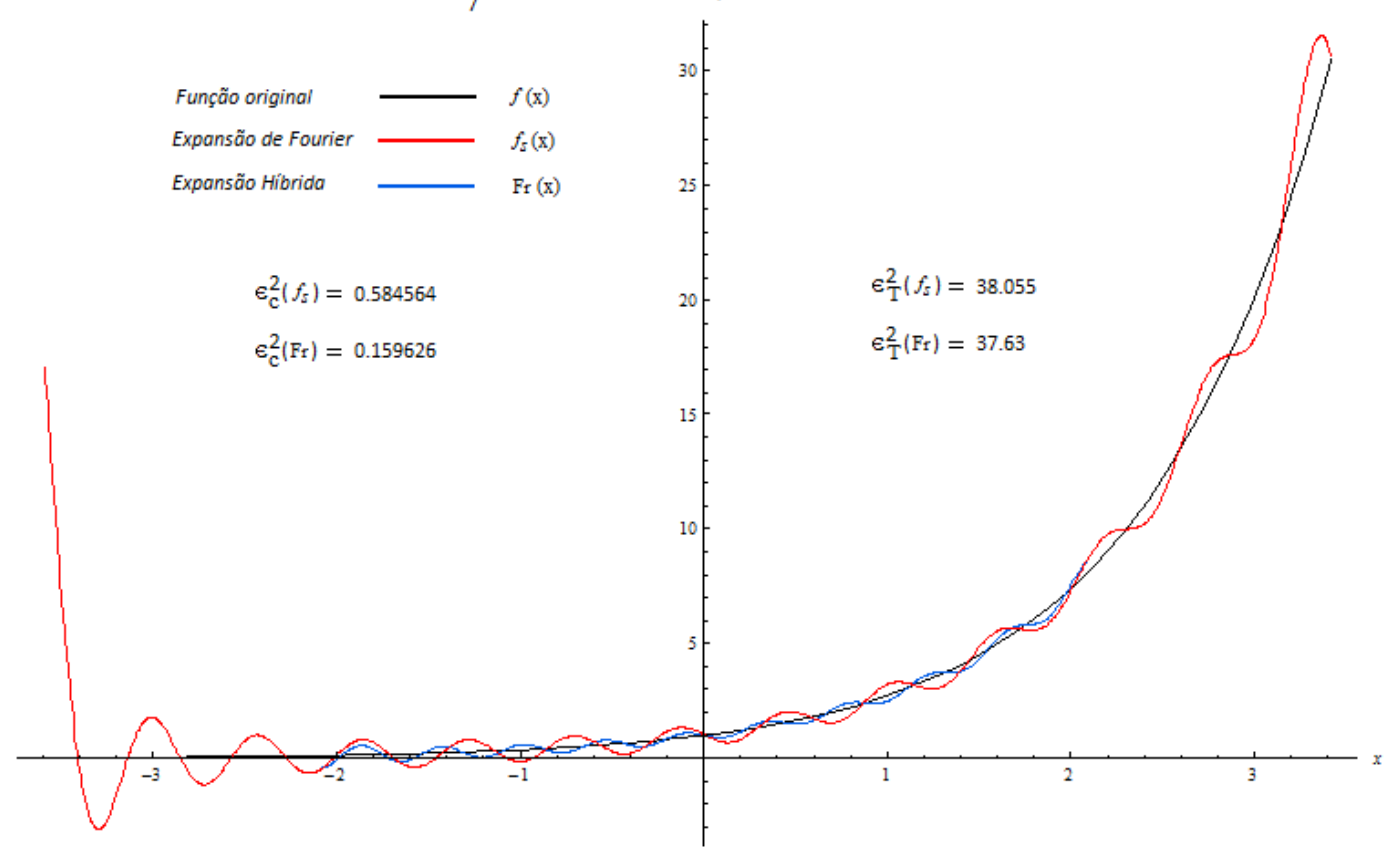

Detalhe da Restrição de Periodo




Figura 30: Séries de Fourier original e associada da wavelet Mexican Hat Mexican Hat - wavelet (Semiperíodo: $\frac{10 \pi}{7}$, range: 5 ) $f, f_{s}, \mathrm{Fr}_{\mathrm{s}}$ $y(x)=\left(1-2 x^{2}\right) \exp \left(-x^{2}\right)$

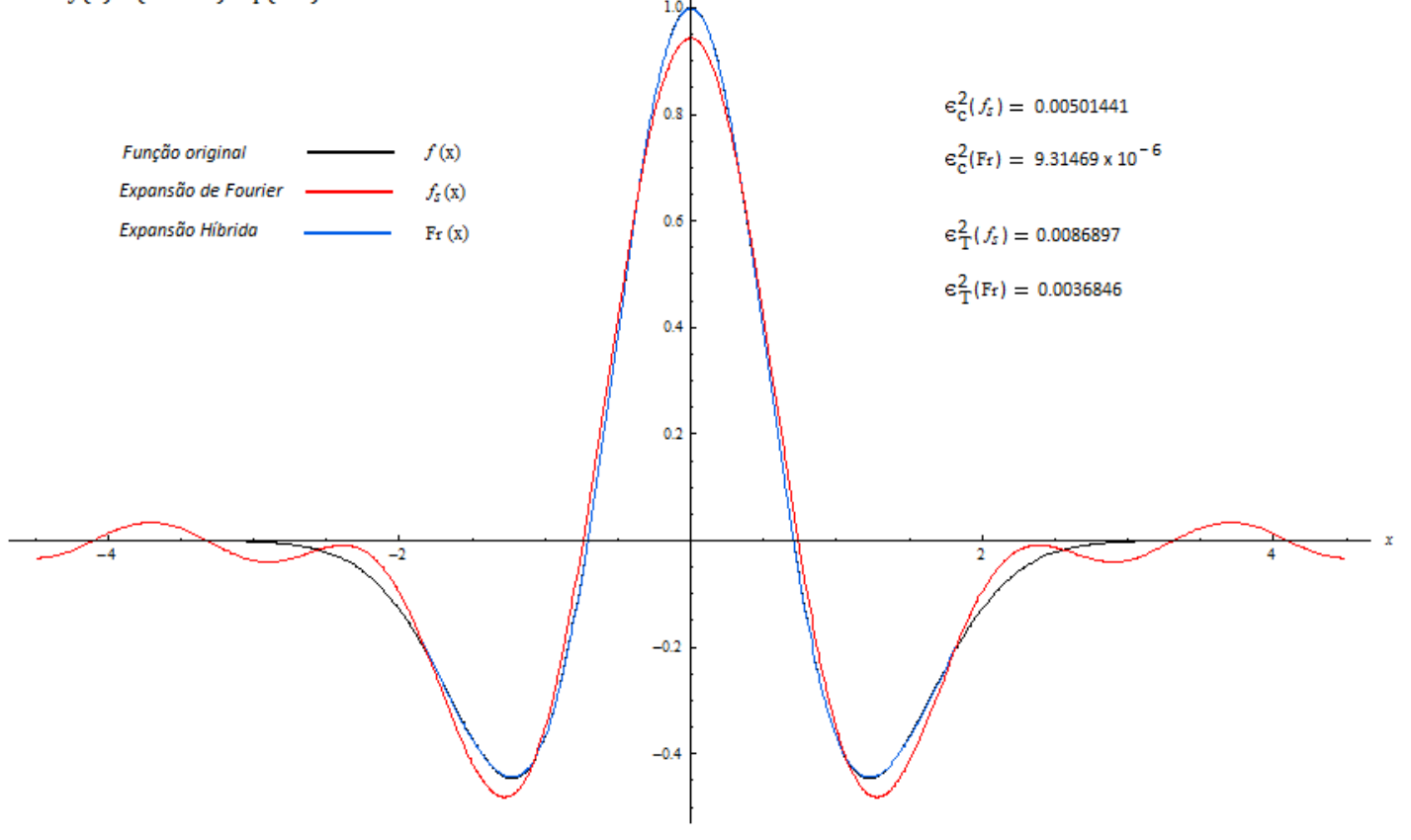

Figura 31: Séries de Fourier original e associada da wavelet de Morlet na restrição Morlet Wavelet (Semiperíodo: $\frac{13 \pi}{7}$, range: 12 ) $y(x)=\cos \left(\pi x \sqrt{\frac{2}{\ln 2}}\right) e^{-x^{2}}$

Função original — $f(\mathrm{x})$

Expansão de Fourier $\longrightarrow f_{s}(\mathrm{x})$

Expansão Híbrida $\longrightarrow \operatorname{Fr}(\mathrm{x})$

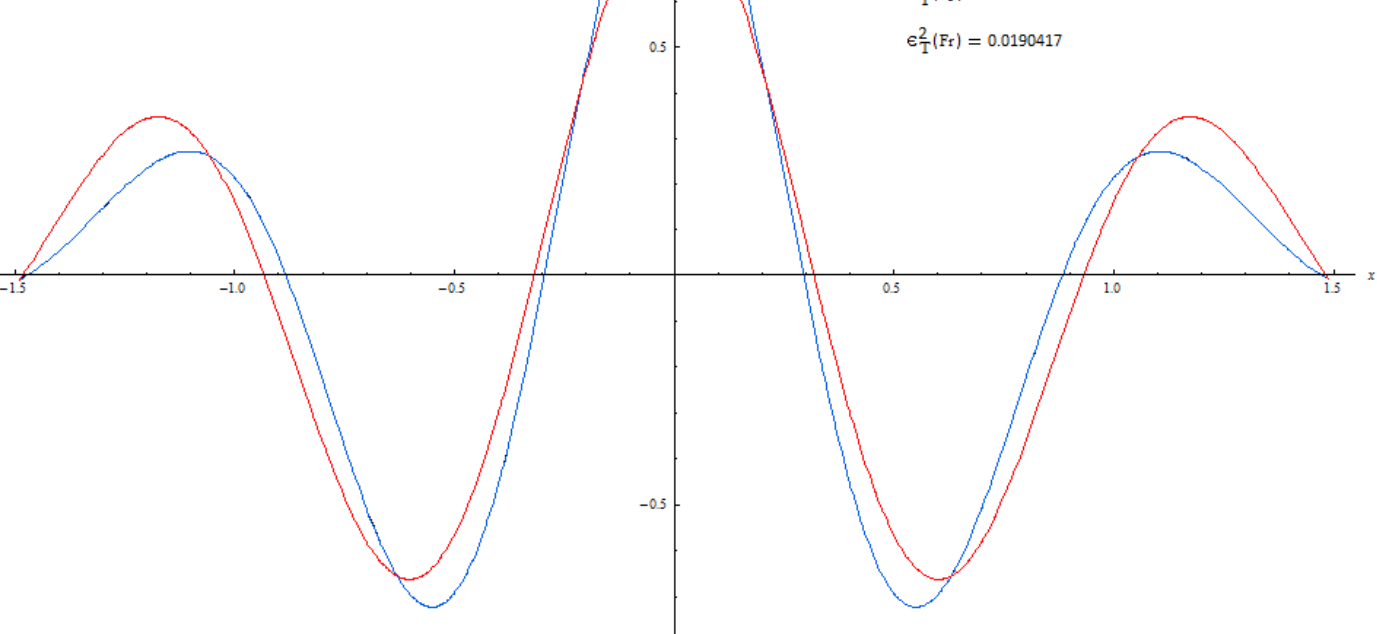


trição de período do que a devida à série de Fourier original. Isso significa que a série associada tende mais rapidamente à função original do que a série de Fourier. Esse fato é ilustrado pela figura (32), na qual aparecem os gráficos da wavelet de Morlet original e as duas expansões, em desenvolvimentos com vários ranges diferentes, com variação unitária, desde $\ell=4$ até $\ell=11$ para um semiperíodo $L=\frac{10 \pi}{7}$.

Como podemos ver, já com $\ell=6$ a série associada se aproxima da função original de modo quase perfeito, enquanto a série de Fourier só mostra um resultado comparável ao obtido no desenvolvimento associado com $\ell=5$ ao atingir o range $\ell=11$, o que revela uma convergência muito mais rápida da série associada. Logo, fica bem ilustrada a vantagem oferecida pelo uso da série associada para períodos inferiores a $2 \pi$.

\subsubsection{Discussão sobre a ausência de Parâmetros Impositivos}

À primeira vista, pode parecer bastante estranha a possibilidade de solucionar uma equação diferencial sem impor nenhuma condição que assegure uma função definida. Naturalmente, no método usual, o processo de integração fornece constantes arbitrárias que devem ser especificadas através da imposição de certas condições às quais a equação deva obedecer. É o que estamos denominando aqui de parâmetros impositivos, quer sejam condições iniciais, no caso de IVPs, quer sejam condições de contorno, para BVPs. No método de modelagem NBV/NIV, contudo, isso não se faz necessário. De fato, o processo de resolução proposto nessa tese, por meio do formalismo bra-ket, possibilita até mesmo soluções de caráter híbrido, como já foi inclusive efetuado em um exemplo anterior. Isto significa não apenas que se pode optar por impor condições, caso isso seja conveniente, ou por não fazê-lo, mas também que podemos, de acordo com a necessidade de cada caso, escolher o número de condições que quisermos.

Para ilustrar de forma mais nítida o que significa isto e por que esse procedimento é possível, vamos utilizar o método em uma EDO extremamente simples, de solução conhecida e muito fácil de interpretar. Com isso, esperamos esclarecer esse ponto tão fundamental.

Consideremos a equação

$$
\frac{d y}{d x}=e^{x}
$$

cuja solução é dada pela própria exponencial mais uma constante arbitrária: $y(x)=e^{x}+c$. Então, para especificar a solução exata, basta eleger uma condição inicial como, por exemplo, $y(0)=0$, já que a EDO é de primeira ordem. Nesse caso, a constante fica especificada por $c=-1$ e a solução exata é dada por $y(x)=e^{x}-1$. Se o problema for de 
Figura 32: Ilustração da convergência mais rápida da série associada Expansão da wavelet de Morlet de semiperíodo $L=10 \pi / 7$
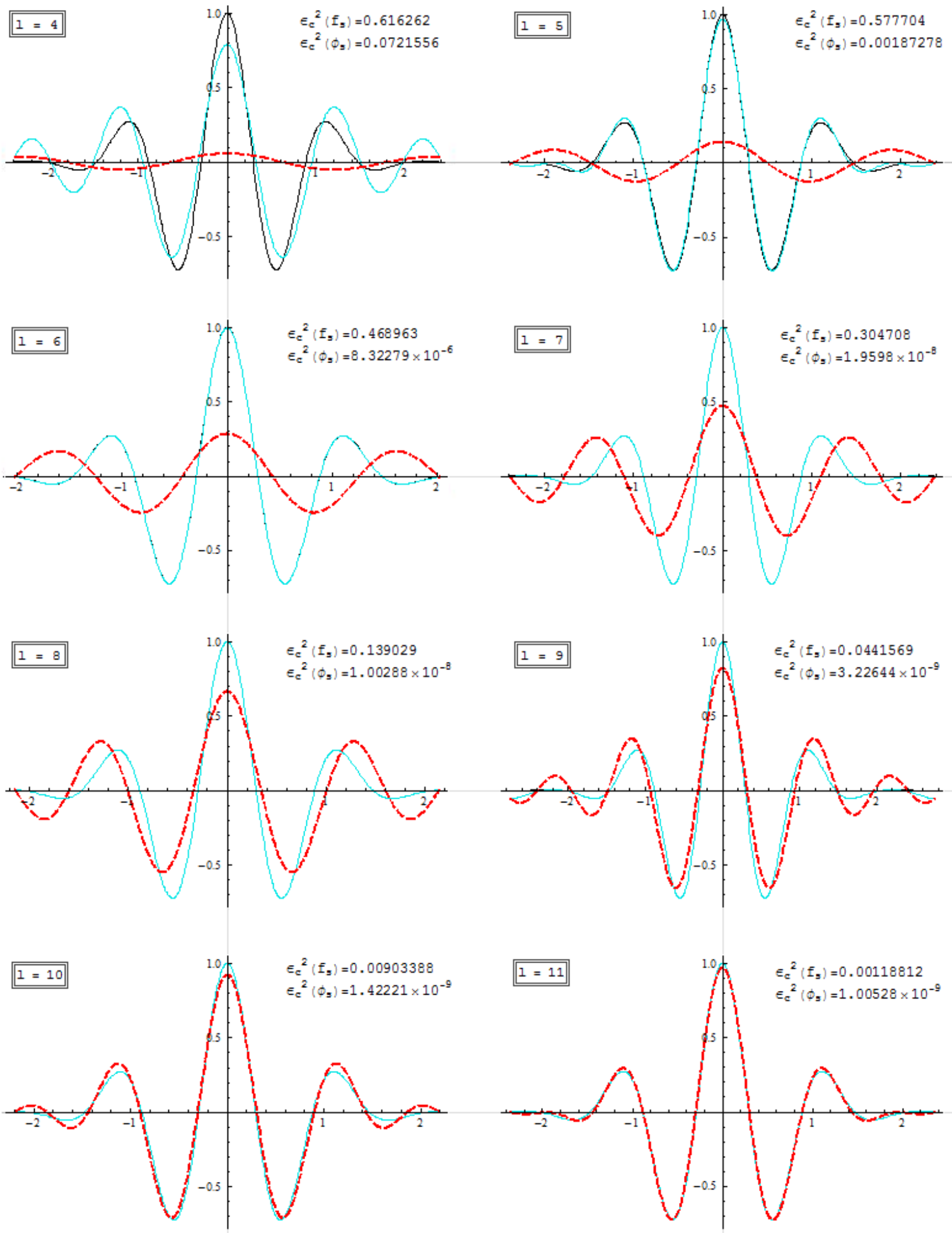
valores de contorno, só uma condição pode ser imposta, pois só há uma constante livre a ser determinada.

No método de modelagem, no entanto, a situação é diferente. A exponencial aqui faz o papel de termo independente $\phi(x)$, enquanto o lado esquerdo da EDO apresenta as funções coeficientes $\mu_{1}(x)=1$ e $\mu_{0}(x)=0$. Há tantos parâmetros quantos são os pontos de eleição do intervalo. Evidentemente, não se pode vinculá-los todos a condições, pois isso impossibilitaria a resolução, a menos que tais condições fossem escolhidas de forma a se adaptarem à EDO, o que, em geral, não pode ser feito, porque não conhecemos a expressão explícita da solução. Apenas em casos específicos, como esse da exponencial que estamos analisando, tal recurso pode ser tentado, a título de estudo, porque a solução é previamente conhecida.

Vamos aplicar o método, inicialmente, sem qualquer condição, para investigar com o que se parece a solução livre. É de se esperar que seja uma exponencial exatamente igual à função $\phi$, sem constantes adicionais, já que nada foi imposto de antemão. Utilizando o algoritmo NBV de resolução no Mathematica ${ }^{\circledR} 6.0$, obtivemos, de fato, o que seria esperado, mas apenas em situações específicas. Vamos detalhar o que ocorre em cada caso estudado:

1. Séries de Fourier + espaçamento constante : a EDO não tem solução;

2. Séries de Fourier + nodos de Chebyshev : a EDO apresenta solução falsa, com amplitude explosiva;

3. Séries de Fourier + nodos de Fourier : a EDO apresenta solução oscilante com precisão relativamente baixa;

4. Séries Associadas + espaçamento constante : a EDO apresenta solução com boa precisão;

5. Séries Associadas + nodos de Chebyshev : a EDO apresenta solução com precisão ainda melhor que a anterior;

6. Séries Associadas + nodos de Fourier : a EDO apresenta solução com altíssima precisão nas vizinhanças do centro do intervalo;

Na figura (33) aparecem os gráficos da solução $f_{15}(x)$ e da sobreposição entre ed $(x)$ e $\phi(x)$ no caso 3 acima, para um semiperíodo $L=\frac{5 \pi}{7}$ e range 15 . Naturalmente, como a EDO fornece a própria função $\phi(x)$, a solução encontrada e $\mathcal{D}_{e q} f_{15}(x)$ terão a mesma 
forma. Em seguida, na figura (34), o gráfico do resto quadrático $\mathcal{R}_{15}^{2}$ para esse mesmo caso. O erro quadrático total é $\mathcal{E}_{15}^{2}=1.3808$.

Figura 33: Solução NBV da EDO exponencial do caso 3 com $\ell=15, L=\frac{5 \pi}{7}$
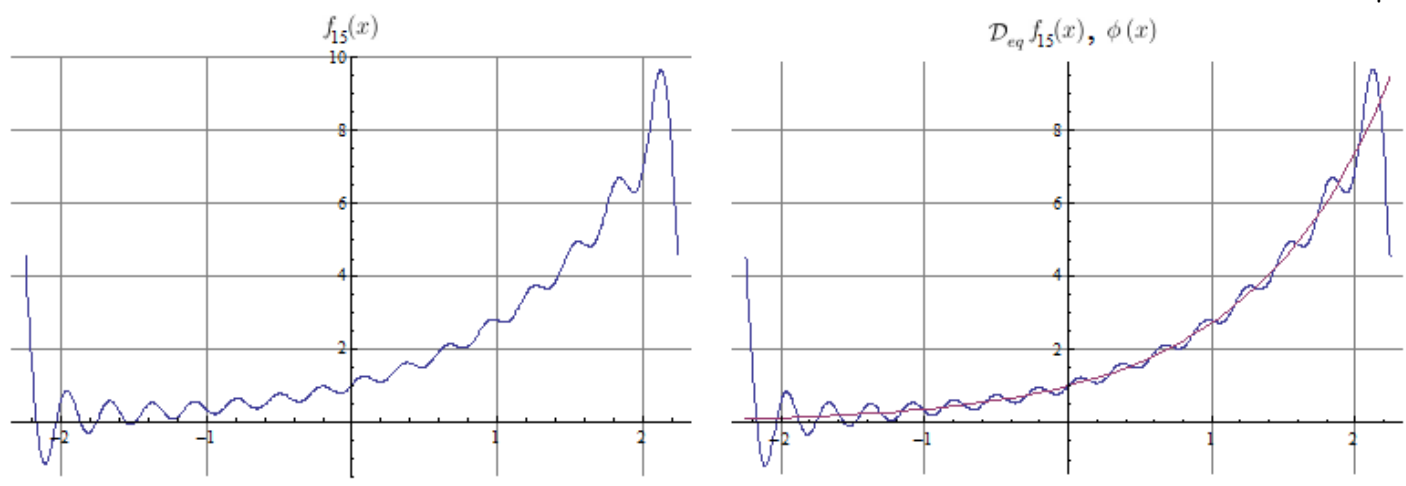

Figura 34: Resto quadrático da solução NBV para o caso 3 com range 15 e $L=\frac{5 \pi}{7}$

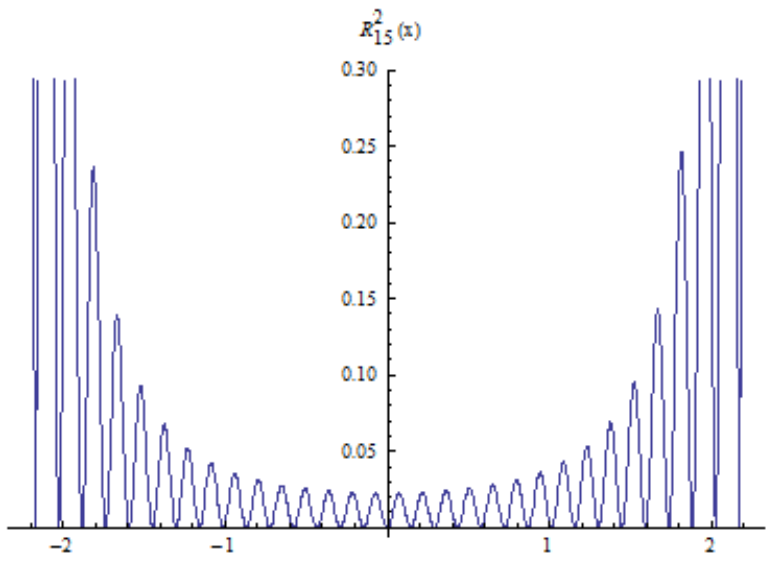

Agora, estudando os casos em que a série associada é utilizada em lugar da de Fourier, constatamos que as soluções tornam-se não apenas satisfatórias, mas muito aprimoradas. Determinamos as soluções e calculamos sua precisão para os casos 4, 5 e 6, com semiperíodo $L=\frac{5 \pi}{7}$, primeiramente para $\ell=2 \mathrm{e}$, em seguida, para $\ell=3$ e $\ell=15$.

Nas figuras (35) e (36) mostram-se os gráficos das sobreposições entre $\mathcal{D}_{e q} f_{\ell}(x)$ e $\phi(x)$ calculados, respectivamente, com espaçamento constante (EC), nodos de Chebyshev (NC) e nodos de Fourier (NF), para os dois primeiros ranges, $\ell=2$ e $\ell=3$.

A seguir, nas figuras (37), (38) e (39), podemos ver os restos quadráticos de cada um dos casos, respectivamente, acompanhados dos valores dos erros totais em três situações diferentes: no período todo $(T)$, na chamada "Área de Segurança", equivalente a $75 \%$ do período $\left(\frac{3 T}{4}\right)$ e no intervalo central correspondente a meio-período $\left(\frac{T}{2}\right)$. Percebe-se pela análise gráfica dos períodos mais restritos que, em todos os casos, o erro próximo à área central é minimizado com o uso dos nodos de Fourier. 
Figura 35: Gráficos de $\mathcal{D}_{e q} f_{2}(x)$ e $e^{x}$ para séries associadas com EC, NC e NF, $L=\frac{5 \pi}{7}$
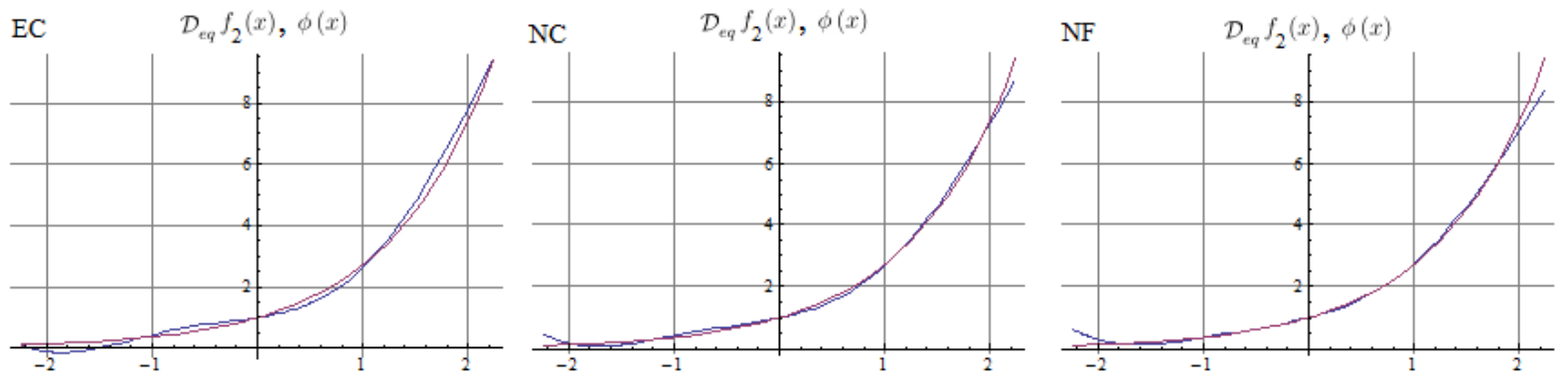

Figura 36: Gráficos de $\mathcal{D}_{e q} f_{3}(x)$ e $e^{x}$ para séries associadas com EC, NC e NF, $L=\frac{5 \pi}{7}$
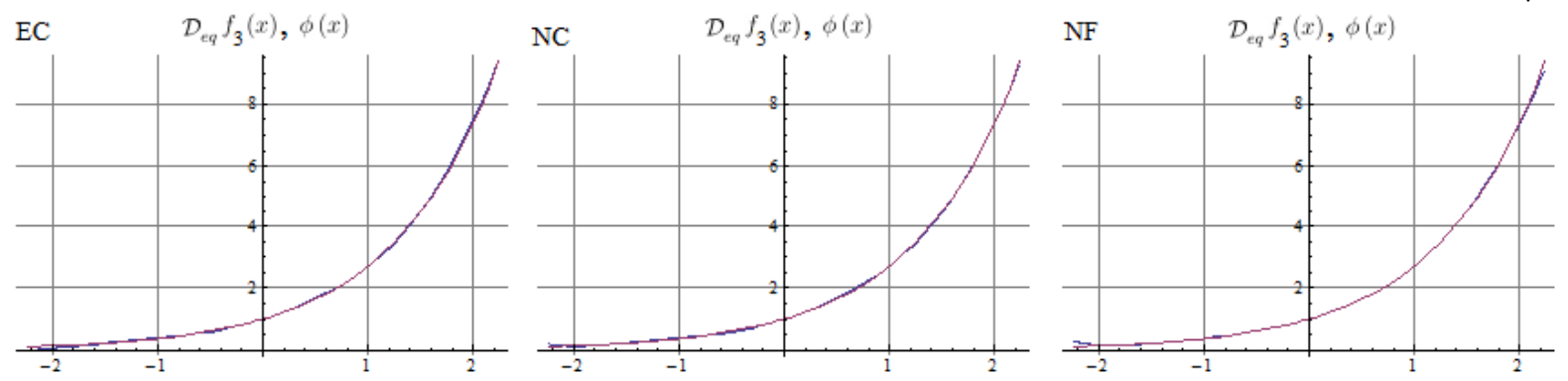

Figura 37: Restos quadráticos de $\mathcal{D}_{e q} f_{2}(x)$ para SA com EC, NC e NF, $L=\frac{5 \pi}{7}$
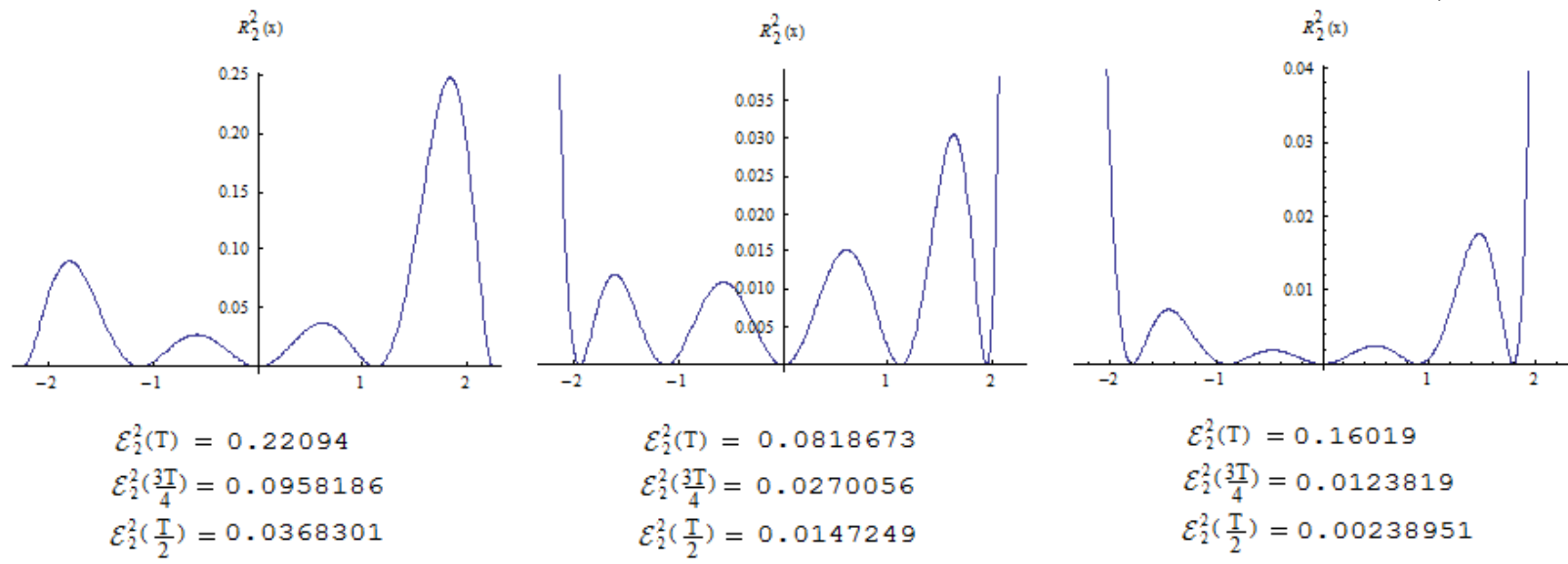

Tabela 9: Erros quadráticos da solução NBV-SA para a exponencial com diferentes espaçamentos e range $\ell=2$

\begin{tabular}{|c||c|c|c|}
\hline $\mathcal{E}_{2}^{2}$ & $\mathrm{EC}$ & $\mathrm{NC}$ & $\mathrm{NF}$ \\
\hline \hline$L$ & 0.22094 & 0.0818673 & 0.16019 \\
\hline $3 L / 4$ & 0.0958186 & 0.0270056 & 0.0123819 \\
\hline$L / 2$ & 0.0368301 & 0.0147249 & 0.00238951 \\
\hline
\end{tabular}


Figura 38: Restos quadráticos de $\mathcal{D}_{e q} f_{3}(x)$ para SA com EC, NC e NF, $L=\frac{5 \pi}{7}$
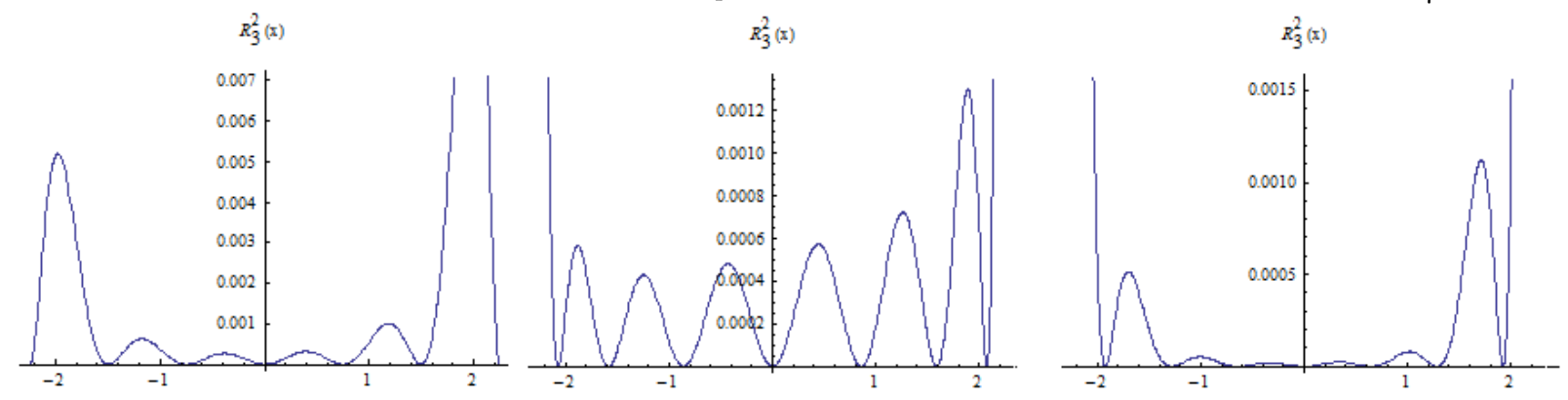

$\mathcal{E}_{3}^{2}(\mathrm{~T})=0.00702567$

$\mathcal{E}_{3}^{2}(\mathrm{~T})=0.00255385$

$\mathcal{E}_{3}^{2}(\mathrm{~T})=0.0106897$

$\mathcal{E}_{3}^{2}\left(\frac{3 \mathrm{~T}}{4}\right)=0.00102209$

$\mathcal{E}_{3}^{2}\left(\frac{3 \mathrm{~T}}{4}\right)=0.000889526$

$\mathcal{E}_{3}^{2}\left(\frac{3 \mathrm{~T}}{4}\right)=0.000307178$

$\mathcal{E}_{3}^{2}\left(\frac{T}{2}\right)=0.000453283$

$\mathcal{E}_{3}^{2}\left(\frac{T}{2}\right)=0.000540378$

$\mathcal{E}_{3}^{2}\left(\frac{T}{2}\right)=0.0000500459$

Figura 39: Restos quadráticos de $\mathcal{D}_{e q} f_{15}(x)$ para SA com EC, NC e NF, $L=\frac{5 \pi}{7}$


$$
\begin{aligned}
& \mathcal{E}_{15}^{2}(\mathrm{~T})=6.4826 \times 10^{-17} \\
& \mathcal{E}_{15}^{2}\left(\frac{3 \mathrm{~T}}{4}\right)=1.12084 \times 10^{-23} \\
& \mathcal{E}_{15}^{2}\left(\frac{\mathrm{T}}{2}\right)=6.67458 \times 10^{-27}
\end{aligned}
$$

$$
\begin{aligned}
& \mathcal{E}_{15}^{2}(\mathrm{~T})=5.78868 \times 10^{-21} \\
& \mathcal{E}_{15}^{2}\left(\frac{3 \mathrm{~T}}{4}\right)=5.61018 \times 10^{-21} \\
& \mathcal{E}_{15}^{2}\left(\frac{\mathrm{T}}{2}\right)=5.03923 \times 10^{-21}
\end{aligned}
$$

$\mathcal{E}_{15}^{2}(\mathrm{~T})=1.42267 \times 10^{-15}$

$\mathcal{E}_{15}^{2}\left(\frac{3 \mathrm{~T}}{4}\right)=8.62579 \times 10^{-24}$

$\mathcal{E}_{15}^{2}\left(\frac{T}{2}\right)=1.11581 \times 10^{-27}$

Tabela 10: Erros quadráticos da solução NBV-SA para a exponencial com diferentes espaçamentos e range $\ell=3$

\begin{tabular}{|c||c|c|c|}
\hline $\mathcal{E}_{2}^{2}$ & EC & NC & NF \\
\hline \hline$L$ & 0.00702567 & 0.00255385 & 0.0106897 \\
\hline $3 L / 4$ & 0.00102209 & 0.000889526 & 0.000307178 \\
\hline$L / 2$ & 0.000453283 & 0.000540378 & 0.0000500459 \\
\hline
\end{tabular}

Tabela 11: Erros quadráticos da solução NBV-SA para a exponencial com diferentes espaçamentos e range $\ell=15$

\begin{tabular}{|c||c|c|c|}
\hline $\mathcal{E}_{2}^{2}$ & $\mathrm{EC}$ & $\mathrm{NC}$ & $\mathrm{NF}$ \\
\hline \hline$L$ & $6.4826 \times 10^{-17}$ & $5.78868 \times 10^{-21}$ & $1.42267 \times 10^{-15}$ \\
\hline $3 L / 4$ & $1.12084 \times 10^{-23}$ & $5.61018 \times 10^{-21}$ & $8.62579 \times 10^{-24}$ \\
\hline$L / 2$ & $6.67458 \times 10^{-27}$ & $5.03923 \times 10^{-21}$ & $1.11581 \times 10^{-27}$ \\
\hline
\end{tabular}


Mas, voltando à solução híbrida, podemos aplicá-la com duas ou mais imposições, mesmo para essa simples EDO, como para os extremos da função, por exemplo, à maneira de condições de contorno. Nossa finalidade agora é, por conseguinte, comparar o método NBV com a solução híbrida associada à fixação de uma única condição, em um dos extremos, para compará-la com a situação já conhecida.

Comparação BVP-NBV A fim de analisar as mudanças na precisão com a introdução das condições de contorno e estudar o range de precisão ótima, construímos uma tabela com os valores do erro total absoluto $\left(\mathcal{E}_{a b s}\right)$ para cada range, de 1 até 15 , para duas situações distintas, quer dizer, estabelecendo uma condição de contorno na borda esquerda e mantendo livre a direita e, em seguida, alterando para a situação oposta, isto é, mantendo a esquerda livre e ficando uma condição de contorno à direita.

Como a função $\phi$ é exponencial e seus valores são conhecidos, introduzimos variações específicas diferentes nos extremos para estudar os efeitos. Sendo o valor da função na borda esquerda menor que o da direita, ou seja, $\phi(-L)=e^{-L}<\phi(L)=e^{L}$, estipulamos uma variação maior na esquerda $\left(\Delta y_{0}=90 \% y_{0}\right)$ que na direita $\left(\Delta y_{f}=10 \% y_{f}\right)$, i.e., $y_{0}=0.1 \phi(-L)$ e $y_{f}=0.9 \phi(L)$ e calculamos $\mathcal{E}_{a b s}$ em cada caso.

A definição do erro real total absoluto no período é

$$
\mathcal{E}_{a b s}:=\int_{-L}^{L}\left\|\Re\left\{\phi(x)-\phi_{s}(x)\right\}\right\| d x
$$

A seguir, a tabela com os resultados, para os cálculos feitos com o uso do método NBV-SA-NC.

Para o caso da condição de contorno à esquerda o range de otimização é 6, enquanto para o da condição à direita, o range vale 3. Isso significa que a solução híbrida para o caso de se fixar apenas uma condição de contorno à esquerda (e especificamente esta), apresenta a máxima precisão possível quanto $\ell=6$. Qualquer outro range tende a piorar a precisão. O mesmo ocorre à direita, sendo que, nessa circunstância, a precisão será necessariamente menor que no primeiro caso, já que o range é inferior. Note-se que, ao alterarmos os valores das condições, não apenas a máxima precisão irá mudar, mas também o range de otimização.

No caso BVP, que apresenta variação em ambos os extremos, o range ótimo também vale 3 , talvez pelo peso da maior imprecisão no extremo direito. De qualquer modo, o erro absoluto total é menor que no caso híbrido com a condição à direita. 
Tabela 12: Erros absolutos totais da exponencial na comparação BVP-NBV e imposição de condições à esquerda e à direita em função do range

\begin{tabular}{|c||c|c|c|c|}
\hline Range & $\mathcal{E}_{a b s}(N B V)$ & $\mathcal{E}_{a b s}\left(\Delta y_{0}\right)$ & $\mathcal{E}_{a b s}\left(\Delta y_{f}\right)$ & $\mathcal{E}_{a b s}(B V P)$ \\
\hline \hline 1 & 6.29875 & 6.31976 & 6.28849 & 6.28180 \\
\hline 2 & 2.19296 & 2.24675 & 2.52724 & 2.40274 \\
\hline 3 & 0.578546 & 0.724453 & 2.33473 & 1.99386 \\
\hline 4 & 0.135785 & 0.462585 & 2.9810 & 2.62347 \\
\hline 5 & $3.00861 \times 10^{-2}$ & 0.396789 & 3.32356 & 2.95241 \\
\hline 6 & $6.46129 \times 10^{-3}$ & 0.38962 & 3.54778 & 3.16397 \\
\hline 7 & $1.36141 \times 10^{-3}$ & 0.397825 & 3.7302 & 3.33364 \\
\hline 8 & $2.83634 \times 10^{-4}$ & 0.410287 & 3.89982 & 3.4898 \\
\hline 9 & $5.86817 \times 10^{-5}$ & 0.424846 & 4.07139 & 3.6466 \\
\hline 10 & $1.20748 \times 10^{-5}$ & 0.441297 & 4.25305 & 3.81176 \\
\hline 11 & $2.47794 \times 10^{-6}$ & 0.460086 & 4.45331 & 3.99316 \\
\hline 12 & $5.07539 \times 10^{-7}$ & 0.481837 & 4.67838 & 4.19653 \\
\hline 13 & $1.03826 \times 10^{-7}$ & 0.506855 & 4.93356 & 4.4267 \\
\hline 14 & $2.12244 \times 10^{-8}$ & 0.53586 & 5.22642 & 4.69056 \\
\hline 15 & $4.33677 \times 10^{-9}$ & 0.57008 & 5.56127 & 4.99201 \\
\hline
\end{tabular}

O mais interessante é o fato de a solução NBV aparentemente não apresentar range ótimo, o que significa que a precisão, para um período fixo, parece depender de fato apenas do range, melhorando indefinidamente. Mas é claro que deve haver uma aproximação assintótica, levando a uma precisão máxima. Para tentar comprovar ou descartar essa hipótese, refizemos os cálculos de NBV com range variando de 5 em 5, de 20 a 65, obtendo os seguintes valores para os erros absolutos totais:

Tabela 13: Variação do erro absoluto total na solução NBV da exponencial em função do range

$$
\begin{aligned}
& \mathcal{E}_{a b s}^{(20)}=1.50428 \times 10^{-12} \\
& \mathcal{E}_{a b s}^{(30)}=4.66511 \times 10^{-14} \\
& \mathcal{E}_{a b s}^{(40)}=4.14415 \times 10^{-14} \\
& \mathcal{E}_{a b s}^{(50)}=6.9932 \times 10^{-14} \\
& \mathcal{E}_{a b s}^{(60)}=4.90667 \times 10^{-14}
\end{aligned}
$$$$
\begin{aligned}
& \mathcal{E}_{a b s}^{(25)}=1.07539 \times 10^{-13} \\
& \mathcal{E}_{a b s}^{(35)}=9.22369 \times 10^{-14} \\
& \mathcal{E}_{a b s}^{(45)}=4.56845 \times 10^{-14} \\
& \mathcal{E}_{a b s}^{(55)}=6.91978 \times 10^{-14} \\
& \mathcal{E}_{a b s}^{(65)}=9.17226 \times 10^{-13}
\end{aligned}
$$

A observação desses valores revela que há, na verdade, uma oscilação na precisão após o range 30, aproximadamente. Isso implica que o primeiro mínimo local do erro deve situar-se nessa faixa, demonstrando também que existe um limite máximo de precisão no método NBV, para um dado range, após o qual o valor do erro começa a oscilar. Calculando os valores do erro para ranges entre 30 e 40, chegamos à conclusão de que o range local de otimização ocorre para $\ell=31$ e vale $\mathcal{E}_{a b s}^{(31)}=1.77894 \times 10^{-14}$. Infelizmente, 
como os elementos que possuímos, não há meios de saber se se trata de um mínimo local ou global.

Como se vê, a precisão cai muito quando inserimos condições determinantes. Uma parte da explicação para isso está na natureza nos nodos de Chebyshev. Neles, a concentração de pontos é maior nas extremidades, mas o miolo fica desguarnecido, além de favorecer mais um lado do que o outro. Um modo de diminuir o erro nas soluções híbridas é deslocar os NC para incluir a condição imposta. Com isso, a aproximação local melhora, embora piore a precisão no lado oposto. Afora isso, há o fato óbvio de "forçar"o modelamento da função-solução pelos vínculos impostos, os quais, muitas vezes, podem se afastar demasiadamente da solução ideal.

Acreditamos que o ideal talvez fosse construir um conjunto de pontos que amalgamasse os dois conceitos, quer dizer, concentrasse os pontos onde essa maior densidade se faz necessária, a fim de minimizar as imprecisões e efeitos de oscilação. Mas tal investigação está além dos nossos objetivos nesse trabalho.

Dito isto, esperamos ter esclarecido esse ponto tão importante, referente à ausência de condições impositivas no método NBV/NIV.

\subsection{Resolução pelo Método NBV}

A seguir, o método NBV, uma vez justificado, é usado com as séries de Fourier original e associada e os resultados obtidos são comparados.

Inicialmente, na figura (40), ilustra-se a eficácia do uso dos nodos de Chebyshev na interpolação da distribuição de Cauchy: $\pi \phi(5 x)=1 /\left(1+25 x^{2}\right)$, pelo método de Lagrange.

Em seguida, o método NBV original (i.e., com séries de Fourier originais) com espaçamento constante, nodos de Chebyshev e nodos de Fourier, respectivamente, é utilizado na solução da mesma EDO não-homogênea de terceira ordem já resolvida anteriormente, mas agora com termo independente dado pela wavelet de Morlet:

$$
\frac{d^{3} y}{d x^{3}}+(x+9) \frac{d^{2} y}{d x^{2}}-(x+1) \frac{d y}{d x}+x^{2} y=\exp \left(-x^{2}\right) \cos (\pi x \sqrt{2 / \ln 2}),
$$

definida no semiperíodo $L=5 \pi / 8$ e extensão $\ell=5$, isto é, resolvida com uma função candidata de 11 termos. Os gráficos das soluções obtidas aparecem nas figuras (41), (42) e (43). 
Figura 40: Interpolação polinomial da distribuição de Cauchy
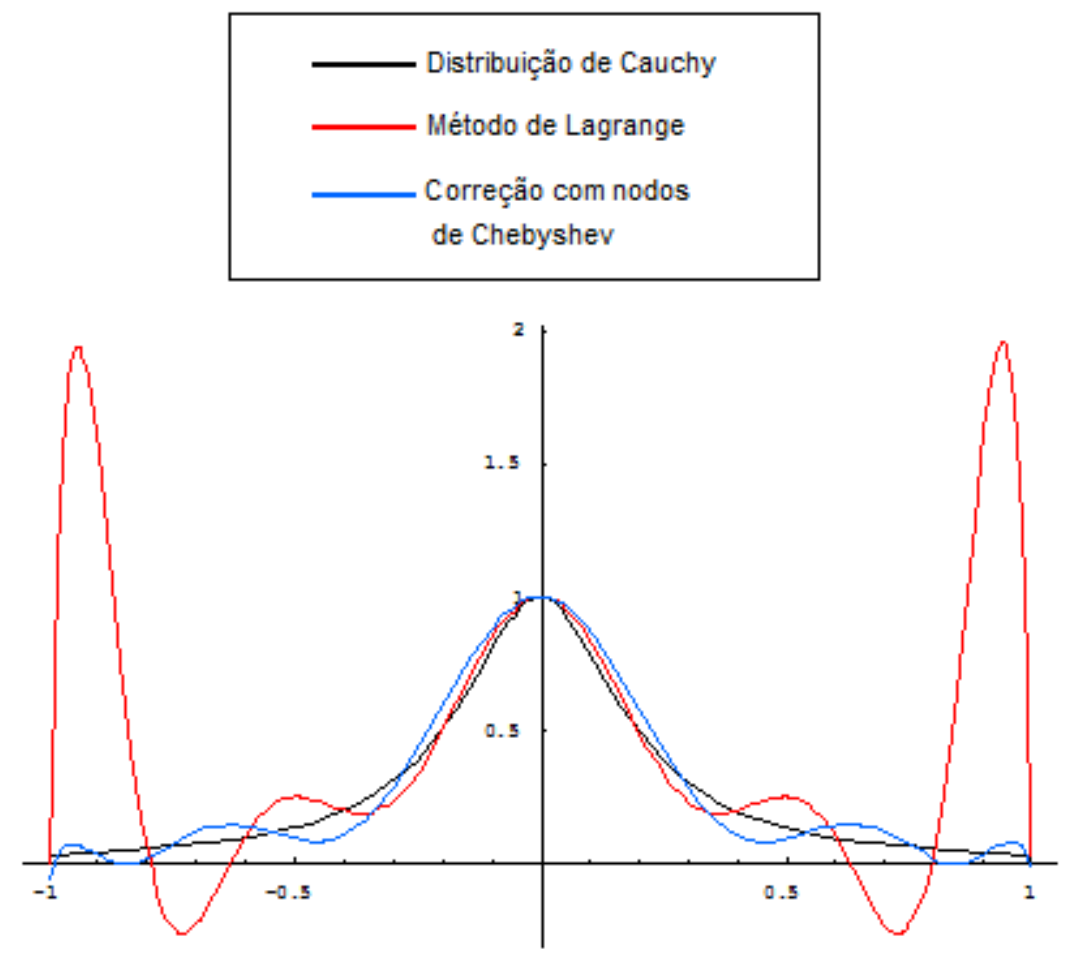

Figura 41: Solução da EDO na base de Fourier original com range 5 e $L=\frac{5 \pi}{8}$

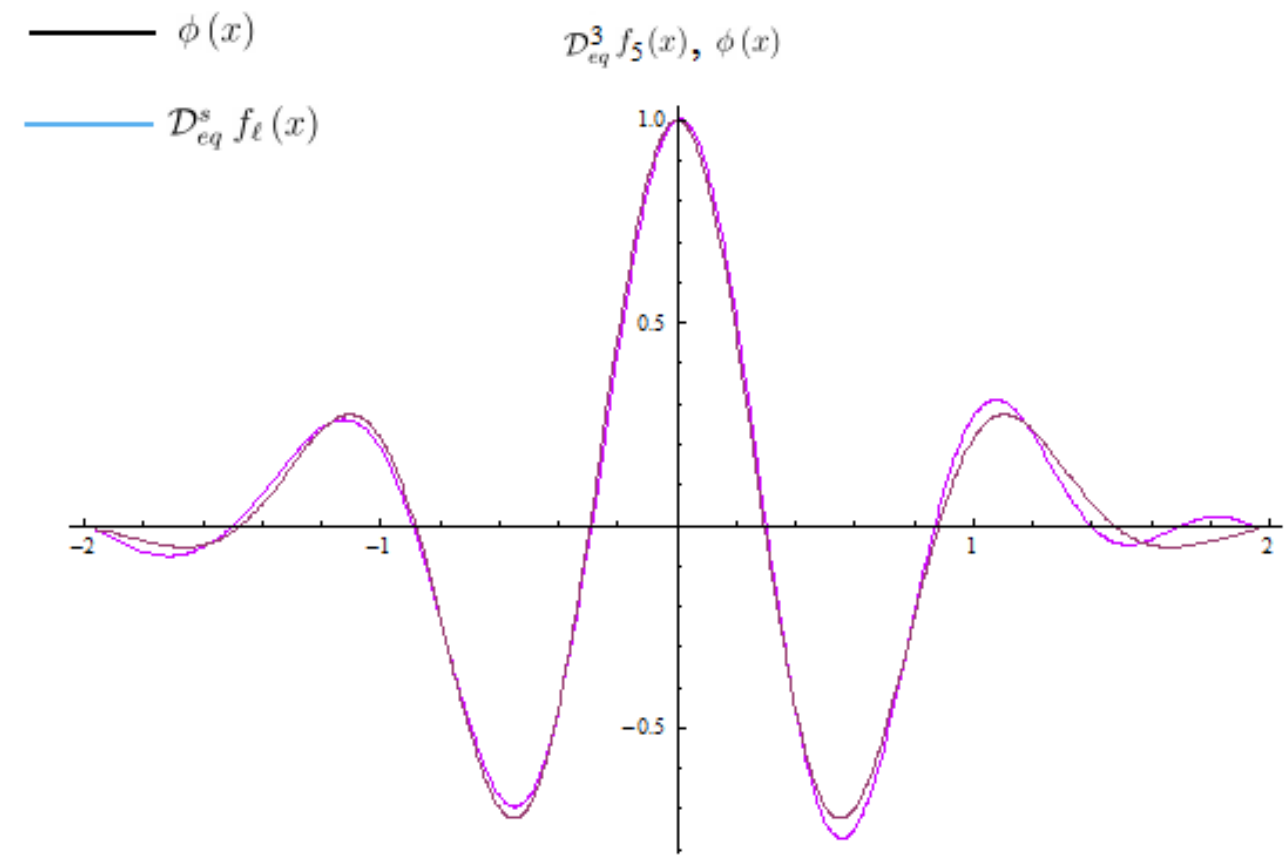


Figura 42: Solução na base original com range 5 e $L=\frac{5 \pi}{8}$ usando os nodos de Chebyshev

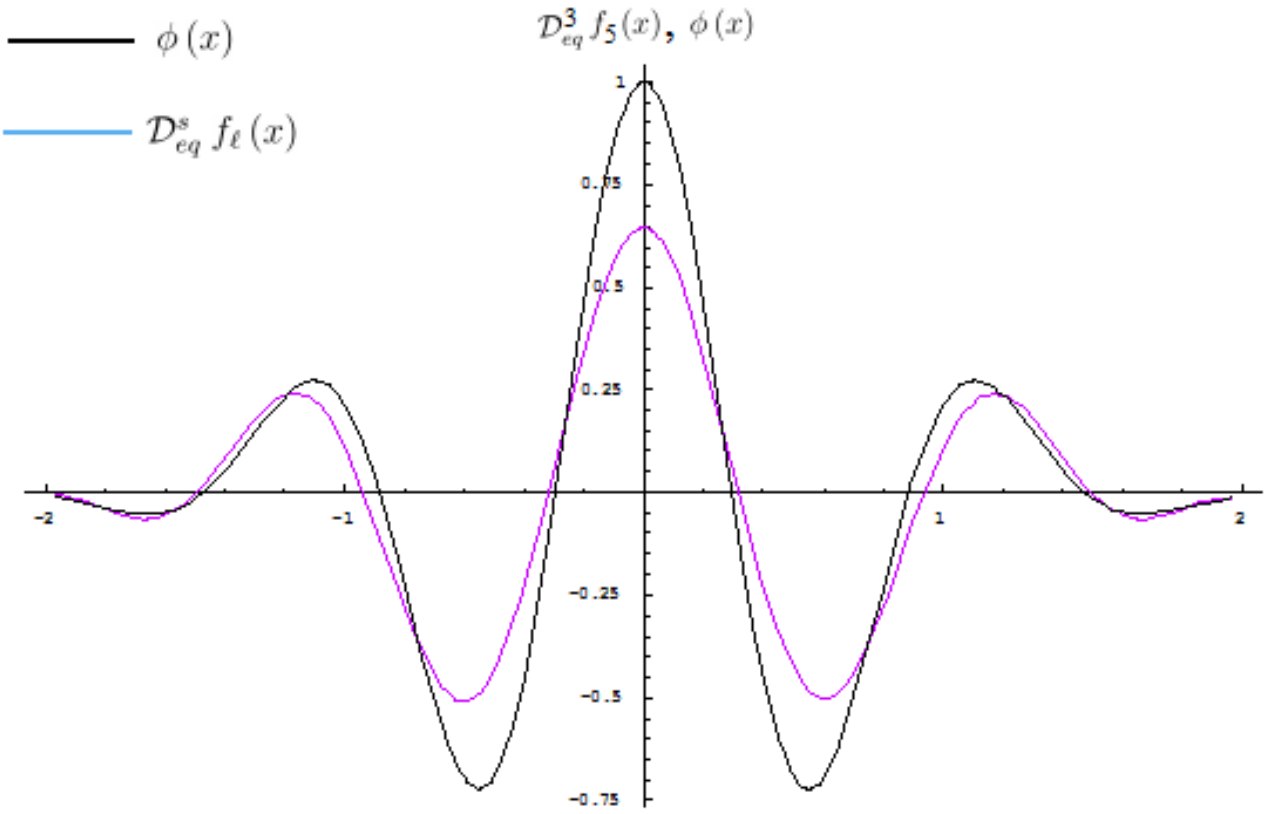

Figura 43: Solução na base original com range 5 e $L=\frac{5 \pi}{8}$ usando os nodos de Fourier

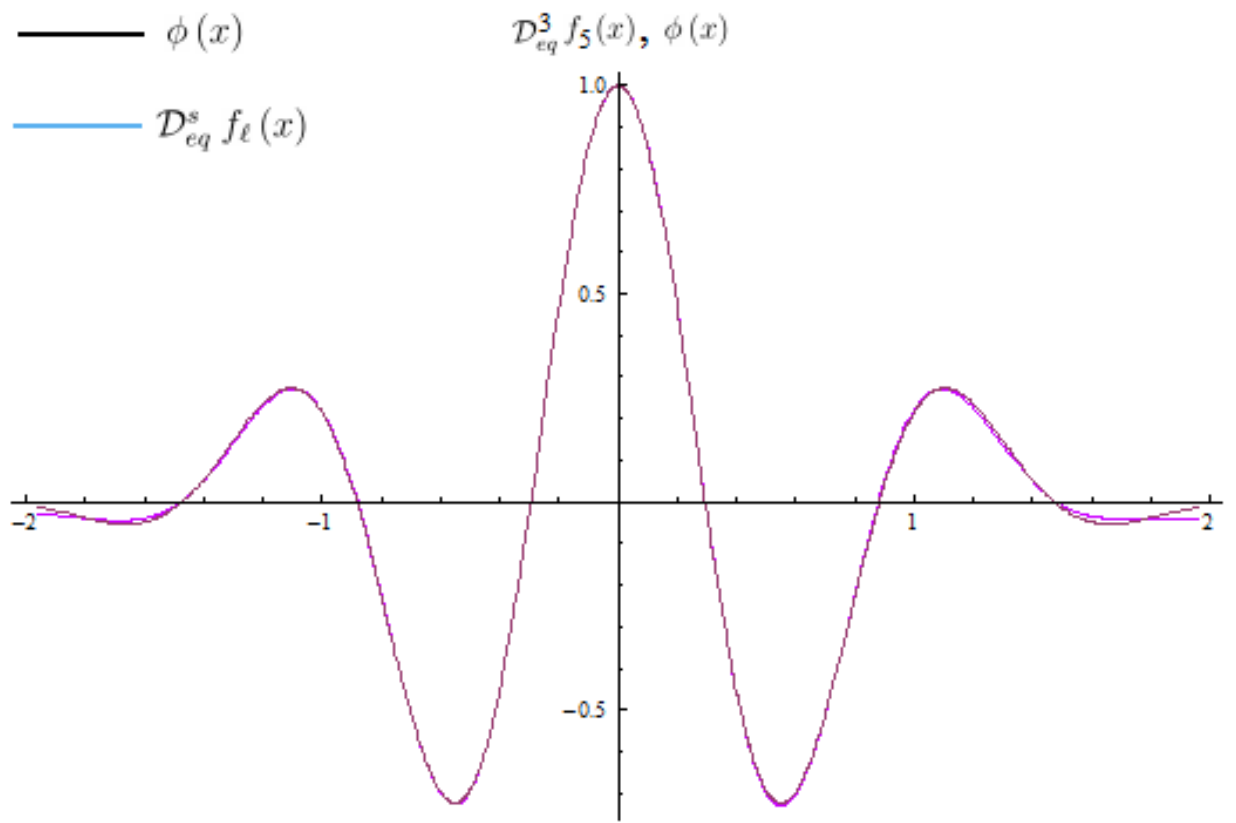


As figuras (44), (45) e (46) apresentam o mesmo tipo de solução acima, mas com extensão 9, ou seja, com uma função candidata de 19 termos, e mostram, respectivamente, a solução NBV original com espaçamento constante, com nodos de Chebyshev e de Fourier.

Figura 44: Solução na base original com range 9 e $L=\frac{5 \pi}{8}$

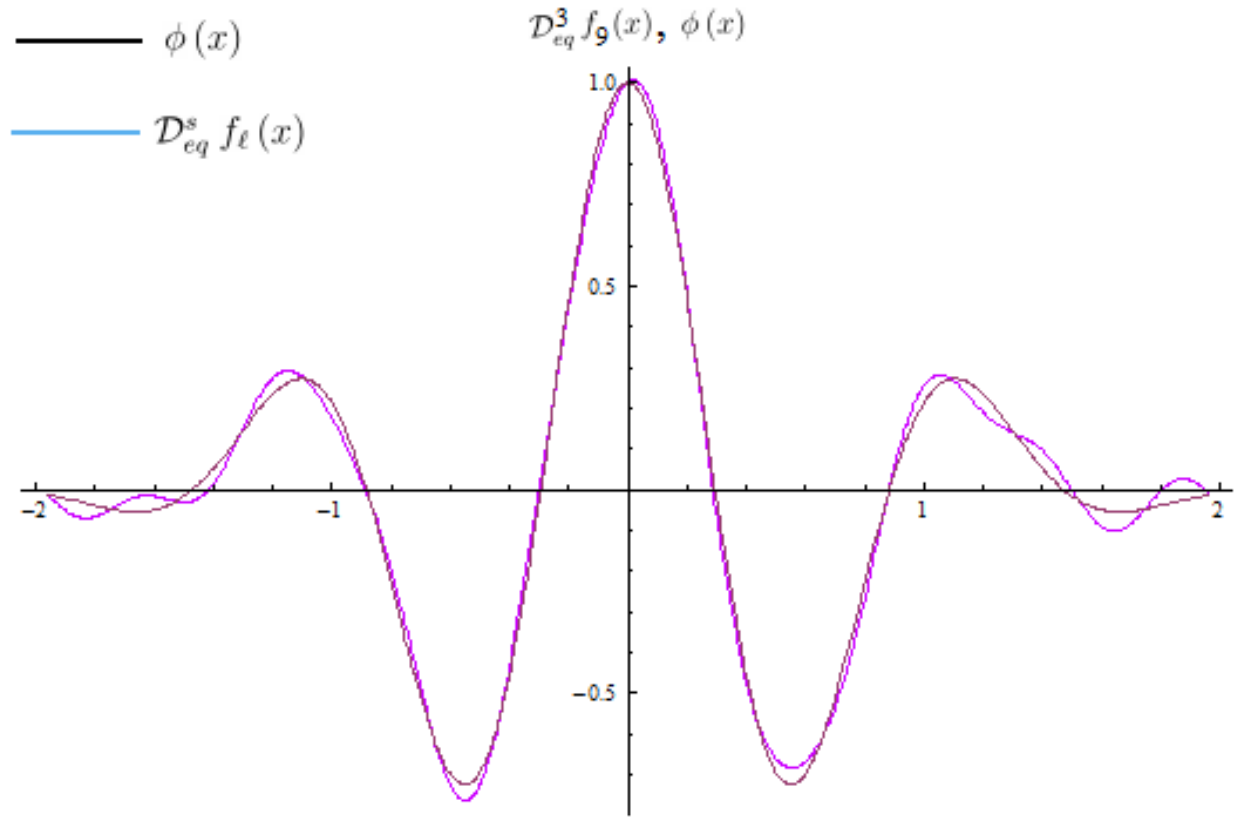

Figura 45: Solução na base original com range 9 e $L=\frac{5 \pi}{8}$ usando os nodos de Chebyshev

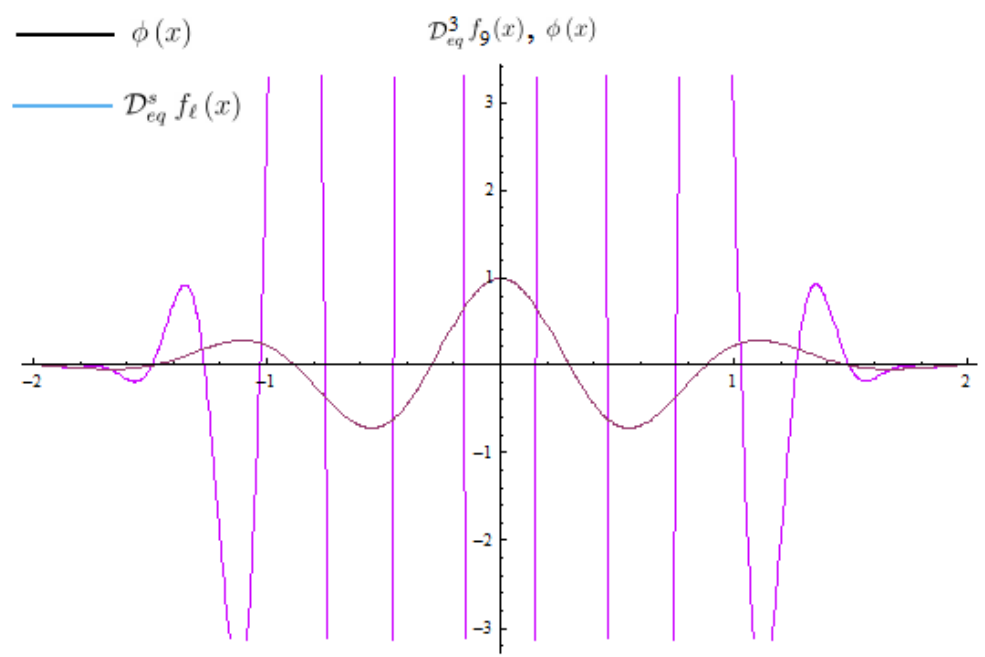

Já as figuras (47), (48) e (49) mostram os gráficos da solução pelo método NBV na base associada, também nos três casos, com espaçamento constante, com os nodos de Fourier e com os nodos de Chebyshev.

As figuras (50) e (51) mostram o resto quadrático das soluções na base original usando 
Figura 46: Solução na base original com range 9 e $L=\frac{5 \pi}{8}$ usando os nodos de Fourier

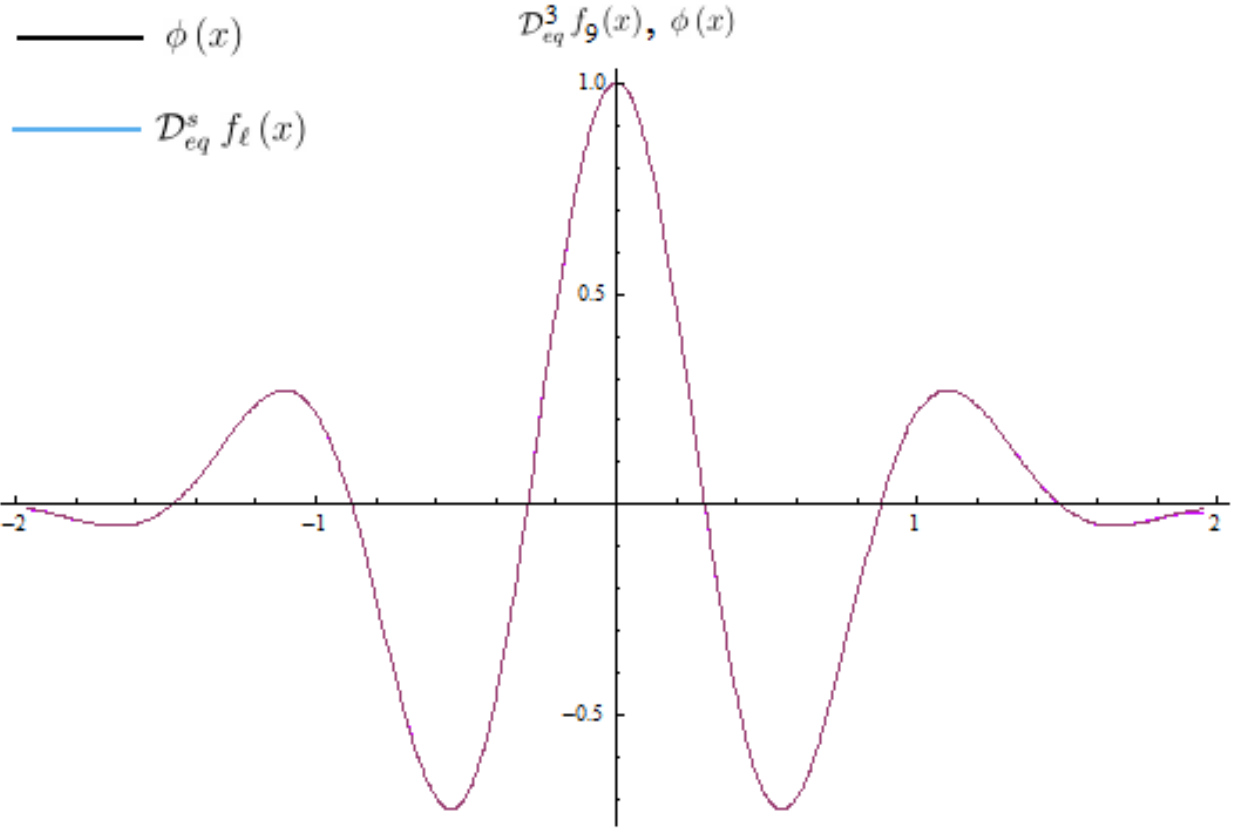

Figura 47: Solução na base associada com range 9 e $L=\frac{5 \pi}{8}$

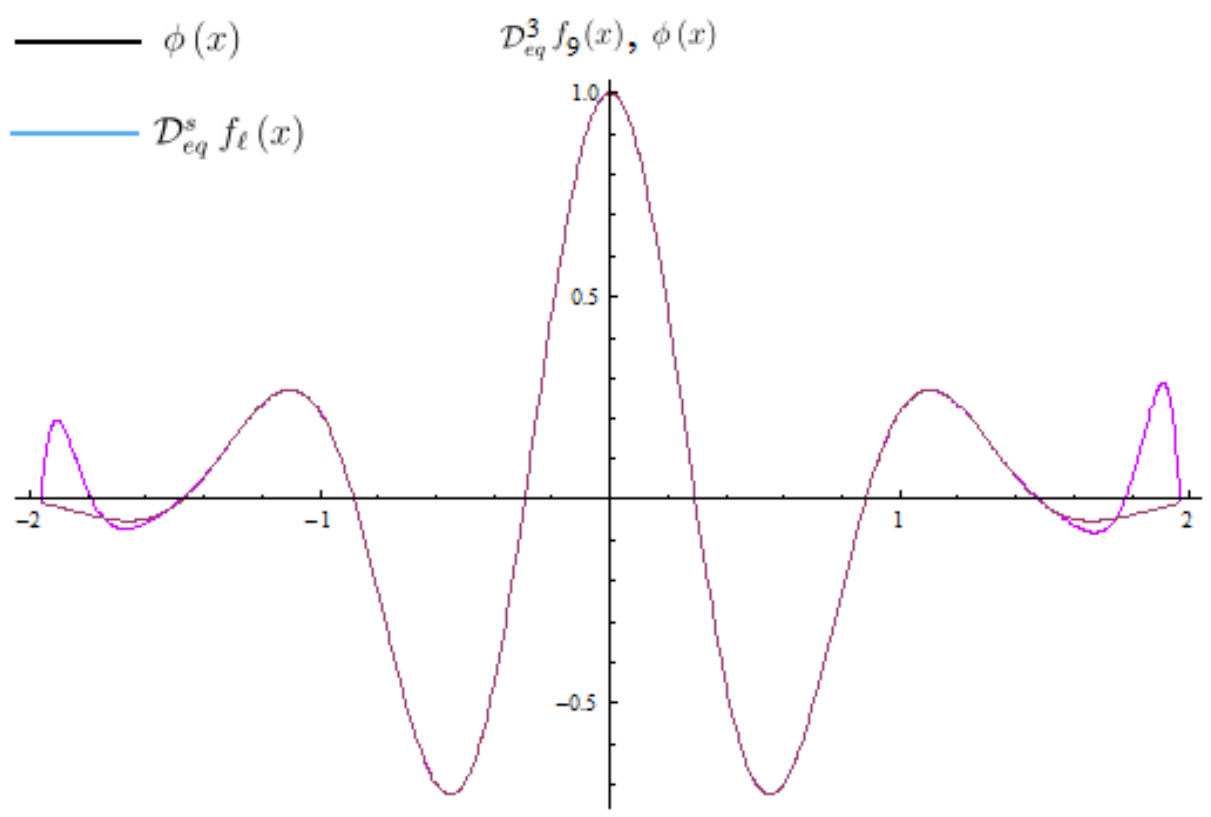


Figura 48: Solução na base associada com range 9 e $L=\frac{5 \pi}{8}$ usando os nodos de Fourier

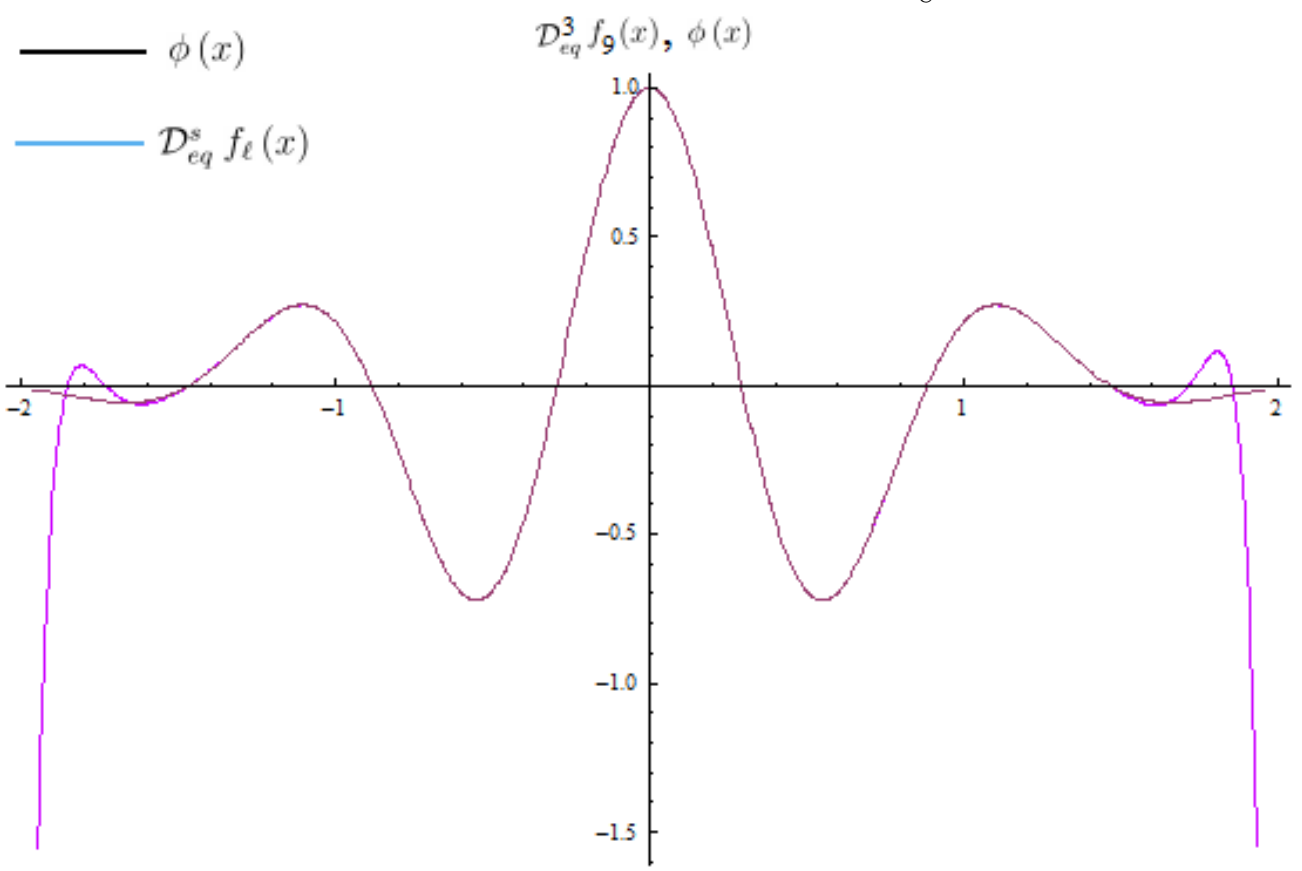

Figura 49: Solução na base associada com range 9 e $L=\frac{5 \pi}{8}$ usando os nodos de Chebyshev

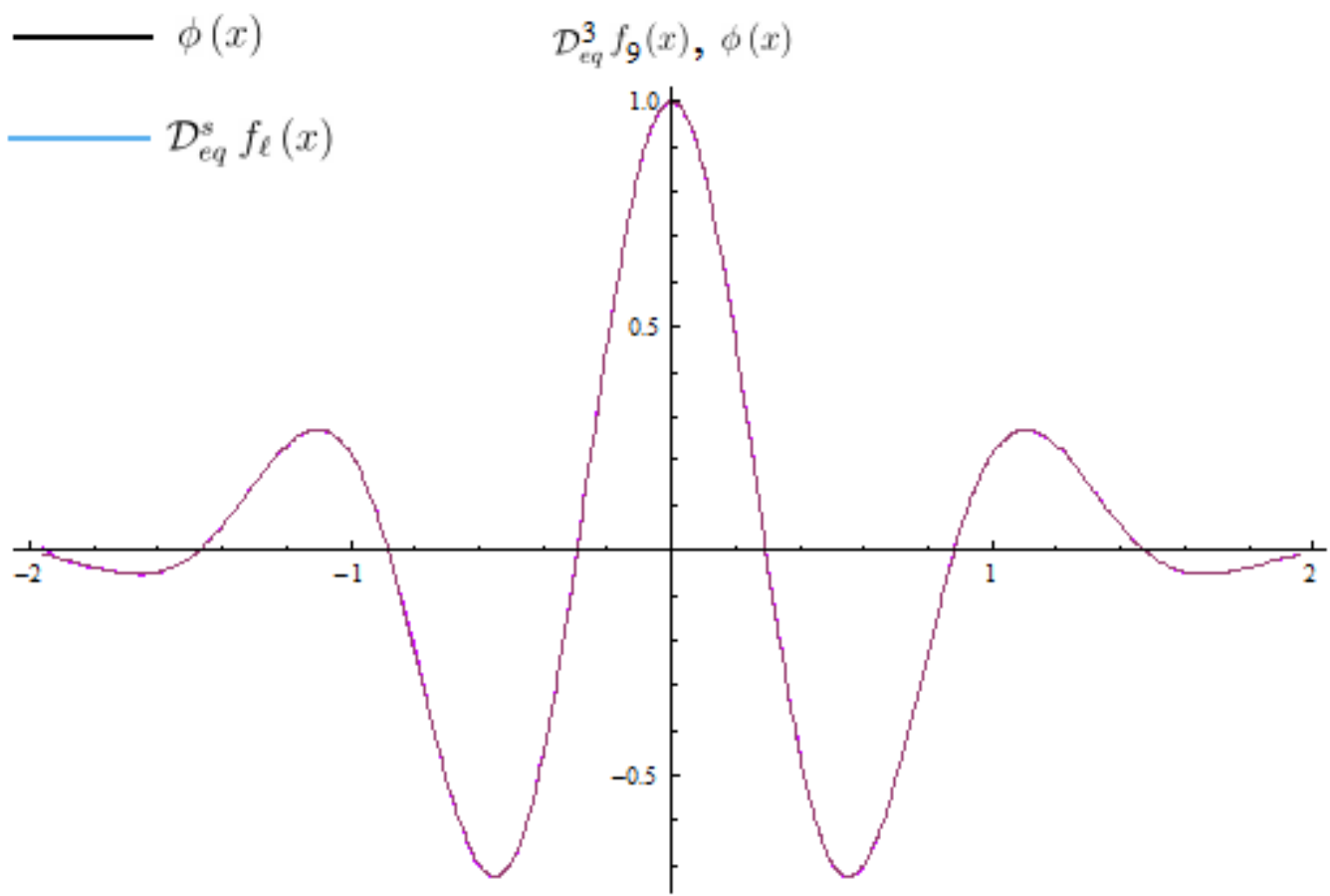


os nodos de Fourier e na base associada com os nodos de Chebyshev, com $\ell=9$ e $L=\frac{5 \pi}{8}$.

Efetuamos ainda uma solução da mesma EDO com range 15 pela série associada e nodos de Chebyshev, cuja função-resto quadrático é apresentada na figura (52), a fim de mostrar quão preciso pode ser o método nessas circunstâncias. A solução pela série original com nodos de Fourier é mostrada em sequência, na figura (53). Apenas para comparação, inserimos também o resto da solução pela série associada com os nodos de Fourier, visto na figura (54).

Por fim, usamos o método das séries associadas para calcular a solução NBV com nodos de Chebyshev dessa mesma EDO, mas agora com termo independente dado pela Mexican Hat Wavelet (MHW):

$$
\phi_{M H}(x)=\left(1-2 x^{2}\right) \exp \left(-x^{2}\right)
$$

O gráfico do resto quadrático dessa solução aparece representado na figura (55). Finalmente, no último gráfico, mostra-se novamente o resto quadrático total dessa mesma EDO, após todo o processo ser repetido para um novo range, de valor 15, o que fornece uma precisão estupenda.

Figura 50: Resto quadrático da solução original com nodos de Fourier, $\ell=9$ e $L=\frac{5 \pi}{8}$

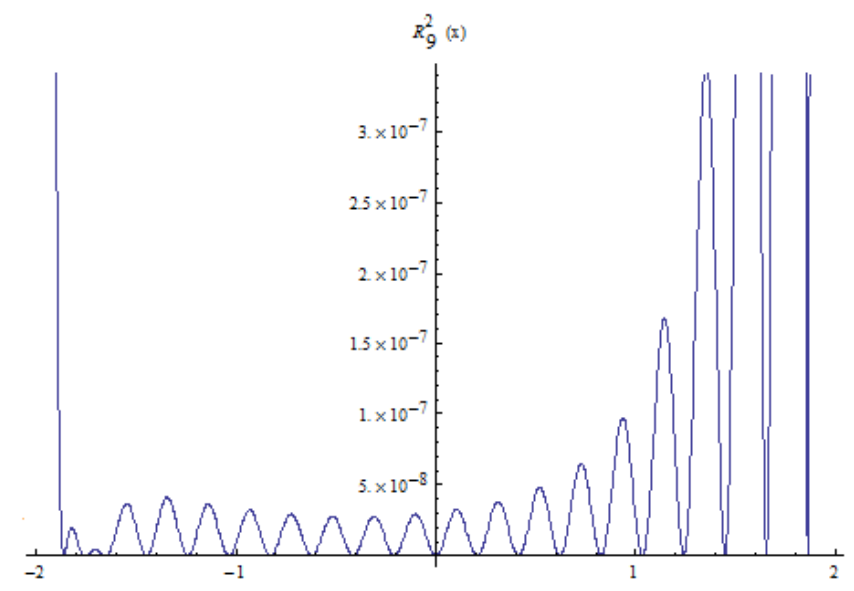

Comentários sobre os resultados Percebe-se que o uso dos nodos de Chebyshev no método das séries de Fourier usuais não necessariamente melhora a solução NBV, pelo contrário, chega a piorá-la, o que pode ser visto nas comparações entre as figuras (41) e (42), onde se nota que a inserção dos nodos parece ajustar melhor a distribuição de frequência, simetrizando o gráfico, mas piorando bastante o ajuste da amplitude.

Já a comparação entre as figuras (44) e (45) mostra que o resultado nesse caso foi muito pior com o uso dos nodos de Chebyshev, quase produzindo uma divergência. 
Figura 51: Resto quadrático da solução associada com nodos de Chebyshev, $\ell=9$ e $L=\frac{5 \pi}{8}$

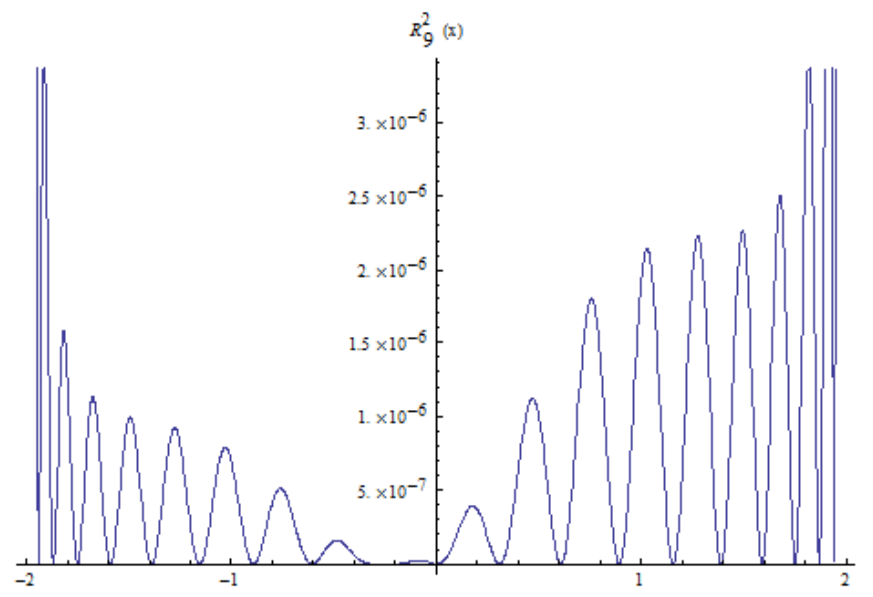

Figura 52: Resto quadrático da solução associada e nodos de Chebyshev, $\ell=15$ e $L=\frac{5 \pi}{8}$

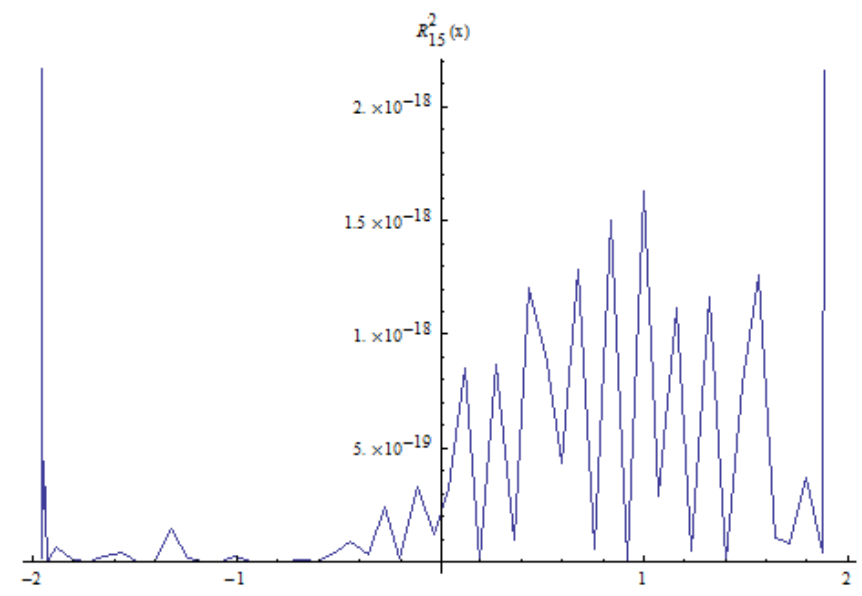

Figura 53: Resto quadrático da solução original com nodos de Fourier, $\ell=15$ e $L=\frac{5 \pi}{8}$

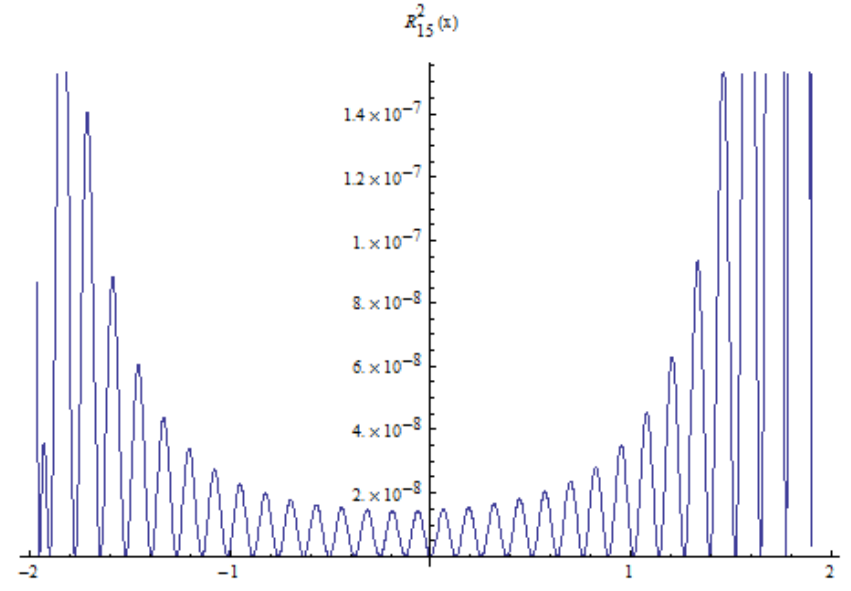


Figura 54: Resto quadrático da solução associada e nodos de Fourier, $\ell=15$ e $L=\frac{5 \pi}{8}$

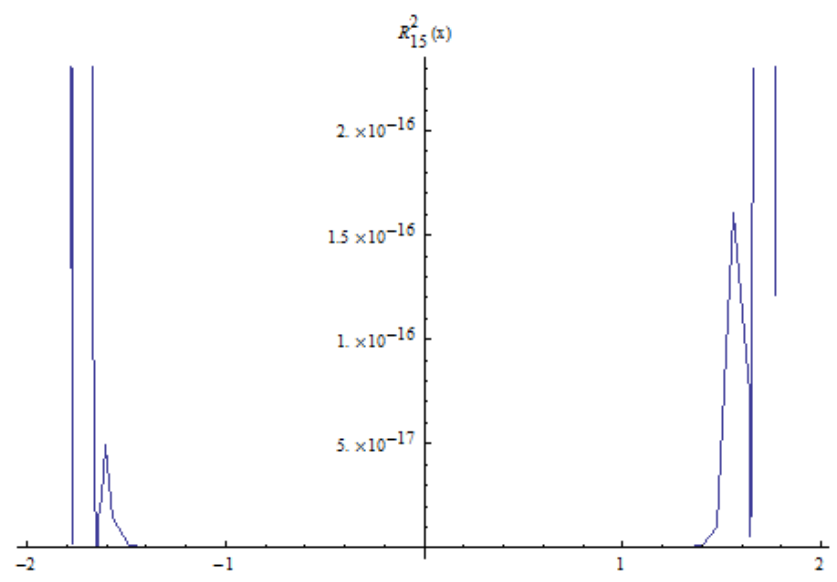

Figura 55: Resto quadr. da sol. NBV assoc. p/ MHW com nodos de Cheb., $\ell=7$

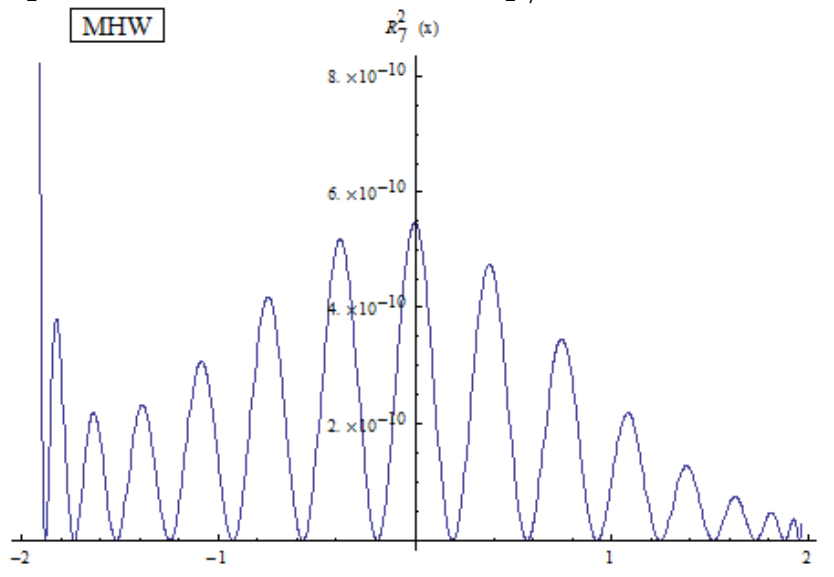

Figura 56: Resto quadr. da sol. NBV assoc. p/ MHW com nodos de Cheb., $\ell=15$




Entretanto, a utilização dos nodos de Fourier tende a melhorar muito o resultado quando aplicamos o processo de solução da série de Fourier original. No exemplo do range 9, a solução da série original com nodos de Fourier chega a ser melhor que o da série associada e nodos de Chebyshev, o que pode ser verificado pela comparação das figuras (50) e (51), referentes às funções-resto de cada caso. Contudo, à medida que o range cresce a precisão na série associada tende a ser maior que na original, devido à sua convergência mais rápida.

No que diz respeito ao método das séries associadas, a primeira coisa que se constata é o intenso aprimoramento da solução, mesmo sem o uso dos nodos de Chebyshev. Todavia, quando passamos a utilizá-los, conseguimos melhorar bastante a precisão obtida. A oscilação do efeito de borda presente no gráfico da figura (47), por exemplo, foi totalmente eliminado com o uso dos nodos, conforme mostra a figura (49). O resto quadrático nesse caso, visto no gráfico (51), é da ordem de $10^{-6}$. O erro quadrático total no período também já é extremamente reduzido, valendo $\mathcal{E}_{9}^{2}=1.02078 \times 10^{-5}$.

O gráfico da figura (52) revela que a NBV associada para a EDO de Morlet de range 15 apresenta um resto quadrático extremamente diminuto, oscilando na faixa de $10^{-18}$. De fato, o próprio erro quadrático total no período é de $1.70461 \times 10^{-18}$. Deve-se considerar o fato notável de que isto é conseguido com uma matriz operacional de ordem 31, que pode ser entendida como de dimensões reduzidas em termos de cálculos algébricos, o que representa um ganho computacional enorme. Efetuando as soluções com os nodos de Fourier para a série original e também para a associada e comparando com a dos nodos de Chebyshev para a série associada, observamos que a maior precisão foi obtida justamente com esta última.

Solução ainda mais impressionante é conseguida com o uso dos nodos de Chebyshev na série associada para a solução NBV da EDO com termo independente MHW, para um modesto range $\ell=7$, no mesmo semiperíodo anterior. Pode-se ver no gráfico da figura (55) que o resto quadrático dessa solução oscila em torno de $10^{-10}$, apresentando $\mathcal{E}_{7}^{2}=1.10245 \times 10^{-9}$, resultado surpreendente para um range tão baixo.

Finalmente, ao repetirmos o mesmo processo com range 15, i.e., com 31 termos, obtivemos, para o erro total, a espantosa marca de $\mathcal{E}_{15}^{2}=2.84234 \times 10^{-32}$, já na ordem da precisão da máquina! O gráfico do resto total aparece na figura (56). Isso mostra o quanto o método pode ser preciso, se for escolhida a variante adequada a cada caso.

O próximo passo consiste em estender um pouco mais o uso do método, considerando a introdução de funções complexas nas EDOs. Dessa forma, um caso adicional será 
abordado, para uma equação bem mais elaborada, de $7^{\mathrm{a}}$ ordem, na qual as funções coeficientes e a do termo homogêneo são estendidas ao corpo complexo. O intuito dessa análise é mostrar o poder do método em casos bem mais complicados e de maior abrangência.

\subsubsection{EDO com Funções Coeficientes e Termo Independente Complexos}

Vamos analisar agora uma EDO bem mais complicada que as anteriores, expressa por

$$
\sum_{k=0}^{7} \mu_{k}(x) D^{k} f(x)=\phi(x),
$$

na qual as funções coeficientes e a função do termo independente passam a ser complexas. A função $\phi$ escolhida foi:

$$
\phi: \mathbb{R} \rightarrow \mathbb{C} \mid \phi(x)=\exp \left\{-(x / \sqrt{L})^{2}+i(x / \sqrt{L})\right\}
$$

e as funções coeficientes, também complexas, são dadas a seguir:

$$
\begin{aligned}
& \mu_{0}(x)=x^{2} \\
& \mu_{1}(x)=1-i x \\
& \mu_{2}(x)=1 \\
& \mu_{3}(x)=-1 \\
& \mu_{4}(x)=-e^{i x} \\
& \mu_{5}(x)=e^{x} \\
& \mu_{6}(x)=0 \\
& \mu_{7}(x)=1
\end{aligned}
$$

Nesse caso obteremos, como solução, funções complexas cuja parte imaginária não pode ser desprezada. Por isso, antes de prosseguir, salientamos que, para analisar graficamente as soluções de modo saisfatório, faz-se necessário definir recursos gráficos adicionais, que serão abordados no próximo parágrafo. 


\subsubsection{Recursos Gráficos Adicionais para Funções com Argumento Com- plexo}

Como agora estaremos lidando com funções complexas como solução, cuja parte imaginária representa papel significativo, ao contrário dos exemplos anteriores, nos quais era desprezível, passa a ser interessante considerar, além dos gráficos das partes real e imaginária, também gráficos do resultado complexo como um todo e não apenas da parte real da solução. Para isso, vamos implementar o gráfico de uma curva complexa através da plotagem paramétrica das coordenadas $(u, v)$ das funções $f_{\ell}(x) \in \mathbb{C}$ obtidas, definidas no intervalo $[-L, L]$ por:

$$
\begin{aligned}
& u(x)=\Re\left\{f_{\ell}(x)\right\} \\
& v(x)=\Im\left\{f_{\ell}(x)\right\}
\end{aligned}
$$

Para ilustrar essa exposição, apresentamos, na figura (57), o arranjo dos gráficos mencionados para a função $\phi$ complexa definida acima. Além disso, apresentaremos também as curvas paramétricas complexas das funções resto, acompanhadas de gráficos separados para a parte real e imaginária.

Figura 57: Partes real e imaginária e curva paramétrica complexa da função $\phi$
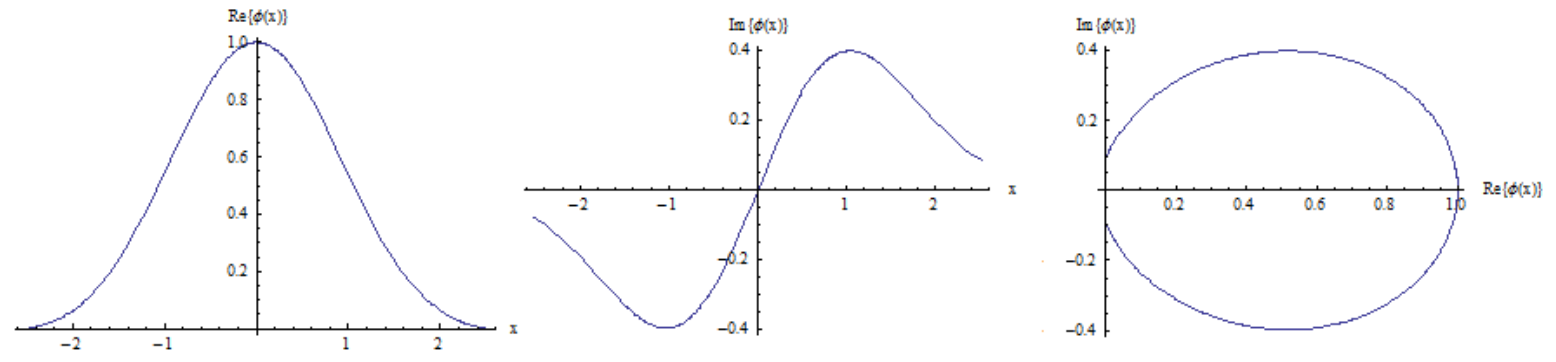

Uma outra análise gráfica será efetivada considerando a extensão das soluções ao domínio complexo, fazendo $x \rightarrow z(x, y):=x+i y \in \mathbb{C}$. Assim, pode-se construir, pelo isomorfismo $\mathbb{R}^{2} \simeq \mathbb{C}$, um gráfico em três dimensões para cada uma das partes (real e imaginária) da solução, usando o mesmo domínio de período $2 L$ para $y$, ou seja, plotando $\Re\left\{f_{\ell}(x, y)\right\}$ e $\Im\left\{f_{\ell}(x, y)\right\}$ para o domínio $A=[-L, L] \times[-L, L] \subset \mathbb{R}^{2}$, onde se entende $f_{\ell}(x, y):=f_{\ell}(z(x, y))$, para $z(x, y)=x+i y$. Dessa maneira, obtêm-se os gráficos complexos de superfície para cada parte: $\Re\{f \ell\}: A \subset \mathbb{R}^{2} \longrightarrow \mathbb{R}$ e $\Im\{f \ell\}: A \subset \mathbb{R}^{2} \longrightarrow \mathbb{R}$.

Um recurso gráfico também bastante interessante são os mapas paramétricos complexos, que revelam, de certa maneira, a geometria envolvida na função. Dada a função sobre o corpo complexo $f(x, y)=u(x, y)+i v(x, y)$, o mapa paramétrico cartesiano no domínio 
$A=[-L, L] \times[-L, L] \subset \mathbb{R}^{2}$ é construído pelo gráfico do par ordenado $(u(x, y), v(x, y))$ para $u, v: \mathbb{R}^{2} \longrightarrow \mathbb{R}$ quando as variáveis $x$ e $y$ percorrem o domínio.

Introduziremos também o mapa paramétrico polar pela transformação da função solução $f_{\ell} \longrightarrow f_{\ell}^{p o l}$, quando $(x, y) \longrightarrow(\rho, \theta)$ e $z(x, y) \longrightarrow z(\rho, \theta) \simeq \rho \exp (i \theta)$, plotando o par ordenado $\left(u_{\text {pol }}(\rho, \theta), v_{\text {pol }}(\rho, \theta)\right)$, onde

$$
\begin{aligned}
& u_{p o l}(\rho, \theta):=\Re\left\{f_{\ell}^{p o l}(\rho, \theta)\right\} \\
& v_{p o l}(\rho, \theta):=\Im\left\{f_{\ell}^{p o l}(\rho, \theta)\right\}
\end{aligned}
$$

sobre o domínio $B=[0, L] \times[-\pi, \pi]$.

Ambos os mapas têm aspectos úteis a serem aproveitados e cada um deles pode ser mais interessante que o outro, de acordo com a função analisada. Observem-se, por exemplo, os mapas paramétricos cartesiano e polar da função $f(x, y)=z(x, y)$, na figura (58) e os da função exponencial na figura (59).

Os mapas da função identidade complexa $f(z)=z$ apresentam, respectivamente, uma geometria cartesiana usual e uma geometria de natureza polar com distância radial constante, quer dizer, sem alteração do fator de escala modular.

Figura 58: Mapas paramétricos cartesiano e polar da função $f(x, y)=z(x, y)$
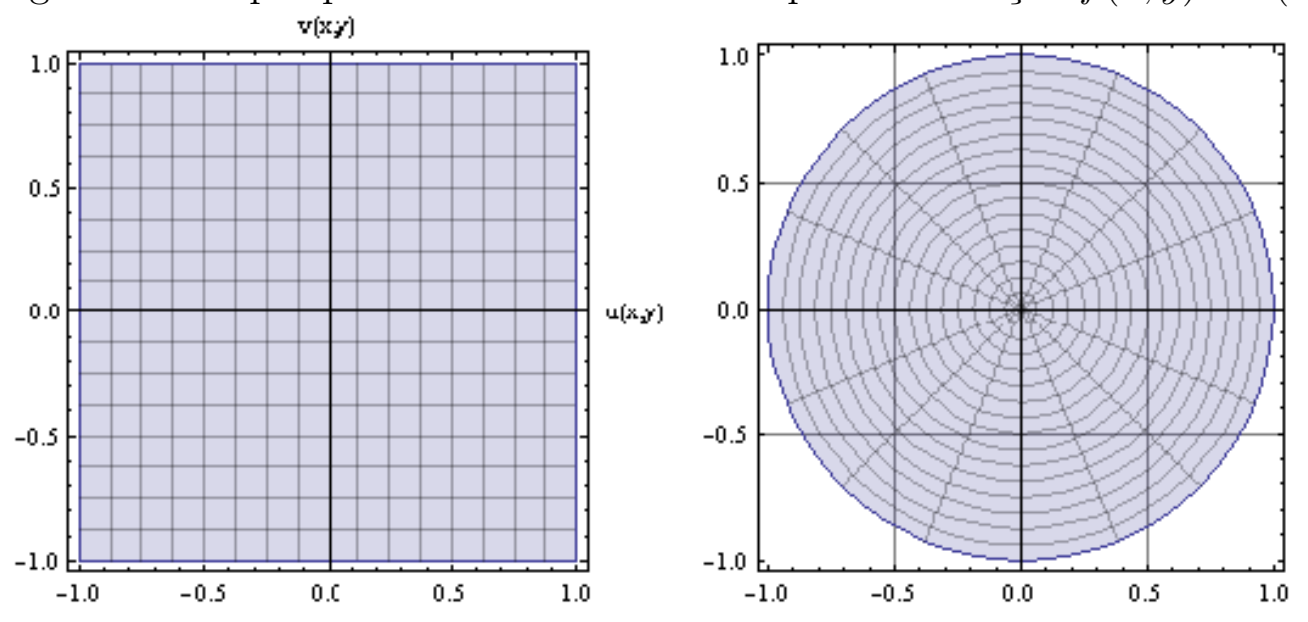

Apesar de o mapa paramétrico cartesiano parecer mais fácil de ser interpretado à primeira vista, isso nem sempre corresponde à verdade. De fato, o mapa paramétrico polar constitui, eventualmente, um recurso mais apropriado no que se refere à visualização de alguns aspectos geométricos da função, como o fator de escala, que têm relação com a distância das linhas concêntricas a partir da origem. Para entender melhor, basta comparar visualmente os mapas polares das funções $z, z^{2}$ e $z^{3}$, mostrados na fig.(60). Neles, podemos perceber o gradativo aumento das distâncias polares, relativas às crescentes or- 
Figura 59: Mapas paramétricos cartesiano e polar da função $f(x, y)=e^{z(x, y)}$

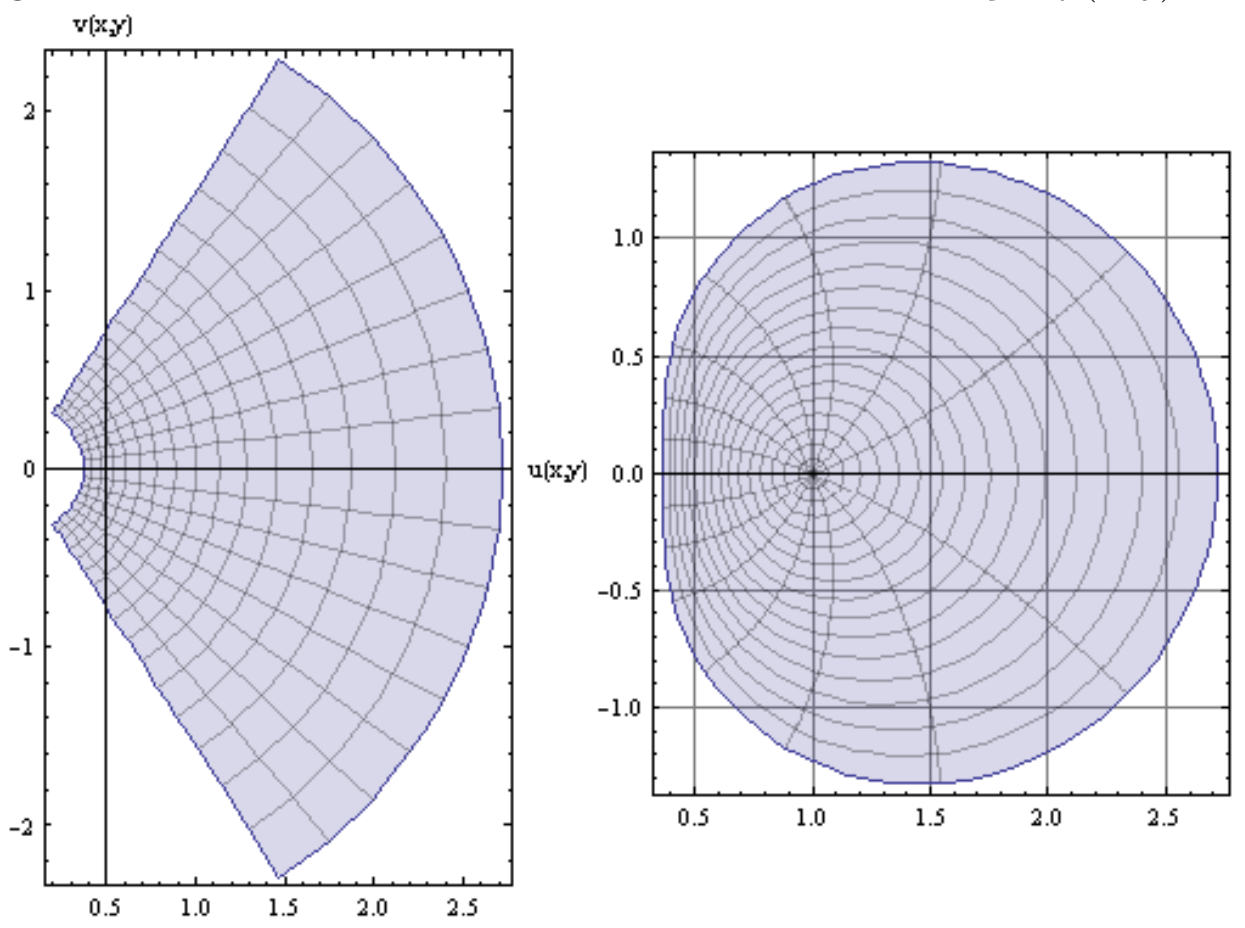

dens dos expoentes. O escurecimento gradual do fundo diz respeito ao número de vezes em que o plano é percorrido angularmente, duas vezes para $z^{2}$ e três vezes para $z^{3}$.

Figura 60: Mapas paramétricos polares das funções $z, z^{2}$ e $z^{3}$


\subsubsection{Modelagem NBV da EDO de $7^{\mathrm{a}}$ Ordem}

Voltando à equação (4.3), com os coeficientes estipulados, o método de resolução utilizado foi o de modelagem NBV pelas séries associadas e nodos de Chebyshev, sinteticamente grafado NBV-SA-NC, para semiperíodo $L=4 \pi / 5$ e extensão $\ell=15$. Essas especificações foram escolhidas por se mostrarem as melhores entre todos os processos, fornecendo o resultado com maior precisão.

Não apresentamos as matrizes de derivação ou do operador diferencial da EDO, nem 
o covetor coeficiente, por motivos de espaço, mas a solução aproximada $f_{15}(x)$ vem na sequência, acompanhada de seus gráficos.

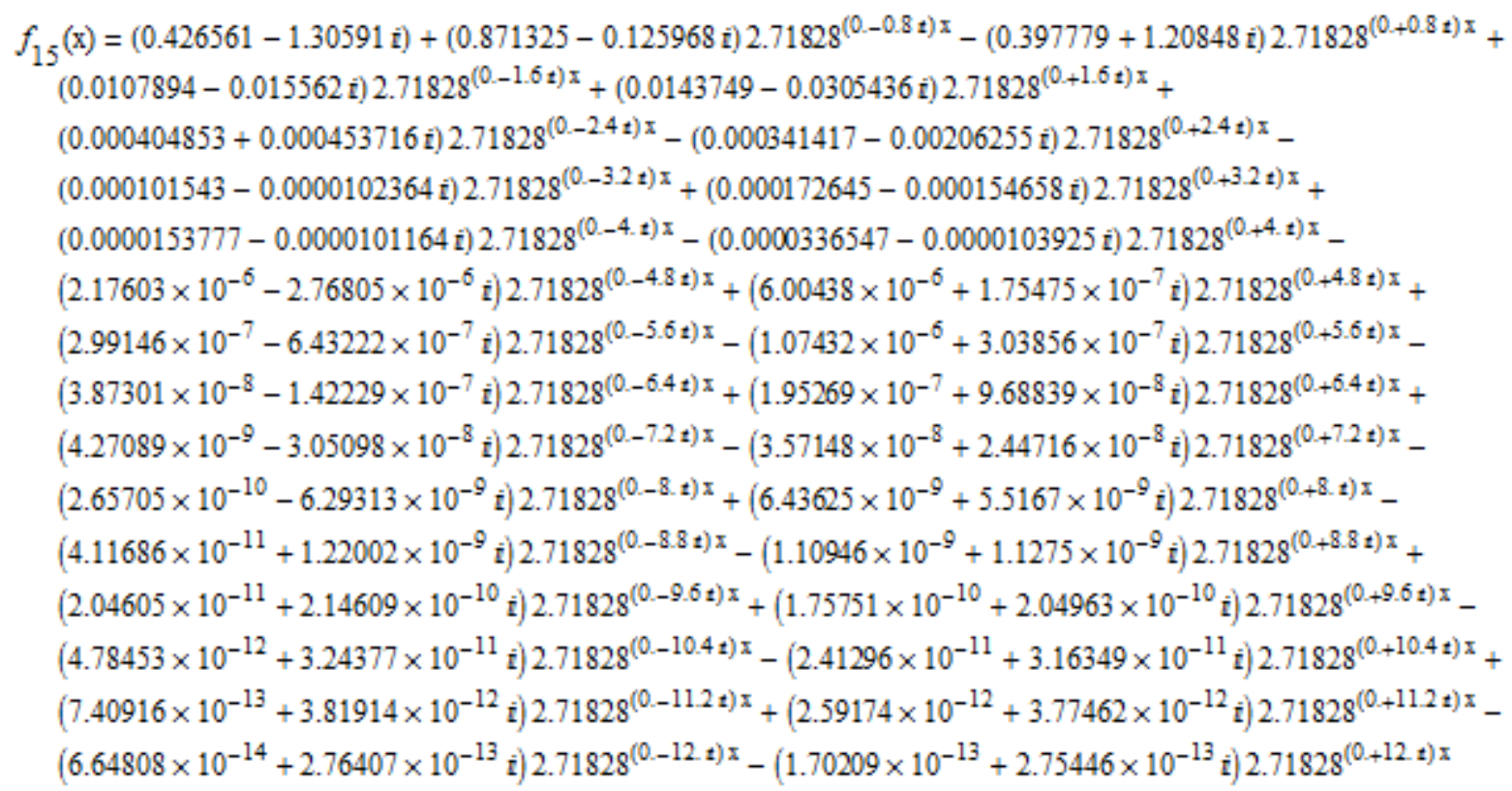

Figura 61: Gráfico da parte real da solução aproximada da EDO de $7^{\mathrm{a}}$ ordem

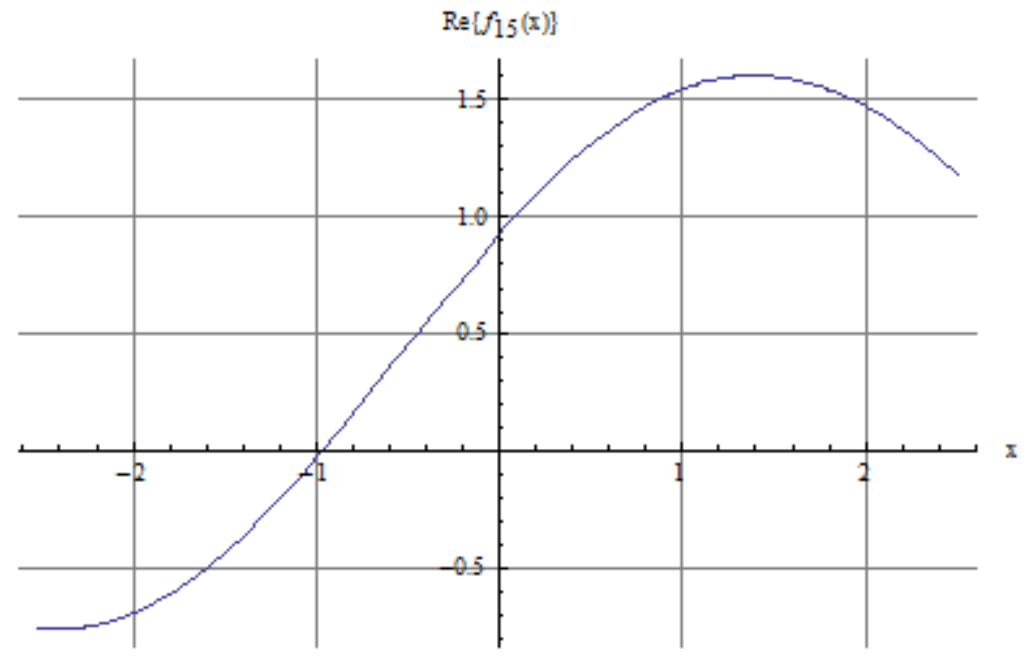

Na figura (64) aparece o gráfico comparativo entre as curvas paramétricas complexas de $\mathcal{D}_{e q}^{15} f_{\ell}(x)$ e $\phi(x)$, mostrando que se ajustam perfeitamente. A simples observação do gráfico nos permite concluir que os restos locais devem ser de ordem muito reduzida, tanto para a parte real quanto para a imaginária, o que pode ser formalmente verificado nos gráficos das figuras (65), (66) e (67). Curiosamente, a ordem do resto real neste exemplo é menor que a do resto imaginário. 
Figura 62: Gráfico da parte imaginária da solução aproximada da EDO de $7^{\mathrm{a}}$ ordem

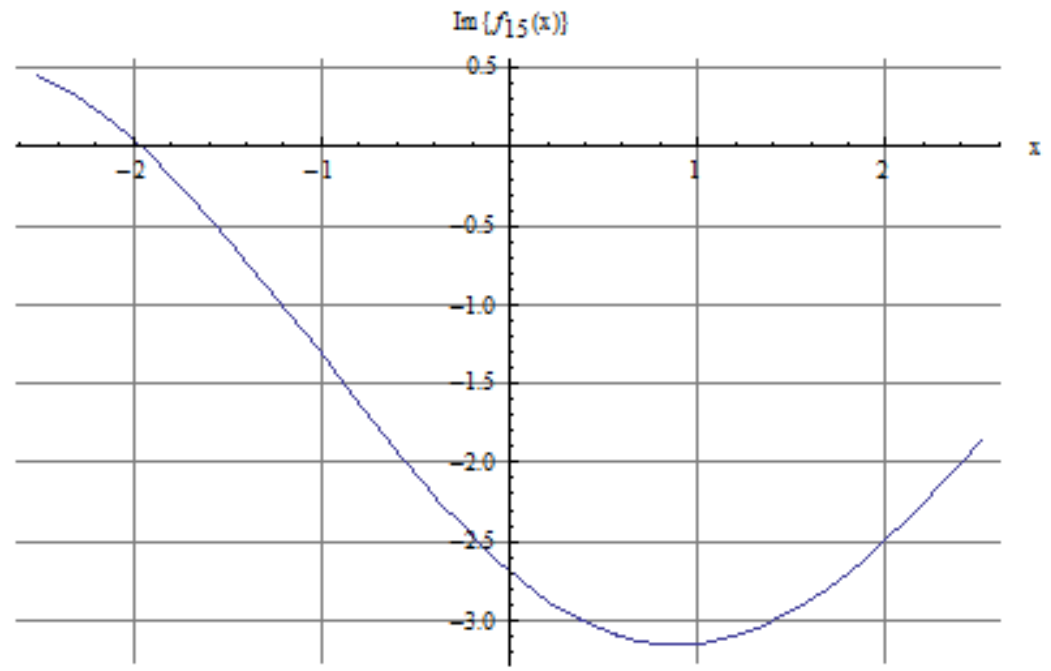

Figura 63: Curva paramétrica complexa da solução aproximada da EDO de $7^{\mathrm{a}}$ ordem

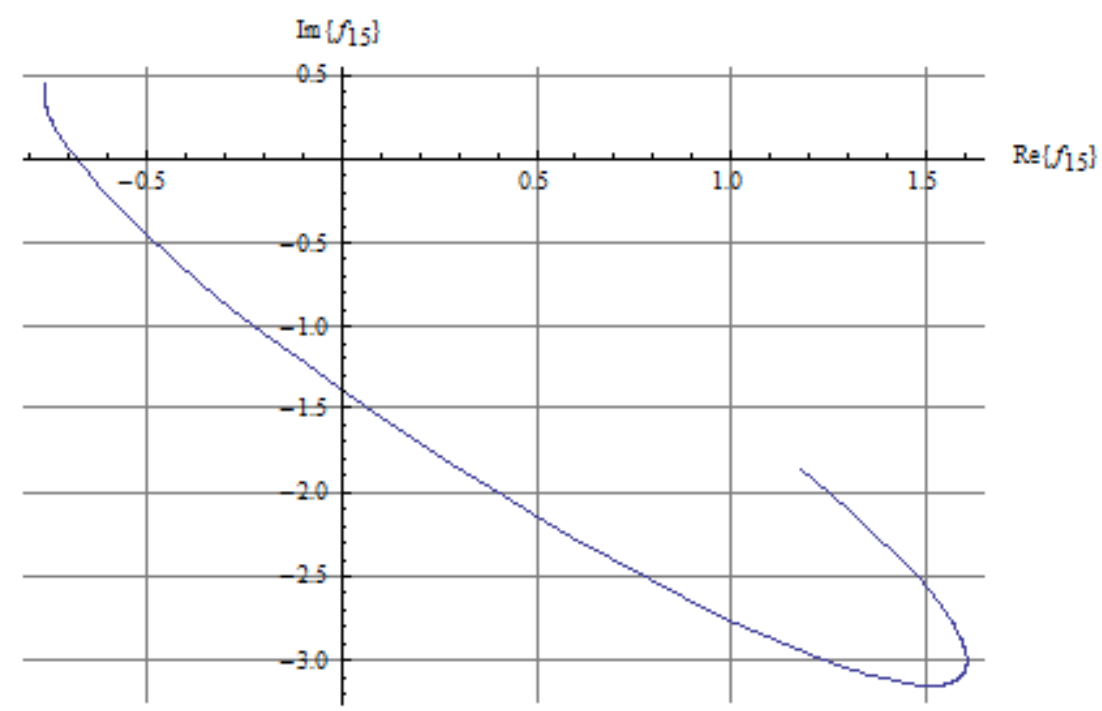


Figura 64: Comparação gráfica entre as curvas paramétricas de $\mathcal{D}_{e q} f_{15}(x)$ e $\phi(x)$

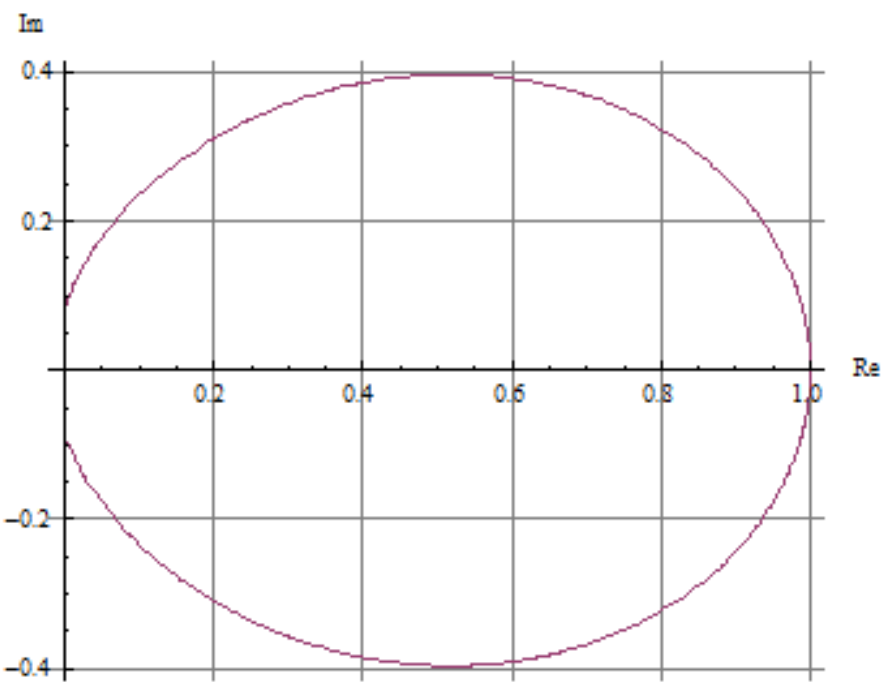

Figura 65: Gráfico da parte real da função-resto $\mathcal{R}_{15}(x)$

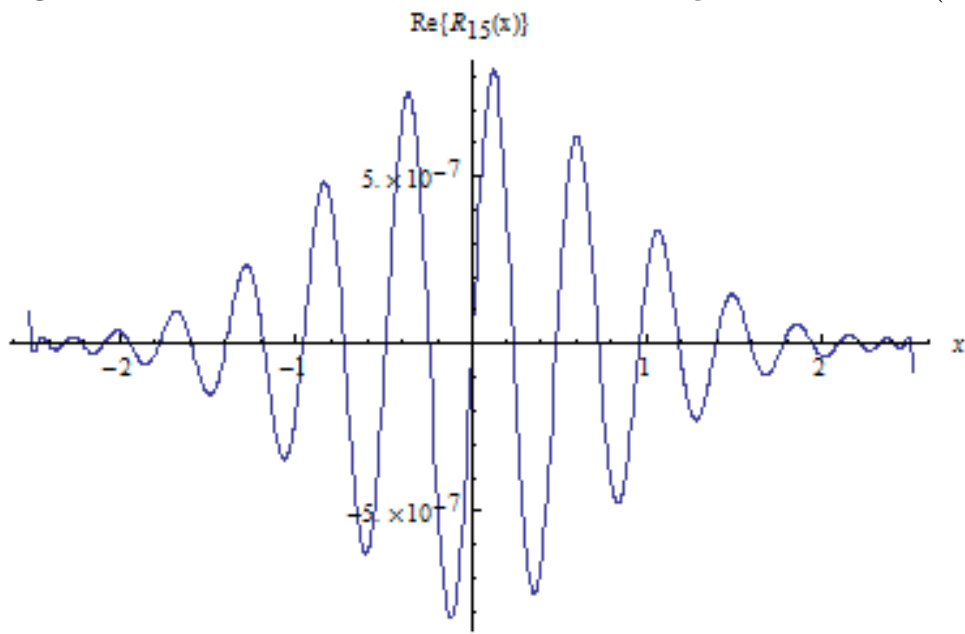

Figura 66: Gráfico da parte imaginária da função-resto $\mathcal{R}_{15}(x)$

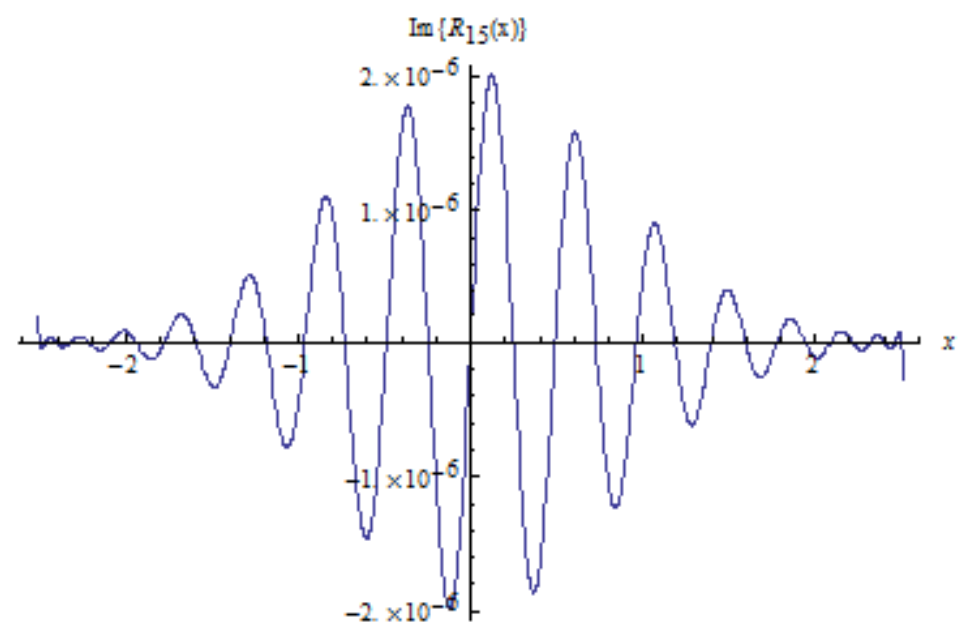


Figura 67: Curva paramétrica complexa da função resto $\mathcal{R}_{15}(x)$

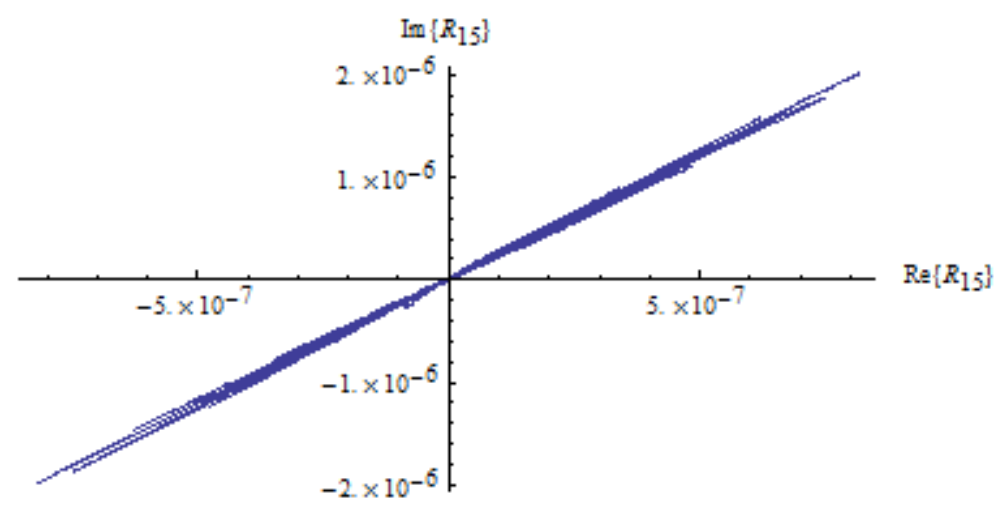

Como dissemos antes, também acrescentamos os gráficos de superfície (fig.68) e, por fim, na figura (69), os mapas paramétricos cartesiano e polar da solução aproximada $f_{15}(x)$, os quais, infelizmente, devido à sua extrema complexidade, não permitem extrair informação suficiente para compreendê-los de maneira clara. De qualquer forma, permanecem como uma adendo enriquecedor sobre a natureza da solução.

Figura 68: Gráficos de superfície das partes real, imaginária e absoluta de $f_{15}(x)$
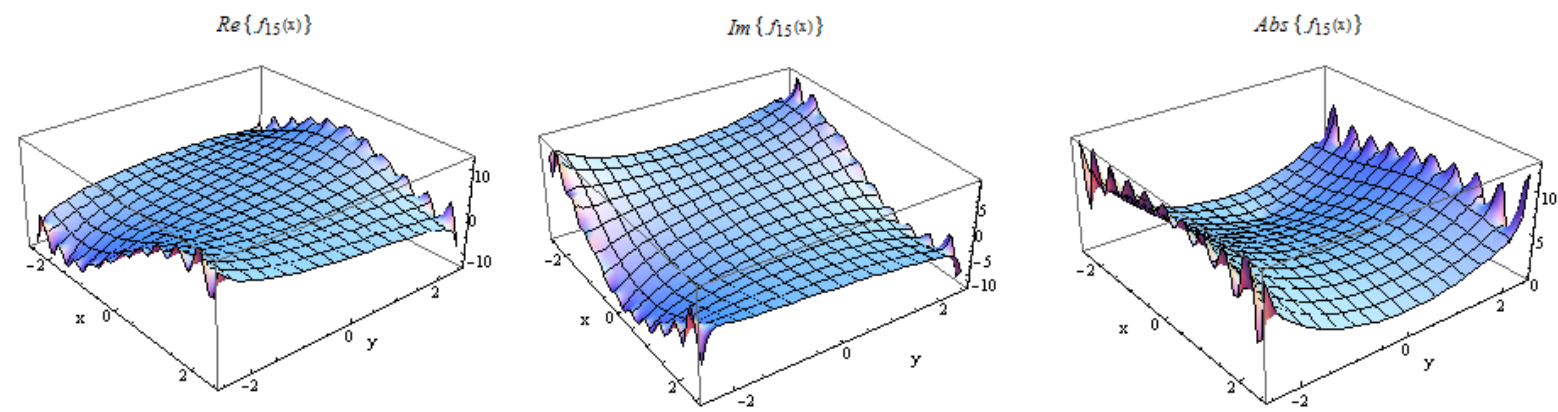

Figura 69: Mapas paramétricos cartesiano e polar da solução aproximada $f_{15}(x)$
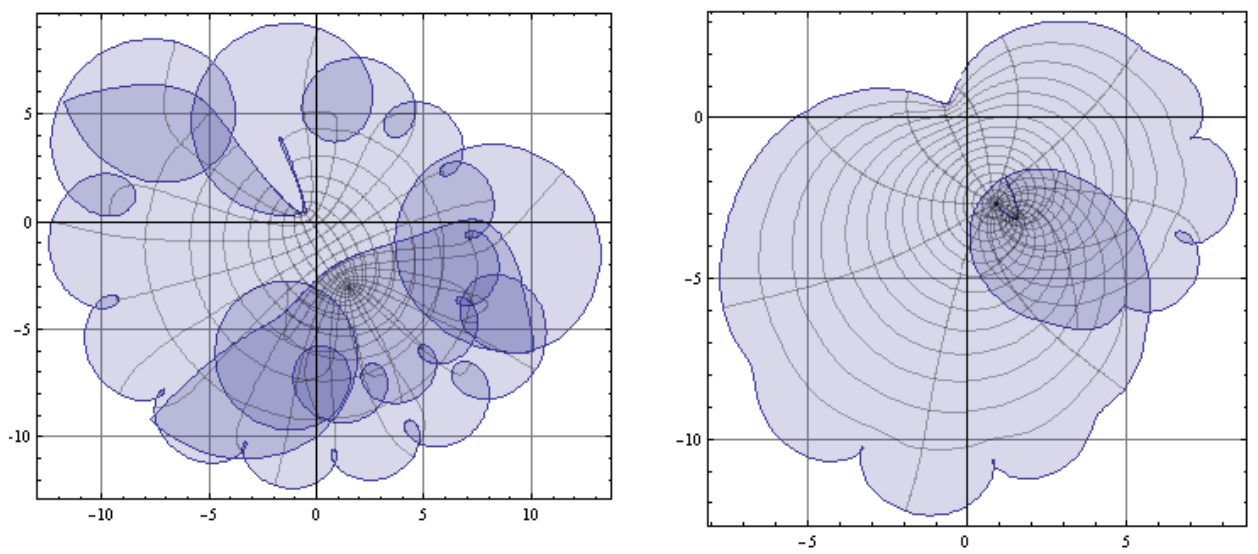


\subsubsection{Comentários sobre os Resultados Obtidos na Resolução de EDOs}

Pesando tudo o que foi visto até agora com relação às EDOs, podemos afirmar que o método proposto afigura-se como um processo extremamente eficiente de resolução, mostrando-se preciso e confiável até para equações de alta ordem, mesmo com ranges relativamente baixos, como os utilizados aqui.

No método NBV a solução apresenta-se mais precisa com range mais reduzido do que nos outros procedimentos, i.e, BVP e IVP, o que é fácil de ser explicado, se levarmos em conta os aspectos já abordados na seção dos nodos, ou seja, a modelagem da solução pelo termo independente é melhor naquele caso, em que nenhuma condição é imposta sobre ela. 


\section{APLICAÇÕES FRACIONAIS E ANÁLISE DOS RESULTADOS}

Neste capítulo, expandimos o método para aplicações no cálculo fracional, desenvolvendo a abordagem dos operadores fracionais de Weyl para funções periódicas genéricas e procedendo a uma extensão das matrizes operacionais a ordens arbitrárias.

Com isso, estamos aptos a resolver equações diferenciais parciais fracionárias nãohomogêneas, aplicando a técnica desenvolvida nos capítulos anteriores pelo formalismo coadjunto no método de modalagem operacional NBV/NIV.

\subsection{Matrizes Operacionais Fracionárias}

Até agora estivemos calculando as matrizes operacionais de derivadas e integrais clássicas, quer dizer, de ordens inteiras. A partir de agora, vamos estender esses cálculos aos casos de ordens fracionárias, baseados na teoria exposta na última parte do capítulo 3. Lá, foi determinada a matriz de integração clássica da base complexa de Fourier e foi discutido o surgimento dos termos constantes adicionais provenientes da presença do termo $C^{0}$.

A matriz de integração clássica calculada no capítulo anterior apresenta, no entanto, aplicação restrita, visto que há questões conceituais envolvidas que só podem ser entendidas e enfrentadas com os recursos da abordagem fracional.

A questão principal resume-se ao seguinte: se quisermos operar sobre funções polinomiais, por exemplo, isto é, derivá-las e integrá-las por meio de matrizes operacionais, é necessário utilizar as IFRL no processo de integração, a fim de evitar singularidades no cálculo, mesmo de ordem inteira. Entretanto, isso acarreta o surgimento de termos adicionais, modificando a forma da MOI anteriormente determinada, por meio de primitivação 
simples, pois a base de Fourier complexa é constituída de exponenciais, cuja IFRL produz constantes. A eliminação dessas constantes na integração fracional é obtida pelo uso das IFL.

Já para operações efetuadas sobre funções exponenciais e trigonométricas complexas correlatas, tanto a IFRL quanto a IFL podem ser utilizadas, com relativa vantagem para esta última, por não gerar constantes adicionais.

Esse fato significa que é preciso recalcular a matriz de integração da base complexa de Fourier para um caso mais geral usando as IFR com terminal anterior $c$ genérico e estabelecer os casos particulares para IFRL e IFL, com $c=0$ e $c \rightarrow-\infty$, respectivamente.

O tipo de operador usado dependerá da natureza da função sobre a qual se deseja realizar a operação.

Um fato notável referente ao ramo negativo das séries de Laurent deve ser ressaltado. Os expoentes negativos exigem o uso de IFL para evitar infinitos no cálculo das integrais. Porém, isso não se aplica ao expoente -1, visto que a função logarítmica resultante da integração ${ }_{c} D_{x}^{-1}$ apresenta a constante adicional $k=-\ln c$, o que gera infinitos, tanto para IFRL quanto para IFL. Isso advém do fato de o resíduo estar associado a uma singularidade essencial. Portanto, é preciso cautela ao tratar de funções desse tipo.

\subsubsection{MOI de Fourier complexa pela IFRL}

Dito isto, vamos recalcular a matriz de integração na base complexa de Fourier usando a IFRL.

Dada a função $f_{\ell}(x)$, a função integrada será

$$
\left|F_{\ell}\right\rangle_{\infty}={ }_{0} D_{x}^{-1}\left|f_{\ell}\right\rangle_{\infty}=\sum_{k=-\ell}^{\ell} c^{k}\left({ }_{0} D_{x}^{-1}\left|e_{k}\right\rangle_{\infty}\right)=\sum_{k=-\ell}^{\ell} c^{k} \int_{0}^{x} e_{k}(u) d u
$$

Essa integração deve ser feita para dois casos distintos: $k \neq 0$ e $k=0$, obtendo-se:

$$
\int_{0}^{x} e_{k}(u) d u=\left\{\begin{array}{cc}
\frac{\exp (i k q x)-1}{i k q}, \quad k \neq 0 \\
x, \quad k=0
\end{array}\right.
$$

Disso, segue

$$
\left|F_{\ell}\right\rangle_{\infty}=\sum_{\substack{k=-\ell \\ k \neq 0}}^{\ell} \frac{c^{k}}{i k q}\left(e^{i k q x}-1\right)+c^{0} x
$$


Agora é preciso desenvolver o termo em $x$ na base complexa de Fourier. Isso já foi feito no capítulo 3 , fornecendo os coeficientes

$$
\alpha^{k}=\frac{(-1)^{k} i}{k q} \quad(k \neq 0), \quad \alpha^{0}=0,
$$

e, portanto,

$$
x \cong \sum_{\substack{k=-\ell \\ k \neq 0}}^{\ell} \frac{(-1)^{k} i}{k q} e^{i k q x}
$$

o que conduz a

$$
\left|F_{\ell}\right\rangle=\sum_{\substack{k=-\ell \\ k \neq 0}}^{\ell} \frac{i}{k q}\left\{\left[(-1)^{k} c^{0}-c^{k}\right]\left|e_{k}\right\rangle+c^{k}\left|e_{0}\right\rangle\right\}
$$

Agora, efetuando uma translação de range: $k \longrightarrow r-\ell-1, r \in[1, n] \subset \mathbb{N}$, com $n=2 \ell+1$, reescrevemos a base e os coeficientes da seguinte maneira: $\left|e_{k}\right\rangle \longrightarrow\left|E_{r}\right\rangle \equiv$ $\exp [i(r-\ell-1) q x]$ e $c^{k} \longrightarrow C^{r}=c^{r-\ell-1}$, conduzindo a

$$
\left|F_{\ell}\right\rangle=\sum_{\substack{r=1 \\ r \neq \ell+1}}^{n} \frac{i}{(r-\ell-1) q}\left\{\left[(-1)^{r-\ell-1} C^{\ell+1}-C^{r}\right]\left|E_{r}\right\rangle+C^{r}\left|E_{\ell+1}\right\rangle\right\}
$$

Mas

$$
\begin{aligned}
\left|F_{\ell}\right\rangle & =\sum_{r=1}^{n} \mathcal{C}^{r}\left|E_{r}\right\rangle=\sum_{\substack{r=1 \\
r \neq \ell+1}}^{n} \mathcal{C}^{r}\left|E_{r}\right\rangle+\mathcal{C}^{\ell+1}\left|E_{\ell+1}\right\rangle \Rightarrow \\
\mathcal{C}^{r} & =\frac{i}{(r-\ell-1) q}\left[(-1)^{r-\ell-1} C^{\ell+1}-C^{r}\right], \quad r \neq \ell+1 \\
\mathcal{C}^{\ell+1} & =\sum_{\substack{r=1 \\
r \neq \ell+1}}^{n} \frac{i}{(r-\ell-1) q} C^{r}
\end{aligned}
$$

Porém, da equação dos coeficientes da matriz de integração

$$
\langle\mathcal{C}|=\langle C| J \Rightarrow \mathcal{C}^{r}=\sum_{s=1}^{n} C^{s} J_{s}^{r}
$$

e das equações dos coeficientes reescritas como

$$
\begin{aligned}
\mathcal{C}^{r} & =C^{\ell+1}\left[\frac{i(-1)^{r-\ell-1}}{(r-\ell-1) q}\right]+\sum_{\substack{s=1 \\
s \neq \ell+1}}^{n} C^{s}\left[\frac{-i \delta_{s}^{r}}{(s-\ell-1) q}\right], \quad(r \neq \ell+1) \\
\mathcal{C}^{\ell+1} & =\sum_{\substack{s=1 \\
s \neq \ell+1}}^{n} C^{s}\left[\frac{i}{(s-\ell-1) q}\right]
\end{aligned}
$$


obtemos os elementos da matriz operacional de integração para $r, s \neq \ell+1$ :

$$
\begin{aligned}
J_{\ell+1}{ }^{r} & =\frac{i(-1)^{r-\ell-1}}{(r-\ell-1) q} \\
J_{s}^{\ell+1} & =\frac{i}{(s-\ell-1) q} \\
J_{s}{ }^{r} & =\frac{-i \delta_{s}{ }^{r}}{(s-\ell-1) q}
\end{aligned}
$$

Portanto, a matriz operacional de ${ }_{0} D_{x}^{-1}$ na base complexa de Fourier é:

$$
J=\frac{i}{q}\left[\begin{array}{ccccccc}
1 / \ell & 0 & \cdots & -1 / \ell & \cdots & 0 & 0 \\
0 & \frac{1}{\ell-1} & \cdots & -\frac{1}{\ell-1} & \cdots & 0 & 0 \\
\vdots & \vdots & \ddots & \vdots & \cdots & \vdots & \vdots \\
-\frac{(-1)^{\ell}}{\ell} & -\frac{(-1)^{\ell-1}}{\ell-1} & \cdots & 0 & \cdots & \frac{(-1)^{\ell-1}}{\ell-1} & \frac{(-1)^{\ell}}{\ell} \\
\vdots & \vdots & \vdots & \vdots & \ddots & \vdots & \vdots \\
0 & 0 & \cdots & \frac{1}{\ell-1} & \cdots & -\frac{1}{\ell-1} & 0 \\
0 & 0 & \cdots & 1 / \ell & \cdots & 0 & -1 / \ell
\end{array}\right]
$$

Comparando esse resultado com o obtido no caso clássico, percebemos que a única diferença consiste no aparecimento de termos não-nulos na coluna central da matriz. Embora esse acréscimo não fosse possível no cálculo clássico, ele é fundamental para se obter resultados válidos na integração mesmo para ordens inteiras.

Vamos voltar ao exemplo da exponencial apresentado na primeira seção do capítulo 4, no qual se registrou uma translação indesejada no gráfico resultante do processo de integração operacional clássica com range 12 para semiperíodo $\pi$. Refazendo-se os cálculos com a nova MOI em lugar da antiga, esse efeito é parcialmente corrigido, conforme se pode ver na figura (70). O erro real total da integração operacional no período cai de 23.76 para 7.938 .

Para que efeitos translacionais desse tipo não surjam no processo de integração, é preciso uma abordagem diferente, apropriada a funções periódicas. O tratamento correto será encontrado no método de Weyl.

A pergunta que pode surgir agora é se as ordens fracionárias de integração poderiam ser obtidas diretamente da matriz acima por simples exponenciação. 
Figura 70: Gráficos comparativos da expansão e das integrais real e operacional por IFRL da função exponencial
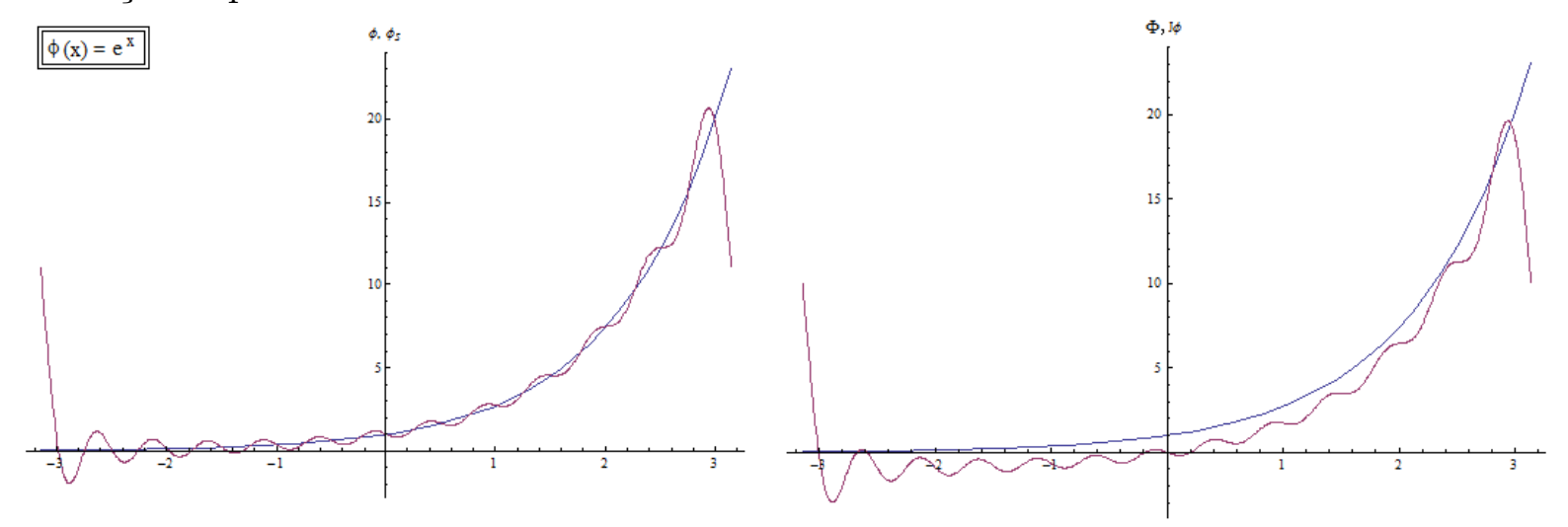

\subsubsection{MOI fracional de Fourier complexa pela IFRL}

Nosso objetivo nessa altura é calcular a matriz operacional de integração de ordem arbitrária. Há mais de um modo de fazer isto, mas vamos considerar o cálculo pela IFRL. Já sabemos que a IFRL de uma função exponencial é

$$
{ }_{0} D_{x}^{-\nu} e^{\lambda x}=\frac{\lambda^{-\nu} e^{\lambda x}}{\Gamma(\nu)} \gamma(\nu, \lambda x)
$$

e a de uma constante é

$$
{ }_{0} D_{x}^{-\nu} k=\frac{k x^{\nu}}{\Gamma(\nu+1)}, \quad(\Re\{\nu\}>0)
$$

Portanto, segue diretamente que

$$
\begin{aligned}
{ }_{0} D_{x}^{-\nu}\left|e_{m}\right\rangle & =\frac{(i m q)^{-\nu} \gamma(\nu, i m q x)}{\Gamma(\nu)}\left|e_{m}\right\rangle, \quad(m \neq 0) \\
{ }_{0} D_{x}^{-\nu}\left|e_{0}\right\rangle & =\frac{x^{\nu}}{\Gamma(\nu+1)}
\end{aligned}
$$

Substituindo $\nu$ por 1, conclui-se que

$$
{ }_{0} D_{x}^{-1}\left|e_{m}\right\rangle=\left\{\begin{array}{cc}
\frac{\exp (i m q x)-1}{i m q}, & m \neq 0 \\
x, & m=0
\end{array}\right.
$$

Este resultado é idêntico ao da eq.(5.1), obtido pelo cálculo direto.

Assim, a IFRL de ordem arbitrária de uma função $\left|f_{\ell}\right\rangle$ será

$$
{ }_{0} D_{x}^{-\nu}\left|f_{\ell}\right\rangle={ }_{0} D_{x}^{-\nu}\langle c \mid e\rangle={ }_{0} D_{x}^{-\nu} \sum_{m=-\ell}^{\ell} c^{m} e_{m}(x)=\sum_{m=-\ell}^{\ell}{ }_{0} D_{x}^{-\nu}\left(c^{m} e_{m}(x)\right)
$$


Mas, pela regra fracional de Leibniz para diferintegrais (HILFER et al., 2000, p.16), obtémse

$$
{ }_{0} D_{x}^{-\nu}\left|f_{\ell}\right\rangle=\sum_{m=-\ell}^{\ell} \sum_{k=0}^{\infty}\left(\begin{array}{l}
\nu \\
k
\end{array}\right){ }_{0} D_{x}^{-\nu-k} e_{m}(x) D^{k} c^{m}=\sum_{m=-\ell}^{\ell} c^{m}{ }_{0} D_{x}^{-\nu} e_{m}(x)
$$

Então, usando a fórmula da IFRL de exponencial, chega-se a

$$
{ }_{0} D_{x}^{-1}\left|f_{\ell}\right\rangle=\sum_{|m|>0} \frac{c^{m}}{i m q}\left(e^{i m q x}-1\right)+c^{0} x
$$

Naturalmente, esse resultado é idêntico ao obtido diretamente. Contudo, isso só significa que a redução da IFRL de ordem arbitrária ao caso particular para ordem 1 é consistente, mas não implica o inverso, ou seja, que a exponenciação fracionária da MOI de ordem 1 forneça, necessariamente, o caso geral. A comprovação dessa hipótese exigiria uma pouco mais de trabalho. Mas o que salta aos olhos aqui é que, de qualquer maneira, evidencia-se uma complicação crescente nas integrais subsequentes, sejam de ordem inteira ou não. Mesmo no caso das derivadas, o cálculo não é trivial, ainda que se use a derivada fracional de Caputo. Como vimos, a DFC de uma exponencial vale

$$
\mathcal{D}^{\nu} e^{\lambda x}=\frac{\lambda^{\nu} e^{\lambda x}}{\Gamma(\lceil\nu\rceil-\nu)} \gamma(\lceil\nu\rceil-\nu, \lambda x),
$$

o que implica

$$
\mathcal{D}^{\nu} e^{i m q x}=\frac{\lambda^{\nu} e^{i m q x}}{\Gamma(\lceil\nu\rceil-\nu)} \gamma(\lceil\nu\rceil-\nu, i m q x),
$$

mostrando que a determinação da MOD fracional deve ser uma função não elementar da variável $x$. Como forma de melhorar a operacionalidade das MOps fracionais faz-se necessário, portanto, investigar caminhos alternativos. Essa é a motivação para estudarmos os operadores de Weyl.

\subsubsection{Operadores Fracionais de Weyl}

Uma vez que estamos trabalhando com funções periódicas, é mais do que justo dedicar uma parte aos operadores fracionais de Weyl, que foram concebidos justamente com esse propósito. O problema de integrar e derivar funções periódicas a ordens arbitrárias foi primeiramente estudado por volta de 1917 pelo matemático, físico e filófoso alemão Hermann Weyl (1885-1955), já que o operador de Riemann-Liouville geralmente não se aplica a funções periódicas, como a de Fourier, sendo mais adequado para funções expandidas em séries do tipo Jacobi.

Weyl estudou funções de Fourier complexas no círculo unitário $\mathbb{G}=\mathbb{R} / 2 \pi \mathbb{Z}$, corres- 
pondentes a um período de $2 \pi$ sobre a reta real (HILFER et al., 2008), mas vamos estender o tratamento a um período genérico $T=2 L$.

\subsubsection{Integral Fracional de Weyl}

Suponhamos $f(x)$ uma função $\mathcal{L}^{1}$. Já sabemos que funções $\mathcal{L}^{1}$ podem ser expandidas em série de Fourier complexas e, portanto, $f(x)$ será

$$
f(x)=\sum_{r=-\infty}^{\infty} c^{r} e_{r}(x)
$$

onde, como de costume, $e_{k}(x)=\exp (i k q x)$. Por outro lado, funções periódicas em $2 \pi$ na reta real correspondem a funções no círculo unitário $\mathbb{G}=\mathbb{R} / 2 \pi \mathbb{Z}$, o que nos permite relacionar as integrais na reta com as do círculo unitário. Todavia, vamos realizar a correspondência para um período mais geral $T=2 L$, no intervalo $I=[-L, L], L \in \mathbb{R}$.

Seja então $f(x)$ uma função $\mathcal{L}^{1}$ periódica definida em $I$, tal que sua integral seja nula nesse período, o que implica $c_{0}=0$, pois, devido à ortogonalidade da base de Fourier no período considerado, $\left\langle e_{0} \mid e_{r}\right\rangle_{\infty}=\frac{1}{2 L} \int_{-L}^{L} e_{r}(x) d x=\delta_{0 r}$, a integral da série no intervalo periódico $I$ fornece $f^{(-1)}(x)=2 L c_{0}=0 \Leftrightarrow c_{0}=0$. Esta condição é importante porque a existência de um termo $c_{0}$ não-nulo implicaria a divergência da série complexa de Fourier da integral da função:

$$
f^{(-1)}(x)=\sum_{r=-\infty}^{\infty} c^{r} \frac{e_{r}(x)}{i r q}
$$

No entanto, com essa condição satisfeita, a integral de $f$ é ela própria uma função periódica; ademais podemos escolher a constante de integração de forma que o valor da segunda integral também seja nula no período considerado. Dessa maneira, substituindo na expansão de $f^{(-1)}$ a expressão explícita para $c^{r}=\frac{1}{2 L} \int_{-L}^{L} \bar{e}_{r}(y) f(y) d y$, tem-se:

$$
f^{(-1)}(x)=\frac{1}{2 L} \int_{-L}^{L} f(y) \sum_{\substack{r=-\infty \\ r \neq 0}}^{\infty} \frac{e_{r}(x-y)}{i r q} d y
$$

De fato, como foi observado antes, para que a integral anterior convirja, devemos ter $r \neq 0$ na somatória. Consequentemente, se esse procedimento for repetido $n$ vezes, então

$$
f^{(-n)}(x)=\sum_{k=-\infty}^{\infty} c^{k} \frac{e_{k}(x)}{(i k q)^{n}}=\frac{1}{2 L} \int_{-L}^{L} f(y) \sum_{\substack{r=-\infty \\ r \neq 0}}^{\infty} \frac{e_{r}(x-y)}{(i r q)^{n}} d y
$$

Lembrando que, na equação (F.56), foi definida uma convolução cíclica para funções 
periódicas $f$ e $g$ num intervalo $I=[-L, L]$, como

$$
(f * g)_{L}(x)=\frac{1}{2 L} \int_{-L}^{L} f(x-u) g(u) d u=\sum_{r=-\infty}^{\infty} \hat{f}^{r} \hat{g}^{r} e^{i r q x},
$$

sendo $\hat{f}^{r}$ e $\hat{g}^{r}$ os coeficientes de Fourier das séries $f$ e $g$, respectivamente, onde usamos a propriedade de ortogonalidade da base de Fourier na última igualdade, vemos que a expressão da $n$-ésima integral pode ser escrita como uma convolução cíclica, i.e.,

$$
f^{(-n)}(x)=\left(\Psi^{n} * f\right)_{L}(x)
$$

onde se definiu

$$
\Psi^{n}(x):=\sum_{|r|>0} \frac{e_{r}(x)}{(i r q)^{n}}
$$

Isso sugere uma expansão para ordens genéricas $\nu \in \mathbb{C}$, através da definição do operador $\Psi^{\nu}(x)$. Logo, define-se o operador de integração de Weyl de ordem $\nu, \Re(\nu) \in(0,1)$, como:

$$
\Psi_{ \pm}^{\nu}(x)=\sum_{|r|>0} \frac{\exp (i r q x)}{( \pm i r q)^{\nu}}
$$

Assim, a ação do operador integral de Weyl sobre uma função $f(x)$ toma a forma

$$
f_{\nu}(x):=\left(\Psi_{ \pm}^{\nu} * f\right)_{L}(x)=\frac{1}{2 L} \int_{-L}^{L} \Psi_{ \pm}^{\nu}(x-y) f(y) d y, \quad(0<\Re(\nu)<1)
$$

onde $f_{\nu}$ é a integral fracional de Weyl de ordem $\nu$.

Devemos observar que a definição aqui introduzida para o operador de Weyl difere da usual, que é construída para funções de período $2 \pi$ e tem a forma

$$
\Psi_{ \pm}^{\nu}(x)=\sum_{|k|>0} \frac{\exp (i k x)}{( \pm i k)^{\nu}}
$$

Miller e Ross (1993) utilizam, para a integral fracional de Weyl, a notação (BUTZER; WESTPHAL, 2000, p.7):

$$
{ }_{x} W_{\infty}^{-\nu} f(x)=\left(\Psi_{-}^{\nu} * f\right)(x) \equiv{ }_{x} D_{\infty}^{-\nu} f(x)
$$

Uma outra notação consagrada é a indicação da ordem da integral ou da derivada diretamente na letra que representa a função, por meio de um índice subscrito para a integral $\left(f_{\nu}(x)\right)$ e sobrescrito para a derivada $\left(f^{\nu}(x)\right)$, conforme utilizado acima. No entanto, não usaremos tal notação para evitar confusão com os índices de range e matriciais. Em vez 
disso, denotaremos a integral de Weyl por ${ }_{L} W_{ \pm}^{-\nu}$ e a derivada de Weyl por ${ }_{L} W_{ \pm}^{\nu}$, onde o subíndice esquerdo $L$ faz menção à definição em um período genérico aqui utilizada. Assim, escreve-se:

$$
{ }_{L} W_{ \pm}^{-\nu} f(x):=\left(\Psi_{ \pm}^{\nu} * f\right)_{L}(x)=\frac{1}{2 L} \int_{-L}^{L} \Psi_{ \pm}^{\nu}(x-y) f(y) d y, \quad(0<\Re(\nu)<1)
$$

Deve-se notar que, devido à periodicidade do integrando e à propriedade periódica da convolução circular, vale também a expressão:

$$
{ }_{L} W_{ \pm}^{-\nu} f(x)=\frac{1}{2 L} \int_{-L}^{L} \Psi_{ \pm}^{\nu}(y) f(x-y) d y
$$

Pode-se mostrar (ZYGMUND, 2002, Vol.2) que, caso a série $\Psi_{ \pm}^{\nu}(x)$ convirja, então a integral fracional de Weyl coincidirá com a de Liouville, ou seja,

$$
\begin{aligned}
& \left(\Psi_{+}^{\nu} * f\right)(x) \equiv{ }_{-\infty} D_{x}^{-\nu} f(x)=\frac{1}{\Gamma(\nu)} \int_{-\infty}^{x}(x-u)^{\nu-1} f(u) d u \\
& \left(\Psi_{-}^{\nu} * f\right)(x) \equiv{ }_{x} D_{\infty}^{-\nu} f(x)=\frac{1}{\Gamma(\nu)} \int_{x}^{\infty}(u-x)^{\nu-1} f(u) d u
\end{aligned}
$$

Mostraremos a seguir, através da abordagem desenvolvida na tese, que o operador de Weyl conforme definido nessa seção satisfaz as mesmas relações para o caso específico de funções expandidas em séries de Fourier complexas.

\subsubsection{Equivalência Weyl-Liouville}

Já foi visto que a aplicação da integral fracional de Liouville de ordem $\nu$ sobre um elemento $\left|e_{m}\right\rangle_{\infty}$ da base complexa de Fourier, para $0<\Re(\nu)<1$ e $m \neq 0$, fornece

$$
\begin{gathered}
D_{-\infty}^{-\nu}\left|e_{m}\right\rangle_{\infty}=\frac{1}{(i m q)^{\nu}}\left|e_{m}\right\rangle_{\infty} \Rightarrow \\
\sum_{s=-\ell}^{\ell}\left(D_{-\infty}^{-\nu}\right)_{m}{ }^{s}\left|e_{s}\right\rangle_{\infty}=\sum_{\substack{s=-\ell \\
s \neq 0}}^{\ell} \frac{\delta_{m}{ }^{s}}{(i m q)^{\nu}}\left|e_{s}\right\rangle_{\infty} \Rightarrow \\
\left(D_{-\infty}^{-\nu}\right)_{m}{ }^{s}=\frac{\delta_{m}^{s}}{(i m q)^{\nu}}, \quad m \neq 0
\end{gathered}
$$

Logo, a aplicação desse operador sobre a expansão de Fourier de dimensão infinita de uma função $\mathcal{L}^{1}$ gera, de acordo com a equação (3.32) e com a regra generalizada de 
Leibniz:

$$
D_{-\infty}^{-\nu}|f\rangle=\left\langle c\left|D_{-\infty}^{-\nu}\right| e\right\rangle=\lim _{\ell \rightarrow \infty} \sum_{r=-\ell}^{\ell} \sum_{s=-\ell}^{\ell} c^{r}\left(D_{-\infty}^{-\nu}\right)_{r}^{s}\left|e_{s}\right\rangle_{\infty}=\lim _{\ell \rightarrow \infty} \sum_{r=-\ell}^{\ell} \sum_{\substack{s=-\ell \\ s \neq 0}}^{\ell} \frac{c^{r} \delta_{r} s}{(i r q)^{\nu}}\left|e_{s}\right\rangle_{\infty}
$$

Efetuando a somatória em $s$, obtém-se

$$
D_{-\infty}^{-\nu}|f\rangle=\sum_{|r|>0} \frac{c^{r}}{(i r q)^{\nu}}\left|e_{r}\right\rangle_{\infty}
$$

Abrindo agora a expressão acima, substituindo $c^{r}$ por sua forma explícita, chega-se a

$$
\begin{aligned}
D_{-\infty}^{-\nu} f(x) & =\sum_{|r|>0} \frac{e^{i r q x}}{(i r q)^{\nu}} \frac{1}{2 L} \int_{-L}^{L} e^{-i r q u} f(u) d u= \\
& =\frac{1}{2 L} \int_{-L}^{L}\left(\sum_{|r|>0} \frac{e^{i r q(x-u)}}{(i r q)^{\nu}}\right) f(u) d u
\end{aligned}
$$

Mas o termo entre parênteses é exatamente o operador fracional de Weyl positivo, i.e.,

$$
D_{-\infty}^{-\nu} f(x)=\frac{1}{2 L} \int_{-L}^{L} \Psi_{+}^{\nu}(x-u) f(u) d u=\left(\Psi_{+}^{\nu} * f\right)_{L}(x)
$$

Logo,

$$
\begin{gathered}
\left(\Psi_{+}^{\nu} * f\right)_{L}(x)=D_{-\infty}^{-\nu} f(x) \equiv{ }_{L} W_{+}^{-\nu} f(x) \Rightarrow \\
{ }_{L} W_{+}^{-\nu} \equiv D_{-\infty}^{-\nu}
\end{gathered}
$$

De maneira análoga, demonstra-se a relação para o operador de Weyl negativo. i.e., ${ }_{L} W_{-}^{-\nu} \equiv{ }_{x} D_{\infty}^{-\nu}$. Com isso, podemos descrever a atuação do operador de Weyl sobre uma função periódica expandida numa série de Fourier finita por

$$
{ }_{L} W_{+}^{-\nu}\left|f_{n}\right\rangle_{\infty} \equiv\left\langle c\left|D_{-\infty}^{-\nu}\right| e\right\rangle_{0_{n}}
$$

\subsubsection{Propriedades do Operador Integral de Weyl}

Vamos enunciar alguns resultados importantes que devem ser utilizados posteriormente, na definição da derivada de Weyl:

1. Periodicidade:

$$
\Psi_{ \pm}^{\nu}(x+2 L)=\Psi_{ \pm}^{\nu}(x)
$$

A demonstração é imediata. 
2. Derivação:

$$
\frac{\partial \Psi_{ \pm}^{1-\nu}(x-y)}{\partial x}=\sum_{|r|>0}( \pm i r q)^{\nu} e^{i r q(x-y)}
$$

A demonstração é imediata.

3. Periodicidade da derivada:

$$
\frac{d \Psi_{ \pm}^{\nu}(x+2 L)}{d x}=\frac{d \Psi_{ \pm}^{\nu}(x)}{d x}
$$

A demonstração é imediata.

4. Transformada de Fourier:

$$
\mathfrak{F}\left\{\Psi_{ \pm}^{\nu}(x)\right\}=\sum_{|r|>0} \frac{\sqrt{2 \pi}}{( \pm i r q)^{\nu}} \delta(y-r q)
$$

$\underline{\text { Dem.: }}$

$$
\begin{aligned}
\mathfrak{F}\left\{\Psi_{ \pm}^{\nu}(x)\right\} & =\frac{1}{\sqrt{2 \pi}} \int_{-\infty}^{\infty} \Psi_{ \pm}^{\nu}(x) e^{-i x y} d x=\frac{1}{\sqrt{2 \pi}} \int_{-\infty}^{\infty} e^{-i x y} \sum_{|r|>0} \frac{e^{i r q x}}{( \pm i r q)^{\nu}} d x= \\
& =\sum_{|r|>0} \frac{\sqrt{2 \pi}}{( \pm i r q)^{\nu}}\left(\frac{1}{2 \pi} \int_{-\infty}^{\infty} e^{i(r q-y) x} d x\right)=\sum_{|r|>0} \frac{\sqrt{2 \pi}}{( \pm i r q)^{\nu}} \delta(y-r q)
\end{aligned}
$$

5. Convolução:

$$
\left(\Psi_{ \pm}^{\alpha} * \Psi_{ \pm}^{\beta}\right)_{L}=\Psi_{ \pm}^{\alpha+\beta}
$$

Dem.: Antes vamos estabelecer uma outra relação importante:

$$
\Psi_{ \pm}^{\alpha}(x-y) \Psi_{ \pm}^{\beta}(y-u)=\sum_{|r|>0} \sum_{|s|>0} \frac{e^{i r q x} e^{-i s q u}}{( \pm i r q)^{\alpha}( \pm i s q)^{\beta}} e^{i q(s-r) y}
$$


Daí segue, usando a relação acima,

$$
\begin{aligned}
\left(\Psi_{ \pm}^{\alpha} * \Psi_{ \pm}^{\beta}\right)_{L}(x) & =\frac{1}{2 L} \int_{-L}^{L} \Psi_{ \pm}^{\alpha}(x-y) \Psi_{ \pm}^{\beta}(y) d y= \\
& =\frac{1}{2 L} \int_{-L}^{L} \sum_{|r|>0} \sum_{|s|>0} \frac{e^{i r q x}}{( \pm i r q)^{\alpha}( \pm i s q)^{\beta}} e^{i q(s-r) y} d y= \\
& =\sum_{|r|>0} \sum_{|s|>0} \frac{e^{i r q x}}{( \pm i r q)^{\alpha}( \pm i s q)^{\beta}}\left(\frac{1}{2 L} \int_{-L}^{L} e^{i q(s-r) y} d y\right)= \\
& =\sum_{|r|>0} \sum_{|s|>0} \frac{e^{i r q x} \delta_{s}^{r}}{( \pm i r q)^{\alpha}( \pm i s q)^{\beta}}= \\
& =\sum_{|r|>0} \frac{e^{i r q x}}{( \pm i r q)^{\alpha+\beta}}= \\
& =\Psi_{ \pm}^{\alpha+\beta}(x)
\end{aligned}
$$

6. Convolução da derivada:

$$
\left(\partial_{x} \Psi_{ \pm}^{1-\alpha} * \partial_{x} \Psi_{ \pm}^{1-\beta}\right)_{L}=\partial_{x} \Psi_{ \pm}^{1-(\alpha+\beta)}
$$

Dem.: Vamos usar a relação:

$$
\partial_{x} \Psi_{ \pm}^{1-\alpha}(x-y) \partial_{y} \Psi_{ \pm}^{1-\beta}(y-u)=\sum_{|r|>0} \sum_{|s|>0} e^{i r q x} e^{-i s q u}( \pm i r q)^{\alpha}( \pm i s q)^{\beta} e^{i q(s-r) y}
$$

Partindo daí, temos

$$
\begin{aligned}
\left(\partial_{x} \Psi_{ \pm}^{1-\alpha} * \partial_{x} \Psi_{ \pm}^{1-\beta}\right)_{L}(x) & =\frac{1}{2 L} \int_{-L}^{L} \partial_{x} \Psi_{ \pm}^{1-\alpha}(x-y) \partial_{y} \Psi_{ \pm}^{1-\beta}(y) d y= \\
& =\frac{1}{2 L} \int_{-L}^{L} \sum_{|r|>0} \sum_{|s|>0} e^{i r q x}( \pm i r q)^{\alpha}( \pm i s q)^{\beta} e^{i q(s-r) y} d y= \\
& =\sum_{|r|>0} \sum_{|s|>0} e^{i r q x}( \pm i r q)^{\alpha}( \pm i s q)^{\beta}\left(\frac{1}{2 L} \int_{-L}^{L} e^{i q(s-r) y} d y\right)= \\
& =\sum_{|r|>0} \sum_{|s|>0} e^{i r q x}( \pm i r q)^{\alpha}( \pm i s q)^{\beta} \delta_{s}^{r}= \\
& =\sum_{|r|>0}( \pm i r q)^{\alpha+\beta} e^{i r q x}= \\
& =\partial_{x} \Psi_{ \pm}^{1-(\alpha+\beta)}(x)
\end{aligned}
$$


Exemplo 2 : Vamos ilustrar a aplicação da integral fracional de Weyl-Liouville efetuando o cálculo para um vetor da base complexa de Fourier. Assim, usando a definição e a propriedade de ortogonalidade da base de Fourier, tem-se:

$$
\begin{aligned}
&{ }_{L} W_{ \pm}^{-\nu}\left|e_{m}\right\rangle_{\infty}=\frac{1}{2 L} \int_{-L}^{L} \Psi_{ \pm}^{\nu}(x-y) e^{i m q y} d y= \\
&=\frac{1}{2 L} \int_{-L}^{L} \sum_{|r|>0} \frac{e^{i r q(x-y)}}{( \pm i r q)^{\nu}} e^{i m q y} d y= \\
&=\sum_{|r|>0} \frac{e^{i r q x}}{( \pm i r q)^{\nu}}\left(\frac{1}{2 L} \int_{-L}^{L} e^{i q(m-r) y} d y\right)= \\
&=\sum_{|r|>0}( \pm i r q)^{-\nu} \delta_{m}^{r}\left|e_{r}\right\rangle_{\infty}= \\
&=( \pm i m q)^{-\nu}\left|e_{m}\right\rangle_{\infty} \Rightarrow \\
&{ }_{L} W_{ \pm}^{-\nu}\left|e_{m}\right\rangle=( \pm i m q)^{-\nu}\left|e_{m}\right\rangle, \quad m \neq 0
\end{aligned}
$$

Obtivemos assim um resultado semelhante ao que já havíamos determinado em seção precedente pela derivada fracional de Liouville, conforme era esperado, dada a equivalência entre ambos.

\subsubsection{Propriedades da Integral Fracional de Weyl}

1. Semigrupo

$$
{ }_{L} W_{ \pm}^{-\alpha}{ }_{L} W_{ \pm}^{-\beta}={ }_{L} W_{ \pm}^{-\beta}{ }_{L} W_{ \pm}^{-\alpha}={ }_{L} W_{ \pm}^{-(\alpha+\beta)}
$$

Dem.:

$$
\begin{gathered}
{ }_{L} W_{ \pm}^{-\alpha}\left({ }_{L} W_{ \pm}^{-\beta} f(x)\right)=\frac{1}{2 L} \int_{-L}^{L} \Psi_{ \pm}^{\alpha}(x-y)\left[{ }_{L} W_{ \pm}^{-\beta} f(y)\right] d y= \\
=\frac{1}{2 L} \int_{-L}^{L} \Psi_{ \pm}^{\alpha}(x-y)\left[\frac{1}{2 L} \int_{-L}^{L} \Psi_{ \pm}^{\beta}(y-u) f(u) d u\right] d y= \\
=\left(\frac{1}{2 L}\right)^{2} \int_{-L}^{L} \int_{-L}^{L} \Psi_{ \pm}^{\alpha}(x-y) \Psi_{ \pm}^{\beta}(y-u) f(u) d u d y
\end{gathered}
$$

Usando a equação (5.15), tem-se

$$
\begin{gathered}
{ }_{L} W_{ \pm}^{-\alpha}\left({ }_{L} W_{ \pm}^{-\beta} f(x)\right)= \\
=\left(\frac{1}{2 L}\right)^{2} \int_{-L}^{L} \int_{-L}^{L} \sum_{|r|>0} \sum_{|s|>0} \frac{e^{i r q x} e^{-i s q u}}{( \pm i r q)^{\alpha}( \pm i s q)^{\beta}} e^{i q(s-r) y} f(u) d u d y= \\
=\frac{1}{2 L} \int_{-L}^{L} \sum_{|r|>0} \frac{e^{i r q x}}{( \pm i r q)^{\alpha}} \sum_{|s|>0} \frac{e^{-i s q u}}{( \pm i s q)^{\beta}} f(u) d u\left(\frac{1}{2 L} \int_{-L}^{L} e^{i q(s-r) y} d y\right)=
\end{gathered}
$$




$$
\begin{gathered}
=\frac{1}{2 L} \int_{-L}^{L} \sum_{|r|>0} \frac{e^{i r q x}}{( \pm i r q)^{\alpha}} \sum_{|s|>0} \frac{e^{-i s q u}}{( \pm i s q)^{\beta}} \delta_{r}{ }^{s} f(u) d u= \\
=\frac{1}{2 L} \int_{-L}^{L} \sum_{|r|>0} \frac{e^{i r q(x-u)}}{( \pm i r q)^{\alpha+\beta}} f(u) d u=\frac{1}{2 L} \int_{-L}^{L} \Psi_{ \pm}^{\alpha+\beta}(x-u) f(u) d u= \\
={ }_{L} W_{ \pm}^{-(\alpha+\beta)} f(x)
\end{gathered}
$$

Logicamente, comutando-se $\alpha$ e $\beta$, obtém-se o mesmo resultado e, portanto, fica demonstrada a propriedade.

2. Transformada de Fourier:

$$
\mathfrak{F}\left\{{ }_{L} W_{ \pm}^{-\nu} f(x)\right\}=\sum_{|r|>0} \frac{\sqrt{2 \pi}}{( \pm i r q)^{\nu}} \delta(y-r q) \hat{f}^{r}
$$

onde $\hat{f}^{r}=\left\langle e^{r} \mid f\right\rangle_{\infty}$ é o coeficiente de $f(x)$ na expansão de Fourier. Observe que o termo da somatória sem o elemento $\hat{f}^{r}$ nada mais é que $\mathfrak{F}\left\{\Psi_{ \pm}^{\nu}(x)\right\}$. Dem.:

$$
\begin{gathered}
\mathfrak{F}\left\{{ }_{L} W_{ \pm}^{-\nu} f(x)\right\}=\frac{1}{\sqrt{2 \pi}} \int_{-\infty}^{\infty} e^{-i y x} \frac{1}{2 L} \int_{-L}^{L} \sum_{|r|>0} \frac{e^{i r q(x-u)}}{( \pm i r q)^{\nu}} f(u) d u d x= \\
=\sum_{|r|>0} \frac{\sqrt{2 \pi}}{( \pm i r q)^{\nu}}\left(\frac{1}{2 \pi} \int_{-\infty}^{\infty} e^{i(r q-y) x} d x\right)\left(\frac{1}{2 L} \int_{-L}^{L} e^{-i r q u} f(u) d u\right)= \\
=\sum_{|r|>0} \frac{\sqrt{2 \pi}}{( \pm i r q)^{\nu}} \delta(y-r q) \hat{f}^{r}
\end{gathered}
$$

Observando que $\left\langle e^{r} \mid e_{m}\right\rangle=\delta_{m}{ }^{r}$, podemos determinar $\mathfrak{F}\left\{{ }_{L} W_{ \pm}^{-\nu}\left|e_{m}\right\rangle\right\}$ como um caso particular desta propriedade:

$$
\mathfrak{F}\left\{{ }_{L} W_{ \pm}^{-\alpha}\left|e_{m}\right\rangle\right\}=\frac{\sqrt{2 \pi}}{( \pm i m q)^{\nu}} \delta(y-m q), \quad(m \neq 0)
$$

De fato, comparando com o cálculo feito para a IFL (eq. F.72), vemos que fornece o mesmo resultado.

Uma outra característica que torna interessante o uso da IFW é o resultado nulo obtido na integração de uma constante:

$$
{ }_{L} W_{ \pm}^{-\nu} C=0
$$


De fato,

$$
\begin{aligned}
{ }_{L} W_{ \pm}^{-\nu} C & =\frac{1}{2 L} \int_{-L}^{L} \Psi_{ \pm}^{\nu}(x-y) C d y= \\
& =\frac{1}{2 L} \int_{-L}^{L} \sum_{|r|>0} \frac{e^{i r q(x-y)}}{( \pm i r q)^{\nu}} C d y= \\
& =\sum_{|r|>0} \frac{C e^{i r q x}}{( \pm i r q)^{\nu}}\left(\frac{1}{2 L} \int_{-L}^{L} e^{-i r q y} d y\right)= \\
& =\sum_{|r|>0}( \pm i r q)^{-\nu} C \frac{\sin (\pi r)}{\pi r}\left|e_{r}\right\rangle_{\infty}= \\
& =0
\end{aligned}
$$

\subsubsection{Derivadas Fracionais de Weyl}

Para estabelecer uma derivada fracional de Weyl, assim como nas outras abordagens, partimos da definição integral, de acordo com a expressão

$$
\left(D_{ \pm}^{\alpha} f\right)(x):= \pm \frac{d}{d x}\left(\Psi_{ \pm}^{1-\alpha} * f\right)_{L}(x), \quad 0<\Re(\alpha)<1
$$

Utilizando (5.8) e a notação antes estabelecida, tem-se

$$
{ }_{L} W_{ \pm}^{\nu} f(x):= \pm \frac{d}{d x}\left({ }_{L} W_{ \pm}^{-(1-\nu)} f(x)\right)
$$

Essa definição é conhecida como derivada de Weyl-Liouville. Derivando o operador integral de Weyl na equação acima, chega-se a

$$
{ }_{L} W_{ \pm}^{\nu} f(x)= \pm \frac{1}{2 L} \int_{-L}^{L} \frac{\partial}{\partial x} \Psi_{ \pm}^{1-\nu}(x-y) f(y) d y
$$

Existe uma outra definição de derivada a partir da integral de Weyl, chamada Derivada de Weyl-Marchaud (HILFER et al., 2008), dada por

$$
\left(D_{ \pm}^{\alpha} f\right)(x):=\frac{1}{2 \pi} \int_{0}^{2 \pi}[f(x-y)-f(x)]\left(D^{1} \Psi_{ \pm}^{1-\alpha}\right)(y) d y, \quad 0<\Re(\alpha)<1
$$

a qual, entretanto, no presente trabalho deve ser reescrita como

$$
\left({ }_{L} D_{ \pm}^{\nu} f\right)(x):=\frac{1}{2 L} \int_{-L}^{L}[f(x-y)-f(x)] D^{1} \Psi_{ \pm}^{1-\nu}(y) d y
$$

Exemplo 3 : Como exemplo de aplicação da derivada de Weyl-Liouville, vamos efetuar o cálculo para sua atuação sobre um vetor da base complexa 
de Fourier. Então, usando a propriedade da derivação do operador de Weyl, equação (5.13), segue que

$$
\begin{aligned}
{ }_{L} W_{ \pm}^{\nu}\left|e_{m}\right\rangle_{\infty}= & \frac{1}{2 L} \int_{-L}^{L} e^{i m q y} \sum_{|r|>0}( \pm i r q)^{\nu} e^{i r q(x-y)} d y= \\
= & \sum_{|r|>0}( \pm i r q)^{\nu} e^{i r q x}\left(\frac{1}{2 L} \int_{-L}^{L} e^{i q(m-r) y} d y\right)= \\
= & \sum_{|r|>0}( \pm i r q)^{\nu} \delta_{m}^{r}\left|e_{r}\right\rangle_{\infty}= \\
= & ( \pm i m q)^{\nu}\left|e_{m}\right\rangle_{\infty} \Rightarrow \\
& { }_{L} W_{ \pm}^{\nu}\left|e_{m}\right\rangle=( \pm i m q)^{\nu}\left|e_{m}\right\rangle
\end{aligned}
$$

Portanto, o resultado confere com o obtido anteriormente pela derivada fracional de Liouville, ilustrando mais uma vez a equivalência.

Exemplo 4 : A seguir, mostra-se o cálculo da derivada de Weyl-Marchaud de um vetor da base de Fourier.

$$
\begin{aligned}
{ }_{L} D_{ \pm}^{\nu}\left|e_{m}\right\rangle= & \frac{1}{2 L} \int_{-L}^{L}\left[e^{i m q(x-y)}-e^{i m q x}\right] \sum_{|r|>0}( \pm i r q)^{\nu} e^{i r q y} d y= \\
= & \frac{1}{2 L} \sum_{|r|>0}( \pm i r q)^{\nu} e^{i m q x} \int_{-L}^{L}\left[e^{i q(r-m) y}-e^{i r q y}\right] d y= \\
= & \frac{1}{2 L} \sum_{|r|>0}( \pm i r q)^{\nu}\left(2 L \delta_{m}^{r}\right)\left|e_{m}\right\rangle= \\
= & ( \pm i m q)^{\nu}\left|e_{m}\right\rangle \Rightarrow \\
& { }_{L} D_{ \pm}^{\nu}\left|e_{m}\right\rangle=( \pm i m q)^{\nu}\left|e_{m}\right\rangle
\end{aligned}
$$

Assim, exatamente como antes, obtivemos outra vez uma expressão idêntica às dos cálculos efetuados pelas derivadas fracionais de Liouville e Weyl-Liouville, demonstrando a equivalência de todas as operações.

Usando esse resultado, pode-se constatar que a derivada de Weyl-Liouville de ordem nula atua como operador identidade:

$$
{ }_{L} W_{ \pm}^{0}\left|e_{m}\right\rangle=( \pm i m q)^{0}\left|e_{m}\right\rangle=\left|e_{m}\right\rangle
$$

Um outro ponto importante é que a equivalência entre a DFW e a DFL vale também 
para as derivadas, uma vez que

$$
{ }_{L} W_{+}^{\nu} \equiv \frac{d}{d x}\left\{{ }_{L} W_{+}^{-(1-\nu)}\right\} \equiv D^{1}\left\{{ }_{-\infty} D_{x}^{-(1-\nu)}\right\} \equiv{ }_{-\infty} D_{x}^{\nu}
$$

a, analogamente,

$$
{ }_{L} W_{-}^{\nu} \equiv \frac{d}{d x}\left\{{ }_{L} W_{-}^{-(1-\nu)}\right\} \equiv D^{1}\left\{{ }_{x} D_{\infty}^{-(1-\nu)}\right\} \equiv{ }_{x} D_{\infty}^{\nu}
$$

\subsubsection{Propriedades da Derivada Fracional de Weyl}

1. Semigrupo

$$
{ }_{L} W_{ \pm}^{\alpha}{ }_{L} W_{ \pm}^{\beta}={ }_{L} W_{ \pm}^{\beta}{ }_{L} W_{ \pm}^{\alpha}={ }_{L} W_{ \pm}^{(\alpha+\beta)}
$$

$\underline{D e m}$.

$$
\begin{aligned}
& { }_{L} W_{ \pm}^{\alpha}\left({ }_{L} W_{ \pm}^{\beta} f(x)\right)=\frac{1}{2 L} \int_{-L}^{L} \partial_{x} \Psi_{ \pm}^{1-\alpha}(x-y)\left[{ }_{L} W_{ \pm}^{\beta} f(y)\right] d y= \\
= & \frac{1}{2 L} \int_{-L}^{L} \partial_{x} \Psi_{ \pm}^{1-\alpha}(x-y)\left[\frac{1}{2 L} \int_{-L}^{L} \partial_{y} \Psi_{ \pm}^{1-\beta}(y-u) f(u) d u\right] d y= \\
= & \left(\frac{1}{2 L}\right)^{2} \int_{-L}^{L} \int_{-L}^{L} \partial_{x} \Psi_{ \pm}^{1-\alpha}(x-y) \partial_{y} \Psi_{ \pm}^{1-\beta}(y-u) f(u) d u d y
\end{aligned}
$$

Usando a equação (5.17), tem-se

$$
\begin{gathered}
{ }_{L} W_{ \pm}^{\alpha}\left({ }_{L} W_{ \pm}^{\beta} f(x)\right)= \\
=\left(\frac{1}{2 L}\right)^{2} \int_{-L}^{L} \int_{-L}^{L} \sum_{|r|>0} \sum_{|s|>0} e^{i r q x} e^{-i s q u}( \pm i r q)^{\alpha}( \pm i s q)^{\beta} e^{i q(s-r) y} f(u) d u d y= \\
=\frac{1}{2 L} \int_{-L}^{L} \sum_{|r|>0}( \pm i r q)^{\alpha} e^{i r q x} \sum_{|s|>0}( \pm i s q)^{\beta} e^{-i s q u} f(u) d u\left(\frac{1}{2 L} \int_{-L}^{L} e^{i q(s-r) y} d y\right)= \\
=\frac{1}{2 L} \int_{-L}^{L} \sum_{|r|>0}( \pm i r q)^{\alpha} e^{i r q x} \sum_{|s|>0}( \pm i s q)^{\beta} e^{-i s q u} \delta_{r}{ }^{s} f(u) d u= \\
=\frac{1}{2 L} \int_{-L}^{L} \sum_{|r|>0}( \pm i r q)^{\alpha+\beta} e^{i r q(x-u)} f(u) d u= \\
=\frac{1}{2 L} \int_{-L}^{L} \partial_{x} \Psi_{ \pm}^{1-(\alpha+\beta)}(x-u) f(u) d u= \\
={ }_{L} W_{ \pm}^{\alpha+\beta} f(x)
\end{gathered}
$$

Exatamente como no caso das integrais, obtemos o mesmo resultado ao comutar $\alpha$ e $\beta$, o que demonstra a propriedade. 
2. Transformada de Fourier:

$$
\mathfrak{F}\left\{{ }_{L} W_{ \pm}^{\nu} f(x)\right\}=\sum_{|r|>0} \sqrt{2 \pi}( \pm i r q)^{\nu} \delta(y-r q) \hat{f}^{r}
$$

sendo $\hat{f}^{r}=\left\langle e^{r} \mid f\right\rangle_{\infty}$ o coeficiente de $f(x)$ na expansão de Fourier.

$\underline{D e m}$.

$$
\begin{gathered}
\mathfrak{F}\left\{{ }_{L} W_{ \pm}^{\nu} f(x)\right\}=\frac{1}{\sqrt{2 \pi}} \int_{-\infty}^{\infty} e^{-i y x}\left\{\frac{1}{2 L} \int_{-L}^{L} \partial_{x}\left(\sum_{|r|>0} \frac{e^{i r q(x-u)}}{( \pm i r q)^{1-\nu}}\right) f(u) d u\right\} d x= \\
=\frac{1}{\sqrt{2 \pi}} \int_{-\infty}^{\infty} e^{-i y x}\left\{\frac{1}{2 L} \int_{-L}^{L} \sum_{|r|>0} \frac{e^{i r q(x-u)}}{( \pm i r q)^{-\nu}} f(u) d u\right\} d x= \\
=\sum_{|r|>0} \frac{\sqrt{2 \pi}}{( \pm i r q)^{-\nu}}\left(\frac{1}{2 \pi} \int_{-\infty}^{\infty} e^{i(r q-y) x} d x\right)\left(\frac{1}{2 L} \int_{-L}^{L} e^{-i r q u} f(u) d u\right)= \\
=\sum_{|r|>0} \sqrt{2 \pi}( \pm i r q)^{\nu} \delta(y-r q) \hat{f}^{r}
\end{gathered}
$$

Novamente, como no caso da integral fracional, notando que $\left\langle e^{r} \mid e_{m}\right\rangle=\delta_{m}{ }^{r}$, podemos determinar o caso particular para um elemento da base de Fourier:

$$
\mathfrak{F}\left\{{ }_{L} W_{ \pm}^{\nu}\left|e_{m}\right\rangle\right\}=\sum_{|r|>0} \sqrt{2 \pi}( \pm i m q)^{\nu} \delta(y-m q)
$$

Esta fórmula, naturalmente, é idêntica à eq. (F.74).

Em suma, com esses resultados, idênticos às eq. (F.71) e (F.73), podemos então expressar as transformadas de Fourier da IFW e DFW pelas fórmulas acima ou pelas obtidas da equivalência com a versão de Liouville, ou seja,

$$
\begin{gathered}
\mathfrak{F}\left\{{ }_{L} W_{+}^{-\nu} f(x)\right\}=(i y)^{-\nu} \mathcal{F}(y) \\
\mathfrak{F}\left\{{ }_{L} W_{+}^{\nu} f(x)\right\}=(i y)^{\nu} \mathcal{F}(y)
\end{gathered}
$$

Além disso, a DFW de uma constante fornece um valor nulo, pois a IFW também o faz e, pela definição da DFW, isso segue imediatamente.

Desse modo, fica estabelecido que a derivada fracional de Weyl-Liouville é adequada a funções periódicas expandidas em séries complexas de Fourier e é equivalente às derivadas fracionais de Liouville e Caputo-Liouville, bem como à derivada fracional de WeylMarchaud. 
Agora estamos prontos para determinar as matrizes operacionais fracionárias na base de Fourier complexa.

\subsubsection{MOps Fracionais de Weyl na Base Complexa de Fourier}

Usando os recursos dos operadores de Weyl, torna-se fácil estabelecer as matrizes operacionais de derivação e integração de ordem arbitrária na base complexa de Fourier, pois, de acordo com as equações (5.18) e (5.26), tem-se

$$
{ }_{L} W_{ \pm}^{\nu}\left|e_{m}\right\rangle=( \pm i m q)^{\nu}\left|e_{m}\right\rangle, \quad \nu \in \mathbb{C}
$$

e, utilizando o operador positivo, que é equivalente à DFL, podemos escrever

$$
{ }_{L} W_{+}^{\nu}\left|e_{m}\right\rangle \equiv D_{-\infty}^{\nu}\left|e_{m}\right\rangle=(i m q)^{\nu}\left|e_{m}\right\rangle, \quad m \neq 0, \nu \in \mathbb{C}
$$

Daí, grafando-se a matriz operacional de derivação por $W^{\nu}$, obtém-se

$$
\sum_{|k|>0}^{\ell}\left(W^{\nu}\right)_{r}{ }^{k}\left|e_{k}\right\rangle_{\infty}=\sum_{|k|>0}^{\ell}(i r q)^{\nu} \delta_{r}{ }^{k}\left|e_{k}\right\rangle_{\infty}
$$

e, levando em conta que a DFCL de uma constante é nula, ou seja,

$$
{ }_{L} W_{+}^{\nu}\left|e_{0}\right\rangle \equiv{ }^{c} D_{-\infty}^{\nu} 1=0
$$

segue que

$$
\sum_{k=-\ell}^{\ell}\left(W^{\nu}\right)_{r}{ }^{k}\left|e_{k}\right\rangle_{\infty}=\sum_{k=-\ell}^{\ell}(\text { irq })^{\nu} \delta_{r}{ }^{k}\left|e_{k}\right\rangle_{\infty}, \quad \Re\{\nu\}>0
$$

e, por completude,

$$
\left(W^{\nu}\right)_{r}^{k}=(i r q)^{\nu} \delta_{r}^{k}
$$

Logo, como esperado, a MOD fracionária assume uma simples forma diagonal. Dessa forma, a MOD fracional de uma função $\mathcal{L}^{1}$ será simplesmente

$$
\left|f_{\ell}^{(\nu)}\right\rangle_{\infty}=\sum_{r=-\ell}^{\ell} c^{r} W^{\nu}\left|e_{m}\right\rangle_{\infty}=\sum_{|k|>0}^{\ell} c^{r}(i m q)^{\nu}\left|e_{m}\right\rangle_{\infty}
$$

Este resultado, contudo, também vale para a MOI, visto que, para uma função

$$
\left|f_{\ell}\right\rangle_{\infty}=\sum_{r=-\ell}^{\ell} c^{r}\left|e_{m}\right\rangle_{\infty}
$$


pela propriedade da IFW de constante ser nula, conclui-se que

$$
\left|f_{\ell}^{(-\nu)}\right\rangle_{\infty}=\sum_{r=-\ell}^{\ell} c^{r} W^{-\nu}\left|e_{m}\right\rangle_{\infty}=\sum_{|k|>0}^{\ell} c^{r}(i m q)^{-\nu}\left|e_{m}\right\rangle_{\infty}
$$

Portanto, as MOps de Weyl de ordem arbitrária $\nu \in \mathbb{C}$ para a base de Fourier podem ser expressas pela fórmula comum $\left(W^{\nu}\right)_{r}{ }^{k}=(i r q)^{\nu} \delta_{r}{ }^{k}$ ou, mais explicitamente:

$$
W^{\nu}=(i q)^{\nu} \operatorname{diag}\left[\begin{array}{lllllllll}
(-\ell)^{\nu} & (-\ell+1)^{\nu} & \cdots & (-1)^{\nu} & 0 & 1^{\nu} & \cdots & (\ell-1)^{\nu} & (\ell)^{\nu}
\end{array}\right]
$$

\subsection{Equações Diferenciais Fracionárias}

Enfim, chegamos à última parte deste capítulo, visando a aplicação do método em equações diferenciais fracionárias. Para iniciar a exposição das EDFs, escolhemos quatro exemplos de caráter ilustrativo e didático e um outro, de maior complexidade, para expor as possibilidades do método, exatamente como procedemos antes, no caso das EDOs. Aliás, a mesma EDO de $7^{\circ}$ grau estudada na seção anterior será revista aqui em uma versão fracional, o que significa que manteremos as mesmas funções coeficientes, mudando as ordens das derivadas e mantendo a função $\phi$ daquele exemplo, que já tinha sido estendida ao corpo complexo. Por fim, analisaremos um caso concreto de aplicação física referente a EDFs, que é uma versão fracional da equação de condução de calor unidimensional com presença de fonte.

Uma EDF pode ser escrita de várias maneiras. Podemos, em princípio, expandir uma EDO linear não-homogênea para ordens arbitrárias de derivação, da seguinte maneira:

$$
\sum_{r=0}^{s} \mu_{r}(x) D^{r \nu} f(x)=\phi(x)
$$

onde $\nu$ é uma ordem arbitrária. Esse tipo de EDF é chamada de sequencial, pois pode ser expressa por uma sequência de derivações fracionárias cujas ordens são múltiplos de um valor fundamental. Nesse caso, as ordens de derivação mantém um vínculo entre si. Uma outra possibilidade consiste em desvincular as ordens de derivação, escrevendo

$$
\sum_{r=0}^{s} \mu_{r}(x) D^{\alpha_{r}} f(x)=\phi(x)
$$

onde as ordens são geralmente tomadas com a condição $r-1<\alpha_{r}<r,(r>0)$ e $\alpha_{0}=0$.

Normalmente, as derivadas fracionais utilizadas nos procedimentos de resolução de EDFs são entendidas no sentido de Caputo. De fato, a fim de evitar condições iniciais 
fracionárias, esse é o procedimento mais adequado. Em nosso caso, no entanto, se estivermos utilizando o método NBV, isso nem seria necessário, sendo porém recomendável, visto que a imposição de condições é, via de regra, exigência física do problema. Ocorre, todavia, que a utilização da base de Fourier complexa, de caráter periódico, impõe o uso dos operadores de derivação de Weyl, os quais, como já demonstramos, equivalem às derivadas fracionais de Liouville e, mais especificamente, às de de Caputo-Liouville, em perfeita adequação ao caso em estudo, cujas bases apresentam forma exponencial. Assim sendo, vamos construir as EDFs com base no operador de Weyl. Entretanto, visando a concisão, vamos grafar ${ }_{L} W_{+}^{\nu}$ apenas por $W^{\nu}$.

\subsubsection{Solução Analítica da Equação de Bagley-Torvik}

Iremos utilizar os métodos expostos e desenvolvidos nos capítulos anteriores para resolver, tanto analítica quanto numericamente, algumas equações diferenciais fracionárias. É instrutivo exemplificar o uso de um processo analítico de resolução de EDFs.

Para tanto, vamos escolher uma EDF relativamente simples, mas de uso generalizado. Vários fenômenos obedecem a esse tipo de equação. O modelo matemático é parecido ao do MHS forçado com amortecimento ou ao do circuito RLC com gerador AC, embora outras equações importantes, como a de Bagley-Torvik também possam ser adaptadas a esse mesmo formato.

Em Herrmann (2011) é apresentado o caso do oscilador harmônico fracional forçado com amortecimento, cuja forma da EDF é semelhante à desse exemplo, mas com ordens mais gerais. A EDF do MHS forçado com amortecimento, de acordo com Herrmann, mas grafada conforme a notação mais adequada às nossas necessidades, poderia ser apresentada na forma:

$$
W^{\alpha} x(t)+\mu W^{\beta} x(t)+\lambda x(t)=\phi(t)
$$

Nessa equação, os coeficientes são constantes e as condições assumidas para as ordens são $0<\beta<1$ e $1<\alpha<2$.

Por outro lado, como foi dito, a EDF não-homogênea de Bagley-Torvik (TORVIK; BAGLEY, 1984), dada por

$$
D^{2} u(x)+D^{3 / 2} u(x)+u(x)=\phi(x)
$$

também se enquadra no modelo, como se pode constatar por simples observação, para $\lambda=\mu=1, \quad \alpha=2$ e $\beta=3 / 2$. 
Assim, podemos escrever

$$
\left[{ }^{c} D_{a}^{2}+{ }^{c} D_{a}^{3 / 2}+1\right] u(x)=\phi(x)
$$

estando a determinação do terminal inferior $a$ condicionada à classe da função $\phi$. Um dos modos de solução da EDF é pelo método das transformadas de Laplace. No caso específico da DFC, segue da equação (F.68) que

$$
\mathcal{L}\left\{{ }^{c} D^{3 / 2} u(x)\right\}=s^{3 / 2} U(s)-s^{1 / 2} u(0)-s^{-1 / 2} u^{\prime}(0)
$$

Portanto,

$$
\begin{gathered}
\mathcal{L}\left\{{ }^{c} D^{2} u(x)+{ }^{c} D^{3 / 2} u(x)+u(x)\right\}=s^{2} U(s)-s u(0)-u^{\prime}(0)+ \\
+\left[s^{3 / 2} U(s)-s^{1 / 2} u(0)-s^{-1 / 2} u^{\prime}(0)\right]+U(s)=\Phi(s) \Rightarrow \\
\Rightarrow U(s)\left(s^{2}+s^{3 / 2}+1\right)=\left(s+s^{1 / 2}\right) u(0)+\left(s^{-1 / 2}+1\right) u^{\prime}(0)+\Phi(s) \Rightarrow \\
\Rightarrow U(s)=\frac{1}{\left(s^{2}+1\right)\left(1+\frac{s^{3 / 2}}{s^{2}+1}\right)}\left[\left(s+s^{1 / 2}\right) u(0)+\left(s^{-1 / 2}+1\right) u^{\prime}(0)+\Phi(s)\right]
\end{gathered}
$$

Mas, para $\left|\frac{s^{3 / 2}}{s^{2}+1}\right|<1$ vale o desenvolvimento binomial:

$$
\left(1+\frac{s^{3 / 2}}{s^{2}+1}\right)^{-1}=\sum_{k=0}^{\infty}(-1)^{k} \frac{s^{3 k / 2}}{\left(s^{2}+1\right)^{k}}
$$

o que conduz a

$$
U(s)=\sum_{k=0}^{\infty}(-1)^{k} \frac{s^{3 k / 2}}{\left(s^{2}+1\right)^{k+1}}\left[\left(s+s^{1 / 2}\right) u(0)+\left(s^{-1 / 2}+1\right) u^{\prime}(0)+\Phi(s)\right]
$$

Separando cada termo, chega-se a

$$
\begin{gathered}
U(s)=u(0) \sum_{k=0}^{\infty}(-1)^{k} \frac{s^{3 k / 2+1}}{\left(s^{2}+1\right)^{k+1}}+u(0) \sum_{k=0}^{\infty}(-1)^{k} \frac{s^{3 k / 2+1 / 2}}{\left(s^{2}+1\right)^{k+1}}+ \\
+u^{\prime}(0) \sum_{k=0}^{\infty}(-1)^{k} \frac{s^{3 k / 2-1 / 2}}{\left(s^{2}+1\right)^{k+1}}+u^{\prime}(0) \sum_{k=0}^{\infty}(-1)^{k} \frac{s^{3 k / 2}}{\left(s^{2}+1\right)^{k+1}}+\Phi(s) \sum_{k=0}^{\infty}(-1)^{k} \frac{s^{3 k / 2}}{\left(s^{2}+1\right)^{k+1}}
\end{gathered}
$$

Usando a TL inversa:

$$
\mathcal{L}^{-1}\left\{\frac{s^{\alpha \gamma-\beta}}{\left(s^{\alpha}+\lambda\right)^{\gamma}}\right\}=t^{\beta-1} E_{\alpha, \beta}^{\gamma}\left(-\lambda t^{\alpha}\right)
$$

obtemos, para cada os quatro primeiros termos acima, os valores

$$
\mathcal{L}^{-1}\left\{\frac{s^{3 k / 2+1}}{\left(s^{2}+1\right)^{k+1}}\right\}=x^{\frac{1}{2} k} E_{2, \frac{1}{2}(k+2)}^{k+1}\left(-x^{2}\right),
$$




$$
\begin{aligned}
& \mathcal{L}^{-1}\left\{\frac{s^{3 k / 2+1 / 2}}{\left(s^{2}+1\right)^{k+1}}\right\}=x^{\frac{1}{2}(k+1)} E_{2, \frac{1}{2}(k+3)}^{k+1}\left(-x^{2}\right), \\
& \mathcal{L}^{-1}\left\{\frac{s^{3 k / 2-1 / 2}}{\left(s^{2}+1\right)^{k+1}}\right\}=x^{\frac{1}{2}(k+3)} E_{2, \frac{1}{2}(k+5)}^{k+1}\left(-x^{2}\right), \\
& \mathcal{L}^{-1}\left\{\frac{s^{3 k / 2}}{\left(s^{2}+1\right)^{k+1}}\right\}=x^{\frac{1}{2}(k+2)} E_{2, \frac{1}{2}(k+4)}^{k+1}\left(-x^{2}\right)
\end{aligned}
$$

e, por convolução para o último termo, tem-se

$$
\mathcal{L}^{-1}\left\{\frac{\Phi(s) s^{3 k / 2}}{\left(s^{2}+1\right)^{k+1}}\right\}=\phi(x) *\left[x^{\frac{1}{2}(k+2)} E_{2, \frac{1}{2}(k+4)}^{k+1}\left(-x^{2}\right)\right],
$$

de modo que a solução geral será:

$$
\begin{gathered}
u(x)=u(0) \sum_{k=0}^{\infty}(-1)^{k} x^{k / 2} E_{2, \frac{1}{2}(k+2)}^{k+1}\left(-x^{2}\right)+u(0) \sum_{k=0}^{\infty}(-1)^{k} x^{\frac{1}{2}(k+1)} E_{2, \frac{1}{2}(k+3)}^{k+1}\left(-x^{2}\right)+ \\
+u^{\prime}(0) \sum_{k=0}^{\infty}(-1)^{k} x^{\frac{1}{2}(k+3)} E_{2, \frac{1}{2}(k+5)}^{k+1}\left(-x^{2}\right)+u^{\prime}(0) \sum_{k=0}^{\infty}(-1)^{k} x^{\frac{1}{2}(k+2)} E_{2, \frac{1}{2}(k+4)}^{k+1}\left(-x^{2}\right)+ \\
+\sum_{k=0}^{\infty}(-1)^{k} \int_{0}^{x} \phi(x-\xi) \xi^{\frac{1}{2}(k+2)} E_{2, \frac{1}{2}(k+4)}^{k+1}\left(-\xi^{2}\right) d \xi
\end{gathered}
$$

Se definirmos um fator $\rho(s):=\frac{1}{2}\left\{1-(-1)^{[4 / s]}\right\}$, onde $[k] \equiv \operatorname{int}(k)$, é possível reescrever a solução acima de modo sintético como

$$
\begin{gathered}
u(x)=\sum_{k=0}^{\infty}(-1)^{k}\left\{\left.\sum_{s=1}^{4} D^{\rho(s)} u(x)\right|_{x=0} x^{\frac{1}{2}(k+s-1)} E_{2, \frac{1}{2}(k+s+1)}^{k+1}\left(-x^{2}\right)+\right. \\
\left.+\int_{0}^{x} \phi(x-\xi) \xi^{\frac{1}{2}(k+2)} E_{2, \frac{1}{2}(k+4)}^{k+1}\left(-\xi^{2}\right) d \xi\right\}
\end{gathered}
$$

Essa é uma solução analítica geral da EDF de Bagley-Torvik em termos das funções de Mittag-Leffler de três parâmetros. Entretanto, essa expressão supõe o uso das integrais de Riemann-Liouville, visto que assume o terminal inferior $a=0$ como condição. Seria possível, no entanto, para certa classe de funções $\phi$, escolher uma condição diferente, como, por exemplo, atribuir às derivadas a natureza das DFCL. Isso forneceria um resultado diverso do obtido, embora não pudesse ser aplicado a funções polinomiais, por gerar infinitos.

\subsubsection{EDFs Ilustrativas}

Passamos a partir de agora a estudar algumas EDFs ilustrativas, resolvendo-as numericamente pelo método de modelagem NBV-SA-NC. Os operadores escolhidos para os 
exemplos básicos foram os seguintes:

1. $W^{0}$

2. $W^{\pi}+1$

3. $W^{2 \nu}+x W^{\nu}+x^{2} W^{0}, \quad \nu=1$

4. $W^{2 \nu}+x W^{\nu}+x^{2} W^{0}, \quad \nu=0.4$

Cada um desses operadores irá atuar sobre uma função candidata $\mathcal{L}_{1}$, de forma a gerar uma EDF para cada caso. Escolhemos, para o termo não-homogêneo das EDFs, a mesma função real usada nas EDOs, i.e., $\phi(x)=\cos (7 x) e^{-x^{2}}$. Porém, para a solução analítica do caso 2 e para uma segunda solução numérica do caso 4, bem como para a EDF de grande ordem do exemplo ilustrativo adicional, elegemos uma função complexa $\phi: \mathbb{R} \rightarrow \mathbb{C} \mid \phi(x)=\exp \left\{-(x / \sqrt{L})^{2}+i(x / \sqrt{L})\right\}$, já empregada no exemplo da EDO de $7^{\text {a }}$ ordem. Os mapas paramétricos das soluções são construídos sobre o domínio complexo $z=x+i y \in \mathbb{C}$.

Todas as equações têm soluções analíticas, embora as fracionárias sejam altamente transcendentes. Para exemplificar o processo de resolução vamos determinar a solução analítica da equação 2 com termo independente complexo, que é uma EDF relativamente simples, através do método das transformadas de Fourier. Essa EDF pode ser escrita

$$
W^{\pi} f(x)+f(x)=\phi(x),
$$

$\operatorname{com} \phi(x)=e^{i x / \sqrt{L}} e^{-x^{2} / L}$. Usando as TF, obtemos

$$
\begin{gathered}
\mathfrak{F}\left\{W^{\pi} f(x)+f(x)\right\}=\mathfrak{F}\{\phi(x)\} \quad \Rightarrow \\
(i y)^{\pi} \mathcal{F}(y)+\mathcal{F}(y)=\Phi(y) \Rightarrow \\
\mathcal{F}(y)=\frac{\Phi(y)}{(i y)^{\pi}+1}
\end{gathered}
$$

Utilizando agora as transformadas inversas e o terorema da convolução, tem-se

$$
\mathfrak{F}^{-1}\{\mathcal{F}(y)\}=f(x)=\mathfrak{F}^{-1}\left\{\frac{\Phi(y)}{(i y)^{\pi}+1}\right\}=\phi(x) * \mathfrak{F}^{-1}\left\{\frac{1}{(i y)^{\pi}+1}\right\}
$$

Mas, usando a linearidade da TF inversa e o resultado

$$
\mathfrak{F}^{-1}\left\{(i y)^{\alpha}\right\}=-\frac{\Gamma(\alpha+1) \sin (\alpha \pi)}{\sqrt{2 \pi}} \frac{(x+|x|)}{|x|^{\alpha+2}}, \quad(x \neq 0)
$$


e expandindo:

$$
\left(1+(i y)^{\pi}\right)^{-1}=\sum_{k=0}^{\infty}(-1)^{k}(i y)^{k \pi}, \quad\left|y^{\pi}\right|<1,
$$

pode-se reescrever a transformada de Fourier inversa da função como

$$
\mathfrak{F}^{-1}\left\{\frac{1}{(i y)^{\pi}+1}\right\}=\sqrt{2 \pi} \delta(x)+\sum_{k=1}^{\infty}(-1)^{k-1} \frac{\Gamma(k \pi+1) \sin \left(k \pi^{2}\right)}{\sqrt{2 \pi}} \frac{(x+|x|)}{|x|^{k \pi+2}},
$$

Assim, a função assume a forma

$$
f(x)=\sqrt{2 \pi} \delta(x)+\frac{1}{\sqrt{2 \pi}} \int_{-\infty}^{\infty} \phi(x-u) \frac{(u+|u|)}{\sqrt{2 \pi}|u|^{2}} \sum_{k=1}^{\infty}(-1)^{k-1} \frac{\Gamma(k \pi+1) \sin \left(k \pi^{2}\right)}{|u|^{k \pi}} d u
$$

Externando a somatória e usando as propriedades modulares, a integral remanescente pode ser reexpressa por

$$
\int_{-\infty}^{\infty} \phi(x-u) \frac{(u+|u|)}{|u|^{k \pi+2}} d u=2 \int_{0}^{\infty} \frac{\phi(x-\xi)}{\xi^{k \pi+1}} d \xi
$$

o que nos permite determinar a solução analítica através da expressão:

$$
f(x)=\sqrt{2 \pi} \delta(x)+\frac{1}{\pi} \sum_{k=1}^{\infty}(-1)^{k-1} \Gamma(k \pi+1) \sin \left(k \pi^{2}\right) \int_{0}^{\infty} \frac{\phi(x-\xi)}{\xi^{k \pi+1}} d \xi
$$

Esta é a solução geral, quer dizer, para uma função $\phi$ genérica. Para nosso problema, no entanto, a resolução da integral em um período $L=\frac{4 \pi}{5}$, escrita em termos da função hipergeométrica $U$, fornece, para $k \neq 0$, o resultado:

$$
\mathcal{I}_{k}(x)=\left(\frac{5}{\pi}\right)^{\frac{k \pi}{2}} \exp \left[\frac{x}{4 \pi}(2 i \sqrt{5 \pi}-5 x)\right] \Gamma(-k \pi) U\left[-\frac{k \pi}{2}, \frac{1}{2},-\frac{(\sqrt{5 \pi}+5 i x)^{2}}{20 \pi}\right]
$$

onde $\mathcal{I}_{k}(x):=\int_{0}^{\infty} \frac{\phi(x-\xi)}{\xi^{k \pi+1}} d \xi$. Logo, se substituirmos esse valor na eq.(5.35), chegaremos à expressão final da solução analítica da EDF do caso 2, obtendo:

$$
f(x)=\sqrt{2 \pi} \delta(x)+\frac{1}{\pi} \sum_{k=1}^{\infty}(-1)^{k-1} \Gamma(k \pi+1) \sin \left(k \pi^{2}\right) \mathcal{I}_{k}(x)
$$

onde $\mathcal{I}_{\phi}(x)$ é dada pela eq.(5.36). Os gráficos das três primeiras integrais, i.e., para $k=1,2,3$, aparecem na figura (71). O inconveniente dessas funções está na nãodiferenciabilidade na origem. A solução específica dessa equação é obtida por uma espécie de soma de harmônicos, representados pelas funções $\mathcal{I}_{k}(x)$, onde as amplitudes $A_{k}$ são 
Figura 71: Gráficos de $\mathcal{I}_{1}(x), \mathcal{I}_{2}(x), \mathcal{I}_{3}(x)$
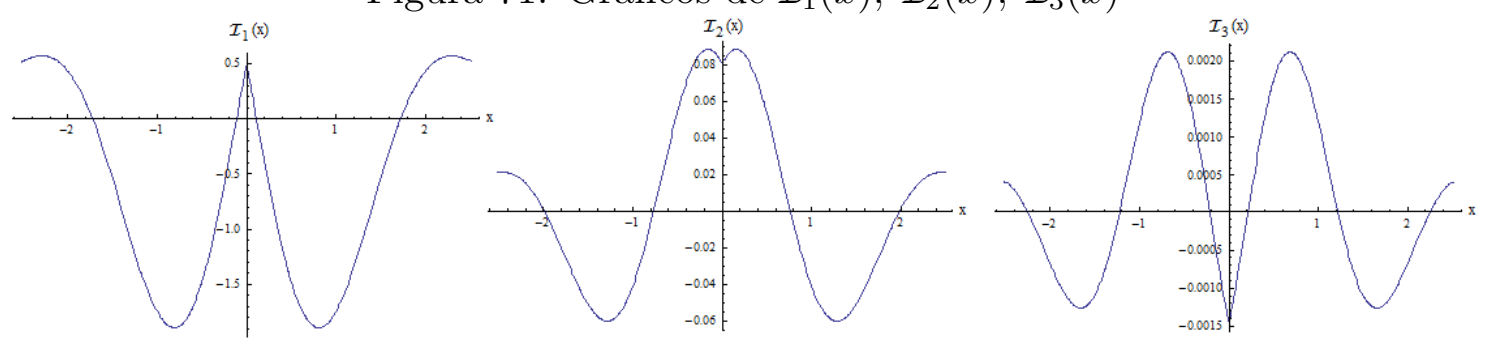

descritas pelos fatores $\frac{(-1)^{k-1}}{\pi} \Gamma(k \pi+1) \sin \left(k \pi^{2}\right)$, de forma que a solução é escrita como

$$
f(x)=\sum_{k=1}^{\infty} A_{k} \mathcal{I}_{k}(x)
$$

Percebe-se claramente que, mesmo para uma EDF tão simples, a solução apresenta considerável complexidade. Por esse motivo é tão importante o critério de mensuração do erro e da função resto através da comparação entre a EDF com a solução aproximada e a função do termo não-homogêneo $\phi$. A comparação direta com a solução analítica demandaria grande dispêndio computacional, aumentando demais o tempo de processamento.

\subsubsection{Resoluções Numéricas}

Procederemos agora aos cálculos numéricos para cada uma das EDFs. Para todas elas usaremos o período $L=4 \pi / 5$ e extensão $l=15$. As soluções serão efetuadas, como já dissemos, pelas séries de Fourier originais (SF) e pelas séries associadas (SA), com três conjuntos distintos de pontos para cada uma: espaçamento constante (EC), nodos de Chebyshev (NC) e nodos de Fourier (NF). Assumimos a mesma função gaussiana real já mencionada. Os resultados dos processos de resolução são mostrados a seguir.

- $W^{0} f_{n}(x)=\phi(x)$

1. fornece solução boa para SF+EC (fig. 72)

2. fornece solução ótima para $\mathrm{SF}+\mathrm{NF}$ (fig. 73)

3. fornece solução errada, hiperamplificada, para $\mathrm{SF}+\mathrm{NC}$

4. fornece soluções ótimas para $\mathrm{SA}+\mathrm{EC}, \mathrm{SA}+\mathrm{NF}$ e $\mathrm{SA}+\mathrm{NC}$, com maior precisão para $\mathrm{SA}+\mathrm{NF}$ (figs. 74, 75 e 76 )

- $\left[W^{\pi}+1\right] f_{n}(x)=\phi(x)$

1. fornece solução boa para SF+EC (fig. 77) 
199

Figura 72: Solução NBV SF-EC da EDO $(\nu=0)$
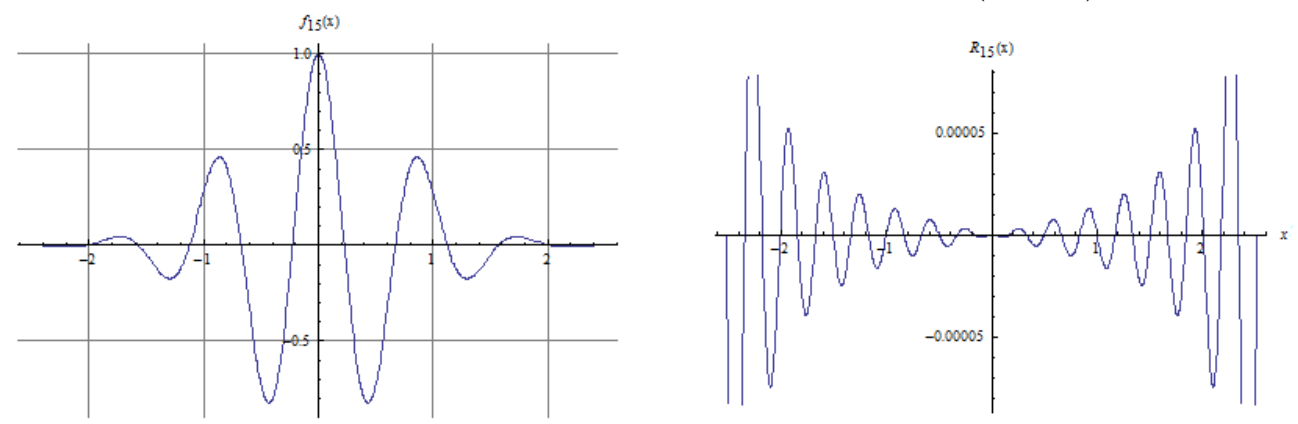

Figura 73: Solução NBV SF-NF da EDO $(\nu=0)$
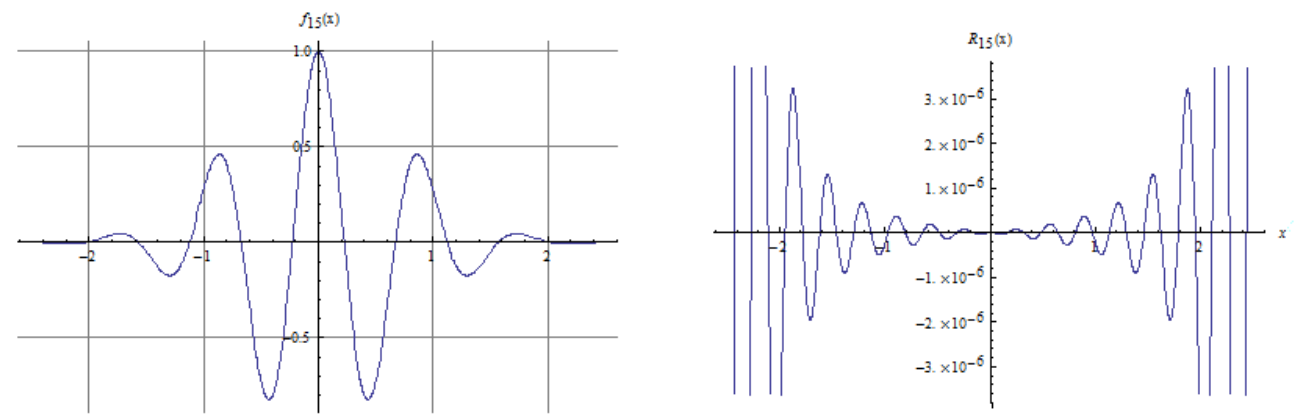

Figura 74: Solução NBV SA-EC da EDO $(\nu=0)$
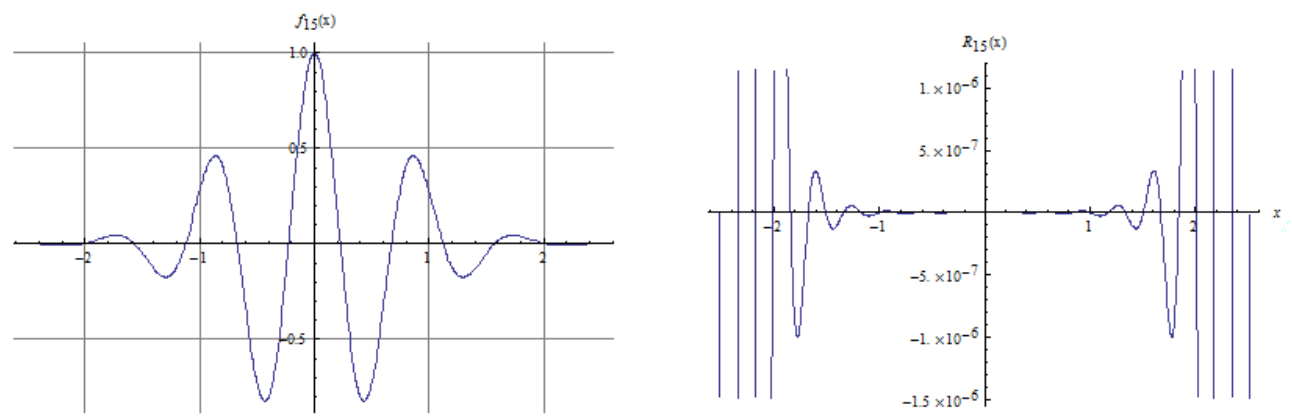

Figura 75: Solução NBV SA-NF da EDO $(\nu=0)$
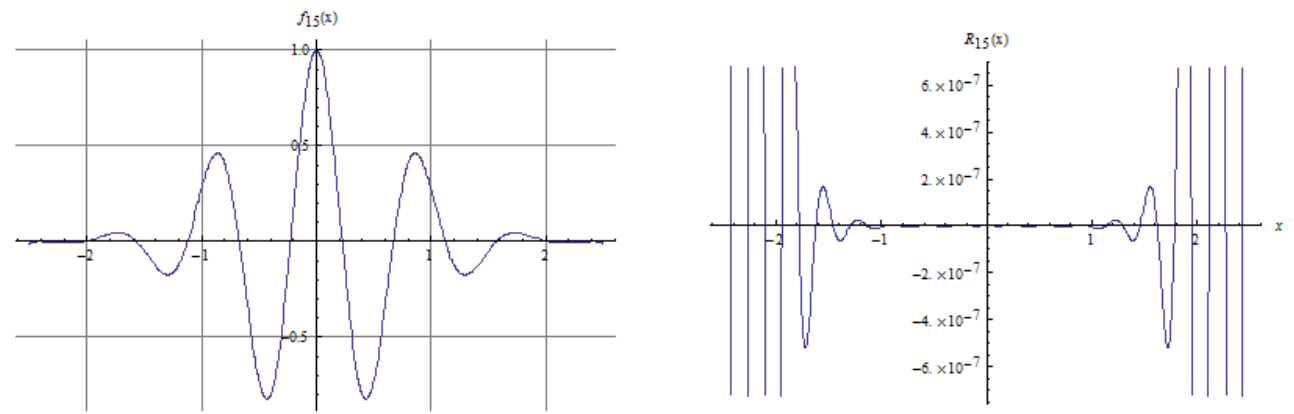
Figura 76: Solução NBV SA-NC da $\operatorname{EDO}(\nu=0)$
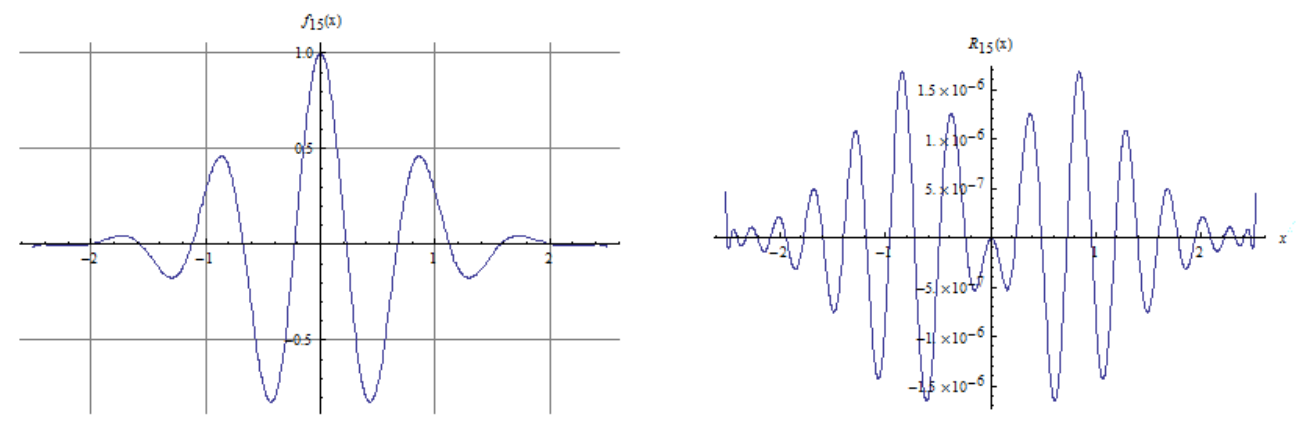

2. fornece solução ótima para $\mathrm{SF}+\mathrm{NF}$ (fig. 78)

3. fornece solução errada, hiperamplificada, para $\mathrm{SF}+\mathrm{NC}$

4. fornece soluções ótimas para $\mathrm{SA}+\mathrm{EC}, \mathrm{SA}+\mathrm{NF}$ e $\mathrm{SA}+\mathrm{NC}$, com maior precisão para $\mathrm{SA}+\mathrm{NF}$ (figs. 79,80 e 81 )

Figura 77: Solução NBV SF-EC da $\operatorname{EDF}(\nu=\pi)$
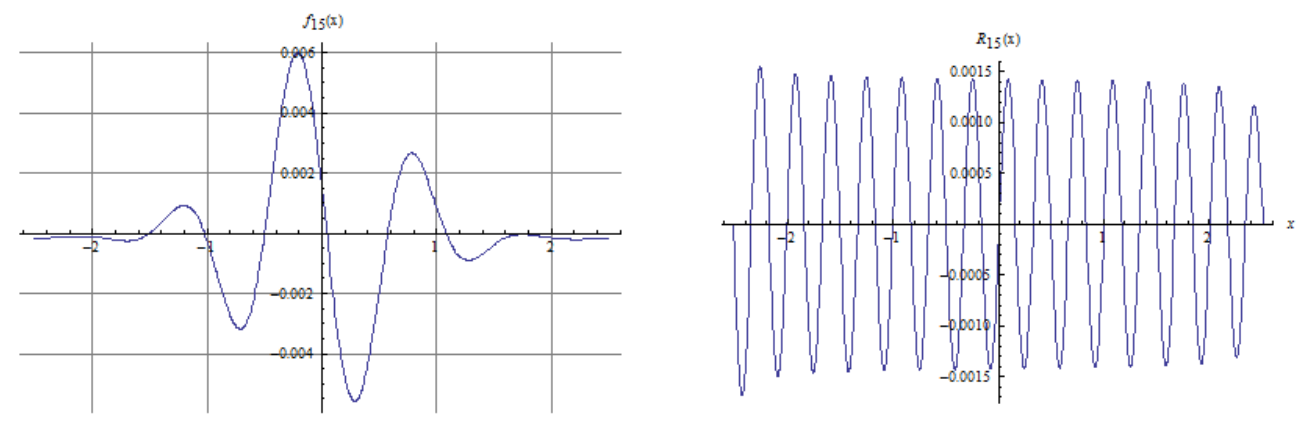

Figura 78: Solução NBV SF-NF da $\operatorname{EDF}(\nu=\pi)$
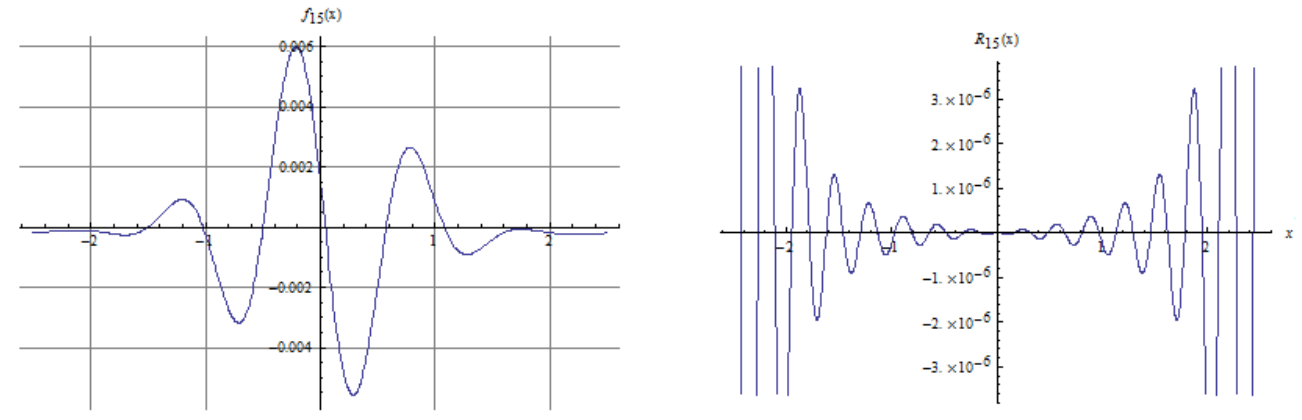

- $\left[W^{2}+x W^{1}+x^{2} W^{0}\right] f_{n}(x)=\phi(x)$

1. fornece solução boa para SF+EC (fig. 82)

2. fornece solução ótima para $\mathrm{SF}+\mathrm{NF}$ (fig. 83)

3. fornece solução errada, hiperamplificada, para $\mathrm{SF}+\mathrm{NC}$ 
Figura 79: Solução NBV SA-EC da $\operatorname{EDF}(\nu=\pi)$
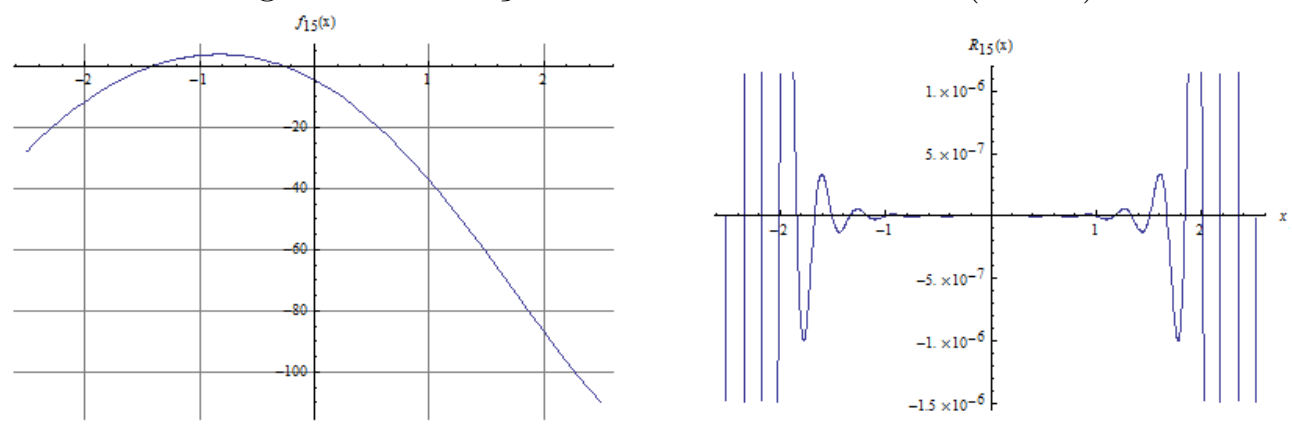

Figura 80: Solução NBV SA-NF da $\operatorname{EDF}(\nu=\pi)$
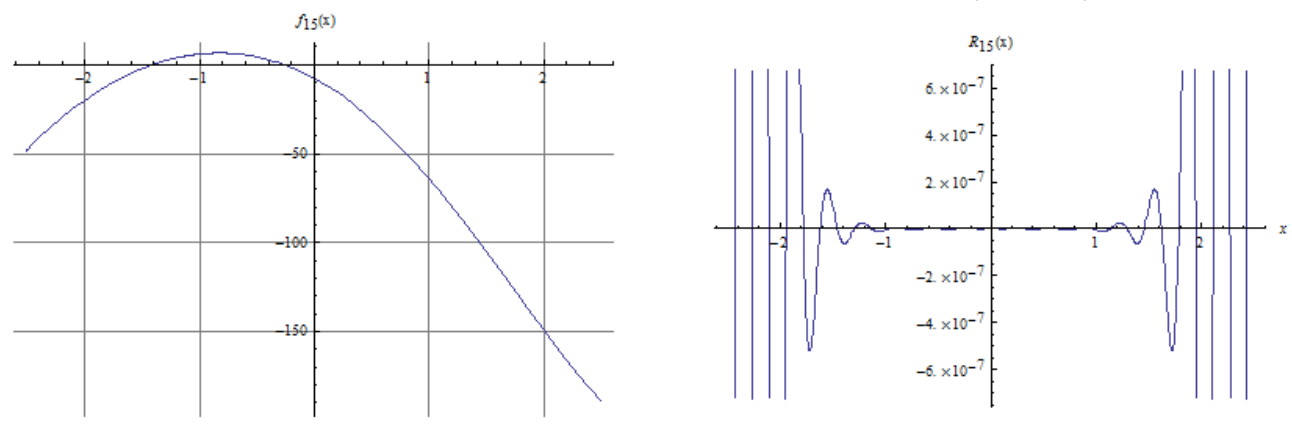

Figura 81: Solução NBV SA-NC da $\operatorname{EDF}(\nu=\pi)$
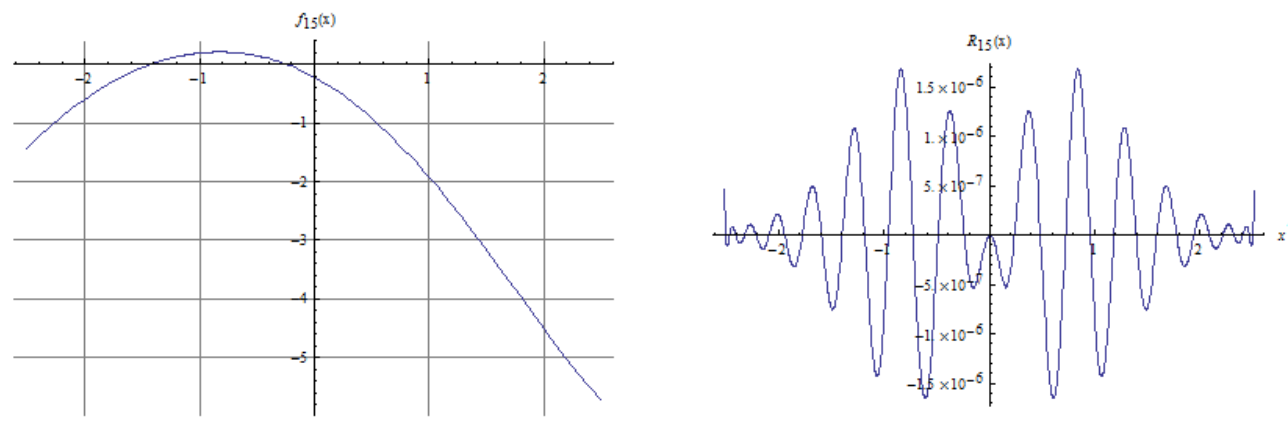
4. fornece soluções ótimas para $\mathrm{SA}+\mathrm{EC}, \mathrm{SA}+\mathrm{NF}$ e $\mathrm{SA}+\mathrm{NC}$, com maior precisão para $\mathrm{SA}+\mathrm{NF}$ (figs. 84, 85 e 86)

Figura 82: Solução NBV SF-EC da EDO $(\nu=1)$
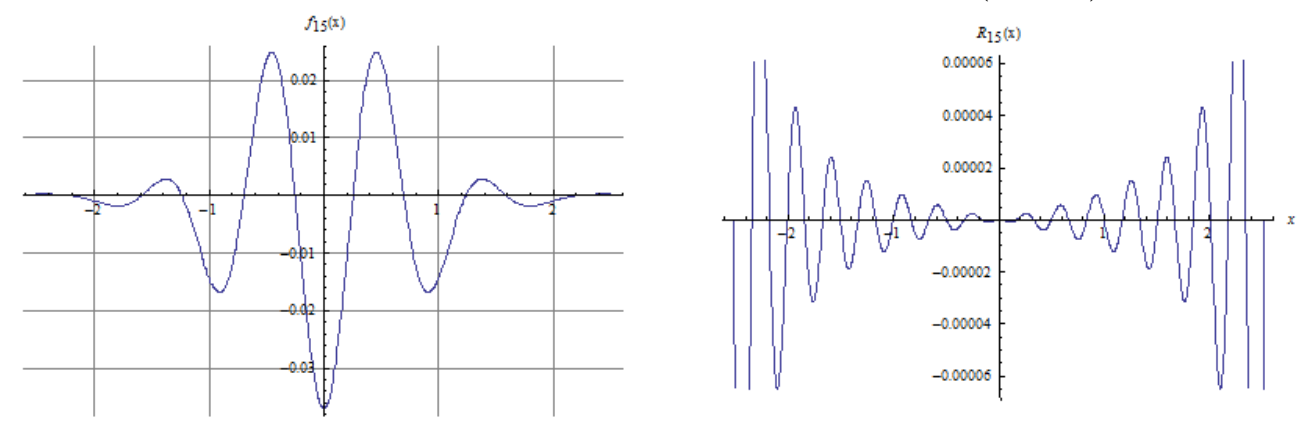

Figura 83: Solução NBV SF-NF da EDO $(\nu=1)$
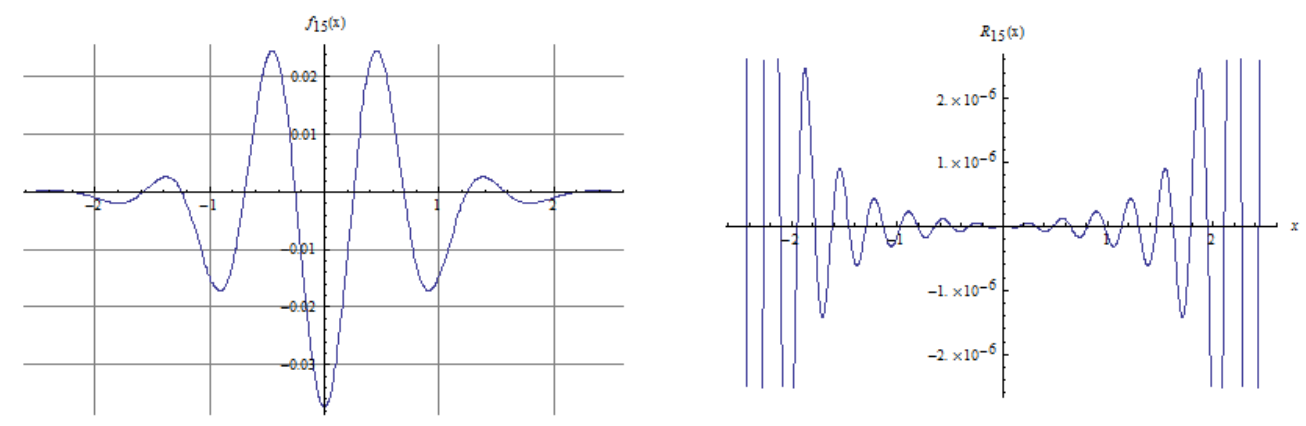

Figura 84: Solução NBV SA-EC da EDO $(\nu=1)$


- $\left[W^{2 \nu}+x W^{\nu}+x^{2} W^{0}\right] f_{n}(x)=\phi(x), \quad \nu=0.4$

1. fornece solução ruim, com baixa precisão para SF+EC (fig. 87)

2. fornece solução boa para $\mathrm{SF}+\mathrm{NF}$ (fig. 88)

3. fornece solução errada, hiperamplificada, para $\mathrm{SF}+\mathrm{NC}$

4. fornece soluções ótimas para $\mathrm{SA}+\mathrm{EC}, \mathrm{SA}+\mathrm{NF}$ e $\mathrm{SA}+\mathrm{NC}$, com maior precisão para $\mathrm{SA}+\mathrm{NC}$ (figs. 89, 90 e 91 ) 
Figura 85: Solução NBV SA-NF da EDO $(\nu=1)$
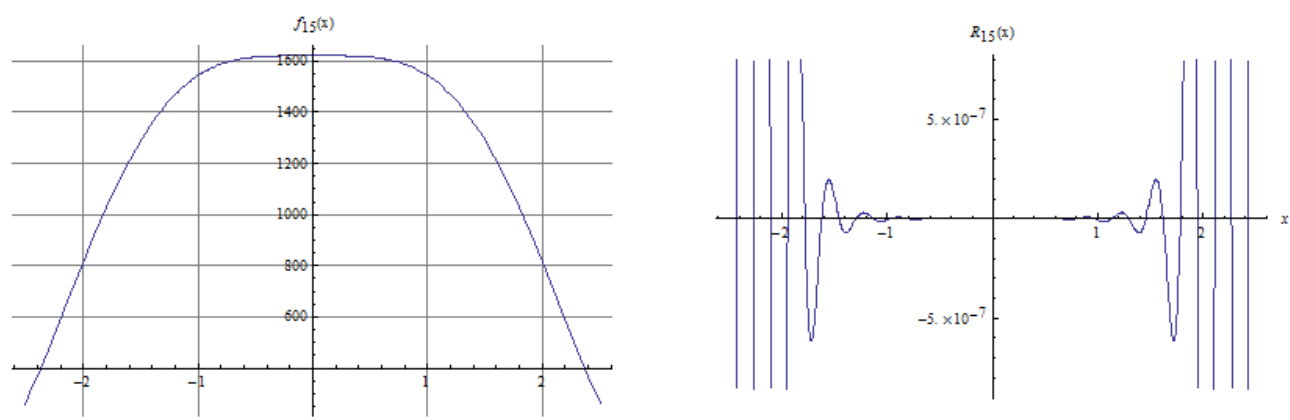

Figura 86: Solução NBV SA-NC da EDO $(\nu=1)$
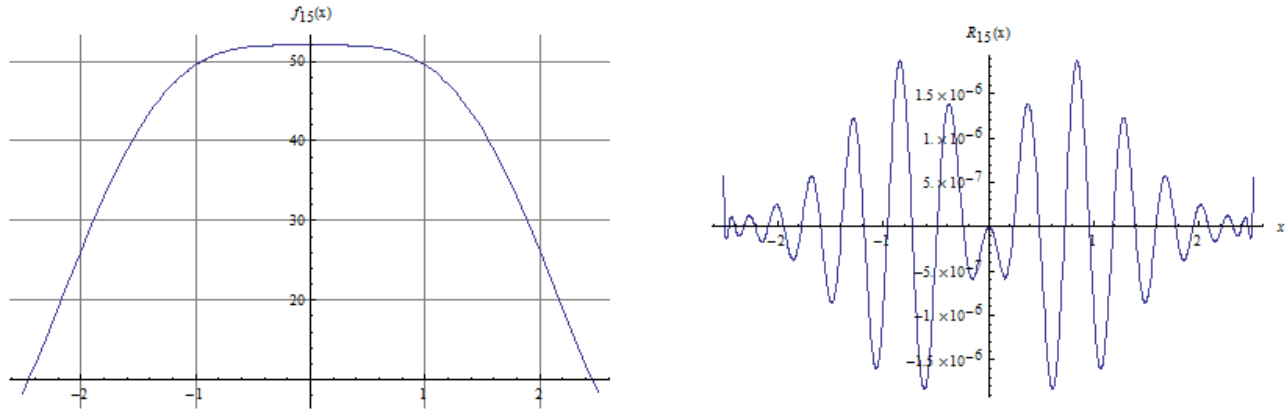

Figura 87: Solução NBV SF-EC da EDF $(\nu=0.4)$
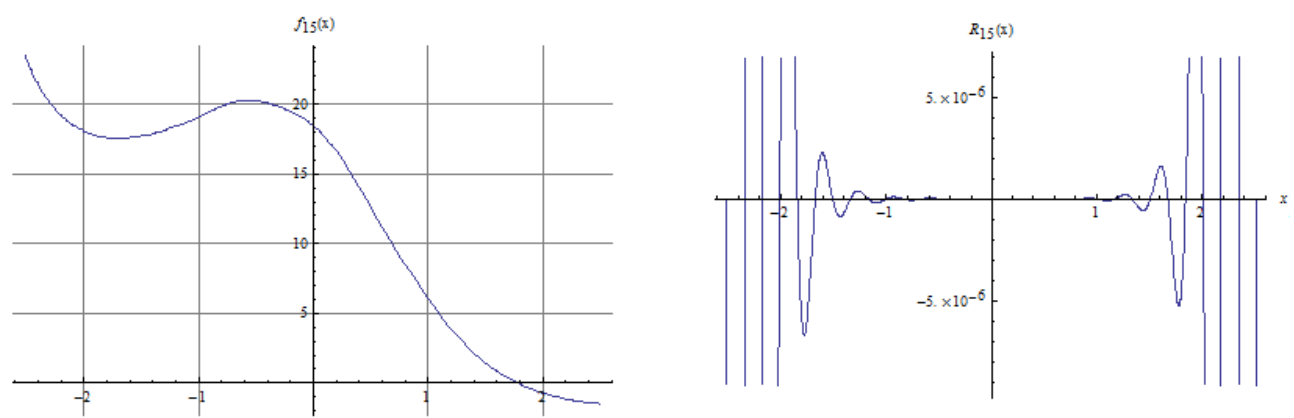

Figura 88: Solução NBV SF-NF da EDF $(\nu=0.4)$
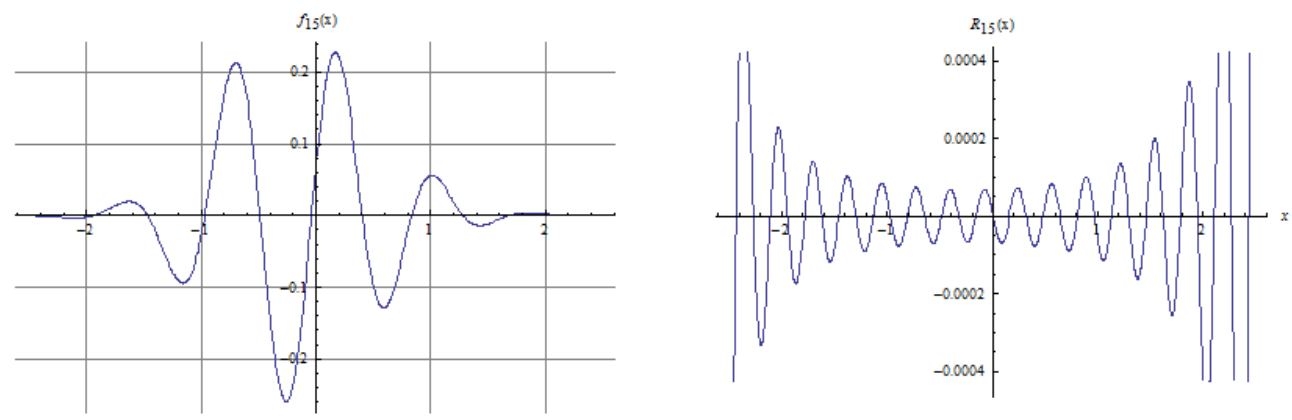
Figura 89: Solução NBV SA-EC da EDF $(\nu=0.4)$
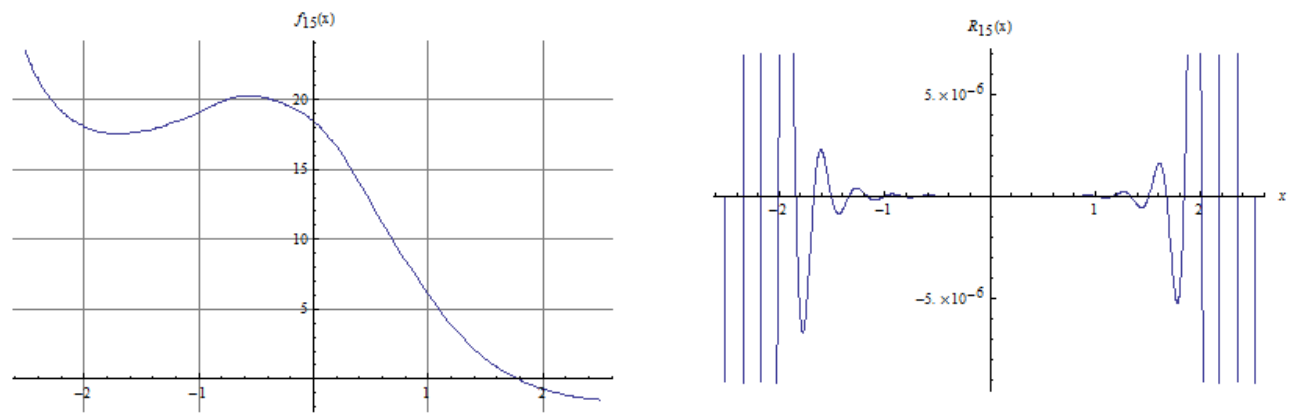

Figura 90: Solução NBV SA-NF da EDF $(\nu=0.4)$
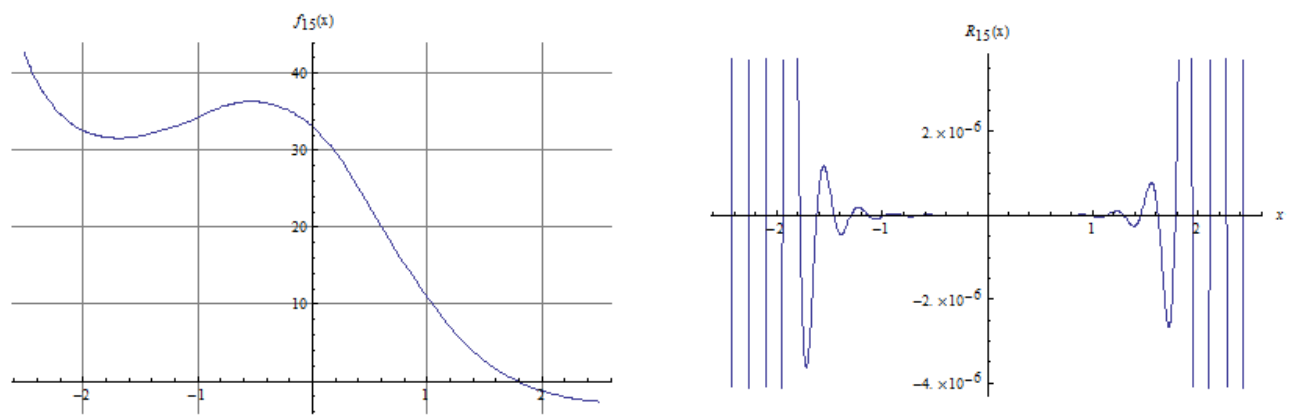

Figura 91: Solução NBV SA-NC da EDF $(\nu=0.4)$
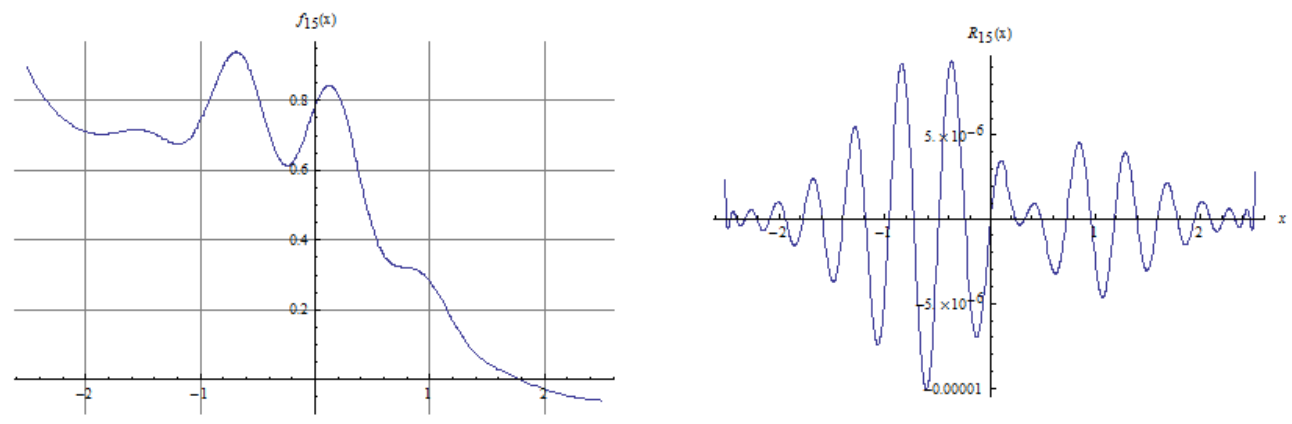


\subsubsection{Aplicação a uma EDF Sequencial de Ordem $7 \nu$}

A partir de agora, usamos o método de modelagem NBV-SA-NC com os operadores de Weyl para resolver uma EDF sequencial de forma similar à do exemplo de $7^{\mathrm{a}}$ ordem na seção das EDOs (4.4.1), quer dizer, com os mesmos coeficientes e mesmo termo independente, mas com ordens arbitrárias de derivação do tipo da equação (5.30) para uma ordem fracional elementar $\nu=0.64$. A solução é efetuada para um semiperíodo $L=4 \pi / 5$ com range $\ell=15$. Escrevendo a EDF na forma explícita, tem-se

$$
\left[W^{7 \nu}+e^{x} W^{5 \nu}-e^{i x} W^{4 \nu}-W^{3 \nu}+W^{2 \nu}+(1-i x) W^{\nu}+x^{2}\right] f(x)=e^{-\left(\frac{x}{\sqrt{L}}\right)^{2}+i \frac{x}{\sqrt{L}}}
$$

As ordens crescentes de derivação têm valores $0.64,1.28,1.92,2.56,3.20,3.84$ e 4.48, sendo que o coeficiente da ordem 3.84 é nulo. O método fornece a seguinte solução:

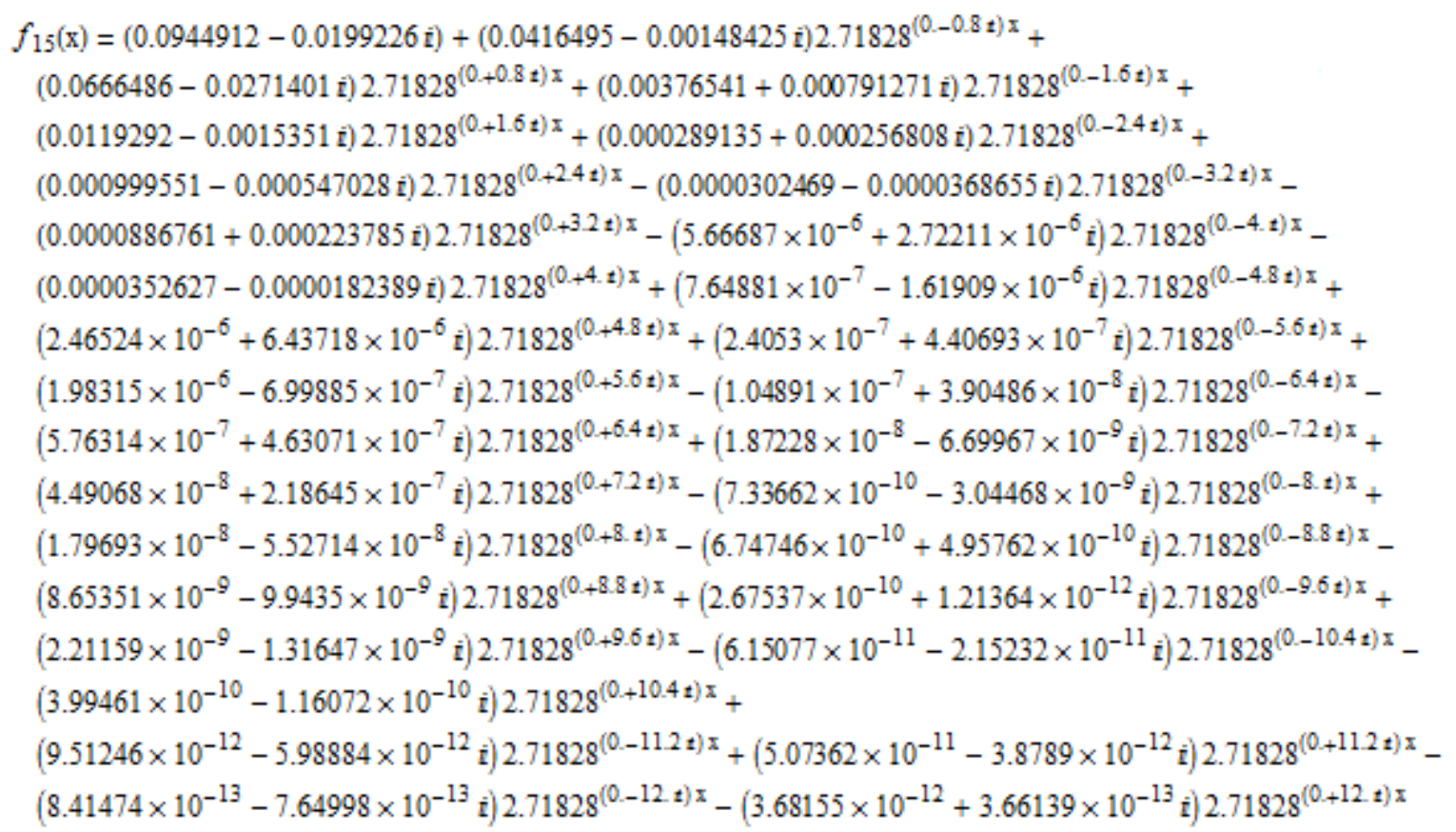

Os gráficos das partes real e imaginária e a curva paramétrica complexa dessa solução aparecem, respectivamente, nas figuras (92), (93) e (94).

São apresentados também os gráficos de superfície (fig.95) e os mapas paramétricos cartesiano e polar da solução aproximada $f_{15}(x)$, na figura (96).

Para finalizar a análise dessa equação, mostramos os gráficos das funções-resto $\mathcal{R}_{15}(x)$ da solução $f_{15}(x)$ encontrada, nas figuras $(97)$ e (98), bem como sua curva paramétrica complexa, na figura (99).

É fácil constatar que mesmo nesse caso, aparentemente bastante complicado quanto à 
Figura 92: Gráfico da parte real da solução $f_{15}$ da $\operatorname{EDF}(7 \nu)$

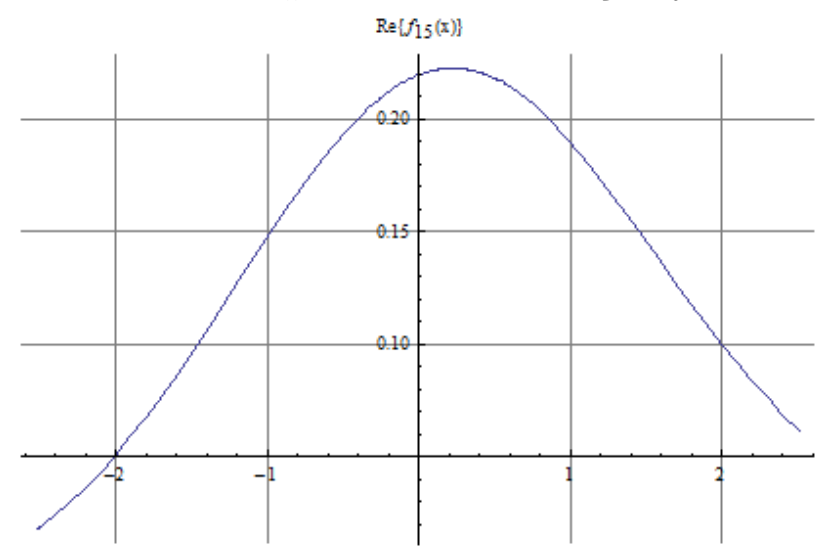

Figura 93: Gráfico da parte imaginária da solução $f_{15}$ da $\operatorname{EDF}(7 \nu)$



Figura 94: Gráfico da curva paramétrica complexa da solução $f_{15}$ da $\operatorname{EDF}(7 \nu)$

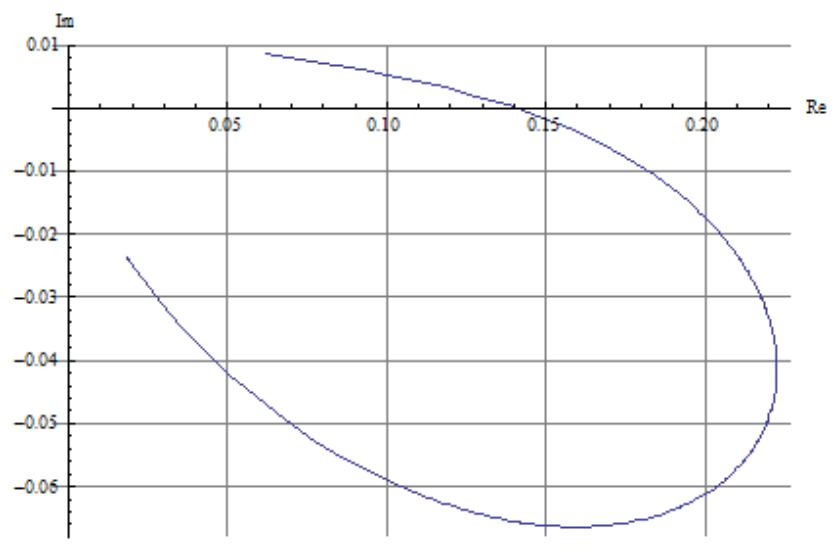


Figura 95: Gráficos complexos de superfície da solução $f_{15}$ da $\operatorname{EDF}(7 \nu)$
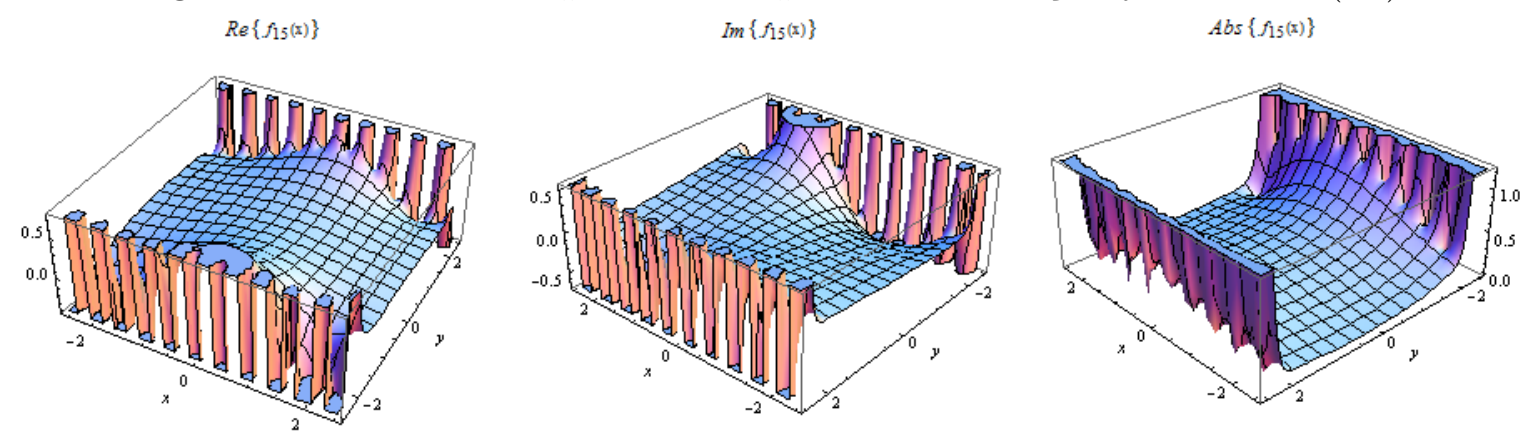

Figura 96: Mapas paramétricos cartesiano e polar da solução $f_{15}$ da $\operatorname{EDF}(7 \nu)$
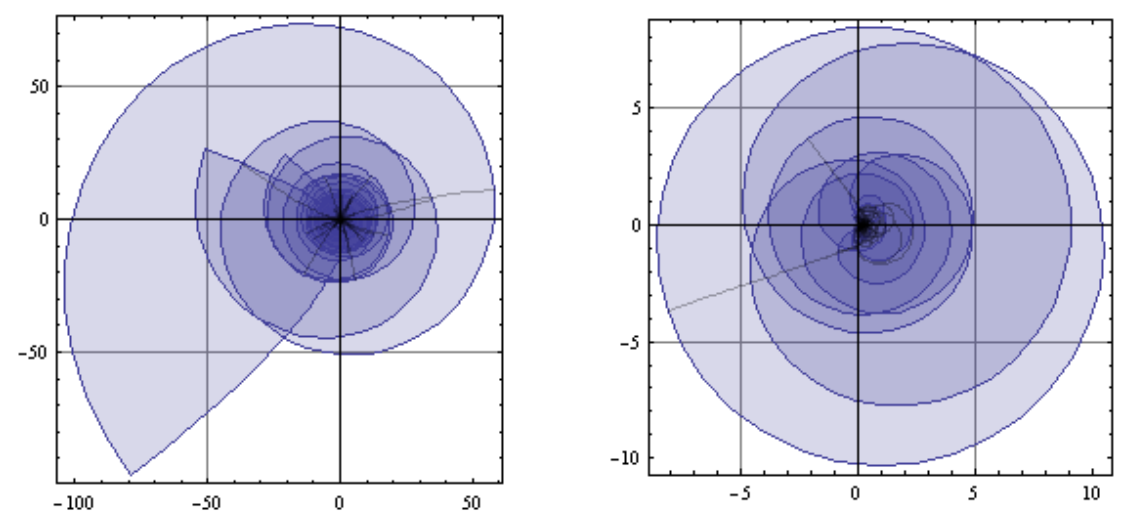

Figura 97: Gráfico da parte real da função-resto $\mathcal{R}_{15}(x)$

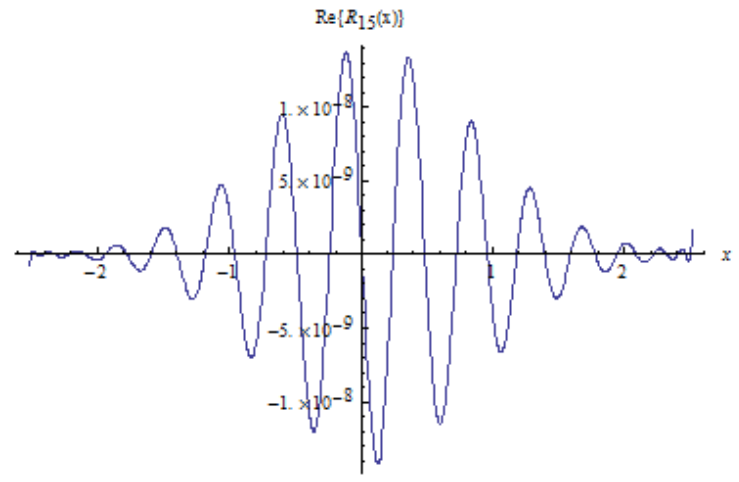

Figura 98: Gráfico da parte imaginária da função-resto $\mathcal{R}_{15}(x)$

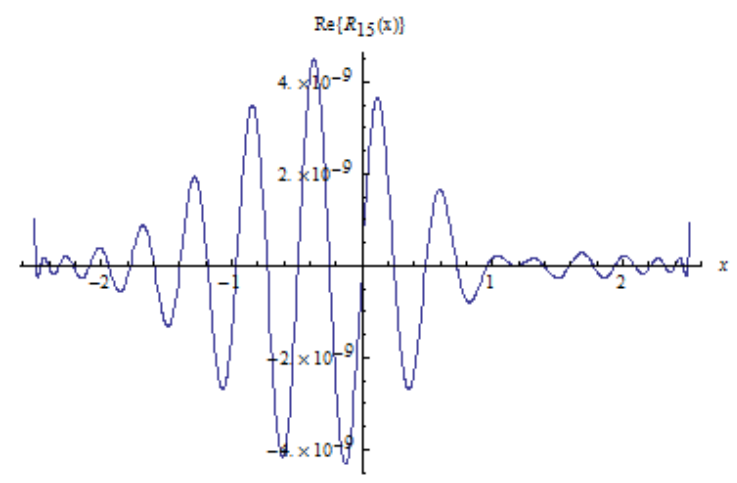


Figura 99: Curva paramétrica complexa da função resto $\mathcal{R}_{15}(x)$

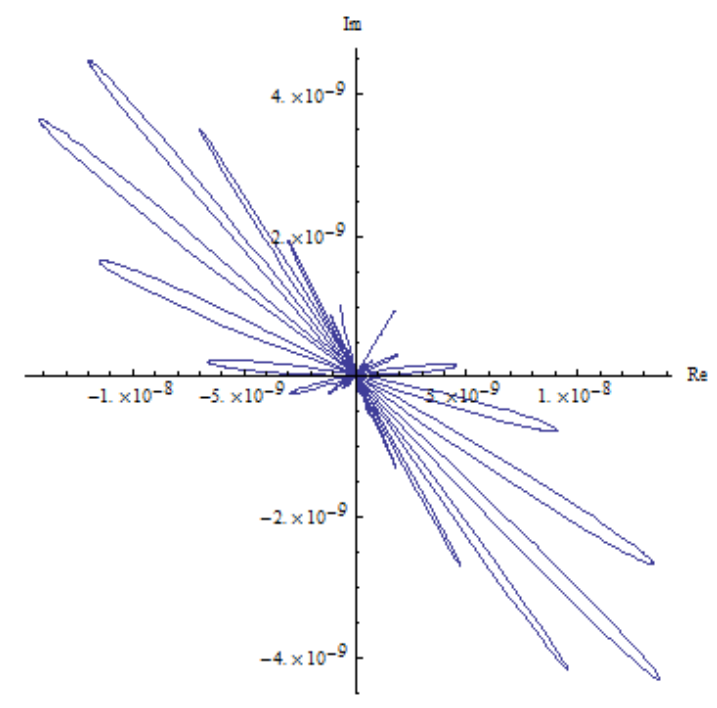

questão da precisão no processo de resolução, que o método de modelagem NBV-SA-NC satisfaz amplamente as expectativas esperadas. Agora, com o intuito de complementar tudo o que foi feito, partimos para um problema prático.

\subsubsection{EDF Unidimensional de Condução do Calor com Fonte}

Após o estudo desses casos ilustrativos, vamos partir para a análise de uma EDF mais significativa para uso prático. Há diversas EDFs interessantes a serem estudadas, especialmente as dos casos clássicos, como a do oscilador harmônico forçado amortecido e a equação de Schrödinger, que usa a derivada fracional de Riesz, bem como as equações de Lane-Emden e Bagley-Torvik, entre várias outras. Herrmann (2011) apresenta, por exemplo, o caso do oscilador harmônico fracional forçado com amortecimento. Sobre a equação de Schrödinger fracional, podemos citar, entre outros, os artigos de Laskin (2000a, 2002) e Oliveira, Costa e Vaz Junior (2010) e, sobre a EDF de Bagley-Torvik, os artigos de Diethelm e Ford (2002), Wang e Wang (2010), Raja, Khan e Qureshi (2011), Mekkaoui e Hammouch (2012) e Staněk (2013). Também a equação fracional de LaneEmden tem recebido maior atenção nos últimos anos (YILDIRIM; ÖZIŞ, 2007; CHOWDHURY; HASHIM, 2007; YILDIRIM; ÖZIŞ, 2009; HARLEY; MOMONIAT, 2010; HEYDARI; HOSSEINI; LOGHMANI, 2011; RISMANI; MONFARED, 2012; BHRAWY; ALOFI, 2012; MECHEE; SENU, 2012; CĂRUNTU; BOTA, 2013; DUPAIGNE; DAVILA; WEI, 2014).

No entanto, escolhemos aqui efetuar o estudo de um outro problema importante, que é a equação do calor. Essa equação é uma EDP e apresenta duas possibilidades de formulação, com ou sem fonte de calor, o que produz uma equação não-homogênea e uma 
homogênea, respectivamente. Como nosso método pressupõe a presença de um termo não-homogêneo, vamos adotar a versão mais completa, com fonte de calor. Entretanto, vamos nos ater ao caso unidimensional, visto que neste trabalho não foram abordadas EDPs. Para isso, precisamos reduzir a equação de forma apropriada.

\subsubsection{Solução NBV da EDF Unidimensional de Condução de Calor com Fonte}

A solução analítica dessa equação pode ser encontrada em Vázquez, Trujillo e Velasco (2011) e Anwar et al. (2013), por exemplo. Outros aspectos interessantes relacionados são encontrados nos artigos de Scherer et al. (2008) e Yang e Baleanu (2013).

A Equação de condução do calor em presença de uma fonte é dada por

$$
\frac{\partial \mathcal{U}}{\partial t}=\left(\frac{k}{c \rho}\right) \nabla^{2} \mathcal{U}+\left(\frac{1}{c \rho}\right) s,
$$

onde $\mathcal{U}$ é a temperatura, $\rho$ é a concentração (densidade de massa), $k$ e $c$ são a condutividade térmica do meio e sua capacidade térmica, respectivamente, e $s$ é a a densidade da fonte, i.e., a taxa de calor criada por unidade de tempo por unidade de volume. Para a redução a uma única dimensão espacial, tem-se:

$$
\frac{\partial \mathcal{U}(x, t)}{\partial t}=a^{2} \frac{\partial^{2} \mathcal{U}(x, t)}{\partial x^{2}}+b s(x, t)
$$

com $a^{2}=\left(\frac{k}{c \rho}\right)$ e $b=\left(\frac{1}{c \rho}\right)$. Sua versão fracional ${ }^{1}$ pode ser escrita como

$$
D_{t} \mathcal{U}(x, t)=a^{2} D^{1+\nu} \mathcal{U}(x, t)+b s(x, t), \quad 0<\nu<1
$$

que é uma equação diferencial parcial fracionária (EDPF). Supondo que a função $\mathcal{U}(x, t)$ seja separável, de modo que tenhamos $\mathcal{U}(x, t)=u(x) \vartheta(t)$ e tomando um intervalo de tempo finito, de maneira a impor uma partição

$$
P_{n}=\bigcup_{i=1}^{n}\left[t_{i-1}, t_{i}\right]
$$

sobre ele, podemos reescrever a EDPF como uma EDF para um instante específico $t_{j}$, obtendo

$$
u(x) \vartheta^{\prime}\left(t_{j}\right)=a^{2} \vartheta\left(t_{j}\right) D^{1+\nu} u(x)+b s\left(x, t_{j}\right),
$$

onde usamos a regra generalizada de Leibniz para derivadas de produtos, dispondo as

\footnotetext{
${ }^{1}$ De fato, apenas uma das versões possíveis, já que a ordem fracional pode ser acoplada à parte temporal em vez da espacial.
} 
funções de forma que o desenvolvimento da somatória forneça apenas as derivadas fracionais em $x$. Redenominando o termo da fonte no instante especificado: $s\left(x, t_{j}\right)=s_{j}(x) \mathrm{e}$ dividindo toda a equação por $a^{2} \vartheta\left(t_{j}\right)$, obtém-se

$$
D^{1+\nu} u(x)+\lambda\left(t_{j}\right) u(x)=\alpha\left(t_{j}\right) s_{j}(x), \quad 0<\nu<1
$$

onde

$$
\begin{aligned}
\lambda\left(t_{j}\right) & =-\left.\frac{1}{\vartheta(t)} \frac{d \vartheta(t)}{d t} \frac{1}{a^{2}}\right|_{t=t_{j}}=-\left.\left(\frac{c \rho}{k}\right) \frac{1}{\vartheta(t)} \frac{d \vartheta(t)}{d t}\right|_{t=t_{j}} \\
\alpha\left(t_{j}\right) & =-\left(\frac{b}{a^{2}}\right) \frac{1}{\vartheta\left(t_{j}\right)}=-\frac{1}{k \vartheta\left(t_{j}\right)}
\end{aligned}
$$

Assim, nosso problema fica restrito à solução da EDF unidimensional (5.37), uma vez que, para efeito de cálculo, os coeficientes são entendidos como constantes, devendo a função $\phi(x)$ do termo não-homogêneo ser especificada de acordo com a natureza da fonte de calor $s_{j}(x)$. Portanto, vamos utilizar o método NBV na solução de uma equação do tipo:

$$
D^{\beta} u(x)+\lambda u(x)=\phi(x), \quad 1<\beta<2
$$

Escolhemos, para a solução NBV, semiperíodo $L=\frac{4 \pi}{5}$, range $\ell=15$ e, naturalmente, o uso dos nodos de Chebyshev na série associada. Elegemos ordem fracional $\nu=0.7$, o que implica em uma EDF de ordem 1.4 e coeficientes unitários $\lambda=\alpha=1$, por simplicidade. A função complexa do termo homogêneo é a mesma já foi empregada nos exemplos da EDO e da EDF de $7^{\mathrm{a}}$ ordem: $\phi(x)=e^{i x / \sqrt{L}} e^{-x^{2} / L}$.

Os gráficos real e imaginário da função solução $f_{15}$ aparecem nas figuras (100) e (101) e sua curva paramétrica complexa, na figura (102). Já as curvas de superfície dessa solução vêm mostradas na figura (107).

Nas figuras (104) a (106) vêem-se as partes real, imaginária e a curva paramétrica da função-resto local, enquanto o gráfico comparativo entre as curvas paramétricas de $\mathcal{D}_{e q} f_{15}(x)$ e $\phi(x)$ é apresentado na figura (103). Por fim, vêm os mapas paramétricos cartesiano e polar da solução obtida, juntos na figura (108).

O que se percebe é que a parte real do resto local é menor que a imaginária, sendo o resto absoluto total dessa mesma ordem, indicando uma precisão excelente.

Para concluir a exposição dos resultados, calculamos o erro total absoluto $\mathcal{E}_{a b s}^{(15)}$, defi- 
nido pela equação:

$$
\mathcal{E}_{a b s}^{(\ell)}=\int_{-L}^{L}\left\|\phi(x)-\mathcal{D}_{e q}^{\beta} f_{\ell}(x)\right\| d x
$$

onde $\mathcal{D}_{e q}^{\beta}$ é o operador diferencial fracionário da EDF e $\beta$ é sua ordem fracional máxima que, no nosso caso, vale $\beta=2 \nu=1.4$.

O resultado do erro absoluto total foi de $\mathcal{E}_{a b s}^{(15)}=2.01088 \times 10^{-11}$, o que vem a comprovar o que foi dito sobre a excelência do método.

Figura 100: Parte real da solução NBV-SA-NC da EDF de Calor

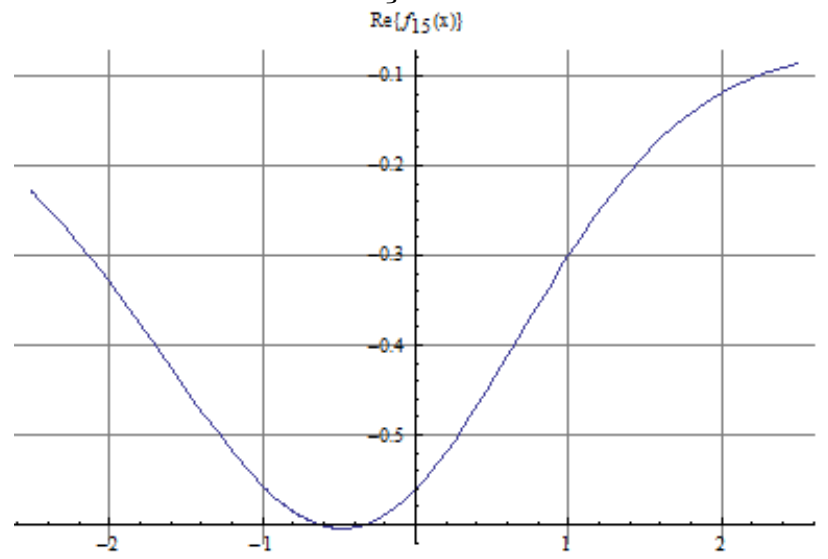

Figura 101: Parte imaginária da solução NBV-SA-NC da EDF de Calor $\operatorname{In}\left\{f_{15}(x)\right\}$

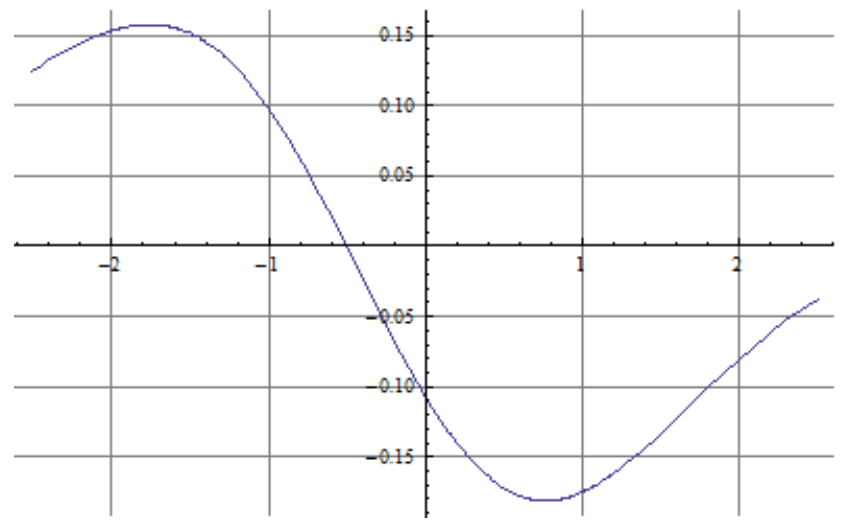

\subsubsection{Conclusão}

Após todas as análises efetuadas sobre os mais variados exemplos apresentados, comprovamos a grande eficiência do formalismo tensorial e do método de modelagem na solução de EDOs e EDFs não-homogêneas. Constatamos também o aprimoramento produzido pela implementação das séries associadas, não apenas na solução de equações diferenciais, mas também na própria expansão de funções em períodos inferiores a $2 \pi$. 
Figura 102: Curva paramétrica complexa da solução NBV-SA-NC da EDF de Calor



Figura 103: Comparação entre as curvas paramétricas de $\mathcal{D}_{e q} f_{15}$ e $\phi$

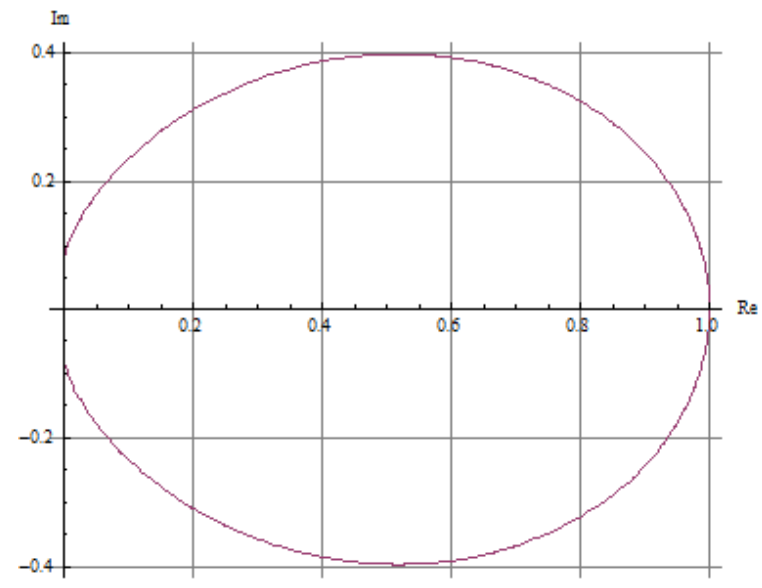

Figura 104: Parte real do resto local $R_{15}(x)$ na solução da EDF de Calor $\operatorname{Re}\left[R_{15}(x)\right]$

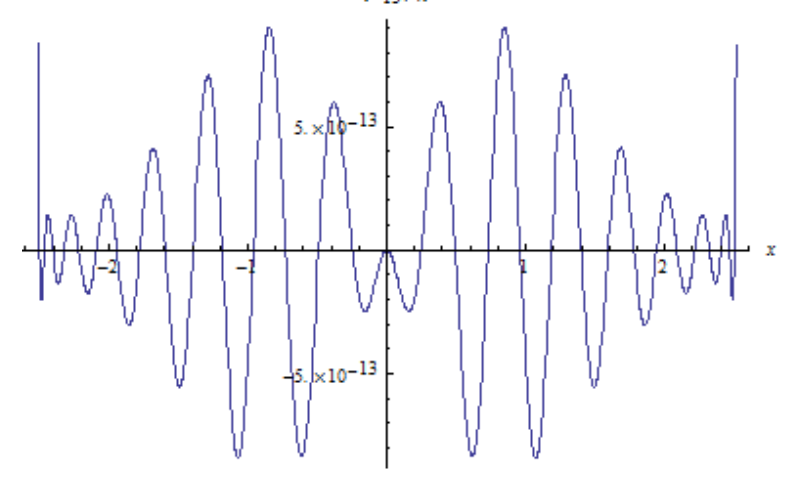


Figura 105: Parte imaginária do resto local $R_{15}(x)$ na solução da EDF de Calor

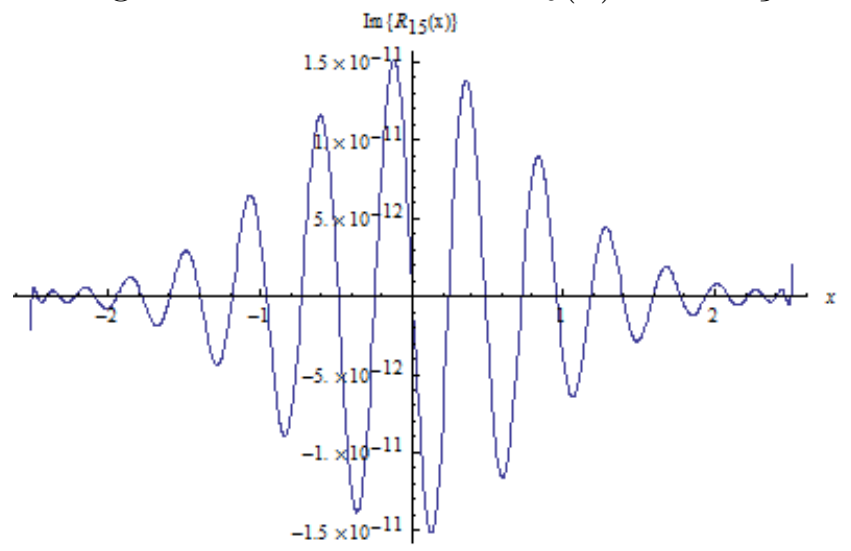

Figura 106: Curva paramétrica complexa do resto local $R_{15}(x)$ na solução da EDF de Calor

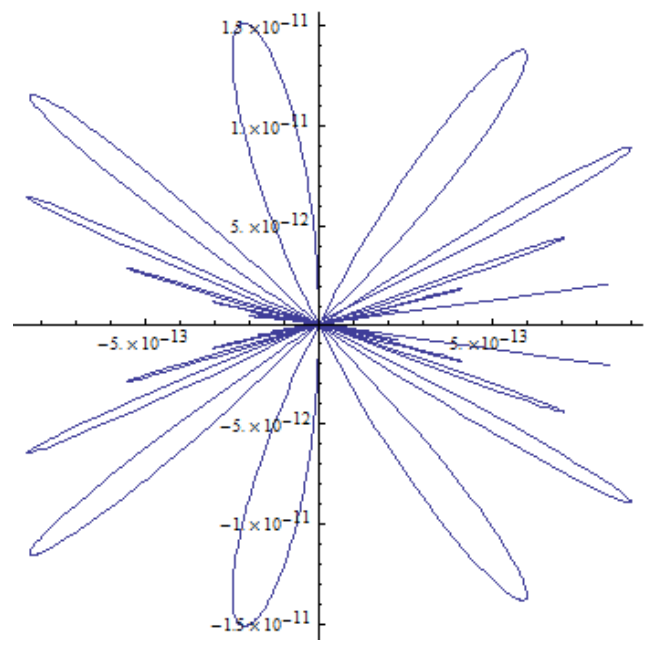

Figura 107: Gráficos complexos de superfície da solução NBV-SA-NC da EDF de Calor $\operatorname{Re}\left\{f_{15}(\mathrm{x})\right\}$
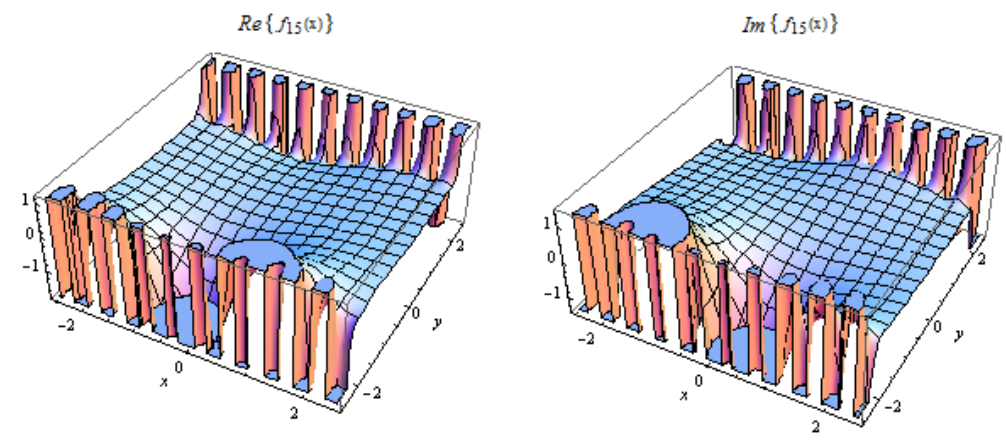
$A b s\left\{f_{15}(\mathrm{x})\right\}$

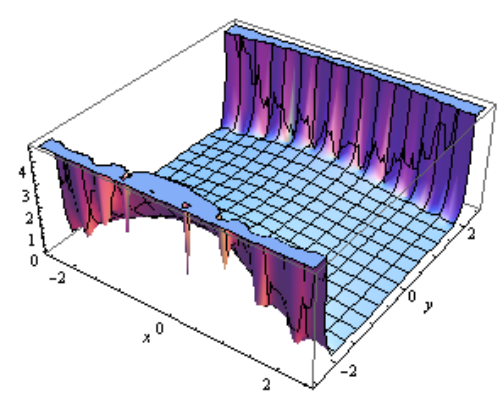


Figura 108: Mapas paramétricos da solução NBV-SA-NC da EDF de Calor
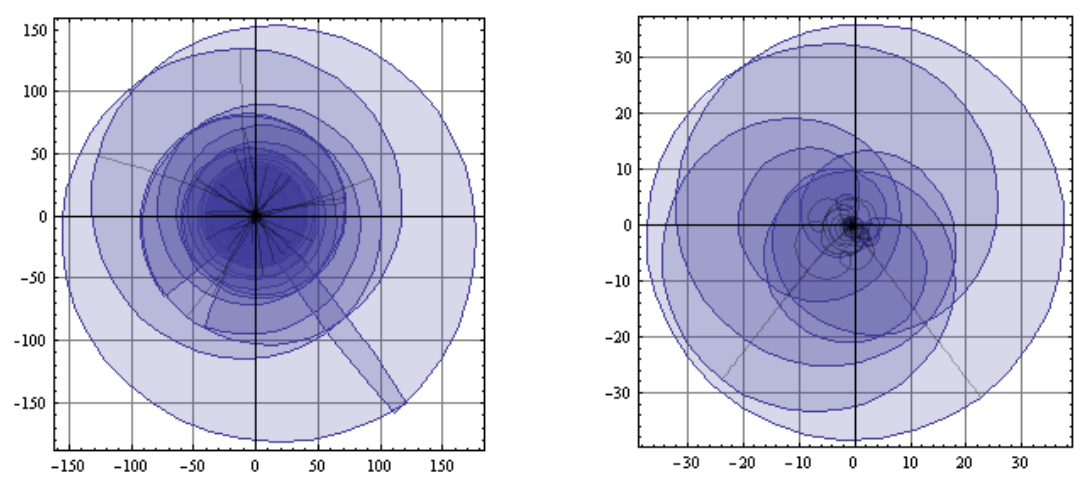

Além disso, concluímos que a utilização do formalismo tensorial de Dirac amplia as possibilidades de compreensão e desenvolvimento da teoria dos espaços de funções, inserindo de forma eficaz os polinômios não-ortogonais nos processos de solução e análise.

E, finalmente, acrescentamos que a maior precisão conseguida está associada ao uso dos nodos de Chebyshev na expansão pelas séries associadas no método NBV/NIV, razão pela qual achamos recomendável, para domínios de definição periódica inferiores a $2 \pi$, a modelagem NBV/NIV-SA-NC.

Todavia, é preciso acrescentar que, para o caso específico de algumas EDFs, o método NBV/NIV-SF-EC também funciona de modo bastante preciso, por vezes até melhor que o SA-NC. O motivo dessa boa adequação exige um estudo formal sistemático, não implementado nessa tese, inclusive para se determinar a aplicação adequada de cada método às situações concretas. 


\section{CONCLUSÃO}

\subsection{Uma Visão Geral}

O objetivo essencial deste trabalho era o de estudar e desenvolver técnicas alternativas de resolução de equações diferenciais ordinárias não-homogêneas e fracionárias. Conseguiu-se atingir essa meta através de uma formulação inovadora e eficaz, com excelentes perspectivas de ampliação.

O método e o algoritmo desenvolvidos para resolver os problemas de condições iniciais ou de contorno por meio de aproximações de séries de Fourier complexas, denominado método de modelagem, foi generalizado a fim de ser utilizado para soluções sem imposição de condições, denominado método NBV para BVPs ou NIV para IVPs.

Além disso, foi introduzida uma nova série, fundamentada na teoria de Fourier, denominada série associada, a qual, como foi demonstrado, alcança resultados ainda melhores e com maior eficiência que a própria série de Fourier, para períodos menores que $2 \pi$, especialmente se for combinada com o uso sistemático dos nodos de Chebyshev.

No que diz respeito à utilização do espaçamento constante ou dos nodos de Fourier e Chebyshev, embora os primeiros desses nodos tenham melhorado a precisão das soluções pela série original, estes últimos mostraram-se totalmente ineficientes na resolução NBV, gerando divergências em alguns casos. Apesar disso, incrementaram significativamente a precisão quando utilizados no método das séries associadas.

Podemos afirmar, após haver estudado outros casos não mostrados aqui, que a melhor escolha de pontos para a solução na base de Fourier original é a dos nodos de Fourier. Para a solução na série associada, contudo, a escolha recai nos nodos de Chebyshev. Ainda mais que isso, é aconselhável o uso desses últimos e da série associada para qualquer caso de período inferior a $2 \pi$, visto que a precisão obtida é significativamente maior à medida que o range aumenta, devido à convergência mais rápida dessa série.

Mostrou-se também que o formalismo de Dirac fornece uma maneira simples de solu- 
cionar este tipo de problema com excelente precisão, se for usado em combinação com os outros recursos adicionais previamente mencionados.

No que tange a resultados originais obtidos, há uma grande variedade de tópicos que acreditamos serem novos. Pelas sondagens que fizemos na bibliografia do assunto, pudemos enumerar uma série extensa de pontos cuja existência não pôde ser verificada, o que nos leva a crer que sejam realmente novos. Como a relação é grande, optamos por apresentá-la na forma de uma lista, exposta a seguir.

\subsubsection{Contribuições da Tese}

Na presente seção é feito um balanço geral de todos os tópicos originais alcançados na consecução deste trabalho.

Incluímos na descrição, tanto as inovações de linguagem e formalismo quanto os resultados matemáticos inéditos atingidos.

1. Construção da Reformulação Coadjunta: adaptação da linguagem bra-ket a espaços de dimensão finita;

2. Desenvolvimento do formalismo tensorial na teoria de funções nessa linguagem;

3. Introdução dos conceitos de IP hermitiano, dual e misto;

4. Introdução das representações correlatas;

5. Implementação do tensor métrico recíproco como um operador tensorial de expansão de funções;

6. Implementação e aplicação do formalismo tensorial a bases de funções não-ortogonais com excelentes resultados;

7. Determinação da forma geral das bases recíprocas de Legendre, de Fourier e polinomial canônica;

8. Extensão do uso de matrizes operacionais ao corpo complexo;

9. Extensão do Teorema da Matriz Operacional de Guimarães ao corpo complexo;

10. Extensão da Matriz Sandwich ao corpo complexo;

11. Determinação da matriz operacional de derivação clássica na base de Fourier complexa; 
12. Determinação da matriz operacional de integração clássica na base de Fourier complexa;

13. Determinação do covetor coeficiente de funções, derivadas e integrais clássicas de qualquer ordem em qualquer base, ortogonal ou não;

14. Determinação das matrizes operacionais de derivação fracionárias na base de Fourier complexa;

15. Determinação das matrizes operacionais de integração fracionárias na base de Fourier complexa;

16. Determinação do covetor coeficiente de funções, derivadas e integrais fracionais em qualquer base, ortogonal ou não;

17. Elaboração do Método de Modelagem Operacional para resolução de EDOs e EDFs não-homogêneas;

18. Elaboração de um procedimento de resolução de EDOs e EDFs não-homogêneas sem a imposição de condições iniciais ou de contorno, denominado Método NBV/NIV;

19. Construção de uma série alternativa do tipo Fourier, denominada Série de Fourier Associada, com convergência mais rápida em períodos inferiores a $2 \pi$;

20. Enunciado e demonstração de dois teoremas de convergência referentes às séries associadas;

21. Aperfeiçoamento do método de modelagem operacional pela introdução das séries associadas coligadas ao uso dos nodos de Chebyshev;

22. Análise de resultados em diferentes versões do método de modelagem, com o uso de séries de Fourier e associadas para pontos com espaçamento constante, nodos de Chebyshev e nodos de Fourier;

23. Extensão do formalismo coadjunto ao cálculo fracional;

24. Extensão do método de modelagem ao cálculo fracional;

25. Adaptação dos Operadores de Weyl a períodos genéricos no formalismo de Dirac;

26. Introdução dos mapas paramétricos na descrição gráfica;

27. Resolução analítica da EDF de Bagley-Torvik em termos das funções de Prabhakar (Mittag-Leffler de três parâmetros); 
28. Resolução numérica da EDF complexa unidimensional de condução do calor com fonte por modelagem operacional NBV-SA-NC;

\subsubsection{Limitações do Método Proposto}

Embora estejamos ressaltando as inegáveis vantagens conseguidas, sabemos que há limitações importantes do método a serem destacadas. A primeira e mais óbvia é comentada a seguir.

O método de modelagem operacional apresenta uma limitação fundamental. O processo se baseia essencialmente na comparação entre o operador da equação diferencial e a função do termo independente. Assim, caso a EDO seja homogênea, não há termo independente com o qual comparar o operador e, desse modo, a única solução encontrada seria, não a solução homogênea, mas a trivial. Logo, o método não funciona para EDOs ou EDFs homogêneas.

Uma outra limitação evidente é o fato de não terem sido abordadas EDOs não-lineares. De fato, a adaptação da linguagem de Dirac aos espaços $\mathcal{O}_{n}$ ainda não foi desenvolvida de forma extensiva para uso efetivo na solução de EDOs não-lineares, pois há algumas complicações inerentes a essa tentativa, das quais não cabe comentar nada aqui. No entanto, já estamos entrevendo caminhos adequados de expansão a tais casos.

Uma perspectiva interessante de desenvolvimento posterior consiste, portanto, em ampliar o tratamento tensorial e o processo de solução operacional de forma a incluir as EDOs homogêneas e não-lineares.

Além disso, a ampliação do método a duas e três variáveis para solucionar EDPs, sejam de ordem inteira ou arbitrária, é altamente desejável e o caminho para atingir esta meta já se apresenta mais ou menos esquematizado. Na verdade, consideramos prioritária essa expansão no momento atual.

A principal ideia a ser explorada com esses objetivos consiste em expandir o formalismo já desenvolvido à teoria geométrica de sistemas, com a qual esperamos alcançar todas as metas propostas.

Sobre isso, discorre-se no próximo parágrafo. 


\subsubsection{Prognósticos de Trabalho Futuro}

Como dissemos antes, a principal perspectiva no momento é a obtenção de uma generalização do método para funções de duas ou mais variáveis, com o intuito de solucionar EDPFs no corpo complexo.

Embora o formalismo desenvolvido aqui, da maneira como foi exposto, não suporte essa expansão, alguns caminhos alternativos parecem bastante promissores. Um deles consiste numa reinterpretação do isomorfismo $\mathbb{R}^{2} \simeq \mathbb{C}$ como um mecanismo para a primeira ampliação a duas dimensões; um outro, repousa numa extensão do formalismo à teoria de sistemas tradicional.

Um outro aspecto essencial que consideramos promissor é a expansão do método a EDOs não-lineares, visto que a interpretação geométrica do formalismo coadjunto sugere uma aplicação relativamente direta à teoria geométrica de sistemas.

Caso tenhamos sucesso, a consecução desse objetivo permitirá a obtenção de resultados muito precisos em EDPs não-lineares importantes, mas de difícil resolução, principalmente as relacionadas a fenômenos termo-hidráulicos e fluidodinâmicos.

Um campo de pesquisa muito interessante diz respeito à associação das EDPFs a sistemas caóticos e geometria fractal. Foi observado, embora não tenhamos feito menção a esse fato na tese, que determinadas soluções com condições específicas, parecem gerar atratores ou gráficos estocásticos, principalmente quando as soluções obtidas são de ordem muito pequena. Entretanto, isso exige estudos mais aprofundados.

De qualquer forma, acreditamos que a própria técnica aqui exposta ainda guarda muitas novidades. Tencionamos dar prosseguimento imediato a esse assunto, expandindo o conceito de transposição de domínios, que foi introduzida na tese de Guimarães (2008), para utilizá-la na solução de equações de difícil abordagem em seus próprios domínios, solucionando-as no domínio transposto e trazendo as soluções de volta ao domínio original.

Além disso, o esquema geral de transformações entre bases genéricas pode ser mais explorado no intuito de obter relações entre essas diferentes bases e as formas das equações diferenciais mais facilmente solúveis em cada base. Isso poderia ser de grande ajuda na resolução de equações ainda sem solução. Queremos dizer que uma EDO com certa forma numa base deve apresentar diferente aspecto em outra e, por meio do processo descrito, poderíamos obter uma simplificação na resolução, de modo similar ao que ocorre com o processo de transformadas, mas de uma natureza diferente, mais ampla nesse caso. 


\subsubsection{Considerações Finais}

Acreditamos, após a análise empreendida no capítulo anterior, que não seja exagero dizer que a técnica desenvolvida neste trabalho produziu resultados extremamente satisfatórios, tanto no que se refere à precisão obtida no processo de resolução de EDOs e EDFs, quanto à simplicidade de exposição, bem como à rapidez dos processos computacionais.

Aliada a esses fatores, está a construção desse formalismo alternativo, o qual abre novos horizontes ao desenvolvimento e expansão na teoria de equações diferenciais, com a futura implementação de uma teoria geométrica de sistemas nessa linguagem.

Chegou-se, comprovadamente, à obtenção de um método ágil, elegante e, mais importante do que tudo, bastante eficiente. 


\section{Referências}

ADOMIAN, G. A review of the decomposition method in applied mathematics. Journal of mathematical analysis and applications, Elsevier, v. 135, n. 2, p. 501-544, 1988. Disponível em: http://www.sciencedirect.com/science/article/ pii/0022247X88901709.

ANWAR, A. M. O. et al. Fractional caputo heat equation within the double laplace transform. Romanian Journal of Physics, v. 58, n. 1-2, p. 15-22, 2013. Disponível em: http://www.ifin.ro/rjp/2013_58_1-2/0015_0022.pdf.

BALEANU, D.; ALIPOUR, M.; JAFARI, H. The Bernstein operational matrices for solving the fractional quadratic Riccati differential equations with the Riemann-Liouville derivative. In: HINDAWI PUBLISHING CORPORATION. Abstract and Applied Analysis. 2013. v. 2013. Disponível em: http://www.hindawi.com/journals/aaa/ 2013/461970/abs/.

BHRAWY, A. H.; ALOFI, A. S. A jacobi-gauss collocation method for solving nonlinear lane-emden type equations. Communications in Nonlinear Science and Numerical Simulation, Elsevier, v. 17, n. 1, p. 62-70, 2012. Disponível em: http://www.sciencedirect.com/science/article/pii/S100757041100219X.

The operational matrix of fractional integration for shifted Chebyshev polynomials. Applied Mathematics Letters, Elsevier, v. 26, n. 1, p. 25-31, 2013. Disponível em: http://www.sciencedirect.com/science/article/pii/ S0893965912000444.

BRENNER, S. C.; SCOTT, R. The mathematical theory of finite element methods. [S.l.]: Springer Science \& Business Media, 2008.

BULGAKOV, B. V. Kolebaniya (vibrations). Gostekhizdat, Moscow, 1954.

BUTZER, P. L.; WESTPHAL, U. An introduction to fractional calculus. World Scientific, 2000. Disponível em: http://www.researchgate.net/profile/Denis_ Matignon/publication/229762111_An_Introduction_to_Fractional_Calculus/ links/5448df2c0cf22b3c14e338d1.pdf.

CAITI, A.; CANNATA, G. Comments on the properties of the operational matrices of integration and differentiation for Fourier trigonometric functions. IEEE transactions on automatic control, Institute of Electrical and Electronics Engineers, v. 38, n. 4, p. 667-671, 1993.

CĂRUNTU, B.; BOTA, C. Approximate polynomial solutions of the nonlinear lane-emden type equations arising in astrophysics using the squared remainder minimization method. Computer Physics Communications, Elsevier, v. 184, n. 7, p. 
1643-1648, 2013. Disponível em: http://www.sciencedirect.com/science/article/ pii/S0010465513000647.

CHALISHAJAR, H. D.; KANTAWALA, P. S. Fractional calculus using wavelet packets. Bulletin of the Marathwada Mathematical Society, v. 9, n. 1, p. 7-17, 2008.

CHANG, R.Y.; WANG, M.L. Parameter identification via shifted Legendre polynomials. International Journal of Systems Science, Taylor \& Francis, v. 13, n. 10, p. 1125-1135, 1982.

CHEN, C.F.; HSIAO, C.H. Walsh series analysis in optimal control. International Journal of Control, Taylor \& Francis, v. 21, n. 6, p. 881-897, 1975.

CHEN, C.F.; TSAY, Y.T.; WU, T.T. Walsh operational matrices for fractional calculus and their application to distributed systems. Journal of the Franklin Institute, Elsevier, v. 303, n. 3, p. 267-284, 1977. Disponível em: http: //www.sciencedirect.com/science/article/pii/0016003277900291.

CHOWDHURY, M. S. H.; HASHIM, I. Solutions of a class of singular second-order ivps by homotopy-perturbation method. Physics Letters A, Elsevier, v. 365, n. 5, p. 439-447, 2007. Disponível em: http://www.sciencedirect.com/science/article/ $\mathrm{pii/S0375960107001752.}$

DIETHELM, K.; FORD, J. Numerical solution of the bagley-torvik equation. BIT Numerical Mathematics, Kluwer Academic Publishers, v. 42, n. 3, p. 490-507, 2002. ISSN 0006-3835. Disponível em: http://dx.doi.org/10.1023/A\%3A1021973025166.

DOHA, E. H.; BHRAWY, A. H. Efficient spectral-Galerkin algorithms for direct solution of fourth-order differential equations using Jacobi polynomials. Applied Numerical Mathematics, Elsevier, v. 58, n. 8, p. 1224-1244, 2008. Disponível em: http://www. sciencedirect.com/science/article/pii/S0168927407001109.

DUPAIGNE, L.; DAVILA, J.; WEI, J. On the fractional lane-emden equation. 2014. Disponível em: https://hal .archives-ouvertes.fr/hal-00978738.

EBRAHIMI, R. et al. Application of Fourier series direct method to the optimal control of singular systems. ICGST-ACSE Journal, v. 7, n. 2, p. 19-24, 2007.

ELBARBARY, E. M. E. Legendre expansion method for the solution of the second-and fourth-order elliptic equations. Mathematics and Computers in Simulation, Elsevier, v. 59, n. 5, p. 389-399, 2002. Disponível em: http: //www.sciencedirect.com/science/article/pii/S0378475401004219.

ENDOW, Y. Optimal control via Fourier series of operational matrix of integration. Automatic Control, IEEE Transactions on, IEEE, v. 34, n. 7, p. 770-773, 1989.

ERJAEE, G. H. The operational matrix of fractional integration for shifted Legendre polynomials. Iranian Journal of Science and Technology (Sciences), v. 37, n. 4, p. 439-444, 2013. Disponível em: http://ijsts.shirazu.ac.ir/article_1778_37.html.

ERN, A.; GUERMOND, J.L. Theory and practice of finite elements. [S.l.]: Springer Science \& Business Media, 2013. 
FIGUEIREDO, D. G. de. Análise de Fourier e equações diferenciais parciais. [S.l.]: Instituto de Matemática Pura and Aplicada, 2000.

GUIMARÃES, J. O. S. Computação evolutiva na resolução de equações diferenciais ordinárias não lineares no espaço de Hilbert. Tese (Doutorado) Universidade de São Paulo, 2008. Disponível em: http://www.teses.usp.br/teses/ disponiveis/3/3142/tde-29062009-154349/en.php.

GUIMARÃES, Osvaldo; PIQUEIRA, J. R. C.; LOBO NETTO, Márcio. Direct computation of operational matrices for polynomial bases. Mathematical Problems in Engineering, Hindawi Publishing Corporation, v. 2010, 2010.

HADAMARD, J. Essai sur l'étude des fonctions données par leur développement de Taylor. [S.l.]: Gauthier-Villars, 1892.

HARLEY, C.; MOMONIAT, E. Efficient boundary value problem solution for a laneemden equation. Mathematical and Computational Applications, v. 15, n. 4, p. 613, 2010. Disponível em: http://mcajournal.cbu.edu.tr/specialissue15_4/8.pdf.

HERRMANN, R. Fractional calculus: An introduction for physicists. [S.l.]: World Scientific, 2011.

HEYDARI, M.; HOSSEINI, S. M.; LOGHMANI, G. B. Numerical solution of singular ivps of lane-emden type using integral operator and radial basis functions. International Journal of Industrial Mathematics, v. 4, n. 2, p. 135-146, 2011. Disponível em: http://ijim.srbiau.ac.ir/pdf_2111_8e5da61133f6fe9b91f457cf8163f8ee.html.

HILFER, R. et al. Applications of fractional calculus in physics. [S.l.]: World Scientific, 2000.

. Threefold introduction to fractional derivatives. Anomalous transport: Foundations and applications, Wiley-VCH Verlag, Weinheim, p. 17-73, 2008. Disponível em: http://www.icp.uni-stuttgart.de/ hilfer/publikationen/pdfa/ ZZ-2008-ATFaA-17-a.pdf.

HORNG, I.R.; CHOU, J.H. Shifted Chebyshev direct method for solving variational problems. International Journal of Systems Science, Taylor \& Francis, v. 16, n. 7, p. 855-861, 1985. Disponível em: http://dx.doi.org/10.1080/00207728508926718.

HWANG, C.; SHIH, Y.P. Solution of integral equations via Laguerre polynomials. Computers \& Electrical Engineering, Elsevier, v. 9, n. 3, p. 123-129, 1982. Disponível em: http://www.sciencedirect.com/science/article/pii/0045790682900180.

IVERSON, K. E. A programming language. In: ACM. Proceedings of the May 1-3, 1962, spring joint computer conference. [S.l.], 1962. p. 345-351.

KARR, C. L.; YAKUSHIN, I.; NICOLOSI, K. Solving inverse initial-value, boundary-value problems via genetic algorithm. Engineering Applications of Artificial Intelligence, Elsevier, v. 13, n. 6, p. 625-633, 2000. Disponível em: http://www.sciencedirect.com/science/article/pii/S0952197600000257. 
KATUGAMPOLA, U. N. New approach to a generalized fractional integral. Applied Mathematics and Computation, Elsevier, v. 218, n. 3, p. 860-865, 2011. Disponível em: http://www.sciencedirect.com/science/article/pii/S0096300311004309.

KAZEM, S.; ABBASBANDY, S.; KUMAR, S. Fractional-order Legendre functions for solving fractional-order differential equations. Applied Mathematical Modelling, Elsevier, v. 37, n. 7, p. 5498-5510, 2013. Disponível em: http: //www.sciencedirect.com/science/article/pii/S0307904X12006312.

KHADER, M. M. Numerical solution of nonlinear multi-order fractional differential equations by implementation of the operational matrix of fractional derivative. Studies in Nonlinear Sciences, v. 2, n. 1, p. 5-12, 2011. Disponível em: http://www.idosi.org/sns/2(1)11/2.pdf.

KHAN, M. A.; AHMED, S. On some properties of the generalized Mittag-Leffler function. SpringerPlus, Springer International Publishing, v. 2, n. 1, p. 1-9, 2013. Disponível em: http://dx.doi.org/10.1186/2193-1801-2-337.

KHELLAT, F.; YOUSEFI, S. A. The linear Legendre mother wavelets operational matrix of integration and its application. Journal of the Franklin Institute, Elsevier, v. 343, n. 2, p. 181-190, 2006. Disponível em: http://www.sciencedirect.com/ science/article/pii/S0016003205001158.

KILBAS, A. A.; SRIVASTAVA, H. M.; TRUJILLO, J. J. Theory and applications of fractional differential equations. [S.l.]: Elsevier Science Limited, 2006.

KILICMAN, A.; ZHOUR, Z. A. A. Al. Kronecker operational matrices for fractional calculus and some applications. Applied Mathematics and Computation, Elsevier, v. 187, n. 1, p. 250-265, 2007. Disponível em: http://www.sciencedirect.com/ science/article/pii/S0096300306011593.

KING, R. E.; PARASKEVOPOULOS, P. N. Parametric identification of discrete-time SISO systems. International Journal of Control, Taylor \& Francis, v. 30, n. 6, p. 1023-1029, 1979.

KREYSZIG, E. Introduction to differential geometry and Riemannian geometry. [S.l.]: University of Toronto Press, 1968.

LABECCA, W.; GUIMARÃES, O.; PIQUEIRA, J. R. C. Dirac's formalism combined with complex Fourier operational matrices to solve initial and boundary value problems. Communications in Nonlinear Science and Numerical Simulation, Elsevier, v. 19, n. 8, p. 2614-2623, 2014. Disponível em: http: //www.sciencedirect.com/science/article/pii/S1007570414000100.

LACROIX, S. F. Traité du calcul différentiel et du calcul intégral. [S.l.]: Courcier, 1810.

LARA, L. P.; GADELLA, M. An approximation to solutions of linear ODE by cubic interpolation. Computers \& Mathematics with Applications, Elsevier, v. 56, n. 6, p. 1488-1495, 2008. Disponível em: http://www.sciencedirect.com/science/ article/pii/S0898122108001752. 
LASKIN, N. Fractals and quantum mechanics. Chaos: An Interdisciplinary Journal of Nonlinear Science, AIP Publishing, v. 10, n. 4, p. 780-790, 2000.

. Fractional quantum mechanics. Physical Review E, American Physical Society, v. 62, n. 3, p. 3135-3145, 2000. Disponível em: http://link.aps.org/doi/10.1103/ PhysRevE.62.3135.

Fractional Schrödinger equation. Physical Review E, American Physical Society, v. 66, n. 5, p. 056108, 2002. Disponível em: http://link.aps.org/doi/10. 1103/PhysRevE. 66.056108.

LIMA, E. L. Curso de análise, Volume 1. [S.l.]: Instituto Nacional de Matemática Pura e Aplicada, 2004.

LIZORKIN, P. I. Fractional integration and differentiation. Encyclopedia of Mathematics, 2001.

LOGHMANI, G. B. Chebyshev cardinal functions for solving Volterra-Fredholm integrodifferential equations using operational matrices. Iranian Journal of Science and Technology (Sciences), v. 36, n. 1, p. 13-24, 2012. Disponível em: http://ijsts.shirazu.ac.ir/article_2050_0.html.

LUCHKO, Y. Operational method in fractional calculus. Fract. Calc. Appl. Anal, v. 2, n. 4, p. 463-488, 1999. Disponível em: http://public.beuth-hochschule.de/ luchko/papers/tmsf_luc.PDF.

MACHADO, J. T. A new method for approximating fractional derivatives: application in non-linear control. 2011. Disponível em: http://lib.physcon.ru/doc?id= a5359dad24ce.

MALEKNEJAD, K.; BASIRAT, B.; HASHEMIZADEH, E. A new method based on operational matrices of Bernstein polynomials for nonlinear integral equations. 2011? Disponível em: http://www.lidi.info.unlp.edu.ar/worldcomp2011-mirror/ CSC2646.pdf.

A Bernstein operational matrix approach for solving a system of high order linear Volterra-Fredholm integro-differential equations. Mathematical and Computer Modelling, Elsevier, v. 55, n. 3, p. 1363-1372, 2012. Disponível em: http://www.sciencedirect.com/science/article/pii/S0895717711006078.

MECHEE, M. S.; SENU, N. Numerical study of fractional differential equations of lane-emden type by method of collocation. Applied Mathematics, Scientific Research Publishing, v. 3, n. 08, p. 851, 2012.

MEKKAOUI, T.; HAMMOUCH, Z. Approximate analytical solutions to the bagley-torvik equation by the fractional iteration method. Annals of the University of CraiovaMathematics and Computer Science Series, v. 39, n. 2, p. 251-256, 2012. Disponível em: http://inf.ucv.ro/ ami/index.php/ami/article/viewFile/476/422.

MILLER, K. S.; ROSS, B. An introduction to the fractional calculus and fractional differential equations. John Wiley \& Sons, 1993. Disponível em: http://www . amazon . com/exec/obidos/redirect?tag=citeulike07-20\\&path=ASIN/0471588849. 
MOHAN, B. M.; KAR, S. K. Comments on "optimal control via Fourier series of operational matrix of integration". Automatic Control, IEEE Transactions on, IEEE, v. 50, n. 9, p. 1466-1467, 2005.

MUNKHAMMAR, J. D. Riemann-Liouville fractional derivatives and the TaylorRiemann series. UUDM project report, v. 7, p. 1-18, 2004. Disponível em: http: //www.researchgate.net/profile/Joakim_Munkhammar/publication/247409680_ Riemann-Liouville_Fractional_Derivatives_and_the_Taylor-Riemann_Series/ links/54ad20370cf2213c5fe0ff95.pdf.

NOUGIER, J.P. Méthodes de calcul numérique: Systèmes d'équations, Dérivées, Interpolations, Racines d'équations, Diagonalisation de matrices, Lissage-Intégrales, Equations différentielles, Equations aux dérivées partielles, Méthode des éléments finis Méthodes de Monte-Carlo. [S.l.]: Masson, 1983.

OLDHAM, K. B.; SPANIER, J. The fractional calculus. [S.l.]: Academic Press, New York, 1974.

OLIVEIRA, E. C. de; COSTA, F. S.; VAZ JUNIOR, J. The fractional Schrödinger equation for delta potentials. Journal of Mathematical Physics, v. 51, n. 12, p. 123517, 2010. Disponível em: http://www.researchgate.net/profile/Edmundo_ Capelas_De_Oliveira2/publication/252653028_The_fractional_Schrdinger_ equation_for_delta_potentials/links/53fb63110cf22f21c2f32097.pdf.

OLIVEIRA, E. C. de; MAINARDI, F.; VAZ JUNIOR, J. Models based on Mittag-Leffler functions for anomalous relaxation in dielectrics. The European Physical Journal Special Topics, Springer, v. 193, n. 1, p. 161-171, 2011.

PARASKEVOPOULOS, P. N. Chebyshev series approach to system identification, analysis and optimal control. Journal of the Franklin Institute, Elsevier, v. 316, n. 2, p. 135-157, 1983.

Legendre series approach to identification and analysis of linear systems. IEEE transactions on automatic control, Institute of Electrical and Electronics Engineers, v. 30, n. 6 , p. 585-589, 1985.

PARASkevopoulos, P. N.; Sklavounos, P. G.; GeOrGiOU, G. Ch. The operational matrix of integration for Bessel functions. Journal of the Franklin Institute, Elsevier, v. 327, n. 2, p. 329-341, 1990. Disponível em: http://www.sciencedirect.com/science/article/pii/001600329090026F.

PARASKEVOPOULOS, P. N.; SPARIS, P. D.; MOUROUTSOS, S. G. The Fourier series operational matrix of integration. International journal of systems science, Taylor \& Francis, v. 16, n. 2, p. 171-176, 1985. Disponível em: http://dx.doi.org/10.1080/00207728508926663.

PODLUBNY, I. Fractional differential equations: An introduction to fractional derivatives, fractional differential equations, to methods of their solution and some of their applications, vol. 198 of. Mathematics in Science and Engineering, 1999.

Matrix approach to discrete fractional calculus. Fractional Calculus and Applied Analysis, Citeseer, v. 3, n. 4, p. 359-386, 2000. 
PRABHAKAR, T. R. et al. A singular integral equation with a generalized MittagLeffler function in the kernel. Yokohama City University, 1971. Disponível em: http: //133.34.170.46/dspace/bitstream/10131/5281/1/YMJ_19_N1_1971_007-015.pdf.

RAHMANI, L.; RAHIMI, B.; MORDAD, M. Numerical solution of Volterra-Fredholm integro-differential equation by block pulse functions and operational matrices. Gen, v. 4, n. 2, p. 37-48, 2011. Disponível em: http://www.emis.ams.org/journals/GMN/ yahoo_site_admin/assets/docs/4_GMN-482-V4N2.160165143.pdf.

RAJA, M. A. Z.; KHAN, J. A.; QURESHI, I. M. Solution of fractional order system of bagley-torvik equation using evolutionary computational intelligence. Mathematical Problems in Engineering, Hindawi Publishing Corporation, v. 2011, 2011.

RAZZAGHI, M.; ARABSHAHI, A.; LIN, S. D. Identification of nonlinear differential equations via Fourier series operational matrix for repeated integration. Applied mathematics and computation, Elsevier, v. 68, n. 2, p. 189-198, 1995. Disponível em: http://www.sciencedirect.com/science/article/pii/009630039400093J.

RAZZAGHI, M.; RAZZAGHI, M. Fourier series direct method for variational problems. International Journal of Control, Taylor \& Francis, v. 48, n. 3, p. 887-895, 1988.

RAZZAGHI, M.; YOUSEFI, S. The Legendre wavelets operational matrix of integration. International Journal of Systems Science, Taylor \& Francis, v. 32, n. 4, p. 495-502, 2001.

RISMANI, A. M.; MONFARED, H. Numerical solution of singular ivps of laneemden type using a modified legendre-spectral method. Applied Mathematical Modelling, Elsevier, v. 36, n. 10, p. 4830-4836, 2012. Disponível em: http: //www.sciencedirect.com/science/article/pii/S0307904X11008043.

SABATIER, J.; AGRAWAL, O. P.; MACHADO, J. T. Advances in fractional calculus: Theoretical developments and applications in physics and engineering. [S.1.]: Springer Publishing Company, Incorporated, 2007.

SCHERER, R. et al. Numerical treatment of fractional heat equations. Applied Numerical Mathematics, Elsevier, v. 58, n. 8, p. 1212-1223, 2008. Disponível em: http://www.sciencedirect.com/science/article/pii/S0168927407001092.

SHUKLA, A. K.; PRAJAPATI, J. C. On a generalization of Mittag-Leffler function and its properties. Journal of Mathematical Analysis and Applications, Elsevier, v. 336, n. 2, p. 797-811, 2007. Disponível em: http://www.sciencedirect.com/ science/article/pii/S0022247X07003174.

SINGH, A. K.; SINGH, V. K.; SINGH, O. P. The Bernstein operational matrix of integration. Appl. Math. Sciences, v. 3, n. 49, p. 2427-2436, 2009. Disponível em: http://www.m-hikari.com/ams/ams-password-2009/ams-password49-52-2009/ singhomAMS49-52-2009.pdf.

SRIVASTAVA, H. M.; TOMOVSKI, Ž. Fractional calculus with an integral operator containing a generalized Mittag-Leffler function in the kernel. Applied Mathematics and Computation, Elsevier, v. 211, n. 1, p. 198-210, 2009. Disponível em: http://www.sciencedirect.com/science/article/pii/S009630030900071X. 
STANĚK, S. Two-point boundary value problems for the generalized bagley-torvik fractional differential equation. Open Mathematics, v. 11, n. 3, p. 574-593, 2013.

SUPRUNENKO, D. A.; TYSHKEVICH, R. I. Perestanovochnye matritsy. [S.l.]: Nauka i Tekhnika, 1966.

TORVIK, P. J.; BAGLEY, R. L. On the appearance of the fractional derivative in the behavior of real materials. Journal of Applied Mechanics, American Society of Mechanical Engineers, v. 51, n. 2, p. 294-298, 1984.

TOUTOUNIAN, F.; TOHIDI, E.; KILICMAN, A. Fourier operational matrices of differentiation and transmission: introduction and applications. In: HINDAWI PUBlishing CORPORATION. Abstract and Applied Analysis. [S.l.], 2013. v. 2013.

VÁZQUEZ, L.; TRUJILLO, J.; VELASCO, M. P. Fractional heat equation and the second law of thermodynamics. Fractional Calculus and Applied Analysis, v. 14, n. 3, p. 334-342, 2011.

WANG, Y. et al. An operational matrix-based algorithm for simulating linear and fractional differential circuits. In: Proceedings of the Conference on Design, Automation and Test in Europe. San Jose, CA, USA: EDA Consortium, 2012. (DATE '12), p. 1463-1466. ISBN 978-3-9810801-8-6. Disponível em: http: //dl . acm.org/citation. cfm?id=2492708. 2493066.

WANG, Z.H.; WANG, X. General solution of the bagley-torvik equation with fractional-order derivative. Communications in Nonlinear Science and Numerical Simulation, Elsevier, v. 15, n. 5, p. 1279-1285, 2010. Disponível em: http://www.sciencedirect.com/science/article/pii/S1007570409003311.

WIMAN, Adders. Über den fundamentalsatz in der teorie der funktionen $\mathrm{E}_{a}(x)$. Acta Mathematica, Springer, v. 29, n. 1, p. 191-201, 1905.

Über die nullstellen der funktionen $\mathrm{E}_{a}(x)$. Acta Mathematica, Springer, v. 29, n. 1, p. 217-234, 1905.

WU, J.L.; CHEN, C.H. A new operational approach for solving fractional calculus and fractional differential equations numerically. IEICE Transactions on Fundamentals of Electronics, Communications and Computer Sciences, The Institute of Electronics, Information and Communication Engineers, v. 87, n. 5, p. 1077-1082, 2004.

YANG, X.J.; BALEANU, D. Fractal heat conduction problem solved by local fractional variation iteration method. Thermal Science, v. 17, n. 2, p. 625-628, 2013. Disponível em: http://www.doiserbia.nb.rs/img/doi/0354-9836/2013/0354-98361200216Y. pdf.

YILDIRIM, A.; ÖZIŞ, T. Solutions of singular ivps of lane-emden type by homotopy perturbation method. Physics Letters A, Elsevier, v. 369, n. 1, p. 70-76, 2007. Disponível em: http://www.sciencedirect.com/science/article/pii/ S0375960107006445. 
. Solutions of singular ivps of lane-emden type by the variational iteration method. Nonlinear Analysis: Theory, Methods \& Applications, Elsevier, v. 70, n. 6, p. 2480-2484, 2009. Disponível em: http://www.sciencedirect.com/science/article/ $\mathrm{pii/S0362546X08002113.}$

ZASLAVSKY, G. M. Chaos, fractional kinetics, and anomalous transport. Physics Reports, Elsevier, v. 371, n. 6, p. 461-580, 2002. Disponível em: http://www.sciencedirect.com/science/article/pii/S0370157302003319. 2008. Hamiltonian chaos and fractional dynamics. [S.l.]: Oxford Univ. Press,

ZHU J.Q., Jianxian; SHU, C.W.; DUMBSER, M. Runge-Kutta discontinuous Galerkin method using WENO limiters II: unstructured meshes. Journal of Computational Physics, Elsevier, v. 227, n. 9, p. 4330-4353, 2008. Disponível em: http://www.sciencedirect.com/science/article/pii/S0021999108000120.

ZYGMUND, A. Trigonometric series. Vol. I, II. With a foreword by Robert A. Fefferman. Cambridge Mathematical Library. [S.l.]: Cambridge University Press, Cambridge, 2002. 


\section{APÊNDICE A - Fundamentos Matemáticos}

Neste apêndice são expostos os fundamentos matemáticos utilizados ao longo do trabalho. Apresenta-se uma recapitulação dos conceitos mais importantes de Álgebra Linear, Topologia e Análise Matemática, os quais fornecem o embasamento necessário ao desenvolvimento da tese.

É importante apresentar claramente os conceitos relativos às noções de espaços em geral e, em particular, dos espaços métricos, conceitos que são amplamente utilizados no texto. Por isso, achamos conveniente iniciar a exposição dos fundamentos matemáticos pela definição formal de espaço topológico para, em seguida, estabelecer as demais definições.

\section{A.1 Espaços Topológicos}

Seja $U$ um conjunto não-vazio. Define-se uma topologia em $U$ por uma família $\tau$ de subconjuntos de $U$ com as propriedades a seguir:

1. $\emptyset, U \in \tau$;

2. A união de uma família arbitrária de membros de $\tau$ pertence a $\tau$;

3. A interseção de uma família finita de membros de $\tau$ pertence a $\tau$.

Um espaço topológico é um par ordenado $(U, \tau)$ sendo $\tau$ uma topologia em $U$. Nestas condições, os membros de $\tau$ são chamados de abertos. Frequentemente $U$ é designado um espaço topológico. 


\section{A.2 Espaços Vetoriais Complexos}

Seja $\mathbb{V}$ um conjunto munido de duas operações, adição e multiplicação por escalar, denotadas por $(+)$ e $(\cdot)$, respectivamente, sendo:

$$
(+): \mathbb{V} \times \mathbb{V} \longrightarrow \mathbb{V} \quad(\cdot): \mathbb{C} \times \mathbb{V} \longrightarrow \mathbb{V}
$$

tais que, para quaisquer $\lambda, \mu \in \mathbb{C}, x, y, z \in \mathbb{V}$, onde $\mathbb{C}$ é o corpo dos números complexos, valham as seguintes propriedades:

Para a adição $(+)$ :

- (A0) fechamento: $x+y \in \mathbb{V}$,

- (A1) comutatividade: $x+y=y+x$,

- (A2) associatividade: $(x+y)+z=x+(y+z)$,

- (A3) elemento neutro aditivo : $\exists 0 \in \mathbb{V}: x+0=0+x=x$,

- (A4) elemento oposto: $\exists(-x) \in \mathbb{V}: x+(-x)=(-x)+x=0$.

Para o produto por escalar $(\cdot)$ :

- (M0) fechamento: $\lambda x \in \mathbb{V}$,

- $(M 1)$ distributividade à esquerda: $(\lambda+\mu) x=\lambda x+\mu x$,

- (M2) distributividade à direita: $\lambda(x+\beta)=\lambda x+\lambda y$,

- (M3) elemento neutro: $1 x=x, 1=1+0 i \in \mathbb{C}$,

- $(M 4)$ associatividade: $\lambda(\mu x)=(\lambda \mu) x$.

Nessas condições, $\mathbb{V}$ é denominado um espaço vetorial sobre o corpo complexo ou, simplesmente, um espaço vetorial complexo, sendo seus elementos constituintes denominados vetores.

Espaço Complexo Conjugado Um espaço complexo conjugado de $\mathbb{V}$, denotado $\mathbb{V}^{*}$, é um espaço que consiste de todos os complexos conjugados dos elementos de $\mathbb{V}$, ou seja, $\mathbb{V}^{*}$ é um espaço vetorial cujos elementos apresentam uma correspondência biunívoca com os elementos de $\mathbb{V}$, de forma que:

$$
\mathbb{V}^{*}=\{\bar{v} \mid v \in \mathbb{V}\}
$$




\section{A.3 Mapeamentos em Espaços Vetoriais}

Uma aplicação entre espaços vetoriais complexos $f: \mathbb{V} \longrightarrow \mathbb{U}$ é chamada de mapeamento linear se, para quaisquer $\forall x, y \in \mathbb{V}, a \in \mathbb{C}, f$ satisfaz as condições:

- aditividade:

$$
f(x+y)=f(x)+f(y)
$$

- homogeneidade de grau 1:

$$
f(a x)=a f(x)
$$

ou, de maneira equivalente, se satisfaz à relação

$$
f(a x+b y)=a f(x)+b f(y), \forall a, b \in \mathbb{C}, \forall x, y \in \mathbb{V}
$$

Por outro lado, uma aplicação similar será chamada de mapeamento antilinear se obedecer a

$$
f(a x+b y)=\bar{a} f(x)+\bar{b} f(y)
$$

onde $\bar{a}$ e $\bar{b}$ são os complexos conjugados de $a$ e $b$, respectivamente.

Funcionais Um caso particular importante de mapeamento é chamado funcional, definido quando o contradomínio da aplicação é o próprio corpo sobre o qual atua o espaço vetorial do domínio, i.e., aplicações do tipo $\varphi: \mathbb{V} \longrightarrow \mathbb{C}$, onde $\mathbb{V}$ é um espaço vetorial sobre $\mathbb{C}$. Analogamente à definição anterior, um mapeamento desse tipo que obedeça a

$$
\varphi(a x+b y)=a \varphi(x)+b \varphi(y), \quad \forall a, b \in \mathbb{C}, \forall x, y \in \mathbb{V}
$$

será chamado de funcional linear, enquanto aqueles que obedecerem à condição

$$
\varphi(a x+b y)=\bar{a} \varphi(x)+\bar{b} \varphi(y)
$$

serão denominados funcionais antilineares.

O terorema de existência e unicidade diz que, se $\mathbb{V}$ é um espaço vetorial de dimensão $n$ sobre um corpo $\mathbb{K}$ e $B=\left\{e_{i}\right\}_{i=1}^{n}$ é uma base de $\mathbb{V}$, então existe um único funcional $\varphi$, tal que $\varphi\left(e_{i}\right)=\alpha_{i}, \quad i=1, \ldots, n, \operatorname{com} \alpha_{i} \in \mathbb{K}$.

Espaços Duais Dado um espaço vetorial $\mathbb{V}$ sobre $\mathbb{C}$, o espaço que consiste de todos os funcionais lineares $\varphi: \mathbb{V} \longrightarrow \mathbb{C}$ também apresenta estrutura de espaço vetorial, sendo 
chamado de espaço dual de $\mathbb{V}$ e denotado $\mathbb{V}^{\star}$. Os elementos do espaço dual são denominados covetores ou um-formas. Ademais, pelo teorema de unicidade, se $\operatorname{dim} \mathbb{V}$ é finita, então $\mathbb{V}^{\star}$ é isomorfo a $\mathbb{V}$.

Bases Duais O teorema da base dual diz que, para um espaço vetorial $\mathbb{V}(\mathbb{C})$ e $\operatorname{dim} \mathbb{V}=$ $n$, se $B=\left\{e_{j}\right\}_{j=1}^{n}$ é uma base de $\mathbb{V}$, então existe apenas uma única base $B^{\star}=\left\{\phi_{i}\right\}_{i=1}^{n}$ de $\mathbb{V}^{\star}$ tal que $\phi_{i}\left(e_{j}\right)=\delta_{i j}$. Tal base é denominada de base dual de $\mathbb{V}$.

\section{A.4 Espaços Vetoriais Normados}

Norma A norma sobre um espaço vetorial $\mathbb{V}$ é um mapeamento $\|\cdot\|: \mathbb{V} \longrightarrow \mathbb{R}_{+}$tal que, para $\forall x, y \in \mathbb{V}, \forall \alpha \in \mathbb{C}$, valem as propriedades:

(i) $\|x\| \geq 0, \quad\|x\|=0 \Leftrightarrow x=0$

(ii) $\|\alpha x\|=|\alpha|\|x\| ;$

(iii) $\|x+y\| \leq\|x\|+\|y\| \quad$ (desigualdade triangular).

Seminorma Um mapeamento no qual apenas as propriedades (ii) e (iii) acima sejam satisfeitas, será denominado uma seminorma.

Tipos de Norma Define-se, para um número real $p \geq 1$, uma $p$-norma (também designada como norma $\mathcal{L}^{p}$ ), num espaço vetorial $\mathbb{V}^{n}$, de dimensão $n$, por:

$$
\|x\|_{p}=\left(\left|x_{1}\right|^{p}+\left|x_{2}\right|^{p}+\cdots+\left|x_{n}\right|^{p}\right)^{1 / p}
$$

Uma norma do tipo $\|x\|_{2}=\left(\left|x_{1}\right|^{2}+\cdots+\left|x_{n}\right|^{2}\right)^{1 / 2}$, para os escalares $x_{1}, x_{2}, \ldots x_{n} \in \mathbb{R}$, é chamada de norma euclidiana em $\mathbb{R}^{n}$.

Chama-se norma máxima ou uniforme à norma $\mathcal{L}^{\infty}$, definida por:

$$
\|x\|_{\infty}=\max \left\{\left|x_{1}\right|,\left|x_{2}\right|, \cdots,\left|x_{n}\right|\right\}
$$


Espaços Normados Espaços vetoriais dotados de norma denominam-se espaços vetoriais normados. Tais estruturas constituem espaços topológicos métricos, definidos adiante.

\section{A.5 Métrica}

Uma métrica sobre um espaço $V$ qualquer, isto é, vetorial ou não, é um mapeamento $d(\cdot, \cdot): V \times V \longrightarrow \mathbb{R}_{+}$tal que, para $\forall x, y, z \in V$, sejam satisfeitas as seguintes propriedades:

(i) $d(x, y)=d(y, x)$;

(ii) $d(x, x)=0$

(iii) $d(x, z) \leq d(x, y)+d(y, z) \quad$ (desigualdade triangular $)$

(iv) $d(x, y)>0, \forall x, y: x \neq y$.

Pseudométrica Define-se uma pseudométrica por um mapeamento que satisfaça as três primeiras condições acima, mas não a última.

\section{A.6 Espaços Métricos}

Agora que já foram expostos todos os conceitos necessários, podemos, finalmente, definir espaços métricos. Formalmente, um espaço métrico é um par ordenado $(V, d)$ no qual $d$ é uma métrica definida em $V \times V$. Em outras palavras, qualquer espaço dotado de uma métrica, seja ou não um espaço vetorial linear, constitui um espaço métrico.

Um espaço linear normado é automaticamente um espaço métrico, com a métrica definida por $d(u, v)=\|u-v\|$, contudo, a recíproca não se verifica, ou seja, nem toda métrica é gerada por uma norma, o que torna possível a existência de espaços métricos não-normados. Em outros termos, os espaços normados são uma subcategoria dos espaços métricos, com a métrica induzida pela norma. Assim, se $\mathcal{N}$ é o conjunto formado por todos os espaços normados e $\mathcal{M}$, o conjunto constituído por todos os espaços métricos, 
então $\mathcal{N} \subset \mathcal{M}$.

Além disso, outra característica importante de um espaço métrico é que ele admite uma estrutura natural de espaço topológico ou, em outros termos, dizemos que é topologizável.

Sequências de Cauchy Uma sequência de elementos $\left(x_{n}\right)$ de um espaço métrico com métrica $d(\cdot, \cdot)$ é chamada sequência de Cauchy se

$$
\forall \varepsilon>0, \exists n_{0} \mid \forall m, p \geq n_{0} \Rightarrow d\left(x_{m}, x_{p}\right)<\varepsilon
$$

Espaços Métricos Completos Um espaço métrico $V$ é chamado completo se toda sequência de Cauchy de elementos de $V$ converge para um limite em $V$. Intuitivamente, pode-se conceber que um espaço seja completo se não apresentar "furos", isto é, se não estiver faltando nenhum ponto ou elemento nesse espaço. Um exemplo clássico de espaço não-completo é dado pelo conjunto dos números racionais $\mathbb{Q}$, pois a sequência de Cauchy definida por $x_{n+1}=\frac{1}{2}\left(x_{n}+\frac{2}{x_{n}}\right)$, com $x_{1}=1$, converge para $\sqrt{2}$, que não pertence a $\mathbb{Q}$, já que é um número irracional (LIMA, 2004). Já o conjunto dos números reais constitui um espaço completo.

\section{A.7 Espaços de Banach}

Um espaço de Banach é um espaço vetorial normado completo. Em outros termos, podemos dizer que um espaço de Banach é um espaço vetorial $\mathbb{V}$ sobre um corpo $\mathbb{K}$ ( $\mathbb{R}$ ou $\mathbb{C}$ ) com uma norma $\|x\|$ e munido de uma métrica $d(x, y)=\|x-y\|$ tal que toda sequência de Cauchy em $\mathbb{V}$, com respeito à métrica, possui um limite bem definido nesse espaço.

Há um teorema importante que faz referência a espaços normados duais. Como o conjunto de todos os funcionais lineares limitados definidos em um espaço normado $\mathcal{V}(\mathbb{K})$ também constitui um espaço normado e $\mathbb{K}$ é um corpo completo, então $\mathcal{V}^{\star}$ é um espaço normado completo. Logo, pode-se enunciar: se $\mathcal{V}$ é um espaço normado, então seu dual $\mathcal{V}^{\star}$ é um espaço de Banach. 


\section{A.8 Produto Interno}

Um produto interno definido sobre um espaço vetorial complexo é uma aplicação $\langle\rangle:, \mathbb{V} \times \mathbb{V} \longrightarrow \mathbb{C}$ a valores complexos de pares ordenados que obedece, para $\forall \lambda \in$ $\mathbb{C}, \forall x, y, z \in \mathbb{V}$, às seguintes propriedades:

1. $\langle y, x\rangle=\overline{\langle x, y\rangle}$

2. $\langle x, \lambda y\rangle=\lambda\langle x, y\rangle$

3. $\langle\lambda x, y\rangle=\bar{\lambda}\langle x, y\rangle$

4. $\langle x+y, z\rangle=\langle x, z\rangle+\langle y, z\rangle$

5. $\langle z, x+y\rangle=\langle z, x\rangle+\langle z, y\rangle$

6. $\langle x, x\rangle \geq 0,\langle x, x\rangle=0 \Leftrightarrow x=0$.

Obs: Note-se que estamos escrevendo a conjugação no primeiro elemento e não no segundo, como é a prática usual em matemática, convenção que, no entanto, é mais comum na Física. $O$ motivo advém do fato de que, no formalismo bra-ket, o primeiro elemento encontra-se no espaço dual e o segundo, no original e, uma vez que vetores constituem grandezas "mais fundamentais"que covetores, no sentido da Álgebra Linear, parece-nos mais lógico e natural preservar as propriedades vetoriais, atribuindo a conjugação aos elementos do espaço dual.

Formas Sesquilineares Uma forma sesquilinear sobre um espaço complexo $\mathbb{V}$ é um mapeamento $\mathbb{V} \times \mathbb{V} \longrightarrow \mathbb{C}$ antilinear no primeiro argumento e linear no segundo, isto é, $\forall \lambda \in \mathbb{C}, \forall u, v, w \in \mathbb{V}$, tem-se $\phi: \mathbb{V} \times \mathbb{V} \longrightarrow \mathbb{C}$ tal que:

$$
\left\{\begin{array}{l}
\phi(u, \lambda v+w)=\lambda \phi(u, v)+\phi(u, w) \\
\phi(\lambda u+v, w)=\bar{\lambda} \phi(u, w)+\phi(v, w)
\end{array}\right.
$$

Uma vez que o produto interno $\phi: \mathbb{V} \times \mathbb{V} \longrightarrow \mathbb{C}$ acima definido satisfaz estas condições, quer dizer, é linear no segundo argumento e antilinear no primeiro, dizemos que constitui uma forma sesquilinear.

Tais estruturas, que correspondem a uma extensão das formas bilineares ao corpo complexo, também podem ser vistas como um mapeamento bilinear $\mathbb{V}^{*} \times \mathbb{V} \longrightarrow \mathbb{C}$, onde $\mathbb{V}^{*}$ é o espaço complexo conjugado de $\mathbb{V}$. 
Formas Hermitianas Além disso, formas sesquilineares simétricas, isto é, que obedecem adicionalmente à condição de simetria conjugada:

$$
\phi(u, v)=\overline{\phi(v, u)}, \quad(\forall u, v \in \mathbb{V})
$$

recebem o nome de formas hermitianas. Devemos notar que o produto interno em espaços complexos constitui uma forma hermitiana. As formas hermitianas também recebem a denominação de formas autoadjuntas.

Formas Antiautoadjuntas No caso em que valha:

$$
\phi(u, v)=-\overline{\phi(v, u)}, \quad(\forall u, v \in \mathbb{V})
$$

dizemos que $\phi$ é uma forma antiautoadjunta.

Ademais, se $\mathbb{V}$ é um espaço vetorial normado completo dotado de um produto interno $\langle\cdot, \cdot\rangle: \mathbb{V} \times \mathbb{V} \rightarrow \mathbb{R}$ com uma norma associada $\|\cdot\|: \mathbb{V} \rightarrow \mathbb{R}$, então, para $\forall x, y \in \mathbb{V}$, valem as propriedades abaixo, conhecidas como desigualdade de Cauchy-Schwarz-Bunyakowski, lei do paralelogramo e identidade de polarização, respectivamente:

(i) $|\langle x, y\rangle| \leq\|x\| \cdot\|y\| ;$

(ii) $\|x+y\|^{2}+\|x-y\|^{2}=2\left(\|x\|^{2}+\|y\|^{2}\right) ;$

(iii) $\langle x, y\rangle=\frac{1}{4}\left(\|x+y\|^{2}-\|x-y\|^{2}-i\|x+i y\|^{2}+i\|x-i y\|^{2}\right)$.

A lei do paralelogramo é uma condição necessária e suficiente para a existência de um produto interno associado a uma dada norma. Se essa condição não for satisfeita, então a norma não é induzida por um produto interno. Se, no entanto, a relação procede, então o produto interno é dado pela última expressão.

Espaços IP Qualquer espaço vetorial dotado da estrutura de um produto interno é denominado um espaço com produto interno ou, resumidamente, espaço IP.

Todo espaço IP possui uma norma definida por $\|x\|=\langle x, x\rangle^{1 / 2}$, que é denominada, nesse caso, de norma induzida pelo IP. 
Figura 109: Representação esquemática das relações entre diversos tipos de espaços

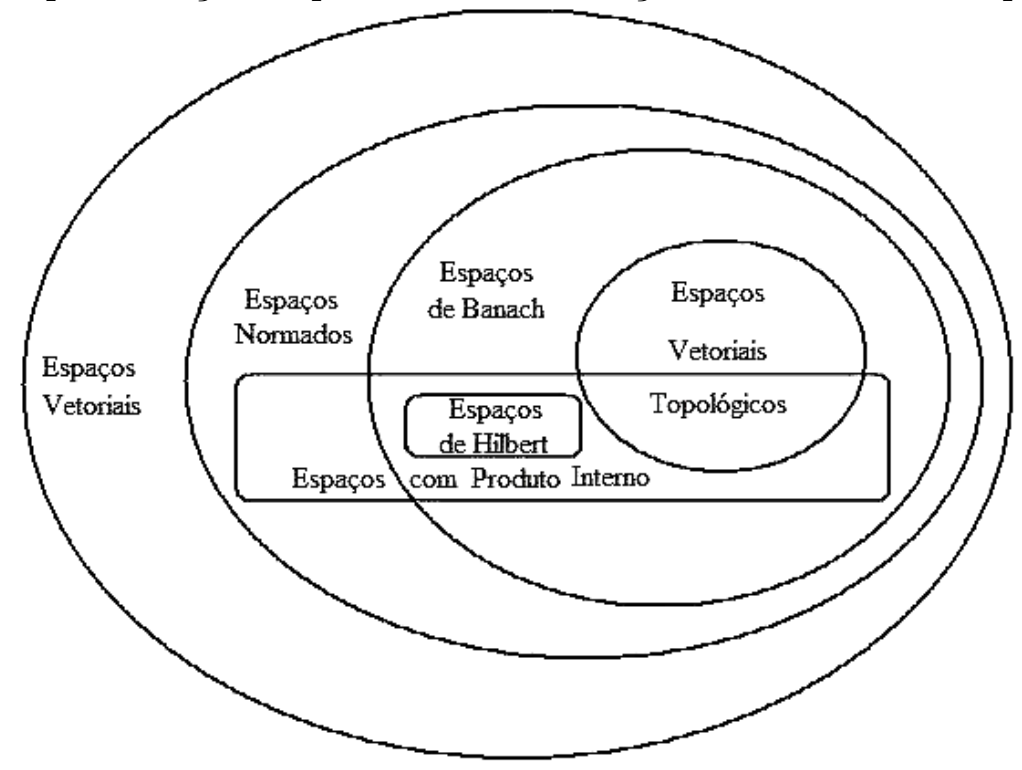

Fonte: Adaptado de Aldrovandi e Pereira (1995)

\section{A.9 Espaços de Hilbert}

Os espaços de Hilbert constituem uma subcategoria dos espaços de Banach, com a particularidade de serem munidos de um produto interno $\langle x, y\rangle$ como o descrito acima e por definirem uma norma $\|x\|: \mathbb{V} \rightarrow \mathbb{R}$ e uma métrica $d(x, y)=\|x-y\|: \mathbb{V} \times \mathbb{V} \rightarrow \mathbb{R}$ induzidas por esse produto interno. Em outros termos, podemos dizer que um espaço de Hilbert é um espaço IP, completo sob a topologia da norma do produto interno.

O caso padrão é constituído pelo conjunto de sequências $x=\left\{x_{i}\right\}_{x_{i} \in \mathbb{N}}$ de números complexos tais que

$$
\sum_{i=1}^{\infty}\left|x_{i}\right|^{2}<\infty
$$

sendo o produto interno definido por

$$
\langle x, y\rangle=\sum_{i=1}^{\infty} x_{i}^{*} y_{i}
$$

e a base descrita pelas bolas abertas com a função distância

$$
d(x, y)=|\langle x, y\rangle|
$$

Se adotarmos, por exemplo, norma e métrica euclidianas $\|x\|:=\langle x, x\rangle^{1 / 2}$ e $\|x-y\|:=$ $\langle x-y, x-y\rangle^{1 / 2}$, o espaço métrico provido dessas entidades constitui um espaço de Hilbert, embora não seja o único possível. 
Os espaços de Hilbert constituem a generalização natural dos espaços euclidianos para o caso de dimensão infinita. As propriedades fundamentais do produto interno definido num espaço de Hilbert $\mathcal{H}$ são as mesmas expostas anteriormente, agora em um mapeamento $\langle\rangle:, \mathcal{H} \times \mathcal{H} \longrightarrow \mathbb{C}$.

É importante ressaltar que, em vista da não-definição de um produto interno em espaços de Banach genéricos, a propriedade de ortogonalidade deixa de fazer sentido nesses espaços, o que impossibilita seu uso em aplicações efetivas à teoria das equações diferenciais. Por esse motivo, restringimo-nos aos espaços de Hilbert.

\section{A.10 Espaços de Funções}

Os espaços de funções, que definiremos a seguir, constituem uma subcategoria dos espaços de Hilbert. Seja $C^{0}[a, b]$, para $a<b$, o conjunto das funções contínuas definidas num intervalo $[a, b]$. Tal conjunto, munido das operações de soma e multiplicação por escalar, constitui um espaço linear de dimensão infinita cujo produto interno, dado por uma aplicação $\langle\rangle:, C^{0}[a, b] \times C^{0}[a, b] \longrightarrow \mathbb{C}$, é definido por:

$$
\langle f, g\rangle=\int_{a}^{b} f^{*}(x) g(x) d x
$$

para $f, g \in C^{0}[a, b]$, onde estamos denotando $[f(x)]^{*}=f^{*}(x)$. Entretanto, como a integral acima pode não convergir, exige-se adicionalmente que $f$ e $g$ sejam funções quadraticamente integráveis, isto é,

$$
\int|f(x)|^{2} d x<\infty, \quad \int|g(x)|^{2} d x<\infty
$$

Demonstra-se que, se $f$ e $g$ obedecem a essa condição, então $\langle f, g\rangle$ é finito. Por concisão, usaremos, para funções quadraticamente integráveis, a denominação abreviada de funções $Q I$.

Espaço de Lebesgue $\mathcal{L}^{2} \quad$ Um espaço de Hilbert dotado do produto interno sobre o corpo complexo conforme definido acima é chamado de espaço $\mathcal{L}^{2}(\mathbb{C})$. Na teoria das séries de Fourier, as funções deste espaço, que são as chamadas quadrado-integráveis (QI), são as protagonistas, desempenhando um papel essencial. O espaço $\mathcal{L}^{2}(\mathbb{C})$ é um caso particular dos espaços de Lebesgue $\mathcal{L}^{p}$. 
Bases Ortonormais Uma base de funções $\left\{\phi_{i}(x)\right\}$ é dita ortonormal se satisfizer a condição:

$$
\left\langle\phi_{i}, \phi_{j}\right\rangle=\delta_{i j}
$$

Como exemplo, consideremos o conjunto de todos os polinômios com grau $\leq n$ definidos no intervalo $[-1,1]$. Eles são descritos por

$$
p_{n}(x)=\sum_{i=1}^{n} a_{i} x^{i}
$$

Como polinômios desse tipo são funções QI dentro do intervalo de definição, então o produto interno pode ser definido. Além disso, a base canônica $\left\{e_{i}(x)\right\}$ é constituída pelos elementos

$$
e_{1}(x)=1, \quad e_{2}(x)=x, \quad e_{3}(x)=x^{2}, \ldots, \quad e_{n}(x)=x^{n-1}
$$

Essa base, contudo, não é ortonormal (estritamente falando, não chega a ser sequer ortogonal), pois, de modo geral, tem-se

$$
\left\langle e_{i}, e_{j}\right\rangle \neq \delta_{i j}
$$

Por exemplo,

$$
\left\langle e_{1}, e_{1}\right\rangle=\int_{-1}^{1} 1 d x=2, \quad\left\langle e_{1}, e_{3}\right\rangle=\int_{-1}^{1} x^{2} d x=\frac{2}{3}
$$

Entretanto, é possível ortonormalizar a base acima pelo processo de Gram-Schmidt. Existe um conjunto de polinômios ortogonais (mas não ortonormais) construídos a partir dessa base; são os conhecidos polinômios de Legendre $P_{n}(x)$, determinados pela fórmula de Rodrigues:

$$
P_{n}(x)=\frac{1}{2^{n} n !}\left(\frac{d}{d x}\right)^{n}\left(x^{2}-1\right)^{n}
$$

Uma base ortonormal pode, todavia, ser obtida a partir dos polinômios de Legendre pela expressão

$$
\left|\hat{e}_{n}\right\rangle=\sqrt{n-\frac{1}{2}} P_{n-1}(x)
$$

\section{A.11 Espaço $\mathcal{L}_{w}^{2}$ e Integral de Lebesgue}

Num espaço de funções, o IP $\langle\rangle:, C^{0}[a, b] \times C^{0}[a, b] \rightarrow \mathbb{C}$ entre funções contínuas $f, g \in C^{0}[a, b]$ pode ser definido através de uma expressão que incorpore uma função peso 
$w(x)$, de modo que, para $w \in C^{0}[a, b]$, tenhamos

$$
\langle f, g\rangle:=\int_{a}^{b} f^{*}(x) g(x) w(x) d x
$$

Evidentemente, pela necessidade de estabelecer um espaço vetorial normado, exige-se que

$$
\langle f, f\rangle:=\int_{a}^{b} f^{*}(x) f(x) w(x) d x=\int_{a}^{b}|f(x)|^{2} w(x) d x
$$

seja finito. A expressão acima é chamada de Integral de Lebesgue e o espaço vetorial formado pelas funções que satisfazem essa condição, é designado $\mathcal{L}_{w}^{2}[a, b]$.

Funções dessa natureza são denominadas quadraticamente integráveis sob o peso w $(x)$. Conforme salientamos antes, espaços vetoriais normados cuja norma seja induzida pelo IP constituem espaços de Hilbert. Logo, como essa condição é satisfeita no presente caso, o espaço $\mathcal{L}_{w}^{2}$ é um espaço de Hilbert.

Demonstramos, a seguir, duas propriedades importantes, as de fechamento para o IP e para a soma, a saber, para duas funções quaisquer $f, g \in \mathcal{L}_{w}^{2}[a, b]$, tem-se:

$$
\begin{gathered}
\langle f, g\rangle \in \mathcal{L}_{w}^{2}[a, b] \\
(f+g) \in \mathcal{L}_{w}^{2}[a, b]
\end{gathered}
$$

Procedemos agora às demonstrações.

Demonstração 2 (eq. A.1):

Notemos, inicialmente, que $\left|f^{*} g\right|=|f||g|$. Por outro lado,

$$
\begin{gathered}
(|f|-|g|)^{2}=|f|^{2}+|g|^{2}-2|f||g| \geq 0 \Rightarrow 2|f||g| \leq|f|^{2}+|g|^{2} \Rightarrow \\
2\left|f^{*} g\right| \leq|f|^{2}+|g|^{2}
\end{gathered}
$$

Daí, como

$$
\langle f, g\rangle=\int_{a}^{b} f^{*}(x) g(x) w(x) d x
$$

e tendo que vista que cada um das partes de um número complexo deve ser menor ou igual a seu módulo, isto é, $\Re\{\langle f, g\rangle\} \leq|\langle f, g\rangle|$ e também $\Im\{\langle f, g\rangle\} \leq$ $|\langle f, g\rangle|, \operatorname{logo}, \Re\{\langle f, g\rangle\}+\Im\{\langle f, g\rangle\} \leq 2|\langle f, g\rangle|$, o que nos permite escrever, no sentido simbólico expresso por essas relações: $\langle f, g\rangle \leq 2|\langle f, g\rangle|$, de onde vem 
que

$$
\langle f, g\rangle \leq \int_{a}^{b} 2\left|f^{*}(x) g(x)\right| w(x) d x
$$

E, usando a relação (A.3), segue que

$$
\langle f, g\rangle \leq \int_{a}^{b}|f(x)|^{2} w(x) d x+\int_{a}^{b}|g(x)|^{2} w(x) d x
$$

Portanto,

$$
\langle f, g\rangle \leq(\langle f, f\rangle+\langle g, g\rangle)
$$

$\log O$

$$
\langle f, g\rangle \in \mathcal{L}_{w}^{2}[a, b]
$$

Demonstração 3(eq. A.2):

Notando que

$$
|f+g|^{2}=(f+g)^{*}(f+g)=|f|^{2}+|g|^{2}+2 \Re\left\{f^{*} g\right\}
$$

e também que

$$
2 \Re\left\{f^{*} g\right\} \leq 2\left|f^{*} g\right|=2|f||g|,
$$

podemos escrever, usando novamente (A.3):

$$
|f+g|^{2} \leq 2|f|^{2}+2|g|^{2}
$$

Multiplicando ambos os lados da desigualdade acima pela função peso e integrando no domínio de validade, obtém-se:

$$
\int_{a}^{b}|f(x)+g(x)|^{2} w(x) d x \leq 2 \int_{a}^{b}|f|^{2} w(x) d x+2 \int_{a}^{b}|g|^{2} w(x) d x<\infty .
$$

Mas

$$
(f+g) \leq|f+g|
$$

Logo,

$$
(f+g) \in \mathcal{L}_{w}^{2}[a, b]
$$

\section{A.12 Isomorfismos em Diversos Tipos de Espaços}

Isomorfismos em Espaços Vetoriais $\operatorname{Sejam~} \mathcal{X}=(\mathcal{X},+, \cdot)$ e $\mathcal{Y}=(\mathcal{Y}, \oplus, *)$ espaços vetoriais sobre um mesmo corpo $\mathbb{K}$. Então, um isomorfismo de $\mathcal{X}$ em $\mathcal{Y}$ é uma aplicação 
bijetiva $T: \mathcal{X} \longrightarrow \mathcal{Y}$ tal que:

$$
T(\alpha \cdot x+\beta \cdot y)=\alpha * T x \oplus \beta * T y, \quad \forall x, y \in \mathcal{X}, \quad \forall \alpha, \beta \in \mathbb{K}
$$

Isomorfismos em Espaços Métricos $\operatorname{Sejam~} \mathcal{X}=(\mathcal{X}, d)$ e $\mathcal{Y}=\left(\mathcal{Y}, d^{\prime}\right)$ espaços métricos. Então, um isomorfismo de $\mathcal{X}$ em $\mathcal{Y}$ é uma aplicação bijetiva $T: \mathcal{X} \longrightarrow \mathcal{Y}$ que preserva a distância, i.e.,

$$
d^{\prime}(T x, T y)=d(x, y), \quad \forall x, y \in \mathcal{X}
$$

Isomorfismos em Espaços Normados $\operatorname{Sejam} \mathcal{X}=\left(\mathcal{X},\|\cdot\|_{\mathcal{X}}\right)$ e $\mathcal{Y}=\left(\mathcal{Y},\|\cdot\|_{\mathcal{Y}}\right)$ espaços normados. Então, um isomorfismo entre $\mathcal{X}$ e $\mathcal{Y}$ é uma aplicação linear bijetiva $T: \mathcal{X} \longrightarrow \mathcal{Y}$ que preserva a norma, i.e.,

$$
\begin{gathered}
T(\alpha x+\beta y)=\alpha T x+\beta T y, \quad \forall x, y \in \mathcal{X}, \quad \forall \alpha, \beta \in \mathbb{K} \\
\|T x\|_{\mathcal{Y}}=\|x\|_{\mathcal{X}}, \quad \forall x \in \mathcal{X}
\end{gathered}
$$

Isomorfismos em Espaços de Hilbert $\operatorname{Sejam} \mathcal{X}=\left(\mathcal{X},\langle,\rangle_{\mathcal{X}}\right)$ e $\mathcal{Y}=\left(\mathcal{Y},\langle,\rangle_{\mathcal{Y}}\right)$ espaços de Hilbert. Então, um isomorfismo entre $\mathcal{X}$ e $\mathcal{Y}$ é uma aplicação linear bijetiva $T: \mathcal{X} \longrightarrow \mathcal{Y}$ que preserva o IP, i.e.,

$$
\begin{gathered}
T(\alpha x+\beta y)=\alpha T x+\beta T y, \quad \forall x, y \in \mathcal{X}, \quad \forall \alpha, \beta \in \mathbb{K} \\
\langle T x, T y\rangle_{\mathcal{Y}}=\langle x, y\rangle_{\mathcal{X}}, \quad \forall x \in \mathcal{X}
\end{gathered}
$$

\section{A.13 Variedades Diferenciáveis}

Finalmente, chegamos à noção de variedade diferenciável. O conceito aqui envolvido está intimamente relacionado à ideia de difeomorfismo, cuja aplicação também é de grande utilidade na teoria exposta.

Uma variedade diferenciável de dimensão $n$ é um conjunto $M$ e uma família de aplicações biunívocas $\mathbf{x}_{\alpha}: U_{\alpha} \subset \mathbb{R}^{n} \rightarrow M$ de abertos $U_{\alpha}$ de $\mathbb{R}^{n}$ em $M$ tais que:

1. $\bigcup_{\alpha} \mathbf{x}_{\alpha}\left(U_{\alpha}\right)=M$;

2. Para todo par $\alpha, \beta$ com $\mathbf{x}_{\alpha}\left(U_{\alpha}\right) \cap \mathbf{x}_{\beta}\left(U_{\beta}\right)=W \neq \emptyset$, os conjuntos $\mathbf{x}_{\alpha}^{-1}(W)$ e $\mathbf{x}_{\beta}^{-1}(W)$ são abertos em $\mathbb{R}^{n}$ e as aplicações $\mathbf{x}_{\beta}^{-1} \circ \mathbf{x}_{\alpha}$ são diferenciáveis; 
3. A família $\left\{\left(U_{\alpha}, \mathbf{x}_{\alpha}\right)\right\}$ é máxima relativamente às condições (1) e (2).

\section{A.14 Definição Algébrica de Corpo}

Uma vez que iremos precisar desse conceito posteriormente, a fim de justificar um resultado que utilizamos no capítulo referente ao desenvolvimento, achamos conveniente expor sua definição matemática aqui.

Um corpo $(\mathbb{K},+, \cdot)$ é um conjunto $\mathbb{K}$, com pelo menos dois elementos, munido de duas operações, uma de adição, denotada por + , e uma de multiplicação, denotada por ·, que satisfazem as seguintes condições:

Com respeito à adição, $\forall x, y, z \in \mathbb{K}$ :

- (A1) Associatividade: $(x+y)+z=x+(y+z)$

- (A2) Comutatividade: $x+y=y+x$

- (A3) Elemento neutro: $\exists \varnothing \in \mathbb{K} \mid x+\varnothing=\varnothing+x=x$

- (A4) Elemento oposto: $\exists \bar{x} \in \mathbb{K} \mid \bar{x}+x=x+\bar{x}=\varnothing$

Com respeito à multiplicação, $\forall x, y \in \mathbb{K}$ :

- (M1) Associatividade: $(x \cdot y) \cdot z=x \cdot(y \cdot z)$

- (M2) Comutatividade: $x \cdot y=y \cdot x$

- (M3) Elemento neutro: $\exists 1 \in \mathbb{K} \mid x \cdot 1=1 \cdot x=x$

- (M4) Elemento oposto: $\forall z \in \mathbb{K} \backslash\{\varnothing\}, \exists z^{\prime} \in \mathbb{K} \mid z \cdot z^{\prime}=1$

Com respeito a ambas (compatibilidade entre operações), $\forall x, y, z \in \mathbb{K}$ :

- $(\mathrm{AM})$ Distributividade: $\quad x \cdot(y+z)=x \cdot y+x \cdot z$

Para este trabalho, a importância desse conceito está no fato, demonstrável pelas definições acima, de que qualquer corpo constitui um espaço vetorial sobre si mesmo, resultado que será mencionado no decorrer da exposição. 


\section{$A P \hat{E} N D I C E B$ - Notação de Dirac}

Em virtude de estarmos lidando aqui com variáveis complexas, consideramos ser a formalização linear na notação bra-ket de Dirac o caminho mais indicado a seguir no presente caso, conforme salientamos antes.

Neste apêndice apresentamos os princípios da linguagem que é utilizada no desenrolar do trabalho. O desenvolvimento formal das ferramentas necessárias à sua implementação total para o uso específico nesta tese é efetuado em detalhes no capítulo 2.

\section{B.1 Definição de Kets, Bras e Operadores}

O engenheiro elétrico e matemático britânico, de origem suíça, Paul Adrien Maurice Dirac (1902-1984), mais conhecido por suas contribuições à Física, estabeleceu, para uso específico no formalismo quântico, uma linguagem apropriada às características fundamentais da teoria, cuja maior virtude consistiu na incorporação da Mecânica Matricial de Heisenberg com a Mecânica Ondulatória de Schrödinger num único formalismo matemático. Essa linguagem é construída para espaços mais gerais de funções sobre o corpo complexo, os espaços de Hilbert, mas também pode ser utilizada num contexto mais restrito, para espaços de dimensão finita.

Na Mecânica Quântica a dimensionalidade é estabelecida de acordo com a natureza do sistema físico considerado. Se a dimensão é infinita, recaímos nos espaços de Hilbert.

A principal característica que diferencia a notação criada por Dirac da tradicional usada na álgebra linear diz respeito ao rigor no tratamento em relação à especificação dos vetores no espaço original e no espaço dual. Os vetores ordinários, isto é, no espaço vetorial n-dimensional usual sobre o corpo complexo, são chamados kets e denotados por $|u\rangle$, em vez da grafia tradicional em negrito $\mathbf{u}$ ou com a seta superposta $\vec{u}$. Já os elementos do espaço dos funcionais lineares sobre o espaço vetorial original, ou seja, os vetores do chamado espaço dual, são denominados bras e denotados por $\langle u|$. Os termos bra e ket surgem em alusão à palavra inglesa "bracket", que significa colchete ou parêntese. 




Do ponto de vista da descrição quântica, os kets devem conter a informação completa sobre o estado físico em questão, sendo chamados portanto de vetores de estado. Além disso, apresentam as mesmas propriedades que os vetores usuais, munidos das duas operações tradicionais: adição e multiplicação por um escalar, complexo, no caso. Garantida a propriedade do fechamento (i.e., a soma de dois kets ou o produto de um ket por um número complexo também deve ser um ket), então, para elementos de um espaço de estados $\mathbb{V}$ valem as mesmas propriedades de um espaço vetorial complexo, listadas na subseção A.2

Como foi dito antes, os bras correspondem aos vetores do espaço dual ao dos kets, sendo também chamados de covetores no formalismo algébrico tradicional. Como estamos operando sobre espaços complexos, é necessário ressaltar que a obtenção de vetores de estado duais a partir dos vetores de estado originais deve levar em conta a operação de conjugação complexa, ou seja,

$$
\langle\alpha|=| \alpha\rangle^{*}
$$

Além disso, pelo fechamento da segunda operação, podemos escrever

$$
\lambda|\alpha\rangle=|\lambda \alpha\rangle,
$$

o que conduz a

$$
|\lambda \alpha\rangle^{*}=\lambda^{*}|\alpha\rangle^{*}=\langle\alpha| \lambda^{*}
$$

O elemento oposto a $|\alpha\rangle$, definido pela propriedade A4 e obtido por $(-1)|\alpha\rangle$ será, por consequência, denotado por $|-\alpha\rangle$. 


\section{B.2 Produto Interno}

Vamos estabelecer agora a definição de produto interno para espaços vetoriais complexos na linguagem do formalismo de Dirac.

Um produto interno entre um bra $\langle\alpha|$ e um ket $|\beta\rangle$, definido sobre um espaço vetorial complexo, é uma aplicação $\langle\mid\rangle: \mathbb{V} \times \mathbb{V} \longrightarrow \mathbb{C}$ a valores complexos de pares ordenados que obedece às mesmas propriedades do produto interno usual, de forma que $\langle\alpha|| \beta\rangle$ é grafado simplesmente $\langle\alpha \mid \beta\rangle$, ou seja, para $\forall \lambda, \mu \in \mathbb{C}, \forall|x\rangle,|y\rangle,|z\rangle \in \mathbb{V}$, vale:

(1) $\langle\alpha \mid \beta\rangle^{*}=\langle\beta \mid \alpha\rangle$

$(2)\langle\alpha \mid \alpha\rangle \geq 0,\langle\alpha \mid \alpha\rangle=0 \Leftrightarrow|\alpha\rangle=|0\rangle$

(3) $\langle\alpha|(\lambda|\beta\rangle+\mu|\gamma\rangle)=\lambda\langle\alpha \mid \beta\rangle+\mu\langle\alpha \mid \gamma\rangle$

Também a norma de um autoestado é definida da maneira usual para os espaços de Hilbert, ou seja, através do produto interno: $\|\alpha\|=\sqrt{\langle\alpha \mid \alpha\rangle}$.

Dois vetores de estado $|\alpha\rangle$ e $|\beta\rangle$, ambos não-nulos, são ditos ortogonais entre si se $\langle\alpha \mid \beta\rangle=\langle\beta \mid \alpha\rangle=0$

No caso de um espaço discreto (i.e., um conjunto enumerável, embora eventualmente de dimensão infinita), é possível também definir bases do tipo $\left\{\left|e_{i}\right\rangle\right\}_{i=1}^{N}$ de forma que um vetor de estado $|\alpha\rangle$ possa ser descrito por suas componentes $\alpha_{i}$ nessa base. Uma base ortonormal é dada pela relação $\left\langle e_{i} \mid e_{j}\right\rangle=\delta_{i j}$, onde $\delta_{i j}$ é o símbolo de Kronecker. Numa base desse tipo as componentes $\alpha_{i}$ de um vetor de estado $|\alpha\rangle=\sum_{i} \alpha_{i}\left|e_{i}\right\rangle$ são dadas por $\alpha_{i}=\left\langle e_{i} \mid \alpha\right\rangle$, pois

$$
\left\langle e_{i} \mid \alpha\right\rangle=\left\langle e_{i}\left|\sum_{j} \alpha_{j}\right| e_{j}\right\rangle=\sum_{j} \alpha_{j}\left\langle e_{i} \mid e_{j}\right\rangle=\sum_{j} \alpha_{j} \delta_{i j}=\alpha_{i}
$$

Em termos de tais coordenadas o produto interno pode ser escrito como

$$
\langle\alpha \mid \beta\rangle=\sum_{i=1}^{N} \alpha_{i}^{*} \beta_{i},
$$

pois

$$
\begin{gathered}
\langle\alpha \mid \beta\rangle=\left\langle\sum_{i} \alpha_{i} e_{i} \mid \sum_{j} \beta_{j} e_{j}\right\rangle=\sum_{i} \sum_{j}\left\langle e_{i}\left|\alpha_{i}^{*} \beta_{j}\right| e_{j}\right\rangle= \\
=\sum_{i} \sum_{j} \alpha_{i}^{*} \beta_{j}\left\langle e_{i} \mid e_{j}\right\rangle=\sum_{i} \alpha_{i}^{*} \sum_{j} \beta_{j} \delta_{i j}=\sum_{i} \alpha_{i}^{*} \beta_{i}
\end{gathered}
$$

Exemplo 5: Como exemplo de aplicação, escreveremos a desigualdade de 
Schwarz em termos da notação de Dirac. A desigualdade de Schwarzé $|u \cdot v| \leq$ $|u||v|$. Na notação de Dirac o produto escalar $u \cdot v$ passa a $\langle\alpha \mid \beta\rangle \in \mathbb{C}$ e a norma quadrática $|u \cdot v|^{2}$ se transforma em $|\langle\alpha \mid \beta\rangle|^{2}$. Entretanto, é preciso lembrar que a norma de um número complexo é dada por $|z|^{2}=z^{*} z$ e, portanto, devemos tomar cuidado nesse ponto, escrevendo

$$
|\langle\alpha \mid \beta\rangle|^{2}=\langle\alpha \mid \beta\rangle^{*}\langle\alpha \mid \beta\rangle=\langle\beta \mid \alpha\rangle\langle\alpha \mid \beta\rangle
$$

Logo, como $\|\alpha\|^{2}=\langle\alpha \mid \alpha\rangle$, segue que

$$
|\langle\alpha \mid \beta\rangle|^{2}=\langle\alpha \mid \beta\rangle\langle\beta \mid \alpha\rangle \leq\langle\alpha \mid \alpha\rangle\langle\beta \mid \beta\rangle
$$

A partir da relação mostrada acima, o ângulo entre dois vetores de estado pode ser definido por

$$
\cos \theta=\sqrt{\frac{\langle\alpha \mid \beta\rangle\langle\beta \mid \alpha\rangle}{\langle\alpha \mid \alpha\rangle\langle\beta \mid \beta\rangle}}
$$

Exemplo 6: Vamos ilustrar o processo através de um exemplo concreto em três dimensões, calculando o ângulo entre $|\alpha\rangle$ e $|\beta\rangle$, para

$$
|\alpha\rangle=(1+i) \widehat{\mathbf{i}}+1 \widehat{\mathbf{j}}+i \widehat{\mathbf{k}} \quad e \quad|\beta\rangle=(4-i) \widehat{\mathbf{i}}+(2-2 i) \widehat{\mathbf{k}}
$$

Calculando $\langle\alpha \mid \beta\rangle$, temos:

$$
\begin{aligned}
& \langle\alpha \mid \beta\rangle=[(1-i) \hat{\mathbf{i}}+1 \widehat{\mathbf{j}}-i \widehat{\mathbf{k}}] \cdot[(4-i) \widehat{\mathbf{i}}+(2-2 i) \widehat{\mathbf{k}}]= \\
& =(1-i)(4-i)-i(2-2 i)=3-5 i-2 i-2=1-7 i
\end{aligned}
$$

As normas quadráticas valem

$$
\begin{gathered}
\langle\alpha \mid \alpha\rangle=[(1-i) \widehat{\mathbf{i}}+1 \widehat{\mathbf{j}}-i \widehat{\mathbf{k}}] \cdot[(1+i) \widehat{\mathbf{i}}+1 \widehat{\mathbf{j}}+i \widehat{\mathbf{k}}]= \\
=2+1+1=4 \\
\langle\beta \mid \beta\rangle=[(4+i) \widehat{\mathbf{i}}+(2+2 i) \widehat{\mathbf{k}}] \cdot[(4-i) \widehat{\mathbf{i}}+(2-2 i) \widehat{\mathbf{k}}]= \\
=17+8=25
\end{gathered}
$$

Logo,

$$
\begin{gathered}
\cos \theta=\sqrt{\frac{\langle\alpha \mid \beta\rangle\langle\beta \mid \alpha\rangle}{\langle\alpha \mid \alpha\rangle\langle\beta \mid \beta\rangle}}= \\
=\sqrt{\frac{(1+7 i)(1-7 i)}{4 \cdot 25}}=\sqrt{\frac{50}{100}}=\frac{\sqrt{2}}{2} \Rightarrow
\end{gathered}
$$




$$
\cos \theta=\frac{\sqrt{2}}{2} \Rightarrow \theta=45^{\circ}
$$

É possível, ademais, definir um vetor de estado normalizado:

$$
|\widetilde{\alpha}\rangle=\frac{1}{\sqrt{\langle\alpha \mid \alpha\rangle}}|\alpha\rangle
$$

\section{B.3 Álgebra de Operadores}

Uma das características mais significativas do formalismo quântico, para a qual a notação de Dirac se adequa notavelmente, é a possibilidade de trabalhar com operadores diferenciais como representantes de grandezas físicas, chamadas de observáveis na teoria. Esses operadores, assim como na álgebra linear, são aplicados sobre os vetores. Em outras palavras, os operadores atuam sobre os kets, isto é, sobre os vetores de estado do sistema, gerando outros kets, ou seja, outros vetores de estado. Seja $\hat{A}$ um operador, então

$$
\hat{A}(|\alpha\rangle)=\hat{A}|\alpha\rangle
$$

é um novo vetor de estado. Na álgebra linear tais operadores podem, geralmente, ser representados por matrizes. Como estamos operando num espaço complexo, os operadores duais devem corresponder aos adjuntos dos originais, sendo obtidos pelas operações de transposição e conjugação complexa combinadas.

O transposto conjugado ou hermitiano conjugado de um operador $\hat{A}$ é denotado pelo símbolo dagger (adaga) † sobrescrito à direita do mesmo: $\hat{A}^{\dagger}$ e corresponde, na álgebra de matrizes, a uma transposição combinada com uma conjugação complexa. As matrizes assim obtidas são chamadas de adjuntas das originais e, por extensão, os operadores eventualmente também recebem esse nome (não se deve confundir essa definição com a de matriz adjunta, i.e., adj $(A)$, usada no processo de inversão de matrizes). A definição matricial dessa operação é dada pela relação seguinte:

$$
A^{\dagger}=\left(A^{*}\right)^{T}=\left(A^{T}\right)^{*}
$$

ou, em termos de seus elementos,

$$
\left(A^{\dagger}\right)_{i j}=A_{j i}^{*}
$$

onde o asterisco denota a conjugação.

Também por analogia com a álgebra de matrizes, um operador $\hat{A}$ é dito hermitiano 
ou autoadjunto, se $\hat{A}^{\dagger}=\hat{A}$. Se, por outro lado, tivermos $\hat{A}^{\dagger}=-\hat{A}$, então o operador $\hat{A}$ é denominado anti-hermitiano.

A álgebra de operadores obedece às propriedades já conhecidas:

(1) $\widehat{A}+\widehat{B}=\widehat{B}+\widehat{A}$

(2) $(\widehat{A}+\widehat{B})+\widehat{C}=\widehat{A}+(\widehat{B}+\widehat{C})$

(3) $\widehat{A}(\lambda|\alpha\rangle+\mu|\beta\rangle)=\lambda \widehat{A}|\alpha\rangle+\mu \widehat{A}|\beta\rangle$

Define-se o operador nulo $\widehat{A}$ no caso $\widehat{A}|\alpha\rangle=|0\rangle, \forall|\alpha\rangle \in \mathbb{V}$. Dois operadores são idênticos se ocorrer o seguinte:

$$
\widehat{A}|\alpha\rangle=\widehat{B}|\alpha\rangle, \forall|\alpha\rangle \Leftrightarrow, \widehat{A}=\widehat{B}
$$

Outra possibilidade que temos é a de definir retrooperadores, quer dizer, operadores que atuem à direita de bras. Define-se

$$
(\widehat{A}|\alpha\rangle)^{*}=\langle\alpha| \hat{A}^{\dagger}
$$

Isso permite postular a ação de um operador sobre um bra.

Define-se também o produto de operadores pelo resultado da atuação de um sobre outro:

$$
(\widehat{A} \widehat{B})|\alpha\rangle=\widehat{A}(\widehat{B}|\alpha\rangle)
$$

A comutatividade não é válida no caso geral, i.e., $\widehat{A} \widehat{B} \neq \widehat{B} \widehat{A}$, mas a associatividade continua valendo: $(\widehat{A} \widehat{B}) \widehat{C}=\widehat{A}(\widehat{B} \widehat{C})$.

Mostraremos agora que, para dois operadores $\widehat{X}$ e $\widehat{Y}$ quaisquer, vale:

$$
(\widehat{X} \widehat{Y})^{\dagger}=\widehat{Y}^{\dagger} \widehat{X}^{\dagger}
$$

O produto dos operadores $\widehat{X} \widehat{Y}$ é um novo operador, ao passo que a atuação do operador $\widehat{Y}$ sobre um estado $|\alpha\rangle$ fornece um novo estado. Seja $|\beta\rangle=\widehat{Y}|\alpha\rangle$. Então

$$
\begin{aligned}
& (\widehat{X} \widehat{Y})|\alpha\rangle=\widehat{X}(\widehat{Y}|\alpha\rangle)=\widehat{X}|\beta\rangle \Rightarrow \\
& {[(\widehat{X} \widehat{Y})|\alpha\rangle]^{*}=(\widehat{X}|\beta\rangle)^{*}=\langle\beta| \widehat{X}^{\dagger}}
\end{aligned}
$$


Porém, como $\langle\beta|=| \beta\rangle^{*}=(\widehat{Y}|\alpha\rangle)^{*}=\langle\alpha| \widehat{Y}^{\dagger}$, segue que

$$
[(\widehat{X} \widehat{Y})|\alpha\rangle]^{*}=\langle\alpha| \widehat{Y}^{\dagger} \widehat{X}^{\dagger}
$$

Por outro lado,

$$
[(\widehat{X} \widehat{Y})|\alpha\rangle]^{*}=\langle\alpha|(\widehat{X} \widehat{Y})^{\dagger}
$$

Logo, pela igualdade dos dois resultados acima, vem

$$
(\widehat{X} \widehat{Y})^{\dagger}=\widehat{Y}^{\dagger} \widehat{X}^{\dagger}
$$

\section{B.4 Autovalores e Autoestados}

Seja $a^{\prime}$ o autovalor referente a um dado operador $\hat{A}$ e seja $\left|\alpha^{\prime}\right\rangle$ seu autovetor correspondente:

$$
\hat{A}\left|\alpha^{\prime}\right\rangle=a^{\prime}\left|\alpha^{\prime}\right\rangle
$$

Então, $\left|\alpha^{\prime}\right\rangle$ recebe o nome de eingenket ou autoket do operador $\hat{A}$. O estado físico correspondente a um eigenket é chamado um autoestado de $\hat{A}$. Um operador tem geralmente tantos autovalores quanto o número de dimensões do espaço de estados. Se houver autoestados diferentes com um mesmo autovalor, dizemos que ocorre degenerescência. O conjunto de autovalores possíveis constitui o espectro do operador. Se um operador apresenta um espectro não-degenerado, então o conjunto de seus autoestados constitui uma base. Seja $\left\{a^{\prime}, a^{\prime \prime}, a^{\prime \prime \prime}, \ldots\right\} \equiv\left\{a^{\prime}\right\}$ o conjunto dos autovalores de um operador $\hat{A}$ nãodegenerado e $\left\{\left|\alpha^{\prime}\right\rangle\right\}$ o conjunto de seus autoestados. Então, podemos escrever o estado $|\alpha\rangle$ em termos de seus autoestados $\left\{\left|\alpha^{\prime}\right\rangle\right\}$ :

$$
|\alpha\rangle=\sum_{\alpha^{\prime}}^{N} c_{\alpha^{\prime}}\left|\alpha^{\prime}\right\rangle, \quad c_{\alpha^{\prime}} \in \mathbb{C}
$$

Disso e das propriedades dos operadores decorre que se $\lambda$ é um autovetor de um operador $\hat{A}$ com autoestado $|\alpha\rangle$, então

$$
(\hat{A}|\alpha\rangle)^{*}=(\lambda|\alpha\rangle)^{*}=\langle\alpha| \lambda^{*}
$$

Mas, por outro lado,

$$
(\hat{A}|\alpha\rangle)^{*}=\langle\alpha| \hat{A}^{\dagger} \Rightarrow
$$




$$
\langle\alpha| \hat{A}^{\dagger}=\langle\alpha| \lambda^{*}
$$

Além disso, para um operador $\widehat{X}$ qualquer,

$$
\begin{gathered}
\langle\beta|(\widehat{X}|\alpha\rangle)=(\langle\beta| \widehat{X})|\alpha\rangle \equiv\langle\beta|\widehat{X}| \alpha\rangle \Rightarrow \\
\langle\beta|\widehat{X}| \alpha\rangle^{*}=[\langle\beta|(\widehat{X}|\alpha\rangle)]^{*}=\left\langle\alpha\left|\widehat{X}^{\dagger}\right| \beta\right\rangle
\end{gathered}
$$

Então, para um operador hermitiano $\widehat{H}$ vale a propriedade:

$$
\langle\alpha|\widehat{H}| \beta\rangle^{*}=\langle\beta|\widehat{H}| \alpha\rangle
$$

Esta expressão é, na verdade, tomada como uma definição de operador hermitiano. Como consequência, há três importantes teoremas relacionados a operadores hermitianos:

A seguir são enunciados três teoremas importantes:

Teorema 5: Os autovalores de um operador hermitiano são reais.

Teorema 6: Os autoestados de um operador hermitiano correspondentes a autovalores distintos são ortogonais.

Teorema 7: Os autoestados de um operador hermitiano formam uma base completa.

Aqui, demonstraremos os dois primeiros.

\section{Demonstração 4 (teorema 5):}

Seja $\widehat{H}$ um operador hermitiano e $|\alpha\rangle$ um de seus autoestados com autovalor correspondente $\lambda$; então, pela definição,

$$
\langle\alpha|\widehat{H}| \alpha\rangle^{*}=\langle\alpha|\widehat{H}| \alpha\rangle
$$

Mas, usando a equação do autovalor $\widehat{H}|\alpha\rangle=\lambda|\alpha\rangle$, decorre que

$$
\begin{gathered}
\langle\alpha|\lambda| \alpha\rangle^{*}=\langle\alpha|\lambda| \alpha\rangle \Rightarrow \\
\lambda^{*}\langle\alpha \mid \alpha\rangle=\lambda\langle\alpha \mid \alpha\rangle \Rightarrow \\
\lambda^{*}=\lambda
\end{gathered}
$$

Logo, $\lambda \in \mathbb{R}$. 
Demonstração 5 (teorema 6):

Seja $\widehat{H}$ um operador hermitiano e sejam $\left|\alpha_{i}\right\rangle$ e $\left|\alpha_{j}\right\rangle$ dois de seus autoestados com autovalores $\lambda_{i}$ e $\lambda_{j}$, respectivamente. Então, pela definição de operador hermitiano, segue que

$$
\left\langle\alpha_{j}|\widehat{H}| \alpha_{i}\right\rangle^{*}=\left\langle\alpha_{i}|\widehat{H}| \alpha_{j}\right\rangle
$$

Usando as equações de autovalor $\widehat{H}\left|\alpha_{i}\right\rangle=\lambda_{i}\left|\alpha_{i}\right\rangle$ e $\widehat{H}\left|\alpha_{j}\right\rangle=\lambda_{j}\left|\alpha_{j}\right\rangle$, temos

$$
\begin{gathered}
\left\langle\alpha_{j}\left|\lambda_{i}\right| \alpha_{i}\right\rangle^{*}=\left\langle\alpha_{i}\left|\lambda_{j}\right| \alpha_{j}\right\rangle \Rightarrow \\
\lambda_{i}^{*}\left\langle\alpha_{j} \mid \alpha_{i}\right\rangle^{*}=\lambda_{j}\left\langle\alpha_{i} \mid \alpha_{j}\right\rangle
\end{gathered}
$$

Mas, sabemos pelo teorema 1 que $\lambda_{i}^{*}=\lambda_{i}$ e portanto a equação acima fica

$$
\begin{gathered}
\lambda_{i}\left\langle\alpha_{i} \mid \alpha_{j}\right\rangle=\lambda_{j}\left\langle\alpha_{i} \mid \alpha_{j}\right\rangle \Rightarrow \\
\lambda_{i}\left\langle\alpha_{i} \mid \alpha_{j}\right\rangle-\lambda_{j}\left\langle\alpha_{i} \mid \alpha_{j}\right\rangle=0 \Rightarrow \\
\left(\lambda_{i}-\lambda_{j}\right)\left\langle\alpha_{i} \mid \alpha_{j}\right\rangle=0
\end{gathered}
$$

Logo, como os autovalores são distintos por hipótese, segue que

$$
\left(\lambda_{i}-\lambda_{j}\right) \neq 0 \Rightarrow\left\langle\alpha_{i} \mid \alpha_{j}\right\rangle=0
$$

Portanto, os autoestados são ortogonais.

\section{B.5 Axioma da Associatividade do Produto}

Podemos nos perguntar quais tipos de operações são possíveis entre bras e kets. Já foi mostrada a definição do produto interno, obtido pela justaposição de um bra sobre um ket, mas será que o contrário teria sentido, isto é, a superposição de um ket sobre um bra? Respondendo positivamente a essa questão, Dirac enunciou o axioma associativo na seguinte forma matemática:

$$
(|\beta\rangle\langle\alpha|) \cdot|\gamma\rangle=|\beta\rangle \cdot(\langle\alpha|| \gamma\rangle)
$$

Nessa expressão o ponto significa simplesmente um produto de alguma natureza. Sabendo que $\langle\alpha|| \gamma\rangle \equiv\langle\alpha \mid \gamma\rangle$, pode-se interpretar o segundo membro da equação como um produto interno multiplicado por um ket, ou seja, um outro ket, já que o resultado do 
produto interno é simplesmente um escalar complexo. Assim, o primeiro membro também deve ser um ket. Como $|\gamma\rangle$ já é um ket, a grandeza $(|\beta\rangle\langle\alpha|)$ pode ser interpretado como um operador. Logo, define-se o produto externo por $|\beta\rangle\langle\alpha|$, fornecendo como resultado um operador.

Denotando-se $|\beta\rangle\langle\alpha|=\widehat{X}$, vemos que a expressão

$$
(|\beta\rangle\langle\alpha|)|\gamma\rangle=\widehat{X}|\gamma\rangle=\langle\alpha \mid \gamma\rangle|\beta\rangle
$$

indica que o operador $\widehat{X}$ assume um significado geométrico equivalente ao de rotacionar o estado $|\gamma\rangle$ na direção do estado $|\beta\rangle$.

Obs: Também se define o equivalente de um produto diádico

$$
|\alpha\rangle \otimes|\beta\rangle
$$

para kets em espaços vetoriais distintos ou de sistemas diferentes, como, por exemplo, $|s\rangle \otimes|l\rangle$ para spin e momento orbital do $e^{-}$, ou $\left|s_{1}\right\rangle \otimes\left|s_{2}\right\rangle$ para spins de partículas distintas 1 e 2 . $O$ costume é grafar $|\alpha\rangle \otimes|\beta\rangle$ simplesmente como $|\alpha \beta\rangle$.

\section{B.6 Processo de ortonormalização de Gram-Schmidt para vetores de estado}

Partido-se de uma base $\left\{\left|e_{i}\right\rangle\right\}$ qualquer, podemos construir uma outra base $\left\{\left|\hat{e}_{i}\right\rangle\right\}$, ortonormalizada.

O primeiro passo é fazer

$$
\left|\hat{e}_{1}\right\rangle=\frac{1}{\sqrt{\left\langle e_{1} \mid e_{1}\right\rangle}}\left|e_{1}\right\rangle
$$

Em seguida, ortogonalizar o segundo eixo:

$$
\left|e_{2}^{\prime}\right\rangle=\left|e_{2}\right\rangle-\left\langle\hat{e}_{1} \mid e_{2}\right\rangle\left|\hat{e}_{1}\right\rangle
$$

e torná-lo unitário, fazendo

$$
\left|\hat{e}_{2}\right\rangle=\frac{1}{\sqrt{\left\langle e_{2}^{\prime} \mid e_{2}^{\prime}\right\rangle}}\left|e_{2}^{\prime}\right\rangle
$$

Então, basta repetir o processo sucessivamente. Para o terceiro elemento da base, tem-se:

$$
\left|e_{3}^{\prime}\right\rangle=\left|e_{3}\right\rangle-\left\langle\hat{e}_{1} \mid e_{3}\right\rangle\left|\hat{e}_{1}\right\rangle-\left\langle\hat{e}_{2} \mid e_{3}\right\rangle\left|\hat{e}_{2}\right\rangle
$$


Define-se o projetor pela expressão

$$
P_{k}=\sum_{i=1}^{k}\left|\hat{e}_{i}\right\rangle\left\langle\hat{e}_{i}\right|
$$

a fim de estabelecer o caso geral para o $n$-ésimo elemento da base ortonormalizada:

$$
\left|e_{n}^{\prime}\right\rangle=\left(\mathbf{1}-P_{n-1}\right)\left|e_{n}\right\rangle, \quad\left|\hat{e}_{n}\right\rangle=\frac{1}{\sqrt{\left\langle e_{n}^{\prime} \mid e_{n}^{\prime}\right\rangle}}\left|e_{n}^{\prime}\right\rangle
$$

\section{B.7 Extensão a Espaços de Funções}

Produto Interno Estamos considerando agora espaços de funções sobre o corpo complexo, ou seja, espaços vetoriais complexos de dimensão infinita. Nessas condições, torna-se necessário generalizar os conceitos anteriores. Como vimos, para funções QI, ou seja, pertencentes ao espaço $\mathcal{L}^{2}$, o IP é definido como

$$
\langle f \mid g\rangle=\int f^{*}(x) g(x) d x
$$

Bases Uma base no espaço de funções é formada por um conjunto de kets de funções complexas, i.e., $\left\{\left|\phi_{i}(x)\right\rangle\right\}$, onde $\phi_{i}(x) \in C^{0}[a, b]$ são funções QI. Em princípio, o número de elementos pode ou não ser limitado, ou seja, a base pode ser de dimensão finita ou infinita. Contudo, devido à característica essencial desse trabalho, que visa a resolução de equações diferenciais por meios computacionais numéricos, nosso enfoque se concentrará em bases de dimensão finita.

Operadores Os operadores no espaço de Hilbert são comumente operadores diferenciais, por exemplo, $\widehat{D} \equiv \frac{d}{d x}$. Os autovetores nesses espaços passam a chamar-se autofunções, obedecendo à equação

$$
\widehat{T} f(x)=\lambda f(x)
$$

Exemplo 7: $f(x)=A \exp (\lambda x)$ é autofunção do operador $\widehat{D}$ definido acima, com autovalor $\lambda$, pois $\widehat{D} f(x)=\lambda f(x)$.

Operadores hermitianos no espaço de Hilbert são definidos de forma análoga:

$$
\langle f \mid \widehat{H} g\rangle=\langle\widehat{H} f \mid g\rangle
$$

Demonstraremos a identidade acima para um operador hermitiano $\widehat{H}$ num intervalo 
$[a, b]$ qualquer.

\section{Demonstração 6:}

De fato,

$$
\begin{gathered}
\langle f \mid \widehat{H} g\rangle=\int_{a}^{b} f^{*}(x)[\widehat{H} g(x)] d x= \\
=\int_{a}^{b}\left[f^{*}(x) \widehat{H}\right] g(x) d x=\int_{a}^{b}\left[\widehat{H}^{\dagger} f(x)\right]^{*} g(x) d x= \\
=\int_{a}^{b}[\widehat{H} f(x)]^{*} g(x) d x=\langle\widehat{H} f \mid g\rangle
\end{gathered}
$$

Exemplo 8: Mostraremos, a título de exemplo, que o operador $\widehat{D} \equiv \frac{d}{d x} n \tilde{a} o$ é hermitiano. Tomemos, para isso, um intervalo $[a, b]$ arbitrário. Então,

$$
\begin{aligned}
\langle f \mid \widehat{D} g\rangle & =\int_{a}^{b} f^{*}(x) \frac{d}{d x} g(x) d x=\int_{a}^{b}\left\{\frac{d}{d x}\left[f^{*}(x) g(x)\right]-g(x) \frac{d f^{*}(x)}{d x}\right\} d x= \\
& =\left[f^{*}(x) g(x)\right]_{a}^{b}-\int_{a}^{b} \frac{d f^{*}(x)}{d x} g(x) d x=\left[f^{*} g\right]_{a}^{b}-\langle\widehat{D} f \mid g\rangle
\end{aligned}
$$

Se $f(a)=f(b)$ e $g(a)=g(b)$ o primeiro termo desaparece, mas o restante seria anti-hermitiano.

Exemplo 9: Também como exemplo, será mostrado agora que o operador $\widehat{\mathcal{D}} \equiv i \widehat{D}$ no intervalo $(-\infty, \infty)$ é hermitiano para funções de quadrado integrável. Usando o fato de que funções $\mathcal{L}^{2}$ devem se anular nos infinitos, i.e.,

$$
\lim _{x \rightarrow \pm \infty} f(x)=\lim _{x \rightarrow \pm \infty} g(x)=0
$$

isso nos permite escrever:

$$
\begin{gathered}
\langle f \mid \widehat{\mathcal{D}} g\rangle=\langle f \mid i \widehat{D} g\rangle=\int_{-\infty}^{\infty} f^{*}(x)\left(i \frac{d}{d x}\right) g(x) d x= \\
=\underbrace{\left[f^{*} g\right]_{-\infty}^{+\infty}}_{=0}+\int_{-\infty}^{\infty}\left[-i \frac{d f^{*}(x)}{d x}\right] g(x) d x= \\
=\int_{-\infty}^{\infty}\left(i \frac{d f(x)}{d x}\right)^{*} g(x) d x=\int_{-\infty}^{\infty}(\widehat{\mathcal{D}} f(x))^{*} g(x) d x=\langle\widehat{\mathcal{D}} f \mid g\rangle
\end{gathered}
$$

Logo, $\langle f \mid \widehat{\mathcal{D}} g\rangle=\langle\widehat{\mathcal{D}} f \mid g\rangle$ e, portanto, o operador $\widehat{\mathcal{D}}$ é hermitiano no intervalo $(-\infty, \infty)$. 


\section{APÊNDICE C - Matrizes Operacionais}

A idéia da substituição de uma equação diferencial por um sistema linear de equações algébricas reside no pressuposto da possibilidade de determinação unívoca de um operador linear $\mathcal{D}$ que represente a operação de derivação ou de integração, conforme o caso, sobre uma função $f(x)$ aproximada por uma série, de tal forma que, para a função aproximada $f_{n}(x)=\sum_{i=1}^{n} c_{i} b_{i}(x)$, sendo $c_{i}$ os coeficientes da série e $b_{i}(x)$ as componentes da base da série escolhida, tenhamos

$$
\frac{d}{d x} f(x) \rightarrow \mathcal{D} f_{n}(x)
$$

onde $f_{n}(x)$ é a série finita de $n$ termos que representa a função aproximada. Nesse caso, o operador linear de derivação $\mathcal{D}$ pode ser representado por uma matriz quadrada de ordem $n$, como em Paraskevopoulos (1985) por exemplo.

A série aproximativa deverá ser descrita pelo produto interno de um vetor, dado pelos coeficientes da soma, por um outro vetor, formado pelos elementos da base da série, que pode ser polinomial, exponencial ou de qualquer outro tipo que se deseje, ou seja,

$$
f_{n}(x)=\langle C, B(x)\rangle
$$

sendo $C$ o vetor dos coeficientes e $B(x)$ o vetor da base.

É importante destacar o fato de que os vetores acima descritos atuam em espaços de natureza diversa, isto é, o vetor dos coeficientes encontra-se num espaço de dimensão finita $n$-dimensional, cujas componentes são elementos de um corpo $\mathbb{K}$ (real ou complexo), ao passo que os elementos da base pertencem a um espaço de Hilbert $\mathcal{H}$, de dimensão infinita. Entretanto, o vetor de base pode ser interpretado como um vetor de elementos de $\mathcal{H}$ num espaço de dimensão finita igual à do vetor dos coeficientes. Daí, a possibilidade da representação em termos de um produto interno.

Matricialmente, pode-se obter o mesmo resultado pela multiplicação de uma matrizlinha de $n$ colunas por uma matriz-coluna de $n$ linhas. O produto dessa operação, embora seja um vetor no espaço de Hilbert, é uma variável escalar do corpo $\mathbb{K}$ no espaço discreto 
de $n$ dimensões da série aproximativa. O mesmo se aplica à sua derivada (ou, alternativamente, à sua integral), a qual pode ser obtida pela aplicação de um operador linear sobre a função aproximada, gerando uma nova função, descrita na base original com outros coeficientes, conforme a equação:

$$
f_{n}^{\prime}(x)=\left\langle C_{D}, B(x)\right\rangle
$$

O ferramental matemático necessário para a obtenção e descrição de matrizes operacionais através do formalismo de Dirac adaptado para esse fim específico é desenvolvido no capítulo 2. 


\section{APÊNDICE D - Panorama Histórico do Cálculo Fracional}

Neste apêndice apresentamos um sucinto panorama do desenvolvimento do Cálculo Fracional, sem maiores detalhes, apenas para situar o tema em um contexto histórico. Uma exposição técnica um pouco mais elaborada encontra-se nos apêndices E e F.

Embora do ponto de vista formal a definição fundamental do processo de derivação fracionária seja dada pela formulação de Grünwald-Letnikov (OLDHAM; SPANIER, 1974):

$$
\mathbb{D}^{q} f(x)=\lim _{h \rightarrow 0} \frac{1}{h^{q}} \sum_{0 \leq m \leq \infty}(-1)^{m}\left(\begin{array}{c}
q \\
m
\end{array}\right) f(x+(q-m) h),
$$

que é uma generalização da expressão para a derivada usual, sob a perspectiva da aplicação prática predominam as fórmulas integrais, em várias principais apresentações. Historicamente, várias definições foram propostas para generalizar derivadas e integrais para ordens não-inteiras.

A primeira menção de uma derivada de ordem arbitrária é devida ao matemático francês Sylvestre François Lacroix (1765-1843) que, em 1819, propôs uma mera extensão da fórmula da $n$-ésima derivada de uma função polinomial em um tratado genérico de 700 páginas sobre tópicos matemáticos diversos (LACROIX, 1810), das quais apenas duas (p.409-410) são dedicadas a esse tema específico. Partindo de $f(x)=x^{m}, m \in \mathbb{N}$, Lacroix utiliza a fórmula:

$$
\frac{d^{n}}{d x^{n}} x^{m}=\frac{m !}{(m-n) !} x^{m-n}
$$

para valores não inteiros de $n$, com a importante restrição $m \geq n$, reescrevendo-a em termos do símbolo de Legendre para o que se chamava, na época, de fatorial generalizado, hoje conhecido como função $\Gamma$ :

$$
\frac{d^{\alpha}}{d x^{\alpha}} x^{m}=\frac{\Gamma(m+1)}{\Gamma(m-\alpha+1) !} x^{m-\alpha}, \quad(\forall m, \alpha, m \geq \alpha)
$$


A partir desta fórmula, tomando $m=1$ e $\alpha=1 / 2$, Lacroix obteve

$$
\frac{d^{1 / 2}}{d x^{1 / 2}} x=\frac{\Gamma(2)}{\Gamma\left(\frac{3}{2}\right)} x^{1-1 / 2}=\frac{2 \sqrt{x}}{\sqrt{\pi}}
$$

Este resultado, mostrado informalmente por Lacroix, é calculado atualmente pela definição da derivada fracional de Riemann-Liouville. O problema principal do método de Lacroix é que ele não fornece pistas para uma derivada de ordem arbitrária de uma função genérica.

Figura 111: As duas páginas do tratado de Lacroix que versam sobre derivada fracional

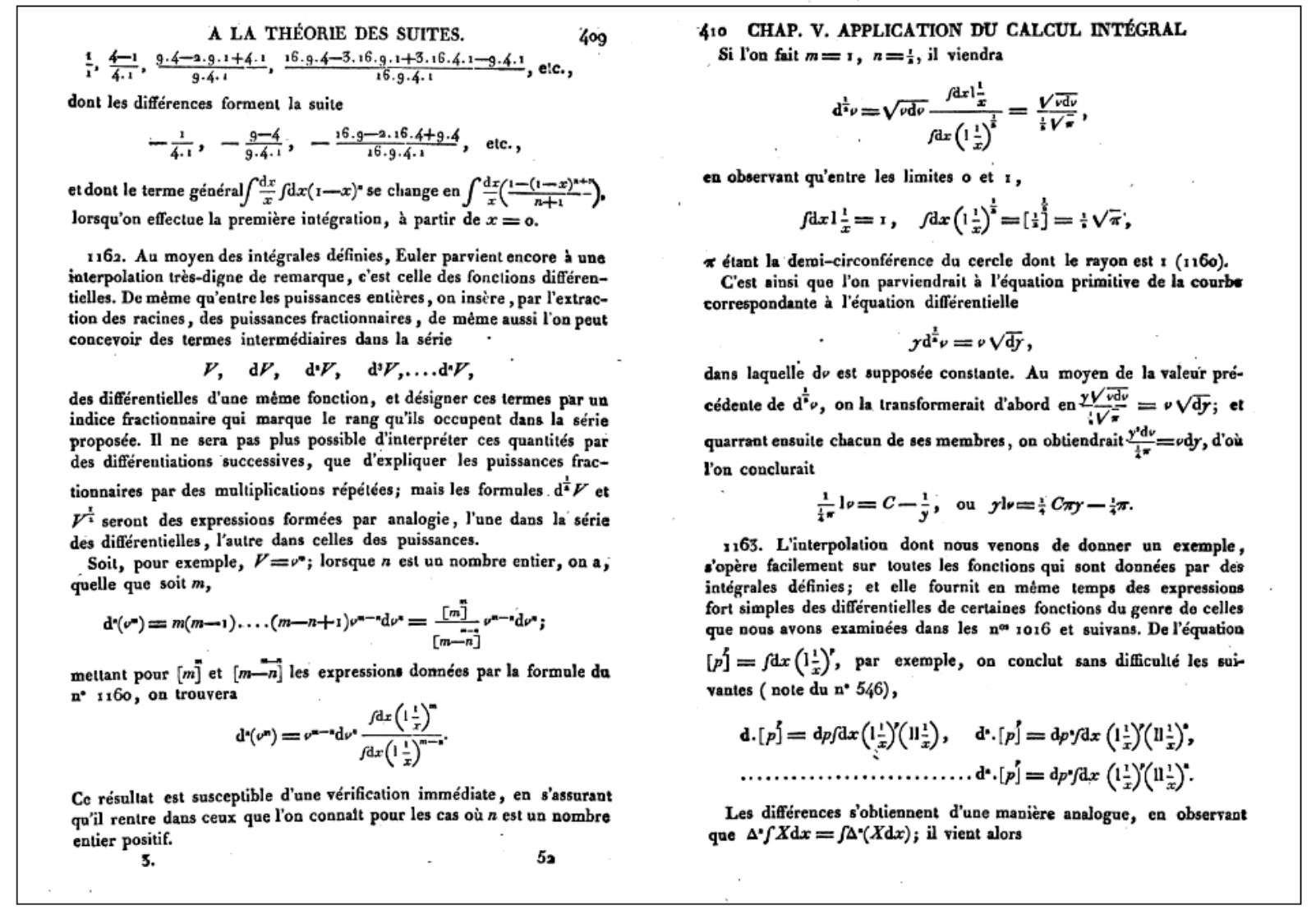

Fourier também tratou do assunto, propondo uma definição de operações fracionais através de sua fórmula para a representação integral de uma função:

$$
\frac{d^{\alpha} f(x)}{d x^{\alpha}}=\frac{1}{2 \pi} \int_{-\infty}^{\infty} f(\xi) \int_{-\infty}^{\infty} p^{\alpha} \cos \left[p(x-\xi)+\frac{1}{2} \alpha \pi\right] d p d \xi
$$

Por outro lado, a partir da n-ésima derivada da fórmula integral de Cauchy:

$$
D^{n} f(z)=\frac{n !}{2 \pi i} \int_{C} \frac{f(\zeta)}{(\zeta-z)^{n+1}} d \zeta
$$

surgiu a forma clássica da definição fracional, dada pela expressão da integral fracionária 
de Riemann (MILLER; ROSS, 1993):

$$
{ }_{c} D_{x}^{-\alpha} f(x)=\frac{1}{\Gamma(\alpha)} \int_{c}^{x} f(\xi)(x-\xi)^{\alpha-1} d \xi, \quad(x>c, \Re(\alpha)>0)
$$

No caso em que o limite inferior $c=0$ e a integral converge para $f\left(\frac{1}{x}\right)=O\left(x^{1-\epsilon}\right), \epsilon>$ 0), a fórmula costuma chamar-se integral fracionária de Riemann-Liouville. Para o caso $c=-\infty$, a expressão ${ }_{-\infty} D_{x}^{\alpha}$ pode receber o nome de $2^{a}$ fórmula de Liouville, dando-se a convergência para $f(-x)=O\left(x^{-\alpha-\epsilon}\right), \epsilon>0, x \rightarrow \infty$.

No entanto, quanto à questão da denominação das várias formulações, infelizmente não existe ainda um consenso. Por exemplo, em Oldham e Spanier (1974) adota-se o nome de Riemann-Liouville para ${ }_{c} D_{x}^{-\alpha}$, denominando-se a expressão ${ }_{-\infty} D_{x}^{\alpha}$ de derivada fracional de Weyl. Já Miller e Ross (1993) denominam essa mesma fórmula por derivada fracional de Liouville, reservando o nome de derivada fracionária de Weyl para a expressão

$$
{ }_{x} W_{\infty}^{\alpha}=\frac{1}{\Gamma(-\alpha)} \int_{x}^{\infty} \frac{f(\xi)}{(x-\xi)^{\alpha+1}} d \xi, \quad(\Re(\alpha)>0)
$$

Enfim, a nomenclatura é assunto ainda não completamente convencionado e devemos salientar que se trata aqui de uma questão de escolha dentre os possíveis conjuntos de nomes para referir-se a cada definição específica. Nesse trabalho, buscamos ser ainda mais específicos nessa questão, redefinindo alguns nomes de acordo com as necessidades. Todavia, é claro que, uma vez escolhida a nomenclatura, não se deve alterá-la indiscriminadamente, para evitar confusão ou equívocos desnecessários.

De forma geral, no atual tratamento faz-se frequentemente uma distinção entre as definições da diferintegral de Riemann-Liouville e a de Caputo, com base na ordem das operações. Explicando melhor, como no processo de derivação usual ocorre perda de informação, uma operação de integração seguida de uma de derivação pode fornecer um resultado diferente daquele obtido em ordem inversa, ou seja, de uma derivação seguida por uma integração da mesma função. Isso diz respeito a uma questão específica, chamada de problema da inicialização no cálculo fracional. Por causa dessa ambiguidade proveniente do limite inferior da integral, já que ${ }_{c} D_{x}^{-\mu}{ }_{\bar{c}} D_{x}^{-\nu} f(x)$ não é geralmente comutativo, gerando um desvio para $c \neq \bar{c}$, Riemann introduziu a sua função complementar $\Psi(x)$, que pode conter uma infinidade de constantes arbitrárias.

Em virtude desse fato, devemos escolher qual operação fazer primeiro, o que desemboca em resultados com características distintas, apesar de a teoria atual ter eliminado esse problema, que foi solucionado por meio das definições de limites no processo de de- 
rivação, o que unifica o processo. Mesmo assim, há propriedades relacionadas às EDOs que são características da forma com que as operações são realizadas no processo de resolução. Por exemplo, se desejarmos uma derivação de ordem $\alpha$ fracionária, podemos escolher um inteiro $m$ tal que $m-1<\alpha<m$, para determinar a $m$-ésima derivada da função, utilizando em seguida a fórmula da integral fracionária de Riemann-Liouville para integrar esse resultado $m-\alpha$ vezes, o que forneceria uma derivação de ordem fracionária $\alpha$, conforme o desejado. Alternativamente, pode-se optar pelo contrário, isto é, fazer primeiro a integração de ordem fracionária $m-\alpha$ para em seguida determinar a $m$ ésima derivada do resultado obtido. Pois bem, o primeiro processo descrito é a definição conhecida como diferintegral de Caputo:

$$
D_{*}^{\alpha} f(x):=\frac{1}{\Gamma(m-\alpha)} \int_{0}^{x} \frac{f^{(m)}(\xi)}{(x-\xi)^{\alpha-m+1}} d \xi, \quad(m-1<\alpha<m \in \mathbb{Z})
$$

Já a segunda forma acima descrita é, provavelmente, a que a maior parte da literatura atual denomina de diferintegral de Riemann-Liouville:

$$
D^{\alpha} f(x):=\frac{d^{m}}{d x^{m}}\left\{\frac{1}{\Gamma(m-\alpha)} \int_{0}^{x} \frac{f(\xi)}{(x-\xi)^{\alpha-m+1}} d \xi\right\}
$$

Cada uma delas apresenta vantagens e desvantagens. A definição de Caputo foi implementada por apresentar uma clara vantagem em relação à de Riemann-Liouville no que se refere à resolução de EDOs. A saber, as condições iniciais naquela definição apresentam ordens inteiras, podendo ser usadas na solução, o que não ocorre nesta última.

Entretanto, a abordagem de Caputo apresenta um inconveniente. Como $f(x)$ deve ser causal, i.e., $f(x)=0, x \leq 0$, deve-se conhecer, neste caso, todas as derivadas nulas até ordem $m$, ou seja, $f(x)=f^{\prime}(x)=\cdots=f^{(m)}(x)$, o que também dificulta a determinação das soluções. Em compensação, na definição de Riemann-Liouville a derivação fracional de uma constante pode gerar um resultado não-nulo, conforme

$$
D^{\alpha} C=\frac{C x^{-\alpha}}{\Gamma(1-\alpha)}, \quad(C=\text { const. })
$$

fato que não ocorre na definição de Caputo, na qual a derivação de uma constante é nula, como na derivada usual de ordem inteira.

Ademais, na teoria de funções periódicas, incluindo as condições de contorno repetidas por período, a formulação predominante é a do operador integral de Weyl. Ele é definido sobre séries de Fourier e requer o desaparecimento do coeficiente constante da série de Fourier, sendo aplicado a funções no círculo unitário com valor de integração 
nulo. Assim como a de Riemann-Liouville, surge da extensão da integral, em contraste com a diferintegral de Grünwald-Letnikov, que tem sua origem na definição da derivada. Para uma função $f(\theta)$ de valor médio nulo num intervalo de período $2 \pi$, dada pela série $\sum_{n=-\infty}^{\infty} c_{n} e^{i n \theta}$, com $c_{0}=0$, o operador integral de Weyl de ordem $\alpha$ é definido sobre uma série de Fourier (LIZORKIN, 2001), de acordo com

$$
f^{\alpha}(\theta)=\sum_{n=-\infty}^{\infty} \frac{c_{n} e^{i n \theta}}{(i n)^{\alpha}}, \quad\left(\alpha>0, c_{0}=0\right)
$$

A condição $c_{0}=0$ é requerida, evidentemente, para evitar o surgimento de uma divisão por zero na expressão acima. Já sua contraparte derivada seria escrita na forma

$$
f_{\beta}(\theta)=\frac{\partial^{n}}{\partial \theta^{n}} f^{n-\beta}(\theta), \quad(\beta>0)
$$

onde $n$ é o menor inteiro maior que $\beta$. Deve-se notar que que $f^{\alpha}(\theta)={ }_{a} D_{x}^{-\alpha} f(\theta)$, onde ${ }_{a} D_{x}^{-\alpha}$ é a integral fracionária de Riemann-Liouville.

Além das definições anteriores, também podemos citar a integral fracional de Hadamard (HADAMARD, 1892):

$$
{ }_{c} D_{x}^{-\alpha} f(x)=\frac{1}{\Gamma(\alpha)} \int_{c}^{x} f(\xi) \frac{1}{\xi}\left(\ln \frac{x}{\xi}\right)^{\alpha-1} d \xi
$$

e o operador de Erdélyi-Kober (KATUGAMPOLA, 2011):

$$
\left({ }^{\rho} \mathcal{I}_{c+}^{\alpha} f\right)(x)=\frac{\rho^{1-\alpha}}{\Gamma(\alpha)} \int_{c}^{x} \frac{\xi^{\rho-1} f(\xi)}{\left(x^{\rho}-\xi^{\rho}\right)^{1-\alpha}} d \xi, \quad(x>c)
$$

que generaliza a integral fracional de Riemann e o operador integral de Weyl.

Funções de Mittag-Leffler De forma análoga às soluções de EDOs que fornecem funções do tipo exponencial, a solução de equações diferenciais de ordem arbitrária costuma gerar um tipo específico de função, conhecida como função de Mittag-Leffler. Existem, de fato, várias classes de funções desse tipo, das quais duas se destacam. A primeira, definida em 1903 pelo matemático sueco Magnus Gösta Mittag-Leffler (1846-1927), tem a forma:

$$
E_{\alpha}(z)=\sum_{k=0}^{\infty} \frac{z^{k}}{\Gamma(\alpha k+1)}, \quad(\alpha \in \mathbb{C}, \Re(\alpha)>0)
$$

A segunda, chamada de função de Mittag-Leffler generalizada, foi, na verdade, definida por outro matemático sueco, Anders Wiman (1865-1959) (WIMAN, 1905a, 1905b), tendo sido posteriormente estudada por vários matemáticos, dos quais se destaca o indiano $R$. 
P. Agarwal (1953), entre outros:

$$
E_{\alpha, \beta}(z)=\sum_{k=0}^{\infty} \frac{z^{k}}{\Gamma(\alpha k+\beta)}, \quad(\alpha, \beta \in \mathbb{C}, \Re(\alpha), \Re(\beta)>0)
$$

Figura 112: Magnus Gösta Mittag-Leffler (1846-1927)

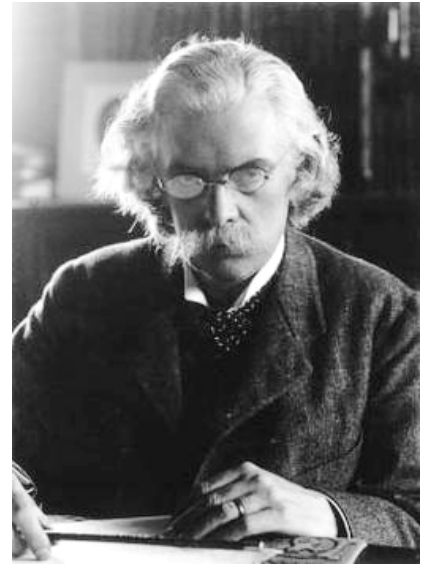

A função generalizada se relaciona à primeira definição por $E_{\alpha}(z)=E_{\alpha, 1}(z)$. A função de Mittag-Leffler constitui uma espécie de generalização para uma vasta gama de outras funções, que assumem, então, o aspecto de casos particulares dela. Por exemplo, a própria exponencial, a série geométrica, a função erro e o cosseno hiperbólico são algumas das funções podem ser escritas como casos particulares da função de Mittag-Leffler. Além disso, ela também está relacionada às conhecidas funções hiperbólicas generalizadas.

Ademais, podemos citar sua representação integral:

$$
E_{\alpha, \beta}(z)=\frac{1}{2 \pi i} \int_{C} \frac{\zeta^{\alpha-\beta} e^{\zeta}}{\left(\zeta^{\alpha}-z\right)^{n+1}} d \zeta,
$$

estabelecida em 1954 por A. Erdélyi et al. e M. M. Dzherbashyan. 


\section{APÊENDICE E - Funções Especiais do Cálculo Fracional}

O desenvolvimento formal do cálculo fracional requer o conhecimento de alguns conceitos pouco usuais, ou mesmo estranhos, ao tratamento clássico do cálculo diferencial e integral, bem como uma série de generalizações de definições e conceitos mais restritos naquele formalismo.

Entre esses novos recursos exigidos, encontram-se algumas funções especiais, as quais guardam uma relação mais profunda com o cálculo a valores arbitrários e que serão estudadas ao longo dessa seção, na qual apresentaremos uma exposição formal resumida com os principais resultados do Cálculo Fracional, cujo conhecimento se faz estritamente necessário para a utilização em aspectos essenciais desta tese.

Entremeados nessa exposição, encontram-se algumas revisões necessárias, de assuntos usualmente conhecidos, mas vistos sob uma outra perspectiva, visando suas devidas extensões a conjuntos mais amplos, sejam a valores reais ou complexos.

\section{E.1 Função Gama}

A função gama foi estabelecida por Euler no século XVIII com o intuito de estender a noção de fatorial aos números reais. De fato, ela é definida para todos os complexos, exceto os inteiros negativos e o zero. Também conhecida como integral de Euler de segunda espécie, sua moderna notação com a letra grega $\Gamma$ é devida a Legendre, tendo sido introduzida em 1811. Há várias formas de expressá-la e apresentaremos aqui todas as que forem importantes para a clara exposição do assunto.

Representação Integral A forma mais usual de se escrever a função gama é por sua representação integral (KILBAS; SRIVASTAVA; TRUJILLO, 2006): 


$$
\Gamma(z)=\int_{0}^{\infty} t^{z-1} e^{-t} d t, \quad z \in \mathbb{C}, \Re(z)>0
$$

Uma propriedade importante da função gama é obtida de sua integração por partes, $\operatorname{com} t^{z}=u$ :

$$
\begin{gathered}
\Gamma(z+1)=\int_{0}^{\infty} t^{z} e^{-t} d t=\left.\left[t^{z}\left(-e^{-t}\right)\right]\right|_{t=0} ^{\infty}+\int_{0}^{\infty} z t^{z-1} e^{-t} d t=z \Gamma(z) \Rightarrow \\
\Gamma(z+1)=z \Gamma(z)
\end{gathered}
$$

Com isso verifica-se que, para o caso de $n \in \mathbb{N}$, a função gama identifica-se com o fatorial, ou seja,

$$
\Gamma(n+1)=n \Gamma(n)=n(n-1) \Gamma(n-1)=\cdots=n(n-1) \ldots 1 . \Gamma(1)
$$

mas, uma vez que

$$
\Gamma(1)=\int_{0}^{\infty} e^{-t} d t=1
$$

chega-se a:

$$
\Gamma(n+1)=n !, \quad \forall n \in \mathbb{N}
$$

Obtém-se daí o valor do fatorial de zero:

$$
\Gamma(1)=0 !=1
$$

Extensão Analítica da Função Gama A partir da relação de recorrência (E.2) é possível estender a função gama ao semieixo real negativo $\Re(z) \leq 0$, fazendo

$$
\Gamma(z)=\frac{\Gamma(z+1)}{z}
$$

Daí, repetindo esse procedimento, obtém-se:

$$
\begin{gathered}
\Gamma(z+1)=\frac{\Gamma(z+2)}{z+1} \Rightarrow \Gamma(z)=\frac{\Gamma(z+2)}{z(z+1)}=\frac{\Gamma(z+3)}{z(z+1)(z+2)}=\cdots= \\
=\frac{\Gamma(z+n)}{z(z+1)(z+2) \ldots(z+n-1)} \Rightarrow \\
\Gamma(z)=\frac{\Gamma(z+n)}{(z)_{n}}, \quad z \in \mathbb{C} \backslash \mathbb{Z}_{0}^{-}, \quad \Re(z)>-n, \quad n \in \mathbb{N}
\end{gathered}
$$

onde $(z)_{n}=z(z+1)(z+2) \ldots(z+n-1)$ é o símbolo de Pochhammer, cuja forma es- 
tendida é

$$
(\alpha)_{r}=\frac{\Gamma(\alpha+r)}{\Gamma(\alpha)}, \quad \alpha \in \mathbb{C}
$$

Percebe-se que $\Gamma(p) \rightarrow \infty$ quando $p \rightarrow 0$. Entretanto, não apenas neste ponto, mas para todo $p$ inteiro negativo ocorre o mesmo, visto que, neste caso, $(p)_{n} \rightarrow 0$ para qualquer $k<n, \quad k \in \mathbb{N}$. Em outros termos, a função gama apresenta infinitos pólos nos inteiros negativos. Portanto, fica determinado seu domínio: $\mathbb{C} \backslash \mathbb{Z}_{0}^{-}$.

Além disso, a extensão negativa da função gama entre cada dois inteiros é alternadamente negativa e positiva, pois observa-se que, para $p \in \mathbb{R}$, com $-n<p<-n+1$, o termo $(p)_{n}$ só possui termos menores que zero e, portanto, $(p)_{n}=p(p+1) \ldots(p+n-1) \Rightarrow$ $(p)_{1}<0,(p)_{2}>0,(p)_{3}<0$ e assim por diante. Mostra-se na figura 113 abaixo o gráfico da função gama no domínio real.

Figura 113: Gráfico da função gama no domínio real

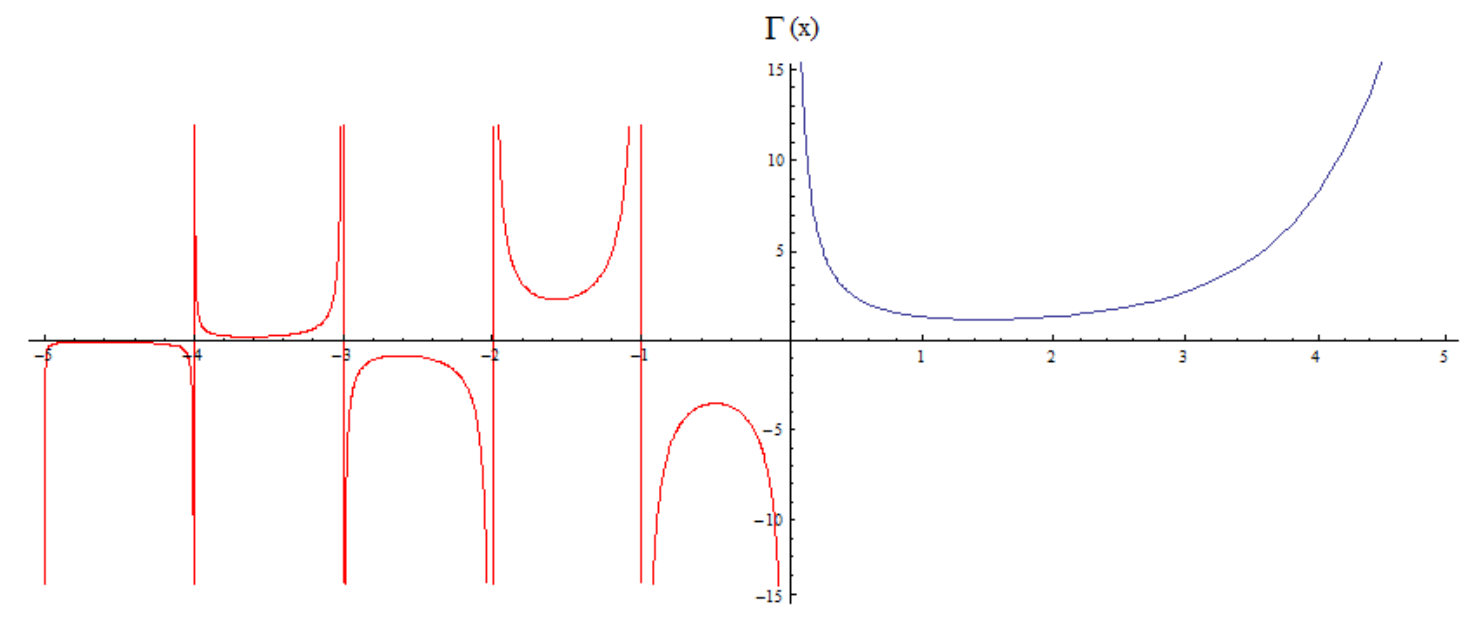

Duplos Fatoriais O duplo fatorial é definido como

$$
n ! != \begin{cases}n(n-2)(n-4) \ldots 2, & p / n \text { par } \\ n(n-2)(n-4) \ldots 1, & p / n \text { ímpar }\end{cases}
$$

O fatorial, escrito em termos do duplo fatorial, para cada um dos casos, torna-se:

$$
n !=\left\{\begin{array}{cc}
{[n(n-2) \ldots 2][(n-1)(n-3) \ldots 1],} & p / n \text { par } \\
\text { ou } & \\
{[n(n-2) \ldots 1][(n-1)(n-3) \ldots 2],} & p / n \text { ímpar }
\end{array}\right.
$$


o que conduz, para ambos os casos, ao resultado

$$
n !=n ! !(n-1) ! !
$$

$\mathrm{Ou}$

$$
(n-1) ! !=\frac{n !}{n ! !}
$$

Para o caso de $n$ ser par, isto é, $n=2 m, m \in \mathbb{N}$, vale

$$
(2 m) ! !=2 m(2 m-2) \ldots 2=2^{m} m !
$$

ou, fazendo $m=n / 2$ :

$$
n ! !=2^{n / 2}\left(\frac{n}{2}\right) ! \quad(p / n \text { par })
$$

Já fazendo $n=2 m$ na equação (E.6) e usando a equação (E.7), vemos que

$$
(2 m-1) ! !=\frac{(2 m) !}{(2 m) ! !}=\frac{(2 m) !}{2^{m} m !}, \quad(m \in \mathbb{N})
$$

ou ainda, escrevendo-se $k=2 m-1$, obtém-se, para o caso ímpar,

$$
k ! !=\frac{(k+1) !}{2^{(k+1) / 2}\left(\frac{k+1}{2}\right) !} \quad(p / k \text { ímpar })
$$

Entretanto, podemos generalizar o conceito de duplo fatorial para o caso de valores não-inteiros, visando sua utilização no cálculo fracional. Isso pode ser conseguido, através de uma relação estabelecida entre o duplo fatorial e a função gama.

Valores Não-inteiros Para o caso de $x=\frac{k}{2}$, onde $k$ é um natural diferente de zero, também não é difícil calcular explicitamente a função gama, desde que saibamos o resultado de $\Gamma\left(\frac{1}{2}\right)$, o que também pode ser feito com uma mudança apropriada de variáveis. A integral

$$
\Gamma\left(\frac{1}{2}\right)=\int_{0}^{\infty} t^{-\frac{1}{2}} e^{-t} d t
$$

transforma-se, através da substituição $t \longrightarrow u^{2}$, em

$$
\int_{0}^{\infty} \frac{e^{-u^{2}}}{u} 2 u d u=2 \int_{0}^{\infty} e^{-u^{2}} d u
$$

Conhece-se, entretanto, o valor da integral gaussiana

$$
\int_{0}^{\infty} e^{-u^{2}} d u=\frac{1}{2} \sqrt{\pi}
$$


o que nos permite escrever

$$
\Gamma\left(\frac{1}{2}\right)=\sqrt{\pi}
$$

Usando agora a propriedade de recorrência (E.2) repetidamente, chega-se a

$$
\Gamma\left(\frac{2 k+1}{2}\right)=\left(\frac{2 k-1}{2}\right)\left(\frac{2 k-3}{2}\right) \cdots\left(\frac{3}{2}\right)\left(\frac{1}{2}\right) \Gamma\left(\frac{1}{2}\right)=\frac{\sqrt{\pi}}{2^{k}}(2 k-1) ! !
$$

ou, invertendo a expressão acima,

$$
(2 k-1) ! !=\frac{2^{k}}{\sqrt{\pi}} \Gamma\left(k+\frac{1}{2}\right)
$$

Agora, chamando-se $n=2 k-1$, tem-se

$$
n ! !=\sqrt{\frac{2^{n+1}}{\pi}} \Gamma\left(\frac{n}{2}+1\right), \quad p / n \text { ímpar }
$$

Por outro lado, diretamente da equação (E.8) vem,

$$
n ! !=\sqrt{2^{n}} \Gamma\left(\frac{n}{2}+1\right), \quad p / n \text { par }
$$

As duas expressões são parecidas, divergindo apenas pelo fator multiplicativo antes da função gama. Uma forma de reduzir as equações a uma única fórmula seria por meio de $\bmod (n, 2)$, porém a função resultante seria somente lisa por partes, apresentando infinitos pontos de descontinuidade nos inteiros pares. A fim estabelecer uma função contínua para o duplo fatorial, é mais adequado utilizar, em vez de mod, uma função contínua periódica, como seno ou cosseno, para representar números pares e ímpares como casos particulares. Assim, como $\cos (\pi x)$ assume os valores \pm 1 , então $\frac{1}{2}[1-\cos (\pi x)]$ é nulo para $x$ par e igual a um para $x$ ímpar, ou seja, $\frac{1}{2}[1-\cos (\pi x)]=\bmod (x, 2)$, sem apresentar a desvantagem das descontinuidades. Portanto, podemos expressar os fatores multiplicativos de cada caso acima por um único fator comum:

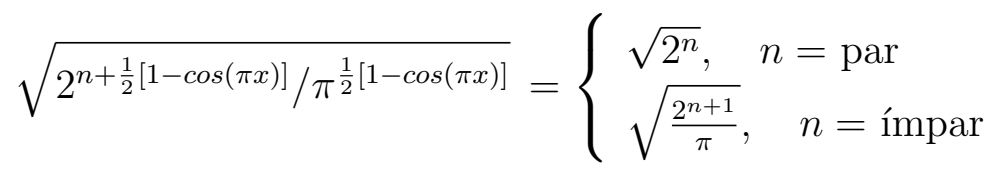

Dessa maneira, como esse fator é definido não apenas para negativos mas também para complexos, então, de modo análogo à função gama, o duplo fatorial também pode ser definido para valores complexos por meio da expressão:




Na figura 114 vê-se o gráfico dessa função para o domínio real.

Figura 114: Gráfico da função duplo fatorial no domínio real

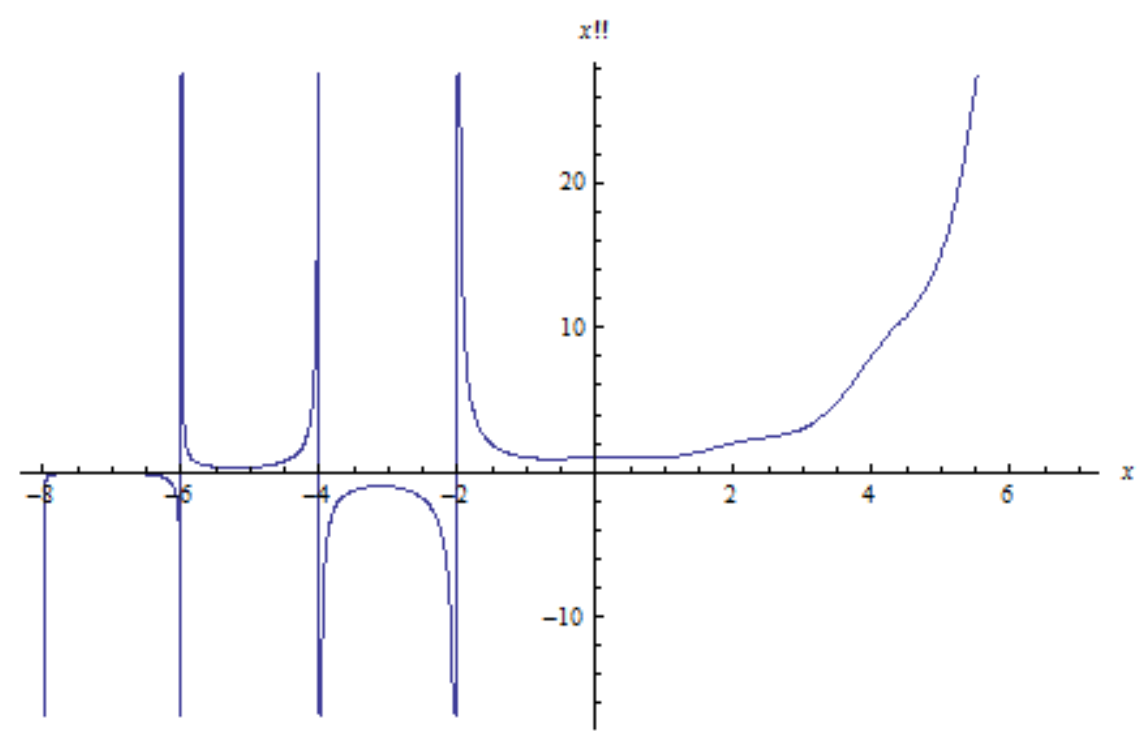

Relação com as Transformadas de Laplace A função gama também torna possível obter mais facilmente o resultado de integrais do tipo das transformadas de Laplace: $\mathcal{L}\left\{t^{x}\right\}=\int_{0}^{\infty} t^{x} e^{-s t} d t$, onde $s$ é uma constante real arbitrária, pela substituição $s t \longrightarrow \lambda$, conforme se vê abaixo:

$$
\mathcal{L}\left\{t^{x}\right\}=\int_{0}^{\infty} t^{x} e^{-s t} d t=\frac{1}{s^{x+1}} \int_{0}^{\infty} \lambda^{x} e^{-\lambda} d \lambda=\frac{\Gamma(x+1)}{s^{x+1}}
$$

Expressão de Gauss A função gama pode ser expressa na forma de um limite, chamado de expressão de Gauss, fórmula que mostra notável aplicação na exposição formal do cálculo fracional. Vamos deduzi-la a partir da representação integral. Introduziremos, com esse fim, uma função auxiliar $f_{n}(z)$. Seja

$$
f_{n}(z)=\int_{0}^{n}\left(1-\frac{t}{n}\right)^{n} t^{z-1} d t, \quad \Re(z)>0
$$

Visto que

$$
\lim _{n \rightarrow \infty}\left(1-\frac{t}{n}\right)^{n}=e^{-t}
$$

é plausível esperar que

$$
\lim _{n \rightarrow \infty} f_{n}(z) \rightarrow \int_{0}^{\infty} e^{-t} t^{z-1} d t=\Gamma(z)
$$

Obs: Esta passagem não é tão trivial e inocente quanto parece à primeira vista e exige, na 
verdade, uma justificativa mais rigorosa. Uma demonstração formal da expressão E.16 pode ser encontrada em Podlubny (1999).

Agora, efetuando a substituição de variáveis $t \rightarrow n \tau$, com $0 \leq \tau \leq 1$, tem-se

$$
f_{n}(z)=\int_{0}^{1}(1-\tau)^{n} n^{z} \tau^{z-1} d \tau
$$

Após uma integração por partes com $u=(1-\tau)^{n}$, obtém-se

$$
\begin{gathered}
f_{n}(z)=-\left.n^{z}(1-\tau)^{n} \frac{\tau^{z}}{z}\right|_{0} ^{1}+n^{z} \int_{0}^{1} n(1-\tau)^{n-1} \frac{\tau^{z}}{z} d \tau= \\
=\frac{n^{z}}{z} n \int_{0}^{1}(1-\tau)^{n-1} \tau^{z} d \tau
\end{gathered}
$$

Repetindo a integração por partes para $I=\int_{0}^{1}(1-\tau)^{n-1} \tau^{z} d \tau$, com $u=(1-\tau)^{n-1}$, reobtém-se

$$
\begin{gathered}
I=-\left.(1-\tau)^{n-1} \frac{\tau^{z+1}}{z+1}\right|_{0} ^{1}+\int_{0}^{1}(n-1)(1-\tau)^{n-2} \frac{\tau^{z+1}}{z+1} d \tau \Rightarrow \\
f_{n}(z)=\frac{n^{z}}{z(z+1)} n(n-1) \int_{0}^{1}(1-\tau)^{n-2} \tau^{z+1} d \tau
\end{gathered}
$$

Prosseguindo nesse mesmo sentido, calcula-se o terceiro passo, chegando-se a

$$
f_{n}(z)=\frac{n^{z}}{z(z+1)(z+2)} n(n-1)(n-2) \int_{0}^{1}(1-\tau)^{n-3} \tau^{z+2} d \tau,
$$

o que nos conduz, por indução, a

$$
\begin{gathered}
f_{n}(z)=\frac{n^{z} n(n-1)(n-2) \ldots(n-n+1)}{z(z+1)(z+2) \ldots(z+n-1)} \int_{0}^{1} \tau^{z+n-1} d \tau= \\
=\left.\frac{n^{z} n !}{z(z+1)(z+2) \ldots(z+n-1)} \frac{\tau^{z+n}}{z+n}\right|_{0} ^{1} \Rightarrow \\
f_{n}(z)=\frac{n^{z} n !}{z(z+1)(z+2) \ldots(z+n)}
\end{gathered}
$$

Usando, então, o limite expresso em (E.16) na fórmula acima, somos conduzidos ao resultado:

$$
\Gamma(z)=\lim _{n \rightarrow \infty} \frac{n^{z} n !}{z(z+1)(z+2) \ldots(z+n)}, \quad \Re(z)>0
$$

É conveniente notar que a expressão de Gauss pode ser escrita em termos do símbolo 
de Pochhammer como

$$
\Gamma(z)=\lim _{n \rightarrow \infty} \frac{n^{z} n !}{(z)_{n+1}}
$$

Expressão de Euler Partindo da representação limite da função gama (E.17) e notando que qualquer número $n \in \mathbb{N}$ pode ser reescrito na forma

$$
n=\left(\frac{2}{1} \frac{3}{2} \frac{4}{3} \cdots \frac{n-1}{n-2} \frac{n}{n-1}\right)
$$

reescrevemos:

$$
\Gamma(z)=\lim _{n \rightarrow \infty} \frac{1}{z} \frac{n !}{(z+1)(z+2) \ldots(z+n)}\left(\frac{2}{1} \frac{3}{2} \cdots \frac{n}{n-1}\right)^{z}
$$

Por outro lado, como

$$
\lim _{n \rightarrow \infty}\left(1+\frac{1}{n}\right)^{z}=1
$$

podemos multiplicar esse fator pela expressão acima, obtendo

$$
\begin{gathered}
\Gamma(z)=\lim _{n \rightarrow \infty} \frac{1}{z} \frac{n !}{(z+1)(z+2) \ldots(z+n)}\left(\frac{2}{1} \frac{3}{2} \cdots \frac{n}{n-1} \frac{n+1}{n}\right)^{z}= \\
=\lim _{n \rightarrow \infty} \frac{1}{z} \frac{n !}{(z+1)(z+2) \ldots(z+n)} \prod_{k=1}^{n}\left(\frac{k+1}{k}\right)^{z}
\end{gathered}
$$

Ainda é possível melhorar a forma da produtória escrevendo-se

$$
\prod_{k=1}^{n}\left(\frac{k+1}{k}\right)^{z}=\prod_{k=1}^{n}\left(1+\frac{1}{k}\right)^{z}
$$

O termo $(z+1)(z+2) \ldots(z+n)$, por sua vez, também pode ser reescrito de um modo mais apropriado:

$$
\begin{aligned}
(z+1)(z+2) \ldots(z+n) & =1\left(1+\frac{z}{1}\right) 2\left(1+\frac{z}{2}\right) \cdots n\left(1+\frac{z}{n}\right)= \\
& =n ! \prod_{k=1}^{n}\left(1+\frac{z}{k}\right)
\end{aligned}
$$

o que nos conduz a

$$
\begin{gathered}
\Gamma(z)=\lim _{n \rightarrow \infty} \frac{1}{z} n ! \prod_{k=1}^{n}\left(1+\frac{1}{k}\right)^{z} / \lim _{n \rightarrow \infty}\left[n ! \prod_{k=1}^{n}\left(1+\frac{z}{k}\right)\right]= \\
=\lim _{n \rightarrow \infty} \frac{1}{z} \prod_{k=1}^{n}\left(1+\frac{1}{k}\right)^{z}\left(1+\frac{z}{k}\right)^{-1}
\end{gathered}
$$


e, finalmente, à expressão de Euler:

$$
\Gamma(z)=\frac{1}{z} \prod_{n=1}^{\infty}\left(1+\frac{1}{n}\right)^{z}\left(1+\frac{z}{n}\right)^{-1}
$$

Expressão de Weierstrass Parte-se agora do recíproco da expressão limite (E.17), efetuando-se o mesmo arranjo anterior:

$$
\frac{1}{\Gamma(z)}=\lim _{n \rightarrow \infty} \frac{z(z+1)(z+2) \ldots(z+n)}{n^{z} n !}=\lim _{n \rightarrow \infty} \frac{z}{n^{z}} \prod_{k=1}^{n}\left(1+\frac{z}{k}\right)
$$

Multiplicando e dividindo essa expressão por $\prod_{k=1}^{n} e^{z / k}=\exp \left(z \sum_{k=1}^{n} \frac{1}{k}\right)$, obtém-se

$$
\frac{1}{\Gamma(z)}=\lim _{n \rightarrow \infty} \exp \left(z \sum_{k=1}^{n} \frac{1}{k}\right) \frac{z}{n^{z}} \prod_{k=1}^{n}\left(1+\frac{z}{k}\right) e^{-z / k}
$$

Reescrevendo $n^{-z}=\exp (-z \ln n)$, segue

$$
\frac{1}{\Gamma(z)}=\lim _{n \rightarrow \infty} \exp \left[z\left(-\ln n+\sum_{k=1}^{n} \frac{1}{k}\right)\right] z \lim _{n \rightarrow \infty} \prod_{k=1}^{n}\left(1+\frac{z}{k}\right) e^{-z / k}
$$

Mas, como a exponencial é contínua, pelas propriedades dos limites, vale

$$
\lim _{n \rightarrow \infty} \exp \left[z\left(-\ln n+\sum_{k=1}^{n} \frac{1}{k}\right)\right]=\exp \left[z \lim _{n \rightarrow \infty}\left(-\ln n+\sum_{k=1}^{n} \frac{1}{k}\right)\right]
$$

Por fim, usando a definição do número harmônico $H_{n}=\sum_{k=1}^{n} \frac{1}{k}$ e denominando

$$
\gamma=\lim _{n \rightarrow \infty}\left(\sum_{k=1}^{n} \frac{1}{k}-\ln n\right) \equiv \lim _{n \rightarrow \infty}\left(H_{n}-\ln n\right),
$$

sendo $\gamma \simeq 0.5772156649$ conhecida como constante de Euler-Mascheroni, chega-se, tomando o limite da produtória, à expressão de Weierstrass:

$$
\frac{1}{\Gamma(z)}=e^{\gamma z} z \prod_{n=1}^{\infty}\left(1+\frac{z}{n}\right) e^{-z / n}
$$

É interessante observar que, embora a função gama apresente infinitos polos no domínio real negativo, sua inversa, denominada função gama recíproca, é uma função contínua, como se vê na figura 115. De fato, ainda melhor do que isso, é uma função inteira no domínio complexo. 
Figura 115: Função gama recíproca no domínio real

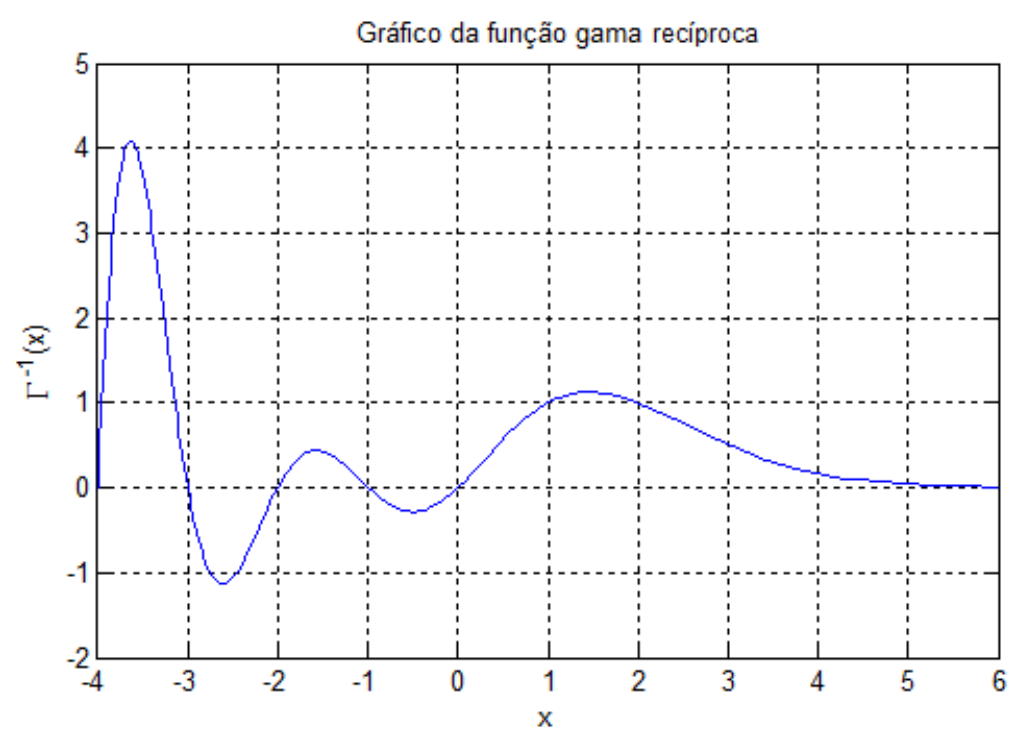

\section{E.2 Fórmula Assintótica de Stirling}

É uma excelente aproximação usada para cálculo de fatoriais de alto valor, mas funciona bem inclusive para valores não tão elevados. Foi parcialmente determinada, a menos de uma constante, pelo matemático francês Abraham de Moivre (1667-1754) e completamente demonstrada pelo matemático escocês James Stirling (1692-1770).

O ponto de partida é a integral

$$
n !=\int_{0}^{\infty} x^{n} e^{-x} d x
$$

Fazendo a mudança de variáveis $x \longrightarrow n y$, a integral se torna

$$
n !=n^{n+1} \int_{0}^{\infty} y^{n} e^{-n y} d y
$$

Reescrevendo-se $y^{n} e^{-n y}=\exp \left[\ln \left(y^{n} e^{-n y}\right)\right]=\exp (n \ln y-n y)$ e denominando $f(y)=$ $\ln y-y$, a integral assume a forma

$$
n !=n^{n+1} \int_{0}^{\infty} \exp [n f(y)] d y
$$

Podemos, então, utilizar o método de Laplace para efetivar o cálculo assintótico da integral

$$
I(n)=\int_{0}^{\infty} \exp [n f(y)] d y
$$


O máximo global de $f(y)$ ocorre em $y_{0}=1$, o que conduz à expansão em Taylor:

$$
\begin{gathered}
f(y)=\left(\ln y_{0}-y_{0}\right)+\left(\frac{1}{y_{0}}-1\right) \frac{\left(y-y_{0}\right)}{1 !}+\left(-\frac{1}{y_{0}^{2}}\right) \frac{\left(y-y_{0}\right)^{2}}{2 !}+O(3) \Rightarrow \\
f(y)=-1-\frac{1}{2}(y-1)^{2}+O(3)
\end{gathered}
$$

Desprezando-se os termos de ordem superior e estendendo o domínio de integração para todo o eixo real, condição que pode ser assumida porque a exponencial decai muito rápido longe de $y_{0}$, obtém-se

$$
I(n) \approx \int_{-\infty}^{\infty} \exp \left[-n-\frac{n}{2}(y-1)^{2}\right] d y=e^{-n} \int_{0}^{\infty} e^{-\frac{n}{2}(y-1)^{2}} d y
$$

Usando o conhecido resultado da integral gaussiana, chegamos à expressão desejada:

$$
\begin{gathered}
I(n) \approx e^{-n} \sqrt{\frac{\pi}{n / 2}} \Rightarrow \\
n ! \approx n^{n+1} e^{-n} \sqrt{\frac{2 \pi}{n}}
\end{gathered}
$$

ou, em sua forma mais conhecida,

$$
n ! \approx\left(\frac{n}{e}\right)^{n} \sqrt{2 \pi n}
$$

Expandindo para a função gama a valores quaisquer:

$$
\begin{aligned}
z ! \longrightarrow \Gamma(z+1) & =z \Gamma(z) \approx\left(\frac{z}{e}\right)^{z} \sqrt{2 \pi z} \Rightarrow \\
\Gamma(z) & \approx \sqrt{2 \pi} e^{-z} z^{z-1 / 2}
\end{aligned}
$$

\section{E.3 Funções Gama Incompletas}

Definem-se, ademais, duas outras espécies de funções gama no corpo complexo, chamadas incompletas, mas em diferentes intervalos. São elas:

$$
\begin{gathered}
\gamma(z, w)=\int_{0}^{w} t^{z-1} e^{-t} d t, \quad \Re(z)>0 \\
\Gamma(z, w)=\int_{w}^{\infty} t^{z-1} e^{-t} d t
\end{gathered}
$$

São imediatas as relações:

$$
\gamma(z, w)+\Gamma(z, w)=\gamma(z, \infty)=\Gamma(z, 0)=\Gamma(z) \quad(\Re(z)>0)
$$


Nas expressões acima, a variável $w$ é, em princípio, real. Entretanto, é possível estender seu domínio aos complexos, desenvolvendo a chamada extensão holomórfica, por meio de uma fórmula de recorrência. Usando integração por partes, cheagamos a duas fórmulas de recorrência para a função $\gamma$ :

$$
\gamma(z, w)=(z-1) \gamma(z-1, w)-w^{z-1} e^{-w}
$$

e

$$
\gamma(z, w)=\frac{1}{z}\left[w^{z} e^{-w}+\gamma(z+1, w)\right]
$$

Utilizar a segunda fórmula de recorrência $n$ vezes, tomando o limite $n \rightarrow \infty$, nos conduz a uma série de potências (MILLER; ROSS, 1993):

$$
\gamma(z, w)=w^{z} \Gamma(z) e^{-w} \sum_{k=0}^{\infty} \frac{w^{k}}{\Gamma(z+k+1)}
$$

Como a série acima converge uniformemente, define-se a extensão holomórfica da função gama incompleta em termos dessa série como

$$
\gamma^{*}(z, w):=e^{-w} \sum_{k=0}^{\infty} \frac{w^{k}}{\Gamma(z+k+1)}
$$

Portanto, essa função e a gama incompleta estão relacionadas por:

$$
\gamma(z, w)=w^{z} \Gamma(z) \gamma^{*}(z, w)
$$

Daí, segue a definição integral da função gama:

$$
\gamma(z, w)=\int_{0}^{w} t^{z-1} e^{-t} d t, \quad \Re(z)>0,
$$

com a propriedade

$$
\int_{u}^{v} t^{z-1} e^{-t} d t=\gamma(z, v)-\gamma(z, u)
$$

desde que o caminho de integração esteja todo contido no domínio do integrando. Desse modo, a função gama incompleta holomórfica assume a forma integral:

$$
\gamma^{*}(\nu, z)=\frac{1}{\Gamma(\nu) z^{\nu}} \int_{0}^{z} t^{\nu-1} e^{-t} d t
$$




\section{E.4 Relação entre as Funções Gama e Logarítmica}

Vamos avaliar a expressão

$$
\int_{x}^{x+1} \frac{\Gamma^{\prime}(z)}{\Gamma(z)} d z
$$

Então,

$$
\begin{aligned}
& \int_{x}^{x+1} \frac{\Gamma^{\prime}(z)}{\Gamma(z)} d z=\left.\ln \Gamma(z)\right|_{x} ^{x+1}=\ln \Gamma(x+1)-\ln \Gamma(x)= \\
&= \ln x \Gamma(x)-\ln \Gamma(x)=\ln x+\ln \Gamma(x)-\ln \Gamma(x) \Rightarrow \\
& \int_{x}^{x+1} \frac{\Gamma^{\prime}(z)}{\Gamma(z)} d z=\ln x
\end{aligned}
$$

Além disso, para valores inteiros, tem-se

$$
\begin{gathered}
\int_{1}^{n+1} \frac{\Gamma^{\prime}(z)}{\Gamma(z)} d z=\ln \Gamma(n+1)-\ln \Gamma(1) \Rightarrow \\
\int_{1}^{n+1} \frac{\Gamma^{\prime}(z)}{\Gamma(z)} d z=\ln (n !)
\end{gathered}
$$

Figura 116: Valor absoluto da função gama com argumento complexo

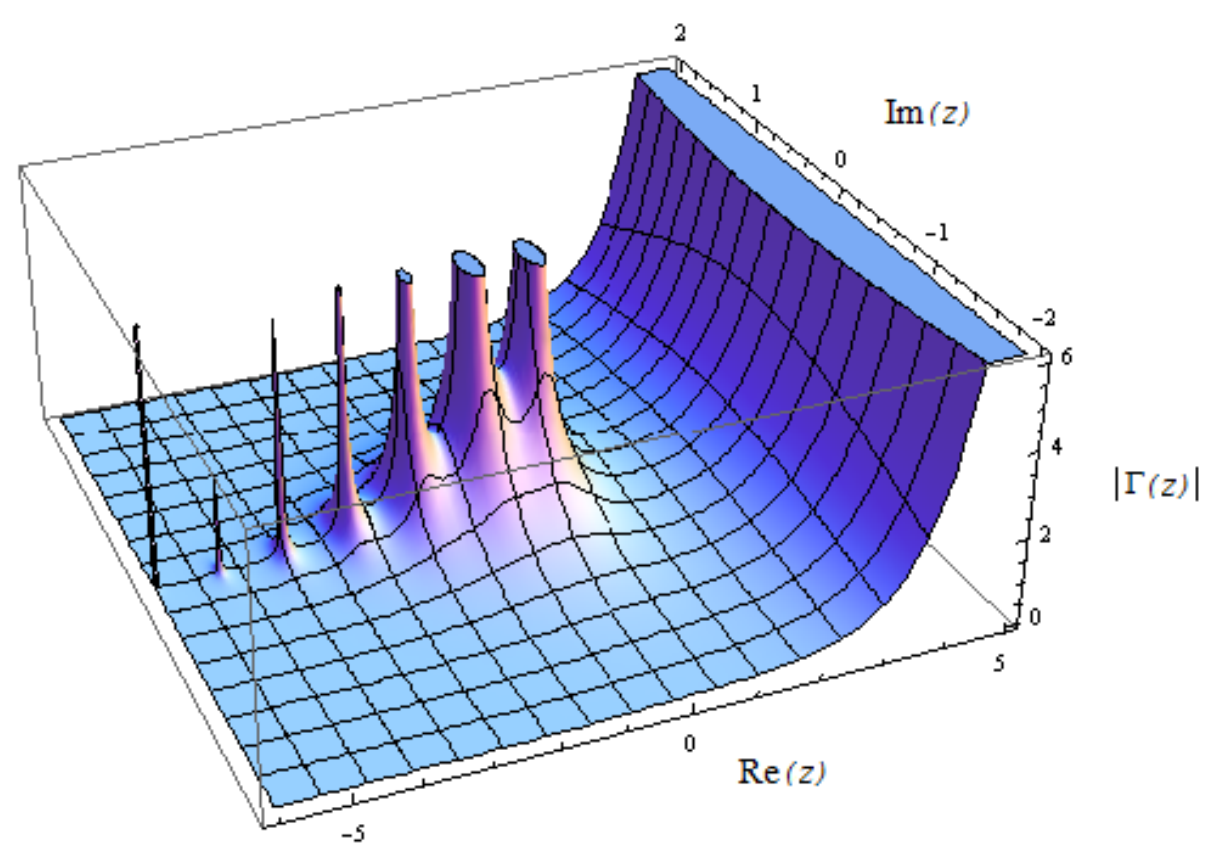

Como se sabe, o argumento da função gama pode assumir valores complexos. É interessante, então, observar o gráfico do valor absoluto da função gama com argumento complexo $z=x+i y$, mostrado na figura 116. 


\section{E.5 Funções Psi}

Função Digama A função psi de Euler ou função digama é definida pela derivada do logaritmo da função gama:

$$
\psi(z)=\frac{d}{d z} \ln \Gamma(z)=\frac{\Gamma^{\prime}(z)}{\Gamma(z)} \quad(z \in \mathbb{C})
$$

Uma definição equivalente é dada pela expressão integral

$$
\int_{0}^{\infty}\left(\frac{e^{-t}}{t}-\frac{e^{-z t}}{1-e^{-t}}\right) d t
$$

\section{Propriedades}

- P1:

$$
\psi(z)=-\gamma-\frac{1}{z}+\sum_{n=1}^{\infty}\left(\frac{1}{n}-\frac{1}{n+z}\right)=\lim _{n \rightarrow \infty}\left(\ln n-\sum_{k=0}^{\infty} \frac{1}{k+z}\right),
$$

onde $\gamma \cong 0.5772156649$ é a constante de Euler-Mascheroni.

\section{Demonstração 7 :}

Partindo da inversa da expressão de Weierstrass

$$
\Gamma(z)=\frac{e^{-\gamma z}}{z} \prod_{n=1}^{\infty}\left(\frac{n}{n+z} e^{z / n}\right)
$$

pode-se expressar a relação $\Gamma^{\prime}(z) / \Gamma(z)$ pela derivação do logaritmo de gama:

$$
\begin{gathered}
\psi(z)=\frac{\Gamma^{\prime}(z)}{\Gamma(z)}=\frac{d}{d z} \ln \Gamma(z)=\frac{d}{d z} \ln \left[e^{-\gamma z} z^{-1} \prod_{n=1}^{\infty}\left(\frac{n}{n+z} e^{z / n}\right)\right]= \\
=\frac{d}{d z}\left\{-\gamma z-\ln z+\ln \prod_{n=1}^{\infty}\left(\frac{n}{n+z} e^{z / n}\right)\right\}= \\
=\frac{d}{d z}\left\{-\gamma z-\ln z+\sum_{n=1}^{\infty}\left(\ln \frac{n}{n+z}+\frac{z}{n}\right)\right\}= \\
=-\gamma-\frac{1}{z}+\sum_{n=1}^{\infty}\left[\frac{-n /(n+z)^{2}}{n /(n+z)}+\frac{1}{n}\right] \Rightarrow \\
\psi(z)=-\gamma-\frac{1}{z}+\sum_{n=1}^{\infty}\left(\frac{1}{n}-\frac{1}{n+z}\right)
\end{gathered}
$$


Já para a segunda parte da demonstração basta aplicar a definição da constante $\gamma$ e notar que $1 / z=1 /(n+z)_{n=0}$ para obter

$$
\begin{gathered}
\psi(z)=-\lim _{n \rightarrow \infty} \sum_{k=1}^{n}\left(\frac{1}{k}-\ln n\right)+\sum_{n=1}^{\infty} \frac{1}{n}-\sum_{n=0}^{\infty} \frac{1}{n+z}= \\
=-\sum_{n=1}^{n} \frac{1}{n}+\sum_{n=1}^{\infty} \frac{1}{n}+\lim _{n \rightarrow \infty}\left(\ln n-\sum_{k=0}^{n} \frac{1}{k+z}\right) \Rightarrow \\
\psi(z)=\lim _{n \rightarrow \infty}\left(\ln n-\sum_{k=0}^{n} \frac{1}{k+z}\right)
\end{gathered}
$$

- P2:

$$
\psi(1)=-\gamma
$$

\section{Demonstração 8:}

Fazendo $z=1$ na propriedade anterior, abrindo o primeiro termo da primeira somatória e reindexando a segunda, obtém-se

$$
\begin{gathered}
\psi(1)=-\gamma-1+\sum_{n=1}^{\infty}\left(\frac{1}{n}-\frac{1}{n+1}\right)= \\
=-\gamma-1+1+\sum_{n=2}^{\infty} \frac{1}{n}-\sum_{n=2}^{\infty} \frac{1}{n} \Rightarrow \\
\psi(1)=-\gamma
\end{gathered}
$$

- P3:

$$
\psi(2)=-\gamma+1
$$

\section{Demonstração 9:}

Usando o mesmo procedimento anterior para os dois primeiros termos com $z=2$, obtemos

$$
\begin{gathered}
\psi(2)=-\gamma-\frac{1}{2}+\sum_{n=1}^{\infty}\left(\frac{1}{n}-\frac{1}{n+2}\right)= \\
=-\gamma-\frac{1}{2}+1+\frac{1}{2}+\sum_{n=3}^{\infty} \frac{1}{n}-\sum_{n=3}^{\infty} \frac{1}{n} \Rightarrow \\
\psi(2)=-\gamma+1
\end{gathered}
$$


Funções Psi Generalizadas É possível expandir o conceito de função psi por derivações sucesivas da função gama, denotando-se:

$$
\begin{aligned}
& \psi^{(0)}(z)=\psi(z)=\frac{\Gamma^{\prime}(z)}{\Gamma(z)} \\
& \psi^{(1)}(z)=\frac{d \psi^{(0)}(z)}{d z}=\left[\frac{\Gamma^{\prime}(z)}{\Gamma(z)}\right]^{\prime} \\
& \psi^{(2)}(z)=\frac{d \psi^{(1)}(z)}{d z}=\left[\frac{\Gamma^{\prime}(z)}{\Gamma(z)}\right]^{\prime \prime}
\end{aligned}
$$

e assim por diante. Tais funções são denominadas funções poligama, podendo receber nomes particulares, como trigama para $\psi^{(1)}$, tetragama para $\psi^{(2)}$ etc.

Derivando-se a expressão (E.28), pode-se obter uma expressão em série para a função trigama:

$$
\psi^{(1)}(z)=\frac{1}{z^{2}}+\sum_{n=1}^{\infty} \frac{1}{(n+z)^{2}}=\sum_{n=0}^{\infty} \frac{1}{(n+z)^{2}}
$$

Calculando a derivada da razão $\Gamma^{\prime}(z) / \Gamma(z)$ obtemos a seguinte propriedade:

$$
\begin{gathered}
{\left[\frac{\Gamma^{\prime}(z)}{\Gamma(z)}\right]^{\prime}=\frac{\Gamma^{\prime \prime}(z) \Gamma(z)-\left[\Gamma^{\prime}(z)\right]^{2}}{[\Gamma(z)]^{2}}=\frac{\Gamma^{\prime \prime}(z)}{\Gamma(z)}-\left[\frac{\Gamma^{\prime}(z)}{\Gamma(z)}\right]^{2} \Rightarrow} \\
\frac{\Gamma^{\prime \prime}(z)}{\Gamma(z)}=\left[\frac{\Gamma^{\prime}(z)}{\Gamma(z)}\right]^{\prime}+\left[\frac{\Gamma^{\prime}(z)}{\Gamma(z)}\right]^{2}=\psi^{(1)}(z)+\psi^{2}(z)
\end{gathered}
$$

\section{E.6 Função Beta}

Forma Padrão A função beta, também conhecida como integral de Euler de primeira espécie, é uma função especial definida nos complexos por

$$
B(p, q):=\int_{0}^{1} x^{p-1}(1-x)^{q-1} d x, \quad \Re(p), \Re(q)>0
$$

Estudada por Euler e Legendre, seu nome foi dado pelo matemático francês Jacques P. M. Binet (1786-1856). Uma importante característica é sua propriedade de simetria:

$$
B(q, p)=B(p, q)
$$




\section{Demonstração 10:}

Partindo-se da expressão

$$
B(q, p)=\int_{0}^{1} t^{q-1}(1-t)^{p-1} d t
$$

e efetuando a mudança de variável $t \rightarrow 1-x$, obtemos:

$$
\begin{gathered}
B(q, p)=\int_{1}^{0}(1-x)^{q-1} x^{p-1}(-d x)= \\
=-\int_{1}^{0} x^{p-1}(1-x)^{q-1} d x=\int_{0}^{1} x^{p-1}(1-x)^{q-1} d x=B(p, q)
\end{gathered}
$$

Além disso, assim como no caso da função gama, também é possível expressar a função beta de várias formas distintas, cada uma com sua aplicabilidade específica. A seguir, são expostas algumas formulações equivalentes.

\section{Segunda Forma}

$$
B(p, q)=a^{1-p-q} \int_{0}^{a} y^{p-1}(a-y)^{q-1} d y
$$

\section{Demonstração 11:}

Partindo-se da definição da função beta, através da mudança de variável $x \rightarrow$ y/a, obtém-se:

$$
\begin{aligned}
& d x \rightarrow \frac{1}{a} d y, \quad x \in[0,1] \rightarrow y \in[0, a] \Rightarrow \\
& B(p, q)=\frac{1}{a} \int_{0}^{a}\left(\frac{y}{a}\right)^{p-1}\left(1-\frac{y}{a}\right)^{q-1} d y= \\
& =\frac{1}{a} \frac{1}{a^{p-1}} \frac{1}{a^{q-1}} \int_{0}^{a} y^{p-1}(a-y)^{q-1} d y \Rightarrow \\
& B(p, q)=a^{1-p-q} \int_{0}^{a} y^{p-1}(a-y)^{q-1} d y
\end{aligned}
$$

\section{Forma Trigonométrica}

$$
B(p, q)=2 \int_{0}^{\pi / 2}(\sin \theta)^{2 p-1}(\cos \theta)^{2 q-1} d \theta
$$

\section{Demonstração 12:}

Partindo-se da primeira forma e efetuando uma mudança de variável: $x \rightarrow$ $\sin ^{2} \theta$, obtemos:

$$
d x=2 \sin \theta \cos \theta d \theta, \quad 1-x=\cos ^{2} \theta, \quad x \in[0,1] \rightarrow \theta \in\left[0, \frac{\pi}{2}\right] \Rightarrow
$$




$$
\begin{gathered}
B(p, q)=\int_{0}^{\pi / 2}\left(\sin ^{2} \theta\right)^{p-1}\left(\cos ^{2} \theta\right)^{q-1} 2 \sin \theta \cos \theta d \theta \Rightarrow \\
B(p, q)=2 \int_{0}^{\pi / 2}(\sin \theta)^{2 p-1}(\cos \theta)^{2 q-1} d \theta
\end{gathered}
$$

\section{Forma Racional}

$$
B(p, q)=\int_{0}^{\infty} \frac{y^{p-1}}{(1+y)^{p+q}} d y
$$

\section{Demonstração 13:}

A mudança de variável $x \rightarrow y /(1+y)$, em $y \in[0, \infty[$ fornece

$$
\begin{gathered}
\left\{\begin{array}{l}
d x=\frac{(1+y)-y}{(1+y)^{2}} d y=(1+y)^{-2} d y \\
1-x=1-\frac{y}{1+y}=\frac{1}{1+y}
\end{array}\right. \\
B(p, q)=\int_{0}^{\infty}\left(\frac{y}{1+y}\right)^{p-1}\left(\frac{1}{1+y}\right)^{q-1}(1+y)^{-2} d y= \\
=\int_{0}^{\infty} y^{p-1} \frac{1}{(1+y)^{p-1}} \frac{1}{(1+y)^{q-1}}(1+y)^{-2} d y \Rightarrow \\
B(p, q)=\int_{0}^{\infty} \frac{y^{p-1}}{(1+y)^{p+q}} d y
\end{gathered}
$$

Acrescentamos que as derivadas parciais da função beta podem ser calculadas em termos de $\Gamma$ e expressas por meio das funções $\psi$ de Euler, de modo que:

$$
\frac{\partial}{\partial x} B(x, y)=B(x, y)[\psi(x)-\psi(x+y)]
$$

Funções Beta Incompletas De maneira análoga à função gama, também se define uma função beta incompleta pela expressão

$$
B(z ; p, q)=\int_{0}^{z} t^{p-1}(1-t)^{q-1} d t
$$

Esta expressão coincide com a função beta no caso $z=1$. Às vezes essa função também é denotada como $B_{z}(p, q)$. Adicionalmente, ainda definimos a função beta regularizada, que é uma variação da função beta incompleta:

$$
I_{z}(p, q)=\frac{B(z ; p, q)}{B(p, q)}
$$




\section{E.7 Relação entre a Função Beta e a Função Gama}

Partindo da definição da função gama

$$
\Gamma(p)=\int_{0}^{\infty} t^{p-1} e^{-t} d t
$$

e fazendo a substituição $t \rightarrow y^{2}$, obtém-se

$$
\Gamma(p)=2 \int_{0}^{\infty} y^{2 p-1} e^{-y^{2}} d y
$$

Disso, segue

$$
\Gamma(p) \Gamma(q)=4 \int_{0}^{\infty} \int_{0}^{\infty} y^{2 p-1} x^{2 q-1} e^{-\left(x^{2}+y^{2}\right)} d x d y
$$

Efetivando agora uma mudança de coordenadas em $\mathbb{R}^{2}$, de cartesianas para polares, i.e, $(x, y) \rightarrow(\rho, \theta)$, tem-se que $\left|\frac{\partial(x, y)}{\partial(\rho, \theta)}\right|=\rho \Rightarrow$

$$
\begin{aligned}
& \Gamma(p) \Gamma(q)=4 \int_{0}^{\infty} \int_{0}^{\pi / 2}(\rho \cos \theta)^{2 q-1}(\rho \sin \theta)^{2 p-1} e^{-\rho^{2}} \rho d \theta d \rho= \\
& =\left(2 \int_{0}^{\infty} \rho^{2 p+2 q-1} e^{-\rho^{2}} d \rho\right)\left(2 \int_{0}^{\pi / 2}(\sin \theta)^{2 p-1}(\cos \theta)^{2 q-1} d \theta\right)
\end{aligned}
$$

Identificamos então a primeira integral com a fórmula (E.39) e a segunda, com a forma trigonométrica de $B(p, q)$, obtendo

$$
\Gamma(p) \Gamma(q)=\Gamma(p+q) B(p, q)
$$

de onde segue, finalmente, que

$$
B(p, q)=\frac{\Gamma(p) \Gamma(q)}{\Gamma(p+q)}
$$

\section{E.8 Fórmula de Duplicação de Legendre}

A função beta para dois argumentos iguais é escrita

$$
B(z, z)=\int_{0}^{1}[\tau(1-\tau)]^{z-1} d \tau, \quad \Re(z)>0
$$

Constata-se que o integrando apresenta uma função $y(\tau)=\tau(1-\tau)$ que é simétrica 
em torno da média do intervalo $[0,1]$, pois

$$
y\left(\frac{1}{2}+\delta\right)=\left(\frac{1}{2}+\delta\right)\left(\frac{1}{2}-\delta\right)=y\left(\frac{1}{2}-\delta\right), \quad 0 \leq \delta \leq \frac{1}{2}
$$

Assim, a integral assume a forma

$$
B(z, z)=2 \int_{0}^{1 / 2}[\tau(1-\tau)]^{z-1} d \tau
$$

Fazendo a substituição $s \longrightarrow 4 \tau(1-\tau)$, obtém-se que $d s=4(1-2 \tau) d \tau$ e $1-2 \tau=$ $\sqrt{1-s}$, o que nos permite reescrever

$$
B(z, z)=2 \int_{0}^{1}\left(\frac{s}{4}\right)^{z-1} \frac{d s}{4 \sqrt{1-s}}=\frac{1}{2^{2 z-1}} \int_{0}^{1} s^{z-1}(1-s)^{-1 / 2} d s=2^{1-2 z} B\left(z, \frac{1}{2}\right)
$$

No entanto, a relação entre as funções beta e gama fornece

$$
B(z, z)=\frac{\Gamma(z) \Gamma(z)}{\Gamma(2 z)} \quad \text { e } \quad B\left(z, \frac{1}{2}\right)=\frac{\Gamma(z) \Gamma\left(\frac{1}{2}\right)}{\Gamma\left(z+\frac{1}{2}\right)}=\frac{\Gamma(z) \sqrt{\pi}}{\Gamma\left(z+\frac{1}{2}\right)}
$$

e, portanto,

$$
\begin{gathered}
\frac{\Gamma(z) \Gamma(z)}{\Gamma(2 z)}=2^{1-2 z} \frac{\Gamma(z) \sqrt{\pi}}{\Gamma\left(z+\frac{1}{2}\right)} \Rightarrow \\
\Gamma(z) \Gamma\left(z+\frac{1}{2}\right)=\sqrt{\pi} 2^{1-2 z} \Gamma(2 z)
\end{gathered}
$$

que é a fórmula de duplicação de Legendre.

Extensão Analítica da Função Beta Uma vez que a função beta pode ser expressa em termos da função gama pela relação (E.40), é possível estender analiticamente o domínio da função beta a valores negativos, ou a valores complexos com parte real negativa, através da definição da função gama estendida, quer dizer, $B(x, y)=$ $\Gamma(x) \Gamma(y) / \Gamma(x+y)$. Assim, para $x, y \in \mathbb{C} \backslash \mathbb{Z}_{0}^{-}$, tem-se, por exemplo,

$$
B(x, y)=\frac{\frac{\Gamma(x+1)}{x} \frac{\Gamma(y+1)}{y}}{\frac{\Gamma(x+y+1)}{x+y}}=\left(\frac{1}{x}+\frac{1}{y}\right) \frac{\Gamma(x+1) \Gamma(y+1)}{\Gamma(x+y+1)}, \quad \Re(x), \Re(y)<0
$$

A seguir, na figura 117, mostra-se o gráfico da função beta no domínio real.

Extensão das Funções Beta Incompletas De modo semelhante ao que foi feito na extensão analítica da função beta, também a beta incompleta e a regularizada podem ser 
Figura 117: Função beta no domínio real



estendidas ao domínio real negativo. Para isso, fazemos,

$$
\begin{aligned}
B(z ; a, b) & =\int_{0}^{1} t^{a-1}(1-t)^{b-1} d t-\int_{z}^{1} t^{a-1}(1-t)^{b-1} d t= \\
& =\int_{0}^{1} t^{a-1}(1-t)^{b-1} d t-\int_{0}^{1-z} u^{b-1}(1-u)^{a-1} d u= \\
& =B(a, b)-B(1-z ; b, a),
\end{aligned}
$$

onde usamos a mudança de variáveis $u=1-t$ na segunda integral.

Outra relação útil na extensão da função beta incompleta é:

$$
B(z ; a, b)=z^{a}(1-z)^{b}+(a+b) B(a+1, b ; z)
$$

\section{E.9 Extensão dos Coeficientes Binomiais}

O número binomial é definido como

$$
\left(\begin{array}{l}
n \\
k
\end{array}\right)=\frac{n !}{(n-k) ! k !}, \quad\left(n, k \in \mathbb{N}_{0}, k \leq n\right)
$$

A generalização para os inteiros negativos, caso que ocorre, por exemplo, no desenvolvimento de $(1+x)^{-n},\left(n \in \mathbb{N}^{*}\right)$, que gera uma série convergente para $|x|<1$, deverá ser

$$
\left(\begin{array}{c}
-n \\
k
\end{array}\right)=\frac{(-n)(-n-1) \ldots(-n-k+1)}{k !}=(-1)^{k} \frac{n(n+1) \ldots(n+k-1)}{k !}
$$


Utilizando a notação do símbolo de Pochhammer, escreve-se

$$
\left(\begin{array}{c}
-n \\
k
\end{array}\right)=(-1)^{k} \frac{(n)_{k}}{k !}
$$

Definindo a notação

$$
\left[\begin{array}{l}
n \\
k
\end{array}\right]:=\frac{(n)_{k}}{k !}, \quad k \in \mathbb{N}, n \in \mathbb{N}^{*}
$$

tem-se

$$
\left(\begin{array}{c}
-n \\
k
\end{array}\right)=(-1)^{k}\left[\begin{array}{l}
n \\
k
\end{array}\right]
$$

Todavia, deve-se notar que

$$
\begin{gathered}
{\left[\begin{array}{l}
n \\
k
\end{array}\right]=\left(\begin{array}{c}
n+k-1 \\
k
\end{array}\right)=\left(\begin{array}{c}
n+k-1 \\
n-1
\end{array}\right) \Rightarrow} \\
{\left[\begin{array}{l}
n \\
0
\end{array}\right]=1, n \in \mathbb{N}^{*}}
\end{gathered}
$$

Além disso, percebe-se que qualquer binômio do tipo $(1+x)^{-n},(n>0)$, deverá se converter numa série infinita, uma vez que os termos negativos dos binomiais na somatória $\sum_{k}\left(\begin{array}{c}-n \\ k\end{array}\right)$ não encontram nenhum limite inferior como no caso positivo, no qual $\left(\begin{array}{c}n \\ n+1\end{array}\right)=0$, ou seja,

$$
\left(\begin{array}{c}
-n \\
0
\end{array}\right)=1,\left(\begin{array}{c}
-n \\
1
\end{array}\right)=-n,\left(\begin{array}{c}
-n \\
2
\end{array}\right)=\frac{n(n+1)}{2}, \ldots
$$

e assim por diante, sem interrupção.

Já para o caso $p \in \mathbb{R}_{+} \backslash \mathbb{Z}$, o binomial $\left(\begin{array}{l}p \\ k\end{array}\right)$ não pode mais ser descrito por meio de fatoriais, os quais devem ser então substituídos pela função gama, isto é,

$$
\left(\begin{array}{l}
p \\
k
\end{array}\right)=\frac{\Gamma(p+1)}{k ! \Gamma(p-k+1)}, \quad p \in \mathbb{R}^{+}, \quad k \in \mathbb{N}_{0}
$$

Se quisermos estender a definição do binomial para valores negativos de $p \in \mathbb{R}$ em $\left(\begin{array}{l}p \\ k\end{array}\right)$, podemos usar a relação estabelecida anteriormente:

$$
\left(\begin{array}{c}
-\alpha \\
k
\end{array}\right)=(-1)^{k}\left[\begin{array}{c}
\alpha \\
k
\end{array}\right] \equiv(-1)^{k}\left(\begin{array}{c}
\alpha+k-1 \\
k
\end{array}\right)=(-1)^{k} \frac{\Gamma(\alpha+k)}{k ! \Gamma(\alpha)}, \quad \alpha \in \mathbb{R}^{+}, \quad k \in \mathbb{N}_{0}
$$


Além disso, fica definida a relação geral:

$$
\left[\begin{array}{l}
\alpha \\
k
\end{array}\right]=\frac{\Gamma(\alpha+k)}{k ! \Gamma(\alpha)}, \quad \alpha \in \mathbb{R}^{+}, \quad k \in \mathbb{N}_{0}
$$

Assim, a extensão para o caso complexo, mais geral que os anteriores, é imediata:

$$
\left(\begin{array}{l}
z \\
k
\end{array}\right)=\frac{\Gamma(z+1)}{k ! \Gamma(z-k+1)}, \quad z \in \mathbb{C} \backslash \mathbb{Z}^{-}, \quad k \in \mathbb{N}_{0}
$$

Ainda é possível estender mais essa definição a fim de incluir valores quaisquer de $\alpha$ e $\beta$ em $\left(\begin{array}{l}\alpha \\ \beta\end{array}\right)$, inclusive complexos, novamente por meio da função gama, uma vez que essa função é definida em $\mathbb{C}$ :

$$
\left(\begin{array}{l}
\alpha \\
\beta
\end{array}\right)=\frac{\Gamma(\alpha+1)}{\Gamma(\alpha-\beta+1) \Gamma(\beta+1)}, \quad \alpha \in \mathbb{C} \backslash \mathbb{Z}^{-}, \beta \in \mathbb{C}
$$

Assim, podemos calcular, por exemplo, os valores seguintes:

$$
\begin{gathered}
\left(\begin{array}{l}
\pi \\
e
\end{array}\right)=1.90357, \quad\left(\begin{array}{l}
e \\
\pi
\end{array}\right)=0.383614, \quad\left(\begin{array}{c}
1+i e \\
1+i \pi
\end{array}\right)=1.37385-1.20415 i, \\
\left(\begin{array}{c}
-\sqrt{2} \\
\sqrt{3}
\end{array}\right)=0.752596, \quad\left(\begin{array}{c}
-i e \\
\pi
\end{array}\right)=4.45565, \quad\left(\begin{array}{c}
-i e-1 / e \\
-i \pi
\end{array}\right)=1.14141
\end{gathered}
$$

\section{E.10 Funções Binomiais}

É interessante, nesse ponto, introduzir uma classe de funções, definidas por uma expressão que denominaremos função binomial de parâmetro $\alpha$, cuja variável é o índice inferior do binômio e que denotaremos pela letra grega $\kappa$, de modo que:

$$
\kappa_{\alpha}(x):=\frac{1}{(\alpha+1) B(\alpha-x+1, x+1)}, \quad \alpha \in \mathbb{C} \backslash \mathbb{Z}^{-}, \quad x \in \mathbb{C}
$$

Conforme se vê na figura 118, a função binomial com parâmetro $\alpha$ positivo ( $\alpha=$ 15) corresponde a uma distribuição binomial contínua, ao passo que, se tomarmos um parâmetro negativo não-inteiro $(\alpha=-\pi)$, a função apresenta características distintas, mostrando uma simetria em torno de um ponto de mínimo negativo, com infinitas raízes nos inteiros negativos, como se pode verificar na figura 119. Outra característica notável é que, embora a função não seja definida para $\alpha \in \mathbb{Z}^{-}$, para quaisquer outros valores, inclusive imaginários puros, ela é não apenas definida, mas também contínua.

Nos casos em que o parâmetro $\alpha$ assume valores complexos, o valor absoluto da função 
Figura 118: Função binomial com parâmetro $\alpha$ real positivo

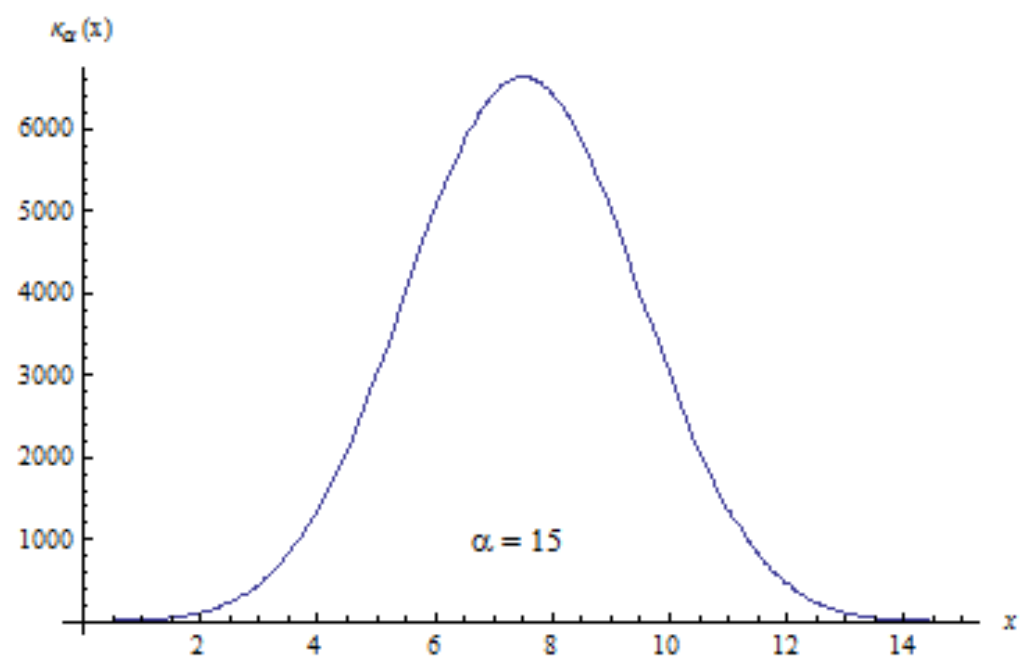

Figura 119: Função binomial com parâmetro $\alpha$ real negativo

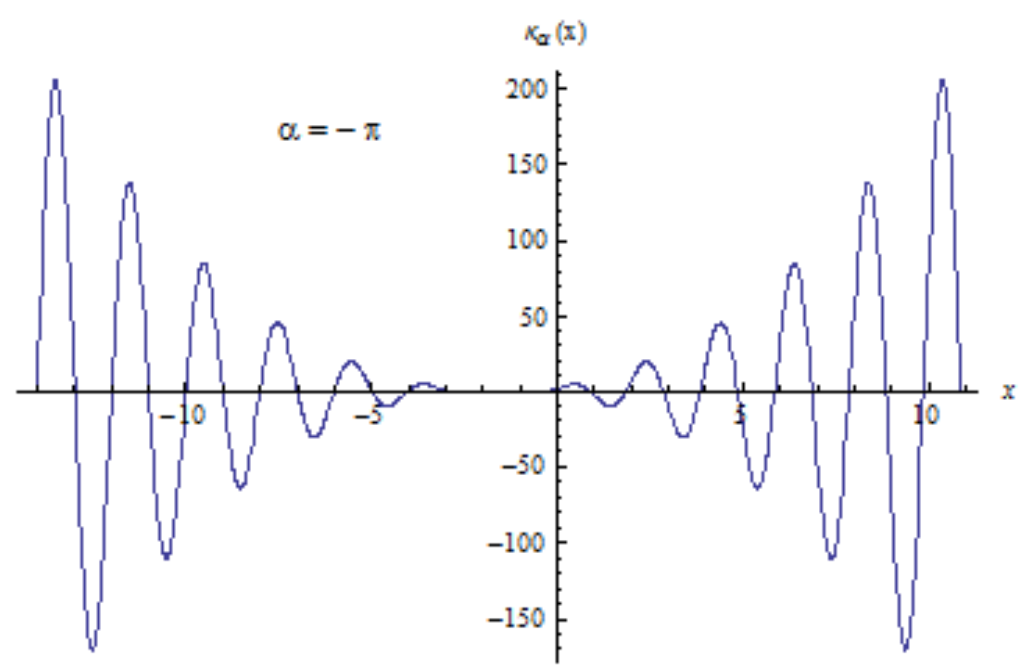


$\kappa_{\alpha}(x)$ apresenta um comportamento que varia, no ramo positivo, entre uma oscilação e um decaimento, de acordo com as grandezas da parte real e imaginária.

Na figura 120 podemos analisar o comportamento de $\left|\kappa_{\alpha}(x)\right|$ para parâmetros imaginários puros. Nota-se que, à medida que a parte imaginária aumenta, a oscilação diminui, predominando o aspecto relacionado ao decaimento puro.

Figura 120: Função binomial com parâmetro $\alpha$ imaginário



A figura 121 apresenta o gráfico da parte real da função binomial com parâmetro $\alpha$ imaginário. Seu comportamento é similar ao do módulo da função, mas com oscilações completas acima e abaixo da abscissa, mostrando um decaimento mais rápido no ramo negativo.

Figura 121: Parte real da função binomial com parâmetro $\alpha$ imaginário

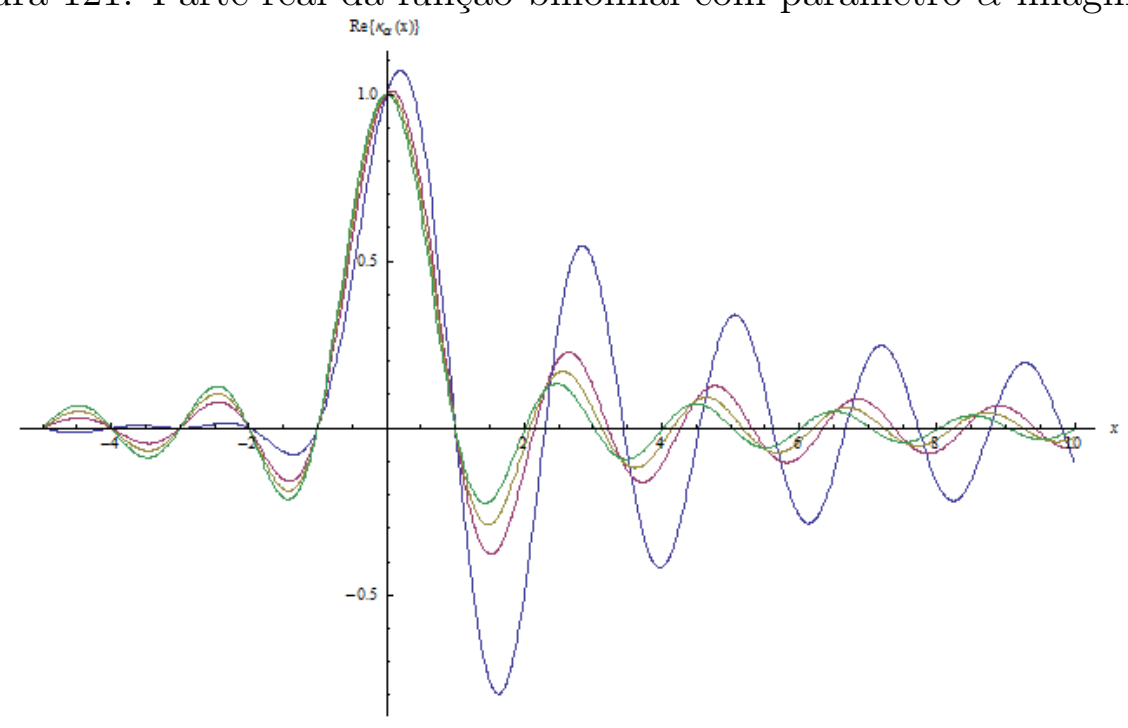

Quando o parâmetro $\alpha$ é complexo, sua parte real é responsável, primordialmente, 
pela concavidade da curva básica, embora a parte imaginária também contribua. Se a parte real for menor que a unidade, o envelope da curva apresenta decaimento, se for idêntico à unidade, a curva tende a uma assíntota horizontal e se for maior, a função cresce indefinidamente e, consequentemente, sua integral diverge no domínio, o que pode ser visto na figura 122 .

Figura 122: Função binomial com parâmetro $\alpha$ complexo

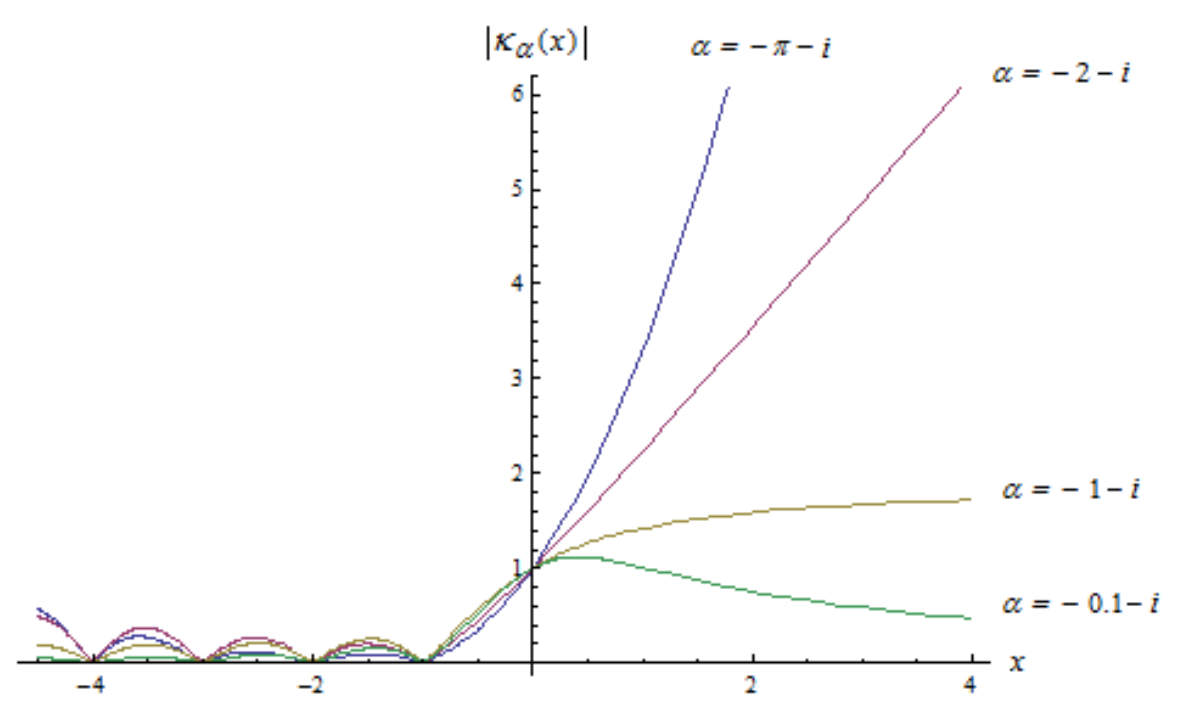

Figura 123: Parte real da função binomial com parâmetro $\alpha$ complexo

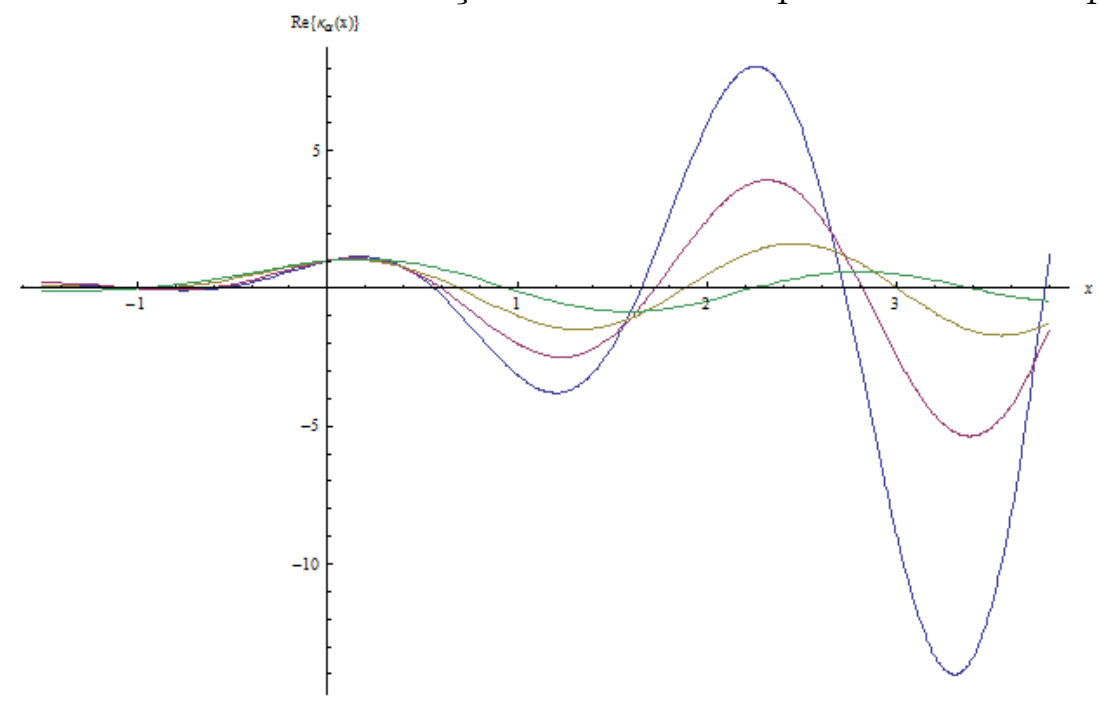

No caso acima, a assíntota da função com parâmetro $\alpha=-1-i$ tem equação

$$
y_{\text {lim }}=\lim _{x \rightarrow \infty}\left[\kappa_{\alpha}(x)\right]_{\alpha=-1-i}=1.9173
$$

que é, simplesmente, o valor limite do próprio binômio generalizado $\left(\begin{array}{c}-1-i \\ x\end{array}\right)$ quando $x \rightarrow \infty$.

Além disso, podemos definir uma função binomial generalizada, com dois parâmetros, 
que representa o binomial generalizado:

$$
\kappa(x, y):=\frac{\Gamma(x+1)}{\Gamma(x-y+1) \Gamma(y+1)}, \quad x \in \mathbb{C} \backslash \mathbb{Z}^{-}, y \in \mathbb{C}
$$

A seguir, nas figuras 124, 125 e 126, aparecem os gráficos da função binomial generalizado nos quadrantes positivo e negativo do plano real $(x, y)$ com pequena amplitude, bem como um gráfico mais genérico e de maior extensão, para ilustrar seu aspecto geral no domínio real.

Figura 124: Função binomial generalizada no domínio real positivo

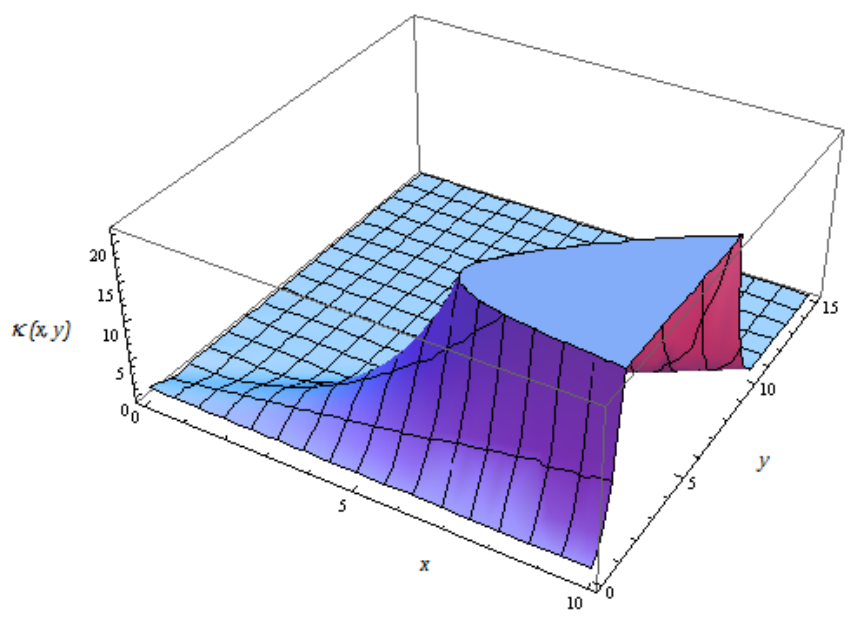

Figura 125: Função binomial generalizada no domínio real negativo

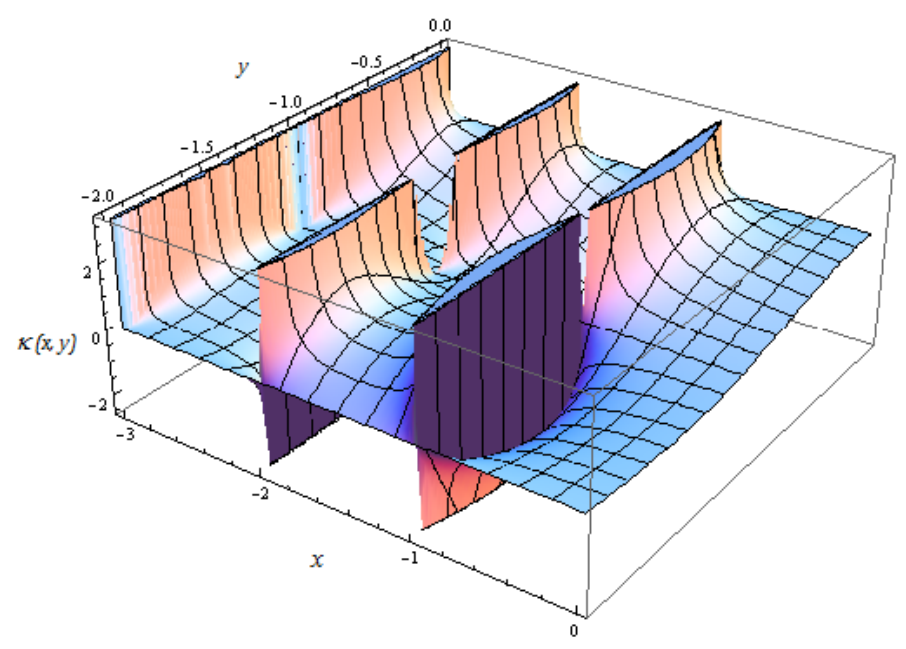

Percebem-se as singularidades nos pontos com valores inteiros negativos de $x$, embora seu aspecto ao longo do eixo y seja mais complicado. 
Figura 126: Função binomial generalizada no domínio real

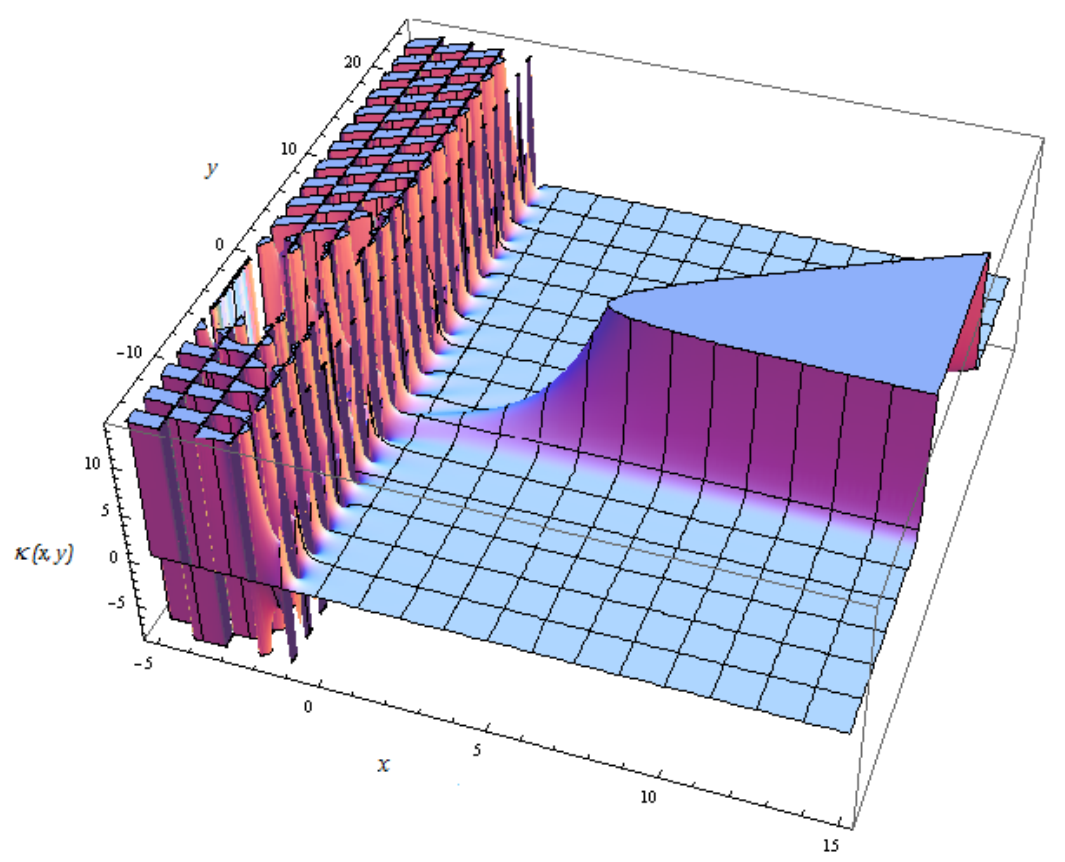

\section{E.11 Relação de Stifel Modificada}

A conhecida relação de Stifel assume uma forma um tanto diferente no caso de binomiais com extensão negativa. Partindo da relação usual, escrita na forma

$$
\left(\begin{array}{c}
n-1 \\
r-1
\end{array}\right)+\left(\begin{array}{c}
n-1 \\
r
\end{array}\right)=\left(\begin{array}{l}
n \\
r
\end{array}\right), \quad 1 \leq r \leq n-1
$$

e tomando $n \longrightarrow-p$, tem-se

$$
\left(\begin{array}{c}
-p-1 \\
r-1
\end{array}\right)+\left(\begin{array}{c}
-p-1 \\
r
\end{array}\right)=\left(\begin{array}{c}
-p \\
r
\end{array}\right)
$$

Então, usando a propriedade

$$
\left(\begin{array}{c}
-p \\
k
\end{array}\right)=(-1)^{k}\left[\begin{array}{l}
p \\
k
\end{array}\right]
$$

pode-se reescrever a relação como

$$
(-1)^{r-1}\left[\begin{array}{c}
p+1 \\
r+1
\end{array}\right]+(-1)^{r}\left[\begin{array}{c}
p+1 \\
r
\end{array}\right]=(-1)^{r}\left[\begin{array}{l}
p \\
r
\end{array}\right]
$$


ou, alternativamente, dividindo toda a expressão por $(-1)^{r}$,

$$
-\left[\begin{array}{c}
p+1 \\
r-1
\end{array}\right]+\left[\begin{array}{c}
p+1 \\
r
\end{array}\right]=\left[\begin{array}{l}
p \\
r
\end{array}\right]
$$

de onde vem:

$$
\left[\begin{array}{c}
p+1 \\
r
\end{array}\right]=\left[\begin{array}{c}
p \\
r
\end{array}\right]+\left[\begin{array}{c}
p+1 \\
r-1
\end{array}\right], \quad p, r \in \mathbb{N}^{*}
$$

A essa expressão, chamamos de relação de Stifel modificada, a qual tem grande aplicabilidade no desenvolvimento do formalismo de Grünwald-Letnikov.

\section{E.12 Expressão Generalizada do Binômio}

Com isso, a somatória $\sum_{k}\left(\begin{array}{l}p \\ k\end{array}\right)$ na expansão do binômio $(1+x)^{p}$, supondo sua validade nesse caso, não mais se reduz a uma quantidade finita de termos, pois o índice $k$ jamais assume o valor exato de qualquer $p$ não-inteiro, como no caso dos fatoriais, o que implica a inexistência do fator nulo como elemento de interrupção da série. Dessa forma, os fatores avançam sem cessar na direção dos números negativos e a expressão deve ser reescrita como uma série infinita:

$$
(1+x)^{p}=\sum_{n=0}^{\infty} \frac{\Gamma(p+1)}{\Gamma(p-n+1) n !} x^{n}, \quad|x|<1
$$

A generalização pode ser efetivada sem maiores problemas, visto que a função gama é definida no domínio real, exceto pelos polos na região negativa, pontos nos quais a expressão pode ser substituída pelo fatorial negativo definido acima. Entretanto, ainda é preciso verificar a convergência da série, o que pode ser feito usando-se o critério da razão:

$$
\begin{gathered}
\lim _{n \rightarrow \infty}\left|\frac{T_{n+1}}{T_{n}}\right|=\lim _{n \rightarrow \infty} \mid \frac{\frac{\Gamma(\alpha+1)}{\frac{\Gamma(\alpha-n)(n+1) !}{\Gamma(\alpha+1)}} \frac{x^{n+1}}{x^{n}}\left|=\lim _{n \rightarrow \infty}\right| \frac{\alpha-n}{n+1}|| x \mid<1 \Rightarrow}{\lim _{n \rightarrow \infty}\left|\frac{T_{n+1}}{T_{n}}\right|=\lim _{n \rightarrow \infty}\left|\frac{\frac{\alpha}{n}-1}{1+\frac{1}{n}}\right||x|<1 \Rightarrow|x|<1}
\end{gathered}
$$

O próximo passo consiste em transformar o resultado acima num binômio qualquer. Para expandir este resultado a um binômio com dois termos distintos $a$ e $b$, devemos escrever $(a+b)^{\alpha},(\alpha \in \mathbb{R})$, numa forma semelhante à obtida acima. Suponhamos, por hipótese, que $|a|>|b|$. Então, $\frac{|b|}{|a|}<1$ permite que reescrevamos a fórmula anterior 
identificando $x \operatorname{com} \frac{b}{a}$, da seguinte maneira:

$$
\begin{gathered}
(a+b)^{\alpha}=a^{\alpha}\left(1+\frac{b}{a}\right)^{\alpha}=a^{\alpha} \sum_{n=0}^{\infty} \frac{\Gamma(\alpha+1)}{\Gamma(\alpha-n+1) n !}\left(\frac{b}{a}\right)^{n} \Rightarrow \\
(a+b)^{\alpha}=\sum_{n=0}^{\infty} \frac{\Gamma(\alpha+1)}{\Gamma(\alpha-n+1) n !} a^{\alpha-n} b^{n}, \quad \alpha, a, b \in \mathbb{R},|a|>|b|,
\end{gathered}
$$

que é a expressão para o teorema do binômio generalizado.

Deve-se notar que, para desenvolvimentos com expoentes não-inteiros negativos, ao se usar a expressão (E.44), o binômio $(1+x)^{-\alpha}$ assume a forma:

$$
(1+x)^{-\alpha}=\sum_{n=0}^{\infty}(-1)^{n}\left[\begin{array}{c}
\alpha \\
n
\end{array}\right]=\sum_{n=0}^{\infty}(-1)^{n} \frac{(\alpha)_{n}}{n !} x^{n}, \quad|x|<1
$$

\section{E.13 Função de Mittag-Leffler}

Finalmente, chegamos à última classe de funções especiais do Cálculo Fracional. A função de Mittag-Leffler desempenha um papel semelhante à exponencial no que diz respeito às soluções de equações diferenciais de ordem arbitrária. Conforme foi adiantado no panorama histórico (subseção D), há mais de um tipo de função de Mittag-Leffler. A primeira, abordada na subseção mencionada, foi definida pelo matemático sueco M.G. Mittag-Leffler em 1903 e é uma função inteira definida por uma série:

$$
E_{\alpha}(z)=\sum_{k=0}^{\infty} \frac{z^{k}}{\Gamma(\alpha k+1)}, \quad(\alpha \in \mathbb{C}, \Re(\alpha)>0)
$$

Por sua vez, a função de Mittag-Leffler generalizada, algumas vezes chamada de função de Wiman, por ter sido introduzida por esse matemático sueco em 1905, assume a forma:

$$
E_{\alpha, \beta}(z)=\sum_{k=0}^{\infty} \frac{z^{k}}{\Gamma(\alpha k+\beta)}, \quad(\alpha, \beta \in \mathbb{C}, \Re(\alpha), \Re(\beta)>0),
$$

tendo sido posteriormente estudada por Agarwal (1953), Humbert (1953), Gorenflo (1987) e Miller (1993). Essa mesma função generalizada também admite uma representação integral, estabelecida em 1954 por A. Erdélyi et al. e M. M. Dzherbashyan, na forma:

$$
E_{\alpha, \beta}(z)=\frac{1}{2 \pi i} \int_{C} \frac{\zeta^{\alpha-\beta} e^{\zeta}}{\left(\zeta^{\alpha}-z\right)^{n+1}} d \zeta
$$

A função de Mittag-Leffler de três parâmetros foi introduzida pelo matemático indiano 
T. R. Prabhakar em um artigo de 1971 (PRABHAKAR et al., 1971) e é definida, em termos do símbolo de Pochhammer $(\gamma)_{k}$, como:

$$
E_{\alpha, \beta}^{\gamma}(z)=\sum_{k=0}^{\infty} \frac{(\gamma)_{k}}{\Gamma(\alpha k+\beta)} \frac{z^{k}}{k !}, \quad(\alpha, \beta, \gamma \in \mathbb{C}, \Re(\alpha), \Re(\beta), \Re(\gamma)>0)
$$

Em 2007 a função foi estendida para quatro parâmetros pelos matemáticos indianos Shukla e Prajapati (2007):

$$
E_{\alpha, \beta}^{\gamma, q}(z)=\sum_{k=0}^{\infty} \frac{(\gamma)_{q k}}{\Gamma(\alpha k+\beta)} \frac{z^{k}}{k !}, \quad(\alpha, \beta, \gamma \in \mathbb{C}, \Re(\alpha), \Re(\beta), \Re(\gamma)>0, q \in(0,1) \cup \mathbb{N})
$$

Dois anos depois essa mesma função teve seu domínio estendido por Srivastava e Tomovski (2009). Nesse mesmo ano, uma outra função de quatro parâmetros foi introduzida por Tariq O. Salim, tendo sido definida como:

$$
E_{\alpha, \beta}^{\gamma, \delta}(z)=\sum_{k=0}^{\infty} \frac{(\gamma)_{k}}{\Gamma(\alpha k+\beta)} \frac{z^{k}}{(\delta)_{k}}, \quad(\alpha, \beta, \gamma, \delta \in \mathbb{C}, \Re(\alpha), \Re(\beta), \Re(\gamma), \Re(\delta)>0)
$$

Existem ainda outros tipos, definidos mais recentemente, com cinco, seis e até dez parâmetros (KHAN; AHMED, 2013). As três primeiras funções estão relacionadas por:

$$
E_{\alpha, \beta}^{1}(z)=E_{\alpha, \beta}(z), \quad E_{\alpha, 1}^{1}(z)=E_{\alpha, 1}(z)=E_{\alpha}(z)
$$

Além disso, como já foi dito, a função de Mittag-Leffler constitui uma generalização da exponencial, de modo que:

$$
E_{1}(z)=\exp (z)
$$

De maneira geral, muitas funções podem ser expressas como casos particulares de Mittag-Leffler. A seguir, apresentamos, além da imediata $E_{1,1}(z)=e^{z}$, uma tabela com algumas delas:

\begin{tabular}{|l|l|}
\hline$E_{0,1}(z)=\frac{1}{1-z}$ & $E_{1,2}(z)=\frac{e^{z}-1}{z}$ \\
\hline$E_{1, \nu+1}(z)=e^{z} \gamma^{*}(\nu, z)$ & $E_{2,1}\left(z^{2}\right)=\cosh (z)$ \\
\hline$E_{2,1}\left(-z^{2}\right)=\cos (z)$ & $E_{2,2}\left(z^{2}\right)=\frac{\sinh (z)}{z}$ \\
\hline$E_{2,2}\left(-z^{2}\right)=\frac{\sin (z)}{z}$ & $E_{\frac{1}{2}, 1}(z)=\exp \left(z^{2}\right) \operatorname{erfc}(-\mathrm{z})$ \\
\hline
\end{tabular}

Para as funções trigonométricas e hiperbólicas de ordem $n, \forall r \in \mathbb{N}$, valem as equações:

$$
K_{r}(z, n)=\sum_{k=0}^{\infty} \frac{(-1)^{k} z^{k n+r-1}}{(k n+r-1) !}=z^{r-1} E_{n, r}\left(-z^{n}\right)
$$




$$
h_{r}(z, n)=\sum_{k=0}^{\infty} \frac{z^{k n+r-1}}{(k n+r-1) !}=z^{r-1} E_{n, r}\left(z^{n}\right)
$$

Ressaltamos também a possibilidade de relacioná-las às funções hiperbólicas generalizadas, introduzidas pelo italiano Vincenzo Riccati em 1757:

$$
F_{n, r}^{\alpha}(x)=\sum_{k=0}^{\infty} \frac{\alpha^{k}}{\Gamma(n k+r)} x^{n k+r}, \quad(\alpha \in \mathbb{C})
$$

de maneira que

$$
E_{\alpha}\left(z^{\alpha}\right)=F_{\alpha, 0}^{1}(z)
$$

Além das precedentes, apresentamos a seguir as notações de Mittag-Leffler das funções de Mellin-Ross, de Rabotnov e a hipergeométrica confluente de Kummer.

Função de Mellin-Ross (MILLER; ROSS, 1993):

$$
E_{t}(\nu, \lambda)=t^{\nu} e^{\lambda t} \gamma^{*}(\nu, \lambda t)=t^{\nu} \sum_{k=0}^{\infty} \frac{(\lambda t)^{k}}{\Gamma(\nu+k+1) !}=t^{\nu} E_{1, \nu+1}(\lambda t)
$$

Função de Rabotnov:

$$
R_{\alpha}(\beta, t)=t^{\alpha} \sum_{k=0}^{\infty} \frac{\beta^{k} t^{k(\alpha+1)}}{\Gamma[(1+\alpha)(k+1)]}=t^{\alpha} E_{\alpha+1, \alpha+1}\left(\beta t^{\alpha+1}\right)
$$

Função hipergeométrica confluente de Kummer (1837):

$$
M(\gamma, \beta, z)={ }_{1} F_{1}(\gamma ; \beta ; z)=\Gamma(\beta) E_{1, \beta}^{\gamma}(z)
$$

É interessante mencionar ainda a função de Wright, aparentada, de certa maneira, à de Mittag-Leffler:

$$
\varnothing(\alpha, \beta ; z)=\sum_{k=0}^{\infty} \frac{z^{k}}{k ! \Gamma(\alpha k+\beta)}, \quad(\alpha>-1, \beta \in \mathbb{C})
$$

Foi definida pelo matemático inglês, recentemente falecido, E.M. Wright (1906-2005). É uma função inteira, quer dizer, analítica em todos os pontos do plano complexo. 


\section{APÊEDICE F - Fundamentos do Cálculo Fracional}

Neste apêndice são expostos os fundamentos do Cálculo Fracional e seus principais resultados, fundamentais para a elaboração deste trabalho. Este resumo não pretende ser uma exposição completa nem exaustiva do tema, constituindo apenas os prolegômenos dos assuntos abordados nesta tese. Assim, partes importantes, como o desenvolvimento de Grünwald-Letnikov, não são incluídos, por não serem necessários para o entendimento do texto.

\section{F.1 Fórmula de Cauchy para Integrações Sucessivas}

A ideia contida na fórmula obtida por Cauchy consiste no fato de se poder reduzir uma integração múltipla de $n$ termos a um único operador integral com um kernel específico, isto é,

$$
\int_{a}^{x} d x_{1} \int_{a}^{x_{1}} d x_{2} \int_{a}^{x_{2}} d x_{3} \cdots \int_{a}^{x_{n-1}} f(t) d t \longrightarrow \int_{a}^{x} K_{n}(x, t) f(t) d t
$$

Isto apresenta, obviamente, uma grande vantagem em termos de cálculo, desde que o kernel da transformada seja uma função conhecida, contínua e integrável. A forma deste operador é semelhante a de uma equação de Volterra de $1^{\circ}$ tipo, com a diferença de que, aqui, a função $f$ do integrando é conhecida e o que queremos determinar é o resultado da aplicação do operador sobre essa função.

A priori, tem-se, para o número de operações, $n \in \mathbb{N}$, mas cabe a pergunta se a expressão poderia ser aplicada também a outros valores. Todavia, uma vez obtida a expressão do kernel, é possível mostrar que a fórmula deduzida será válida, após algumas considerações importantes, para índices $n$ genéricos, ou seja, pertencente a outros conjuntos mais amplos.

O primeiro passo consiste em determinar a expressão da integral múltipla por cálculo direto. Assim, seja $f(x)$ uma função Riemann-integrável. Calculemos a integral dessa 
função, definida em um intervalo $I$ limitado inferiormente por $a \in \mathbb{R}$. Então, denota-se, para $x \in I=[a, b]$,

$$
\mathfrak{I}[f(x)]=(\mathfrak{I} f)(x)=\int_{a}^{x} f(t) d t
$$

Nosso interesse está direcionado ao cálculo de integrais múltiplas do tipo

$$
\left(\mathfrak{I}^{n} f\right)(x)=\int_{a}^{x} d t_{n} \int_{a}^{t_{n}} d t_{n-1} \cdots \int_{a}^{t_{2}} f\left(t_{1}\right) d t_{1}
$$

Daí, segue diretamente que

$$
\left(\mathfrak{I}^{n} f\right)(a)=0
$$

A fim de estabelecer uma fórmula geral, é aconselhável iniciar com um caso simples. O mais simples possível é

$$
\left(\mathfrak{I}^{2} f\right)(x)=\int_{a}^{x} d t \int_{a}^{t} f(\tau) d \tau
$$

já que o caso imediatamente anterior é a integral ordinária

$$
(\mathfrak{I} f)(x)=\int_{a}^{x} f(t) d t
$$

Inicialmente, denotando

$$
\int_{a}^{t} f(\tau) d \tau=u(t)
$$

que fornece $\frac{d u}{d t}=f(t)$, e usando a regra de Leibniz generalizada:

$$
\frac{d}{d x} \int_{g(x)}^{h(x)} f(x, t) d t=f(x, h(x)) \frac{d h(x)}{d x}-f(x, g(x)) \frac{d g(x)}{d x}+\int_{g(x)}^{h(x)} \frac{\partial}{\partial x} f(x, t) d t
$$

podemos reescrever a expressão prévia para $\mathfrak{I}^{2} f(x)$ fazendo uma integração por partes, obtendo

$$
\begin{gathered}
\left(\mathfrak{I}^{2} f\right)(x)=\left.\left(t \int_{a}^{t} f(\tau) d \tau\right)\right|_{t=a} ^{x}-\int_{a}^{x} t f(t) d t= \\
=x \int_{a}^{x} f(t) d t-\int_{a}^{x} t f(t) d t \Rightarrow \\
\left(\mathfrak{I}^{2} f\right)(x)=\int_{a}^{x}(x-t) f(t) d t
\end{gathered}
$$

Então, usando

$$
\frac{d}{d t}\left(\mathfrak{I}^{2} f\right)(t)=\frac{d}{d t} \int_{a}^{t}(\mathfrak{I} f)(\tau) d \tau=(\mathfrak{I} f)(t)
$$


e, notando que

$$
\left.\left(\mathfrak{I}^{2} f\right)(x)\right|_{x=a}=0
$$

podemos fazer a mesma coisa para uma ordem superior de integração:

$$
\left(\mathfrak{I}^{3} f\right)(x)=\int_{a}^{x} d t \int_{a}^{t} d s \int_{a}^{s} f(\tau) d \tau
$$

ou, de modo equivalente, usando (F.2), integrando por partes novamente:

$$
\begin{gathered}
\left(\mathfrak{I}^{3} f\right)(x)=\int_{a}^{x} \mathfrak{I}^{2} f(t) d t \stackrel{\text { por partes }}{\Longrightarrow} \\
\left(\mathfrak{I}^{3} f\right)(x)=\left.t\left(\mathfrak{I}^{2} f\right)(t)\right|_{t=a} ^{x}-\int_{a}^{x} t(\mathfrak{I} f)(t) d t= \\
=x\left(\mathfrak{I}^{2} f\right)(x)-\int_{a}^{x} t(\mathfrak{I} f)(t) d t= \\
=x \int_{a}^{x} d t \int_{a}^{t} f(\tau) d \tau-\int_{a}^{x} t d t \int_{a}^{t} f(\tau) d \tau \Rightarrow \\
\left(\mathfrak{I}^{3} f\right)(x)=\int_{a}^{x}(x-t) d t \int_{a}^{t} f(\tau) d \tau
\end{gathered}
$$

Agora, redenominando de novo $\int_{a}^{t} f(\tau) d \tau=u(t)$, temos

$$
\left(\mathfrak{I}^{3} f\right)(x)=\int_{a}^{x} u(t)(x-t) d t
$$

Assim, integrando por partes, com $(x-t) d t=d v$ e lembrando que $u(a)=0$, obtemos

$$
\begin{gathered}
\left(\mathfrak{I}^{3} f\right)(x)=-\left.u(t) \frac{(x-t)^{2}}{2}\right|_{t=a} ^{x}+\int_{a}^{x} \frac{(x-t)^{2}}{2} f(t) d t= \\
=-\left.\int_{a}^{t} f(\tau) d \tau \frac{(x-t)^{2}}{2}\right|_{t=a} ^{x}+\int_{a}^{x} \frac{(x-t)^{2}}{2} f(t) d t= \\
=\int_{a}^{x} \frac{(x-t)^{2}}{2} f(t) d t
\end{gathered}
$$

já que o primeiro termo é nulo. Logo, chega-se a

$$
\left(\mathfrak{I}^{3} f\right)(x)=\frac{1}{2} \int_{a}^{x}(x-t)^{2} f(t) d t
$$

É possível continuar com esse procedimento tantas vezes quantas se fizerem necessárias para induzir uma fórmula geral. A fim de sugerir a expresssão correta, vamos repetir o 
processo uma vez mais. Então,

$$
\left(\mathfrak{I}^{4} f\right)(x)=\int_{a}^{x} d t \int_{a}^{t} d s \int_{a}^{s} d w \int_{a}^{w} f(\tau) d \tau
$$

ou, alternativamente,

$$
\left(\mathfrak{I}^{4} f\right)(x)=\int_{a}^{x}\left(\mathfrak{I}^{3} f\right)(t) d t
$$

Agora, usando a expressão geral:

$$
\frac{d}{d x}\left(\mathfrak{I}^{n} f\right)(x)=\frac{d}{d x} \int_{a}^{x}\left(\mathfrak{I}^{n-1} f\right)(t) d t=\left(\mathfrak{I}^{n-1} f\right)(x),
$$

obtemos, na integração por partes da expressão anterior,

$$
\begin{gathered}
\left(\mathfrak{I}^{4} f\right)(x)=\left.t\left(\mathfrak{I}^{3} f\right)(t)\right|_{t=a} ^{x}-\int_{a}^{x} t\left(\mathfrak{I}^{2} f\right)(t) d t= \\
=x\left(\mathfrak{I}^{3} f\right)(x)-\int_{a}^{x} t\left(\mathfrak{I}^{2} f\right)(t) d t= \\
=x \int_{a}^{x}\left(\mathfrak{I}^{2} f\right)(t) d t-\int_{a}^{x} t\left(\mathfrak{I}^{2} f\right)(t) d t= \\
=\int_{a}^{x}(x-t)\left(\mathfrak{I}^{2} f\right)(t) d t=\int_{a}^{x}(x-t) d t \int_{a}^{t} d s \int_{a}^{s} f(\tau) d \tau
\end{gathered}
$$

Após a integração por partes, com $u(t)=\left(\mathfrak{I}^{2} f\right)(t)=\int_{a}^{t} d s \int_{a}^{s} f(\tau) d \tau$ e $d v=$ $(x-t) d t$, notando que $\frac{d u}{d t}=\int_{a}^{t} f(\tau) d \tau$, podemos reescrever a equação acima na forma

$$
\left(\mathfrak{I}^{4} f\right)(x)=-\left.\frac{(x-t)^{2}}{2} \int_{a}^{t} d s \int_{a}^{s} f(\tau) d \tau\right|_{t=a} ^{x}+\int_{a}^{x} \frac{(x-t)^{2}}{2} d t \int_{a}^{t} f(\tau) d \tau
$$

Todavia, como o primeiro termo é nulo, temos

$$
\left(\mathfrak{I}^{4} f\right)(x)=\int_{a}^{x} \frac{(x-t)^{2}}{2} d t \int_{a}^{t} f(\tau) d \tau
$$

Integrando este termo por partes de novo, com $d v=\frac{(x-t)^{2}}{2} d t$, obtém-se

$$
\left(\mathfrak{I}^{4} f\right)(x)=\left.\left(-\frac{(x-t)^{3}}{3 !} \int_{a}^{t} f(\tau) d \tau\right)\right|_{t=a} ^{x}+\int_{a}^{x} \frac{(x-t)^{3}}{3 !} f(t) d t
$$


E, visto que o termo inicial se anula, obtemos, finalmente,

$$
\left(\mathfrak{I}^{4} f\right)(x)=\frac{1}{3 !} \int_{a}^{x}(x-t)^{3} f(t) d t
$$

Nessas condições, não é difícil supor, por indução, uma fórmula geral da forma:

$$
\left(\mathfrak{I}^{n} f\right)(x)=\frac{1}{(n-1) !} \int_{a}^{x}(x-t)^{n-1} f(t) d t, \quad n \in \mathbb{N},
$$

Entretanto, ainda é preciso demonstrar a validade da expressão proposta. Para isso, usaremos o princípio da indução finita. Para $n=1$, a fórmula satisfaz $(\mathfrak{I} f)(x)=$ $\int_{a}^{x} f(t) d t$. Assim, suponhamos válida a fórmula para $n \in \mathbb{N}, n \geq 2$. Então, derivando-a, devemos encontrar

$$
\frac{d}{d x}\left(\mathfrak{I}^{n} f\right)(x)=\left(\mathfrak{I}^{n-1} f\right)(x)
$$

De fato, pela regra de Leibniz, temos

$$
\begin{gathered}
\frac{d}{d x}\left(\mathfrak{I}^{n} f\right)(x)=\frac{1}{(n-1) !}\left\{\left.(x-t)^{n-1} f(t)\right|_{t=x}+\int_{a}^{x} \frac{\partial}{\partial x}\left[(x-t)^{n-1} f(t)\right] d t\right\}= \\
=\frac{1}{(n-1) !} \int_{a}^{x}(n-1)(x-t)^{n-2} f(t) d t= \\
=\frac{1}{(n-2) !} \int_{a}^{x}(x-t)^{n-2} f(t) d t=\left(\mathfrak{I}^{n-1} f\right)(x)
\end{gathered}
$$

Portanto, a fórmula proposta é realmente válida. Isto significa que a hipótese assumida, de reduzir uma integração múltipla de ordem $n$ a um operador integral, pode ser satisfeita, quer dizer, é possível escrever:

$$
\int_{a}^{x} d t_{n} \int_{a}^{t_{n}} d t_{n-1} \cdots \int_{a}^{t_{2}} f\left(t_{1}\right) d t_{1}=\int_{a}^{x} K_{n}(x, t) f(t) d t
$$

onde o kernel do operador é dado por

$$
K_{n}(x, t)=\frac{(x-t)^{n-1}}{(n-1) !}=\frac{(x-t)^{n-1}}{\Gamma(n)}
$$

Logo, reescrevendo a expressão (F.5) em termos da função gama, chegamos finalmente à fórmula de Cauchy:

$$
\left(\mathfrak{I}^{n} f\right)(x)=\frac{1}{\Gamma(n)} \int_{a}^{x}(x-t)^{n-1} f(t) d t
$$




\section{F.2 Extensão a Ordens Fracionárias}

A expressão para a integral múltipla pode ser reescrita em termos da notação utilizada no cálculo fracional:

$$
\int_{a}^{x} d t_{n} \int_{a}^{t_{n}} d t_{n-1} \cdots \int_{a}^{t_{2}} f\left(t_{1}\right) d t_{1}={ }_{a} \mathcal{J}_{x}^{n} f(x)
$$

Na notação da integral fracionária de Riemann, tem-se, para a expansão a valores não-naturais de $\nu$ :

$$
{ }_{a} \mathcal{J}_{x}^{\nu} f(x) \rightarrow{ }_{a} D_{x}^{-\nu} f(x), \quad \nu>0
$$

Como estamos tratando de operações de integração, o valor de $\nu$ é restrito aos estritamente positivos. Os índices subscritos da notação semelhante à derivada são chamados terminais. Na extensão a valores $\nu$ quaisquer, escreve-se, portanto,

$$
{ }_{a} D_{x}^{-\nu} f(x)=\frac{1}{\Gamma(\nu)} \int_{a}^{x}(x-t)^{\nu-1} f(t) d t, \quad \Re(\nu)>0
$$

A condição $\Re(\nu)>0$ é exigida também para a convergência da integral. O valor nulo não é definido, pois a função gama não existe nesse ponto. Entretanto, pode-se assumir, por convenção, ${ }_{a} D_{x}^{0} f(x)=f(x)$.

\section{F.3 Abordagem Fracional por Transformadas de La- place}

Seja $y(x)$ uma função diferenciável de classe $C^{m}, m \in \mathbb{N}$. Então, sua derivada de ordem $n<m$, pode ser escrita $D^{n} y(x)=f(x)$. Essa expressão pode ser interpretada como uma equação diferencial de ordem $n$. Suponhamos, ademais, que suas condições iniciais no ponto $x=0$ sejam escolhidas todas nulas, isto é, $D^{k} y(0)=0, \quad$ com $0 \leq$ $k \leq n-1$. Nesses termos, um conjunto fundamental de soluções para a EDO homogênea $D^{n} y(x)=0$ é dado por $\left\{1, x, x^{2}, \ldots, x^{n-1}\right\}$. Usando a notação da derivada fracional de modo mais rigoroso, a equação diferencial com as condições descritas antes, deve ser escrita

$$
\left\{\begin{array}{c}
{ }_{0} D_{x}^{n} y(x)=f(x) \\
\left\{{ }_{0} D_{x}^{k} y(x)_{x=0}=0, \quad(k=1, \ldots, n-1)\right.
\end{array}\right.
$$


Entretanto, utilizando transformadas de Laplace na EDO acima, segue,

$$
\begin{gathered}
\mathcal{L}\left\{D^{n} y(x)\right\}=\mathcal{L}\{f(x)\} \Rightarrow \\
s^{n} Y(s)-\sum_{k=0}^{n-1} s^{n-k-1} D^{k} y(0)=F(s) \Rightarrow \\
s^{n} Y(s)=F(s) .
\end{gathered}
$$

Disso, vem

$$
Y(s)=s^{-n} F(s)
$$

e, pelo teorema da convolução,

$$
\begin{gathered}
\mathcal{L}^{-1}\{Y(s)\}=\mathcal{L}^{-1}\left\{s^{-n} F(s)\right\}=\left(\frac{t^{n-1}}{\Gamma(n)} * f(t)\right) \Rightarrow \\
y(t)=\frac{1}{\Gamma(n)} \int_{0}^{t}(t-\xi)^{n-1} f(\xi) d \xi
\end{gathered}
$$

Entretanto, $D^{n} y(t)=f(t) \Rightarrow y(t)=D^{-n} f(t)$ e, portanto,

$$
{ }_{0} D_{t}^{-n} f(t)=\frac{1}{\Gamma(n)} \int_{0}^{t}(t-\xi)^{n-1} f(\xi) d \xi
$$

Se considerarmos válida a extensão a valores $\nu$ não-inteiros, então chega-se a

$$
{ }_{0} D_{x}^{-\nu} f(x)=\frac{1}{\Gamma(\nu)} \int_{0}^{x}(x-t)^{\nu-1} f(t) d t
$$

Ora, essa é exatamente a definição da integral fracionária de Riemann-Liouville. Consequentemente, mostramos que, através da abordagem das transformadas de Laplace, é possível derivar esta expressão.

\section{F.4 Abordagem Fracional Complexa pela Integral de Cauchy Generalizada}

O ponto de partida é a fórmula integral de Cauchy para derivadas no plano complexo:

$$
f^{(n)}(z)=\frac{n !}{2 \pi i} \oint_{C} \frac{f(\zeta)}{(\zeta-z)^{n+1}} d \zeta
$$

Para valores naturais de $n$, o ponto $z$ é um polo do integrando. No entanto, para valores fracionários de $n$, o mesmo ponto $z$ passa a ser agora um ponto de ramificação. 
Nessas circunstâncias, a generalização da integral acima exige que se determine um caminho de integração apropriado à situação, para se evitar duplicidade de valores. Assim, uma curva fechada não constitui um contorno de integração adequado. Se fizermos um corte de $x=-\infty$ até um ponto $z \in \mathbb{R}_{+}$, uma curva que parta do argumento $\theta=-\pi$ e termine em $\theta=\pi$ evitará a duplicidade nestes pontos, visto que os pontos de partida e de chegada passam a pertencer a folhas de Riemann distintas. Vamos, então, tomar um ponto $z=x>0$ no eixo real e eleger um limite inferior de integração $a<0$. Então, uma curva $\mathfrak{L}$ em torno desse ponto deve percorrer duas folhas de Riemann, visto que $z=x$ passa a ser um ponto de ramificação. Já a generalização do fatorial para a função gama é imediata. Desse modo, a integral assume a forma

$$
D^{\nu} f(z)=\frac{\Gamma(\nu+1)}{2 \pi i} \oint_{\mathfrak{L}}(\zeta-z)^{-\nu-1} f(\zeta) d \zeta
$$

sendo o caminho $\mathfrak{L}$ a união de $C_{1}:[a, x-\rho], \gamma:|\zeta-z|=\rho$ e $C_{2}:[x-\rho, a]$. O ponto $\zeta$ encontra-se sobre a curva $\gamma$, que circula $z=x$. O contorno aparece esquematizado na figura 127.

Figura 127: Caminho de integração

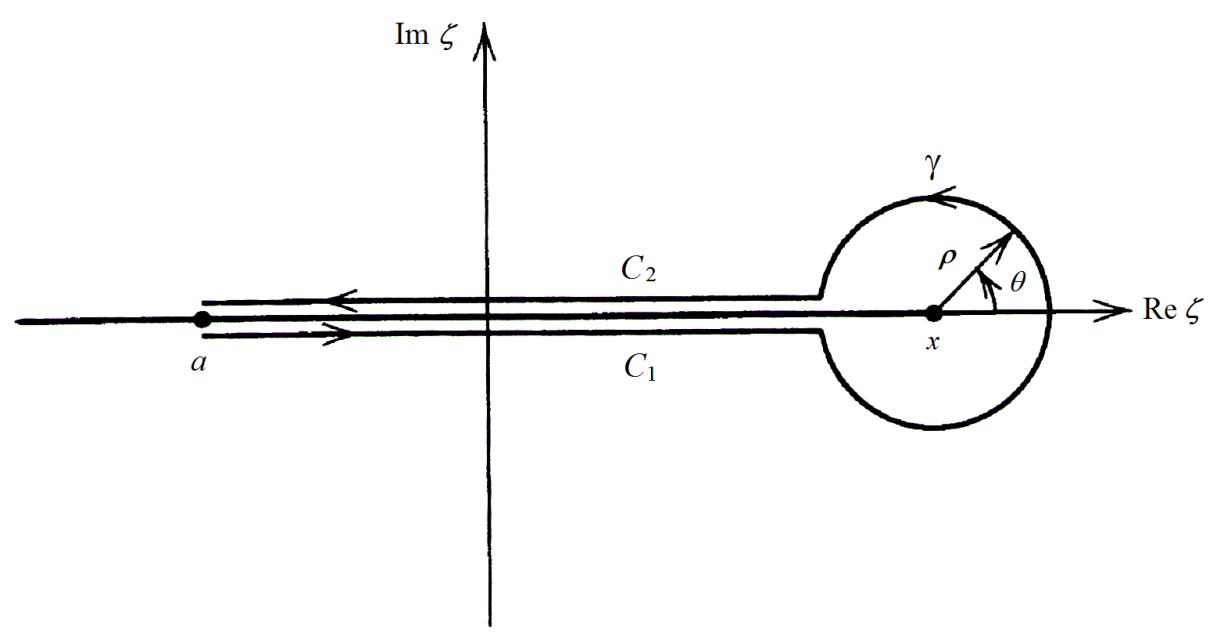

Para calcular os valores de $(\zeta-z)^{-\nu-1}$ sobre cada uma das trajetórias, fazemos:

$$
\begin{gathered}
(\zeta-z)^{-\nu-1}=\exp \left[\ln \left((\zeta-z)^{-\nu-1}\right)\right]= \\
=\exp \left[-(\nu+1) \ln \left(|\zeta-z| e^{i \theta}\right)\right]=\exp [-(\nu+1)(\ln |\zeta-z|+i \theta)]
\end{gathered}
$$


Agora, calculando o valor assumido em cada trecho do caminho $\mathfrak{L}$, temos:

$$
\begin{gathered}
C_{1}:\left\{\begin{array}{c}
\theta=-\pi, \zeta \subset C_{1} \Leftrightarrow \zeta<x \Rightarrow(\zeta-z)^{-\nu-1}= \\
=\exp \{-(\nu+1)[\ln (x-\zeta)-i \pi]\} \Rightarrow \\
(\zeta-z)^{-\nu-1}=e^{i(\nu+1) \pi}(x-\zeta)^{-(\nu+1)}
\end{array}\right. \\
\gamma:\left\{\begin{array}{c}
(\zeta-z)=\rho e^{i \theta},-\pi \leq \theta<\pi \Rightarrow \\
(\zeta-z)^{-\nu-1}=\rho^{-(\nu+1)} e^{-i(\nu+1) \theta}
\end{array}\right. \\
C_{2}:\left\{\begin{array}{c}
\theta=\pi, \zeta \subset C_{2} \Leftrightarrow \zeta<x \Rightarrow(\zeta-z)^{-\nu-1}= \\
=\exp \{-(\nu+1)[\ln (x-\zeta)+i \pi]\} \Rightarrow \\
(\zeta-z)^{-\nu-1}=e^{-i(\nu+1) \pi}(x-\zeta)^{-(\nu+1)}
\end{array}\right.
\end{gathered}
$$

Portanto, a integral sobre o caminho $\mathfrak{L}$ torna-se:

$$
\begin{gathered}
\oint_{\mathfrak{L}}(\zeta-z)^{-\nu-1} f(\zeta) d \zeta=\int_{a}^{x-\rho} e^{i(\nu+1) \pi}(x-t)^{-(\nu+1)} f(t) d t+ \\
+\int_{\gamma}(\zeta-z)^{-(\nu+1)} f(\zeta) d \zeta+\int_{x-\rho}^{a} e^{-i(\nu+1) \pi}(x-t)^{-(\nu+1)} f(t) d t= \\
=\left[e^{i(\nu+1) \pi}-e^{-i(\nu+1) \pi}\right] \int_{a}^{x-\rho}(x-t)^{-(\nu+1)} f(t) d t+ \\
+\int_{-\pi}^{\pi} \rho^{-(\nu+1)} e^{-i(\nu+1) \theta} \rho i e^{i \theta} f\left(x+\rho e^{i \theta}\right) d \theta \Rightarrow \\
\oint_{\mathfrak{L}}(\zeta-z)^{-\nu-1} f(\zeta) d \zeta=2 i \sin [(\nu+1) \pi] \int_{a}^{x-\rho}(x-t)^{-(\nu+1)} f(t) d t+ \\
+\int_{-\pi}^{\pi} i \rho^{-\nu} e^{-i \nu \theta} f\left(x+\rho e^{i \theta}\right) d \theta
\end{gathered}
$$

Trabalhando a integral sobre $\gamma$ e tomando o limite para $\rho \rightarrow 0$ vem

$$
\begin{gathered}
\left|\int_{\gamma}(\zeta-z)^{-(\nu+1)} f(\zeta) d \zeta\right| \leq \int_{-\pi}^{\pi}\left|i \rho^{-\nu} e^{-i \nu \theta} f\left(x+\rho e^{i \theta}\right)\right| d \theta= \\
=\int_{-\pi}^{\pi}\left|\rho^{-\nu}\right|\left|f\left(x+\rho e^{i \theta}\right)\right| d \theta=\rho^{-\Re(\nu)} \int_{-\pi}^{\pi}\left|f\left(x+\rho e^{i \theta}\right)\right| d \theta \Rightarrow \\
\lim _{\rho \rightarrow 0}\left|\int_{\gamma}(\zeta-z)^{-(\nu+1)} f(\zeta) d \zeta\right| \leq \lim _{\rho \rightarrow 0}\left\{\rho^{-\Re(\nu)} \int_{-\pi}^{\pi}\left|f\left(x+\rho e^{i \theta}\right)\right| d \theta\right\}=0
\end{gathered}
$$


E, do limite $\rho \rightarrow 0$ sobre os caminhos no eixo real, vem

$$
\begin{gathered}
\lim _{\rho \rightarrow 0} 2 i \sin [(\nu+1) \pi] \int_{a}^{x-\rho}(x-t)^{-(\nu+1)} f(t) d t= \\
=2 i \sin [(\nu+1) \pi] \int_{a}^{x}(x-t)^{-(\nu+1)} f(t) d t
\end{gathered}
$$

Substituindo esse resultado em (F.8) chega-se a

$$
\begin{gathered}
{ }_{a} D_{x}^{\nu} f(z)=\frac{\Gamma(\nu+1)}{2 \pi i} 2 i \sin [(\nu+1) \pi] \int_{a}^{x}(x-t)^{-(\nu+1)} f(t) d t \Rightarrow \\
{ }_{a} D_{x}^{\nu} f(z)=\frac{\Gamma(\nu+1) \sin [(\nu+1) \pi]}{\pi} \int_{a}^{x}(x-t)^{-(\nu+1)} f(t) d t
\end{gathered}
$$

Utilizando agora a equação funcional de Euler (v. Apêndice $G$ ):

$$
\Gamma(\alpha) \Gamma(1-\alpha)=\frac{\pi}{\sin (\alpha \pi)},
$$

fazendo $\alpha=(\nu+1)$, segue

$$
\frac{\Gamma(\nu+1) \sin [(\nu+1) \pi]}{\pi}=\frac{1}{\Gamma(-\nu)}
$$

e, por conseguinte,

$$
{ }_{a} D_{x}^{\nu} f(z)=\frac{1}{\Gamma(-\nu)} \int_{a}^{x}(x-t)^{-(\nu+1)} f(t) d t, \quad \Re(\nu)<0
$$

E obtemos, assim, a fórmula da derivada fracionária da função $f(z)$. Se for possível estender esse resultado para integrais fracionárias com $\Re(\nu)>0$, seremos conduzidos a

$$
{ }_{a} D_{x}^{-\nu} f(z)=\frac{1}{\Gamma(\nu)} \int_{a}^{x}(x-t)^{\nu-1} f(t) d t, \quad \Re(\nu)>0
$$

que é idêntico ao resultado obtido pela fórmula da integral recorrente de Cauchy e similar àquele com a abordagem pelas transformadas de Laplace, mas com limite inferior $a \mathrm{em}$ vez de 0. Portanto, visto que chegamos, por meio de três caminhos diferentes, ao mesmo resultado, justifica-se a adoção deste como definição do operador fracional de integração. É o que será estabelecido a seguir.

\section{F.5 Integrais Fracionais de Ordem Arbitrária}

O símbolo ${ }_{a} D_{x}^{\nu}$, que representa o operador conhecido como diferintegral de ordem $\nu$, engloba as noções de ambas as operações do Cálculo tradicional, ou seja, integral e 
derivada, em uma única notação. Para ordens de diferintegração com parte real negativa, i.e., para o caso de $\operatorname{Re}\{\nu\}<0$, estamos diante de uma integral fracional ou, como seria mais correto denominá-la, uma integral de ordem arbitrária, já que a ordem pode assumir valores não apenas fracionários, mas também complexos. Tal operação é dada pela expressão:

$$
{ }_{a} D_{x}^{-\nu} f(x)=\frac{1}{\Gamma(\nu)} \int_{a}^{x}(x-t)^{\nu-1} f(t) d t, \quad(\Re(\nu)>0)
$$

Os sub-índices da diferintegral, correspondentes aos extremos de integração, são chamados de terminais.

A nomenclatura apropriada é aplicada de acordo com valores específicos dos terminais e do sinal da parte real da ordem de diferintegração em cada caso. Considerando o caso de $\Re(\nu)$ negativo, para um valor genérico do primeiro terminal $(a)$, a expressão acima recebe o nome de integral fracional de Riemann (IFR), por ser semelhante à definição dada por Riemann, mas sem a presença da função complementar. Já para os casos $a=0$ e $a=-\infty$, ainda para ordem real negativa, teremos uma Integral Fracional de Riemann-Liouville (IFRL), na terminologia introduzida por Holmgren (1863/64) (HILFER et al., 2000) e uma Integral Fracional de Liouville (IFL) ou $2^{a}$ fórmula de Liouville, respectivamente. Ainda há a definição das integrais fracionais de Weyl, relacionadas, de certo modo às de Liouville, mas definidas para uma classe diferente de funções. Resumindo o que foi dito, tem-se:

- ${ }_{a} D_{x}^{-\nu} f(x)$ : Integral fracional de Riemann;

- ${ }_{0} D_{x}^{-\nu} f(x)$ : Integral fracional de Riemann-Liouville;

- ${ }_{-\infty} D_{x}^{-\nu} f(x) \equiv{ }_{-\infty} W_{x}^{\nu} f(x)$ : Integral fracional de Liouville;

- ${ }_{x} D_{\infty}^{-\nu} f(x) \equiv{ }_{x} W_{\infty}^{\nu} f(x)$ : Integral fracional de Weyl.

Habitualmente, por razões de concisão, a versão de Riemann-Liouville será denotada simplesmente $D^{-\nu} f(x)$, seguindo o costume de boa parte da literatura relacionada ao tema. Além disso, tomaremos uma outra liberdade de notação, que consiste em escrever abreviadamente $D_{a}^{\nu} f(x)$ em lugar de ${ }_{a} D_{x}^{\nu} f(x)$, quando a variável $x$ (ou qualquer outra) aparecer explicitamente, uma vez que não existe ambiguidade nesse caso.

Propriedades da Integral Fracional de Riemann Partindo da definição da Integral Fracional de Riemann,

$$
\mathcal{J}_{a}^{\nu} f(x)=\frac{1}{\Gamma(\nu)} \int_{a}^{x}(x-t)^{\nu-1} f(t) d t
$$


onde estamos usando a notação ${ }_{a} D_{x}^{-\nu} \equiv \mathcal{J}_{a}^{\nu}$ (MUNKHAMMAR, 2004), vamos calcular aplicações sucessivas desse operador sobre uma função $f(x)$ :

$$
\begin{gathered}
\mathcal{J}_{a}^{\alpha} \mathcal{J}_{a}^{\beta} f(x)=\frac{1}{\Gamma(\alpha)} \int_{a}^{x}(x-t)^{\alpha-1} \mathcal{J}_{a}^{\beta} f(t) d t= \\
=\frac{1}{\Gamma(\alpha) \Gamma(\beta)} \int_{a}^{x}(x-t)^{\alpha-1}\left\{\int_{a}^{t}(t-u)^{\beta-1} f(u) d u\right\} d t
\end{gathered}
$$

Usando o teorema de Fubini para o domínio de integração acima, podemos reescrever a equação como

$$
\mathcal{J}_{a}^{\alpha} \mathcal{J}_{a}^{\beta} f(x)=\frac{1}{\Gamma(\alpha) \Gamma(\beta)} \int_{a}^{x} f(u) \int_{u}^{x}(x-t)^{\alpha-1}(t-u)^{\beta-1} d t d u
$$

Faremos agora uma substituição de variáveis: $t \rightarrow s: t=u+s(x-u)$, de modo que $d t=(x-u) d s$ e, portanto, como

$$
\begin{aligned}
& (x-t)=(1-s)(x-u) \\
& (t-u)=s(x-u),
\end{aligned}
$$

segue que

$$
\int_{u}^{x}(x-t)^{\alpha-1}(t-u)^{\beta-1} d t=(x-u)^{\alpha+\beta-1} \int_{0}^{1}(1-s)^{\alpha-1} s^{\beta-1} d s
$$

Mas, pela definição da função beta, vem

$$
\int_{u}^{x}(x-t)^{\alpha-1}(t-u)^{\beta-1} d t=(x-u)^{\alpha+\beta-1} B(\alpha, \beta)
$$

e, portanto,

$$
\mathcal{J}_{a}^{\alpha} \mathcal{J}_{a}^{\beta} f(x)=\frac{B(\alpha, \beta)}{\Gamma(\alpha) \Gamma(\beta)} \int_{a}^{x}(x-u)^{\alpha+\beta-1} f(u) d u
$$

Finalmente, usando a relação entre a função beta e a função gama, resulta

$$
\mathcal{J}_{a}^{\alpha} \mathcal{J}_{a}^{\beta} f(x)=\frac{1}{\Gamma(\alpha+\beta)} \int_{a}^{x}(x-u)^{\alpha+\beta-1} f(u) d u,
$$

que é identificado com a integral fracional de ordem $\alpha+\beta$ da função $f(x)$, mostrando que

$$
\mathcal{J}_{a}^{\alpha} \mathcal{J}_{a}^{\beta} f(x)=\mathcal{J}_{a}^{\alpha+\beta} f(x), \quad \forall \alpha, \beta: \Re(\alpha), \Re(\beta)>0
$$

Naturalmente, se houvéssemos realizado a operação em ordem inversa, quer dizer, com o segundo operador antes do primeiro, teríamos obtido:

$$
\mathcal{J}_{a}^{\beta} \mathcal{J}_{a}^{\alpha} f(x)=\mathcal{J}_{a}^{\beta+\alpha} f(x), \quad \forall \alpha, \beta: \Re(\alpha), \Re(\beta)>0
$$


Mas, como a adição dos expoentes é comutativa, segue daí que

${ }_{a} D_{x}^{-\alpha}\left[{ }_{a} D_{x}^{-\beta} f(x)\right]={ }_{a} D_{x}^{-\beta}\left[{ }_{a} D_{x}^{-\alpha} f(x)\right]={ }_{a} D_{x}^{-(\alpha+\beta)} f(x), \quad \Re(\alpha), \Re(\beta)>0$

Isso significa que o operador integral de Riemann de ordem arbitrária apresenta a propriedade de semigrupo, responsável pela preservação da soma de ordem apresentada acima.

\section{F.6 Exemplos de Integração Fracional}

Exemplo 10: Vamos determinar a integral fracional de ordem $\nu$ da função $f(x)=x^{\mu}, \mu>-1$, na versão de Riemann-Liouville:

$$
D^{-\nu} x^{\mu}=\frac{1}{\Gamma(\nu)} \int_{0}^{x}(x-t)^{\nu-1} t^{\mu} d t=\frac{1}{\Gamma(\nu)} \int_{0}^{x} x^{\nu-1}\left(1-\frac{t}{x}\right)^{\nu-1} t^{\mu} d t
$$

Fazendo a mudança de variáveis $t / x=u$, obtém-se:

$$
\begin{aligned}
D^{-\nu} x^{\mu} & =\frac{1}{\Gamma(\nu)} \int_{0}^{1} x^{\nu}(1-u)^{\nu-1}(x u)^{\mu} d u= \\
& =\frac{1}{\Gamma(\nu)} x^{\nu+\mu} \int_{0}^{x}(1-u)^{\nu-1} u^{\mu} d u= \\
& =\frac{1}{\Gamma(\nu)} x^{\nu+\mu} B(\nu, \mu+1)= \\
& =\frac{1}{\Gamma(\nu)} x^{\nu+\mu} \frac{\Gamma(\nu) \Gamma(\mu+1)}{\Gamma(\nu+\mu+1)}= \\
& =\frac{\Gamma(\mu+1)}{\Gamma(\nu+\mu+1)} x^{\nu+\mu}
\end{aligned}
$$

Logo,

$$
D^{-\nu} x^{\mu}=\frac{\Gamma(\mu+1)}{\Gamma(\nu+\mu+1)} x^{\nu+\mu}, \quad(\Re(\nu)>0, \mu>-1)
$$

De posse desse resultado, pode-se determinar a IFRL de ordem $\nu$ de uma constante $k \in \mathbb{C}:$

$$
D^{-\nu} k \equiv D^{-\nu}\left(k x^{0}\right)=\frac{k x^{\nu}}{\Gamma(\nu+1)}, \quad(\Re(\nu)>0)
$$

Exemplo 11: A seguir, calculamos a integral fracional de Riemann-Liouville de uma função exponencial, que é dada em termos da função gama incompleta inferior:

$$
D^{-\nu} e^{\lambda x}=\frac{1}{\Gamma(\nu)} \int_{0}^{x}(x-t)^{\nu-1} e^{\lambda t} d t, \quad(\Re(\nu)>0)
$$


Fazendo a mudança de coordenadas $(x-t)=y$, segue:

$$
D^{-\nu} e^{\lambda x}=\frac{1}{\Gamma(\nu)} e^{\lambda x} \int_{0}^{x} y^{\nu-1} e^{-\lambda y} d y
$$

A expressão acima pode ser reescrita em termos da função gama incompleta da seguinte maneira:

$$
D^{-\nu} e^{\lambda x}=\frac{\lambda^{-\nu}}{\Gamma(\nu)} e^{\lambda x} \gamma(\nu, \lambda x), \quad(\Re(\nu)>0)
$$

Também é interessante expressar o resultado acima em termos da extensão holomórfica da função gama incompleta, conforme a fórmula (E.20), obtendo:

$$
D^{-\nu} e^{\lambda x}=x^{\nu} e^{\lambda x} \gamma^{*}(\nu, \lambda x)
$$

Devido à sua grande importância e aplicação na teoria das equações diferenciais fracionais, essa função, conhecida como função de Mellin-Ross, recebe uma notação especial (MILLER; ROSS, 1993):

$$
E_{x}(\nu, \lambda):=x^{\nu} e^{\lambda x} \gamma^{*}(\nu, \lambda x)=x^{\nu} E_{1, \nu+1}(\lambda x),
$$

onde $E_{\mu, \nu}(x)$ é a função de Mittag-Leffler generalizada. Portanto, podemos escrever:

$$
E_{x}(\nu, \lambda):=D^{-\nu} e^{\lambda x}=x^{\nu} e^{\lambda x} \gamma^{*}(\nu, \lambda x)
$$

Também recebem notação especial as funções obtidas pela IFRL das funções seno e cosseno:

$$
\begin{aligned}
C_{x}(\nu, \omega) & :=D^{-\nu} \cos (\omega x)=\frac{1}{\Gamma(\nu)} \int_{0}^{x}(x-u)^{\nu-1} \cos (\omega u) d u \\
S_{x}(\nu, \omega) & :=D^{-\nu} \sin (\omega x)=\frac{1}{\Gamma(\nu)} \int_{0}^{x}(x-u)^{\nu-1} \sin (\omega u) d u
\end{aligned}
$$

Como são integrais fracionais, deve-se ter $\Re(\nu)>0$ em todos os casos acima.

É interessante notar ainda que é possível escrever uma espécie de extensão integral, por assim dizer, da fórmula de Euler para o caso fracional, que pode ser expressa como:

$$
E_{x}(\nu, i \omega)=C_{x}(\nu, \omega)+i S_{x}(\nu, \omega)
$$

O que podemos notar nos resultados obtidos é que a IFRL de uma exponencial é uma função transcendente, o que torna sua utilização bastante complicada em alguns casos. A razão para isso se encontra na própria definição da IFRL, cujo terminal inferior nulo gera o 
surgimento de termos adicionais para funções exponenciais, fenômeno que não deve ocorrer para terminais inferiores tendendo a $-\infty$. Por esse motivo, torna-se interessante calcular a IFL para a exponencial, com vistas a uma simplificação do resultado. É exatamente o que vamos fazer a seguir.

Exemplo 12: Efetuaremos agora o cálculo da integral fracional de uma função exponencial $e^{\lambda x}$ na versão de Liouville:

$$
{ }_{-\infty} D_{x}^{-\nu} e^{\lambda x}=\frac{1}{\Gamma(\nu)} \int_{-\infty}^{x}(x-t)^{\nu-1} e^{\lambda t} d t, \quad(\Re(\nu)>0)
$$

Fazendo a substituição de variáveis $\lambda(x-t)=y$, segue:

$$
D_{-\infty}^{-\nu} e^{\lambda x}=\frac{1}{\Gamma(\nu)} \int_{0}^{\infty}\left(\frac{y}{\lambda}\right)^{\nu-1} e^{\lambda x-y} \frac{d y}{\lambda}=\frac{e^{\lambda x}}{\lambda^{\nu} \Gamma(\nu)} \int_{0}^{\infty} y^{\nu-1} e^{-y} d y
$$

Porém, como a integral é a própria função $\Gamma(\nu)$, então:

$$
D_{-\infty}^{-\nu} e^{\lambda x}=\lambda^{-\nu} e^{\lambda x}
$$

Consequentemente, constata-se que a IFL de uma exponencial fornece o resultado que seria o esperado na extensão direta do conceito de uma integral clássica com extremo de integração inferior igual a $-\infty$, quer dizer, sem constantes adicionais.

O exemplo do caso seguinte é útil no desenvolvimento de funções em séries de Taylor.

Exemplo 13: Vamos determinar a IFR de um polinômio fora da origem:

$$
D_{a}^{-\nu}(x-a)^{\mu}=\frac{1}{\Gamma(\nu)} \int_{a}^{x}(x-u)^{\nu-1}(u-a)^{\mu} d u
$$

Efetuando a mudança de variáveis $u=a+(x-a) y$, reescrevemos:

$$
\begin{aligned}
D_{a}^{-\nu}(x-a)^{\mu} & =\frac{(x-a)^{\mu+\nu}}{\Gamma(\nu)} \int_{0}^{1}(1-y)^{\nu-1} y^{\mu} d y= \\
& =\frac{(x-a)^{\mu+\nu}}{\Gamma(\nu)} B(\nu, \mu+1)= \\
& =\frac{\Gamma(\mu+1)}{\Gamma(\mu+\nu+1)}(x-a)^{\mu+\nu}, \quad \Re(\mu)>-1
\end{aligned}
$$

Obtivemos, portanto, um resultado compativel ao da IFRL de um polinômio na origem. 


\section{F.7 Derivada de uma Integral Fracional}

Utilizando a fórmula obtida para a integral fracional, vamos determinar o efeito de uma operação de derivação clássica sobre a versão de Riemann-Liouville. Então, usando a regra de derivação de integrais de Leibniz sobre a equação (F.9), com $c=0$, tem-se:

$$
\begin{aligned}
\frac{d}{d x}\left\{D^{-\nu} f(x)\right\} & =\frac{d}{d x}\left\{\frac{1}{\Gamma(\nu)} \int_{0}^{x}(x-u)^{\nu-1} f(u) d u\right\}= \\
& =\frac{1}{\Gamma(\nu)} \int_{0}^{x} \frac{\partial}{\partial x}(x-u)^{\nu-1} f(u) d u= \\
& =\frac{1}{\Gamma(\nu-1)} \int_{0}^{x}(x-u)^{\nu-2} f(u) d u= \\
& =D^{-(\nu-1)} f(x)
\end{aligned}
$$

ou seja, a derivação usual, nesse caso, pode ser representada pelo operador de RiemannLiouville de ordem $\nu=1$, i.e., $\frac{d}{d x} \equiv D$, o que nos leva à propriedade:

$$
D\left\{D^{-\nu} f(x)\right\}=D^{-(\nu-1)} f(x)
$$

Por outro lado, se o extremo inferior de integração assumisse um valor genérico $a$, o resultado acima não se modificaria, o que significa que a derivada clássica usual de ordem inteira pode ser representada de forma geral por um operador diferintegral de Riemann, isto é, $\frac{d}{d x} \equiv{ }_{a} D_{x}^{1}$, o que vai facilitar o estabelecimento das derivadas fracionais.

Naturalmente, a partir dessa fórmula, obtém-se, por indução:

$$
D_{c}^{n}\left\{D_{c}^{-\nu} f(x)\right\}=D_{c}^{-(\nu-n)} f(x), \quad(\Re(\nu)>0, n \in \mathbb{N})
$$

Logo, verificamos que a derivada genérica clássica de ordem inteira de uma IFR apresenta a propriedade de poder ser escrita como uma IFR de ordem inferior à primeira, para $n \in \mathbb{N}, \forall n<\nu$, pois, nesse caso, a expressão continua sendo uma integral fracional. Contudo, para valores de $n$ maiores que $\nu$, o resultado se transforma numa derivada não-inteira, o que nos permitirá definir derivadas fracionais a partir desse fato.

Funções Piso, Teto e Dente de Serra A fim de ajudar no entendimento da exposição referente às derivadas fracionais, vamos fazer uso de algumas funções especiais, listadas abaixo, com os nomes e notações introduzidos por Iverson (1962). A seguir, damos os nomes originais das funções, em inglês, com suas respectivas definições:

- Floor: $\lfloor x\rfloor=\max \{m \in \mathbb{Z} \mid m \leq x\}$ 
- Ceiling: $\lceil x\rceil=\min \{n \in \mathbb{Z} \mid n \geq x\}$

- Sawtooth: $\{x\}=x-\lfloor x\rfloor$

Estas funções recebem, em português, os nomes de função piso, função teto e função dente-de-serra e fornecem, respectivamente, o inteiro imediatamente inferior e posterior ao valor de $x$ e a sua parte fracionária. Para $x>0$, a função piso nada mais é que a sua parte inteira, cuja notação por colchetes, $\operatorname{int}(x)=[x]$, foi primeiramente utilizada por Gauss em 1808.

Figura 128: Funções piso, teto e dente-de-serra
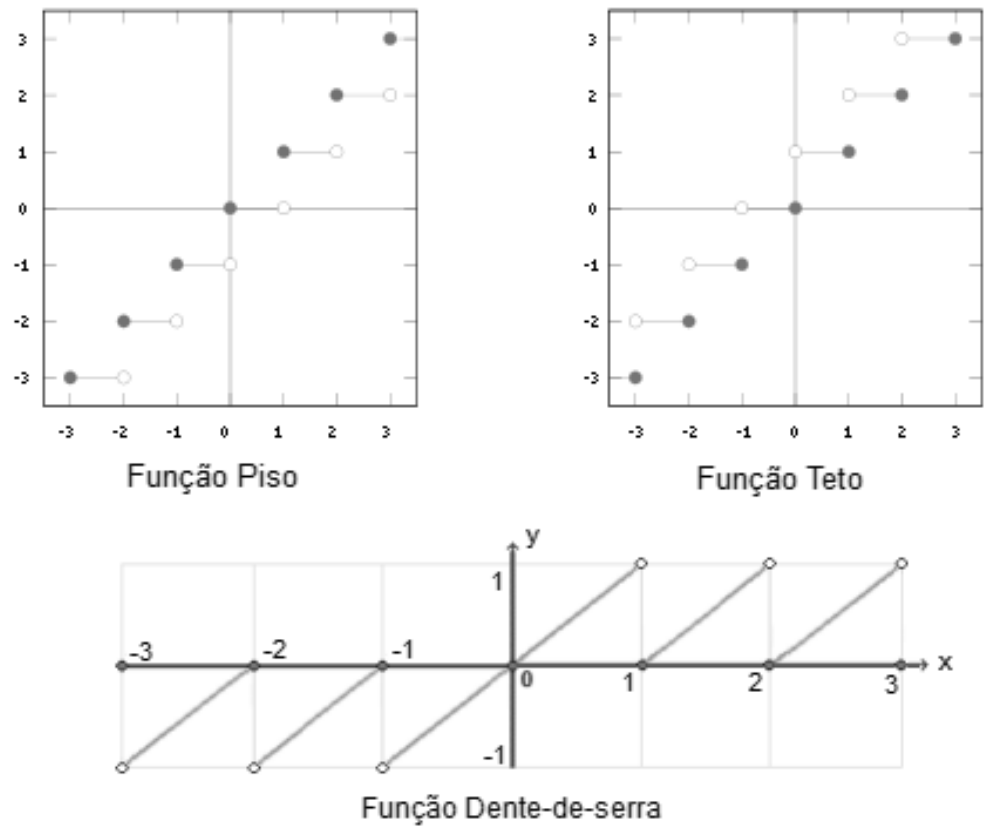

\section{F.8 Derivadas Fracionais}

Uma questão um pouco mais delicada que a anterior é a definição da derivada fracional, uma vez que as fórmulas encontradas são referentes a integrais de ordem arbitrária, não existindo uma fórmula análoga para as derivadas. Assim, somos naturalmente levados a definir a derivada fracional a partir das fórmulas obtidas para as integrais e dos resultados conhecidos das derivadas clássicas usuais.

O caminho formal para o tratamento de derivadas fracionárias é aquele seguido pelo matemático tcheco Anton Carl Grünwald (1838-1920) e pelo russo Alexei Vassilievitch Letnikov (1837-1888), que construíram, durante os anos de 1867 e 1868, o ferramental matemático hoje conhecido como formalismo de Grünwald-Letnikov, que consiste numa 
generalização da abordagem tradicional do cálculo diferencial, por meio da utilização de limites e somas de Riemann, a ordens arbitrárias de derivação, conforme já abordamos no panorama histórico.

Entretanto, como já frisamos antes, embora esta abordagem constitua o caminho formal mais esperado, o desenvolvimento foi diferente do ponto de vista histórico, partindo inicialmente da definição de integrais fracionárias, para ser, posteriormente, estendido a derivadas fracionárias, com base nas fórmulas integrais. É preciso que se diga, todavia, que há mais de uma maneira de se fazer isto.

Derivada Fracional de Riemann-Liouville Suponhamos que se queira calcular uma derivada de ordem $\nu$, não-inteira. Como já dispomos da definição de integral fracionária e da propriedade (F.22), podemos combiná-las com derivações de ordens inteiras para chegarmos ao conceito de derivada fracional. Assim, se quisermos calcular uma derivada de ordem 2.7, por exemplo, podemos realizar uma integração de ordem 0.3 e, em seguida, derivar 3 vezes, atingindo o resultado desejado.

Contudo, também podemos derivar primeiro e, somente então, efetuar a integração de ordem 0.3. A questão que emerge é se a ordem importa e, nesse caso, qual seria a melhor opção. Esses processos aparecem esquematizados na figura 129.

Figura 129: Definições esquerda e direita da derivada fracional

Definição esquerda

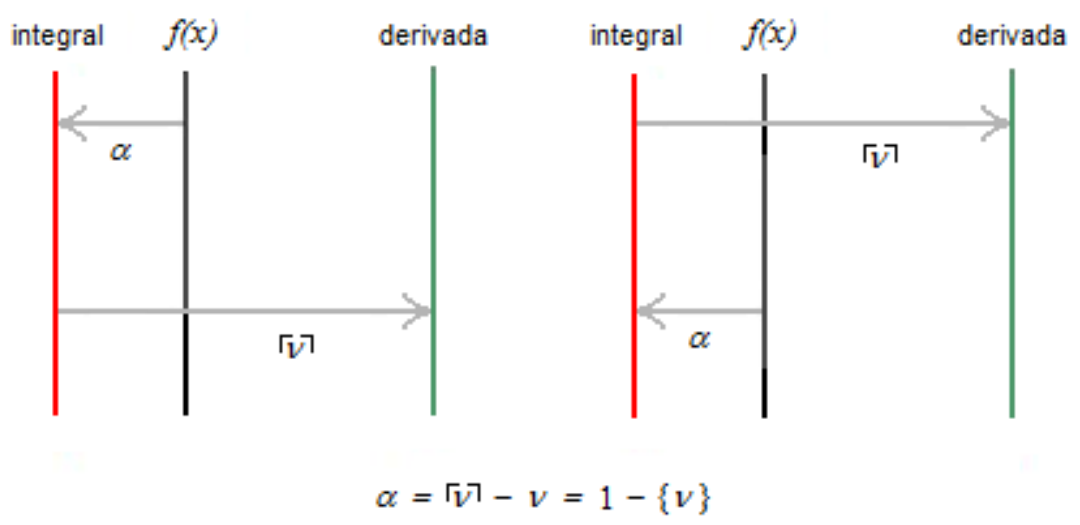

Então, generalizando esse conceito, digamos que, para o cálculo de uma derivada de ordem não-inteira $\nu>0$, efetuemos antes uma integração de ordem $\lceil\nu\rceil-\nu$, seguida de uma derivada de ordem $\lceil\nu\rceil$. Com esse processo, visto que $\nu=\lfloor\nu\rfloor+\{\nu\}$ e $\lceil\nu\rceil=\lfloor\nu\rfloor+1$, 
obteríamos

$$
D^{\nu} f(x)=D^{\lfloor\nu\rfloor} D^{\{\nu\}} f(x)=D^{\lceil\nu\rceil-1} D^{\{\nu\}} f(x)=D^{\lceil\nu\rceil} D^{-1+\{\nu\}} f(x)
$$

e, uma vez que $1-\{\nu\}=\lceil\nu\rceil-\nu>0$, então $-1+\{\nu\}<0$, o que implica que a segunda diferintegral acima é, de fato, uma integral fracional de ordem $\lceil\nu\rceil-\nu$, i.e.,

$$
\begin{gathered}
D^{\nu} f(x)=D^{\lceil\nu\rceil}\left\{D^{-(\lceil\nu\rceil-\nu)} f(x)\right\} \Rightarrow \\
D^{\nu} f(x)=D^{\lceil\nu\rceil}\left\{\frac{1}{\Gamma(\lceil\nu\rceil-\nu)} \int_{0}^{x}(x-u)^{-\{\nu\}} f(u) d u\right\}, \quad \nu \in \mathbb{R}_{+}^{*} \backslash \mathbb{N}
\end{gathered}
$$

Essa fórmula é conhecida na literatura do cálculo fracional como definição esquerda ou derivada fracional de Riemann-Liouville (DFRL) .

Note-se que a fórmula não é válida para números naturais. Todavia, se $\nu$ for um número natural não há necessidade de usar a definição acima, bastando realizar a operação de derivada diretamente, ou seja,

$$
D^{\nu} f(x)=\frac{d^{\nu}}{d x^{\nu}} f(x), \quad \nu \in \mathbb{N}
$$

Naturalmente, se partirmos das definições das integrais fracionais de Riemann, mais gerais, a derivada fracional de Riemann (DFR) se torna:

$$
D_{a}^{\nu} f(x)=D^{\lceil\nu\rceil}\left\{\frac{1}{\Gamma(\lceil\nu\rceil-\nu)} \int_{a}^{x} \frac{f(u)}{(x-u)^{\{\nu\}}} d u\right\}, \quad \nu \in \mathbb{R}_{+}^{*} \backslash \mathbb{N}
$$

E, evidentemente, a versão de Liouville (DFL) adota, como antes, $a=-\infty$.

Resumindo as diferentes derivadas:

- DFRL : $D^{\nu} f(x)=D^{\lceil\nu\rceil}\left[D_{0}^{-\alpha} f(x)\right]$

- DFR : $D_{a}^{\nu} f(x)=D^{\lceil\nu\rceil}\left[D_{a}^{-\alpha} f(x)\right]$

- DFL : $D_{-\infty}^{\nu} f(x)=D^{\lceil\nu\rceil}\left[D_{-\infty}^{-\alpha} f(x)\right]$

Em todos os casos tem-se $\nu>0, \quad \alpha=\lceil\nu\rceil-\nu>0$ e, naturalmente, $D^{\lceil\nu\rceil} \equiv \frac{d^{\lceil\nu\rceil}}{d x^{\lceil\nu\rceil}}$.

Uma observação importante diz respeito à condição de $\nu$ ser um número complexo. Nesse caso, a função teto deve tomar apenas a parte real, uma vez que a derivação está sendo feita pela abordagem clássica, quer dizer, com ordem inteira. Desse modo, a parte imaginária deve ser mantida na integral, que já foi definida para complexos. Em suma, quando escrevemos $\lceil\nu\rceil$, estamos, na verdade, considerando $\lceil\nu\rceil \equiv \Re(\lceil\nu\rceil)$ 
Exemplo 14: Neste exemplo vamos calcular, usando a propriedade (F.22), a derivada fracional de grau $\nu$, com $\Re(\nu)>0$, da função $x^{\alpha}$ pela versão de Riemann-Liouville:

$$
D^{\nu} x^{\alpha}=D^{\lceil\nu\rceil}\left[D^{-(\lceil\nu\rceil-\nu)} x^{\alpha}\right]=D^{\lceil\nu\rceil}\left[\frac{\Gamma(\alpha+1)}{\Gamma(\alpha+\lceil\nu\rceil-\nu+1)} x^{\alpha+\lceil\nu\rceil-\nu}\right]
$$

Porém, pelo resultado da derivação clássica

$$
D^{n} x^{\alpha}=\frac{\Gamma(\alpha+1)}{\Gamma(\alpha-n+1)} x^{\alpha-n}
$$

obtém-se

$$
\begin{aligned}
& D^{\nu} x^{\alpha}=D^{\lceil\nu\rceil}\left[\frac{\Gamma(\alpha+1)}{\Gamma(\alpha+\lceil\nu\rceil-\nu+1)} x^{\alpha+\lceil\nu\rceil-\nu}\right]= \\
& =\frac{\Gamma(\alpha+1)}{\Gamma(\alpha+\lceil\nu\rceil-\nu+1)} \frac{\Gamma(\alpha+\lceil\nu\rceil-\nu+1)}{\Gamma(\alpha-\nu+1)} x^{\alpha-\nu},
\end{aligned}
$$

ou seja, para $\nu \in \mathbb{C}$, vale:

$$
D^{\nu} x^{\alpha}=\frac{\Gamma(\alpha+1)}{\Gamma(\alpha-\nu+1)} x^{\alpha-\nu}, \quad(\Re(\nu)>0, \Re(\alpha)>-1)
$$

Logo, a DFRL de uma função polinomial segue a mesma regra que a IFRL dessa função, o que nos leva a estabelecer uma fórmula geral para a diferintegral de qualquer ordem de uma função polinomial, unificando as equações (F.12) e (F.25) na forma:

$$
D^{\nu} x^{\alpha}=\frac{\Gamma(\alpha+1)}{\Gamma(\alpha-\nu+1)} x^{\alpha-\nu}
$$

Exemplo 15: É conveniente, conforme foi feito no caso da IFR, efetuar o cálculo da DFR de um polinômio fora da origem:

$$
D_{a}^{\nu}(x-a)^{\mu}=D_{a}^{\lceil\nu\rceil}\left[D_{a}^{-\alpha}(x-a)^{\mu}\right]
$$

onde $\alpha=\lceil\nu\rceil e_{a} D_{x}^{\lceil\nu\rceil} \equiv\left(\frac{d}{d x}\right)^{\lceil\nu\rceil}$, pois a derivada é de ordem inteira. Então,

$$
\begin{aligned}
D_{a}^{\nu}(x-a)^{\mu} & =D_{a}^{\lceil\nu\rceil}\left[\frac{\Gamma(\mu+1)}{\Gamma(\mu+\nu+1)}(x-a)^{\mu+\nu}\right]= \\
& =\frac{\Gamma(\mu+1)}{\Gamma(\mu-\nu+1)}(x-a)^{\mu-\nu}, \quad \Re(\mu)>-1
\end{aligned}
$$

De acordo com o esperado, o resultado obtido é consistente com os anteriores. Assim, podemos reduzir ambas as expressões, a da IFR e a da DFR, a uma fórmula comum:

$$
D_{a}^{\nu}(x-a)^{\mu}=\frac{\Gamma(\mu+1)}{\Gamma(\mu-\nu+1)}(x-a)^{\mu-\nu}, \quad \Re(\mu)>-1, \forall \nu \in \mathbb{C}
$$


Usando esse resultado, obtemos, de forma natural, que $D^{0} x^{\mu}=x^{\mu}$. Com isso, também podemos calcular a DFRL de qualquer ordem de uma constante $k \in \mathbb{C}$, obtendo:

$$
D^{\nu} k \equiv D^{\nu}\left(k x^{0}\right)=\frac{k \Gamma(1)}{\Gamma(1-\nu)} x^{-\nu}
$$

Constata-se aqui uma divergência importante em relação ao resultado clássico, pois a derivada fracional de uma constante não se anula, como ocorre no caso de uma derivada inteira usual. Este ponto constituirá uma desvantagem para a DFRL no processo de solução de equações diferenciais fracionais (EDFs).

Derivada Fracional de Caputo Vimos que na DFR a derivação fracional é obtida através de uma derivação clássica sobre uma integral fracional. Por outro lado, também é possível estabelecer esse resultado efetuando-se as operações em ordem inversa, quer dizer, derivando primeiro a função em uma ordem inteira para depois integrá-la na ordem fracionária, conforme esquematizado na figura 129. Como a operação assim definida difere da anterior, é preciso denotá-la por um outro símbolo. Usaremos a notação ${ }^{c} D$. Então, nesse caso, para $\nu \in \mathbb{C}, \Re(\nu)>0$, tem-se:

$$
{ }^{c} D^{\nu} f(x):=D^{-(\lceil\nu\rceil-\nu)}\left\{D^{\lceil\nu\rceil} f(x)\right\}
$$

Abrindo a notação acima, chegamos a

$$
{ }^{c} D^{\nu} f(x)=\frac{1}{\Gamma(\lceil\nu\rceil-\nu)} \int_{0}^{x}(x-u)^{-\{\nu\}} f^{(\lceil\nu\rceil)}(u) d u, \quad \nu \in \mathbb{R}_{+}^{*} \backslash \mathbb{N}
$$

Essa segunda maneira de realizar a operação é chamada de definição direita ou derivada fracional de Caputo (DFC). Naturalmente, para valores inteiros de $\nu$, continua valendo a regra tradicional da derivada clássica:

$$
{ }^{c} D^{\nu} f(x)=\frac{d^{\nu} f(x)}{d x^{\nu}}, \quad \nu \in \mathbb{N}
$$

Para um intervalo mais genérico, com terminal inferior $a$, reescrevemos a derivada fracional de Caputo como

$$
{ }^{c} D_{a}^{\nu} f(x)=\frac{1}{\Gamma(\lceil\nu\rceil-\nu)} \int_{a}^{x} \frac{f^{(\lceil\nu\rceil)}(u)}{(x-u)^{\{\nu\}}} d u, \quad \nu \in \mathbb{R}_{+}^{*} \backslash \mathbb{N}
$$

designando-a por derivada fracional de Caputo-Riemann (DFCR). Lembramos que o resultado é válido também para $\nu \in \mathbb{C} \operatorname{com} R e(\nu)>0$, desde que seja mantida a condição de $\lceil\nu\rceil$ englobar apenas sua parte real. 
Uma vez vez que as derivadas de caputo são efetuadas conjuntamente com as integrais de Riemann, isto é, na versão de Liouville ou de Riemman-Liouville, ou mesmo numa versão mais geral, com terminal a qualquer, usaremos, em prol da clareza, uma terminologia mais específica, de acordo com a versão utilizada na integração, reservando a denominação de derivada de Caputo apenas para a versão com a IFRL. Designaremos, então:

- ${ }^{c} D_{0}^{\nu}$ : Derivada fracional de Caputo (DFC)

- ${ }^{c} D_{a}^{\nu}$ : Derivada fracional de Caputo-Riemann (DFCR)

- ${ }^{c} D_{-\infty}^{\nu}$ : Derivada fracional de Caputo-Liouville (DFCL)

- ${ }_{x}^{c} D_{\infty}^{\nu} \equiv{ }^{c} W_{\infty}^{\nu}$ : Derivada fracional de Caputo-Weyl (DFCW)

Exemplo 16: Procedemos agora ao cálculo da DFC de uma função exponencial. Já sabemos que a DFC de uma função $f(x)$ é

$$
{ }_{0}^{c} D_{x}^{\nu} f(x)=\frac{1}{\Gamma(\lceil\nu\rceil-\nu)} \int_{0}^{x}(x-u)^{-\{\nu\}} f^{(\lceil\nu\rceil)}(u) d u
$$

Utilizando essa fórmula para calcular a DFC de $e^{\lambda x}$, temos

$$
{ }^{c} D^{\nu} e^{\lambda x}=\frac{1}{\Gamma(\lceil\nu\rceil-\nu)} \int_{0}^{x}(x-u)^{-\{\nu\}} \frac{d^{\lceil\nu\rceil}}{d u^{\lceil\nu\rceil}} e^{\lambda u} d u
$$

Porém,

$$
\frac{d^{\lceil\nu\rceil}}{d u^{\lceil\nu\rceil}} e^{\lambda u}=\lambda^{\lceil\nu\rceil} e^{\lambda u} \Rightarrow{ }^{c} D^{\nu} e^{\lambda x}=\frac{\lambda^{\lceil\nu\rceil}}{\Gamma(\lceil\nu\rceil-\nu)} \int_{0}^{x}(x-u)^{-\{\nu\}} e^{\lambda u} d u
$$

Fazendo a substituição $x-u=t$, segue

$$
{ }^{c} D^{\nu} e^{\lambda x}=\frac{\lambda^{\lceil\nu\rceil} e^{\lambda x}}{\Gamma(\lceil\nu\rceil-\nu)} \int_{0}^{x} t^{-\{\nu\}} e^{-\lambda t} d t
$$

Agora, redenominando $\lambda t=y$, obtém-se

$$
{ }^{c} D^{\nu} e^{\lambda x}=\frac{\lambda^{\lceil\nu\rceil} e^{\lambda x}}{\Gamma(\lceil\nu\rceil-\nu)} \lambda^{\{\nu\}-1} \int_{0}^{\lambda x} y^{(\lceil\nu\rceil-\nu)-1} e^{-y} d y
$$

Reconhecendo na integral a expressão da função gama incompleta, podemos então escrever:

$$
{ }^{c} D^{\nu} e^{\lambda x}=\frac{\lambda^{\nu}}{\Gamma(\lceil\nu\rceil-\nu)} e^{\lambda x} \gamma(\lceil\nu\rceil-\nu, \lambda x), \quad \nu \in \mathbb{R}_{+}^{*} \backslash \mathbb{N}
$$


Essa expressão é parecida com a fórmula (F.14), obtida no caso da integração por IFRL, exceto pelo surgimento do termo $\lceil\nu\rceil$ no argumento das funções gama. Contudo, assim como ocorre naquela expressão, esta última também é uma função transcendente de difícil operacionalidade. Por essa razão, torna-se conveniente averiguar a existência de um resultado mais apropriado para a derivação de funções exponenciais. Com esse objetivo, vamos calcular a DFRL de uma integral exponencial conforme dada na equação (F.20).

Exemplo 17: Usando o resultado (F.20), vamos determinar a DFL de uma função exponencial, quer dizer, a partir de uma derivação clássica sobre uma IFL. Então, definindo:

$$
D_{-\infty}^{\nu} e^{\lambda x}:=D^{\lceil\nu\rceil}\left[D_{-\infty}^{-(\lceil\nu\rceil-\nu)} e^{\lambda x}\right]
$$

segue que

$$
\begin{gathered}
D^{\lceil\nu\rceil}\left[D_{-\infty}^{-(\lceil\nu\rceil-\nu)} e^{\lambda x}\right]=D^{\lceil\nu\rceil}\left(\lambda^{-(\lceil\nu\rceil-\nu)} e^{\lambda x}\right)= \\
=\lambda^{-(\lceil\nu\rceil-\nu)}\left(D^{\lceil\nu\rceil} e^{\lambda x}\right)=\lambda^{-(\lceil\nu\rceil-\nu)} \lambda^{\lceil\nu\rceil} e^{\lambda x}=\lambda^{\nu} e^{\lambda x} \Rightarrow \\
\Rightarrow D_{-\infty}^{\nu} e^{\lambda x}=\lambda^{\nu} e^{\lambda x}, \quad \forall \nu \in \mathbb{R} \backslash \mathbb{N}
\end{gathered}
$$

Em outras palavras, para funções exponenciais, existe uma equivalência entre a integral convencional $\left(\frac{d}{d x}\right)^{n} e^{\lambda x},(n \in \mathbb{N})$ e o resultado obtido da definição acima, i.e., $D_{-\infty}^{\nu} e^{\lambda x}, \forall \nu \in \mathbb{R} \backslash \mathbb{N}$, onde $D_{-\infty}^{\nu} \equiv D^{\lceil\nu\rceil}\left(D_{-\infty}^{-(\lceil\nu\rceil-\nu)}\right)$. De fato, o resultado acima é válido não apenas para todos os reais, mas também para os complexos. Ademais, é semelhante à fórmula da IFL de exponenciais. Portanto, podemos escrever:

$$
D_{-\infty}^{\nu} e^{\lambda x}=\lambda^{\nu} e^{\lambda x} \quad(\forall \lambda, \nu \in \mathbb{C})
$$

Além dessas funções, o cálculo da integração e derivação fracionais da função $\ln x$ fornece os seguintes resultados (HILFER et al., 2000):

$$
\begin{aligned}
D^{-\nu} \ln x & =\frac{x^{\nu}}{\Gamma(\nu+1)}[\ln x-\psi(\nu+1)+\psi(1)] \\
D^{\nu} \ln x & =\frac{x^{-\nu}}{\Gamma(1-\nu)}[\ln x-\psi(1-\nu)-\gamma]
\end{aligned}
$$

onde $\gamma$ é a constante de Euler-Mascheroni.

Relação entre DFR e DFCR Uma das características distintivas do Cálculo Fracional em relação ao cálculo tradicional é a variedade de modos possíveis para a derivação. 
Se todas fornecessem o mesmo resultado não haveria problema, mas não teria sentido distinguir entre as várias abordagens. No entanto, não é o que ocorre, pois o resultado da derivação pelo método de Caputo fornece resultado diverso do obtido pela derivação de Riemann. Por esse motivo, torna-se fundamental escolher o método mais adequado, conforme as necessidades e limitações inerentes a cada caso. Em suma, nosso intuito nessa seção é estabelecer a diferença entre a DFR e a DFCR para uma função $f(x)$ genérica.

Seja $f(x)$ de classe $C^{\lceil\nu\rceil}$, com $f^{(\lceil\nu\rceil)}$ integrável, para um dado $\nu \in \mathbb{C}$. Então, a DFR da função $f(x)$ é

$$
D_{a}^{\nu} f(x)=D^{\lceil\nu\rceil}\left[D_{a}^{-\alpha} f(x)\right]=D^{\lceil\nu\rceil}\left\{\frac{1}{\Gamma(\alpha)} \int_{a}^{x}(x-u)^{\alpha-1} f(u) d u\right\}
$$

onde $\alpha=\lceil\nu\rceil-\nu$. Integrando por partes $\operatorname{com} \int_{a}^{x}(x-u)^{\alpha-1} d u=\frac{1}{\alpha}(x-a)^{\alpha}$, obtemos:

$$
D_{a}^{\nu} f(x)=\frac{1}{\Gamma(\alpha)} D^{\lceil\nu\rceil}\left\{\frac{(x-a)^{\alpha}}{\alpha} f(a)+\frac{1}{\alpha} \int_{a}^{x}(x-u)^{\alpha} f^{\prime}(u) d u\right\}
$$

Repetindo a integração por partes, tem-se

$D_{a}^{\nu} f(x)=\frac{1}{\Gamma(\alpha)} D^{\lceil\nu\rceil}\left\{\frac{(x-a)^{\alpha}}{\alpha} f(a)+\frac{(x-a)^{\alpha+1}}{\alpha(\alpha+1)} f^{\prime}(a)+\frac{1}{\alpha(\alpha+1)} \int_{a}^{x}(x-u)^{\alpha+1} f^{\prime \prime}(u) d u\right\}$

Se dermos prosseguimento a esse processo, após um total de $\lceil\nu\rceil$ integrações por partes obteremos

$$
\begin{gathered}
D_{a}^{\nu} f(x)=\frac{1}{\Gamma(\alpha)} D^{\lceil\nu\rceil}\left\{\frac{(x-a)^{\alpha}}{\alpha} f(a)+\frac{(x-a)^{\alpha+1}}{\alpha(\alpha+1)} f^{\prime}(a)+\frac{(x-a)^{\alpha+2}}{\alpha(\alpha+1)(\alpha+2)} f^{\prime \prime}(a)+\cdots\right. \\
\left.\cdots+\frac{(x-a)^{\alpha+\lceil\nu\rceil-1} f^{(\lceil\nu\rceil-1)}(a)}{\alpha(\alpha+1) \ldots(\alpha+\lceil\nu\rceil-1)}+\frac{1}{\alpha(\alpha+1) \ldots(\alpha+\lceil\nu\rceil-1)} \int_{a}^{x}(x-u)^{\alpha+\lceil\nu\rceil-1} f^{(\lceil\nu\rceil)}(u) d u\right\}
\end{gathered}
$$

Reescrevendo os produtos em termos das funções gama e substituindo o valor de $\alpha$, chega-se a

$D_{a}^{\nu} f(x)=D^{\lceil\nu\rceil}\left[\sum_{k=0}^{\lfloor\nu\rfloor} \frac{(x-a)^{\lceil\nu\rceil-\nu+k} f^{(k)}(a)}{\Gamma(\lceil\nu\rceil-\nu+k+1)}+\frac{1}{\Gamma(2\lceil\nu\rceil-\nu)} \int_{a}^{x}(x-u)^{2\lceil\nu\rceil-\nu-1} f^{(\lceil\nu\rceil)}(u) d u\right]$

Efetuando em seguida a derivação, segue:

$$
D_{a}^{\nu} f(x)=\sum_{k=0}^{\lfloor\nu\rfloor} \frac{(x-a)^{k-\nu} f^{(k)}(a)}{\Gamma(k-\nu+1)}+\frac{1}{\Gamma(\lceil\nu\rceil-\nu)} \int_{a}^{x}(x-u)^{\lceil\nu\rceil-\nu-1} f^{(\lceil\nu\rceil)}(u) d u
$$

Entretanto, observando o termo dado pela integral, é possível identificá-lo com a 
DFCR de ordem $\alpha$ da função $f^{(\lceil\nu\rceil)}(x)$, ou seja, $D_{a}^{-(\lceil\nu\rceil-\nu)}\left[f^{(\lceil\nu\rceil)}(x)\right]$, o que nos remete, finalmente, à relação

$$
D_{a}^{\nu} f(x)={ }^{c} D_{a}^{\nu} f(x)+\sum_{k=0}^{\lfloor\nu\rfloor} \frac{(x-a)^{k-\nu}}{\Gamma(k-\nu+1)} f^{(k)}(a)
$$

Uma consequência interessante ocorre no caso limite $a \rightarrow-\infty$, pois

$$
\lim _{a \rightarrow-\infty} \sum_{k=0}^{\lfloor\nu\rfloor} \frac{(x-a)^{k-\nu}}{\Gamma(k-\nu+1)} f^{(k)}(a)=0,
$$

uma vez que, por hipótese, $f^{(k)}(x)$ é limitada e $k-\nu<0, \forall k \in[0,\lfloor\nu\rfloor] \subset \mathbb{N}$. Isto significa, simplesmente, que no caso de o terminal inferior tender a $-\infty$, i.e., quando a DFR se transforma em DFL e a DFCR em DFCL, ambas passam a fornecer o mesmo resultado. Em outros termos, nesse limite as abordagens da derivada fracional de Caputo e da derivada fracional de Liouville coincidem, ou seja,

$$
{ }^{c} D_{-\infty}^{\nu} \equiv D_{-\infty}^{\nu}
$$

Esse fator, em princípio, constitui uma grande vantagem, mas apresenta, por outro lado, o problema da exigência de integrabilidade da função $f(x)$ num intervalo semiinfinito, o que nem sempre é pertinente, principalmente em se tratando de funções periódicas, como é caso essencial do assunto dessa tese.

Além disso, na situação específica de funções polinomiais no ramo principal das séries de Laurent, essa abordagem conduz a infinitos. Com vistas a problemas dessa natureza, é interessante passar a abordar o tratamento dos operadores de Weyl. Antes, no entanto, vamos rever e ampliar para o cálculo fracional alguns conceitos fundamentais sobre transformadas.

Relação entre derivadas e integrais fracionais Vamos demonstrar aqui que a derivada fracional e a integral fracional de Riemann não são inversas perfeitas uma da outra, ou seja, não comutam, ao contrário do que ocorre no caso clássico.

De fato, o que ocorre é que a DFR de uma IFR fornece o operador identidade, como seria de se esperar, mas a IFR de uma DFR não obedece a essa condição, produzindo um resultado diferente e um pouco mais complicado. Em outros termos, os operadores fracionais de Riemann de derivação e operação não são comutativos. 
Mostremos primeiro o caso mais simples:

$$
D_{a}^{\nu}\left[D_{a}^{-\nu} f(x)\right]=f(x)
$$

\section{Demonstração 14:}

De fato, usando a definição de derivação fracional e a propriedade de semigrupo, respectivamente, temos

$$
D_{a}^{\nu}\left[D_{a}^{-\nu} f(x)\right]=D_{a}^{\lceil\nu\rceil}\left\{D_{a}^{-(\lceil\nu\rceil-\nu)}\left[D_{a}^{-\nu} f(x)\right]\right\}=D_{a}^{\lceil\nu\rceil}\left[D_{a}^{-\lceil\nu\rceil} f(x)\right]=f(x),
$$

para $\forall \nu \in \mathbb{C}$, onde devemos lembrar que estamos assumindo tacitamente que $\lceil\nu\rceil: \equiv\lceil\Re(\nu)\rceil . \log O$,

$$
D_{a}^{\nu} D_{a}^{-\nu} \equiv I
$$

Por outro lado, a demonstração de que a inversa não procede constitui tarefa um pouco mais difícil. Vamos então efetuar o cálculo para a IFR da DFR a fim de mostrar que fornece:

$$
D_{a}^{-\nu}\left[D_{a}^{\nu} f(x)\right]=f(x)-\left.\sum_{k=1}^{\lceil\nu\rceil} \frac{(x-a)^{\nu-k}}{\Gamma(\nu-k+1)} D_{a}^{\nu-k} f(u)\right|_{u=a}
$$

\section{Demonstração 15:}

$$
\begin{aligned}
D_{a}^{-\nu}\left[D_{a}^{\nu} f(x)\right] & =\frac{1}{\Gamma(\nu)} \int_{a}^{x}(x-u)^{\nu-1} D_{a}^{\nu} f(u) d u= \\
& =\frac{d}{d x}\left\{\frac{1}{\Gamma(\nu+1)} \int_{a}^{x}(x-u)^{\nu} D_{a}^{\lceil\nu\rceil}\left[D_{a}^{-(\lceil\nu\rceil-\nu)} f(u)\right] d u\right\}
\end{aligned}
$$

Chamando $\alpha=\lceil\nu\rceil-\nu$ e $D_{a}^{-\alpha} f(u)=g(u)$ e fazendo uma integração por partes, reescrevemos:

$$
\begin{aligned}
D_{a}^{-\nu}\left[D_{a}^{\nu} f(x)\right] & =\frac{d}{d x}\left\{\left.\frac{-(x-a)^{\nu}}{\Gamma(\nu+1)} D_{a}^{\lceil\nu\rceil-1} g(u)\right|_{u=a}+\right. \\
& \left.+\frac{1}{\Gamma(\nu)} \int_{a}^{x}(x-u)^{\nu-1} D_{a}^{\lceil\nu\rceil-1} g(u) d u\right\}
\end{aligned}
$$

Efetuando uma segunda integração por partes acima, obtém-se:

$$
\begin{aligned}
D_{a}^{-\nu}\left[D_{a}^{\nu} f(x)\right] & =\frac{d}{d x}\left\{-\left.\frac{(x-a)^{\nu}}{\Gamma(\nu+1)} D_{a}^{\lceil\nu\rceil-1} g(u)\right|_{u=a}-\left.\frac{(x-a)^{\nu-1}}{\Gamma(\nu)} D_{a}^{\lceil\nu\rceil-2} g(u)\right|_{u=a}+\right. \\
& \left.+\frac{1}{\Gamma(\nu-1)} \int_{a}^{x}(x-u)^{\nu-2} D_{a}^{\lceil\nu\rceil-2} g(u) d u\right\}
\end{aligned}
$$


Prosseguindo, após $\lceil\nu\rceil$ integrações por partes, obteremos

$$
\begin{aligned}
D_{a}^{-\nu}\left[D_{a}^{\nu} f(x)\right] & =\frac{d}{d x}\left\{-\left.\sum_{k=1}^{\lceil\nu\rceil} \frac{(x-a)^{\nu-k+1}}{\Gamma(\nu-k+2)} D_{a}^{\lceil\nu\rceil-k} g(u)\right|_{u=a}+\right. \\
& \left.+\frac{1}{\Gamma(\nu-\lceil\nu\rceil+1)} \int_{a}^{x}(x-u)^{\nu-\lceil\nu\rceil} g(u) d u\right\}
\end{aligned}
$$

Voltando a substituir o valor de $g(u)$, tem-se que o termo da integral se torna

$$
\begin{gathered}
\frac{1}{\Gamma(\nu-\lceil\nu\rceil+1)} \int_{a}^{x}(x-u)^{\nu-\lceil\nu\rceil} D_{a}^{-(\lceil\nu\rceil-\nu)} f(u) d u= \\
=D_{a}^{-(\lceil\nu\rceil-\nu+1)}\left[D_{a}^{-(\lceil\nu\rceil-\nu)} f(x)\right]=D_{a}^{-1} f(x),
\end{gathered}
$$

onde usamos a propriedade de semigrupo na última igualdade. Já o termo da somatória assume a forma

$$
\begin{gathered}
-\sum_{k=1}^{\lceil\nu\rceil} \frac{(x-a)^{\nu-k+1}}{\Gamma(\nu-k+2)} D_{a}^{\lceil\nu\rceil-k}\left[D_{a}^{-(\lceil\nu\rceil-\nu)} f(u)\right]_{u=a}= \\
=-\left.\sum_{k=1}^{\lceil\nu\rceil} \frac{(x-a)^{\nu-k+1}}{\Gamma(\nu-k+2)} D_{a}^{\nu-k} f(u)\right|_{u=a}
\end{gathered}
$$

Logo, efetuando a derivada que antecede esses termos, chega-se a

$$
D_{a}^{-\nu}\left[D_{a}^{\nu} f(x)\right]=f(x)-\left.\sum_{k=1}^{\lceil\nu\rceil} \frac{(x-a)^{\nu-k}}{\Gamma(\nu-k+1)} D_{a}^{\nu-k} f(u)\right|_{u=a}
$$

E isto demonstra a equação (F.39).

Portanto, conclui-se que a DFR é a inversa à esquerda da IFR, mas não à direita, mostrando que não vale a comutatividade.

A partir desses resultados e das propriedades estudadas, podemos analisar certos casos específicos:

- $0 \leq \nu \leq \sigma$ :

$$
D_{a}^{\nu}\left[D_{a}^{-\sigma} f(x)\right]=D_{a}^{\nu}\left\{D_{a}^{-\nu}\left[D_{a}^{-(\sigma-\nu)} f(x)\right]\right\}=D_{a}^{-(\sigma-\nu)} f(x)
$$


- $0 \leq \sigma<\nu$ :

$$
\begin{aligned}
D_{a}^{\nu}\left[D_{a}^{-\sigma} f(x)\right] & =D_{a}^{\lceil\nu\rceil}\left\{D_{a}^{-(\lceil\nu\rceil-\nu)}\left[D_{a}^{-\sigma} f(x)\right]\right\}= \\
& =\left(\frac{d}{d x}\right)^{\lceil\nu\rceil}\left[D_{a}^{-(\lceil\nu\rceil-(\nu-\sigma))} f(x)\right]= \\
& =D_{a}^{\lceil\nu\rceil}\left\{D_{a}^{-\lceil\nu\rceil}\left[D_{a}^{\nu-\sigma} f(x)\right]\right\}= \\
& =D_{a}^{\nu-\sigma} f(x)
\end{aligned}
$$

Como ambos os casos levam ao mesmo resultado, podemos resumi-los como

$$
D_{a}^{\nu}\left[D_{a}^{-\sigma} f(x)\right]=D_{a}^{\nu-\sigma} f(x), \quad \Re(\nu), \Re(\sigma) \geq 0
$$

No entanto, também devem ser considerados os outros casos, i.e., $D_{a}^{-\nu}\left[D_{a}^{\sigma} f(x)\right]$. Como a subdivisão dos casos aqui também segue o que foi mostrado acima, a análise pode ser reduzida a uma única situação:

$$
\begin{aligned}
D_{a}^{-\nu}\left[D_{a}^{\sigma} f(x)\right] & =D_{a}^{\sigma-\nu}\left\{D_{a}^{-\sigma}\left[D_{a}^{\sigma} f(x)\right]\right\}= \\
& =D_{a}^{\sigma-\nu}\left\{f(x)-\left.\sum_{k=1}^{\lceil\nu\rceil} \frac{(x-a)^{\sigma-k}}{\Gamma(\sigma-k+1)}\left[D_{a}^{\sigma-k} f(x)\right]\right|_{x=a}\right\}= \\
& =D_{a}^{\sigma-\nu} f(x)-\sum_{k=1}^{\lceil\nu\rceil} \frac{(x-a)^{\nu-k}}{\Gamma(\nu-k+1)}\left[D_{a}^{\sigma-k} f(x)\right]_{x=a},
\end{aligned}
$$

onde usamos

$$
D_{a}^{\sigma-\nu}\left\{\frac{(x-a)^{\sigma-k}}{\Gamma(\sigma-k+1)}\right\}=\frac{(x-a)^{\nu-k}}{\Gamma(\nu-k+1)}
$$

Portanto,

$$
D_{a}^{-\nu}\left[D_{a}^{\sigma} f(x)\right]=D_{a}^{\sigma-\nu} f(x)-\sum_{k=1}^{\lceil\nu\rceil} \frac{(x-a)^{\nu-k}}{\Gamma(\nu-k+1)}\left[D_{a}^{\sigma-k} f(x)\right]_{x=a}
$$

\section{F.9 Transformadas, Convoluções e Correlações}

\section{F.9.1 Transformadas de Fourier}

Partindo da série de Fourier:

$$
f(x)=\sum_{r=-\infty}^{\infty} c^{r} e^{i r q x}
$$


onde

$$
c^{r}=\frac{1}{2 L} \int_{-L}^{L} f(\xi) e^{-i r q \xi} d \xi
$$

e escrevendo-a em termos da mudança de variável $r q=y$, utilizando uma variação indicial inteira $\Delta r$, tem-se

$$
f(x)=\sum_{r=-\infty}^{\infty} \frac{1}{q} c^{r} e^{i(r q) x}(q \Delta r)
$$

com

$$
c^{r} \longrightarrow c(y)=\frac{1}{2 L} \int_{-L}^{L} f(\xi) e^{-i y \xi} d \xi
$$

Definindo-se uma função $g(y):=\frac{c(y)}{q}$ e tomando seu limite para $L \rightarrow \infty$, obtém-se uma nova função $\mathcal{F}(y)$ tal que

$$
\mathcal{F}(y):=\lim _{L \rightarrow \infty} g(y)=\frac{1}{2 \pi} \int_{-\infty}^{\infty} f(\xi) e^{-i y \xi} d \xi
$$

Então, usando esse resultado na expansão em série, no limite $L \rightarrow \infty$ e $\Delta y \rightarrow 0$, chegamos a uma soma de Riemann, de forma que

$$
f(x)=\lim _{\substack{\Delta y \rightarrow 0 \\ L \rightarrow \infty}} \sum_{y=-\infty}^{\infty} g(y) e^{i y x} \Delta y \longrightarrow \int_{-\infty}^{\infty} \mathcal{F}(y) e^{i y x} d y
$$

o que nos conduz às equações conhecidas como Transformadas de Fourier:

$$
\begin{aligned}
\mathcal{F}(y) & =\frac{1}{2 \pi} \int_{-\infty}^{\infty} f(x) e^{-i y x} d x \\
f(x) & =\int_{-\infty}^{\infty} \mathcal{F}(y) e^{i x y} d y
\end{aligned}
$$

Vamos denotar a transformada de Fourier de uma função $f(x)$ por

$$
\mathfrak{F}\{f(x)\}=\mathcal{F}(y)
$$

É comum ocorrerem transformadas nos domínios de tempo e frequência angular, $t$ e $\omega$, de forma que tenhamos pares $\mathfrak{F}\{f(t)\}=\mathcal{F}(\omega)$ e $\mathfrak{F}^{-1}\{\mathcal{F}(\omega)\}=f(t)$.

Há uma outra definição, mais em voga na atualidade, baseada em uma simetrização do fator de normalização $\frac{1}{2 \pi}$, que fornece:

$$
\begin{aligned}
\mathcal{F}(y) & =\frac{1}{\sqrt{2 \pi}} \int_{-\infty}^{\infty} f(x) e^{-i y x} d x \\
f(x) & =\frac{1}{\sqrt{2 \pi}} \int_{-\infty}^{\infty} \mathcal{F}(y) e^{i x y} d y
\end{aligned}
$$


Se recombinarmos as expressões anteriores, teremos

$$
f(x)=\frac{1}{2 \pi} \int_{-\infty}^{\infty} e^{i x y} d y \int_{-\infty}^{\infty} f(\xi) e^{-i y \xi} d \xi=\int_{-\infty}^{\infty} f(\xi) d \xi \frac{1}{2 \pi} \int_{-\infty}^{\infty} e^{i(x-\xi) y} d y
$$

Porém, como essa expressão deve valer para uma função genérica, conclui-se, comparando com as propriedades da função delta de Dirac, que a segunda integral deve ser uma representação daquela, isto é,

$$
\begin{aligned}
f(x)=\int_{-\infty}^{\infty} f(\xi) \delta(x-\xi) d \xi & =\int_{-\infty}^{\infty} f(\xi)\left[\frac{1}{2 \pi} \int_{-\infty}^{\infty} e^{i(x-\xi) y} d y\right] d \xi \Rightarrow \\
\delta(x-\xi) & =\frac{1}{2 \pi} \int_{-\infty}^{\infty} e^{i(x-\xi) y} d y
\end{aligned}
$$

Essa expressão é a chamada representação integral da função delta. Naturalmente, devido à simetria da função delta, essa equação vale também para o expoente com sinal negativo, i.e.,

$$
\delta(x-\xi)=\frac{1}{2 \pi} \int_{-\infty}^{\infty} e^{ \pm i(x-\xi) y} d y
$$

Podemos reescrever essa expressão como

$$
\delta(y-a)=\frac{1}{\sqrt{2 \pi}}\left(\frac{1}{\sqrt{2 \pi}} \int_{-\infty}^{\infty} e^{ \pm i a x} e^{-i x y} d x\right)=\frac{1}{\sqrt{2 \pi}} \mathfrak{F}\left\{e^{ \pm i a x}\right\}
$$

e, consequentemente,

$$
\mathfrak{F}\left\{e^{ \pm i a x}\right\}=\sqrt{2 \pi} \delta(y-a)
$$

Mas, da propriedade da função delta

$$
\int_{-\infty}^{\infty} f(x) \delta(x-y) d x=f(y)
$$

segue, imediatamente, que

$$
\mathfrak{F}\{\delta(x-a)\}=\frac{1}{\sqrt{2 \pi}} \int_{-\infty}^{\infty} \delta(x-a) e^{-i x y} d x=\frac{e^{-i a y}}{\sqrt{2 \pi}}
$$

Da transformação (F.46) conclui-se, por substituição direta, que

$$
\mathfrak{F}\left\{\left|e_{m}\right\rangle\right\}=\sqrt{2 \pi} \delta(y-m q)
$$

Seguindo o mesmo procedimento, obtemos uma variedade de transformadas fundamentais para a resolução de equações diferenciais. A seguir, uma pequena tabela com as transformações mais importantes: 
Tabela 14: Principais transformadas de Fourier

\begin{tabular}{|c||c|}
\hline$f(x)$ & $\mathcal{F}(y)$ \\
\hline \hline 1 & $\sqrt{2 \pi} \delta(y)$ \\
\hline$\delta(x-a)$ & $\frac{e^{-i a y}}{\sqrt{2 \pi}}$ \\
\hline $\cos (\alpha x)$ & $\sqrt{\frac{\pi}{2}}[\delta(y+\alpha)+\delta(y-\alpha)]$ \\
\hline $\sin (\alpha x)$ & $i \sqrt{\frac{\pi}{2}}[\delta(y+\alpha)-\delta(y-\alpha)]$ \\
\hline$e^{-a|x|}$ & $\sqrt{\frac{2}{\pi}} \frac{a}{y^{2}+a^{2}}$ \\
\hline$e^{-\frac{a x^{2}}{2}}$ & $\frac{1}{\sqrt{a}} e^{-\frac{y^{2}}{2 a}}$ \\
\hline
\end{tabular}

\section{F.9.2 Transformadas de Laplace a partir de Fourier}

As transformadas de Fourier são extremamente úteis na resolução de EDOs mas, infelizmente, há casos frequentes nos quais sua integral de definição não converge, o que pode ocorrer para algumas funções relativamente simples, como $f(x)=x^{2}$, por exemplo. Nesse caso, pode-se aplicar o recurso de multiplicar tais funções por um fator exponencial $e^{-\sigma x},(\sigma \in \mathbb{R})$, que obrigue a convergência no ramo positivo do domínio. Isso, por outro lado, tende a piorá-la no ramo negativo. Entretanto, é frequente estarmos interessados apenas no domínio positivo, principalmente para equações em função do tempo. Nesse caso, é conveniente fazer uso da função de Heaviside:

$$
H(x)= \begin{cases}0, & x<0 \\ 1, & x>0\end{cases}
$$

Nessas condições, a nova função $f(x) e^{-\sigma x} H(x)$ apresenta o comportamento esperado para convergência da integral e, portanto, sua transformada de Fourier deve existir e ser igual a

$$
g(y)=\int_{-\infty}^{\infty}\left[f(x) e^{-\sigma x} H(x)\right] e^{-i y x} d x=\int_{0}^{\infty} f(x) e^{-(\sigma+i y) x} d x
$$

onde o limite inferior da integral foi mudado para 0, por efeito da função de Heaviside. Chamando então $\sigma+i y=s$ e definindo $F(s): \equiv g(y)$, a expressão resultante:

$$
F(s)=\int_{0}^{\infty} f(x) e^{-s x} d x
$$

é a conhecida Transformada de Laplace. A transformada de Laplace inversa é obtida da equação precedente a esta última, pela aplicação da transformada de Fourier inversa, ou seja,

$$
f(x) e^{-\sigma x} H(x)=\frac{1}{2 \pi} \int_{-\infty}^{\infty} g(y) e^{i x y} d y \quad \Rightarrow \quad f(x) H(x)=\frac{1}{2 \pi} \int_{-\infty}^{\infty} g(y) e^{x(\sigma+i y)} d y
$$


Adotando o critério de escrever a função $f(x) H(x)$ apenas como $f(x)$, considerando, portanto, apenas seu valor no domínio positivo, o que equivale a considerá-la como causal, e fazendo a substituição $y \rightarrow s$, chega-se à Integral de Bromwich ou fórmula de inversão de Mellin:

$$
f(x)=\frac{1}{2 \pi i} \int_{\sigma-i \infty}^{\sigma+i \infty} F(s) e^{s x} d s
$$

onde $\sigma=\Re(s)$.

\section{F.9.3 Convolução Complexa e Correlação Cruzada}

O estabelecimento do produto de convolução parte da ideia de simetria entre a transformada de um produto de duas funções e o produto de suas transformadas. Assim, a convolução dependerá do tipo de transformada envolvida.

Produto de Convolução para a TL Vamos operar primeiro com as transformadas de Laplace sobre duas funções $f$ e $g$ causais, isto é, tais que $f(t)=g(t)=0, \forall t<0$. A condição exigida será o estabelecimento de um produto de funções $f * g$, tal que

$$
\mathcal{L}\{f\} \mathcal{L}\{g\}:=\mathcal{L}\{f * g\}
$$

Então, usando a definição,

$$
\begin{aligned}
\mathcal{L}\{f\} \mathcal{L}\{g\} & =\int_{0}^{\infty} f(\tau) e^{-s \tau} d \tau \int_{0}^{\infty} g(u) e^{-s u} d u= \\
& =\int_{0}^{\infty} \int_{0}^{\infty} f(\tau) g(u) e^{-s(\tau+u)} d \tau d u= \\
& =\int_{\tau}^{\infty} \int_{0}^{\infty} f(\tau) g(t-\tau) d \tau e^{-s t} d t
\end{aligned}
$$

onde fizemos a substituição $\tau+u \rightarrow t$. Agora, usando o teorema de Fubini e a causalidade das funções, tem-se $g(t-\tau)=0, \forall t<\tau$, o que implica

$$
\mathcal{L}\{f\} \mathcal{L}\{g\}=\int_{0}^{\infty} \int_{0}^{\infty} f(\tau) g(t-\tau) d \tau e^{-s t} d t
$$

Por outro lado, pelo mesmo motivo anterior, tem-se

$$
g(t-\tau)=0, \forall \tau>t \Rightarrow \int_{0}^{\infty} f(\tau) g(t-\tau) d \tau=\int_{0}^{t} f(\tau) g(t-\tau) d \tau
$$


o que nos permite escrever

$$
\mathcal{L}\{f\} \mathcal{L}\{g\}=\int_{0}^{\infty}\left(\int_{0}^{t} f(\tau) g(t-\tau) d \tau\right) e^{-s t} d t
$$

Logo, se definirmos o produto de convolução por

$$
(f * g)(t):=\int_{0}^{t} f(\tau) g(t-\tau) d \tau
$$

obteremos a condição arbitrada. Ressaltamos que também se pode escrever

$$
(f * g)(t)=\int_{0}^{\infty} f(\tau) g(t-\tau) d \tau
$$

Produto de Convolução para a TF Partindo do mesmo pressuposto anterior, vamos calcular o produto $\mathfrak{F}\{f\} \mathfrak{F}\{g\}$ e relacioná-lo com a transformada de um produto de convolução de Fourier $\mathfrak{F}\{f * g\}$, a fim de determinar este último. Utilizando a definição das TF, tem-se

$$
\begin{aligned}
\mathcal{F}(\omega) \mathcal{G}(\omega) & =\frac{1}{2 \pi} \int_{-\infty}^{\infty} \int_{-\infty}^{\infty} f(\tau) g(u) e^{-i \omega(\tau+u)} d \tau d u= \\
& =\frac{1}{\sqrt{2 \pi}} \int_{-\infty}^{\infty}\left(\frac{1}{\sqrt{2 \pi}} \int_{-\infty}^{\infty} f(\tau) g(t-\tau) d \tau\right) e^{-i \omega t} d t
\end{aligned}
$$

onde foi efetuada a mudança de variáveis $\tau+u \rightarrow t$.

Por conseguinte, definindo-se o produto de convolução (BUTZER; WESTPHAL, 2000) como:

$$
(f * g)(t):=\frac{1}{\sqrt{2 \pi}} \int_{-\infty}^{\infty} f(\tau) g(t-\tau) d \tau
$$

obtém-se

$$
\mathfrak{F}\{f * g\}=\mathfrak{F}\{f\} \mathfrak{F}\{g\}
$$

ou, alternativamente,

$$
\mathfrak{F}^{-1}\{\mathcal{F} \mathcal{G}\}=f * g
$$

Propriedades da Convolução De modo geral, uma integral de convolução para funções $f, g: I=[a, b] \rightarrow \mathbb{C}$ é definida

$$
(f * g)_{I}=\lambda \int_{a}^{b} f(\tau) g(t-\tau) d \tau
$$

onde o fator $\lambda$ exerce papel semelhante ao de um coeficiente de normalização, podendo ser tomado como unidade para efeito de cálculo genérico, sem perda de generalidade. 
De forma geral, fazendo $t-\tau \longrightarrow \tau^{\prime}$ para funções periódicas em $[a, b]$, vem

$$
f * g=-\int_{t-a}^{t-b} f\left(t-\tau^{\prime}\right) g\left(\tau^{\prime}\right) d \tau^{\prime}=\int_{-b}^{-a} f(t-\tau) g(\tau) d \tau
$$

Se, além disso, o intervalo periódico for simétrico em torno da origem, i.e., $I=[-a, a]$, então

$$
f * g=\int_{-a}^{a} f(\tau) g(t-\tau) d \tau=\int_{-a}^{a} f(t-\tau) g(\tau) d \tau
$$

Um aspecto importante sobre a convolução para Laplace e Fourier diz respeito à sua comutatividade, pois, fazendo-se $t-\tau \longrightarrow u$ nas respectivas definições, obtém-se

$$
f * g=g * f
$$

Em outros termos, nos casos específicos dos produtos de convolução para Laplace e Fourier, tem-se

$$
(f * g)_{\mathcal{L}}=\int_{0}^{t} f(\tau) g(t-\tau) d \tau=\int_{0}^{t} f(t-\tau) g(\tau) d \tau
$$

e

$$
(f * g)_{\mathfrak{F}}=\frac{1}{\sqrt{2 \pi}} \int_{-\infty}^{\infty} f(\tau) g(t-\tau) d \tau=\frac{1}{\sqrt{2 \pi}} \int_{-\infty}^{\infty} f(t-\tau) g(\tau) d \tau
$$

Convolução Cíclica Além disso, para funções periódicas de período $2 \pi$, define-se a convolução cíclica ou circular como:

$$
(f * g)_{\theta}:=\frac{1}{2 \pi} \int_{0}^{2 \pi} f(\theta) g(\alpha-\theta) d \theta=\frac{1}{2 \pi} \int_{0}^{2 \pi} f(\alpha-\theta) g(\theta) d \theta
$$

No caso dessa tese, porém, vamos redefini-la para funções periódicas de período genérico $T=2 L$ na forma

$$
(f * g)_{L}:=\frac{1}{2 L} \int_{-L}^{L} f(\xi) g(x-\xi) d \xi=\frac{1}{2 L} \int_{-L}^{L} f(x-\xi) g(\xi) d \xi
$$

De fato, se for realizada a mudança de variáveis $x-\xi \rightarrow u$ na primeira definição integral acima, segue que

$$
\int_{-L}^{L} f(\xi) g(x-\xi) d \xi \rightarrow \int_{x-L}^{x+L} f(x-u) g(u) d u
$$

No entanto, devido à periodicidade das funções, vale $\int_{x-L}^{x+L} \equiv \int_{-L}^{L}$, o que demonstra a validade da segunda definição integral para a convolução cíclica, ou seja, sua comutatividade. 
Correlação Cruzada A integral de correlação cruzada em um intervalo $[a, b] \in \mathbb{R}$ é definida:

$$
f \star g=\int_{a}^{b} \bar{f}(\tau) g(t+\tau) d \tau
$$

Fazendo $\tau \longrightarrow-\tau^{\prime}$ :

$$
f \star g=\int_{-b}^{-a} \bar{f}(-\tau) g(t-\tau) d \tau
$$

Para intervalos simétricos $[-a, a]$ vale

$$
\begin{gathered}
f \star g=\int_{-a}^{a} \bar{f}(\tau) g(t+\tau) d \tau=\int_{-a}^{a} \bar{f}(-\tau) g(t-\tau) d \tau \Rightarrow \\
{[f \star g](t)=\bar{f}(-t) * g(t)}
\end{gathered}
$$

Portanto, a correlação circular é escrita:

$$
(f \star g)_{L}=\int_{-L}^{L} \bar{f}(\tau) g(t+\tau) d \tau=\int_{-L}^{L} \bar{f}(-\tau) g(t-\tau) d \tau
$$

Ocupar-nos-emos agora em estender tais conceitos ao cálculo fracional.

\section{F.10 Transformadas no Cálculo Fracional}

O intuito dessa seção é generalizar o uso das transformadas de Laplace (TL) e de Fourier (TF) para sua utilização efetiva no cálculo fracional.

A grande vantagem de uma transformada integral reside na propriedade que ela apresenta de transformar equações diferenciais num espaço vetorial das variáveis originais em equações algébricas num outro espaço vetorial, o espaço das transformadas (de " $t$ " em " $s$ ", no caso de Laplace ou de " $t$ " em " $\omega$ ", no de Fourier). A solução torna-se muito mais simples no caso algébrico e, através da transformada inversa, pode-se obter também a solução da equação diferencial. Esse tipo de solução é particularmente apropriado para EDs com condições iniciais, dispensando o cálculo das soluções geral e particular e das constantes arbitrárias.

De modo geral, a TL de uma função $f(t)$ é uma transformada integral com kernel exponencial $K_{\mathcal{L}}(t, s)=\exp (-s t)$, cuja existência depende da classe da função $f$ :

$$
\mathcal{L}\{f(t)\}:=\int_{0}^{\infty} f(t) e^{-s t} d t=F(s), \quad s \in \mathbb{C}
$$

A existência da TL está condicionada à convergência da integral assim definida. Essa integral converge para funções $f$ de ordem exponencial em $t \rightarrow \infty$, i.e., se $\exists M, c, T>0$ : 
$|f(t)| \leq M e^{c t}, t \geq T$. Já o kernel da transformada de Fourier é dado por $K_{\mathcal{F}}(t, \omega)=$ $\exp (-i \omega t)$.

Duas propriedades muito importantes das transformadas integrais são a transformação da derivada de qualquer ordem de uma função e a operação de convolução entre duas funções. Como já falamos sobre a convolução e correlação, vamos nos limitar aqui a expor as transformadas de Laplace e Fourier das derivadas de funções:

1. TL da derivada ordem $n$ :

$$
\mathcal{L}\left\{f^{(n)}(t)\right\}=s^{n} F(s)-\sum_{k=0}^{n-1} s^{n-k-1} f^{(k)}(0)=s^{n} F(s)-\sum_{k=0}^{n-1} s^{k} f^{(n-k-1)}(0)
$$

2. TF da derivada de ordem $n$ :

$$
\mathfrak{F}\left\{f^{(n)}(t)\right\}=(i \omega)^{n} \mathcal{F}(\omega)
$$

Além disso, TL e TF obedecem à linearidade, quer dizer, $\forall a, b \in \mathbb{R}$ :

$$
\begin{aligned}
& \mathcal{L}\{a f(t)+b g(t)\}=a \mathcal{L}\{f(t)\}+b \mathcal{L}\{g(t)\}, \\
& \mathfrak{F}\{a f(t)+b g(t)\}=a \mathfrak{F}\{f(t)\}+b \mathfrak{F}\{g(t)\}
\end{aligned}
$$

TL da Integral Fracional de Riemann-Liouville Partindo da definição da integral fracional de Riemann-Liouville,

$$
{ }_{0} D_{t}^{-\nu} f(t)=\frac{1}{\Gamma(\nu)} \int_{0}^{t}(t-u)^{\nu-1} f(u) d u
$$

nota-se que a integral constitui um produto de convolução:

$$
\int_{0}^{t}(t-u)^{\nu-1} f(u) d u=t^{\nu-1} * f(t)
$$

e, portanto, reinterpretando-se a expressão como um operador integral cujo kernel é

$$
\mathcal{K}_{\nu}(t)=\frac{t^{\nu-1}}{\Gamma(\nu)}
$$

podemos escrever

$$
D^{-\nu} f(t)=\mathcal{K}_{\nu}(t) * f(t)
$$

Em outras palavras, a aplicação do operador de integração fracional sobre uma função $f$, corresponde ao produto de convolução entre essa função e o kernel do operador integral 
$\mathcal{K}_{\nu}$, que vamos denominar kernel fracional. Daí, como $\mathcal{L}\left\{t^{\nu-1}\right\}=\Gamma(\nu) s^{-\nu}$, então,

$$
\begin{aligned}
\mathcal{L}\left\{D^{-\nu} f(t)\right\} & =\mathcal{L}\left\{\mathcal{K}_{\nu}(t) * f(t)\right\}=\frac{1}{\Gamma(\nu)} \mathcal{L}\left\{t^{\nu-1} * f(t)\right\}= \\
& =\frac{1}{\Gamma(\nu)} \mathcal{L}\left\{t^{\nu-1}\right\} \mathcal{L}\{f(t)\}=s^{-\nu} \mathcal{L}\{f(t)\}
\end{aligned}
$$

Logo,

$$
\mathcal{L}\left\{D^{-\nu} f(t)\right\}=s^{-\nu} \mathcal{L}\{f(t)\}
$$

Assim, podemos escrever:

$$
\mathcal{L}\left\{D^{-\nu} f(t)\right\}=s^{-\nu} F(s), \quad \Re(\nu)>0
$$

Munidos desse recurso, podemos obter as TL da IFRL de várias funções, como, por exemplo:

$$
\begin{aligned}
\mathcal{L}\left\{\mathcal{D}^{-\nu} t^{\alpha}\right\} & =\frac{\Gamma(\alpha+1)}{s^{\nu+\alpha+1}} \\
\mathcal{L}\left\{\mathcal{D}^{-\nu} e^{\lambda t}\right\} & =\frac{1}{s^{\nu}(s-\lambda)} \\
\mathcal{L}\left\{\mathcal{D}^{-\nu} \cos (\omega t)\right\} & =\frac{1}{s^{\nu-1}\left(s^{2}+\omega^{2}\right)} \\
\mathcal{L}\left\{\mathcal{D}^{-\nu} \sin (\omega t)\right\} & =\frac{\omega}{s^{\nu}\left(s^{2}+\omega^{2}\right)} \\
\mathcal{L}\left\{\mathcal{D}^{-\nu} \cosh (\omega t)\right\} & =\frac{1}{s^{\nu-1}\left(s^{2}-\omega^{2}\right)} \\
\mathcal{L}\left\{\mathcal{D}^{-\nu} \sinh (\omega t)\right\} & =\frac{\omega}{s^{\nu}\left(s^{2}-\omega^{2}\right)}
\end{aligned}
$$

TL da Derivada Fracional de Riemann-Liouville Já tendo calculado as transformadas de Laplace das integrais fracionais e conhecendo as transformações sobre as derivadas clássicas, podemos ampliar sua aplicação às derivadas fracionais. Sabe-se que, para uma derivada de ordem $n$ inteira:

$$
\begin{aligned}
\mathcal{L}\left\{\frac{d^{n}}{d t^{n}} f(t)\right\} & =s^{n} F(s)-\sum_{k=0}^{n-1} s^{n-k-1} f^{(k)}\left(0_{+}\right)= \\
& =s^{n} F(s)-\sum_{k=0}^{n-1} s^{k} f^{(n-k-1)}\left(0_{+}\right)
\end{aligned}
$$


Portanto, usando a transformada de uma derivada clássica, obtém-se

$$
\begin{aligned}
\mathcal{L}\left\{D^{\nu} f(t)\right\} & =\mathcal{L}\left\{D^{\lceil\nu\rceil}\left[D^{-1+\{\nu\}} f(t)\right]\right\}= \\
& =s^{\lceil\nu\rceil} \mathcal{L}\left\{D^{-1+\{\nu\}} f(t)\right\}-\sum_{k=0}^{[\nu]} s^{k} D^{[\nu]-k}\left[D^{-1+\{\nu\}} f(t)\right]_{t=0}
\end{aligned}
$$

Agora, como $-1+\{\nu\}<0$, utilizando o resultado (F.60), pode-se escrever

$$
\begin{aligned}
\mathcal{L}\left\{D^{\nu} f(t)\right\} & =s^{\lceil\nu\rceil} s^{-1+\{\nu\}} F(s)-\sum_{k=0}^{[\nu]} s^{k} D^{[\nu]-k-1+\{\nu\}} f\left(0_{+}\right)= \\
& =s^{\nu} F(s)-\sum_{k=0}^{[\nu]} s^{k} D^{\nu-k-1} f\left(0_{+}\right)= \\
& =s^{\nu} F(s)-\sum_{k=1}^{\lceil\nu\rceil} s^{k-1} D^{\nu-k} f\left(0_{+}\right)
\end{aligned}
$$

Portanto, a TL de uma derivada fracional de Riemann-Liouville fornece:

$$
\mathcal{L}\left\{D^{\nu} f(t)\right\}=s^{\nu} F(s)-\sum_{k=1}^{\lceil\nu\rceil} s^{k-1} D^{\nu-k} f\left(0_{+}\right)
$$

TL da Derivada Fracional de Caputo Vamos manter, para a derivada fracional de Riemann-Liouville, a notação $D$ e, para a de Caputo, ${ }^{c} D$. Sua definição para uma ordem arbitrária $\nu$ é:

$$
{ }^{c} D^{\nu} f(x)=D^{-\lceil\nu\rceil+\nu}\left\{D^{\lceil\nu\rceil} f(x)\right\}
$$

Então, uma vez que $-\lceil\nu\rceil+\nu<0$, podemos usar a equação (F.60) para calcular sua TL:

$$
\mathcal{L}\left\{{ }^{c} D^{\nu} f(x)\right\}=\mathcal{L}\left\{D^{-\lceil\nu\rceil+\nu} f^{(\lceil\nu\rceil)}(x)\right\}=s^{-\lceil\nu\rceil+\nu} \mathcal{L}\left\{f^{(\lceil\nu\rceil)}(x)\right\}
$$

Agora, recorrendo à equação da TL de uma derivada clássica:

$$
\mathcal{L}\left\{{ }^{c} D^{\nu} f(x)\right\}=s^{-\lceil\nu\rceil+\nu}\left[s^{\lceil\nu\rceil} \mathcal{L}\{f(x)\}-\sum_{k=0}^{\lfloor\nu\rfloor} s^{\lceil\nu\rceil-k-1} f^{(k)}(0)\right]
$$

Logo, a TL da DFC será, após um rearranjo indicial:

$$
\mathcal{L}\left\{{ }^{c} D^{\nu} f(x)\right\}=s^{\nu} F(s)-\sum_{k=1}^{\lceil\nu\rceil} s^{\nu-k} f^{(k-1)}(0)
$$

Observando as equações (F.67) e (F.68), notamos uma diferença muito importante entre elas. Na primeira, as derivadas da somatória são de ordem fracionária, o que implica a 
necessidade de conhecimento de condições iniciais de ordem não-inteira, cuja interpretação física não é compreendida. Já na segunda, as derivadas são de ordem inteira, o que significa que as condições iniciais do problema se reduzem a interpretações de situações bem conhecidas. Essa característica revela uma considerável vantagem da derivada fracional de Caputo em relação à de Riemann-Liouville no processo de resolução de equações diferenciais de ordem arbitrária.

Entretanto, ainda há um outro detalhe a ser considerado, que diz respeito às derivadas fracionais de constantes. Por vezes, na resolução de EDFs, precisamos que essas derivadas assumam valores nulos, uma condição não-satisfeita pela DFRL, mas sim pela DFC, o que constitui mais uma vantagem desta última.

\section{Cálculo das Transformadas de Laplace Inversas de Funções de Ordem Fra-}

cionária Vamos implementar o cálculo de algumas funções importantes que aparecem na resolução de equações diferenciais de ordem arbitrária pelo método das transformadas de Laplace. No entanto, em vez de partirmos das funções de Mittag-Leffler e calcularmos suas transformadas pela definição, efetuaremos os cálculos pela transformada de Laplace inversa de determinadas funções arbitradas com expoentes não-inteiros, utilizando o conceito do teorema do binômio generalizado, pois essa abordagem simplifica o processo.

Os resultados aqui obtidos serão úteis na resolução de equações diferenciais fracionais.

1. Seja $F(s)=\frac{1}{s^{\alpha}-\lambda}$. Então, sua transformada de Laplace inversa deverá ser calculada através das relações conhecidas. Antes, porém, é melhor reescrever a função numa forma mais apropriada:

$$
F(s)=\frac{1}{s^{\alpha}\left(1-\frac{\lambda}{s^{\alpha}}\right)}=s^{-\alpha}\left(1-\frac{\lambda}{s^{\alpha}}\right)^{-1}
$$

Usando o desenvolvimento binomial estendido para $\left|\frac{\lambda}{s^{\alpha}}\right|<1$, lembrando que $\left(\begin{array}{c}-1 \\ r\end{array}\right)=$ $(-1)^{r}$, obtém-se

$$
F(s)=s^{-\alpha} \sum_{r=0}^{\infty}\left(\begin{array}{c}
-1 \\
r
\end{array}\right)\left(-\frac{\lambda}{s^{\alpha}}\right)^{r}=\sum_{r=0}^{\infty} \lambda^{r} s^{-\alpha-\alpha r}
$$

Disso e da linearidade da transformada inversa, segue que

$$
\mathcal{L}^{-1}\{F(s)\}=\mathcal{L}^{-1}\left\{\sum_{r=0}^{\infty} \lambda^{r} s^{-\alpha-\alpha r}\right\}=\sum_{r=0}^{\infty} \lambda^{r} \mathcal{L}^{-1}\left\{s^{-\alpha-\alpha r}\right\}=
$$




$$
\begin{gathered}
=\sum_{r=0}^{\infty} \frac{\lambda^{r}}{\Gamma(\alpha r+\alpha)} \mathcal{L}^{-1}\left\{\frac{\Gamma(\alpha r+\alpha)}{s^{\alpha r+\alpha}}\right\}=\sum_{r=0}^{\infty} \frac{\lambda^{r}}{\Gamma(\alpha r+\alpha)} t^{\alpha r+\alpha-1}= \\
=t^{\alpha-1} \sum_{r=0}^{\infty} \frac{\left(\lambda t^{\alpha}\right)^{r}}{\Gamma(\alpha r+\alpha)}
\end{gathered}
$$

Mas a expressão da somatória é uma função de Mittag-Leffler generalizada dada por $E_{\alpha, \alpha}\left(\lambda t^{\alpha}\right)$. Logo, chega-se a

$$
\mathcal{L}^{-1}\left\{\frac{1}{s^{\alpha}-\lambda}\right\}=t^{\alpha-1} E_{\alpha, \alpha}\left(\lambda t^{\alpha}\right)
$$

2. Agora, vamos determinar a transformada inversa de uma função $F(s)=\frac{s^{\alpha-1}}{s^{\alpha}+\lambda}$.

Novamente, reescrevemos a função em termos do desenvolvimento do binômio generalizado:

$$
F(s)=\frac{s^{\alpha}}{s}\left(s^{\alpha}+\lambda\right)^{-1}=\frac{1}{s}\left(1+\frac{\lambda}{s^{\alpha}}\right)^{-1}
$$

Para $\left|\frac{\lambda}{s^{\alpha}}\right|<1$, vale

$$
F(s)=\frac{1}{s} \sum_{r=0}^{\infty}\left(\begin{array}{c}
-1 \\
r
\end{array}\right)\left(\frac{\lambda}{s^{\alpha}}\right)^{r}=\sum_{r=0}^{\infty}(-\lambda)^{r} s^{-\alpha r-1}
$$

Portanto, a transformada inversa será

$$
\begin{gathered}
\mathcal{L}^{-1}\{F(s)\}=\mathcal{L}^{-1}\left\{\sum_{r=0}^{\infty}(-\lambda)^{r} s^{-\alpha r-1}\right\}=\sum_{r=0}^{\infty}(-\lambda)^{r} \mathcal{L}^{-1}\left\{s^{-\alpha r-1}\right\}= \\
=\sum_{r=0}^{\infty} \frac{(-\lambda)^{r}}{\Gamma(\alpha r+1)} \mathcal{L}^{-1}\left\{\frac{\Gamma(\alpha r+1)}{s^{\alpha r+1}}\right\}=\sum_{r=0}^{\infty} \frac{(-\lambda)^{r}}{\Gamma(\alpha r+1)} t^{\alpha r}= \\
=\sum_{r=0}^{\infty} \frac{\left(-\lambda t^{\alpha}\right)^{r}}{\Gamma(\alpha r+1)}=E_{\alpha}\left(-\lambda t^{\alpha}\right)
\end{gathered}
$$

Logo,

$$
\mathcal{L}^{-1}\left\{\frac{s^{\alpha-1}}{s^{\alpha}+\lambda}\right\}=E_{\alpha}\left(-\lambda t^{\alpha}\right)
$$

3. Generalizando um pouco mais, calcularemos a transformada de Laplace inversa da função $F(s)=\frac{s^{\alpha \mu-\beta}}{\left(s^{\alpha}+\lambda\right)^{\mu}}$. Reescrevendo a função na forma $F(s)=s^{-\beta}\left(1+\frac{\lambda}{s^{\alpha}}\right)^{-\mu}$ e desenvolvendo o binômio generalizado do termo entre parênteses para $\left|\frac{\lambda}{s^{\alpha}}\right|<1$, obtemos:

$$
F(s)=s^{-\beta} \sum_{r=0}^{\infty}\left(\begin{array}{c}
-\mu \\
r
\end{array}\right)\left(\frac{\lambda}{s^{\alpha}}\right)^{r}
$$


Usando a expressão (E.44) reescrevemos

$$
F(s)=\sum_{r=0}^{\infty}\left(-\lambda^{r}\right) \frac{(\mu)_{r}}{r !} s^{-\alpha r-\beta}
$$

Daí,

$$
\begin{gathered}
\mathcal{L}^{-1}\{F(s)\}=\mathcal{L}^{-1}\left\{\sum_{r=0}^{\infty}\left(-\lambda^{r}\right) \frac{(\mu)_{r}}{r !} s^{-\alpha r-\beta}\right\}=\sum_{r=0}^{\infty}\left(-\lambda^{r}\right) \frac{(\mu)_{r}}{r !} \mathcal{L}^{-1}\left\{s^{-\alpha r-\beta}\right\}= \\
=\sum_{r=0}^{\infty}\left(-\lambda^{r}\right) \frac{(\mu)_{r}}{r !}\left\{\frac{t^{\alpha r+\beta-1}}{\Gamma(\alpha r+\beta)}\right\}=t^{\beta-1} \sum_{r=0}^{\infty}\left(-\lambda^{r}\right) \frac{(\mu)_{r}}{r !} \frac{t^{\alpha r}}{\Gamma(\alpha r+\beta)} \Rightarrow \\
\Rightarrow \mathcal{L}^{-1}\{F(s)\}=t^{\beta-1} \sum_{r=0}^{\infty} \frac{(\mu)_{r}}{\Gamma(\alpha r+\beta)} \frac{\left(-\lambda t^{\alpha}\right)^{r}}{r !}
\end{gathered}
$$

Mas o termo da somatória é identificado com a função de Mittag-Leffler de três parâmetros e, portanto,

$$
\mathcal{L}^{-1}\left\{\frac{s^{\alpha \mu-\beta}}{\left(s^{\alpha}+\lambda\right)^{\mu}}\right\}=t^{\beta-1} E_{\alpha, \beta}^{\mu}\left(-\lambda t^{\alpha}\right)
$$

Deve-se notar que a expressão obtida no segundo cálculo aparece como um caso particular deste último para os valores $\mu=\beta=1$, ou seja,

$$
\mathcal{L}^{-1}\left\{\frac{s^{\alpha-1}}{s^{\alpha}+\lambda}\right\}=t^{0} E_{\alpha, 1}^{1}\left(-\lambda t^{\alpha}\right)=E_{\alpha}\left(-\lambda t^{\alpha}\right)
$$

Já para o caso $\mu=1$, obtém-se

$$
\mathcal{L}^{-1}\left\{\frac{s^{\alpha-\beta}}{s^{\alpha}+\lambda}\right\}=t^{\beta-1} E_{\alpha, \beta}^{1}\left(-\lambda t^{\alpha}\right)=t^{\beta-1} E_{\alpha, \beta}\left(-\lambda t^{\alpha}\right)
$$

Além disso, fazendo-se $\beta=\alpha$ nessa equação obtém-se o resultado do primeiro cálculo como um caso particular deste.

TL da função de Mittag-Leffler de 3 Parâmetros Usando a expressão integral da função gama e o desenvolvimento binomial:

$$
\left(1+\frac{\lambda}{s^{\alpha}}\right)^{-\gamma}=\sum_{k=0}^{\infty}(-1)^{k} \frac{(\gamma)_{k}}{k !}\left(\frac{\lambda}{s^{\alpha}}\right)^{k}, \quad\left|\frac{\lambda}{s^{\alpha}}\right|<1,
$$

vamos calcular, pela definição, a transformada de Laplace da função de Mittag-Leffler de três parâmetros $E_{\alpha, \beta}^{\gamma}\left(-\lambda t^{\alpha}\right)$ multiplicada por um fator semelhante ao que temos obtido 
nos cálculos da transformada inversa, ou seja, $t^{\beta-1}$. Então,

$$
\begin{aligned}
\mathcal{L}\left\{t^{\beta-1} E_{\alpha, \beta}^{\gamma}\left(-\lambda t^{\alpha}\right)\right\} & =\int_{0}^{\infty} e^{-s t} t^{\beta-1} \sum_{k=0}^{\infty} \frac{(\gamma)_{k}\left(-\lambda t^{\alpha}\right)^{k}}{\Gamma(\alpha k+\beta) k !} d t= \\
& =\sum_{k=0}^{\infty} \frac{(-\lambda)^{k}(\gamma)_{k}}{\Gamma(\alpha k+\beta) k !} \int_{0}^{\infty} e^{-s t} t^{\alpha k+\beta-1} d t= \\
& =\sum_{k=0}^{\infty} \frac{(-\lambda)^{k}(\gamma)_{k}}{\Gamma(\alpha k+\beta) k ! s^{\alpha+\beta}} \int_{0}^{\infty} e^{-y} y^{\alpha k+\beta-1} d y= \\
& =\frac{1}{s^{\beta}} \sum_{k=0}^{\infty}(-1)^{k} \frac{\Gamma(k+\gamma)}{k ! \Gamma(\gamma)}\left(\frac{\lambda}{s^{\alpha}}\right)^{k}= \\
& =\frac{1}{s^{\beta}} \sum_{k=0}^{\infty}(-1)^{k} \frac{(\gamma)_{k}}{k !}\left(\frac{\lambda}{s^{\alpha}}\right)^{k}= \\
& =\frac{1}{s^{\beta}} \frac{1}{\left(1+\frac{\lambda}{s^{\alpha}}\right)^{\gamma}}= \\
& =\frac{s^{\alpha \gamma-\beta}}{\left(s^{\alpha}+\lambda\right)^{\gamma}}
\end{aligned}
$$

onde usamos a mudança de variáveis $s t \rightarrow y$. Logo,

$$
\mathcal{L}\left\{t^{\beta-1} E_{\alpha, \beta}^{\gamma}\left(-\lambda t^{\alpha}\right)\right\}=\frac{s^{\alpha \gamma-\beta}}{\left(s^{\alpha}+\lambda\right)^{\gamma}}
$$

Essa expressão será de extrema utilidade no processo de determinação de soluções analíticas para EDFs. Se considerarmos certas situações especiais, reobteremos as TLs para as funções de Mittag-Leffler e de Wiman anteriormente determinadas. Sejam os casos particulares:

- $\gamma=1$ :

$$
\mathcal{L}\left\{t^{\beta-1} E_{\alpha, \beta}\left(-\lambda t^{\alpha}\right)\right\}=\frac{s^{\alpha-\beta}}{s^{\alpha}+\lambda}
$$

- $\gamma=\beta=1$ :

$$
\mathcal{L}\left\{E_{\alpha}\left(-\lambda t^{\alpha}\right)\right\}=\frac{s^{\alpha-1}}{s^{\alpha}+\lambda}
$$

- $\gamma=\beta=\alpha=1$ :

$$
\mathcal{L}\left\{e^{-\lambda t}\right\}=\frac{1}{s+\lambda}
$$

Quer dizer, recaímos nas funções de Wiman, de Mittag-Leffler e exponencial. 
TF de Funções de Ordem Fracionária Recordamos que estamos utilizando a versão simétrica das transformadas de Fourier:

$$
\begin{gathered}
\mathfrak{F}\{f(t)\}=\frac{1}{\sqrt{2 \pi}} \int_{-\infty}^{\infty} e^{-i \omega t} f(t) d t \\
\mathfrak{F}^{-1}\{\mathcal{F}(\omega)\}=\frac{1}{\sqrt{2 \pi}} \int_{-\infty}^{\infty} e^{i \omega t} \mathcal{F}(\omega) d \omega
\end{gathered}
$$

TF da IFL Da função gama na forma integral vem

$$
s^{-\nu}=\frac{1}{\Gamma(\nu)} \int_{0}^{\infty} t^{\nu-1} e^{-s t} d t
$$

Fazendo a mudança de variáveis $s=i \omega, \omega \in \mathbb{R}$, nessa expressão, segue

$$
(i \omega)^{-\nu}=\int_{0}^{\infty} \frac{t^{\nu-1}}{\Gamma(\nu)} e^{-i \omega t} d t=\sqrt{2 \pi}\left(\frac{1}{\sqrt{2 \pi}} \int_{-\infty}^{\infty} \mathcal{K}_{\nu}^{+}(t) e^{-i \omega t} d t\right)=\sqrt{2 \pi} \mathfrak{F}\left\{\mathcal{K}_{\nu}^{+}(t)\right\}
$$

$\mathrm{ou}$

$$
\mathfrak{F}\left\{\mathcal{K}_{\nu}^{+}(t)\right\}=\frac{(i \omega)^{-\nu}}{\sqrt{2 \pi}}
$$

onde $\mathcal{K}_{\nu}^{+}(t)$ é o kernel fracional causal:

$$
\mathcal{K}_{\nu}^{+}(t)=\left\{\begin{array}{cc}
\frac{t^{\nu-1}}{\Gamma(\nu)}, & (t>0) \\
0, & (t \leq 0)
\end{array}\right.
$$

Porém, da expressão da IFL:

$$
D_{-\infty}^{-\nu} f(t)=\frac{1}{\Gamma(\nu)} \int_{-\infty}^{t}(t-\tau)^{\nu-1} f(\tau) d \tau, \quad 0<\Re\{\nu\}<1,
$$

pode-se escrever

$$
\begin{aligned}
D_{-\infty}^{-\nu} f(t) & =\int_{-\infty}^{t} \mathcal{K}_{\nu}^{+}(t-\tau) f(\tau) d \tau= \\
& =\int_{0}^{\infty} \mathcal{K}_{\nu}^{+}(u) f(t-u) d u= \\
& =\int_{-\infty}^{\infty} \mathcal{K}_{\nu}^{+}(u) f(t-u) d u= \\
& =\sqrt{2 \pi}\left(\mathcal{K}_{\nu}^{+} * f\right)_{\mathfrak{F}}(t)
\end{aligned}
$$

Logo, pode-se expressar a IFL por uma convolução definida para Fourier como:

$$
D_{-\infty}^{-\nu} f(t)=\sqrt{2 \pi}\left(\mathcal{K}_{\nu}^{+} * f\right)_{\mathfrak{F}}(t)
$$


Por outro lado, da fórmula acima, do teorema da convolução para Fourier e da expressão (F.70), extrai-se

$$
\mathfrak{F}\left\{D_{-\infty}^{-\nu} f(t)\right\}=\mathfrak{F}\left\{\sqrt{2 \pi} \mathcal{K}_{\nu}^{+}(t) * f(t)\right\}=\sqrt{2 \pi} \mathfrak{F}\left\{\mathcal{K}_{\nu}^{+}(t)\right\} \mathfrak{F}\{f(t)\}=(i \omega)^{-\nu} \mathcal{F}(\omega)
$$

e, portanto,

$$
\mathfrak{F}\left\{D_{-\infty}^{-\nu} f(x)\right\}=(i y)^{-\nu} \mathcal{F}(y)
$$

Dessa expressão e da equação (F.48) pode-se determinar diretamente a TF da IFL de um elemento da base de Fourier:

$$
\mathfrak{F}\left\{D_{-\infty}^{-\nu} e^{i m q x}\right\}=(i y)^{-\nu} \mathfrak{F}\left\{e^{i m q x}\right\}=(i y)^{-\nu} \sqrt{2 \pi} \delta(y-m q)
$$

Logo, essa equação pode ser expressa como

$$
\mathfrak{F}\left\{D_{-\infty}^{-\nu} e^{i m q x}\right\}=\frac{\sqrt{2 \pi}}{(i m q)^{\nu}} \delta(y-m q)
$$

TF da DFL e DFCL A expressão deduzida aqui serve tanto para a DFL quanto para a DFCL, visto que ambas assumem o mesmo valor, conforme foi mostrado em seção precedente. Além do mais, como será explicado adiante, a abordagem da derivada fracional de Weyl ${ }_{L} W_{+}^{\nu}$ fornece resultados idênticos à de Liouville ${ }_{-\infty} D_{x}^{\nu}$ e, por essa razão, a expressão aqui obtida também será a mesma da TL da DFW. Assim, partindo de

$$
D_{-\infty}^{\nu} f(t)={ }^{c} D_{-\infty}^{\nu} f(t)=D_{-\infty}^{-(\lceil\nu\rceil-\nu)} f^{(\lceil\nu\rceil)}(t)=\frac{1}{\Gamma(\lceil\nu\rceil-\nu)} \int_{-\infty}^{t} \frac{f^{(\lceil\nu\rceil)}(\tau)}{(t-\tau)^{\nu+1-\lceil\nu\rceil}} d \tau
$$

e usando (F.71), vem

$$
\begin{gathered}
\mathfrak{F}\left\{D_{-\infty}^{\nu} f(t)\right\}=\mathfrak{F}\left\{D_{-\infty}^{-(\lceil\nu\rceil-\nu)} f^{(\lceil\nu\rceil)}(t)\right\}=(i \omega)^{-(\lceil\nu\rceil-\nu)} \mathfrak{F}\left\{f^{(\lceil\nu\rceil)}(t)\right\}= \\
=(i \omega)^{-(\lceil\nu\rceil-\nu)}(i \omega)^{(\lceil\nu\rceil)} \mathfrak{F}\{f(t)\}=(i \omega)^{-(\lceil\nu\rceil-\nu)} \mathcal{F}(\omega)
\end{gathered}
$$

Logo,

$$
\mathfrak{F}\left\{D_{-\infty}^{\nu} f(t)\right\}=(i \omega)^{\nu} \mathcal{F}(\omega)
$$

Vale dizer que esse resultado se aplica também à derivada fracional de GrünwaldLetnikov, que não estamos abordando nesse trabalho.

Finalmente, calculando de novo o caso que nos interessa, para os elementos da base de Fourier, a eq. (F.73) conduz a

$$
\mathfrak{F}\left\{D_{-\infty}^{\nu} e^{i m q x}\right\}=(i y)^{\nu} \mathfrak{F}\left\{e^{i m q x}\right\}=(i y)^{\nu} \sqrt{2 \pi} \delta(y-m q)
$$


e, portanto,

$$
\mathfrak{F}\left\{D_{-\infty}^{\nu} e^{i m q x}\right\}=\sqrt{2 \pi}(i m q)^{\nu} \delta(y-m q)
$$

No capítulo 4 é desenvolvida detalhadamente a abordagem de Weyl, apropriada a funções periódicas, na qual se demonstra a equivalência de resultados relativos às derivadas fracionais e transformadas dos elementos da base de Fourier naquela formulação e as apresentadas aqui pela versão de Liouville. Isso possibilita a determinação das MOps fracionais de integração e derivação na base de Fourier para uso sistemático no processo de resolução de equações diferenciais fracionais (EDFs). 


\section{APÊENICE G - Demonstração da Fórmula de Reflexão de} Euler

$$
B(p, 1-p)=\Gamma(p) \Gamma(1-p)=\frac{\pi}{\sin \pi p}
$$

Vamos partir da forma racional da função beta

$$
B(p, q)=\int_{0}^{\infty} \frac{y^{p-1}}{(1+y)^{p+q}} d y
$$

para calcular a integral

$$
\int_{0}^{\infty} \frac{t^{p-1}}{1+t} d t, \quad 0<p<1
$$

Notemos que a integral acima é, na verdade, $B(p, 1-p)$. Vamos efetuar o cálculo no plano complexo:

$$
\oint \frac{z^{p-1}}{1+z} d z, \quad 0<p<1
$$

O contorno escolhido será $\mathcal{C}=\gamma_{r} \cup C_{1} \cup \gamma_{R} \cup C_{2}$, onde $\gamma_{r}$ é a circunferência de raio $r$ percorrida em sentido positivo em torno da origem, $C_{1}$ é o percurso sobre o eixo real desde o ponto $r$ até um outro ponto $R, \gamma_{R}$ é a circunferência de raio $R$ percorrida em sentido negativo em torno da origem e $C_{2}$ é o percurso sobre o eixo real desde o ponto $R$ de volta a $r$. Então, o polo $z=-1=e^{i \pi}$ encontra-se dentro da curva $\mathcal{C}$ e

$$
\oint_{\mathcal{C}} \frac{z^{p-1}}{1+z} d z=\oint_{\gamma_{r}}+\int_{C_{1}}+\oint_{\gamma_{R}}+\int_{C_{2}}
$$

A primeira e a terceira integrais assumem a forma

$$
\oint_{\gamma_{\rho}} \frac{\left(\rho e^{i \theta}\right)^{p-1}}{1+\rho e^{i \theta}} \rho i e^{i \theta} d \theta=\oint_{\gamma_{\rho}} i \frac{\rho^{p} e^{i p \theta}}{1+\rho e^{i \theta}} d \theta
$$

onde $\rho=r$ para $\gamma_{r}$ e $\rho=R$ para $\gamma_{R}$. Entretanto, para calcular a integral sobre o eixo real 
desde a origem a $\infty$, é preciso fazer o raio maior crescer ilimitadamente e o raio menor tender a zero, ous seja, $r \rightarrow 0$ e $R \rightarrow \infty$. Para o primeiro caso obtém-se:

$$
\mathcal{I}_{r}=\left|\oint_{\gamma_{r}} i \frac{r^{p} e^{i p \theta}}{1+r e^{i \theta}} d \theta\right| \leq \oint_{\gamma_{r}} \frac{r^{p}}{\left|1+r e^{i \theta}\right|} d \theta
$$

Mas

$$
\lim _{r \rightarrow 0} r^{p} \oint_{\gamma_{r}} \frac{1}{\mid 1+r e^{i \theta \mid}} d \theta=0 \Rightarrow \mathcal{I}_{r} \rightarrow 0
$$

Para o segundo caminho, tem-se

$$
\mathcal{I}_{R}=\left|\oint_{\gamma_{R}} i \frac{R^{p} e^{i p \theta}}{1+R e^{i \theta}} d \theta\right| \leq R^{p} \oint_{\gamma_{R}} \frac{1}{\mid 1+R e^{i \theta \mid}} d \theta
$$

e, como

$$
\lim _{r \rightarrow \infty} R^{p} \oint_{\gamma_{r}} \frac{1}{\mid 1+R e^{i \theta \mid}} d \theta=0,
$$

pois, por eleição, $0<p<1$, então as duas integrais ao longo dos caminhos $\gamma_{r}$ e $\gamma_{R}$ se anulam. Resta calcular as outras duas. Para manter todos os valores na mesma folha de Riemann, a variável $z$ em $C_{1}$ deve ter argumento $\theta=0$ e em $C_{2}$ deve apresentar $\theta=2 \pi$. Assim,

$$
\int_{C_{1}} \frac{z^{p-1}}{1+z} d z \rightarrow \int_{0}^{\infty} \frac{\rho^{p-1}}{1+\rho} d \rho
$$

e

$$
\int_{C_{2}} \frac{z^{p-1}}{1+z} d z \rightarrow \int_{\infty}^{0} \frac{\left(\rho e^{2 \pi i}\right)^{(p-1)}}{1+\rho e^{2 \pi i}} e^{2 \pi i} d \rho=-\int_{0}^{\infty} \frac{\rho^{(p-1)} e^{2 \pi i p}}{1+\rho} d \rho
$$

Assim, a soma das integrais restantes fornece

$$
\begin{gathered}
\oint_{\mathcal{C}} \frac{z^{p-1}}{1+z} d z=\int_{0}^{\infty} \frac{\rho^{p-1}}{1+\rho} d \rho-\int_{0}^{\infty} \frac{\rho^{(p-1)} e^{2 \pi i p}}{1+\rho} d \rho \Rightarrow \\
\oint_{\mathcal{C}} \frac{z^{p-1}}{1+z} d z=\left(1-e^{2 \pi i p}\right) \int_{0}^{\infty} \frac{\rho^{p-1}}{1+\rho} d \rho
\end{gathered}
$$

Todavia, a integral complexa pode ser calculada pelo método de resíduos:

$$
\oint_{\mathcal{C}} \frac{z^{p-1}}{1+z} d z=2 \pi i \sum_{n} \operatorname{Res}\left(f, z_{n}\right)
$$

Mas no interior da curva $\mathcal{C}$ só há a singularidade $z=-1=e^{i \pi}$, o que fornece

$$
\oint_{\mathcal{C}} \frac{z^{p-1}}{1+z} d z=2 \pi i e^{i(p-1) \pi}=-2 \pi i e^{i \pi p}
$$


Logo,

$$
\begin{gathered}
\oint_{\mathcal{C}} \frac{z^{p-1}}{1+z} d z=-2 \pi i e^{i \pi p}=\left(1-e^{2 \pi i p}\right) \int_{0}^{\infty} \frac{\rho^{p-1}}{1+\rho} d \rho \Rightarrow \\
\int_{0}^{\infty} \frac{t^{p-1}}{1+t} d t=\frac{-2 \pi i e^{i \pi p}}{1-e^{2 \pi i p}}=\frac{-2 \pi i e^{i \pi p}}{e^{\pi i p}\left(e^{-\pi i p}-e^{\pi i p}\right)}= \\
=\frac{2 \pi i}{\left(e^{i \pi p}-e^{-i \pi p}\right)}=\frac{\pi}{\sin \pi p}
\end{gathered}
$$

Então, lembrando que a integral calculada pode ser expressa em termos da função beta $B(p, 1-p)$, e que

$$
B(p, q)=\frac{\Gamma(p) \Gamma(q)}{\Gamma(p+q)},
$$

chega-se a

$$
B(p, 1-p)=\frac{\Gamma(p) \Gamma(1-p)}{\Gamma(1)}=\int_{0}^{\infty} \frac{t^{p-1}}{1+t} d t=\frac{\pi}{\sin \pi p}
$$

e, finalmente,

$$
\Gamma(p) \Gamma(1-p)=\frac{\pi}{\sin \pi p}
$$

